\title{
Philosophische Tugenden
}

Eine Verteidigung von J. S. Mills Philosophie der praktischen Philosophie 
Wissenschaftliche Beiträge aus dem Tectum Verlag

Philosophie | Band 40 
Joachim Toenges-Hinn

\section{Philosophische Tugenden}

Eine Verteidigung von J. S. Mills Philosophie der praktischen Philosophie

Tectum Verlag 
Die Deutsche Nationalbibliothek verzeichnet diese Publikation in der Deutschen Nationalbibliografie; detaillierte bibliografische Angaben sind im Internet über http://dnb.d-nb.de abrufbar.

1. Auflage 2020

(C) Joachim Toenges-Hinn

Zugleich Dissertation an der Universität Potsdam, 2020

Erstgutachten: Prof. Dr. Logi Gunnarsson

Zweitgutachten: Prof. Dr. Róbert Haraldsson

Publiziert von

Tectum - ein Verlag in der

Nomos Verlagsgesellschaft mbH \& Co. KG

Waldseestraße 3-5 | 76530 Baden-Baden

www.tectum-verlag.de

Gesamtherstellung:

Tectum - ein Verlag in der

Nomos Verlagsgesellschaft mbH \& Co. KG

Waldseestraße 3-5 |76530 Baden-Baden

ISBN (Print) 978-3-8288-4556-5

ePDF 978-3-8288-7609-5

\section{(c) (1) (8) $\Theta$ \\ BY NC ND}

Dieses Werk ist lizenziert unter einer

Creative Commons Namensnennung - Nicht kommerziell -

Keine Bearbeitungen 4.o International Lizenz. 


\section{Vorwort}

Das vorliegende Buch ist als meine Dissertation an der Universität Potsdam entstanden. Sicherlich treffen viele der Schwächen, die solchen Texten nachgesagt werden, auch auf mein Buch zu. Zusätzlich mag sich die wohlwollende Leser*in nach der Lektüre fragen, ob die Form der akademischen Abhandlung überhaupt die richtige Form für das ist, worum es in diesem Buch geht. Das liegt daran, dass es auch eine hinterfragende Perspektive auf akademische Philosophie nahelegt. John Stuart Mill, die Hauptperson meines Buches, ist dafür natürlich prädestiniert, weil er sich philosophisch zu weiten Teilen außerhalb der akademischen Philosophie bewegte. Und die Probleme, auf die Mills Philosophie eine Antwort sucht, sind solche philosophischen Probleme, die sich beim Führen seines konkreten Lebens - als Individuum und politisch - stellen; und seine Antworten sind solche, die beanspruchen, sich an diesem Test messen zu lassen.

Bei allen Schwächen glaube ich, dass mein Buch in Bezug auf diese beiden Punkte einen Wert hat: Es erarbeitet erstens einen wesentlichen Aspekt von Mills Philosophie der Philosophie. Zweitens glaube ich, dass ich mit der Verteidigung seiner Vorstellung eine wichtige Frage zur Philosophie aufwerfe, mit der die Beschäftigung lohnt, selbst wenn ich am Ende nicht alle Leser*innen überzeuge.

Natürlich wäre nichts von dem ohne die Ermutigung und Unterstützung anderer möglich gewesen. Zunächst seien hier die Mitglieder und Gäste des Forschungskolloquiums von Logi Gunnarsson in Potsdam erwähnt. Ich habe es als einen Ort erlebt, in dem ohne Eitelkeit und Konkurrenzverhalten auf höchstem Niveau diskutiert wird. Dies liegt sicherlich einerseits an der Art, wie es gestaltet wird, und andererseits an seinen Mitgliedern. In besonderer Weise möchte ich unter diesen Lena Ljucovic, Dennis Wildfeuer, Karsten Schoellner, Emanuel John, Antonia Fuhr, Sarah Wellan, Pax Abad, Sandra Schmidt und Svenja Mentler für ihre Ermutigung, ihre konstruktive Kritik und bisweilen ihre Skepsis hervorheben. Auch den Mitarbeiter*innen und Professor*innen aller anderen Lehrstühle am Insti- 
tut für Philosophie in Potsdam gebührt mein Dank, nicht nur für die Unterstützung der Dissertation, sondern auch für ihre Unterstützung und Ihre Offenheit für mich als Kollegen. Aus den vielen möchte ich hier nur Till Hoeppner, Ise Raters, Alina Omerbasic, Adem Mulamustafic und Till Hopfe erwähnen.

Neben Martina Herrmann, Lutz Wingert und Claus Beisbart, die mich nicht nur entscheidend geprägt, sondern auch unterstützt und gefördert haben, möchte ich auch den Mitgliedern ihrer Forschungsseminare danken. Besonders danken möchte ich unter diesen Martin Hurni, Christiane Borchel, Karl Becker, Ulrich Kröger und Peter Rosch für zahlreiche konstruktive philosophische Gespräche.

Meine Frau, Laura Hinn, hätte ich an vielen dieser Stationen erwähnen können. Ihr gebührt unschätzbarer Dank nicht nur für die konstruktiven Gespräche, die wir geführt haben, sondern auch dafür, dass sie dadurch, dass sie immer etwas früher als ich an den gleichen Stationen ihrer Dissertation angekommen ist als ich an denen der meinen, eine unschätzbare Mitstreiterin, ja eigentlich Vorkämpferin war.

Am allermeisten bin ich meinen Betreuern zu Dank verpflichtet: Róbert Haraldsson, dessen Seminar mir neue Sichtweisen auf und eine neue Begeisterung für John Stuart Mill eröffnet hat, und Logi Gunnarsson. Die vielfältige und für die jeweiligen Phasen meiner Dissertation immer genau richtige Art der Betreuung, die ich durch Logi Gunnarsson erfahren habe, lässt sich nur schwer kurz zusammenfassen. Aber vielleicht ist die Tatsache, dass mir während der Zeit der Dissertation - in der von Promovierenden häufig vor allem eine besondere Zielstrebigkeit in Bezug auf ihr Forschungsprojekt verlangt wird - radikale philosophische Lernprozesse ermöglicht wurden, selbst wenn dies einen Umweg auf dem Weg zum Abschluss bedeuten sollte, an dieser Stelle Zeugnis genug. 


\section{Inhaltsverzeichnis}

1. Einleitung 1

I. Erarbeitung der beiden sozialphilosophischen Tugenden und methodische Überlegungen 15

Einleitung zu Teil $1 \ldots \ldots$. . . . . . . . . . 17

2. Eine vorläufige Formulierung der sozialphilosophischen Tugenden und methodische Überlegungen 19

2.1. Eine vorläufige Formulierung von Mills Tugenden . . 20

2.2. Bemerkungen zum Status der Tugenden . . . . . . . 22

2.3. Metametaphilosophie . . . . . . . . . . . . . . 28

2.4. Begründung meiner Lesart von „Bentham" und „Coleridge" . . . . . . . . . . . . . . . 33

3. "Coleridge“ 41

3.1. Coleridge versus "Coleridge“ . . . . . . . . . . 42

3.2. Der erfahrbare, intrinsische Wert von Institutionen und Praktiken nach Mills Coleridge . . . . . . . . . 44

3.3. Die Beispiele . . . . . . . . . . . . . . . . . . . . . 47

3.3.1. Moral und Wissenschaft . . . . . . . . . 48

3.3.2. Die Unitarier*innen und das Christentum . . 51

3.4. Coleridge als Konservativer? . . . . . . . . . . 57

3.4.1. Die ,intuitive Schule" und Konservativismus 61

3.4.2. "Coleridge" versus "Whewell on Moral Philosophy" ................. 64

4. „Bentham“ 71

4.1. Bentham als Kritiker . . . . . . . . . . . . . . 74

4.2. Benthams Bemühen um Objektivität . . . . . . 81

4.3. Qualifikationen von Benthams Tugend . . . . . . . 86

4.3.1. Benthams Tugend und Wissenschaftlichkeit . 87

4.3.2. Ein einzelner Maßstab . . . . . . . . . . . 91 
Zusammenfassung von Teil 1 . . . . . . . . . . . 101

\section{Die Tugenden in Mills eigener Philosophie 105}

Einleitung zu Teil $2 \ldots \ldots$. . . . . . . . . 107

5. Mills Philosophie des Wohlergehens $\mathbf{1 1 5}$

5.1. Anderson über Mill und Experiments in Living . . . 116

5.1.1. Mills philosophischer Lernprozess in seiner Krise116

5.1.2. Die Natur des Guten nach Mill . . . . . . . . 120

5.1.3. Wie wird Benthams Philosophie durch Mills Krisenerfahrung widerlegt? . . . . . . . . . . 124

5.2. Experimente mit neuen Lebensweisen und die beiden Tugenden . . . . . . . . . . . . . . . . . . . . . . . . . . . . 129

5.2.1. Die Natur des Guten . . . . . . . . . . . . 129

5.2.2. Das Urteil der Erfahrenen . . . . . . . . 136

5.2.3. Das Freiheitsprinzip . . . . . . . . . . . . 139

5.3. Fünf Fehleranfälligkeiten von Mills Vorgehen . . . . 144

6. Zwei Grenzen von Mills moralphilosophischem Ansatz als Versuch, den Tugenden gerecht zu werden $\quad 157$

6.1. Unparteilichkeit im Utilitarismus . . . . . . . . . . 158

6.1.1. Die Kritik am „Intuitionismus“ . . . . . . . . 163

6.1.2. Der „Beweis" des Utilitarismus . . . . . . . . 168

6.1.3. Die Rolle des utilitaristischen Maßstabs für die Kritik an der common sense Moral . . . 181

6.2. Mills praktischer Psychologismus . . . . . . . . . 196

6.2.1. Psychologismus im engen Sinn . . . . . . . . 199

6.2.2. Psychologismus in Mills Vorstellungen vom Handeln . . . . . . . . . . . . . . . . . 203

6.2.3. „The Utility of Religion“ . . . . . . . . . . 208

Zusammenfassung von Teil $2 \ldots \ldots$. . . . . . . 215

\section{Allgemeine Überlegungen, Abgrenzung, Aus-} blick

Einleitung zu Teil $3 \ldots \ldots$. . . . . . . . . . . . . . . 219 
7. Moral und Technik: Expertise in Moral und Moralphilosophie und Mills Tugenden

7.1. Mill und Platon . . . . . . . . . . . . . . . . 228

7.2. Expert*innentum und Mills Tugenden . . . . . . . 238

7.3. Lehrbarkeit und Expert*innentum als Zugang zu ethischem Wissen in den platonischen Frühdialogen . . 243

7.3.1. Zum Status der Auseinandersetzung mit den Frühdialogen . . . . . . . . . . . . . 243

7.3.2. Verortung von Benthams Kritik an gängiger philosophischer Moralauffassung im Orakelspruch . . . . . . . . . . . . . 248

7.3.3. Der Aufbau des „Protagoras" und Mills Tugenden . . . . . . . . . . . . 252

7.3.4. Die Handwerker*innen und Benthams Tugend 260

8. Abgrenzung, offene Probleme, Ausblick 285

8.1. Verhältnis zu anderen Ansätzen . . . . . . . . . . 286

8.1.1. Hegelianische Ansätze: Jaeggi . . . . . . . 287

8.1.2. Ausdehnung auf weitere Ansätze? . . . . . . 300

8.2. Offene Probleme und Ausblick . . . . . . . . . . . . 301 


\section{Einleitung}

John Stuart Mill war ein außergewöhnlich guter Philosoph. Der Rest der vorliegenden Arbeit kann als Versuch gesehen werden, diesen Satz zu erläutern und verteidigen. Indem ich den Satz verteidige, werde ich zwei philosophische Ideale, denen Mill explizit (Teil I) und implizit (Teil II) verpflichtet ist, formulieren. Im Nachweis der Verpflichtung Mills auf diese Ideale, in der Bestimmung des Grades, in dem Mill ihnen gerecht wird, und der systematischen Verteidigung der Ideale wird letztlich der Beleg der Aussage, Mill sei ein außergewöhnlich guter Philosoph gewesen, liegen. Die Arbeit ist also eine Ausarbeitung und Verteidigung von Mills Philosophie der praktischen Philosophie.

Dass eine solche Behauptung überhaupt einer Erläuterung und Verteidigung bedarf, liegt nicht nur an der philosophischen Landschaft in Deutschland: Mill wird in Einführungswerken und Einführungsseminaren in die Ethik gelegentlich als wohlmeinender Verteidiger einer am Ende absurden moralphilosophischen Position des Utilitarismus - behandelt. Mill wird gebraucht, dieser Eindruck könnte sich aufdrängen, um gute Philosophie von außen abzugrenzen; er wird oft als das Paradigma eines schlechten Philosophen behandelt. Auf der anderen Seite liegt natürlich die Tatsache, dass die notwendig skizzenhaften Darstellungen in Überblickstexten und die Rhetorik der Kontrastierung, die solchen Texten eigen ist, den oft viel komplexeren Positionen nicht immer gerecht werden kann, in der Natur der Sache und ist kaum Anlass für eine Richtigstellung.

Im Falle von Mill gibt es aber ein Problem über seine undankbare Rolle als Kontrastfolie in der für ihn unvorteilhaften Landschaft der deutschen universitären Philosophie hinaus. Dieses Problem ist, dass seine philosophischen Positionen, selbst wenn die Feinheiten, die in Überblickstexten und Skizzen notwendig ignoriert werden, berücksichtigt werden, oft immer noch entweder zweifelhaft oder unklar zu sein scheinen. Und das bezieht sich nicht nur auf De- 


\section{Einleitung}

tails, meine ich. ${ }^{1}$ Natürlich freue ich mich, wenn diejenigen unter den Leser*innen, die meinen Enthusiasmus für Mill teilen, mir an den Stellen dieser Arbeit, an denen ich Mills Positionen letztlich als unhaltbar darstellen werde, widersprechen; der Punkt, um den es mir hier geht, ist ein anderer. Wie kann Mill von einer Person wie mir, die das alles zugesteht, trotzdem für einen guten Philosophen gehalten werden? Das, was ich meine, wenn ich Mill als außergewöhnlich guten Philosophen bezeichne, ist jedenfalls nicht, dass Mill außergewöhnlich gute philosophische Positionen vertreten hätte.

Wenn die Qualität Mills nicht in den inhaltlichen Ergebnissen seines philosophischen Tuns liegt, so liegt es nahe, sie in der Art und Weise zu suchen, wie Mill zu diesen Ergebnissen kommt. Folgt Mill konsequent einer bestimmten philosophischen Methode? Ist es, wo er einer solchen Methode folgt, die richtige (oder zumindest eine gute) Methode? Argumentiert er besonders sauber? Ist seine Terminologie klar? - Auch wenn "philosophische Methode“ vielleicht ein etwas besserer Ausdruck für die Stärke von Mill als Philosoph ist, als „philosophische Positionen“2, sind die meisten von uns, so denke ich, nicht geneigt, außergewöhnlich viele der Fragen dieses Typs affirmativ zu beantworten: Es ist nicht immer klar, ob es Mill gelingt, seine Schlussfolgerungen aus der „Philosophie der Erfahrung“ - so eine der Bezeichnungen, die Mill für seine Methode wählt abzuleiten. Seine Argumente bedürfen oft umfangreicher Klarstellungen, bevor sie überzeugend oder in manchen Fällen auch nur verständlich werden, und seine Terminologie wird durch Eklekti-

1 Dies ist kompatibel mit der Aussage, der ich mich ebenfalls verschreibe, dass Mills philosophische Positionen oft unterschätzt werden. Hier will ich lediglich sagen, dass Mill nicht außergewöhnlich oft richtig liegt. Mill war aus anderen Gründen ein außergewöhnlich guter Philosoph.

2 Mill selbst sagt zum Beispiel über Bentham, seine Hauptstärke seien nicht seine Thesen, sondern die Art und Weise, wie er zu diesen Thesen komme. (Siehe John Stuart Mill. „Bentham“. In: The Collected Works of John Stuart Mill, Volume X - Essays on Ethics, Religion, and Society. Hrsg. von J. M. Robson. Toronto und andere: University of Toronto Press und Routledge \& Kegan Paul, 1969, S. 78-115, S. 83.) Dieser Text ist auch für mich zentral bei der Erarbeitung von Mills expliziten Idealen. Trotzdem ist "Methode“, so meine ich wird es sich zeigen, (Abschnitt 4) auch im Falle Benthams eigentlich nicht der richtige Ausdruck.

Dies hängt natürlich davon ab, was mit „Methode“ gemeint ist. Aber in dem Sinne, in dem zum Beispiel Alltagssprachanalyse oder Phänomenologie Methoden sind, handelt es sich nicht um Methoden. 
zismus und geschliffene Rhetorik oft so überlagert, dass klassische philosophische Probleme weniger gelöst als dadurch verdeckt werden. Dies alles ist nicht primär als Kritik gemeint. Von bestimmten Vorgehensweisen der akademischen Philosophie (seiner Zeit) hat sich Mill ganz bewusst abgegrenzt. ${ }^{3}$ Es zeigt aber, dass wir auch in diesem Bereich nicht danach suchen können, was Mill zu einem außergewöhnlich guten Philosophen macht.

Das im ersten Satz dieser Arbeit von mir implizierte philosophische Ideal ist nicht das Ideal, wahre philosophische Positionen zu vertreten, und es ist auch nicht das Ideal beim Philosophieren, den richtigen Regeln zu folgen. Freilich ist es ein Ideal, gute oder wahre philosophische Positionen zu formulieren, und genauso ist es ein Ideal, guten oder den richtigen Regeln philosophischen Handelns zu folgen - jeweils vorausgesetzt es gibt „wahre" philosophische Positionen und „die richtigen" Regeln des Philosophierens. Beide Ideale sind aber nicht diejenigen, welche im ersten Satz dieser Arbeit impliziert werden; beide sind es nicht, welche Mill zu einem außergewöhnlich guten Philosophen machen. Nach der üblichen Unterteilung von Ergebnisidealen (Werten), Prozessidealen (Prinzipien) und Akteursidealen (Tugenden) bleiben Tugenden übrig. Mill wäre demnach ein guter Philosoph, weil er bestimmte philosophische Tugenden besitzt. ${ }^{4}$

3 William Whewell ist für Mill ein paradigmatischer Vertreter der akademischen Philosophie zu seiner Zeit in England und wird von Mill scharf kritisiert. (Siehe John Stuart Mill. "Whewell on Moral Philosophy“. In: The Collected Works of John Stuart Mill, Volume X - Essays on Ethics, Religion, and Society. Hrsg. von J. M. Robson. Toronto und andere: University of Toronto Press und Routledge \& Kegan Paul, 1969, S. 167-201, S. 167f.) Einer der Kritikpunkte, die er auch andernorts gegen die Art und Weise vorbringt, wie seiner Meinung nach in England philosophiert wird, ist, dass dort technische Terminologie und superfeine Argumente (,superfine new arguments“) zur Verteidigung dogmatischer Wahrheiten gesucht würden. (Siehe John Stuart Mill. "Coleridge". In: The Collected Works of John Stuart Mill, Volume X - Essays on Ethics, Religion, and Society. Hrsg. von J. M. Robson. Toronto und andere: University of Toronto Press und Routledge \& Kegan Paul, 1969, S. 119-164, S. 126.) Weder in sauberer Terminologie - zumindest nicht in technischer -, noch in superfeinen Argumenten liegt nach Mill ein besonderer Wert. Ich arbeite die hier angedeuteten Punkte weiter unten (Abschnitte 3 beziehungsweise 6.1.1) aus.

4 Sowohl in der Alltagssprache als auch in der Philosophie gibt es natürlich mehr oder weniger erfolgreiche Übersetzungsstrategien für die verschiedenen 


\section{Einleitung}

In dieser Arbeit möchte ich behaupten, dass es zwei philosophische Tugenden sind, die Mill zu einem guten Philosophen machen. Vereinfacht ausgedrückt besteht die erste dieser philosophischen Tugenden darin, sich um einen sprachlichen Ausdruck dessen, was der Witz unserer sozialen Praktiken ist, zu bemühen. Die zweite dieser Tugenden besteht darin, sich um eine Möglichkeit des Hinterfragens unserer Praktiken zu bemühen vor dem Hintergrund, dass wir die Dinge ganz anders machen könnten. Die erste dieser Tugenden werde ich „Coleridges Tugend“ nennen, die zweite „Benthams Tugend", weil ich sie an zwei Aufsätzen Mills ${ }^{5}$ John Stuart Mill. „Preface to Dissertations and Discussions". In: The Collected Works of John Stuart Mill, Volume X-Essays on Ethics, Religion, and Society. Hrsg. von J. M. Robson. Toronto und andere: University of Toronto Press und Routledge \& Kegan Paul, 1969, S. 493-494, S. 493. über diese beiden Personen erarbeiten werde. ${ }^{6}$

Typen von Idealen: Eine Konsequentialist*in mag Fälle von deontologischen Regelverstöße beispielsweise in ihre Konzeption integrieren, indem sie sagt, es sei eine Ungerechtigkeit in die Welt gesetzt worden; das Gleiche gilt analog für die anderen Fälle. Ich hoffe, es wird im Laufe der Arbeit deutlich, warum ich von sozialphilosophischen Tugenden spreche. Sollte meine Leser*in daran dennoch festhalten, dass sich alles, was ich sage, in der in dieser Fußnote angedeuteten Weise umformulieren lässt, bitte ich sie die Redeweise von philosophischen Tugenden einfach als harmlose Redeweise zu akzeptieren: Viele der Thesen meiner Arbeit bleiben davon unberührt.

5 Mill, "Coleridge" und Mill, „Bentham". Ich beziehe mich während der gesamten Arbeit, außer an den Stellen, wo ich dies explizit mache, auf den stellenweise von Mill inhaltlich korrigierten Text der Aufsätze, wie er in Dissertations and Discussions 1867 erschienen ist. Mill schreibt zu seinen Änderungen: „Every one whose mind is progressive, or even whose opinions keep up with the changing facts that surround him, must necessarily, in looking back to his own writings during a series of years, find many things which, if they were to be written again, he would write differently, and some, even, which he has altogether ceased to think true. From these last I have endeavoured to clear the present pages."

6 Streng genommen verleiten diese Bezeichnungen der Tugenden zu einem Missverständnis. Ich habe nicht den Anspruch zu zeigen, dass Coleridge und Bentham tatsächlich Träger der Tugenden sind, die Mill ihnen in den entsprechenden Aufsätzen zuschreibt. Statt von „Coleridges Tugend" zu sprechen, wäre es richtiger von "der Tugend, die Mill in Coleridge sieht" zu sprechen und analog im Falle von „Benthams Tugend“. Da ich mich in dieser Arbeit aber sehr oft auf die Tugenden beziehe, habe ich mich für die unpräzise, aber leichter lesbare Kurzform entschieden. 
Nachdem Gegenstand und Ziel meiner Arbeit nun benannt sind, hat diese Einleitung noch drei Aufgaben: Zunächst möchte ich das Ziel meiner Arbeit vorläufig qualifizieren, um nicht zu viel Widerstand hervorzurufen. Die eigentliche Qualifizierung erfolgt dann in Kapitel 2. Die zweite Aufgabe ist es, die Rolle, die die Koautorinnenschaft von Harriet Taylor Mill für diese Arbeit spielt, zu beleuchten. Und die dritte Aufgabe ist es, einen kurzen Überblick über diese Arbeit zu geben. Ausführlichere Überblicke über das, was in den einzelnen Teilen und Kapiteln im Detail geleistet wird, finden sich in den Einleitungen und Zusammenfassungen zu den entsprechenden Kapiteln und Teilen.

Qualifikation des Ziels der Arbeit. Eine, die über das Wesen einer Praxis redet, provoziert Widerspruch, insbesondere wenn es sich um die Praxis des Philosophierens handelt. Erstens möchte ich aber nicht sagen, dass beide Tugenden das Wesen der Philosophie vollständig erfassen. Es mag andere philosophische Tugenden geben, nach denen Mill vielleicht kein guter Philosoph ist. Es mag auch andere Tugenden geben, nach denen er ein guter Philosoph ist, die ich aber nicht erwähne: Mill schreibt sich selbst beispielsweise eine gewisse geistige Offenheit $\mathrm{zu}^{7}$ die zwar vielleicht mit den Tugenden, wie ich sie vorschlage, irgendwie zu tun hat, ${ }^{8}$ die aber sicherlich nicht in ihnen aufgeht.

Zweitens möchte ich nicht sagen, dass jedes philosophische Ideal die Form einer Tugend hat.

Drittens möchte ich mit meinem Hinweis auf Mills philosophische Tugenden nur sagen, dass Mill ein guter Philosoph war, nicht, dass er ein tugendhafter Mensch war. Es gibt neben allem, was wir

7 Zum Beispiel in John Stuart Mill. The Collected Works of John Stuart Mill, Volume I - Autobiography and Literary Essays. Hrsg. von John M. Robson und Jack Stillinger. Toronto und andere: University of Toronto Press und Routledge \& Kegan Paul, 1981, S. 5. Inwieweit wir Mill diese auch zuschreiben wollen, ist natürlich eine andere Frage. Vgl. Francis Sparshot. „Introduction to Vol. XI". In: The Collected Works of John Stuart Mill, Volume XI. Essays on Philosophy and the Classics. Hrsg. von J. M. Robson. Toronto und andere: University of Toronto Press und Routledge \& Kegan Paul, 1978, S. vii-lxxv, S. lxxiv.

8 Mill scheint in der Tat zu denken, dass von den beiden Paradigmen für die Tugenden Coleridge, aber nicht Bentham, eine solche geistige Offenheit besitzt. Siehe Mill, „Coleridge“, S. 120. 


\section{Einleitung}

an ihm schätzen mögen, viele problematische Seiten an Mill, die wir nicht ohne Weiteres ignorieren sollten: Es gibt Textstellen, die sich apologetisch zum Kolonialismus und Imperialismus verhalten ${ }^{9}$; vielleicht rationalisiert er damit auch seine eigene Anstellung in der East India Company; seine Vorschläge zur Reform des Wahlrechts von 1859 beinhaltet den Vorschlag, Stimmen nach Bildungsgrad zu gewichten, was als Furcht vor der erstarkenden Arbeiterbewegung und ihrem Ziel, wählen zu dürfen, gesehen werden kann; ${ }^{10}$ und schließlich gibt es auch berechtigte Kritik an seiner Spielart des Feminismus. ${ }^{11}$

Der vierte Punkt, den ich hier erwähnen will, ist eigentlich bereits im ersten Punkt enthalten. Er ist mir aber so wichtig, dass ich ihn eigens erwähnen will. John Stuart Mill hat die Gegenstände seiner philosophischen Betrachtung insbesondere in der praktischen Philosophie, aber auch in der theoretischen stets auch als soziale Institutionen in den Blick genommen: Moral etwa ist eben nicht nur praktische Orientierung für mich als philosophisch danach Fragende*r, sondern auch eine gewachsene Institution u.s.w. Dieser Blick wird insbesondere in seiner Auseinandersetzung mit Jeremy Bentham und Samuel Taylor Coleridge deutlich, mit der ich mich im ersten Teil beschäftigen werde: Grob übernimmt Mill von Bentham, dass diese Sicht Anlass bietet, eine Reform der entsprechenden Institution, etwa der Institution der Moral, vorzuschlagen; von Coleridge, dass sie Anlass bietet, ihrer Bedeutung für uns auf den Grund zu gehen. Für eine philosophische Herangehensweise, die die Gegenstände der eigenen Betrachtung in diesem Sinne zunächst

9 Siehe z. B. Eileen Sullivan. „Liberalism and Imperialism: J. S. Mill's Defense of the British Empire". In: Journal of the History of Ideas 44.4 (1983), S. 599617.

10 John Stuart Mill. „Thoughts on Parliamentary Reform“. In: The Collected Works of John Stuart Mill, Volume XIX - Essays on Politics and Society Part 2. Hrsg. von J. M. Robson. Toronto und andere: University of Toronto Press und Routledge \& Kegan Paul, 1977, S. 311-340. Dies liegt insbesondere nahe, da Mill in demselben Text darauf hinweist, dass er in jungen Jahren als die Arbeiterbewegung sehr viel schwächer war - dafür war, jeder Person das gleiche Stimmgewicht zuzusprechen.

11 Ein Überblick über die heutige Rezeption findet sich in Mariana Szapuová. „Mill's Liberal Feminism: Its Legacy and Current Criticism“. In: Prolegomena 5.2 (2006), S. 179-191. Zu seinem Verhalten gegenüber Harriet Taylor Mill siehe unten. 
als soziale Praktiken oder Institutionen in den Blick nimmt, wäre das Wort Sozialphilosophie bestens geeignet, wenn es sich nicht im allgemeinen philosophischen Jargon bereits mit einer bestimmten philosophischen Tradition assoziiert wäre, der Mill natürlich nicht einfach zugeordnet werden kann. Um keine Verwirrung zu stiften, habe ich das Wort im Titel dieses Buches daher nicht verwendet.

Es geht aber trotzdem - und das ist die letzte Qualifikation - bei den philosophischen Tugenden eigentlich um Sozialphilosophie im obigen Sinne. Ich spreche daher im Folgenden von sozialphilosophischen Tugenden. Dies ist als weitere Einschränkung des Skopus der in dieser Arbeit getätigten Aussagen gemeint, in dem Sinne, dass eine Einschränkung auf eine bestimmte Betrachtungsweise philosophischer Fragen gemeint ist, nämlich unter ihrem sozialen Aspekt. Der sozialphilosophische Blick ist also ein Blick auf die Gegenstände philosophischer Tätigkeit, der die Rolle, die diese Gegenstände in unseren Arten und Weisen, zusammen zu leben, spielen und spielen sollten, in den Blick nimmt. Da Kommunikationsformen zweifellos zu diesen philosophischen Gegenständen zählen, ist damit auch eine bestimmte Form der Sprachphilosophie mitgemeint. In der obigen skizzenhaften Formulierung der Tugenden ist dies bereits durch den Verweis auf „unsere sozialen Praktiken“ angezeigt worden. Alternativ kämen natürlich auch andere soziale Entitäten außer unseren sozialen Praktiken in Frage: Der soziale Diskurs, Institutionen, Lebensformen u.s.w. In dieser Arbeit wird es weniger darum gehen, welche soziale Entität wir wählen oder wählen sollten, als um das in den beiden Tugenden ausgedrückte Ideal, dass gute Philosophie einerseits dem Witz dieser sozialen Entitäten Ausdruck verleihen soll und ihr Gut- oder Schlechtsein andererseits vor dem Hintergrund evaluieren soll, dass wir die Dinge auch ganz anders machen könnten.

Damit wäre Sozialphilosophie, so wie ich sie hier verstehe, nicht auf bestimmte Themenfelder beschränkt: Auch etwa die Mathematik lässt sich als Institution betrachten; wir tun etwas auf eine bestimmte Weise, wenn wir mathematische Sätze beweisen, rechnen, mathematische Forschung betreiben. Eine Philosophie der Mathematik, die den in dieser Arbeit vertretenen Tugenden gerecht wird, müsste sich einerseits der Frage stellen, was der Witz unserer Rede von mathematischen Wahrheiten, Ontologien, Begriffen und so weiter ist; andererseits müsste sie deutlich machen, was gut daran 


\section{Einleitung}

ist, die Praxis der Mathematik und nicht zum Beispiel eine andere Praxis mit anderen Institutionen u.s.w. anzunehmen.

Trotzdem stehen in dieser Arbeit Fragen, die auch thematisch näher an der Sozialphilosophie im alltäglicheren Sinne liegen, im Vordergrund. Um solche Fragen geht es in den Aufsätzen „Coleridge" und "Bentham" im ersten Teil dieser Arbeit; und um solche Fragen geht es auch in den Texten Mills, die ich im zweiten Teil der Arbeit behandele. Es wird aber nicht immer möglich sein, Fragen der theoretischen Philosophie auszuschließen. Um nur eines der Beispiele zu nennen, die in dieser Arbeit wichtig werden: Mill vergleicht das Verdienst Benthams in Bezug auf Sozial- und Moralphilosophie einerseits mit dem Verdienst Francis Bacons in Bezug auf die Naturwissenschaften und spricht im Utilitarismus von so etwas wie einer Wissenschaft der Moral. Andererseits ordnet er die Frage letzter Zwecke der Kunst zu, die von der Wissenschaft verschieden sei. Um Mills Aussagen über die Institution der Moral und die Moralphilosophie zu verstehen, ist es natürlich unumgänglich, auch Mills wissenschaftstheoretische Vorstellungen und seine Auffassung von der Institution der Wissenschaft - und den Veränderungen, die diese seiner Ansicht nach seit Bacon durchlaufen hat - zu verstehen.

Harriet Taylor Mill. Natürlich kann eine Arbeit zu John Stuart Mill nicht ohne Würdigung von Harriet Taylor Mill auskommen. Für eine Arbeit wie diese ist dies in einer Hinsicht noch wichtiger. Dadurch nämlich, dass ich behaupte, die philosophischen Ideale, die ich anhand von Mill erarbeite, seien Person-Ideale, Tugenden, werden die Personen in den Vordergrund gerückt: Wenn es sich stattdessen um Text-Ideale handelte, dann wäre die Frage, wer die entsprechenden Texte geschrieben hat, letztlich primär aus externen Gründen wichtig - um die Frage zu klären, wem Anerkennung für die Autor*innenschaft zugesprochen werden sollte, beispielsweise - oder, um zu klären, wie bestimmte Textstellen zu verstehen sind. Indem ich aber sage, es sei die philosophierende Person von Bedeutung, die sich mitunter nicht nur in Texten zeige, die normalerweise als philosophisch gelten, sondern zum Beispiel eben auch in der Autobiographie, wird die Frage zusätzlich auf eine andere Weise wichtig. Diese Weise kann in Form eines Zweifels ausgedrückt werden: Angenommen nämlich, es gebe bei den Texten, die ich unter- 


\section{Einleitung}

suche, gar nicht eine philosophierende Person, sondern zwei, würde das die Thesen dieser Arbeit zerstören? Ich glaube nicht.

Bevor ich dafür argumentiere, sei allerdings in Grundzügen das Problem der Autor*innenschaft der Texte von Mill und Taylor Mill skizziert. Mill schreibt in der Autobiography:

When two persons have their thoughts and speculations completely in common; when all subjects of intellectual or moral interest are discussed between them in daily life, and probed to much greater depths than are usually or conveniently sounded in writings intended for general readers; when they set out from the same principles and arrive at their conclusions by processes pursued jointly, it is of little consequence in respect to the question of originality which of them holds the pen; the one who contributes least to the composition may contribute most to the thought; the writings which result are the joint product of both, and it must often be impossible to disentangle their respective parts and affirm that this belongs to one and that to the other. In this wide sense, not only during the years of our married life, but during many of the years of confidential friendship which preceded, all my published writings were as much her work as mine; her share in them constantly increasing as years advanced. ${ }^{12}$

Was auf den ersten Blick wohlwollend klingt, ist aus heutiger Sicht natürlich - auch gerade deshalb - höchst problematisch: Es ist eben John Stuart Mills Name und nicht Harriet Taylor Mills Name, der mit den Texten, die Mill hier meint, in Verbindung gebracht wird. Und auch wenn Mill meint, es sei letztlich nicht wichtig, die Autor*innenschaft kenntlich zu machen, bleibt ein schaler Beigeschmack angesichts dessen, dass er als Mann die Anerkennung als Autor erhält, die Harriet Taylor Mill auf diese Weise verwehrt bleibt. ${ }^{13}$ Tatsächlich ist ein kontroverser Forschungsbereich zu der Frage entstanden, wie groß der Einfluss von Taylor Mill auf Mill tatsächlich war, ohne dass allerdings die Frage endgültig geklärt werden könnte. Denn, wie Dale Miller bemerkt, wir bräuchten dazu

12 Mill, Autobiography, S. 251.

13 Vgl. Jo Ellen Jacobs. The Voice of Harriet Taylor Mill. Bloomington: Indiana University Press, 2002, z. B. S. 129-31. Der Fairness halber sei gesagt, dass John Stuart Mill sich im Anschluss an die soeben zitierte Passage dennoch bemüht, spezifischere Beiträge von Harriet Taylor Mill aufzuzählen. 


\section{Einleitung}

nicht nur Taylor Mills zerstörte Briefe, sondern auch die Inhalte ihrer Gespräche und so weiter. ${ }^{14}$

Vor diesem Hintergrund wäre es vielleicht in einer Hinsicht am korrektesten, das obige Zitat Mills beim Wort zu nehmen und konsequente Koautor*innenschaft anzunehmen. Auch dies ist aber problematisch: Immerhin gibt es, wie bereits erwähnt, einige Stellen, an denen Mill äußerst problematische Dinge sagt - oder zumindest Dinge, die falsch sind oder die wir für falsch halten mögen. Sollen wir auch die Schwächen Mills, wo wir es nicht genau wissen, ${ }^{15}$ Taylor Mill zuschreiben? In diesem Zusammenhang stellt sich noch eine zweite Frage, die geklärt werden müsste, unabhängig von einer bloß quantitativen Einschätzung von Taylor Mills Einfluss auf Mill: Hat Harriet Taylor Mill John Stuart Mills Philosophie eigentlich besser oder schlechter gemacht? ${ }^{16}$

In Reaktion auf dieses Problem könnten wir auch hier eine Äußerung Mills wörtlich nehmen, die Widmung von „On Liberty“ nämlich, nach der wir Taylor Mill als Mitautorin von „all that is best" in den entsprechenden Texten sehen sollten. ${ }^{17}$ Einer Konvention zu folgen, die das berücksichtigt, hieße, was wir für Stärken halten, beiden und, was wir für Schwächen halten, lediglich Mill zuzuschreiben. Dies hat natürlich erstens eine ganz eigene Problematik - gerade vor dem Hintergrund des Tugendcharakters der Ideale, um die es in dieser Arbeit geht: Nur von John Stuart Mill wür-

14 Siehe Dale E. Miller. „Harriet Taylor Mill“. In: The Stanford Encyclopedia of Philosophy. Hrsg. von Edward N. Zalta. Metaphysics Research Lab, Stanford University, 2018, Abschnitt 4.4.

15 Wir wissen es nur selten, da ihre Briefe nicht erhalten sind. Aus den Antworten Mills können wir es manchmal schließen: Beispielsweise hat sie imperialistische Tendenzen in seiner Rezension zu Grotes History of Greece kritisiert. (Siehe John Stuart Mill. The Collected Works of John Stuart Mill, Volume XIV - The Later Letters of John Stuart Mill 1849-1873 Part I. Hrsg. von Francis E. Mineka und Dwight N. Lindley. Toronto: University of Toronto Press, 1972, S. 17f) Dies könnten wir zum Anlass nehmen, anzunehmen, dass sie auch solche Tendenzen in "On Liberty" kritisiert hätte, hätte sie zum Zeitpunkt der Veröffentlichung noch gelebt.

16 Gertrude Himmelfarb beispielsweise scheint, was sie für Harriet Taylor Mills Einfluss auf „On Liberty“ hält, kritisch zu sehen. Siehe Gertrude Himmelfarb. On Liberty 8 Liberalism: The Case of John Stuart Mill. New York: Knopf, 1974, S. 208-72.

17 John Stuart Mill. On Liberty/Über die Freiheit. Deutsch/Englisch. Stuttgart: Reclam, 2009, S. 216. 


\section{Einleitung}

de das Bild einer wirklichen philosophierenden Person entstehen, die Fehler macht, manchmal aus diesen Fehlern lernt, offen ist für bestimmte neue Gedanken, aber einigen Gedanken auch keine Berechtigung einräumt. Von Harriet Taylor Mill würde das Bild eines seltsam abstrakten Ideals entstehen, das natürlich der wirklichen Person auch nicht entsprochen haben kann. Der Fluchtpunkt einer auf solchen philosophischen Idealperson kann genauso wenig im Besitz philosophischer Tugenden sein, wie der göttliche Wille durch Imperative genötigt werden kann. ${ }^{18}$ Zweitens ist die Konvention gar nicht so leicht umzusetzen: denn zwischen dem ausgesprochenen Für-Falsch-Halten einer Aussage in einem der Texte, um die es geht, und dem ausgesprochenen Für-Wahr-Halten gibt es viele Nuancen. Wem sollen wir eine Aussage zuschreiben, wenn wir uns des Urteils über ihre Falschheit enthalten wollen; wem, wenn wir unsicher sind; wem, wenn wir meinen, eine Aussage sei irrelevant?

Die Frage der Autor*innenschaft ist ein ernsthaftes Problem. Ich glaube, was meine Arbeit angeht, kann ich mich trotz dieser Punkte auf John Stuart Mill beziehen, ohne Harriet Taylor Mill explizit oder implizit ihre philosophische Autorinnenschaft abzusprechen. Das, was ich sage, scheint mir kompatibel zu sein damit, dass viele, ja sogar die entscheidendsten der Gedanken aus dem reifem Werk der beiden eigentlich ihr zugesprochen werden müssten - zumindest, solange John Stuart Mill sie sich zu Herzen genommen hat und nicht nur wiederholt hat. Dafür gibt es vier Gründe, die ich in aufsteigender Wichtigkeit nun nennen werde:

1. Die in der Erarbeitung der Tugenden entscheidenden Texte sind sehr viel früher entstanden als das, was die reife Philosophie der Taylor-Mills genannt werden könnte. John Stuart Mill selbst schreibt, dass Harriet Taylor Mills Einfluss auf sein Denken mit der Zeit gestiegen ist, die früheren Texte also weniger von ihr enthalten.

18 Z. B. Immanuel Kant. Kritik der praktischen Vernunft. Hamburg: Meiner, 2003, S. AA 79. Anders als es Kant mit dem göttlichen Willen sieht, ist es, so meine ich, eine Konsequenz dieser Arbeit, dass die Vorstellung von idealen Philosophierenden nicht erhellend dafür ist, was es heißt, unter den beiden hier vorgestellten philosophischen Tugenden zu philosophieren. Dies wird aus dem vierten Punkt in der nun folgenden Aufzählung (Seite 13) deutlich. 
2. Für diese Texte gibt es andere Einflüsse, die wahrscheinlich entscheidender sind: „Bentham" ist sicherlich eine Auseinandersetzung mit der Philosophie die Mills Erziehung und Jugend prägte, mit der er sich aber nach seiner ersten großen psychischen Krise nicht mehr identifizieren konnte und wollte. Der Aufsatz hat außerdem einen anonym veröffentlichten Vorläufer, „Remarks on Bentham's Philosophy“ von 1833, auf den das noch mehr zutrifft, und der Benthams Philosophie in vielen Hinsichten noch schärfer kritisiert. Auch die intellektuellen Einflüsse, die zu „Coleridge" führen, sind relativ klar: Es ist Mills Auseinandersetzung mit Frederick Maurice und John Sterling und anderen Anhängern der Philosophie Coleridges, mit denen er sich im Debattierklub anfreundet. Beides hängt natürlich zusammen und dies ist einer der Gründe, die Aufsätze als Einheit zu betrachten: Dadurch, dass Mill in seiner psychischen Krise den Glauben ${ }^{19}$ an die benthamitische Philosophie verliert, ist er offener für die Gegenseite im Debattierklub. Und dadurch, dass er Sterling und Maurice zu diesem Zeitpunkt auch als Personen überzeugend findet, fühlt er sich in seiner Kritik an Bentham ermutigt. (Später relativiert er dies etwas, siehe Abschnitt 3.4.)

3. Für das Spätwerk will ich Elizabeth Andersons Interpretation (mit einer leichten Modifikation) verteidigen. (Siehe Abschnitt 5.1.) Nach Anderson spielt Mills Autobiographie und besonders seine psychische Krise eine entscheidende Begründungsrolle für wichtige Gedanken seiner späteren Philosophie in "On Liberty" und „Utilitarianism". Die Autobiography wird in dieser Interpretation zu einem genuin philosophischen Text. Dies ist, so meine ich, vollständig kompatibel damit, dass wir Harriet Taylor Mill als Miturheberin oder sogar alleinige Urheberin dieser Gedanken sehen. Was diese Arbeit angeht, ist es nur entscheidend, dass Anderson damit Recht hat, dass der Grund, warum Mill diese Gedanken wichtig sind, und der Grund, sie für wahr zu halten, in seiner Biographie und insbesondere in seinen psychischen Krisen liegt. Die Ori-

19 Mill spricht von einer Art „Religion“, die Benthams Philosophie in seiner Jugend für ihn darstellt. Siehe Mill, Autobiography, S. 69. 
ginalität dieser Gedanken Mills muss dafür explizit nicht behauptet werden.

4. Es ist ein entscheidender Teil von meiner These zu Mills sozialphilosophischen Tugenden, dass gute Philosophie aus der Sicht einer in kontingente soziale Gepflogenheiten verwickelten Person betrieben wird, die diese Gepflogenheiten für bedeutsam hält, aber an deren Bedeutsamkeit zugleich zweifelt. Daraus ergibt sich Coleridges Tugend, sich um einen dieser Bedeutung adäquaten sprachlichen Ausdruck zu bemühen, und Benthams Tugend, sich um ein objektives Hinterfragen dieser Bedeutung zu bemühen. Um das Gelingen guter Philosophie entsprechend einschätzen zu können, ist eine Geschichte des Lebens eine*r Philosoph*in nötig. Ich beschäftige mich in dieser Arbeit mit John Stuart Mills diesbezüglicher Geschichte - und das schließt natürlich nicht aus, dass sich für Harriet Taylor Mill auch eine solche Geschichte erzählen ließe, hätten wir die entsprechenden Quellen. Diese Geschichte ist für die Arbeit entscheidender, als die philosophischen Thesen, die Mill aufgrund dieser Geschichte aufstellt. Tatsächlich werde ich mich von einigen der wichtigsten Thesen Mills distanzieren, insbesondere, aber nicht ausschließlich, in Kapitel 6.

Übersicht über die Arbeit. Im ersten Teil der Arbeit werde ich die beiden komplementären philosophischen Ideale erarbeiten. Kapitel 3 behandelt dabei Coleridges Tugend, Kapitel 4 Benthams Tugend. Den beiden Kapiteln ist ein Kapitel vorangestellt, in dem ich die Vorgehensweise der Arbeit methodisch begründe und eine vorläufige Formulierung der beiden Tugenden anstrebe. Außerdem werde ich in diesem Kapitel 2 etwas zum Status der beiden Tugenden sagen, um mögliche Missverständnisse zu vermeiden. Ich werde die Tugenden dort als komplementäre, nicht-ableitbare, jedoch nicht exklusive konstitutive Ideale von Philosophie bezeichnen und erklären, was damit gemeint ist.

Nachdem im ersten Teil Mills Philosophie der Sozialphilosophie in einem bestimmten Lebensabschnitt anhand der Aufsätze "Coleridge" und „Bentham" erarbeitet wurde, geht es im zweiten Teil darum, zu zeigen, dass Mill sich in seiner eigenen praktischen Philo- 


\section{Einleitung}

sophie auch in seiner reifen Phase an den Tugenden orientiert. Kapitel 5 soll dabei einen Grundzug von Mills Philosophie erarbeiten, der den beiden Tugenden gerecht wird. Ich orientiere mich dabei an Elizabeth Anderson, die für die Beschreibung dieses Grundzuges die Phrase ,experiments in living" von Mill übernimmt. Experiments in living sind aber möglicherweise nicht die einzige Art und Weise, den Tugenden gerecht zu werden, und sie sind sicherlich nicht die einzige Art und Weise, in der Mill sich bemüht, den Tugenden gerecht zu werden. In gewisser Weise scheinen mir Mills andere Versuche, den Tugenden gerecht zu werden, weniger überzeugend. In dieser Hinsicht kann gesagt werden, Mill scheitere an den Tugenden. Mit dieser Formulierung wird natürlich impliziert, dass er sich trotzdem an den Tugenden orientiert. Dies nachzuweisen und zu zeigen, inwiefern er trotzdem scheitert, ist Aufgabe von Kapitel 6.

Der dritte Teil hat zwei Aufgaben: Zum einen möchte ich einen Gedanken herausarbeiten, der impliziert wird, wenn Mills philosophische Tugenden auf einen Bereich unserer geteilten Praxis angewendet werden. Ich erarbeite diesen am Beispiel unserer moralischen Praxis. Es geht um Expertise und die Frage, inwiefern eine philosophische Beschäftigung, die sich an den beiden Tugenden orientiert, eine solche Expertise über den Gegenstandsbereich ermöglicht. Ich werde zeigen, dass eine bestimmte Art von Expertise dabei ausgeschlossen ist. Ausgehen werde ich dabei von einem Missverständnis Mills der Frühdialoge Platons und zwar aus zwei Hauptgründen: Erstens benutzt Mill auch seine Texte über Platon, um sein Ideal von Philosophie zu erarbeiten; diese Auseinandersetzung sollte also nicht fehlen. Zweitens will ich behaupten, dass der Grund, warum eine bestimmte Art der Expertise über einen Bereich unserer geteilten Praxis durch eine an den beiden sozialphilosophischen Tugenden orientierte Beschäftigung mit dieser Praxis nicht erlangt werden kann, auch Gegenstand der Frühdialoge ist - zumindest einer bestimmten Lesart dieser Dialoge, die ich mit Ursula Wolf assoziiere. Dies geschieht in Kapitel 7.

Die zweite Aufgabe des dritten Teiles ist es, Mills Position abzugrenzen und ins Verhältnis zu anderen Positionen zu setzen sowie offene Fragen und Probleme, die in der Arbeit nicht gelöst oder nicht einmal angesprochen werden konnten, in Form eines Ausblicks zu markieren. Dies geschieht in Kapitel 8. 


\section{Teil I.}

\section{Erarbeitung der beiden sozialphilosophischen Tugenden und methodische Überlegungen}




\section{Einleitung zu Teil 1}

Der folgende Teil der Arbeit (Teil I) dient dazu, die beiden Tugenden inhaltlich zu erarbeiten. Hauptsächlich ist das exegetische Arbeit: Ich werde versuchen zu zeigen, dass das Kernanliegen (oder zumindest eines der Kernanliegen) der Aufsätze „Bentham“20 und "Coleridge“21 die Formulierung zweier philosophischer Tugenden ist. Dies passiert im Wesentlichen in den Kapiteln 3 (für Coleridges Tugend) und 4 (für Benthams Tugend).

Diese Deutung ist aber nur unter drei Voraussetzungen plausibel, die ich in Kapitel 2 ausarbeiten werde. Erstens muss zum Beispiel klar werden, warum Mill, indem er über zwei Philosophen schreibt, zwei philosophische Tugenden erarbeitet. Dies ist eine methodische Überlegung zur Philosophie der Philosophie, die ich einerseits für zutreffend für die beiden Aufsätze zum anderen aber auch für systematisch zutreffend halte. Weil es eine systematische Überlegung ist, ist dieser Gedanke natürlich auch für den nächsten Teil relevant, in welchem ich systematisch für die Tugenden werben will, indem ich über Mill in der Weise schreibe, in welcher dieser über Bentham und Coleridge schreibt. Solche methodischen Überlegungen finden in Kapitel 2 statt.

Zweitens muss gezeigt werden, dass die beiden Aufsätze tatsächlich so zu verstehen sind, wie ich sie verstehe. Formal bedeutet dies zu zeigen, dass sie als komplementäre Teile einer Einheit gedacht sind. Dies tue ich ebenfalls in Kapitel 2. Inhaltlich muss meine Deutung gegen alternative Deutungen verteidigt werden. Dies erfolgt nicht in Kapitel 2, weil diese inhaltliche Frage erst in der detaillierteren Auseinandersetzung mit den Aufsätzen beantwortet werden kann. Ich verteidige meine Deutung gegen alternative Deutungen am Ende von Kapitel 3. („Coleridge“ legt die alternativen Deutungen sehr viel näher als „Bentham“, deshalb findet die Verteidigung auch im Kapitel zu Coleridge statt.)

Drittens schicke ich in Kapitel 2 einige Überlegungen zum Status der Tugenden vorweg, damit verständlich wird, was es eigentlich ist, das ich im ersten Teil exegetisch Mill unterstelle und für das ich in Teil II systematisch werben will. Ich bezeichne die Tugenden dort als „komplementär", „nicht ableitbar", aber „nicht exklusiv"

20 Mill, „Bentham".

21 Mill, "Coleridge“. 
und als „konstitutive Ideale“; und ich erkläre, was damit gemeint ist.

Wie deutlich geworden sein sollte, sind einige der Überlegungen auch systematisch relevant. Der Hauptanspruch dieses ersten Teils liegt aber in einer exegetischen, inhaltlichen Erarbeitung der Tugenden in den Kapiteln 3 und 4. Die Idee ist, dass nachdem diese inhaltlich erarbeitet sind, im nächsten Teil systematisch für sie geworben werden kann. Unter Berücksichtigung der methodischen Überlegungen zur Philosophie der Philosophie, die ich bereits in Teil I anstelle, bedeutet dies, dass ich zu zeigen versuche, dass Mill, indem er sich an den Tugenden orientiert, einer Tätigkeit nachgeht, von der sich zeigt, dass sie durch diese Orientierung ein Philosophieren ist, und dass er je nachdem, inwiefern er scheitert oder erfolgreich ist, dieser Tätigkeit besser oder schlechter nachgeht. 


\section{Eine vorläufige Formulierung der sozialphilosophischen Tugenden und methodische Überlegungen}

In diesem Kapitel werde ich zunächst (Abschnitt 2.1) eine vorläufige Formulierung von Mills Tugenden geben. Ich habe zwei Gründe, es so anzugehen:

Erstens möchte ich etwas zum Status meiner Überlegungen (Abschnitt 2.2) sagen. Dies liegt vor allem daran, dass das Aufstellen philosophischer Tugenden geeignet ist, großen Widerstand hervorzurufen. Dies ist zwar in der Philosophie durchaus nichts Besonderes und in gewissem Maße natürlich auch erwünscht. Allerdings möchte ich vermeiden, unnötig Widerstand hervorzurufen. Daher erkläre ich die Geltung von dem, was ich „Mills Tugenden“ nenne, genauer und schränke den Skopus meiner These ein. Diese Überlegungen zum Status werden sehr viel verständlicher, wenn ungefähr klar ist, worin die Tugenden inhaltlich bestehen.

Zweitens möchte ich meiner Beschäftigung mit „Coleridge"1 und „Bentham" ${ }^{\prime 2}$ in Kapitel 3 und Kapitel 4, den Kapiteln, in denen die Tugenden eigentlich erarbeitet werden, einige methodische Überlegungen (Abschnitt 2.3) vorausschicken. Der wichtigste Punkt dieser methodischen Überlegungen ist, für eine bestimmte Art des Schreibens über andere Philosoph*innen als Argumentationsmethode der Philosophie der Philosophie zu argumentieren. Und natürlich werde ich behaupten, dass das, was Mill in den beiden Aufsätzen tut, wesentlich Philosophie der Philosophie ist. Es ist eine komplexe Frage, was eigentlich einen Versuch des sprachlichen Ausdruck dessen, was wesentlich an unserer philosophischen Praxis ist, besser macht als einen anderen solchen Versuch und welche Begründungen dafür in Frage kommen. Und schlimmer noch: Die Antwort auf diese Frage muss sich im Verfahren schon dessen bedienen, was sie eigentlich ausdrückt - sie muss gute Philosophie sein. Diese Zirkularität, auf

\footnotetext{
1 Mill, "Coleridge".

2 Mill, „Bentham".
} 
die ich unten näher eingehen werde, und der Umgang damit, den ich vorschlagen werde, machen es nötig, bereits ungefähr anzugeben, auf welche der wesentlichen Aspekte unserer philosophischen Praxis ich eigentlich abziele: Nur so lässt sich verstehen, warum eine bestimmte Art des Schreibens über andere Philosoph*innen als Argumentationsmethode in der Philosophie der Philosophie dienen kann.

Außer der vorläufigen Formulierung der Tugenden, den Überlegungen zum Status der Tugenden und den methodischen Überlegungen möchte ich in diesem Kapitel auch meine Lesart von Mills Aufsätzen „Coleridge" und „Bentham" als zusammengehörig rechtfertigen (Abschnitt 2.4).

Die Frage, warum ich den Maßstab für gute Philosophie als Tugenden und nicht zum Beispiel als Anforderungen an philosophische Texte formuliere, gehört nicht zu den Überlegungen, die ich der Verständlichkeit halber vorausschicken muss. Ich werde die Frage in diesem ganzen Teil nicht explizit ansprechen. Es wird im Rahmen der Beschäftigung mit „Coleridge“ und „Bentham" in Kapitel 3 und Kapitel 4 lediglich deutlich werden, dass Mill (auch) auf Personideale abzielt. Den eigentlichen Wert darin, es so zu sehen, werde ich erst in Teil II herausstellen.

\subsection{Eine vorläufige Formulierung von Mills Tugenden}

Bentham ist für Mill ein Paradigma für die eine Tugend ${ }^{3}$; dies werde ich in Kapitel 4 versuchen zu belegen. Hier jedoch, in Abschnitt 2.1, bemühe ich mich nur um eine vorläufige Formulierung, die lediglich dazu dienen soll, die Vorbemerkungen in diesem Kapitel verständlich zu machen. Weder erhebe ich mit diesem Kapitel den systematischen Anspruch, für die Adäquatheit von Mills erster Tugend zu argumentieren, noch den exegetischen Anspruch, dafür zu argumentieren, dass Mill diese Tugend in Bentham verwirklicht sieht. Gemäß Benthams Tugend müssen wir uns in der Sozialphilosophie um Objektivität bemühen. Natürlich ist Objektivität ein höchst problematischer Begriff, ich werde daher in Kapitel 4 und beispielhaft auch in Teil II ausführen, was hier darunter zu verstehen ist.

3 Mill, „Bentham“. 
Hier sei dies nur provisorisch angedeutet: Nach Benthams Tugend müssen wir uns um Gründe für oder gegen eine in Frage stehende soziale Praxis bemühen, die vor dem Hintergrund gültig bleiben, dass wir die Dinge auch ganz anders machen könnten. Für Bentham und auch für Mill selbst (siehe Kapitel 5) bedeutet das ein Bekenntnis dazu, Philosophie auch als empirisch informierten Lernprozess zu begreifen - wobei Mill ganz andere Dinge unter „Erfahrung" versteht als Bentham. Mill legt aber Wert darauf, dass Benthams Tugend nicht auf den Empirismus beschränkt ist, wie ich zeigen werde. Obwohl Mill also selbst der ersten Tugend nachkommt, indem er bestimmten Schlüsselerfahrungen in seinem Leben eine Rolle in der Philosophie einräumt, meint er nicht, dass eine Philosophie der Erfahrung direkt aus Benthams Tugend folgt. (Siehe Abschnitt 4.3.1.)

Coleridge ist für Mill ein Paradigma für die andere Tugend, wie ich in Kapitel $3 \mathrm{zu}$ belegen versuche. Auch für die folgende vorläufige Formulierung dieser Tugend, die ich in diesem Abschnitt 2.1 gebe, beanspruche ich weder, sie am Ende des Abschnittes systematisch begründet zu haben, noch belegt zu haben, dass Mill die Tugend in Coleridge verwirklicht sieht. Beides erfolgt in Abschnitt 3. Coleridges Tugend besteht darin, sich an dem Ideal eines sprachlichen Ausdrucks bezüglich unserer sozialen Praktiken zu orientieren, den eine kompetente Teilnehmer*in an dieser Praxis als adäquaten Ausdruck dessen, was ihr an dieser Praxis wichtig ist, identifizieren würde. Auch das, was hierfür nötig ist, bezeichnet Mill als Erfahrung: Nur eine, die selbst kompetente Teilnehmer*in an der entsprechenden Praxis ist und erlebt hat, worum es geht, kann dies primär entscheiden - wobei es nach Mill durchaus Möglichkeiten gibt, die Erfahrungen andere zu berücksichtigen, auch wenn dies immer nur der zweitbeste Weg ist.

Beide Tugenden stehen in einem Spannungsverhältnis zueinander. Dies wird bei ihrer eigentlichen Erarbeitung deutlicher. An dieser Stelle kann es aber schon durch eine skizzenhafte Gegenüberstellung der jeweiligen Verfallsformen der Tugenden klargemacht werden: Während die Verfallsform der ersten Tugend in einem umfassenden Reduktionismus philosophischer Gegenstände auf empirische Begriffe besteht, der letztlich das, was uns an diesen Begriffen wichtig ist, nicht zu fassen vermag, besteht die Verfallsform der zweiten Tugend in einem unkritischen Quietismus, der die Vernünf- 
tigkeit einer Veränderung unserer Praxis grundsätzlich verneint ${ }^{4}$. Wie dieses Spannungsverhältnis jeweils gelöst wird, ist ein interessanter Gesichtspunkt, anhand dessen sich philosophische Ansätze vergleichen lassen. Ich werde dies in Ansätzen in Abschnitt 8.1 tun, wenn ich versuche, Mills Ansatz von anderen Ansätzen abzugrenzen.

\subsection{Bemerkungen zum Status der Tugenden}

Komplementarität: Beide Tugenden sind, was ihre Rolle als philosophische Tugenden angeht, komplementär zu verstehen: Dies soll erstens heißen, dass der philosophische Wert, den ein durch sie orientiertes Philosophieren hat, insofern es um den philosophischen Wert geht, nur erreicht werden kann, wenn sich das Philosophieren auch an der jeweils anderen Tugend orientiert - und diese auch in einem Mindestmaß verwirklicht.

Zwar mag es durchaus auch einen Wert haben, sich lediglich um einen sprachlichen Ausdruck zu bemühen, den eine Teilnehmer*in an einer Praxis als adäquaten Ausdruck dessen, was ihr an dieser Praxis wichtig ist, identifizieren würde. Zum philosophischen Wert wird dies, so meine Behauptung, aber erst, wenn wir uns auch um Benthams Tugend, nämlich die Möglichkeit der Kritik dieser Praxis bemühen. Umgekehrt gilt dies auch: Kritik des Etablierten hat mit Sicherheit einen Wert, selbst wenn sie nichts anderes ist. Wir sollten kritische Menschen sein. Alleine dass wir kritisch sein sollten, macht Kritik aber noch nicht zur Philosophie, so die Behauptung - auch nicht, wenn wir besondere Maßstäbe der argumentativen Strenge anlegen oder thematische Einschränkungen auf das konventionelle Themengebiet der Philosophie vornehmen.

Zweitens soll dies bedeuten, dass die Tugenden substantiell verschieden sind, also zum Beispiel nicht ableitbar aus einer allgemeinen philosophischen Tugend, die sie beide umfasst. Die Frage der Ableitbarkeit wird im nächsten Abschnitt genauer beleuchtet.

4 Womit natürlich nicht gesagt ist, dass damit die Vernünftigkeit der Beibehaltung unserer Praxis behauptet werden muss. Eine grundlegende Veränderung unserer Praxis würde sich der quietistischen Verfallsform der zweiten Tugend entsprechend einfach der Frage nach ihrer Vernünftigkeit entziehen. 
Vor allem der erste dieser Punkte erinnert natürlich an die bekannte Frage, ob „die gute Weise menschlichen Lebens ${ }^{\text {"5 }}$ eines sei oder aus mehreren Komponenten bestehe. Bereits im Protagoras ${ }^{6}$ wird diesbezüglich die nicht unplausible Position diskutiert, dass das menschliche Gutsein zwar aus Einzeltugenden (zum Beispiel Tapferkeit, Gerechtigkeit, etc.) bestehe, dass aber diese nur als Konstituenten des menschlichen Gutseins und nicht für sich betrachtet gut seien. Das führt zu der einigermaßen plausiblen Feststellung, dass, falls es möglich ist, Tapferkeit ohne Gerechtigkeit zu erwerben, das Gute, das eine Person damit erwirbt, was auch immer es ist, jedenfalls nicht das menschliche Gutsein oder ein Teil davon ist. Auch sehr schlechte Menschen können nach dieser Position tapfer sein; ihre Tapferkeit ist aber keine Verwirklichung des menschlichen Gutseins. Ich halte es für eine eher scholastische Diskussion, ob wir stattdessen lieber sagen sollten, was auch immer schlechte Menschen erwerben können, es kann jedenfalls keine Tapferkeit sein, da Tapferkeit der Name einer Tugend sei und ihre Träger*in somit gut mache. Der dahinter liegende Punkt, den ich gerne auf Mills philosophische Tugenden übertragen will, bleibt der gleiche:

Ich will nicht sagen, dass es keinen Wert haben kann, jeweils eine der beiden Tugenden zu verwirklichen und sich um die andere nicht zu bemühen. Ich will aber erstens sagen, dass philosophischer Wert erst in der Orientierung des eigenen Philosophierens an beiden Tugenden besteht. Genau genommen schwäche ich dies noch einmal ab. Da ich, wie unten in diesem Abschnitt deutlich wird, nicht beanspruche die exklusive Bestimmung von Philosophie gegeben zu haben, will ich auch nicht ausschließen, dass es andere Bestimmungen von Philosophie geben mag, nach der die eine oder die andere der Tugenden alleine wertvoll ist. Das heißt nicht, dass ich den Punkt dieses Abschnittes aufgebe: Das Eingewendete widerspricht nämlich nicht der Aussage, dass es auch einen solchen

5 „Die gute Weise menschlichen Lebens" soll hier gemäß Ursula Wolfs Vorschlag eine Übersetzung von arete sein. Vgl. Ursula Wolf. Die Suche nach dem guten Leben. Platons Frühdialoge. Reinbek bei Hamburg: Rowohlt Taschenbuch Verlag, 1996, S. 25. Ich spreche zwar von philosophischen Tugenden und so könnte eingewendet werden, ich solle auch von der menschlichen Tugend sprechen. Ich glaube aber, es ist zur Unterscheidung der Ebenen sogar sinnvoll, verschiedene Wörter zu verwenden.

6 Ursula Wolf, Hrsg. Platon. Sämtliche Werke, Band 1. 32. Aufl. Reinbek bei Hamburg: Rowohlt Taschenbuchverlag, 2011, S. 271-337. 
philosophischen Wert gibt, der in der Verwirklichung der beiden philosophischen Tugenden liegt und der nur erreicht werden kann, wenn das Philosophieren sich an jeweils beiden Tugenden orientiert. Und zweitens will ich sagen, dass es keine abstraktere Bestimmung gibt, die beide Tugenden umfasst und nicht einfach in der Konjunktion beider Tugenden besteht.

Nicht-Ableitbarkeit: Die beiden Tugenden sind nicht aus anderen Bestimmungen der Philosophie oder der Aufgaben der Philosophie ableitbar. Diese Behauptung muss sicherlich genauer qualifiziert werden: Natürlich ist es für einen beliebigen Satz $p$ möglich, diesen aus anderen Sätzen „abzuleiten“, indem ich zum Beispiel willkürlich die beiden Sätze $q \rightarrow p$ und $q$ formuliere. In vielen Fällen ist dies natürlich eine unnütze Übung: Die beiden Sätze $q \rightarrow p$ und $q$ mögen gänzlich unplausible Behauptungen sein. Aber ich möchte auch keineswegs ausschließen, dass es auch plausible Behauptungen über die Philosophie oder philosophische Tugenden gibt, aus denen ein (mehr oder weniger streng) gültiger Schluss auf meine Formulierungen von Mills philosophischen Tugenden gezogen werden kann.

Dies wird vielleicht am besten an einer Abgrenzung, die weiter unten in der Dissertation (Abschnitt 8.1.1) noch einmal wichtig wird, deutlich. Es geht um die Abgrenzung zwischen interner Kritik $^{7}$ (und ihrem Wert) und der zweiten philosophischen Tugend (und ihrem Wert). Dazu sei folgendes Argument betrachtet:

(1) Alle Formen legitimer Kritik haben einen philosophischen Wert.

(2) Interne Kritik ist eine Form legitimer Kritik.

(3) Interne Kritik setzt voraus, dass das, was den Teilnehmer*innen an einer Praxis wichtig ist, in Form eines Normsatzes sprachlich zum Ausdruck gebracht wird.

7 Ich orientiere mich dabei an dem Begriff interner Kritik, den Rahel Jaeggi (in Anlehnung an Michael Walzer) zur Abgrenzung von immanenter Kritik verwendet. (Vgl. Rahel Jaeggi. Kritik von Lebensformen. Berlin: Suhrkamp, 2014, S. 257-276.) In dem gleichen Abschnitt (Abschnitt 8.1.1) werde ich Mills Tugenden natürlich auch auf Jaeggis eigenes Programm der immanenten Kritik beziehen und von diesem abgrenzen. 
(4) Also ist es auch von philosophischem Wert, uns um einen sprachlichen Ausdruck zu bemühen, den eine Teilnehmer*in an einer Praxis als adäquaten Ausdruck dessen, was ihr an dieser Praxis wichtig ist, identifizieren würde.

Sicherlich ist dieses skizzenhafte Argument an einigen Stellen problematisch. ${ }^{8}$ Ich verwende es nur, um das Zugeständnis zu machen, dass ein Argument in dieser Richtung (klarer formuliert und sorgfältiger ausgedrückt) für die erste und/oder die zweite von Mills Tugenden möglich und sogar sinnvoll sein mag. Dies würde bedeuten, dass es möglich sein mag, eine Formulierung einer oder beider von Mills Tugenden abzuleiten aus anderen Bestimmungen der Philosophie. Das Argument kann so verstanden werden, dass Coleridges Tugend deshalb eine philosophische Tugend ist, weil Kritik einen philosophischen Wert hat. Es liegt daher auf der Hand, dass ein ganz ähnliches Argument auch für Benthams Tugend möglich sein könnte. ${ }^{9}$

Die mit dem Zugeständnis, dass solche Ableitungen gültig sein könnten, verbundene Einsicht ermöglicht es nun, meine These der Nicht-Ableitbarkeit klarer zu formulieren: Aus welchen Gründen auch immer sie sonst noch einen Wert haben mögen, die beiden Tugenden haben auch einen solchen philosophischen Wert, den sie nicht deshalb haben, weil etwas (substantiell) anderes einen philosophischen Wert hat und sich dieser Wert überträgt. Wir könnten das so Bezeichnete ihren intrinsischen Wert nennen; ich habe mich stattdessen dafür entschieden, von der Nicht-Ableitbarkeit der philosophischen Tugend aus anderen philosophischen Tugenden zu sprechen, weil dies eine methodische Schwierigkeit deutlicher macht, auf die ich weiter unten (Abschnitt 2.3) näher eingehen will.

Nicht-Exklusivität und Geltungsbereich: Aus dem unter "Nicht-Ableitbarkeit" Geschriebenen wird schon deutlich, dass ich nicht

8 Zum Beispiel könnte eingewendet werden, dass interne Kritik offensichtlich nur einen Wert haben kann, wenn die internen normativen Festlegungen, die ihr zu Grunde liegen, einen Wert haben. (Vgl. ebd., S. 269-70.) In anderen Fällen mag offensichtlich die praktische Überschreitung einer gesellschaftlichen Norm besser sein als die Kritik dieser Überschreitung.

9 Tatsächlich glaube ich, dass das für Benthams Tugend auch der Fall ist. Ich gehe darauf in Kapitel 4 in Abschnitt 4.1 ein. 
behaupten will, dass mit den philosophischen Tugenden Mills die einzig mögliche Bestimmung der Philosophie gegeben ist. ${ }^{10}$ Die allgemeinste Ebene, auf der dies gilt, ist diejenige, dass ich mit dieser Arbeit überhaupt kein Interesse daran verfolge, den Gebrauch des Wortes „Philosophie“ zu sanktionieren. Dieser Punkt ist relativ unspektakulär und mit Nicht-Exklusivität möchte ich auch mehr sagen: Ich möchte sagen, dass es selbst unter denjenigen, mit denen ich mir einig bin, dass wir über das Gleiche reden, wenn wir "Philosophie“ sagen, Personen geben mag, die informierter und gerechtfertigter Weise eine andere Bestimmung der Philosophie geben würden.

Es mag einerseits einfach noch mehr Maßstäbe philosophischer Güte - Tugenden, aber auch Normen - geben, die problemlos neben den (meiner Behauptung nach) von Mill vorgeschlagenen bestehen können. Andererseits mag es Bereiche der Philosophie geben, die unter anderen Tugenden stehen und die sich an anderen Tugenden anstelle von Mills Tugenden orientieren. Um dies etwas weniger beliebig wirken zu lassen, möchte ich Folgendes vorschlagen: Erstens hoffe ich, dass die Tugenden, wenn auch nicht notwendig, so doch zumindest hinreichend sind. Eine die etwas tut, das sich an beiden Tugenden gleichzeitig orientiert, philosophiert zumindest. Zweitens möchte ich zumindest für den Bereich der Sozialphilosophie vorschlagen, dass beide Tugenden auch notwendig sind. „Sozialphilosophie" möchte ich dabei sehr weit verstanden wissen als alle philosophischen Richtungen, in denen die von uns geteilte soziale Praxis (Lebensform, soziale Wirklichkeit, Institutionen u.s.w.) in irgendeiner Form der Gegenstand des philosophischen Interesses ist. Dieser letzte Punkt bedeutet, dass ich in Abschnitt 8.1, wo ich Mills Tugenden von anderen philosophischen Ansätzen abgrenze, zeigen muss, dass diese Ansätze trotzdem als Realisierungen (oder zumindest Realisierungsbemühungen) von Mills Tugenden aufgefasst werden können.

10 Dies wäre angesichts der Fülle von Bestimmungsversuchen dessen, was Philosophie ist, auch kaum angemessen. Ohne Anspruch auf Vollständigkeit sind einige beispielsweise aufgelistet in Max Horkheimer. „Die gesellschaftliche Funktion der Philosophie". In: Traditionelle und kritische Theorie. 6. Aufl. Frankfurt am Main: Fischer, 2005, S. 270-290, S. 270-272. 
Konstitutive Ideale: Sind die Tugenden nun konstitutiv für einen bestimmten Bereich der sozialphilosophischen Praxis oder sind sie Ideale für gute Sozialphilosophie in diesem Bereich? Hinter einer solchen Frage steckt eine klassische Unterscheidung zwischen konstitutiven und regulativen oder normativen Regeln: Eine Schachspieler*in, die ihre Bauern rückwärts bewegt, verstößt gegen konstitutive Regeln des Schachs - und spielt somit eigentlich nicht Schach. „Springer am Rand bringt Kummer und Schand'“ hingegen ist eine regulative Regel: Eine, die ihre Springer an den Rand des Schachfeldes stellt, spielt zwar Schach, aber möglicherweise schlecht. Allerdings gibt es eine Zwischenform: Eine, der es zum Beispiel egal ist, ob sie gewinnt oder verliert, spielt auch nicht Schach. ${ }^{11}$ Natürlich ist zu gewinnen keine konstitutive Regel für das Schachspiel auch eine, die verliert, spielt Schach. Aber Gewinnenwollen ist konstitutiv. Gewinnenwollen ist, was ich hier als „konstitutives Ideal“ für das Schachspiel bezeichnen will. ${ }^{12}$ Eine die Schach spielt, muss zumindest für das Spiel akzeptieren, dass zu gewinnen gut wäre. Es mag natürlich zahlreiche externe Gründe für eine Spieler*in geben, nicht gewinnen zu wollen.

Schachanalogien sind problematisch; aber sie haben in diesem Fall eine gewisse Tradition. In Wirklichkeit sind aber konstitutive Ideale sehr viel verbreiteter, als es die oben geschilderte Alternative zwischen regulativen und konstitutiven Regeln erscheinen lassen mag. Sie sind keine Kuriosität: Von einem kleinen Kind beispielsweise, das Geld unterschiedslos in seinem Umfeld verteilt, würden wir unter Umständen nicht sagen, es sei großzügig, sondern es habe nicht verstanden, dass unsere Praxis der Geldverwendung mit einer generalisierten Annahmebereitschaft ${ }^{13}$ einhergeht und dass das Gegenstück zur generalisierten Annahmebereitschaft eben eine in-

11 Beispielsweise eine Person, die, wo es möglich ist, immer die Figur setzt, die am weitesten links oben am Spielfeld steht.

12 Für Donald Davidson ist Rationalität beispielsweise in diesem Sinne ein „konstitutives Ideal" für Intentionalität. Siehe Donald Davidson. „Mental Events“. In: Essays on Actions and Events. Philosophical Essays Volume I. Oxford und andere: Oxford University Press, 2001. Kap. 11, S. 207-225, S. 223 und siehe Michael Rescorla. „Rationality as a Constitutive Ideal“. In: A Companion to Donald Davidson. Oxford und andere: Wiley Blackwell, 2013. Kap. 27, S. $472-488$.

13 In Niklas Luhmanns Sprache ist Geld eines der generalisierten Kommunikationsmedien, die Kommunikation auch dort ermöglichen, wo sie sonst un- 
dividuelle Abgabehemmung ${ }^{14}$ ist. Eine, die die Praxis des Geldes verstanden hat, weiß, dass sich Geld zu wünschen und es nicht unterschiedslos zu verteilen, dazugehört. Wie beim Schachspiel gibt es natürlich auch hier gute Gründe, nicht beliebig viel Geld anzuhäufen, und es gibt gute Gründe, großzügig zu sein, aber diese Gründe sind der Praxis unserer Geldverwendung extern; es sind zum Beispiel Klugheitsgründe oder moralische Gründe. Das „Ideal“, Geld lieber haben als nicht haben zu wollen, ist in diesem Sinne konstitutiv für die Institution des Geldes.

Dadurch, dass ich die beiden philosophischen Tugenden als konstitutive Ideale auffasse, entsteht die Möglichkeit, Philosophie besser und schlechter zu machen - nämlich dann, wenn eine Philosoph*in sich an den Tugenden orientiert, ihnen aber mehr oder weniger gerecht wird. Trotzdem bleiben die Tugenden konstitutiv für eine bestimmte philosophische Tätigkeit: Es besteht nämlich auch die Möglichkeit, - unter Umständen, nämlich, wenn es nicht aus anderen Gründen Philosophie ist (siehe Nicht-Exklusivität) - gar nicht Philosophie mehr zu betreiben; dies wäre der Fall, wenn eine Person sich gar nicht an den Tugenden orientiert (und ihr Tun nicht aus anderen Gründen Philosophie ist; siehe Nicht-Exklusivität).

\subsection{Metametaphilosophie}

Die zweite der Fragen, von denen ich angekündigt hatte, sie in diesem Kapitel zu bearbeiten, ist noch offen. Es ist die Frage, warum ich mich dafür entschieden habe, meine Thesen anhand von Mill zu erarbeiten und mich damit, was die akademische Selbstbehauptung angeht, angreifbarer zu machen, entweder, weil ich eigentlich zwei Thesen vertrete, eine systematische und eine exegetische, oder weil ich eine ungünstige und unklare Vermischung von systematischer und exegetischer Herangehensweise praktiziere. Hinter der konkreten Frage steckt natürlich eine allgemeinere Frage, nämlich die, wie überhaupt davon überzeugt werden kann, dass etwas wirklich philosophische Tugenden oder Ideale sind.

wahrscheinlich ist. Siehe Niklas Luhmann. Die Gesellschaft der Gesellschaft. 1. Aufl. Bd. 1. Frankfurt am Berlin: Suhrkamp, 1998, S. 316ff.

14 Geld muss darum knapp sein. Siehe ebd., S. 374. 
Zunächst aber zum Titel dieses Unterabschnittes: Es ist vielleicht eine Unart moderner akademischer Philosophie, die Gegenstandsbereiche, zu denen gearbeitet wird, mit Komposita aus traditionellen Gegenstandsbezeichnungen und ein oder gar mehreren Vorkommnissen der griechischen Vorsilbe „meta“ zu bezeichnen. Dem, dass dies oft problematisch ist, will ich emphatisch zustimmen. ${ }^{15}$ Trotzdem hatte ich weiter oben die Bestimmung der Philosophie, die durch die beiden Tugenden möglich wird, als „Mills metaphilosophische Position" bezeichnet. Dies erlaubt mir jedenfalls, das, was ich in diesem Abschnitt vorhabe, als Metametaphilosophie zu bezeichnen. Die Idee ist: Wenn die Erarbeitung der philosophischen Tugenden Metaphilosophie ist, dann ist ein Reflexionsabschnitt darüber, wie ich eigentlich von einer metaphilosophischen Behauptung überzeugen kann, sicherlich Metametaphilosophie. Dies wiederum erlaubt mir das Problem, dem sich dieser Abschnitt widmet, iterativ und durch die verschiedenen „Meta“-Ebenen hindurch zu bearbeiten.

Angenommen (1) nämlich, Mill hätte Recht und Philosophie orientiere sich idealerweise an den beiden Tugenden, und angenommen, (2) die Behauptung, dass das so ist, die metaphilosophische Behauptung also, sei selbst eine philosophische Behauptung. ${ }^{16}$ Es würde dann folgen, dass der Versuch, von der Behauptung zu überzeugen, dass Philosophie sich an Mills beiden Tugenden orientiert, sich selbst an eben diesen beiden Tugenden zu orientieren hat:

15 Der Grund, wie unten noch deutlicher werden wird, ist, dass hier im Interesse philosophischer Arbeitsteilung und vielleicht vermeintlicher Klarheit eine Trennung von Bereichen vorgenommen wird, die möglicherweise nicht trennbar sind. Zur Kritik Foots an Hare gehört es zum Beispiel, dass die Bestimmung dessen, was überhaupt der Gegenstandsbereich der Metaethik ist, selbst eine ethische Frage ist - sich in der Konsequenz also Metaethik nicht völlig neutral bezüglich ethischer Inhalte verhalten kann: Vgl. Philippa Foot. „Moral Arguments“. In: Mind 67.268 (1958), S. 502-513, S. $511 f$.

16 Natürlich ist nicht jede Behauptung über die Philosophie eine philosophische Behauptung: Es gibt wissenschaftssoziologische, institutionell-juristische, historische u.s.w. Aussagen über die Philosophie. Behauptungen dieser Art sind keine philosophischen Behauptungen und sie sind auch nicht das, was normalerweise als "Metaphilosophie" bezeichnet wird. Metaphilosophie in diesem Sinne ist immer auch Philosophie. Ob wir die Behauptung, dass metaphilosophische Behauptungen auch immer philosophische Behauptungen sind, als eine „metametaphilosophische“, eine „metaphilosophische“ oder eine „philosophische Behauptung“" bezeichnen, ist also letztlich einerlei. 
Es müsste erstens im Verlaufe des Versuches nachgewiesen werden, dass es sich um einen sprachlichen Ausdruck handelt, den eine Philosoph*in als adäquaten Ausdruck dessen, was ihr an der Philosophie wichtig ist, identifizieren würde. Zweitens müsste gezeigt werden, dass es auch gut ist, sich dieser Praxis im Gegensatz zu anderen alternativen Praktiken zu verpflichten.

Was wäre aber erreicht, wenn dies beides gezeigt wäre? Die Art und Weise, wie in der Philosophie die Meta-Ebenen in der angedeuteten Weise kollabieren, führt dazu, dass es sich um ein Selbstanwendungsproblem handelt und streng genommen ein solcher Versuch zirkulär ist. ${ }^{17}$ (Im Fall der obigen Rekonstruktion zeigt sich das daran, dass in der Annahme (1) von der Richtigkeit der Mill'schen Position ausgegangen wurde.) In gewisser Hinsicht sollte uns dies nach Unterabschnitt 2.2 auch nicht überraschen: „Nicht-Ableitbarkeit" war eine der Qualifikationen dessen, wie ich glaube, dass wir Mills sozialphilosophische Tugenden verstehen sollten. Ein rein deduktives Argument dafür, dass das, was ich Mills Tugenden nenne, Tugenden der Philosophie sind, wäre daher in der Tat überraschend. ${ }^{18}$

17 Eine wissenschaftssoziologische Aussage über die Wissenschaftssoziologie ist zum Beispiel im Gegensatz dazu normalerweise nicht zirkulär.

18 Ich bin mir nicht ganz sicher, wie bewusst Mill die Erarbeitung seiner philosophischen Tugenden in der Form betreibt, in der er sie betreibt. Das Zirkularitätsproblem dürfte ihm jedoch bewusst gewesen sein: Eine etwas andere Art und Weise, das Problem zu sehen, ist nämlich, die philosophischen Tugenden innerhalb der Dichotomie „Wissenschaft" und „Kunst" aus Mills System of Logic einzuordnen, vgl. John Stuart Mill. The Collected Works of John Stuart Mill, Volume VIII - A System of Logic Ratiocinative and Inductive (Books $I V$-VI and Appendices). Hrsg. von J. M. Robson. Toronto und andere: University of Toronto Press und Routledge \& Kegan Paul, 1974, Buch VI, Kapitel 12. Von philosophischen Tugenden zu sprechen nimmt Philosophie natürlich als „Kunst" in den Blick, die nicht rein deduktiv sein kann. Die Unmöglichkeit eines deduktiven Beweises für letzte Zwecke wird im ersten Kapitel von "Utilitarianism" wiederholt. Dort ist natürlich die Moral gemeint - aber Gleiches gilt natürlich auch für andere letzte Zwecke. Kapitel 4 wird gelegentlich, jedoch fälschlich (siehe Abschnitt 6.1.2), so gelesen, als ob eben ein solcher Beweis unternommen werden sollte, vgl. John Stuart Mill. „Utilitarianism“. In: The Collected Works of John Stuart Mill, Volume X - Essays on Ethics, Religion, and Society. Hrsg. von J. M. Robson. Toronto und andere: University of Toronto Press und Routledge \& Kegan Paul, 1969, S. 203-259, Kapitel 1 und 4 . 
Wie kann aber stattdessen vorgegangen werden, um zu zeigen, dass das, was ich "Mills philosophische Tugenden" nenne, tatsächlich philosophische Tugenden sind? Offensichtlich müssen wir unser Repertoire an legitimen Argumentationstypen erweitern. Angesichts traditioneller Versuche, die Grenzen des Deduktiven zu sprengen, wäre es vielleicht naheliegend zu versuchen, ein transzendentales Argument zu finden, das etabliert, warum die Tugenden Tugenden sind. Aber transzendentale Argumente sind nur dann überzeugend, wenn die Praxis, deren Bedingungen der Möglichkeit untersucht werden, unausweichlich ist - zum Beispiel weil diejenige, die sie bestreitet, sie sich selbst zunutze machen muss - oder zumindest weil sie pragmatisch unverzichtbar erscheint. Dies trifft auf die Philosophie wohl nicht zu: Denn selbst wenn die Fragen der Philosophie solche sind, denen wir auf irgendeine Weise nicht ausweichen können, ${ }^{19}$ bedeutet dies noch nicht, dass der Versuch einer systematischen Beantwortung notwendig oder überhaupt sinnvoll ist.

Ich will, was ich für Mills eigene Antwort auf diese Frage halte, vorschlagen. Mill, so meine Behauptung, argumentiert nicht deduktiv, sondern beispielhaft: nämlich, indem er auf zwei Personen, Coleridge und Bentham, hinweist, die Träger jeweils einer dieser Tugenden sind, und indem er darauf hinweist, was an der Art zu philosophieren dieser Menschen gut und schlecht ist. Dies gesteht gewissermaßen zu, dass wir dafür, dass es sich wirklich um eine Form philosophischen Gutseins handelt, die an den beiden jeweils deutlich wird, nicht deduktiv argumentieren werden können: Eher umgekehrt, dadurch, dass uns Mill mit Hilfe verschiedener Beispiele den Wert der Art zu philosophieren, die Coleridge beziehungsweise Bentham eigen ist, hervorhebt, erkennen wir, dass es sich wirklich um zwei (1) komplementäre, (2) nicht ableitbare, wenn auch (3) vielleicht nicht exklusive Formen philosophischen Gutseins handelt.

19 Es wurde zum Beispiel öfter gesagt, die Philosophie beschäftige sich mit "questions which the reflective human mind finds naturally puzzling" (Thomas Nagel. What Does It All Mean? A Very Short Introduction To Philosophy. Oxford und andere: Oxford University Press, 1987, S. 3) oder mit Fragen, die ihr „durch die Natur der Vernunft selbst aufgegeben [sind], die sie aber auch nicht beantworten kann." Immanuel Kant. Kritik der reinen Vernunft. Hamburg: Meiner, 1956, A VII. 
Dies, so könnte eingewendet werden, erkläre zwar, warum Mill seine philosophischen Tugenden in zwei Essays über Coleridge und Bentham entwickele, nicht aber, warum ich sie anhand der Essays von Mill und nicht meinerseits direkt an zwei Philosoph*innen, vielleicht sogar Coleridge und Bentham, entwickelt. Dies ist richtig. Zum einen aber ist es in diesem Fall hilfreich, um die Methode darzustellen, sie in ihrer beispielhaften Ausführung bei Mill zu betrachten. Vor allem aber dient mir Mill auch selbst als Beispiel für einen Philosophen, dessen Tun wir als an diesen Tugenden ausgerichtet sehen können. Dies wird in Teil II nachgewiesen. Wenn es plausibel ist, dass Mills Tätigkeit, indem er sie an den Tugenden orientiert, von meinen Leser*innen als adäquater Ausdruck einer Pointe auch ihrer philosophischen Tätigkeit aufgefasst werden sollte, hätte ich die halbe Arbeit geleistet: Coleridges Tugend wäre etabliert. Wenn ich es weiterhin schaffe zu zeigen, dass dies für Mill ein durch Erfahrung informierter Lernprozess war ${ }^{20}$ - und nicht etwa die bloße Verinnerlichung einer zufällig entstandenen historischen Praxis -, hätte ich damit den anderen Teil der Arbeit geleistet. Dies ist der methodische Kern der Arbeit und dies erklärt, warum die Auseinandersetzung mit Mill unverzichtbar zur systematischen Argumentation dieser Arbeit gehört.

Die Methode ist zirkulär, insofern nur kompetente Philosophietreibende (aber natürlich nicht nur professionelle Philosoph*innen) überzeugt werden könnten: Die Argumente reichen nicht, um die Gültigkeit der philosophischen Tugenden aus einem der Philosophie externen Maßstab ${ }^{21}$ abzuleiten. Es ist aber keine schlimme -

20 Wie oben angedeutet, ist Mill stark darum bemüht, nicht nahezulegen, es folge aus Benthams Tugend, dass Philosophie empirisch sein müsse. Auch die Kritiker*innen des Empirismus akzeptierten Benthams Tugend, so behauptet Mill. (Siehe Abschnitt 4.3.1.) Aber nachzuweisen, dass eine Entwicklung als durch Erfahrung informierter Lernprozess verstanden werden kann, ist in jedem Fall eine - und (zumindest im Falle der Tugenden) Mills eigene - Art und Weise, Benthams Tugend gerecht zu werden.

21 Auf das gleiche Problem gehe ich in Abschnitt 6.1.3 ausführlicher ein: Das Problem, das sich Mill stellt, besteht, so behaupte ich dort, in einer auf den ersten Blick absurden Forderung: dass es nämlich auf der einen Seite in einer Hinsicht einen externen Maßstab für die Ethik geben muss, anhand dessen wir unseren eigenen historisch kontingenten ethischen Standpunkt in Frage stellen können; dass es aber auf der anderen Seite einen solchen Maßstab nicht geben kann. 
im Sinne einer sich selbst notwendig validierenden - Form von Zirkularität: Es ist nämlich nicht so, dass eine, die an einer beliebigen Stelle in den Zirkel einsteigt, bereits das Ergebnis akzeptiert hat; vielmehr wird, ob sie es akzeptiert, davon abhängen, ob wirklich plausibel wird, dass Mills Tätigkeit unter dem Lichte, unter dem ich sie betrachten werde, tatsächlich als in den Hinsichten philosophisch wertvoll betrachtet werden kann, in denen sie als Verwirklichung der Tugenden aufgefasst werden kann - ob sie also wirklich sprachlicher Ausdruck eines der Dinge ist, die eine kompetente Leser*in als Witz der Philosophie auffassen würde; und ob sie wirklich (für Mill) Ergebnis eines empirisch informierten Lernprozesses ist: Beides ist nicht bereits durch die Beschreibung der Methode garantiert.

Wenn es stimmt, dass das, was ich Mills Tugenden nenne, wirklich sozialphilosophische Tugenden sind, dann sollte es außerdem möglich sein, mit Hilfe der Formulierungen, die ich von ihnen gebe, ein Gutsein verschiedener philosophischer Ansätze sichtbar zu machen. Gute philosophische Ansätze im Sinne der Tugenden zeichnen sich demnach dadurch aus, dass sie mit der Spannung, die zwischen beiden Tugenden herrscht, überzeugend umgehen. Ein Kriterium des Erfolges meiner Ausarbeitung dessen, was ich Mills Tugenden nenne, ist also, dass sie dazu taugt, einen Weise des Gutsein verschiedener sozialphilosophischer Ansätze zum Ausdruck zu bringen - und also dazu taugt, verschiedene sozialphilosophische Ansätze dahingehend zu vergleichen, wie sie dieses Gutsein realisieren. Dies kann ich in dieser Arbeit natürlich nur sehr unvollständig leisten. Ich streife diese Frage jedoch in Abschnitt 8.1, in dem ich Mills Position von anderen Positionen abgrenze.

\subsection{Begründung meiner Lesart von „Bentham“ und "Coleridge“}

Bevor ich im nächsten und übernächsten Kapitel auf die Texte getrennt voneinander eingehen werde und die Tugenden jeweils herausarbeiten beziehungsweise ihr implizites Vorhandensein in Mills Texten nachweisen werde, möchte ich in diesem Abschnitt rechtfertigen, dass es erstens überhaupt sinnvoll ist, je eine Tugend in 
„Bentham“22 und „Coleridge“23 zu sehen und dass zweitens diese beiden Tugenden von Mill als im obigen Sinne komplementär zueinander verstanden wurden.

Sicherlich erlaubt jede Auseinandersetzung einer Philosoph*in mit anderen Philosoph*innen Rückschlüsse auf die Position ersterer: So können wir trivialerweise annehmen, dass Mill das, was er an Bentham oder Coleridge kritisiert, auch selbst ablehnt, und dass er das, was er an den beiden lobt, auch selbst gutheißt. Und es ist ebenso wenig spektakulär darauf hinzuweisen, dass Mill, insofern er über Qualitäten (und Mängel) von Bentham und Coleridge als Philosophen schreibt, offenbart, was er für gutes und was er für schlechtes Philosophieren hält. Mills Auseinandersetzung mit Bentham und Coleridge ist aber nicht nur eine zufällige Rezension zweier Werke; er schreibt über die beiden: „Whoever could master the premises and combine the methods of both, would possess the entire English philosophy of their age."24 Natürlich müssen Zitate wie dieses mit Vorsicht genossen werden. Mills Schreibstil, wenn er über Personen schreibt, die er schätzt, ist oft enthusiastisch und oft voll des - zum Teil vergifteten - Lobes. Was aber auf jeden Fall gesagt werden kann, ist, dass Mill in dem Zitat zum Ausdruck bringt, dass er die Grundzüge von Coleridges und Benthams Art und Weise zu philosophieren, ihre jeweiligen Methoden und Prämissen, als sich ergänzende Teile dessen sieht, wie Philosophie idealerweise sein sollte.

Dafür, dass die Aufsätze als Einheit gelesen werden können, sprechen noch andere Gründe. Erstens sind die Aufsätze zeitlich verhältnismäßig kurz nacheinander veröffentlicht worden und haben eine ähnliche Struktur. Zweitens wird in beiden Essays das, was er an den Sujets der beiden Essays jeweils lobt, mit den Schwächen des Namensgebers des jeweils anderen Essays kontrastiert und das, was er an seinen Sujets jeweils tadelt, mit den Stärken des jeweils anderen. Mill meint offenbar, dass, was er an Bentham und Coleridge lobt, in ihren Trägern mit Defiziten in den Eigenschaften, wegen derer er den jeweils anderen lobt, assoziiert ist. Hierin wird die Spannung zwischen den Tugenden deutlich, die ich bereits behauptet habe. Und drittens verwendet Mill in beiden Fällen die

22 Mill, „Bentham“.

23 Mill, "Coleridge".

24 Ebd., S. 121. 
gleichen Dichotomien (konservativer versus progressiver Philosoph, intuitive Schule versus Schule der Erfahrung, Außen- versus Innenperspektive), um Bentham und Coleridge zu charakterisieren. ${ }^{25}$

Dies mag dazu ausreichen, zu sagen, die Ansätze ergänzten sich laut Mill. Meint Mill aber, dass sie komplementär sind in dem stärkeren Sinne, den ich in Abschnitt 2.2 erläutert habe? Dazu müsste ich zeigen, dass Mill meint, dass das, was gut an dem ist, wie Coleridge denkt, eben so lange keine philosophische Güte im eigentlichen Sinne ist, wie er Defizite in der Tugend hat, die Mill Bentham zuschreibt. Und für Bentham gilt das Analoge. Tatsächlich legt Mill in Coleridge nahe, dass der Versuch, konservative Philosophie zu betreiben, absurd sein mag. ${ }^{26}$ Im Falle von Bentham spricht er sogar davon, dass Bentham als Philosoph disqualifiziert sei. ${ }^{27}$ In diesem Sinne sind die Tugenden auch für Mill in dem von mir vorgeschlagenen stärkeren Sinne komplementär. ${ }^{28}$

25 Für die erste Dichotomie siehe z. B. Mill, „Bentham“, S. 78 und z. B. Mill, "Coleridge“, S. 146; für die zweite z. B. ebd., S. 125-127; und für die dritte z. B. ebd., S. 119.

26 „And even if a Conservative philosophy [wie die von Coleridge] were an absurdity, it is well calculated to drive out a hundred absurdities worse than itself." ebd., S. 163. Das Zitat legt natürlich nahe, dass Coleridges Philosophie laut Mill trotzdem einen Wert hat. (Tatsächlich geht es unter anderem um die oben bereits angesprochene Möglichkeit interner Kritik, die durch Coleridge möglich wird.) Ich muss nicht bestreiten, dass dieser Wert auch ein philosophischer Wert sein kann, solange es nicht der intrinsische philosophische Wert ist, den Coleridges Art zu philosophieren laut Mill habe könnte, würde sie sich auch an der Tugend orientieren, die Mill in Bentham sieht. Siehe Abschnitt 2.2.

27 „Bentham's contempt, then, of all other schools of thinkers; his determination to create a philosophy wholly out of the materials furnished by his own mind, and by minds like his own; was his first disqualification as a philosopher. His second, was the incompleteness of his own mind as a representative of universal human nature." Mill, „Bentham“, S. 91.

28 Natürlich bezeichnet Mill Coleridge und Bentham durchaus als philosopher[s]. Ich möchte vorschlagen, dass dies einfach daran liegt, dass es durchaus möglich ist, etwas zu tun, das unter einer Tugend steht, ohne diese Tugend vollständig zu besitzen. Insofern wir sagen können, dass eine Tätigkeit (wie das Philosophieren) notwendig unter einer Tugend steht, sagen wir eher, dass es notwendig ist, dass die Tugend einen Maßstab der Güte und damit auch der Defektivität für diese Tätigkeit darstellt, als dass wir sagen würden, dass die Tätigkeit notwendig diesem Maßstab entspricht. In diesem Sinne ist eine Tätigkeit deren Urheber*in nur eine von den beiden komplementären Tugenden besitzt, deswegen nicht notwendig keine Philosophie mehr: Es ist eben 
Für den größeren Kontext meiner Arbeit ist es auch wichtig, dass Mill sich als unter beiden Tugenden stehend betrachtet (siehe Abschnitt 2.3). Auf den ersten Blick ist natürlich die Nähe zu Bentham offensichtlicher. Denn natürlich macht Mill keinen Hehl daraus, dass er in der Tradition Benthams, der Tradition, die Mill als die „progressive“ ${ }^{“ 29}$ bezeichnet, steht, und auch politisch stehen Mill und Coleridge auf unterschiedlichen Seiten des Diskurses: Coleridge unterstützt in seinen Aufsätzen offen die Tory-Regierungen; Mill rechnet sich selbst den Philosophical Radicals zu und sitzt später bekanntermaßen für die Liberal Party im Parlament.

Vor diesem Hintergrund ist es die Art und Weise der Kritik interessant, die in den jeweiligen Aufsätzen zum Ausdruck kommt: In "Coleridge" beschränkt sich Mill darauf, kurz anzudeuten, warum er trotz seiner Wertschätzung desselben inhaltlich anderer Meinung ist als Coleridge. Rhetorisch stärkere Kritiken an den Institutionen, die Coleridge verteidigt, finden wir in anderen Texten, die ungefähr zur gleichen Zeit entstanden sind. Mit Bentham hingegen geht er sehr viel härter - fast bis zur Grenze der persönlichen Beleidigung - ins Gericht. ${ }^{30}$ Die von mir favorisierte Deutung dieses Phänomens ist, dass Mill, dessen liberale Referenzen innerhalb seiner Leser*innenschaft ${ }^{31}$ unumstritten waren, sich eher bemüht hat, diesen den Wert Tugend, die er in Coleridge verwirklicht sah, nahezubringen. ${ }^{32}$ Diese Deutung muss gegenüber einer einer alternativen Deutung verteidigt werden, nach der es zum Zeitpunkt der Veröffentlichung der Aufsätze Mills Strategie war, weitere Personen für sein progressives Projekt zu gewinnen, indem er die Offenheit guter progressi-

schlechte Philosophie in dem Maße, wie ihr die jeweils andere Tugend fehlt, obwohl die Gedanken in irgendeiner anderen Hinsicht (selbst in einer anderen philosophischen Hinsicht) gut sein können. Lediglich eine Tätigkeit, die gar nicht unter beiden Tugenden steht, wäre keine Philosophie mehr - und wir könnten ihrer Urheber*in daher auch keinen Vorwurf dafür machen, nicht beide Tugenden zu realisieren.

29 Vgl. z. B. Mill, „Coleridge“, S. 128, S. 160.

30 Er sei als Philosoph disqualifiziert, (Siehe Mill, „Bentham“, S. 91) unfähig von anderen zu lernen, (Siehe ebd., S. 90) und bis zum Ende seines Lebens eigentlich ein Junge geblieben, (Siehe ebd., S. 92) um nur einige Beispiele zu nennen.

31 "Coleridge" und „Bentham" wurden zunächst im London and Westminster Review, einer von Bentham gegründeten Zeitschrift, veröffentlicht.

32 Vergleiche dazu Mills Einschätzung in der Autobiographie Mill, Autobiography, S. 227. 
ver Philosophie auch für bestimmte konservative Anliegen deutlich machte. Dies werde ich im Abschnitt 3.4.2 ansprechen. Keine der Deutungen aber bestreitet, dass Mill sich - und zwar nicht nur verbal - der benthamitischen, „progressiven“ Strömung zurechnet.

Diese inhaltliche und methodische ${ }^{33}$ Abgrenzung von Coleridge sollte uns aber, so meine ich, nicht davon abbringen, daran festzuhalten, dass das Desideratum guter Philosophie für Mill eine Synthese beider Ansätze ist:

A true thinker can only be justly estimated when his thoughts have worked their way into minds formed in a different school; have been wrought and moulded into consistency with all other true and relevant thoughts; when the noisy conflict of half-truths, angrily denying one another, has subsided, and ideas which seemed mutually incompatible, have been found only to require mutual limitations. This time has not yet come for Coleridge. ${ }^{34}$

Wir dürfen wohl annehmen, dass Mill unter die „minds formed in a different school" auch sich selbst (und seine liberale Leser*innen) zählt; und die Tatsache, dass er schreibt, die Zeit für Coleridge sei noch nicht gekommen, kann fast wie einen Vorsatz gelesen werden, zumindest jedoch als Ausdruck einer Überzeugung, dass es gut wäre, würde diese Zeit kommen. In diesem Sinne ist es wohl keine Dehnung der Tatsachen zu behaupten, auch Mill würde sich selbst unter den Tugenden stehend betrachten, die er Bentham und Coleridge zuschreibt.

Worin bestehen nun die beiden Ansätze, die jeder für sich defizitär sind, aber zusammen für Mill das Wesen guter Philosophie ausmachen? Ein Zugang kann über die Dichotomien gefunden werden, mithilfe derer Mill die Ansätze abgrenzt. Die erste Dichotomie, die ich oben benannt hatte, war die Dichotomie zwischen intuitiver Schule und Schule der Erfahrung. Sie bezieht sich vor allem auf die methodische Einordnung und es verzerrt, wie ich weiter

33 Mill spricht insbesondere die Transzendentalphilosophie der der „GermanoColeridgians" an, hinter deren technischer Sprache er einen tief sitzenden Mystizismus vermutet und in deren a priorischer Methode, er die Gefahr eines ungehemmten Philosophierens gemäß überlieferter Vorurteile sieht. Vgl. Mill, "Coleridge", S. 129. Siehe unten.

34 ebd., S. 122. Vgl. auch Mill, „Bentham“, S. 94. 
unten (Abschnitt 3.2) argumentieren werde, das, worum es Mill geht, wenn wir diese Dichotomie zur Grundlage der Interpretation von "Coleridge" und "Bentham" machen. Eine Interpretation, die diese Dichotomie zur Grundlage macht, werde ich im Folgenden als eine „methodische Interpretation“ bezeichnen. Die zweite Dichotomie ist die zwischen Bentham und Coleridge als „progressivem" beziehungsweise „konservativem" Philosophen. Diese Dichotomie und das, was daran auch vor dem Hintergrund von Mills späteren Texten problematisch ist, diskutiere ich in Abschnitt 3.4. Eine Interpretation, die diese Dichotomie zur Grundlage macht, werde ich im Folgenden als ,gesellschaftspolitische Interpretation“ bezeichnen. Auch diese halte ich für ungenügend. Ich möchte daher, auch wenn es nur aus Verlegenheit ist, meine Leser*innen bitten, sich zunächst einfach auf die metaphorischen Dichotomie zwischen Innen- und Außenperspektive als Interpretationsvorschlag einzulassen, auch wenn dies im Moment nicht viel mehr als eine Abgrenzung zu den anderen Dichotomien ist:

[Coleridge] has been, almost as truly as Bentham, "the great questioner of things established;" for a questioner needs not necessarily be an enemy. By Bentham, beyond all others, men have been led to ask themselves, in regard to any ancient or received opinion, Is it true? and by Coleridge, What is the meaning of it? The one took his stand outside the received opinion, and surveyed it as an entire stranger to it: the other looked at it from within, and endeavoured to see it with the eyes of a believer in it; to discover by what apparent facts it was at first suggested, and by what appearances it has ever since been rendered continually credible-has seemed, to a succession of persons, to be a faithful interpretation of their experience. ${ }^{35}$

Was mit Innen- und Außenperspektive gemeint ist und inwiefern diese Dichotomie sich auf die beiden Tugenden bezieht, bedarf jeweils einer genaueren Erklärung. Entsprechend wird, was diese beiden Perspektiven im einzelnen auszeichnet, Gegenstand der folgenden beiden Kapitel sein.

Zunächst sei jedoch noch auf eine terminologische Schwierigkeit hingewiesen: Mill spricht hier von „received opinion“; für meine

35 Mill, „Coleridge“, S. 119. 
Arbeit ist es aber wichtig, dass es sich auch um geteilte Institutionen oder Praktiken handelt. Ich meine, ich bin gerechtfertigt in der Annahme, dass es in "Coleridge" und „Bentham" auch, nein, sogar vorrangig um geteilte Praktiken und Institutionen und nicht um Meinungen geht: Die beiden Hauptbeispiele, die Mill in dem Aufsatz, aus dem das Zitat stammt, zur Illustration des Ansatzes von Coleridge bespricht, sind die Institution der englischen Staatskirche und die Institution der ungeschriebenen englischen Verfassung. Auch terminologisch benutzt er später im Aufsatz, wie aus den Zitaten im nächsten Unterabschnitt deutlich wird, häufig das Wort „Institution“" ${ }^{36}$ Warum differenziert Mill aber terminologisch nicht sauberer? Ein Grund ist, dass Mill sozialontologisch einen psychologischen Individualismus ${ }^{37}$ vertritt: Letztlich seien alle sozialen Tatsachen auf psychologische Zustände zurückzuführen. ${ }^{38}$ Dies ist zwar in vielen Hinsichten problematisch; ich werde diese Probleme aber bis Abschnitt 6.2, wo ich Mills Psychologismus explizit anspreche, weitgehend ignorieren. Für den ersten und zweiten Teil dieser Arbeit reicht es, festzuhalten, dass "received opinion" als psychologistische Chiffre bei Mill durchaus auch für tradierte Institutionen und Praktiken steht.

Zusammenfassung. Ich habe in diesem Kapitel eine vorläufige Formulierung von Mills philosophischen Tugenden gegeben: Erstens philosophieren wir dann gut, wenn wir (auch) objektive Ansprüche in dem Sinne begründen, dass die unserem philosophischen Gegenstand zugrundeliegende Praxis in entscheidenden Hinsichten besser ist, als die Dinge ganz anders zu machen - zum Beispiel, indem deutlich gemacht wird, inwiefern die Praxis Ergebnis eines empirisch informierten Lernprozesses ist. Zweitens philosophieren

36 Vgl. z. B. ebd., S. 142, S. 147.

37 Vgl. Lars Udehn. „The Changing Face of Methodological Individualism“. In: Annual Review of Sociology 28 (2002), S. 479-507, S. 482.

38 ,[T]he general science of society [...] can proceed in no other manner than by making [...] generalizations-afterwards to be confirmed by connecting them with the psychological and ethological laws on which they must really depend." Mill, The Collected Works of John Stuart Mill, Volume VIII - A System of Logic Ratiocinative and Inductive (Books IV-VI and Appendices), S. 908. „Ethologie“ ist dabei die Wissenschaft vom Charakter, um die es Mill auch andernorts in Buch VI geht. Der psychologische Individualismus Mills wird in Kapitel 6 behandelt. 
wir nach Mill gut, wenn wir uns an dem Ideal eines sprachlichen Ausdrucks orientieren, den eine kompetente Teilnehmer*in an einer Praxis als adäquaten Ausdruck dessen, was ihr an dieser Praxis wichtig ist, identifizieren könnte. Außerdem habe ich Behauptungen darüber gemacht, wie wir die Tugenden nach Mill, wie ich meine, auffassen sollen: D.h. ich habe die beiden Tugenden als „komplementär", „nicht ableitbar" aus allgemeineren Vorstellungen philosophischer Güte, „nicht exklusiv“ und als „konstitutive Ideale“ bezeichnet und jeweils erklärt, was ich mit diesen Bezeichnungen meine. Ich habe außerdem nahegelegt, über andere Philosoph*innen zu schreiben könne als Methode in der Philosophie der Philosophie gelten, und versucht, das zu begründen. Damit habe ich einerseits erklärt, warum ich mich im ersten Teil dieser Arbeit mit Mills Aufsätzen „Coleridge“ und „Bentham“ beschäftige; andererseits will ich damit auch einen systematischen Anspruch betonen, den die Auseinandersetzung mit Mill selbst in Teil II hat. Ich habe außerdem, soweit dies vor der eigentlichen Auseinandersetzung mit den Texten möglich ist, dafür argumentiert, dass es legitim ist, die Aufsätze als Mills Formulierung zweier, komplementärer philosophischer Tugenden auffasst, die er auch für sein eigene philosophische Tätigkeit akzeptiert. Schließlich habe ich, indem ich auf Mills psychologischen Individualismus hingewiesen habe, dafür argumentiert, dass wir Mill sozialphilosophisch lesen sollten, d.h., dass es oft, wenn Mill scheinbar psychologische Begriffe wie "opinion”, „conviction“ etc. verwendet, in Wirklichkeit (auch) um Institutionen, Praktiken u.s.w. geht. Ich habe nicht nicht nachgewiesen, dass meine vorläufigen Formulierungen inhaltlich auch das darstellen, was Mill vertritt. Dies ist Aufgabe der nächsten beiden Kapitel. Ich habe auch nicht dafür argumentiert, dass der Maßstab guter Philosophie, um den es hier geht, in Form von Tugenden und nicht etwa in Form von Anforderungen an philosophische Texte formuliert werden sollte. Dies wird erst in Teil II geschehen. 


\section{3. "Coleridge“}

In diesem Kapitel wird es darum gehen, die erste der beiden sozialphilosophischen Tugenden zu erarbeiten. Zunächst werde ich etwas zum Unterschied zwischen Coleridge als Person und dem Bild, das Mill von ihm zeichnet, sagen und erklären, warum für diese Arbeit das Bild, das Mill von Coleridge zeichnet, entscheidender ist. Der Hauptteil des Kapitels wird darin bestehen, die philosophische Tugend, die Mill in Coleridge sieht - die Tugend, von der ich sagen will, dass sie in Kombination mit ihrer Komplementärtugend aus Kapitel 4 wesentlich für Sozialphilosophie ist - zunächst exegetisch zu erarbeiten. Mill interpretiert Coleridge mit Bezug auf Erfahrung oder Erleben: Den Erfahrungen einer Teilnehmer*in an einer sozialen Praxis, das heißt, dem, was sie an dieser Praxis als wichtig erlebt, bemüht sich eine Philosoph*in, die sich wie Coleridge an dieser Tugend orientiert, adäquaten Ausdruck zu verleihen. Um deutlich zu machen, was das heißt, beziehe ich mich näher auf zwei Beispiele aus dem Aufsatz selbst: Das erste Beispiel ist „Moral und Wissenschaft", das im Wesentlichen sehr ähnlich gelagert ist, wie heutige Kritik am Reduktionismus auf diesen Gebieten. Das zweite Beispiel ist Coleridges Verteidigung der christlichen Mysterien gegen die Unitarier*innen. An diesem Beispiel wird besonders deutlich, dass es dem Atheisten Mill nicht darum ging, für Coleridges Schlussfolgerungen zu werben, sondern um die Art und Weise, wie Coleridge philosophiert. Im letzten Abschnitt des Kapitels werde ich meine Interpretation gegen andere Interpretationen verteidigen. Den drei Dichotomien, die ich bereits in Abschnitt 2.4 erwähnt habe, entsprechen drei Interpretationen, je nachdem, welche der Dichotomien wir für grundlegend halten, und falls die Dichotomien nicht gleichbedeutend sind: die gesellschaftspolitische Interpretation, die methodische Interpretation und die von mir vertretene sozialphilosophische Interpretation. Ich werde im letzten Abschnitt dafür argumentieren, dass die drei Dichotomien nicht gleichbedeutend sind und dass wir unter Voraussetzung des in den anderen 
Abschnitten dieses Kapitels Gezeigten die sozialphilosophische Interpretation favorisieren sollten.

\subsection{Coleridge versus „Coleridge“}

Es scheint mir wichtig, Mills Bild von Coleridge von Coleridge selbst zu unterscheiden, und vor allem Mills Bild von der „GermanoColeridgian school ${ }^{\text {11 }}$ von den Personen, die Mill damit meint: Coleridge wird häufig für seine entscheidende Rolle in der Verbreitung einiger Ideen des deutschen Idealismus im englischsprachigen Raum erwähnt. ${ }^{2}$ Mill hebt insbesondere kantische Gedanken in seiner Darstellung von Coleridge hervor und zwar sowohl, was praktische als auch was theoretische Fragestellungen angeht. ${ }^{3}$ Sicherlich finden sich aber auch rechtshegelianische Gedanken bei Coleridge - insbesondere bezüglich der These, dass Sozialphilosophie Geschichtsphilosophie sein müsse, die Mill ebenfalls hervorhebt. ${ }^{4}$ Für Mills Bild von Coleridge sind unzweifelhaft neben seiner Lektüre auch die Gespräche mit Frederick Maurice und John Sterling von Bedeutung, deren Bedeutung für ihn er in seiner Autobiographie betont und dort als Schüler von Coleridge bezeichnet. ${ }^{5}$ Das Werk Coleridges, mit dem sich Mill hauptsächlich beschäftigt ist On the Constitution of Church and State . $^{6}$

Wie deutlich werden wird, hat Mill weder für die Methoden des deutschen Idealismus noch für die technische Sprache, in der diese seiner Meinung nach Ausdruck finden ${ }^{7}$, viel übrig. Es ist etwas anderes, das Mill an Coleridge schätzt, und entsprechend geht es im Folgenden auch nicht darum, Coleridge systematisch zu untersu-

1 Für den Ausdruck vgl. Mill, „Coleridge“, S. 138.

2 "Samuel Taylor Coleridge is the thinker chiefly responsible for introducing German Idealism to English-speaking readers [...]." Andy Hamilton. „Conservatism“. In: The Stanford Encyclopedia of Philosophy. Hrsg. von Edward N. Zalta. Fall 2015. 2015. Auch Mill hebt diesen Einfluss hervor, wenn er von der "Germano-Coleridgian doctrine" spricht. Mill, „Coleridge", S. 126.

3 Ebd., S. $159 f$ bzw. S. $125 f$.

4 Vgl. ebd., S. $138 f$.

5 Mill, Autobiography, S. 159ff.

6 Samuel Taylor Coleridge. On the Constitution of Church and State. London: William Pickering, 1839. URL: https : / / archive . org / details / cu31924105501906 (besucht am 05. 05. 2019).

7 Mill, „Coleridge“, S. 126. 
chen oder seine genaue ideengeschichtliche Verbindung zum deutschen Idealismus herauszuarbeiten, sondern darum, die philosophische Tugend, die Mill durch ihm repräsentiert sieht, zu formulieren. Natürlich ist die Verwandtschaft Coleridges zum deutschen Idealismus oft auch in Mills Darstellung unverkennbar; aber er stellt einige Gedanken Coleridges - insbesondere diejenigen, von denen er sich nicht distanzieren will - auch in einem eigenen, sehr die menschliche Erfahrung betonenden Lichte dar.

Für die Zwecke dieser Arbeit ist Mills Bild von Coleridge natürlich entscheidender als Coleridge selbst. Was Mill für die Stärken von Coleridge hält, ist entscheidender, wenn es darum geht, Mills Gedanken zur Philosophie der Philosophie zu erarbeiten, als das, was Coleridge tatsächlich gedacht hat. Die methodische Frage aber, ob das Schreiben über andere Philosoph*innen als Methode der Philosophie der Philosophie voraussetzt, dass diese fair dargestellt werden, ist dennoch interessant. Beim Schreiben über andere Philosoph*innen als Methode der Philosophie der Philosophie geht es darum, ostentativ auf ein Stück philosophischer Praxis als Beispiel für gute (oder auch schlechte) Philosophie zu verweisen. Dies darf dementsprechend nicht gänzlich fiktiv sein - auch wenn es sicherlich Freiheiten darin gibt, welche Aspekte etwa betont werden. Dafür, dass "Coleridge“ als Darstellung einer philosophischen Tugend überzeugend ist, ist es also notwendig, dass Mill etwas Wesentliches an einer tatsächlich ausgeübten Art zu philosophieren richtig erfasst. Es ist aber zum Beispiel weniger entscheidend, ob der Besitzer der Tugend tatsächlich Coleridge war oder Maurice oder Sterling. Betrachtet man Coleridges Texte, wird Mill Coleridge aber im Wesentlichen gerecht - und ich habe mich bemüht, an den Stellen, an denen Mills Darstellung dem Original nicht ganz treu ist, darauf hinzuweisen.

Allerdings wird das Hauptaugenmerk dieser Arbeit auf Mill liegen. Mills Leben als Philosoph und seine Philosophie sind das Stück philosophischer Praxis, auf das nun ich meinerseits (in Teil II) ostentativ verweisen will. Deshalb ist für die Überzeugungskraft dieser Arbeit als ganzer weniger entscheidend, ob Mill Coleridge in seiner Darstellung gerecht wird, sondern eher, ob die Perspektive, die ich auf Mills philosophisches Tun richte, eine ist, unter der etwas, das tatsächlich wesentlich für seine Philosophie war, in seiner allgemeinen philosophischen Bedeutung deutlich wird. 


\subsection{Der erfahrbare, intrinsische Wert von Institutionen und Praktiken nach Mills Coleridge}

Oben (Abschnitt 3.1) habe ich behauptet, Mill stelle Coleridge sehr die menschliche Erfahrung betonenden Lichte dar. Dies wird schon in obigem Zitat, zu dem Fußnote 35 des letzten Kapitels gehört, deutlich, in dem behauptet wird, Coleridge sei bemüht zu zeigen, inwiefern tradierte Meinungen (aber gemeint sind auch die damit verbundenen gesellschaftliche Institutionen und so weiter) eine ,getreue Interpretation“ menschlicher Erfahrungen seien. Er sagt ebenso über Coleridge und Bentham: „They employed, indeed, for the most part, different materials; but as the materials of both were real observations, the genuine product of experience - the results will in the end be found not hostile, but supplementary, to one another." ${ }^{* 8}$ Diese Betonung der Erfahrung scheint auf den ersten Blick in Spannung damit zu stehen, dass er Coleridge der intuitiven Schule zuordnet und davon die Schule der Erfahrung abgrenzt. ${ }^{9}$

Diese Spannung kann leicht aufgelöst werden, wenn wir den Status der Äußerungen betrachten: Dass Coleridge in der Lage ist, komplexen menschlichen Erfahrungen Ausdruck zu verleihen, wird von Mill lobend erwähnt; dass er der intuitiven Schule angehört, ist lediglich eine Charakterisierung seines geistesgeschichtlichen Hintergrunds. Das, was Mill an Coleridge schätzt, ist folglich nicht, dass er zur intuitiven Schule gehört. Dies hat Auswirkungen dar-

8 Mill, „Coleridge“, S. 121.

9 Die Textstelle in "Coleridge“, in der er das tut, beginnt mit einer Beschreibung der Schule der Erfahrung, von der Coleridge dann abgegrenzt wird: „There is no knowledge à priori; no truths cognizable by the mind's inward light, and grounded on intuitive evidence. [...] From this doctrine, Coleridge, with the German philosophers since Kant (not to go farther back) and most of the English since Reid, strongly dissents. [...] He claims for the human mind a capacity, within certain limits, of perceiving the nature and properties of 'Things in themselves.' [...] These perceptions are not indeed innate, nor could ever have been awakened in us without experience; but they are not copies of it: experience is not their prototype, it is only the occasion by which they are irresistibly suggested." Ebd., S. 125. Die Bezeichnungen „intuitive school" und "school of experience" sind Bezeichnungen, die Mill andernorts verwendet. Vgl. z. B. Mill, „Utilitarianism“, Kapitel 1. Gemeint sind aber im Wesentlichen die gleichen Strömungen; in beiden Fällen wird zum Beispiel Kant paradigmatisch genannt. 
auf, wie wir das philosophische Ideal, dass durch die Kombination der Weisen zu philosophieren von Bentham und Coleridge entsteht, verstehen sollten. Das philosophische Ideal, von dem Mill spricht, besteht dementsprechend nicht oder zumindest nicht vorrangig darin, die intuitive Schule und die Schule der Erfahrung zu vereinen.

Dass es bei dem, was Mill an Coleridge schätzt, darum geht, wie dieser komplexen menschlichen Erfahrungen Ausdruck zu verleihen vermag, wird auch dadurch plausibel gemacht, dass Mill viel später in der Autobiography im Kapitel über seine Krise zum Beispiel an zwei Stellen Gedichtzeilen von Coleridge zitiert: diese beschrieben genau seinen Fall ${ }^{10}$, beziehungsweise seien eine treffende Beschreibung seiner Gefühle. ${ }^{11}$ Es mag an einigen Stellen problematisch sein, die Autobiographie zur Deutung von „Bentham“ und „Coleridge" zu verwenden - ich werde weiter unten auf Bemerkungen in der Autobiographie zu den von mir behandelten Aufsätzen eingehen -, in diesem Fall ist Mills Meinung über Coleridge in beiden Texten jedoch konsistent.

Hier geht es aber nicht darum, wie gut Coleridge Mill zufolge dazu in der Lage ist, krisenhaften Erfahrungen Ausdruck zu verleihen. Es geht stattdessen darum, was Coleridges Stärke für seine Art und Weise, sozialphilosophische Fragen anzugehen, bedeutet. Was ich meine, wird in folgendem Zitat deutlich, das auch gleichzeitig Ausgangspunkt meiner Beschäftigung damit dient, was Mill damit meint, dass Coleridge im Gegensatz zu Bentham eine Innenperspektive vertrete:

[Coleridge] considered the long or extensive prevalence of any opinion as a presumption that it was not altogether a fallacy; that, to its first authors at least, it was the result of a struggle to express in words something which had a reality to them, though perhaps not to many of those who have since received the doctrine by mere tradition. The long duration of a belief, he thought, is at least proof of an adaptation in it to some portion or other of the human mind; and if, on digging down to the root, we do not find, as is generally the case, some truth, we shall find some natural want or requirement

10 Siehe Mill, Autobiography, S. 139.

11 Siehe ebd., S. 143. 
of human nature which the doctrine in question is fitted to satisfy $[\ldots] .^{12}$

In überlieferten Meinungen und - wie gesagt - Praktiken und Institutionen findet mitunter etwas (something) Ausdruck, das Realität für ihre ersten Autor*innen hatte. (Dabei sollte sicherlich die Phrase der Autor*innenschaft nicht im Sinne einer absichtlichen Gestaltung der sozialen Wirklichkeit verstanden werden.) Dieses Etwas, dessen linguistischer Ausdruck offenbar mit einer gewissen Mühe (struggle) verbunden ist, meine ich auch praktisch deuten zu dürfen: Nicht irgendeine Wahrheit wird ausgedrückt, sondern etwas von ethischem oder vielleicht ästhetischem Wert. Dies wird ebenfalls deutlich, wenn wir die Beispiele berücksichtigen, um die es bei Coleridge tatsächlich geht. Interessant ist, dass von zwei Möglichkeiten gesprochen wird, wie dieser Wert mit der opinion zusammenhängt: Erstens mag sie direkt Ausdruck dieses Wertes sein oder sie mag nützlich zur Erfüllung menschlicher Bedürfnisse sein.

Für uns ist natürlich besonders die erste dieser Möglichkeiten interessant. Die zweite dieser Möglichkeiten ließe sich, wenn wir Mill hier wörtlich nehmen, auch gänzlich in empiristischen Begriffen im traditionelle Sinne fassen - wenn auch mitunter nur, wenn wir bereit sind, den Preis einer self-effacing theory ${ }^{13}$ zu zahlen: Wenn wir beim Freilegen der Wurzel keine Wahrheit, sondern nur ein menschliches Bedürfnis finden, sieht es so aus, als könne eine philosophische Theorie uns nahelegen, etwas zu glauben, dass wir aber nur glauben können, solange wir die Theorie nicht gleichzeitig auch für wahr halten. ${ }^{14}$ Ausführlicher diskutiere ich die Frage, ob Mill in Teilen eine self-effacing theory vertreten haben könnte, in Abschnitt 5.1.3. Eine self-effacing theory zu vertreten mag zwar philosophisch unattraktiv sein - sie mag sogar gegen das verstoßen, was

12 Mill, „Coleridge“, S. 120.

13 Der Begriff stammt aus Derek Parfit. Reasons and Persons. New York: Oxford University Press, USA, 1986, S. 23.

14 Dass das ungünstig ist, schreibt Mill zum Beispiel in der Autobiographie, wenn er von „burthen, so heavy to one who aims at being a reformer in opinions, of thinking one doctrine true, and the contrary doctrine morally beneficial" spricht, von der ihn seine nun entwickelte kompatibilistische Position zur Willensfreiheit befreie. Mill, Autobiography, S. 177, vgl. auch Mill, The Collected Works of John Stuart Mill, Volume VIII - A System of Logic Ratiocinative and Inductive (Books IV-VI and Appendices), Buch VI, Kapitel 2, auf das sich die zitierte Stelle aus der Autobiographie bezieht. 
es heißt, eine gute philosophische Theorie aufzustellen - aber eine solche Theorie ist nicht besonders kompliziert zu verstehen. Naturwissenschaftliche Thesen, etwa aus Psychologie oder Soziologie, dürfen selbstverständlich self-effacing sein - und werden dadurch nicht unverständlich.

Vielleicht ist diese wörtliche Lesart aber eine ungeschickte Lesart von dem, was Mill mit "natural want" oder "requirement of human nature" im Gegensatz zu „truth" eigentlich sagen will: Vielleicht geht es ihm nur darum, dass das mit „something which had a reality to them" Bezeichnete auch ein praktischer (im Gegensatz zu einem theoretischen Wert, d.h. Wahrheit) sein kann. In jedem Fall ist das eigentlich Interessante an Mills Coleridge nicht, dass er einen instrumentellen Nutzen von überlieferten Überzeugungen oder Institutionen hervorhebt. ${ }^{15}$

Wenn wir uns mit sozialen Institutionen philosophisch beschäftigen, geht es nach Mills Coleridge nun darum, diesem „Etwas“ nachzuspüren: „His mode of treating any institution is to investigate what he terms the Idea of it, or what in common parlance would be called the principle involved in it." ${ }^{16}$ Auch an dieser und anderen Stellen zeigt sich wieder die im letzten Absatz diskutierte Deutungsfrage: An anderer Stelle spricht Mill auch von den Zwecken der existierenden Institutionen ${ }^{17}$, die (nach dem Zitat, zu dem Fußnote 12 gehört) wiederum extrinsisch oder intrinsisch sein könnten.

\subsection{Die Beispiele}

Als Hauptbeispiele dienen Mill Coleridges Behandlung der ungeschriebenen Verfassung Großbritanniens ${ }^{18}$, sowie die anglikanische Kirche $^{19}$. Bessere Beispiele für intrinsische Ideen, bei denen es klarer

15 Dies tun die konservativen „Utilitaristen“ des 18. Jahrhunderts, die Mill in "Whewell on Moral Philosophy" erwähnt und die dort von eben genau der Richtung der „deutschen Metaphysiker" abgegrenzt werden, der er in "Coleridge" auch Coleridge zuordnet. Siehe Mill, "Whewell on Moral Philosophy“, S. 170 .

16 Mill, „Coleridge", S. 147.

17 ,the ends of existing institutions, vgl. ebd., S. 142 ".

18 Vgl. ebd., S. 151-159.

19 Vgl. ebd., S. 147-151. 
wird, dass sie an die entsprechende Institution geknüpft sind, finden sich aber unter den Beispielen, die Mill eher beiläufig anführt: beispielsweise das Christentum sowie Moral und Wissenschaft. Aus diesen Gründen und weil für heutige Leser*innen Diskussionen um die Verfassung Großbritanniens und die anglikanische Kirche, wie sie im 19. Jahrhundert geführt wurden, fremd sein dürften, behandele ich die Beispiele von Moral und Wissenschaft und das Christentum. ${ }^{20}$

\subsubsection{Moral und Wissenschaft}

Den Germano-Coleridgians wird von Mill eine Kritik an einem metaethischen und einem wissenschaftstheoretischen Reduktionismus der empiristischen Philosophie zugesprochen, namentlich Hume an der im Folgenden besprochenen Textstelle kurz vor dem hier zitierten Ausschnitt:

It is further said that the same doctrine [die empiristische nämlich] annihilates moral obligation; reducing morality either to the blind impulses of animal sensibility, or to a calculation of prudential consequences, both equally fatal to its essence. Even science, it is affirmed, loses the character of science in this view of it, and becomes empiricism; a mere enumeration and arrangement of facts, not explaining nor accounting for them $\left[\ldots . .{ }^{21}\right.$

Der Reduktionismus laufe, so die Germano-Coleridgians, deren Kritik in der Textstelle wiedergegeben wird, Gefahr, das Wesen, beziehungsweise den Charakter von Moral und Wissenschaft aus den Augen zu verlieren.

Mill geht es an dieser Stelle des Textes vornehmlich darum, die beiden Schulen - die er in Bezug auf die Moralphilosophie später die „induktive“ und die ,intuitive Schule“ nennt ${ }^{22}$ - zu unterscheiden. Er verhält sich nicht explizit zu der hier dargestellten Kritik; es geht ihm zunächst nur darum, eine Debatte darzustellen - nicht darum

20 Für Mill sind zumindest in dieser Phase nicht alle praktischen Werte moralische Werte. Vgl. Mill, „Bentham“, S. 112, siehe auch Mill, The Collected Works of John Stuart Mill, Volume VIII - A System of Logic Ratiocinative and Inductive (Books IV-VI and Appendices), S. 949.

21 Vgl. Mill, „Coleridge“, S. 127.

22 Siehe z. B. Mill, „Utilitarianism“, § 1.3 
einzugreifen. Textintern ist es daher schwierig zu entscheiden, ob Mill sich der hier dargestellten Kritik anschließen will: Immerhin ordnet er sich selbst der induktiven Schule zu. Auf der anderen Seite ist die Passage so aufgebaut, dass er die Wichtigkeit von Kritikpunkten der Coleridgianer in der dargestellten Debatte deutlich machen will, indem unter anderem Defizite der induktiven Schule aufgezählt werden, auch wenn sich Mill zu letzterer zählt. ${ }^{23}$

Ein Blick in andere Texte legt aber nahe, dass Mill die Kritik durchaus teilt. Was die theoretische Seite angeht, so verwendet Mill zum Beispiel das Wort empiricism, mithilfe dessen er auch die Kritik im Zitat formuliert, immer, wenn er es verwendet, in kritischer Absicht $^{24}$ und oft auch um damit genau die Kritik zu äußern, um die es in dem Zitat geht. ${ }^{25}$ (Dies ist auch interessant, weil die Kri-

23 Vgl. Mill, „Coleridge“, S. 129f. Es mag bei dem Zitat zu dem Fußnote 21 gehört, textintern nicht klar sein, ob Mill die Kritik teilt; völlig klar ist es aber bei dem folgenden Beispiel im nächsten Unterabschnitt.

24 Vgl. McRae. „Introduction“. In: The Collected Works of John Stuart Mill, Volume VII - A System of Logic Ratiocinative and Inductive (Books I-III and Appendices). Hrsg. von J. M. Robson. Toronto und andere: University of Toronto Press und Routledge \& Kegan Paul, 1974, S. xxi-xlviii, xxif

25 Noel Annan ist sogar der Meinung, Mill verwende das Wort durchgehend konsistent - das heißt, immer für die gleiche Form der Kritik. Für diese Behauptung und den Nachweis anhand mehrerer Textstellen vgl. R. P. Anschutz. „The Logic of J. S. Mill“. In: Mill - A Collection of Critical Essays. Hrsg. von Jerome Schneewind. London u.a.: Palgrave Macmillan, 1968, S. 46-83, S. 60.

Besonders deutlich wird dies anhand der folgenden, auch von Anschutz zitierten Fußnote aus An Examination of William Hamilton's Philosophy. Diese Fußnote ist auch deshalb interessant für die Diskussion in diesem Abschnitt, weil sie rhetorische Ähnlichkeit mit dem hier diskutierten Text hat: Nachdem er die kontinentale Philosophie charakterisiert und kritisiert hat, charakterisiert und kritisiert er die englische: „It is but just to add, that the English mode of thought has suffered in a different, but almost equally injurious manner, by its exclusive following of what it imagined to be the teaching of Bacon, being in reality a slovenly misconception of him, leaving on one side the whole spirit and scope of his speculations. The philosopher who laboured to construct a canon of scientific Induction, by which the observations of mankind, instead of remaining empirical, might be so combined and marshalled as to be made the foundation of safe general theories, little expected that his name would become the stock authority for disclaiming generalization, and enthroning empiricism, under the name of experience, as the only solid foundation of practice." Vgl. John Stuart Mill. The Collected Works of John Stuart Mill, Volume IX - An Examination of William Hamilton's Philosophy and of The Principal Philosophical Questions Discussed in his Writings. Hrsg. von J. M. 
tik an Mill selbst oft mithilfe des Wortes empiricism formuliert wird. ${ }^{26}$ Wenn er seine eigene Position beschreibt, verwendet Mill hingegen das Wort „experiential“" ${ }^{27}$ ) Was die praktische Seite angeht, dürfte es noch selbstverständlicher sein, dass Mill die Kritik, die er hier darstellt, teilt. Weder glaubt er, Moral ließe sich auf egoistisch-kontraktualistische Weise rekonstruieren, noch meint er, blinde Impulse oder tierisches Empfindungsvermögen gäben inhaltlich vor, was moralisch gut ist. ${ }^{28}$

Inhaltlich sind die Probleme, die Mill hier anspricht, natürlich bekannt. Die Frage, was eigentlich unter einer ,wissenschaftlichen Erklärung" in einer rein experimentell begründeten (oder gar rein statistisch gefassten) Theorie verstanden werden sollte, oder ob wir das Bedürfnis nach Erklärung als eigentlich unwissenschaftlich abtun sollten und uns vielleicht auf die Zuverlässigkeit von Prognosen beschränken sollten, ist keineswegs gelöst. ${ }^{29}$ (Für Mill stellt sich dieses Problem vergleichsweise allgemein, da selbst die (wenig ehrgeizige) Vorstellung von Erklärung als deduktive Ableitung des Explanandums aus einem allgemeinen (kausalen) Satz Schwierigkeiten zu bereiten schien: In Bezug auf die Möglichkeit wissenschaftlicher Allaussagen kann die Logic nach Anschutz auch als Versuch gelesen werden, zwischen Realismus und Nominalismus hindurch zu navigieren. ${ }^{30}$ ) Und natürlich wird auch die praktische Seite der Kritik, die Mill darstellt, in Diskussionen, die wir auch im 21. Jahrhundert noch führen, widergespiegelt: Zum Beispiel kritisiert Gunnarsson bestimmte Formen des Reduktionismus, insbesondere solche, die versuchen, moralischen Gründen eine nicht-moralische, zum Bei-

Robson. Toronto und andere: University of Toronto Press und Routledge \& Kegan Paul, 1979, S. 485.

26 Vgl. McRae, „Introduction“, S. xxi. Ich diskutiere diese Kritik in Abschnitt 6.2.

27 Vgl. ebd., S. xxii und vgl. Anschutz, „The Logic of J. S. Mill“, S. 60.

28 Hier unterscheidet er sich nicht von Bentham. Siehe Jeremy Bentham. An Introduction to the Principles of Morals and Legislation. Oxford: Clarendon Press, 1907, § 24; und noch klarer: „Hume acknowledges the dominion of utility but so he does of the moral sense: which is nothing more than a fiction of ipsedixitism". Siehe Jeremy Bentham. Deontology together with A Table of the Springs of Action and The Article on Utilitarianism. Oxford und andere: Oxford University Press, 1983, S. 57.

29 Vgl. Wesley Salmon. Four Decades of Scientific Explanation. Pittsburgh: University of Pittsburgh Press, 1990, S. 185f.

30 Vgl. Anschutz, "The Logic of J. S. Mill“, S. 82f. 
spiel bloß rationalistische Grundlage ${ }^{31}$ zu geben, wie es Habermas' und Gauthiers Ziel laut ihm ist. ${ }^{32}$

Mill ist an dieser Stelle, was diese Beispiele angeht, eher unspezifisch. Er sagt nicht viel zu der Kritik außer, dass der Vorwurf ist, dass der Reduktionismus dem Charakter oder Wesen von Wissenschaft beziehungsweise Moral nicht gerecht wird und dass es Menschen wie Coleridge braucht, die dem Abhilfe verschaffen.

\subsubsection{Die Unitarier*innen und das Christentum}

Etwas klarer wird der Punkt beim zweiten Beispiel aus dem Text, das ich besprechen möchte. In Bezug auf das Christentum sind es nicht die Empirist*innen, sondern die Unitarier*innen, deren Position Coleridge zufolge droht, reduktionistisch zu werden:

He thus goes quite as far as the Unitarians in making man's reason and moral feelings a test of revelation; but differs toto caelo from them in their rejection of its mysteries, which he regards as the highest philosophic truths, and says that "the Christian to whom, after a long profession of Christianity, the mysteries remain as much mysteries as before, is in the same state as a schoolboy with regard to his arithmetic, to whom the facit at the end of the examples in his cypheringbook is the whole ground for his assuming that such and such figures amount to so and so." ${ }^{33}$

Die Strategie, die Coleridge hier unterstellt wird, ist ähnlich wie die Strategie von Philippa Foot, auf die in Fußnote 31 angespielt wird: Um sagen zu können, dass Moral rational sei, ohne dass Moral an einem der Moral externen Rationalitätsmaßstab gemessen wer-

31 Gemeint ist eine nicht-moralische aber rationale Grundlage. Insofern nur gesagt wird, moralische Gründe seien lediglich (vielleicht unter anderen Gründen) mitkonstitutiv für das, was wir als rational bezeichnen, könnten wir zwar sagen, die Moral habe eine rationale Grundlage; aber es läge sicher kein Reduktionismus vor. Siehe z. B. Foot im Anschluss an Quinn: Philippa Foot. Natural Goodness. Oxford: Clarendon Press (OUP), 2001, S. $62 \mathrm{f}$.

32 Siehe Logi Gunnarsson. Making Moral Sense - Beyond Habermas and Gauthier. Cambridge et al.: Cambridge University Press, 2003, Kapitel 6 bzw. Kapitel 8.

33 Vgl. Mill, „Coleridge“, S. 159. 
den müsste, behauptet Foot an der zitierten Stelle ${ }^{34}$ unter anderem, moralische Gründe seien konstitutiv für das, was als „vernünftig“ bezeichnet wird. Ebenso Coleridge: Um sagen zu können, das Christentum sei vernünftig, ohne dass es, so wie die Unitarier*innen es versuchen, an einem externen Maßstab gemessen wird, nach dem die Mysterien beispielsweise nicht mehr vernünftig sind, behauptet Coleridge eine Vernünftigkeit, die sich der christlich Praktizierenden erschließt - die aber vermutlich ebenso wenig einer NichtPraktizierenden einsichtig gemacht werden kann, wie die Vernünftigkeit moralischer Handlungen der berühmten Amoralist*in einsichtig zu machen sind.

Denn den Vergleich mit der Mathematik verstehe ich so: Zwar spielt Vernunft nach Mills Coleridge eine entscheidende Rolle im christlichen Glauben, aber es reicht nach Coleridge nicht, auf Vernunft zu verweisen, wenn es um das Wesentliche am Christentum geht: Vielmehr sei der einzige Weg, Zugang zu den Mysterien des Christentums zu erhalten - ihre Vernünftigkeit zu begreifen -, das Christentum zu praktizieren, genauso, wie die einzige Art und Weise $\mathrm{zu}$ verstehen, was es heißt, dass das Ergebnis einer Rechenaufgabe aus dieser mathematisch folgt, ist, das Rechnen zu praktizieren. Dort, wo sich praktizierenden Christ*innen wie den Unitarier*innen, die Mysterien nicht aus einer Innenperspektive erschließen, entgehe ihnen etwas am Christentum. Genauso, wie einem Schulkind, das Rechnen übt, etwas entgeht, wenn es meint, die Tatsache, dass im Rechenbuch ein Gleichheitszeichen abgedruckt sei, sei der Grund, warum das Ergebnis auf die Übungsaufgabe folgt. Dass im Buch ein Gleichheitszeichen steht, ist nicht der Grund, sondern der mathematische Zusammenhang zwischen Aufgabe und Ergebnis. Die Vernünftigkeit der Mysterien des Christentums wären also nach Mills Coleridge genauso wenig reduzierbar auf säkulare Vernunft, wie die Vernünftigkeit richtigen Rechnens auf etwas außerhalb dieser Praxis reduziert werden kann - und genauso wenig wie die Vernünftigkeit der Moral zum Beispiel auf blinde Impulse oder prudentielle Erwägung reduziert werden kann.

Ich glaube, dass es legitim ist, die Analogie zwischen Mathematik und Christentum im Sinne von Mills Coleridge etwas besser heraus-

34 Dies unterscheidet sich von dem, was sie in älteren Texten, z. B. Philippa Foot. „Morality as a System of Hypothetical Imperatives". In: The Philosophical Review 81.3 (1972), S. 305-316, stellenweise zu behaupten scheint. 
zuarbeiten, indem wir nicht das Gleichheitszeichen, sondern einen zumindest etwas plausibleren externen Maßstab für die Vernünftigkeit der Rechnung vorschlagen: Die Unitarier*innen versuchen die Vernünftigkeit des Christentums so zu erweisen wie ein Schuljunge, der die Vernünftigkeit arithmetischer Rechnungen durch ihre funktionale Rolle in der Naturwissenschaft zu erweisen versucht.

Etwas weiter hergeholt, aber trotzdem verwandt und vielleicht erhellend ist der folgende, ebenfalls auf einer Analogie mit der Mathematik beruhende Interpretationsvorschlag: Nehmen wir an, das, was im Rechenbuch steht, wären die durchschnittlichen Reaktionen von Menschen auf Rechenaufgaben - oder präziser: Ersetzen wir in dem Beispiel das Rechenbuch durch die dispositionalen Reaktionen als kompetent angesehener Rechner*innen. Dann hätten wir ungefähr die Antwort, die Kripkes Wittgenstein auf die Frage gibt, was damit gemeint ist, dass es korrekt ist, an der entsprechenden Stelle 125 und nicht 5 für die Antwort auf die Rechnung zu halten. ${ }^{35}$ Die Kritik daran, zum Beispiel der systematische Teil der Kritik von McDowell, entspräche dann der Kritik von Coleridge an den Unitarier*innen. ${ }^{36}$ Die Analogie dieser beiden Kontroversen wird allerdings getrübt: Mit Recht könnte McDowells Kritik an Kripke als Kritik an einer psychologistischen - oder soziologistischen - Position aufgefasst werden; genau dies wird Mill selbst aber zum Teil zu Recht vorgeworfen. Inwiefern dieser Vorwurf zu Recht erhoben wird, diskutiere ich in Abschnitt 6.2.

Auch wenn ich glaube, dass Mills Psychologismus am Ende ein Problem dabei darstellt, solche Reduktionsprobleme in ihren letzten Konsequenzen richtig aufzufassen, können wir verstehen, worum es ihm geht, ohne ihn auf eine Theorie dazu festzulegen, worin die Bedeutung einzelner Handlungen und Sprechakte innerhalb einer sozialen Praxis letztlich besteht. Was er nämlich sagen will - und dies wird noch deutlicher, wenn er über Bentham schreibt -, ist, dass es wichtig ist, das, was den Teilnehmer*innen einer solchen Praxis selbst an dieser Praxis liegt, in einer philosophischen Behandlung dieser Praxis ernstzunehmen. Dafür braucht es eine philosophische

35 Vgl. Saul Kripke. Wittgenstein on Rules and Private Language: An Elementary Exposition. Cambridge, Massachusetts: Harvard University Press, 1984, $8 f$.

36 Vgl. John McDowell. „Wittgenstein on Following a Rule“. In: Synthese 58 (1984). 
Tugend, die Mill Coleridge, aber zum Beispiel nicht Bentham zugesteht.

Das Beispiel des Christentums ist auch deshalb interessant, weil Mill hier selbst Außenstehender ist und mit der Textstelle sicherlich nicht sagen will, dass wir trinitarische Christen werden sollten. Mill bekennt sich an mehreren Stellen zum Atheismus. ${ }^{37}$ Ihm dürfte persönlich egal gewesen sein, ob Unitarismus oder Trinitätslehre bessere sprachliche Ausdrücke von etwas Wesentlichem im Christentum sind. Insofern stellt sich auch hier die Frage, die sich schon im letzten Abschnitt gestellt hat, inwiefern Mill hier eigentlich sagen will, dass Coleridge etwas richtig macht, und inwiefern es lediglich eine neutrale Darstellung von Gedanken von Coleridge ist. Textintern ist hier die Lage etwas klarer als im vorigen Abschnitt: Die Textstelle ist, anders als die Textstelle zu Moral und Wissenschaft, nicht in erster Linie die Darstellung philosophischer Schulen (und ihrer Stärken und Schwächen), sondern steht inmitten eines Kontextes, in welchem die Stärken von Coleridge aufgezählt werden. Mill will hier etwas an Coleridge lobend hervorheben. Dies kann aber natürlich nicht den Inhalt betreffen, von dem Mill selbst als Atheist ja nicht überzeugt ist. Mill schreibt dazu selbst, nachdem er seine inhaltliche Skepsis geäußert hat, eine Seite später: „The question, however, is not whether Coleridges attempts are successful, but whether it is desirable or not that such attempts should be made. " 38

Dass Mill meint, der Versuch, das, was wesentlich an der Religion ist, philosophisch zu fassen, sei es wert, unternommen zu werden, zeigt sich auch an seinem eigenen Versuch, dies zu tun. In „The

37 Siehe Mill, Autobiography, S. 41ff, oder John Stuart Mill. „Three Essays on Religion". In: The Collected Works of John Stuart Mill, Volume X-Essays on Ethics, Religion and Society. Hrsg. von J. M. Robson. London: Routledge und Kegan Paul, 1969; in Letzterem möchte er die metaphysischen Implikationen der Religion aufgeben (siehe Abschnitt 6.2.3). Zum Atheismus bekennt sich Mill aber auch direkt im Anschluss an die hier besprochene Textstelle.

38 Mill, „Coleridge“, S. 160. Mill liefert an dieser Stelle auch pragmatische Argumente für seine liberale Leser*innenschaft, warum sie Coleridges Auseinandersetzung mit Religion schätzen sollten. Religiöse Philosophie erleichtere es der Philosophie Anschluss zu finden in einer religiösen Gesellschaft (S. 160). Coleridge richte sich innerhalb des Christentums gegen die zunehmende Tendenz, die Bibel wörtlich zu verstehen, und würde Konservative, wenn sie ihn läsen, sicherlich eher liberaler als konservativer machen. 
Utility of Religion" bemüht sich Mill im zweiten Teil das, was eine „säkulare Religion“ genannt werden könnte, zu formulieren: Diese soll eben das, was gut an der Religion ist, fassen, ohne die fragwürdige Metaphysik dabei zu übernehmen. Auch hier wird deutlich, dass Mills Psychologismus ihm eine philosophische Grenze ist. Ich werde daher in Abschnitt 6.2 den Aufsatz genauer diskutieren.

Die Gemeinsamkeit all dieser Beispiele der Wissenschaft, der Moral und des Christentums ist, dass Mill in Coleridge einen Kritiker einer bestimmten Art von reduktionistischer Tendenz ${ }^{39}$ sieht: Reduktionismus in diesem Sinne besteht in einer Art Versuch, eine soziale Praxis oder Institution wie die Wissenschaft, die Moral oder das Christentum dadurch zu erklären, dass sie in anderen Begriffen eines bestimmten Typs reformuliert wird mit dem Anspruch, das Wesentliche der Praxis oder Institution dabei zu erhalten. Besonders deutlich wird dies im Kontrast zum Aufsatz über Bentham, den ich in Kapitel 4, diskutiere: Bentham behandelt Institutionen wie die herkömmliche Moral und das Christentum vornehmlich als Ausdruck von Interessen der Mächtigen. Statt Interessen könnten aber auch andere Begriffe herangezogen werden. Gemein ist reduktionistischen Tendenzen allerdings, dass ihre Begriffe sich dadurch auszeichnen, dass die Zusammenhänge zwischen ihnen (mutmaßlich) einfacher verständlich (und daher nicht mysteriös) sind oder dass ihre Gegenstände (mutmaßlich) leichter objektiv und/oder empirisch verifizierbar sind oder Ähnliches. Nun ist sicher nicht jede Abstraktion problematisch: Jede Form der feststellenden Rede und sicherlich jede Form der Philosophie ist auf Abstraktionen angewiesen. Problematisch ist ein solcher Reduktionismus, wenn das, was der Witz oder das Wesentliche der Institution oder Praxis ist, in der neuen Beschreibung nicht ausdrückbar ist. Genau diese Schwäche sieht Mill in der philosophischen Tradition, der er sich selbst zugehörig sieht.

39 Natürlich kann Mills Psychologismus seine Versuche, philosophische Fragestellungen auf psychologische Begriffe zu reduzieren, auch als eine Art von Reduktionismus bezeichnet werden. Und, wie bereits gesagt, ist dies nicht ganz unproblematisch. Aber, wie hoffentlich spätestens im nächsten Kapitel (Kapitel 4) deutlich wird, kann diese metaphysische Frage bis zu einem gewissen Grade unabhängig von dem Problem gesehen werden, das Mill in Bentham sieht und auf das "Coleridge" Mills Antwort ist. (Siehe auch Abschnitt 6.2.) 
Der Witz oder das Wesentliche zeigt sich aber nur einer Teilnehmer*in an der Praxis - möglicherweise erst „after a long profession“. Und ihn zu erkennen, ist für Mill bedingt durch die Erfahrungen der Teilnehmer*innen an einer Praxis; es ist eine Frage dessen, was sie als wichtig erleben.

Die philosophische Tugend, die Mill in Coleridge sieht, besteht nun darin, das, was eine Teilnehmer*in an einer Praxis an dieser Praxis wertvoll findet, in Worte zu fassen, die dem gerecht werden. Dies heißt nicht einfach, irgendwie für diesen Wert zu argumentieren. Eine besondere Argumentationsfähigkeit scheint, was diese Tugend angeht, nicht einmal besonders wichtig zu sein. (Adjektive, die in die Richtung analytischer oder argumentativer Fähigkeiten gehen, verwendet Mill tatsächlich eher für Bentham als für Coleridge. Siehe Kapitel 4.) Mill wirft den anderen konservativen englischen Philosophen seiner Zeit vor, ,superfine new arguments “40 $\mathrm{zu}$ entwickeln, um alte Vorurteile zu bestätigen. Der Wert, so kann etwas spekulativ rückgeschlossen werden, den etablierte Institutionen und Praktiken gegebenenfalls haben, kann sich nach Mill nicht dadurch zeigen, dass er als Schlussfolgerung ausgeklügelter Argumente in Erscheinung tritt. Coleridge jedenfalls überzeugt Mill sicher nicht durch bloße intellektuelle Stärke, wie eine Einschätzung Mills bezüglich Frederic Maurice' aus der Autobiographie belegt: „The nearest parallel to him, in a moral point of view, is Coleridge, to whom, in merely intellectual power, apart from poetical genius, I think him decidedly superior. "41

Möglicherweise würden die ausgeklügelten Argumente, um die es geht, das, was sie etablieren sollen, nämlich den Wert einer überlieferten Praxis, genauso reduktionistisch rekonstruieren, wie die kritischen Rekonstruktionsversuche derjenigen, die sie kritisieren

40 Eine Phrase, die in diesem Kontext kritisch gemeint ist; zwar referiert Mill hier Kritik anderer Empiristen, aber er will sich davon mit Sicherheit nicht distanzieren. Vgl. Mill, „Coleridge“, S. 126. Vergleiche (diesmal in Bezug auf die Methode, nicht auf die politische Ausrichtung bezogen) auch den in eine ähnliche Richtung gehenden Vorwurf der Einführung einer ,technical terminology $[. .$.$] for the double purpose of giving logical precision to doctrines$ which we do not admit, and of marking a relation between those abstract doctrines and many concrete experimental truths, which this language, in our judgment, serves not to elucidate, but to disguise and obscure." Vgl. ebd., S. 122.

41 Mill, Autobiography, S. 161. 
sollen. Sie würden dann zu bloßen Sophismen. Der Versuch, genau anzugeben, was Mill an dem, was er mit „superfine new arguments" und "technical terminology" kritisiert, stört, ist vielleicht etwas spekulativ. In jedem Fall aber kann das, was Mill an Coleridge schätzt, nicht seine Fähigkeit zu raffinierter Argumentation sein, sondern seine Fähigkeit, Worte für Wertvolles zu finden, das in einer Philosophie, wie Bentham sie betreibt, ansonsten keinen Ausdruck finden könnte. ${ }^{42}$ Damit dies aber nicht reaktionär wird, braucht es eine Möglichkeit, wie dieses Wertvolle in der Philosophie in Erscheinung treten kann, ohne dass es einfach die Schlussfolgerung einer Untersuchung der Tradition ist. Bevor ich im nächsten Abschnitt den anderen Ansatz vorstelle, aus dessen Synthese mit Coleridges Ansatz in die Richtung einer solchen Philosophie der erste Schritt gemacht werden kann, möchte ich diese Interpretation der Stärke Coleridges im Umgang mit sozialen Praktiken und Institutionen gegen alternative Interpretationsvorschläge verteidigen.

\subsection{Coleridge als Konservativer?}

Ich hatte oben (Abschnitt 3.2) dafür argumentiert, dass Mills Unterscheidung zwischen intuitiver Schule und Schule der Erfahrung als Interpretation für die Tugenden, die in ihrer Kombination gute Philosophie ergeben sollen, den eigentlichen Kern der Tugenden nicht treffe. Ich habe also, was die „methodische Interpretation" genannt werden könnte, zurückgewiesen. ${ }^{43}$ Sie sei, so hatte ich behauptet, eine Beschreibung des geistesgeschichtlichen Hintergrunds, die aber nicht das trifft, was jeweils speziell an Coleridges und Benthams Art zu philosophieren wertzuschätzen sei. Ein anderer häufiger Interpretationsvorschlag besteht in der zweiten Dichotomie, die Mill verwendet: der Dichotomie zwischen konservativer und progressiver Philosophie. ${ }^{44}$ Diese Interpretation werde ich

42 „Bentham should continually miss the truth which is in the traditional opinions, and Coleridge that which is out of them, and at variance with them. But it was also likely that each would find, or show the way to finding, much of what the other missed." Mill, "Coleridge", S. 120.

43 Siehe auch S. 129, wo Mill sich explizit zu distanzieren scheint: ebd., S. 129.

44 So schreibt Mill beispielsweise am Anfang von „Bentham“, alle Philosoph*innen ließen sich in die Dichotomie zwischen progressiv und konservativ einordnen. Siehe Mill, „Bentham“, S. 77. Obwohl dies, falls die Dichotomie den 
„die gesellschaftspolitische Interpretation“ nennen, um sie und die „methodische Interpretation" von meiner sozialphilosophischen Interpretation abzugrenzen.

Auch diese Interpretation trifft den Punkt nicht genau. Dies sollte auch bereits deutlich geworden sein: Es ist sehr unplausibel, dass Mill im gleichen Atemzug, in dem er seine Anhänger noch einmal erinnert, dass er Atheist ist, sagen will, dass es einen Wert hat, eine trinitarische (im Gegensatz zur unitarischen) Version des Christentums zu goutieren. Es muss vielmehr Coleridges Umgang mit dem Christentum sein, den Mill interessant findet.

Aber trotzdem ist es natürlich vielleicht möglich, beide Interpretationen $\mathrm{zu}$ vereinen, um zu einer plausiblen Interpretation $\mathrm{zu}$ gelangen. Meine Argumente dagegen, dass jede der beiden Interpretation für sich betrachtet sinnvoll ist, würden ihre Plausibilität verlieren, wenn sie eben nicht für sich betrachtet werden könnten. Und tatsächlich: Es wirkt stellenweise so, als wolle Mill sagen, dass die Denker*innen seiner Zeit entweder der kontinentalen, intuitiven Schule angehörten und Philosophie im Dienste der Orthodoxie betrieben oder der Schule der Erfahrung angehörten und progressive Philosophie betrieben; dass also die ersten beiden Dichotomien letztlich bedeutungsgleich seien. ${ }^{45}$ Dafür zu argumentieren, dass dies nicht so ist, ist die Aufgabe dieses Abschnittes.

Einen sehr simplen Grund gegen die gesellschaftspolitische Interpretation möchte ich direkt vorweg schicken: Maurice und Sterling, die Mill die Gedanken Coleridges im Debattierklub nahebringen,

logischen Raum abdecken soll, trivial erscheinen mag, so legt es doch nahe, dass Mill auf diese Eigenschaft von Bentham und Coleridge abzielt. Allerdings sagt er eine Seite später, dass sich so große Denker*innen, wie es Bentham und Coleridge sind, dieser Einordnung bis zu einem gewissen Grade entziehen. Siehe Mill, „Bentham“, S. 77.

45 Beispielsweise spricht Mill häufig einfach von der "Gegenseite“ - so, als ob es nur eine kohärente Position gibt, die zu Mills Ansichten entgegengesetzt ist. Es mag im Debattierklub in den 1830ern sinnvoll gewesen sein, von „our Coleridgian adversaries“ zu sprechen (siehe Mill, Autobiography, S. 159), aber auch in anderem Kontext bezeichnet er Coleridge als „contrary side“. Ebd., S. 227. Interessanterweise differenziert Mill in einer früheren Fassung - anders als in der Columbia MS - der Autobiographie an der entsprechenden Stelle klar zwischen den "Coleridgians" und der "Tory party" im Debattierklub. Ebd., S. 158. Alles, was solche Gegenüberstellungen zeigen, ist, dass Mill ideengeschichtlich vielleicht aus rhetorischen Gründen ein zweipoliges Bild favorisiert. 
werden von ihm in der Autobiography sowohl in der Frühversion als auch in der späten Fassung als eine zweite liberale und sogar radikale Partei bezeichnet. ${ }^{46}$ Dieser erste Kontakt Mills mit coleridgianischer Philosophie fand durch Personen statt, die diese in einer Weise vertreten haben, die Mill auch sehr viel später nicht als konservativ bezeichnet. Das Bild, das „coleridgianisch“ automatisch „konservativ" bedeutet, will Mill also zumindest zu dem Zeitpunkt, an dem er Autobiographie schreibt, nicht zeichnen.

Ich werde nun zunächst darstellen, warum Mill meint, dass die intuitive Methode in besonderem Maße für den Konservativismus anfällig sei (Abschnitt 3.4.1). Dann werde ich jedoch meine Zweifel ausdrücken erstens daran, dass die Zuordnung von philosophischen Schulen zu politischen Ausrichtungen so klar ist - oder für Mill selbst so eindeutig war, wie dies oft dargestellt wird. Zweitens möchte ich gegen ein Bild im Zusammenhang mit der gesellschaftspolitischen Interpretation argumentieren, dass alles, was Mill an Positivem über "Coleridge" zu sagen weiß, einfach taktisches politisches Kalkül gewesen sei (beides in Abschnitt 3.4.2).

Zunächst muss aber zugestanden werden: Coleridge ist ein konservativer Denker - und Mill behandelt ihn als solchen. Mill weist darauf hin, dass trotz des Konservativismus Coleridges Art zu philosophieren Gesellschaftskritik zulässt und zwar, insofern eine tatsächlich gelebte Institution nicht ihrer Idee entspricht. Diese Art der Gesellschaftskritik weist natürlich viele Parallelen auf zu dem, was Rahel Jaeggi „interne Kritik" nennt. ${ }^{47}$ Am Beispiel der anglikanischen Kirche macht Mill klar, inwiefern Coleridge zur internen Kritik herangezogen werden kann:

Coleridge's merit in this matter consists, as it seems to us, in two things. First, that by setting in a clear light what a national church establishment ought to be, and what, by the very fact of its existence, it must be held to pretend to be,

46 Siehe ebd., S. 160 bzw. 133. „Radikal“ spielt hier auf die Bezeichnung der Gruppe von Benthamiten an, zu der auch Mill gehört.

47 Jaeggi, Kritik von Lebensformen, S. 261f. So scheint jedenfalls Mill Coleridge zu lesen. Es könnte aber auch argumentiert werden, dass Coleridge bereits auf etwas, das Jaeggis immanenter Kritik ähnlich ist, abzielt. Siehe Kapitel 8. 
he has pronounced the severest satire upon what in fact it is. ${ }^{48}$

Allerdings sind Coleridges konservativen gesellschaftlichen Vorstellungen natürlich eigentlich etwas, das Mill ausdrücklich ablehnt: Coleridge verteidigt konservative Institutionen wie die Kirche, das viktorianische Christentum, eine Verfassung, nach der im House of Lords die (unterstellte) Verantwortlichkeit, die mit Landbesitz einhergeht, direkt zur Legitimation politischen Einflusses genutzt wird. ${ }^{49}$ Nichts davon unterstützt Mill inhaltlich.

Die gesellschaftspolitische Interpretation berücksichtigt ebenso wie das, was ich über die Essays sage, deren offensichtliche Einheit und Komplementarität - interpretiert diese aber vor dem Hintergrund der politischen Strömungen zu ihrer Entstehungszeit. ${ }^{50}$ Und natürlich würden sich die gesellschaftspolitische Interpretation, die methodische und die sozialphilosophische Interpretation unter einer Voraussetzung nicht ausschließen: Wenn es nämlich kein Zufall wäre, dass Coleridges Art zu philosophieren einen gesellschaftspolitischen Konservativismus nahelegt und dass Benthams Art zu philosophieren eine progressive politische Ausrichtung nahelegt.

Mill jedenfalls will dies zumindest zum Zeitpunkt der Veröffentlichung von „Bentham" und "Coleridge" nicht als reinen Zufall erscheinen lassen - auch wenn er später eine differenziertere Ansicht vertritt. Auf der einen Seite liegt das auch nahe: Bentham und Coleridge sind eben nicht hauptamtlich Philosophen, die privat zufällig verschiedene politische Weltanschauungen vertreten, sondern Bentham und Coleridge sind praktische Philosophen, deren Philosophie

48 Mill, „Coleridge“, S. 150. Interessant für das Verständnis von Mill ist auch der zweite Verdienst von Coleridge, der im Herausarbeiten der Idee der Kirche Englands besteht: Diese soll so etwas wie eine spirituelle oder geistige Führung für alle ermöglichen, indem sie bestimmte Menschen von der Notwendigkeit, für die eigene Existenz zu arbeiten, ausnimmt. (Ebd.) Mills lobende Erwähnung dieses Gedankens kann sicherlich in Zusammenhang mit der Unterstellung eines säkularen Elitismus gebracht werden, die oft gegen Mill erhoben wird. (So schlägt Mill im Zuge der zweiten großen Reform des Wahlrechts im 19. Jahrhundert beispielsweise unter anderem die Einführung einer Gewichtung der Stimmen nach Bildung vor. Vgl. Mill, „Thoughts on Parliamentary Reform".)

49 Vgl. Mill, „Coleridge“, S. 152.

50 Wie sie sich beispielsweise im Debattierklub Mills abzeichneten. Vgl. Mill, Autobiography, S. 159. 
eng daran geknüpft ist, die soziale und politische Wirklichkeit ihrer Zeit unter einem bestimmten Blickwinkel zu sehen und zu kritisieren. $^{51}$

Wenn ich im Folgenden gegen die gesellschaftspolitische Interpretation argumentiere, dann argumentiere ich dementsprechend selbstverständlich nicht dagegen, dass die gesellschaftliche Relevanz von Philosophie für Mill wichtig ist. Diese ist Mill außerordentlich wichtig. Dies impliziert auch, dass die Dichotomie „konservative versus progressive Philosophie" für Mill wichtig und auch in seiner Beschäftigung mit Coleridge und Bentham wichtig ist und vieles in den Aufsätzen verständlich macht. Ich argumentiere lediglich dagegen, dass das, was Mill jeweils in Bentham und Coleridge schätzt - was er als ihre philosophischen Tugenden erkennt - durch diese Dichotomie erfasst wird.

\subsubsection{Die „intuitive Schule“ und Konservativismus}

Oben hatte ich dafür argumentiert, dass die gesellschaftspolitische Interpretation von vornherein nur plausibel sein kann, wenn sie in Kombination mit der methodischen vertreten wird. Und tatsächlich scheint der Konservativismus Coleridges aus Mills Sicht nicht nur in seiner Wahl der Institutionen, deren Wert er herausstellen will, zu liegen; er liegt zumindest zum Zeitpunkt, zu dem „Coleridge“ geschrieben wurde, auch in dem, was Mill unter der ,intuitiven Schule" versteht. Paradigmatisch für diesen Zusammenhang ist, was Mill unter Transzendentalphilosophie versteht:

They [die Empiristen, zu denen Mill sich selbst auch zählt] in their turn allege that the transcendentalists [Mill meint die Transzendentalphilosophie, nicht die amerikanische Bewegung] make imagination, and not observation, the criterion of truth; that they lay down principles under which a man may enthrone his wildest dreams in the chair of philosophy, and impose them on mankind as intuitions of the pure reason $[\ldots]$. And even if $[\ldots]$ the private revelations of

51 Neben der oberflächlichen Kompromissfreudigkeit der britischen Philosophie seiner Zeit macht Mill auch die Erosion der gesellschaftlichen Legitimation der existierenden Institutionen dafür verantwortlich, dass die großen Hinterfrager (the great questioners) - gemeint sind Coleridge und Bentham hervorgebracht werden konnten. Vgl. Mill, „Coleridge“, S. $142 \mathrm{ff}$. 
any individual [...] be disowned, or, in other words, outvoted [...], this is still only substituting, as the test of truth, the dreams of the majority for the dreams of each individual. Whoever form a strong enough party, may at any time set up the immediate perceptions of their reason, that is to say, any reigning prejudice, as a truth independent of experience; [...] for no less authority than this is claimed by some transcendentalists for their à priori truths. And thus a ready mode is provided, by which whoever is on the strongest side may dogmatize at his ease $\left[\ldots . .{ }^{52}\right.$

Weil, so können wir versuchen, Mills Argument zu rekonstruieren, transzendentale Argumente die Bedingungen der Möglichkeit einer Praxis (Begriff, Institution) etablieren sollen, wird, was sie zeigen, letztlich immer an der Autorität hängen, mit der diese Praxis behauptet werden kann. „Die Träume der Mehrheit" sind dann letztlich die fragwürdige Grundlage philosophischer Wahrheiten. Natürlich sind die Träume der Mehrheit nicht unbedingt konservativ aber es wird ja, anders als es in diesem kurzen Abschnitt verkürzt dargestellt ist, auch nicht garantiert, dass es die Träume der Mehrheit sind, die in philosophischen Abhandlungen den Test der Wahrheit bilden, sondern die Träume der Mehrheit der Philosophierenden. Diese sind in privilegierten gesellschaftlichen Positionen und diese erhalten zu wollen ist konservativ. Mill ist dies durchaus bewusst. In „Whewell on Moral Philosophy“ macht Mill zum Beispiel sehr viel deutlicher, warum er meint, dass besonders die Kombination der intuitiven Methode mit der Institution der englischen Universität zu seiner Zeit zu Konservativismus führe. ${ }^{53}$

Die Kritik Mills erinnert natürlich an die Kritik Schopenhauers an Kant, die er unter dem Vorwurf der "umgekehrten Theologie“ fasst, und kann durch diese illustriert werden: Es sei nicht verwunderlich, dass Kant, wenn er den „im Schwange gehenden“ Moralbegriff ${ }^{54}$ analysiert, letztlich auf die Annahme Gottes (natürlich ausschließlich zu praktischem „Behuf“) als Garant für das höchste Gut $^{55}$ komme, weil die im Schwange befindliche Moral nun einmal

52 Mill, „Coleridge“, S. 127.

53 Siehe Mill, „Whewell on Moral Philosophy“, $167 f$.

54 Vgl. Immanuel Kant. Grundlegung zur Metaphysik der Sitten. Hamburg: Felix Meiner, 1999, AA 445.

55 „Ist dieses nun einmal geschehen, so wird dem Begriffe des Objekts eines moralisch bestimmten Willens (dem des höchsten Guts) und mit ihm den 
zu Kants Zeit eine christlich geprägte Moral sei, die in ihrer säkularisierten Form genau dort eine Leerstelle aufweise, wo Kant die Idee Gottes einfügt. ${ }^{56}$ Wenn man den „im Schwange gehenden“ Moralbegriff als "Moraltraum der Mehrheit" bezeichnet, und die (aus Sicht des atheistischen Mill konservative) Schlussfolgerung bedenkt, wird verständlich, wieso Mill meint, ein solches Vorgehen lege einen Konservativismus nahe.

Natürlich könnte gesagt werden, Mill habe transzendentalphilosophische Argumente nicht richtig verstanden. Wir könnten zum Beispiel darauf hinweisen, dass ein transzendentales Argument zu formulieren nicht heißt, für irgendeine Praxis die Bedingungen ihrer Möglichkeit herauszustellen, sondern, dass dies auf Praktiken eingeschränkt werden muss, an denen die Teilnahme in gewissem Sinne nicht optional ist, weil wir zum Beispiel mit Jürgen Habermas derjenigen, die ihre Teilnahme an unserer Praxis des Gründegebens hinterfragt, einen performativen Widerspruch nachweisen könnten. ${ }^{57}$ Interessant ist natürlich, dass Habermas Mill insofern eingeschränkt Recht geben könnte, als er den Status der Schlussfolgerung seines eigenen Argumentes nicht in Unabhängigkeit von den empirischen Gegebenheiten unserer tatsächlich verwendeten "Begriffssysteme“ sehen will. ${ }^{58}$

Mill jedenfalls würde der Verweis auf die Notwendigkeit nicht überzeugen. Bereits in Abschnitt 3.3 wurde darauf hingewiesen, dass Mill nicht glaubt, dass das, was an dem, was die GermanoColeridgians tun, auf die sich die Textstelle am Anfang dieses Abschnitte bezieht, gut ist, ausgeklügelte Argumentationen sind. Mill übersieht auch nicht einfach, dass ein vollständiges transzendentales

Bedingungen seiner Möglichkeit, den Ideen von Gott, Freiheit und Unsterblichkeit, auch Realität, aber immer nur in Beziehung auf die Ausübung des moralischen Gesetzes (zu keinem spekulativen Behuf), gegeben." Vgl. Kant, $K p V$, AA 138. Natürlich ist der Status dieser Idee komplex.

56 „In abstracto ausgesprochen ist Kants Verfahren dieses, daß er zum Resultat machte, was das Princip oder die Voraussetzung hätte seyn müssen (die Theologie), und zur Voraussetzung nahm, was als Resultat hätte abgeleitet werden sollen (das Gebot)." Arthur Schopenhauer. Preisschrift über das Fundament der Moral. Hamburg: Meiner, 1979, S. 23.

57 Vgl. z. B. Jürgen Habermas. „Diskursethik - Notizen zu einem Begründungsprogramm". In: Moralbewusstsein und kommunikatives Handeln. Frankfurt am Main: Suhrkamp, 1983, S. 53-125, S. 96-104.

58 Vgl. ebd., S. 104-108. 
Argument auf die Bedingung der Möglichkeit einer Praxis ein Argument für die Nicht-Optionalität der Teilnahme an dieser Praxis beinhalten sollte. Er ist gegenüber Formulierungen, die eben diese Nicht-Optionalität ausdrücken sollen, einfach äußerst skeptisch, wie an anderer Stelle noch deutlicher wird: Beispiele für Formulierungen, die eine solche Notwendigkeit ausdrücken sollen, denen Mill gegenüber aber sehr skeptisch ist, sind „our idea of man as man“, „all men think“, und „we cannot help feeling“. ${ }^{\circ 9}$

\subsection{2. "Coleridge“ versus „Whewell on Moral Philosophy“}

Aus der Sicht, die Mill in „Coleridge“ darstellt, besteht also ein Zusammenhang zwischen intuitiver Schule und Konservativismus. Wie bereits angedeutet, beschränkt sich diese Ansicht auch nicht auf "Coleridge" oder die Zeit, in der dieser Aufsatz geschrieben wurde. Die Frage ist, wie eng dieser Zusammenhang für Mill wirklich war. Selbst aber, wenn die mit „intuitive Schule" bezeichnete Methode für Mill eine intrinsische Tendenz hätte, konservative Positionen zu rechtfertigen, wäre beides nicht gleichzusetzen: Weder in „Coleridge" noch sonst scheint Mill Zugeständnisse bezüglich methodischer Entscheidungen der intuitiven Schule zu machen. Genauso wenig kommt er Coleridge inhaltlich in seinem Konservativismus entgegen. Wenn überhaupt, müsste die philosophische Tugend, die Mill an Coleridge verdeutlicht, also ein Beiprodukt einer methodischen (oder gesellschaftspolitischen) Präferenz sein.

Auch das ist aber nicht nicht der Fall. Egal, wie plausibel die Verbindung transzendentaler Argumente mit einer konservativen Weltanschauung Mill zum Veröffentlichungszeitpunkt von Coleridge geschienen haben mag, er sieht es später differenzierter. In „Whewell on Moral Philosophy" schreibt Mill (Whewell kritisierend):

During the greater part of the eighteenth century, the received opinions in religion and ethics were chiefly attacked, as by Shaftesbury, and even by Hume, on the ground of instinctive feelings of virtue, and the theory of a moral taste or sense. As a consequence of this, the defenders of established opinions, both lay and clerical, commonly professed utilitarianism. [...] But a change ensued, and the utilitarian

59 Vgl. Mill, „Whewell on Moral Philosophy“, S. 193. 
doctrine, which had been the favourite theory of the defenders of orthodoxy, began to be used by its assailants. In the hands of the French philosophers, and in those of Godwin and of Bentham,--who, though earlier than Godwin in date, was later in acquiring popular influence,-a moral philosophy founded on utility led to many conclusions very unacceptable to the orthodox. ${ }^{60}$

Dieses Zitat legt zunächst nur nahe, dass der Utilitarismus (beziehungsweise das, was Mill zu diesem Zeitpunkt so bezeichnet ${ }^{61}$ ) sowohl in konservativer als auch in progressiver Absicht vertreten wurde. Dies trübt die Chancen für die Gleichsetzung der gesellschaftspolitischen Interpretation mit den anderen beiden Interpretationen bereits insofern, als die Elemente einer der Seiten der beiden Dichotomien offensichtlich nicht gleichbedeutend sind. Bedenkt man weiter, dass Mill an anderer Stelle meint, die intuitive Schule sei, was die praktische Philosophie angeht, so etwas wie die logische Konsequenz der hier angesprochenen moral sense-Theorie, wären sogar beide Seiten der Dichotomie in Frage gestellt. ${ }^{62}$ Noch etwas deutlicher wird das wenig später in „Whewell on Moral Philosophy“, wenn Mill explizit auf die „deutschen Metaphysiker" eingeht, die laut Mill die ideengeschichtliche Vorlage der "Orthodoxie" in England zu seiner Zeit bilden:

It was not without reluctance that they found themselves engaged in this path; for German metaphysics in Germany lay under as grave a suspicion of religious scepticism, as the rival philosophy in England or France. But it was found on trial, that philosophy of this cast admitted of easy adaptation, and would bend to the very Thirty-nine Articles; as it

60 Ebd., S. 170.

61 Einige der Denker, die Mill in der Auslassung aufzählt, vertreten zum Beispiel keine Maximierung des Gesamtnutzens als Maßstab für individuelles Handeln. Dies ist an dieser Stelle aber nicht wichtig.

62 ,[T]hose believers in [a moral sense] have been obliged to abandon the idea that it discerns what is right or wrong in the particular case in hand, as our other senses discern the sight or sound actually present. Our moral faculty, according to all those of its interpreters who are entitled to the name of thinkers, $[\ldots]$ is a branch of our reason, not of our sensitive faculty [...]. The intuitive, no less than what may be termed the inductive, school of ethics, insists on the necessity of general laws.“ Mill, „Utilitarianism“, § 1.3. 
is the essence of a philosophy which seeks its evidence in internal conviction, that it bears its testimony with equal ease for any conclusions in favour of which there is a predisposition, and is sceptical with the sceptical, and mystical with the mystical. ${ }^{63}$

Hier wird deutlich, dass die Kritik an der intuitiven Methode (zumindest zu dem Zeitpunkt als Mill "Whewell on Moral Philosophy“ verfasst) weniger darin begründet liegt, dass sie Konservativismus zur Folge hätte, sondern dass sie zur Verteidigung beliebiger Konklusionen taugt. Sie ist dann konservativ, wenn sie von in bestimmter Weise Privilegierten betrieben wird - wie Mill dies beispielsweise in den englischen Universitäten seiner Zeit sieht. Die Frage ist, ob er dies vor dem Hintergrund dessen, was er kurz vorher zum Utilitarismus gesagt hat, nicht auch für den Utilitarismus zugeben müsste. (In diesem Sinne lässt sich zum Beispiel Mills Aussage aus „Bentham" über Benthams Verpflichtung auf den Utilitarismus deuten: "It was by mere accident that this opinion became connected in Bentham with his peculiar method.“64) In jedem Fall sind auch für Mill die Dichotomien nicht bedeutungsgleich. Dass sie von Mill an anderen Stellen oft so behandelt werden, mag, wie Roger Crisp nahelegt, damit zu tun haben, dass er in Personen wie Whewell oft gegen Intuitionismus und Konservativismus in Personalunion zu kämpfen hatte. ${ }^{65}$

Nun könnte eingewandt werden, „Whewell on Moral Philosophy“ dürfe zur Interpretation von „Coleridge“ nicht herangezogen werden, weil Mill inzwischen - immerhin zwölf Jahre später - seine Taktik geändert habe. Die gesellschaftspolitische Interpretation sei richtig, die als Interpretationsausgangspunkt entscheidende Dichotomie sei diejenige zwischen konservativer und progressiver Philosophie, nur dass Mill inzwischen nicht mehr die Strategie verfolge,

63 Mill, „Whewell on Moral Philosophy“, S.170f.

64 Mill, „Bentham“, S. 87.

65 Crisp legt an der gleichen Stelle sogar nahe, Mill sei eigentlich selbst Intuitionist: zumindest die Unparteilichkeitsannahme, die Mill für sein Argument für den Utilitarismus in Kapitel 4 von „Utilitarianism" voraussetze, sei letztlich nur intuitionistisch zu begründen. Dass Mill das nicht wahrhaben wolle, liege eher daran, dass er sich nicht mit Personen wie Whewell gemein machen wolle, als am Intuitionismus. Siehe Roger Crisp. Mill on Utilitarianism. London und New York: Routledge, 1997, S. 82 f. 
seinen Widersachern entgegenzukommen. Mill legt dies auf der anderen Seite selbst etwas nahe, da er sich in der Autobiographie etwas unspezifisch von den hier behandelten Aufsätzen zu distanzieren scheint:

In both cases, the impetus with which I had detached myself from what was untenable in the doctrines of Bentham [...], may have carried me, though in appearance rather than in reality, too far on the contrary side. But as far as relates to the article on Coleridge, my defence is, that I was writing for Radicals and Liberals, and it was my business to dwell most on that in writers of a different school, from the knowledge of which they might derive most improvement. ${ }^{66}$

Zum einen aber distanziert sich Mill nur von dem erweckten Anschein wirklich - was die Meinungen von Coleridge angeht, hatte er schon in "Coleridge" selbst keinen Zweifel gelassen, dass er sie für reaktionär hält. Zum anderen aber scheint er das, was er an der Art und Weise Coleridges zu philosophieren schätzt, weiterhin wichtig zu finden, sonst würde die „Entschuldigung“ Coleridge als Korrektiv für „Radicals" und „Liberals" einzusetzen, keine Entschuldigung sein. Dafür spricht auch, dass er „Coleridge“ und „Bentham“ 1859 und 1867 noch einmal in Dissertations and Discussions publiziert. ${ }^{67}$

Das Hauptargument aber, das dagegen spricht, die gesellschaftspolitische Interpretation unter Voraussetzung einer Änderung der

66 Mill, Autobiography, S. 227.

67 Außer der bereits zitierten Autobiographie lassen sich Mills Einstellungen zu diesem Zeitpunkt gegenüber seinen früheren Texten auch aus dem Vorwort dieser Ausgabe entnehmen: John Stuart Mill. „Preface to Dissertations and Discussions". In: The Collected Works of John Stuart Mill, Volume X - Essays on Ethics, Religion, and Society. Hrsg. von J. M. Robson. Toronto und andere: University of Toronto Press und Routledge \& Kegan Paul, 1969, S. 493-494. Er befürchtet dort zwar, dass "Review on Sedgwick's Discourse" eine zu große Sympathie für die Philosophie des 18. Jhd. erkennen lasse, was durch „Coleridge" und „Bentham" korrigiert werde, die aber ihrerseits eine zu große Sympathie für die Reaktion des 19. Jhd. auf das 18. Jhd. nahelege, was wiederum durch "Whewell on Moral Philosophy" korrigiert werde. Er sagt aber auch, dass der Wert, den „Coleridge" und „Bentham" haben, nicht bloß temporär ist. Stellen, wo er seine Meinung wirklich geändert hat (und nicht bloß den Tonfall ändern würde) hat Mill außerdem nach eigenen Angaben in der Ausgabe, die in dieser Arbeit, wenn nicht anders angegeben, verwendet wird, ab 1859 geändert. (Siehe Fußnote 1 in Kapitel 1.) 
Taktik zur Grundlage der Interpretation von "Coleridge“ zu machen, ist, dass diese voraussetzen würde, dass Mill, würde er vor die durch seine eigene Differenzierung in „Whewell on Moral Philosophy" möglich gewordene Wahl gestellt, welche Eigenschaften von Coleridge er nun schätzen würde, die konservativen Einstellungen wählen würde. Dies ist aber selbst vor dem Hintergrund taktischer Überlegungen unplausibel. Wie ich versucht habe deutlich zu machen, distanziert sich Mill auch in "Coleridge" bereits sowohl von dessen konservativen Überzeugungen als auch von der intuitiven Methode - und lobt ihn nicht etwa dafür. Damit ist die sozialphilosophische Interpretation die einzig plausible.

Zusammenfassung. In diesem Kapitel habe ich gezeigt, dass für Mill Coleridge ein Paradigma für ein philosophisches Ideal ist, das ich eine „sozialphilosophische Tugend“ genannt habe. Wie bereits im letzten Kapitel angedeutet, wird der systematische Wert, der darin liegt, dieses Ideal als Tugend auszulegen, erst in Teil II deutlich. (Auch wenn einige Zitate in diesem Kapitel schon deutlich gemacht haben dürften, dass Mill zumindest unter anderem auf Dispositionen und Fähigkeiten der Person Coleridge hinweisen will. Dies wird besonders im nächsten Kapitel in Bezug auf Bentham und seine Schwächen als Person noch deutlicher.) Die Tugend ist charakterisiert durch Coleridges Vermögen und sein Interesse daran, dem, was kompetente Teilnehmer*innen an einer sozialen Praxis oder Institution als wertvoll erfahren oder als wichtig erleben, adäquaten Ausdruck zu verleihen. Ich hatte betont, dass es Mill hier um den adäquaten Ausdruck einer Erfahrung geht und nicht etwa darum, für diesen Wert durch geschickte Argumentation zu argumentieren. Insbesondere reduktionistische Versuche, den Wert einer Praxis stattdessen zu bestimmen, sind daher zum Scheitern verurteilt: Anhand von zwei Negativbeispielen aus "Coleridge" habe ich versucht deutlich zu machen, wieso ein solcher Reduktionismus kein adäquater Ausdruck der Erfahrung einer Teilnehmer*in sein kann, und die Kritik von Mills Coleridge daran erläutert und zu modernen Fragestellungen ins Verhältnis gesetzt. Diese Interpretation von "Coleridge" (und die von „Bentham" im folgenden Kapitel) schließt aus, dass es Mill in den beiden Aufsätzen lediglich darum geht, dass Vertreter*innen zweier politischer Strömungen oder Anhänger*innen zweier philosophischer Methoden voneinander lernen können: 
Ich habe daher im letzten Teil dieses Kapitels gegen das, was ich die "gesellschaftspolitische Interpretation" genannt habe, und das, was ich die „methodische Interpretation“ genannt habe, argumentiert. 


\section{4. „Bentham“}

Nachdem ich im letzten Kapitel Coleridges Tugend erarbeitet habe, wird es nun um Benthams Tugend gehen. (Auch zwischen Bentham und dem Bild, das Mill von Bentham zeichnet, muss natürlich unterschieden werden. Ich werde aber diesmal auf diese Selbstverständlichkeit nicht gesondert eingehen, weil Mill hier nicht wie im Falle von "Coleridge" den eigenartigen Spagat unternimmt, diesen einerseits dem, was er "die intuitive Schule" nennt, zuzuordnen, ihn dann aber dafür zu loben, wie er besondere menschliche Erfahrungen in seiner Philosophie berücksichtigt. ${ }^{1}$ ) Eine, die sich in der Philosophie um Benthams Tugend bemüht, bemüht sich um eine bestimmte Form der Objektivität. Speziell für die Sozialphilosophie bedeutet das, dass wir uns bei der Evaluation sozialer Praktiken darum bemühen müssen, ihr Gutsein (oder ihr Schlechtsein) gegenüber möglichen Alternativen nachzuweisen.

Ein erster Schritt dazu ist das Infragestellen des Bestehenden. (In „Coleridge" hatte Mill auch Bentham „the great questioner of things established“2 bezeichnet.) Diese Charakterisierung, die ich in Abschnitt 4.1 erarbeiten werde, legt die gesellschaftspolitische Interpretation nahe. Vielleicht noch offensichtlicher als in "Coleridge“ macht Mill aber sehr deutlich, dass in dessen progressiven Einstellung seiner Meinung nach nicht die Hauptstärke von Bentham liegt. Es geht vor allem darum, das Gutsein sozialer Praktiken vor dem Hintergrund von Alternativen zu prüfen. Es liegt nahe, zu vermuten, Mill ziele damit auf den Utilitarismus Benthams ab: Darauf, dass wir mithilfe des Utilitarismus das Bessersein einer Praxis gegenüber einer anderen nachweisen können. Dies ist aber nicht der Fall: Obwohl Bentham Utilitarist ist und sich auch Mill in „Bentham" (mehr oder weniger) zum Utilitarismus bekennt, geht es Mill

1 Es gilt weiterhin: Wenn ich der Kürze halber von „Benthams Tugend“ spreche, dann sind damit nicht tatsächliche philosophische Stärken Benthams gemeint, sondern Stärken des Bildes, das Mill von Bentham zeichnet.

2 Mill, „Coleridge“, S. 119. Auch Coleridge könne, wenn auch in anderer Weise, so bezeichnet werden. Siehe Kapitel 3. 
um die funktionale Rolle, die der Utilitarismus in Überlegungen dieser Art spielt. Diesen Gedankengang und die Rolle werde ich in Abschnitt 4.2 erarbeiten. Bevor Benthams Tugend aber endgültig erarbeitet ist, ist es nötig, diese zu qualifizieren: Zwei Fragen sind dabei wichtig: Wie sollten wir die „wissenschaftlichen Dispositionen" verstehen, die nach Mill mit dieser Tugend Einzug in die Philosophie erhalten? Welche Rolle spielt für Mill die Tatsache, dass es letztlich einen Maßstab geben muss? Diese beiden Fragen diskutiere ich in Abschnitt 4.3.

Bevor ich mit dem ersten dieser drei Schritte beginne, folgt eine kurze Übersicht über den Text von „Bentham“: Mill beginnt „Bentham" wie auch „Coleridge“, indem er die Komplementarität beider Denker und ihre Beispielhaftigkeit für das, wofür sie stehen, hervorhebt. ${ }^{3}$ Auch in Bentham bieten sich alle drei im letzten Kapitel unterschiedenen Arten, diese Komplementarität zu verstehen an; und auch in diesem Kapitel werde ich, wie in Abschnitt 3.4, dafür argumentieren, dass wir das, was ich die „sozialphilosophische Interpretation" genannt habe, akzeptieren sollten.

Nach dieser Einleitung würdigt Mill zwei seiner Ansicht nach zusammenhängende Stärken von Bentham: Erstens sei er (wie Hume) ein kritischer Denker; er weite aber Kritik in Humes Stil als erste Philosoph*in auf die Sozialphilosophie aus. ${ }^{4}$ Zweitens, und wichtiger für Mill, bringe Bentham das Verlangen nach Verifikation in die Philosophie ein, sodass diese nicht bloßer Ausdruck individueller Sichtweisen sei. ${ }^{5}$ Durch die Ausarbeitung dieser zweiten von Mill erwähnten Stärke Benthams werde ich die Formulierung von Benthams Tugend erarbeiten.

Es folgen Benthams Schwächen. Diese wurden stärker rezipiert, einerseits, weil es überraschend ist, mit welcher Schärfe Mill hier sein ohne Zweifel wichtigstes philosophisches Vorbild ${ }^{6}$ kritisiert (insbesondere im Gegensatz zur viel sanfter formulierten Kritik an Coleridge in "Coleridge"); andererseits, weil es scheint, dass Mill diese aus unterschiedlichen Gründen in „Bentham“ tatsächlich wichtiger sind. Vor allem aber ist die Kritik stark auf die Person bezogen:

3 Siehe Mill, „Bentham“, S. 77-78.

4 Siehe ebd., S. 78-82.

5 Siehe ebd., S. 82-88.

6 Wie in einer Fußnote in Kapitel 1 erwähnt, bezeichnet Mill die Rolle, die Bentham für ihn spielt, als religionsähnlich. Siehe Mill, Autobiography, S. 69. 
Bentham hat Mill zufolge als Mensch Schwächen, die sich auf seine Philosophie auswirken. Es ist kein Zufall, dass Mills Kritik ad hominem ist: Es zeigt, dass die Ideale, um die es in „Bentham" und "Coleridge" geht, Ideale für philosophierende Personen - was ich also philosophische Tugenden nenne - sind. Benthams erste Schwäche ist, dass er unfähig ist, die Erfahrungen anderer Leute zu berücksichtigen beziehungsweise von anderen zu lernen. ${ }^{7}$ Benthams zweite Schwäche ist, dass ihm selber wichtige menschliche Erfahrungen unzugänglich sind. ${ }^{8}$

Diese Schwächen wurden im letzten Kapitel zum Teil schon angesprochen; sie sind der Gegensatz zu den Stärken Coleridges. Es sollte also nicht verwundern, dass Mill hier auch das Wort „Erfahrung" verwendet, um die methodische Stärke von Bentham und die entsprechende Schwäche von Coleridge zu beschreiben; er verwendet das Wort „Erfahrung" genauso, um die Stärke von Coleridge ${ }^{9}$ und die Schwäche von Bentham zu beschreiben: Wenn Benthams Empirismus derjenige eines Mannes ist, der wenig Erfahrung hatte, ${ }^{10}$ so ist Coleridges Intuitionismus derjenige eines Mannes, der echte menschliche Erfahrungen ernst nimmt und ihnen Ausdruck verleihen kann. ${ }^{11}$

Diese Schwächen Benthams führen nach Mill dazu, dass Bentham ein sehr verarmtes Bild der menschlichen Natur hat, dass Mill danach beschreibt. ${ }^{12}$ Dies wiederum führe zu einer sehr beschränkten Moralphilosophie ${ }^{13}$ und einer beschränkten Sozialphilosophie und

7 Siehe Mill, „Bentham“, S. 89-91.

8 Siehe ebd., S. 91-94.

9 Siehe Abschnitt 3.2.

10 Dies sagt Mill über Bentham: siehe Mill, „Bentham“, S. 92.

11 Eine der Erfahrungen, die Bentham laut Mill fehlt, ist die Erfahrung der seelischen Verzweiflung. Siehe ebd., S. 92. Dass Mill meint, Coleridge habe solche Erfahrungen und könne ihnen Ausdruck verleihen, wird in der Autobiographie deutlich, wo Mill, wie bereits erwähnt, Gedichte von Coleridge benutzt, um seine eigene Krisenerfahrung verständlich zu machen. Siehe Mill, Autobiography, S. 139 und S. 145.

12 Siehe Mill, „Bentham“, S. 94-97.

13 Mills Kritik ist zum Teil verheerend: „It is fortunate for the world that Bentham's taste lay rather in the direction of jurisprudential than of properly ethical inquiry. Nothing expressly of the latter kind has been published under his name, except the Deontology-a book scarcely ever, in our experience, alluded to by any admirer of Bentham without deep regret that it ever saw the light." Ebd., S. 98. 
politischen Philosophie, weil beide laut Mill natürlich auf dem Bild des Menschen aufbauen müssten. ${ }^{14}$

Wie auch in Coleridge soll der überwiegende Teil des Restes des Aufsatzes anhand von Beispielen die Stärken von Bentham verdeutlichen: „We turn with pleasure from what Bentham could not do, to what he did. [...] The unpleasing part of our work is ended."15 In Wirklichkeit ist es aber keineswegs so, dass Mill in dem, was folgt, Bentham nicht mehr (zum Teil auch grundlegend) kritisieren würde. Es scheint eher so zu sein, dass die Kritik, die Mill nun übt, nicht so sehr auf die persönlichen Schwächen von Bentham abzielt, sondern Benthams Schwächen anderweitig entschuldbar sind. In Bereich des Recht und der Rechtsphilosophie einerseits ${ }^{16}$ und Benthams Theorie der Regierung andererseits ${ }^{17}$ scheinen es nicht Benthams persönliche Schwächen zu sein, die seine Theorien defizitär machen. Außerdem hat Mill - vor allem vor dem Hintergrund des (vor Bentham) vorherrschenden Rechtsverständnisses ${ }^{18}$ - hier auch viel zu loben. Es folgen in „Bentham" abschließende, zum Teil kritische Bemerkungen Mills.

\subsection{Bentham als Kritiker}

Mill verwendet eine eigenartige Terminologie, um die beiden Stärken Benthams zu beschreiben: Er sei einerseits ein „negativer oder destruktiver Philosoph"19 gewesen, andererseits aber ein „positiver“20. Den „positiven“ Aspekt werde ich im nächsten Abschnitt diskutieren. Was „negativ“ hier bedeutet, ist zunächst leichter zu verstehen: Dass Bentham für Mill komplementär zu Coleridge ist, zeigt sich zunächst nämlich daran, dass er nicht konservativ, sondern kritisch gegenüber der „received opinion on any subject“21 ist: "he is the great subversive, or, in the language of continental philosophers, the great critical, thinker of his age and country." ${ }^{\text {"2 }}$

14 Siehe Mill, „Bentham“, S. 97-100.

15 Ebd., S. 100.

16 Siehe ebd., S. 100-105.

17 Siehe ebd., S. 105-110.

18 Siehe ebd., S. 100-103.

19 Siehe ebd., S. 79.

20 Siehe ebd., S. 82.

21 Siehe ebd., S. 82.

22 Ebd., S. 79. 
Mill stellt Bentham dazu in eine Reihe mit Hume: Zwar sei er $\mathrm{Hu}-$ me, was Humes Qualitäten diesbezüglich angeht, weit unterlegen gewesen, aber

there was a wide field left vacant for him by Hume, and which he has occupied to an unprecedented extent; the field of practical abuses. This was Bentham's peculiar province: to this he was called by the whole bent of his disposition: to carry the warfare against absurdity into things practical. ${ }^{23}$

Wie sieht Benthams Methode der Kritik aus? Sie wird in „Coleridge" charakterisiert als "short and easy method of referring all to the selfish interests of aristocracies, or priests, or lawyers, or some other species of impostors “24. Benthams Kritik an der Parteilichkeit der Institutionen wird in „Bentham“ ausführlicher ausgeführt und die Interessen herrschender Klassen herausgestellt. ${ }^{25}$ In „Coleridge“ charakterisiert Mill diese Perspektive Benthams, wie bereits zitiert, als Außenperspektive ${ }^{26}$ - gemeint hatte ich, dass Bentham auf Institutionen und Praktiken die Perspektive einer Außenstehenden einnimmt. In Bentham wird diese Perspektive an die Darstellung des Bentham'schen Menschenbildes geknüpft und sie erscheint weniger als Außenperspektive, sondern sehr viel mehr als eine sehr verarmte Innenperspektive:

Man is conceived by Bentham as a being susceptible of pleasures and pains, and governed in all his conduct partly by the different modifications of self-interest, and the passions commonly classed as selfish, partly by sympathies, or occasionally antipathies, towards other beings. And here Bentham's conception of human nature stops. ${ }^{27}$

Diese Perspektive ist keine Außenperspektive im Sinne einer naturwissenschaftlichen Betrachtung: Menschliche Handlungen, das Festhalten an unfairen Institutionen durch diejenigen, die davon profitieren, beispielsweise, erscheinen in dieser Perspektive als ra-

\footnotetext{
23 Ebd., S. 80f.

24 Mill, „Coleridge“, S. 120.

25 Vgl. Mill, „Bentham“, S. $109 f$.

26 Mill, „Coleridge“, S. 119.

27 Vgl. Mill, „Bentham“, S. 94.
} 


\section{4. „Bentham"}

tional. ${ }^{28}$ Sie ist aber eine Außenperspektive in dem Sinne, dass die Rationalisierung, die hier angeboten wird, keine ist, die den Beteiligten an der Institution offen stünde. Die Perspektive Benthams gleicht in dieser Hinsicht vielleicht Foucault oder Bourdieu: Die Behauptung, menschliches Handeln sei symbolischer Ausdruck von Macht oder menschliches Handeln könne stets als Akkumulation verschiedener Arten von Kapital begriffen werden, liefern ein allgemeines $^{29}$ Rationalisierungsmodell, das sich zur Kritik bestehender Institutionen eignet, indem es herausstellt, wem diese letztlich dienen. Dieses kommt aber nicht für die Teilnehmer*innen an den entsprechenden Institutionen zur Selbstbeschreibung in Frage. Um ein naheliegendes Beispiel zu nehmen: Wir können zwar die Arbeit an einer philosophischen Dissertation als den Erwerb institutionalisierten kulturellen Kapitals in Bourdieus Sinne verstehen - wenn es zum Beispiel unser Ziel ist, eine Präferenz derjenigen, die im Besitz dieses Kapitals sind, für die Institution der Universität beziehungsweise die Form von Bildung, die in einer philosophischen Dissertation zum Ausdruck kommen soll, zu verstehen. Wir können aber kaum unser eigenes Arbeiten an unseren akademischen Projekten ausschließlich unter dem Gesichtspunkt der Kapitalakkumulation rationalisieren. ${ }^{30}$

28 Es ist stattdessen - obwohl weder Mill noch Bentham diese Idee noch nicht kannten - die Perspektive einer verstehenden Sozialwissenschaft (im Gegensatz zur erklärenden Naturwissenschaft). Vgl. z. B. Max Weber. Wirtschaft und Gesellschaft. Grundriß der verstehenden Soziologie [1921]. Tübingen: Mohr, 1976, Kap. 1, § 1.

29 Die Allgemeinheit ist sicher an das kritische Potential geknüpft: Weil wir alle oder fast alle Handlungen tatsächlich im Lichte dieser Theorien sehen können, taugen sie zur kritischen Beleuchtung eben dieser Handlungen. In diesem Sinne kann nicht gesagt werden, Benthams Theorie sei nicht umfassend: Er hat „one sort of comprehensiveness, but not [...] another." Mill, „Bentham", S. 88.

30 Wir können und sollten natürlich unser eigenes Tun auch unter Perspektiven wie der von Bentham, Foucault oder Bourdieu sehen - und zwar auch aus praktischen Gesichtspunkten: Die dadurch eröffnete Perspektive auf das eigene Tun ermöglicht es, sich auf eine besondere Weise vom eigenen Tun $\mathrm{zu}$ distanzieren und letztlich das eigene Verhalten anzupassen. Gemeint ist stattdessen nur: Wir können unser Tun nicht ohne Weiteres ausschließlich in Begriffen dieser Perspektive wertschätzen. 
Und wie bei Bourdieu und Foucault ist auch für Bentham unser tatsächlicher politischer und moralischer Diskurs eines der Hauptziele des Angriffes:

Proceeding on this principle, Bentham makes short work with the ordinary modes of moral and political reasoning. These, it appeared to him, when hunted to their source, for the most part terminated in phrases. In politics, liberty, social order, constitution, law of nature, social compact, \&c., were the catch-words: ethics had its analogous ones. ${ }^{31}$

Diese leeren Phrasen und Schlagworte dienen nach Bentham einfach dazu, der Adressat*in die Meinung der Autor*in aufzuzwingen, wobei die politischen und moralischen Phrasen sowohl der Verschleierung dieses Vorgangs gegenüber den Adressat*innen als auch der Selbsttäuschung über das, was wirklich geschieht, dienen. ${ }^{32}$ Menschen, insbesondere Philosoph*innen, verwendeten moralische Phrasen, um andere Menschen dazu zu bringen, die eigene mora-

31 Mill, „Bentham“, S. 84.

32 Siehe ebd., S. 84. Neben der asketischen Moral lassen sich alle anderen Moraltheorien nach Bentham auf das Prinzip der Sympathie und Antipathie zurückführen, egal, ob sie kognitivistische oder non-kognitivistische Ansprüche hätten: „In looking over the catalogue of human actions (says a partizan of this principle) in order to determine which of them are to be marked with the seal of disapprobation, you need but to take counsel of your own feelings: whatever you find in yourself a propensity to condemn, is wrong for that very reason." Bentham, An Introduction to the Principles of Morals and Legislation, § II.XIII. In diesem Zusammenhang steht auch Benthams Überlegung zu moralischen Phrasen, auf die Mill im obigen Zitat anspielt: „They consist all of them in so many contrivances for avoiding the obligation of appealing to any external standard, and for prevailing upon the reader to accept of the author's sentiment or opinion as a reason for itself. The phrases different, but the principle the same." Ebd., § II.XIV. Dies wird noch beispielhaft erläutert durch eine lange Fußnote, die Mill eine Seite später fast vollständig zitiert, und die die Benutzung solcher moralischer Sprache als Verschleierung und als Selbsttäuschung beschreibt: „It is curious enough to observe the variety of inventions men have hit upon, and the variety of phrases they have brought forward, in order to conceal from the world, and, if possible, from themselves, this very general and therefore very pardonable self-sufficiency. [...] The mischief common to all these ways of thinking and arguing (which, in truth, as we have seen, are but one and the same method, couched in different forms of words) is then serving as a cloke, and pretense, and aliment, to despotism [...]." Ebd., § II.XIVn. 


\section{4. „Bentham“}

lische Meinung anzunehmen; auf der Oberfläche sehe das, was geschieht, wie eine Begründung aus; aber, was in Wirklichkeit geschehe, sei, dass die eigenen Sympathien und Antipathien gegenüber bestimmten Handlungen durchgesetzt werden.

Wir könnten natürlich hinterfragen, ob es wirklich gerechtfertigt ist, eine so beschriebene Perspektive (und Foucaults und Bourdieus) als kritisch $\mathrm{zu}$ bezeichnen: Bentham bezeichnet das, was im tatsächlichen moralischen Diskurs passiert, wie in Fußnote 32 belegt, beispielsweise als „Despotismus" - und es scheint, als funktioniere dieses Wort bei ihm sehr ähnlich, wie er es seinen Gegner*innen unterstellt. ${ }^{33}$ Wieso sollten wir, falls wir seine Beschreibung der Funktion moralischer Phrasen akzeptieren, uns seiner Bewertung anschließen? Eine Alternative wäre ja, diese Beschreibung bereitwillig als wesentlich für die Moral anzunehmen: In der Tat klingt die Funktion moralischer Äußerungen in Stevensons Version des Emotivismus, wenn von der Bewertung dieser Funktion abgesehen wird, sehr ähnlich wie das, was Bentham beschreibt. ${ }^{34}$ Wenn Mills Bentham also nicht mehr dazu sagt, droht seine Position, sich selbst zu unterminieren. Natürlich sagt er mehr, dazu komme ich in Kürze.

Auch in Bezug auf Foucault wurde diese Kritik geäußert: So legt Habermas nahe, die Art und Weise, wie Foucault die Grundlage der aufklärerischen Tradition in Begriffen der Macht rekonstruiere, unterminiere letztlich die Position der Kritiker*in selbst. ${ }^{35}$ Nicht anders als die Aristokraten, Anwälte und Priester, in deren Interesse soziale Praktiken und Institutionen laut Bentham zu seiner Zeit oft gewesen seien, läuft auch Benthams Kritik an ihnen Gefahr, nicht mehr als Ausdruck eines Interesses zu sein. So lange Bentham nichts dazu sagen kann, was sein Interesse der Kritik an den Institutionen von dem Interesse der Aristokraten, Anwälte und

33 Es ist interessant, dass Mill diese Bewertung Benthams im Zitat der Fußnote, die er ansonsten fast vollständig zitiert, auslässt. Mill, „Bentham“, S. 86.

34 Vgl. Charles Leslie Stevenson. „Die emotive Bedeutung ethischer Ausdrücke“. In: Sprache und Ethik - Zur Entwicklung der Metaethik. Hrsg. von Günther Grewendorf und Georg Meggle. Frankfurt am Main: Suhrkamp, 1974, S. 116139.

35 Z. B. „How can Foucault's selfunderstanding as a thinker in the tradition of the enlightenment be compatible with his unmistakable criticism of this very form of knowledge of modernity?" Jürgen Habermas. „Taking Aim at the Heart of the Present". In: Foucault - A Critical Reader. Hrsg. von David C. Hoy. Oxford und andere: Basil Blackwell, 1986, S. 103-108, S. 106. 
Priester unterscheidet, so lange kann er uns zwar etwas über die soziale Wirklichkeit beibringen, aber nichts von dem, was wir so lernen können, sagt uns, ob wir diese gut oder schlecht finden sollen. So könnten wir Habermas' Kritik an Foucault versuchen auf das bisher Gesagte zu übertragen.

Natürlich müssen wir mit der Bezeichnung einer Position als „kritisch" nicht so streng sein. Warum sollten wir nicht eine theoretische Position, die in kritischer Absicht formuliert wird und dabei stellenweise durchaus effektiv ist, nicht als kritisch bezeichnen, bloß weil diese es unterlässt, gleichzeitig den Standpunkt der Kritikerin als rationalen Standpunkt zu rekonstruieren? Es gibt viele Hinsichten, in denen solche Positionen effektiv bleiben: Selbst, wenn sie nichts anderes machen, so entfremden sie uns immer noch von gewohnten Auffassungen über soziale Institutionen und Praktiken und liefern uns so Beschreibungen einer Realität, mit der wir dann nicht zufrieden sind - auch wenn die Unzufriedenheit zunächst lediglich in der Fremdheit liegt: Darin nämlich, dass sie zumindest auch etwas anderes sind, als wir bisher dachten, und sich so zumindest die Frage eröffnet, ob sie vielleicht nur das sind. Trotzdem will Bentham Mill zufolge mehr, wie in Abschnitt 4.2 deutlich werden sollte.

Damit, dass Benthams kritisches Potential hier mit Foucault und Bourdieu verglichen wurde, soll übrigens keineswegs gesagt werden, dass es egal sei, auf Grundlage welcher dieser Vorstellungen wir die Institutionen und Praktiken in kritischer Absicht rekonstruieren. Der Begriff „Interesse“ hat sicherlich durch seine Verwendung im klassischen Liberalismus und in den Wirtschaftswissenschaften eine Geschichte, die ihn ungeeignet macht, wenn es zum Beispiel darum geht, kapitalistisch geprägte Institutionen und Praktiken in einem ihnen fremden Licht erscheinen zu lassen. Darüber hinaus mag "Interesse“ ein sehr viel defizitäreres Konzept sein als die angesprochenen Alternativen, wenn es darum geht, die subtilen symbolischen Aspekte der gesellschaftlichen Kontrolle herauszustellen, die mit unseren Institutionen einhergehen. Auf der anderen Seite sollte Bentham hier auch nicht unterschätzt werden. Außer der in meiner Fußnote 32 angesprochenen Idee des concealment hebt Mill zum Beispiel hervor, dass Bentham mit dem Begriff des „interest-begotten prejudice" durchaus den Anspruch hatte, par- 


\section{4. „Bentham"}

teiische Machtausübung, die denjenigen, die diese Macht ausüben, zum Beispiel als Moral erscheint, zu entlarven. ${ }^{36}$

Eine Möglichkeit wäre es, den Wert, den Bentham für Mill hat, in der Effektivität der gesellschaftlichen Kritik oder der Tatsache, dass er sich überhaupt um radikale Kritik bemüht, zu sehen. Dies würde der Dichotomie konservativ-progressiv entsprechen und die gesellschaftspolitische Interpretation der Aufsätze nahelegen. Dies wäre analog dem Argument, das auf Seite 25 für einen abgeleiteten Wert der zweiten Tugend diskutiert wurde. Dies die sozialphilosophische Interpretation unterminieren, die ich favorisiere. Meine Strategie hier ist ähnlich, wie ich mit dem analogen Einwand im vorigen Kapitel umgegangen bin: Ich stimme auf systematischer Ebene emphatisch zu, dass kritische Perspektiven in dieser Hinsicht ihre eigene Berechtigung haben. (Und zwar auch, wenn sie sich aus der Sicht der Teilnehmer*in an einer Praxis nicht zur Begründung ihrer Handlungen eignen. Dieser Vorwurf läuft in gewisser Hinsicht einfach ins Leere: Dies ist bereits berücksichtigt, insofern die Perspektive der Handelnden bei Bentham immer eine bereits korrumpierte Perspektive ist.) Ich stimme auch exegetisch zu, dass auch Mill dem emphatisch zustimmt. Dies lässt aber, wie oben, den Punkt unberührt, dass es zusätzlich zum Wert, den Kritik dieser Sicht zufolge immer hat, eine philosophische Tugend gibt, die

36 Vgl. Mill, „Bentham“, S. 109. Der Begriff „interest-begotten prejudice“ stammt aus The Book of Fallacies und wird dort so erklärt: „If by interest in some shape or other-that is, by a motive of some sort or other-every act of the will, and thence every act of the hand, is produced, so, directly or indirectly, must every act of the intellectual faculty: though in this case the influence of the interest, of this or that motive, is neither so perceptible, nor in itself so direct as in the other.

But how (it may be asked) is it possible that the motive a man is actuated by can be secret to himself? Nothing is more easy-nothing more frequent: indeed the rare case is, not that of his not knowing, but that of his knowing it." (S. 477)

Kurz: Nicht nur was wir tun, auch, wovon wir überzeugt sind, wird durch unser Interesse verursacht, auch wenn wir uns dessen oft nicht bewusst sind. Neben dem von Mill zitierten ,interest-begotten prejudice“ gibt es noch das "self-conscious sinister interest", das "authority-begotten prejudice" und etwas, dass ich als intellektuellen Opportunismus (mitunter aus guter Absicht) bezeichnen würde. Vgl. Jeremy Bentham. „The Book of Fallacies“. In: The Works of Jeremy Bentham, Volume II. Hrsg. von John Bowring. London: Simpkin, Marshall \& Co., 1843, S. 374-487, S. 475-480. 
Mill in Bentham sieht, deren philosophisches Gutsein nur zu tragen kommen könnte, wenn Bentham auch die Tugend von Coleridge besäße.

Worauf es Mills Bentham nämlich eigentlich ankommt, ist, dass Bentham nach der objektiven Grundlage für unsere moralischen, politischen und philosophischen Diskurse fragt und so eine Unterscheidung ziehen will zwischen philosophischen (oder ethischen oder politischen) Gedanken, die uns aufgrund unserer Prägung als wahr (oder gut) erscheinen, und solchen, die es wirklich sind. Die Möglichkeit von Kritik der entsprechenden Diskurse ist eine Konsequenz dieses Vorgehens; aber Benthams Vorgehen hätte auch einen philosophischen Wert, wenn sich die untersuchte philosophische Meinung an der Erfahrung als wahr herausstellen würde. Dies werde ich im folgenden Abschnitt genauer erläutern.

\subsection{Benthams Bemühen um Objektivität}

Mill scheint in der Tatsache, dass Bentham nicht ein rein negativer Philosoph gewesen ist, die eigentliche Stärke gesehen zu haben. Zum Teil hat das vielleicht auch damit zu tun, dass Bentham anderen kritischen Philosoph*innen laut Mill nicht das Wasser reichen kann:

If Bentham had merely continued the work of Hume, he would scarcely have been heard of in philosophy; for he was far inferior to Hume in Hume's qualities, and was in no respect fitted to excel as a metaphysician. We must not look for subtlety, or the power of recondite analysis, among his intellectual characteristics. In the former quality, few great thinkers have ever been so deficient [...]. Bentham had not these peculiar gifts; but he possessed others $\left[\ldots . .{ }^{37}\right.$

37 Mill, „Bentham“, S. 80. Die anderen Begabungen, die Mill hier meint, werden an dieser Textstelle nicht eindeutig genannt. In der Abgrenzung zu Hume scheint es Mill schon darum zu gehen, dass Bentham "positive“ Philosophie betreibe, während Hume „nur" negative Philosophie betreibe. Andererseits beziehen sie sich vielleicht auch auf die Ausdehnung auf das Praktische, das Mill auch in den Begriffen von Disposition und Begabung beschreibt: „This was Bentham's peculiar province: to this he was called by the whole bent of his disposition: to carry the warfare against absurdity into things practical. His was an essentially practical mind." Ebd., S. 80. 


\section{4. „Bentham“}

Aber Mill meint nicht nur, dass Benthams Leistung dadurch verbessert wird, dass er sich nicht auf ein Feld beschränkt, in dem er nicht sonderlich begabt ist. Auch das Feld selbst ist weniger wert als das, worin Benthams eigene Stärke liegt. Positive Philosophie scheint für Mill mehr wert zu sein als „bloß" negative Philosophie - selbst wenn sie mit Humes Fähigkeiten betrieben wird:

We consider this, however, to be not his highest title to fame. Were this all, he were only to be ranked among the lowest order of the potentates of mind-the negative, or destructive philosophers; those who can perceive what is false, but not what is true; who awaken the human mind to the inconsistencies and absurdities of time-sanctioned opinions and institutions, but substitute nothing in the place of what they take away. ${ }^{38}$

Was ist aber mit Benthams „positivem“, offenbar wichtigerem Beitrag gemeint? Mill schreibt, es gehe hier um Benthams Methode: Er habe in die Moralphilosophie und die politische Philosophie Denkgewohnheiten und Arten der Untersuchung eingeführt, die für die Wissenschaften wesentlich seien und deren Abwesenheit diese Bereiche $\mathrm{zu}$,a field of interminable discussion, leading to no result" gemacht hätten. ${ }^{39}$ Es liegt vielleicht nahe, hinter der Methode, die die praktische Philosophie der Wissenschaft ähnlicher mache, Benthams utilitaristisches Verfahren zur Evaluation sozialer Institutionen zu vermuten. Immerhin wird der Utilitarismus oft als das Bindeglied zwischen Mill und Bentham gesehen.

Das oben angesprochene Problem, dass sich Benthams kritische Perspektive systematisch selbst unterminiert, würde dieser Idee zufolge dadurch behoben, dass Bentham seine eigene Perspektive utilitaristisch untermauert: „Despotismus“ mag eine moralische Phrase sein und damit besteht die Gefahr, dass der Ausdruck selbst unter das fällt, was er kritisieren soll. Aber, ob eine moralische Phrase despotisch ist oder nicht, hängt nach dieser Idee davon ab, ob sie sich utilitaristisch begründen lässt. (Selbstverständlich wäre dies nur eine Lösung, wenn sich das, was Bentham mit „despotisch" meint, utilitaristisch rekonstruieren lässt. Das lässt sich natürlich mit guten Gründen bezweifeln. Für die Zwecke dieses Kapitels ist

38 Mill, „Bentham“, S. 79.

39 Ebd., S. 79. 
es aber unerheblich, ob Benthams Kritik des moralischen Diskurses als „despotisch“ utilitaristisch rekonstruiert werden kann.) Die Idee, dass der Utilitarismus ein Maßstab sein könnte, der nicht bloß formale, sondern auch materiale Kritik zulassen könnte, geht auch bereits in Richtung dessen, was Mill wichtig ist.

Aber Mill meint den Utilitarismus selbst offensichtlich hier nicht: Erstens spricht Mill den Utilitarismus nur auf knapp zwei Seiten ganz am Ende des Aufsatzes an und, so sind seine Bemerkungen wohl zu verstehen, eher weil er meint, seine Leser*innen erwarteten von ihm, etwas dazu zu sagen; ${ }^{40}$ dort bekennt er sich zwar im Allgemeinen zu Benthams Prinzip, distanziert sich aber auch von Bentham bis zu einem gewissen Grade; ${ }^{41}$ er spielt zumindest in der 1838 veröffentlichten Originalfassung außerdem die Bedeutung des utilitaristischen Prinzips in der hier angedeuteten Funktion herunter:

All we intend to say at present is, that we are much nearer to agreeing with Bentham in his principle, than in the degree of importance which he attached to it. [...] As mankind are much more nearly of one nature, than of one opinion about their own nature, they are much more easily brought to agree in their intermediate principles [...] than in their first principles [...]. We consider, therefore, the utilitarian controversy as a question of arrangement and logical subordination rather than of practice; important principally in a purely scientific point of view, for the sake of the systematic unity and coherency of ethical philosophy. Whatever be our own opinion on the subject, it is from no such source that we look for the great improvements which we believe are destined to take place in ethical doctrine. ${ }^{42}$

40 Siehe ebd., S. $110 \mathrm{f}$.

41 ,[W]e do not hold with him that all right thinking on the details of morals depends on its express addertion." Ebd., S. 110. Es werden in diesem Zusammenhang auch bereits „,secondary ends" und „secondary principles" erwähnt. Siehe dazu Abschnitt 5.1.3 dieser Arbeit.

42 Ebd., 110f. Dies widerspricht natürlich der Rolle, die Mill dem utilitaristischen Prinzip in „Utilitarianism" zugesteht (ich diskutiere dies in Abschnitt 6.1) und ist in den Ausgaben ab 1859 von Mill entsprechend verändert worden: Mill erwähnt in Ausgaben ab diesem Zeitpunkt an der Textstelle die Rolle des Utilitarismus bei der Entscheidung zwischen sich widersprechenden sekundären Prinzipien; er streicht außerdem erstens die Behauptung, er hal- 


\section{4. „Bentham"}

Der zweite Gedanke, der dagegen spricht, dass es sich bei Benthams positiven Beitrag um den Utilitarismus oder das utilitaristische Verfahren handeln könnte, ist, dass Mill an mehreren Stellen betont, es seien nicht seine „doctrines" oder „opinions"43 die er in „Bentham" hervorheben wolle; der Utilitarismus sei keine originelle Idee Benthams gewesen; ${ }^{44}$ es sei ein bloßer Zufall, dass Bentham auch Utilitarist sei ${ }^{45}$ er habe seine Tugend an die Intellekte vieler Denker*innen weitergegeben, die seine „opinions“ niemals angenommen oder aufgegeben hätten und habe so auch seine philosophischen Gegenspieler*innen gelehrt; ${ }^{46}$ u.s.w.

Es ist also nicht die utilitaristische Methode der kritischen Bewertung gegebener sozialer Institutionen selbst, in der Benthams Tugend besteht. Aber die utilitaristische Methode kann als Beispiel dienen, um was es geht. Irgendetwas, das die Funktion erfüllt, die die utilitaristische Methode in den hier angedeuteten Überlegungen hat, brauchen wir laut Mill. ${ }^{47}$ Wir müssen uns, so meint Mill, also bemühen, die Philosophie der Wissenschaft in der Hinsicht ähnlicher zu machen, dass sie nicht in endlosen Diskussionen, die zu keinem Ergebnis führen, endet. Ein Beispiel für ein solches Bemühen wurde mit Benthams Kritik der moralischen Phrasen gegeben. Ein Streit zwischen Philosoph*innen, die der von Bentham

te die Frage der Wahrheit des Utilitarismus nicht für wichtig und eine rein wissenschaftliche Frage und er streicht zweitens die Behauptung, er erwarte keinen ethischen Fortschritt durch die Akzeptanz des utilitaristischen Prinzips. Aber obwohl die Textstelle nach diesen Änderungen den Äußerungen in „Utilitarianism" nicht mehr widerspricht, spielt Mill die Wichtigkeit seiner utilitaristischen Gemeinsamkeit mit Bentham dem Tonfall nach immer noch herunter. Vgl. Mill, „Bentham“, $110 \mathrm{f}$.

43 Beides: ebd., S. 83.

44 ,The generalities of his philosophy itself have little or no novelty: to ascribe any to the doctrine that general utility is the foundation of morality, would imply great ignorance of the history of philosophy, of general literature, and of Bentham's own writings." Siehe ebd., S. 86.

45 Siehe ebd., S. 87.

46 Siehe ebd., S. 87.

47 Vgl. dazu ebd., S. 111. Ob es dafür wirklich einen Maßstab geben muss und Moral konsequentialistisch - wenn auch nicht utilitaristisch - aufgefasst werden muss, wie Mill hier anzudeuten scheint, diskutiere ich in Abschnitt 4.3. Vergleiche ähnliche Überlegungen Mills dazu in Mill, The Collected Works of John Stuart Mill, Volume VIII - A System of Logic Ratiocinative and Inductive (Books IV-VI and Appendices), S. 951 und im ersten Kapitel von Mill, "Utilitarianism". 
kritisierten Schule der Sympathie und Antipathie angehören, würde letztlich in despotischen Überredungsversuchen beider Seiten enden. Wieso ist die Kritik an diesen Philosoph*innen als despotisch aber nicht einfach eine dritte Position, die wiederum in eine endlose Diskussion mit den beiden anderen einsteigen müsste? Weil - unter der Voraussetzung, dass der Utilitarismus selbst oder irgendetwas, das diese Funktion erfüllt, irgendwie ${ }^{48}$ begründet werden kann, und unter der Voraussetzung, dass das, was mit „despotisch“ gemeint ist, utilitaristisch rekonstruiert werden kann - ein Verfahren zur Entscheidung zwischen den Positionen gefunden wurde. Natürlich stehen beide Voraussetzungen auf dünnem Eis. In der frühen Version des Aufsatzes legt Mill aber keinen Wert darauf, dass dieses Verfahren der Utilitarismus sein müsse; alles was feststeht, ist, dass wird eine Art und Weise finden müssen, nicht bloß subjektiv zwischen philosophischen Position zu entscheiden.

Aber: In den späteren Fassungen des Aufsatzes (ab 1859) spricht Mill dem Utilitarismus dann doch genau diese Rolle zu. Wenn nämlich „secondary ends“49 - zu denen wahrscheinlich einerseits zählt, anderen gegenüber nicht despotisch aufzutreten, aber andererseits sicher auch das, was durch die moralischen Phrasen der „ordinary modes of moral and political reasoning " 50 bezeichnet wird - in Konflikt geraten, dann dient das utilitaristische Prinzip dazu, eine Antwort $\mathrm{zu}$ finden. ${ }^{51}$ Trotzdem ist das utilitaristische Prinzip auch, wenn wir nur die spätere Fassung berücksichtigen würden, nur ein Spezialfall oder eine Anwendung eines allgemeineren Ideals - und zwar aus den genannten Gründen.

Die Rolle, die Benthams Tugend zukommt, kann präzisiert werden, wenn wir einen Aspekt von Mills Charakterisierung von Benthams „positiver" Philosophie im Kontrast zur „negativen“ Philosophie von Hume u.s.w. berücksichtigen, auf die ich mich später in dieser Arbeit mit dem Begriff „Benthams Herausforderung“ an die Philosoph*innen beziehen möchte:

Their [gemeint sind die negativen Philosophen] character was exclusively analytic, his [gemeint ist Bentham] was synthetic. They took for their starting-point the received opinion on

48 Siehe zu diesem ernsthaften Problem Abschnitt 6.1.2.

49 Mill, „Bentham“, S. 110.

50 Ebd., S. 84.

51 Ebd., S. 111. 
any subject, dug round it with their logical implements, pronounced its foundations defective, and condemned it: he began de novo, laid his own foundations deeply and firmly, built up his own structure, and bade mankind compare the two $[\ldots] .^{52}$

Die Textstelle zeigt nicht nur den revisionären Charakter der benthamitischen Philosophie: Benthams Utilitarismus ist nach Mill nicht als Analyse unserer moralischen Begriffe gedacht, sondern als deren Ersatz, auch wenn bereits Bentham meint, dass das Prinzip der Sympathie und Antipathie oft, ohne dies zu beabsichtigen, mit dem Utilitarismus deckungsgleich ist. ${ }^{53}$ Hier geht es aber, wie gesagt, Mill nicht darum, Benthams utilitaristisches System selbst zu verteidigen, sondern darum, ein Anliegen seiner Philosophie deutlich zu machen: Benthams Herausforderung ist es, dass unsere überlieferten Meinungen (und hier sind auch soziale Praktiken gemeint, siehe Abschnitt 2.4) in der philosophischen Untersuchung nicht bloß daran scheitern können, in sich widerspruchsfrei zu sein - dies kann auch mit „negativer" Philosophie nachgewiesen werden. Eine philosophische Untersuchung muss auch ein materiales Scheitern nachweisen können, indem sich unsere Praktiken als schlechter erweisen als (mitunter radikale) Alternativen.

Anders ausgedrückt: Unsere Praktiken (oder überlieferten Meinungen) könnten auch sehr anders sein. Benthams Herausforderung besteht vor dem Hintergrund dieser Feststellung darin, sich vor dem Hintergrund dieser Alternativen um eine Antwort zu bemühen, die die Frage tatsächlich entscheidet. Wohl auch aus diesem Grund habe sich Bentham, so Mill, auch darum bemüht, die größten Selbstverständlichkeiten zu beweisen: Das Mord, Aufwiegelung, Raub schlecht sei, dürfe nicht ohne Nachweis akzeptiert werden. ${ }^{54}$

\subsection{Qualifikationen von Benthams Tugend}

Die Grundidee von Benthams Tugend ist damit dargestellt. Ich möchte aber zwei Punkte ansprechen, die vielleicht als besonders kontrovers empfunden werden: zum einen Mills lobende Behaup-

52 Mill, „Bentham“, S. 82.

53 Bentham, An Introduction to the Principles of Morals and Legislation, S. II.V.

54 Siehe Mill, „Bentham“, S. 83. 
tung, die Philosophie werde, wenn sich die Philosophie mehr an Benthams Tugend orientiere, der Wissenschaft ähnlicher; zum anderen Mills Behauptung ganz am Ende des Aufsatzes, es müsse einen Maßstab geben, damit Benthams Tugend überhaupt realisiert werden kann. Die erste Behauptung durchzieht weite Teile des gesamten Aufsatzes. Die zweite Behauptung findet sich nur ganz am Ende (im Zusammenhang mit der Diskussion zum Utilitarismus); sie spielt dafür auch in anderen wichtigen Texten aus allen Lebensabschnitten Mills eine Rolle. Es erscheint mir daher wichtig, auf beide einzugehen. Meine Strategie im ersten Falle ist es, die Kontroverse zu entschärfen: Mills Behauptung, die Philosophie solle mehr wie die Wissenschaft werden, ist weniger radikal, als es den Anschein hat. Meine Strategie im zweiten Fall ist es, Mill zu widersprechen: Benthams Tugend setzt nicht voraus, dass wir einen Maßstab gefunden haben, anhand dessen wir unterschiedliche Praktiken bewerten können. Ich möchte also in diesem Falle behaupten, dass es möglich ist, Mills Bestimmung der Tugend von seiner persönlichen Überzeugung dazu, welche Anforderungen an eine philosophische Theorie mit dieser Tugend verbunden sind, zu trennen.

\subsubsection{Benthams Tugend und Wissenschaftlichkeit}

Ich werde drei Argumente anführen, warum die Behauptung Mills, durch Benthams Tugend werde die Philosophie eher wie die Wissenschaft, nicht als so kontrovers aufgefasst werden sollte, wie es vielleicht auf den ersten Blick den Anschein hat. Erstens ist gar nicht genau klar, welche Verpflichtungen Mill mit der Verwendung von „Wissenschaft" eingehen will. Zweitens sollten wir „wissenschaftlich“ hier nicht mit „empirisch“ gleichsetzen - auch wenn Mill natürlich glaubt, dass nur mit Erfahrung Begründetes letztlich wissenschaftlich sein kann, ist er für den Zweck, die Tugend Benthams zu erarbeiten, nicht daran interessiert, beides gleichzusetzen. Drittens ist Benthams Tugend in letzter Instanz nur als Komplement zu Coleridges Tugend ein vollständiges sozialphilosophisches Ideal.

Erstens. Mill vertritt darin, ob Moralphilosophie oder politische Philosophie als Wissenschaft bezeichnet werden sollte, keineswegs eine einheitliche Meinung: In dem in diesem Kapitel diskutierten Aufsatz schreibt er beispielsweise die Frage danach, ob der Utilita- 


\section{4. „Bentham"}

rismus wahr sei, sei eine Frage, die hauptsächlich aus „wissenschaftlicher" Perspektive interessant sei. ${ }^{55}$ Fünf Jahre später schreibt er im System of Logic, die Beschäftigung mit der Moral würde, sofern sie nicht die Wissenschaft des menschlichen Charakters betreffe, um die es in den ersten Kapiteln des Buches geht, zwar konventioneller-, aber streng genommen unzulässigerweise als Wissenschaft bezeichnet. ${ }^{56}$ In „Utilitarianism“ scheint Mill uneinheitlich zu sprechen. Auf der einen Seite scheint Mill die Dichotomie zwischen Kunst und Wissenschaft aus A System of Logic dort aufrecht erhalten zu wollen: „But though in science the particular truths precede the general theory, the contrary might be expected to be the case with a practical art, such as morals or legislation. "57 (Dieser Unterschied zwischen Kunst und Wissenschaft ist sogar der Grund dafür, warum wir die Frage, ob das utilitaristische Prinzip wahr ist, überhaupt klären müssen.) Auf der anderen Seite schreibt Mill einen Absatz später: ,[T]he intuitive school affirm as strongly as the inductive, that there is a science of morals. "58 Der Beweis ist allerdings kein Beweis in der gängigen Bedeutung dieses Wortes, weil Fragen nach letzten Zwecken solchen Beweisen nicht zugänglich sind. ${ }^{59}$ Wenn nach Mill Benthams Tugend die Philosophie also „wissenschaftlicher" macht, ist damit nicht unbedingt eine klar umrissene Vorstellung gemeint; eine „doctrine of ends" fällt möglicherweise auch darunter - angenommen Mill benutzt in „Bentham“, wie anscheinend zum Teil in „Utilitarianism“, die konventionelle und nicht die streng genommen nur zulässige Verwendungsweise des Wortes.

Zweitens. Mill meint zweifellos, dass Wahrheiten letztlich auf Erfahrung beruhen müssen. (Mills diesbezügliches Bekenntnis in „Coleridge ${ }^{600}$ wurde bereits in Kapitel 3 angesprochen. Ebenso ist aber zum Beispiel seine Zuordnung zur ,induktiven Schule“ der Moralphilosophie in „Utilitarianism“61 ${ }^{* u}$ verstehen und natürlich die

55 Vgl. Mill, „Bentham“, S. 111.

56 Vgl. Mill, The Collected Works of John Stuart Mill, Volume VIII - A System of Logic Ratiocinative and Inductive (Books IV-VI and Appendices), S. 943. Der damaligen Kapitelzählung zufolge wird dieses spätere 12. Kapitel noch

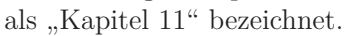

57 Mill, „Utilitarianism“, § 1.2.

58 Ebd., $\$ 1.3$.

59 Siehe ebd., § 1.5. Ich behandele den „Beweis" in Abschnitt 6.1.2.

60 Siehe Mill, „Coleridge“, S. 128

61 Siehe Mill, „Utilitarianism“, § 1.3. 
die theoretische Philosophie betreffenden Hauptideen aus System of Logic 62.) In „Bentham" verwendet er aber „empirical" nicht zur Selbstbeschreibung, sondern zur Beschreibung von Bentham - und charakterisiert dessen Methode im gleichen Atemzug als „empiricism"63. Der Begriff „Erfahrung“ wird in „Bentham" hauptsächlich verwendet, um einen Mangel bei Bentham auszudrücken, nicht, um eine seiner Stärken zu charakterisieren: Bentham fehlt die Fähigkeit zu bestimmten Erfahrungen. ${ }^{64}$ In der gleichen Weise wird „Erfahrung" verwendet, um eine Stärke von Coleridge zu charakterisieren, siehe Kapitel 3. Vor allem aber scheint Mill explizit zu meinen, dass Benthams Tugend auch von Nicht-Empirist*innen verwirklicht werden kann: Es gehört zu Benthams Tugend nach Mill zum Beispiel, vage Allgemeinheiten auf „facts (whether of experience or of consciousness)" zurückzuführen ${ }^{65}$.; mit Bewusstseinstatsachen soll sicherlich grob in Richtung nicht-empirischer Philosophie gedeutet werden, zumal Mill meint, dies sei ,a sound maxim, and one which all close thinkers have felt" - also nicht nur Empirist*innen. Dass Benthams Tugend auch von Nicht-Empirist*innen verwirklicht werden kann, zeigt auch Mills Würdigung des Einflusses Benthams: „This method Bentham has finally installed in philosophy; has made it henceforth imperative on philosophers of all schools. [...] He has taught the method to men of the most opposite schools to his [...]." "66. Wenn Mill also Benthams Tugend in „Bentham“ als wissenschaftlich charakterisiert, sollten wir ihn trotz seiner eigenen, heute als „empiristisch“ charakterisierbaren Überzeugungen nicht so lesen, dass hier empirische Wissenschaften oder gar Naturwissenschaften als Vorbild gemeint sind: Benthams Tugend ist nach

62 Siehe beispielsweise in der Zusammenfassung der Dinge, die bezeichnet werden können: John Stuart Mill. The Collected Works of John Stuart Mill, Volume VII - A System of Logic Ratiocinative and Inductive (Books I-III and Appendices). Hrsg. von J. M. Robson. Toronto und andere: University of Toronto Press und Routledge \& Kegan Paul, 1974, S. 75-77.

63 Siehe Mill, „Bentham“, S. 92. Zur kritischen Absicht, die bei Mill (stets) hinter der Verwendung dieses Wortes steht, siehe Anschutz, „The Logic of J. S. Mill“, S. 60. Diskutiert wurde dies in Abschnitt 3.3.1, siehe auch Fußnote 25 in diesem Abschnitt.

64 Siehe vor allem Mill, „Bentham“, S. 91-94.

65 Ebd., S. 84.

66 Ebd., S. 87. 
Mill allgemein genug, um nicht auf den Empirismus festgelegt $\mathrm{zu}$ sein.

Drittens. Selbst wenn wir Benthams Tugend so verstünden, dass die Philosophie sich, indem sich Philosoph*innen an ihr orientieren, in einer Hinsicht hin zu einer empirischen Wissenschaft oder sogar einer Naturwissenschaft bewegte, würde das nicht bedeuten, dass die Philosophie dem Wesen nach eine Naturwissenschaft würde: Benthams Tugend ist nur eines der Komplemente guter Philosophie. Coleridges Tugend macht das andere Komplement aus - und von dieser Tugend könnte mit gleichem Recht gesagt werden, sie bewege die Philosophie weg von der Wissenschaft; vielleicht wäre es in Mills Sinne zu sagen, sie bewege sie hin zur Poesie. ${ }^{67}$ Werden also beide Tugenden berücksichtigt, könnte die Spannung zwischen ihnen so ausgedrückt werden, dass die Philosophie ein bisschen wie die Wissenschaft und ein bisschen wie die Poesie sein sollte. Dies, so mag eingewendet werden, ist etwas dunkel, aber ich nehme an, es ist wesentlich weniger kontrovers als die erste Hälfte des Vergleichs alleine.

Wir sollten also die Tatsache, dass Mill Benthams Tugend mit Wissenschaftlichkeit der Philosophie assoziiert, nicht als zu radikal auffassen, weil erstens nicht ganz klar ist, welche Ansprüche Mill hier genau im Blick hat; zweitens aber klar ist, dass nicht die Ansprüche einer empirischen Wissenschaft oder gar Naturwissenschaft gemeint sind; und drittens damit keine vollständige Charakterisierung eines Wesenszuges der Philosophie gemeint ist, sondern nur ein Komplement eines philosophischen Ideals. Stattdessen sollten wir davon ausgehen, dass „Wissenschaftlichkeit“ in „Bentham“ zunächst nicht mehr bedeutet als die Ansprüche an die Sozialphilosophie, die ich mit der Formulierung von Benthams sozialphilosophischer Tugend explizit machen will: Die Sozialphilosophie muss sich auch darum bemühen, das Gutsein oder Schlechtsein einer sozialen Praxis vor dem Hintergrund zu prüfen, dass unsere Praxis auch ganz anders beschaffen sein könnte.

Dies mag immer noch für kontrovers gehalten werden, denn diese Charakterisierung schließt zum Beispiel sicher aus, dass Philosophie ausschließlich im Explizitmachen analytischer Sätze bestehen

67 Dies wird in Mill, „Bentham“, S. 113f, bereits angesprochen. Wie ich an mehreren Stellen in Kapitel 5 anhand anderer Texte ausführlicher argumentieren werde, spielt Poesie für Mill tatsächlich diese Rolle. 
könnte. Analytische Sätze sind alternativlos wahr; ihre Wahrheit kann nicht vor dem Hintergrund geprüft werden, dass die Dinge anders sein könnten. ${ }^{68}$ (Und es könnte im Sinne der Gedanken aus Abschnitt 3.4.1 argumentiert werden, dass analytische Wahrheiten daran gebunden sind, unsere begriffliche Praxis so zu akzeptieren, wie sie ist; also bloß Ausdruck unserer Praxis sein können. Mill schreibt auch explizit über das, was ich hier „analytische Sätze“ genannt habe, dass „such propositions are not, strictly speaking, susceptible of truth or falsity, but only of conformity or disconformity to usage or convention; and all the proof they are capable of, is proof of usage; proof that the words have been employed by others in the acceptation in which the speaker or writer desires to use them."69) Sobald aber zumindest für Philosoph*innen, die zugestehen, dass entweder synthetische Urteile nicht mit empirischen Urteilen zusammenfallen oder empirische Urteile (im Sinne eines weiten Erfahrungsbegriffs) nicht mit wissenschaftlichen Urteilen zusammenfallen, dürfte Benthams Tugend nach dieser Qualifikation weniger kontrovers sein.

\subsubsection{Ein einzelner Maßstab}

Wie deutlich geworden sein sollte, bemüht sich Mill Benthams Tugend so zu fassen, dass möglichst viele philosophische Richtungen („all schools“) darunter fallen können. Auch wenn dies sicherlich nicht auf alle philosophischen Richtungen zutrifft, hatte ich argumentiert, dass wir „Wissenschaftlichkeit" bei Mill nicht zu streng auslegen sollten und Benthams Tugend dann tatsächlich weniger kontrovers sein dürfte. Die Strategie, seine Redeweise durch wohlwollende Auslegung akzeptabel zu machen, ist, so meine ich, bei Mills Überlegungen dazu, warum es ein einzelnes höchstes Prinzip oder einen einzelnen letzten Zweck geben müsse, nicht erfolgreich. $^{70}$ Stattdessen will ich dafür argumentieren, dass wir Benthams Tugend systematisch nicht an diese Voraussetzung knüpfen sollten -

68 Vergleiche dazu die Diskussion um Moores Missverständnis von Mills Beweis des utilitaristischen Prinzips in Abschnitt 6.1.2.

69 Mill, The Collected Works of John Stuart Mill, Volume VII - A System of Logic Ratiocinative and Inductive (Books I-III and Appendices), S. 109.

70 Auch hier meint Mill aber, dass „rational persons of all schools" ihm zustimmen müssen. Mill, „Bentham“, S. 111. 
auch wenn Mill dieser Meinung war. Bevor ich dafür argumentiere, will ich aber das Argument skizzieren und auf einen Unterschied zwischen den verschiedenen Stellen, an denen Mill das Argument präsentiert, eingehen. Anschließend werde ich argumentieren, dass wir, unabhängig davon, ob Mills Argument für die Notwendigkeit letzter Zwecke gültig ist oder nicht, Benthams Tugend nicht an letzte Zwecke knüpfen sollten.

Das Argument für die Notwendigkeit letzter Zwecke wird zum Beispiel am Ende von „Bentham“ angedeutet ${ }^{71}$ es ist ein eigener Paragraph im zwölften Kapitel des sechsten Buches von A System of Logic ${ }^{72}$ und es wird im ersten Kapitel von „Utilitarianism" wiederholt $^{73}$. Mill meint offenbar, um die Rationalität von Handlungen oder von mittleren Prinzipien nachzuweisen, müsse gezeigt werden, dass diese abgeleitet („deduced“74) beziehungsweise bestimmt („determined“75) werden können durch ein gültiges höheres Prinzip, oder auf dieses verweisen („refer" ${ }^{\text {“76) }}$ müssen. Dass es dann am Ende ein Prinzip sein muss, aus dem alle anderen abgeleitet werden, liegt daran, dass ansonsten Konflikte zwischen mittleren Prinzipien nicht entschieden ${ }^{77}$ beziehungsweise mit Hilfe von Argumentation und Diskussion gelöst ${ }^{78}$ werden können. In allen drei Fällen soll das Argument nicht begründen, dass der Utilitarismus gilt, sondern nur, dass es einen letzten Zweck oder ein höchstes Prinzip geben muss. In A System of Logic und in "Bentham" hat Mill auch nicht den Anspruch, seine Überzeugung, dass das utilitaristische Prinzip diese Rolle einnehmen muss, zu begründen - obwohl er in beiden Fällen

71 Mill, „Bentham“, S. 111.

72 Mill, The Collected Works of John Stuart Mill, Volume VIII - A System of Logic Ratiocinative and Inductive (Books IV-VI and Appendices), S. 951f.

73 Mill, „Utilitarianism", § 1.3 .

74 Ebd., § 1.3.

75 Mill, The Collected Works of John Stuart Mill, Volume VIII - A System of Logic Ratiocinative and Inductive (Books IV-VI and Appendices), S. $951 \mathrm{f}$.

76 Mill, „Bentham“, S. 111.

77 Siehe Mill, „Utilitarianism“, § 1.3 bzw. siehe Mill, The Collected Works of John Stuart Mill, Volume VIII - A System of Logic Ratiocinative and Inductive (Books IV-VI and Appendices), S. 951

78 Mill, „Bentham“, S. 111. 
diese Überzeugung äußert. ${ }^{79}$ In „Utilitarianism“ folgt die Begründung an anderer Stelle. ${ }^{80}$

Bevor ich auf das Argument für die Notwendigkeit letzter Zwecke eingehe, möchte ich auf einen interessanten Unterschied im Skopus des Argumentes hinweisen. Die Anordnung des Textes bei Bentham legt nahe, dass das Argument sich ausschließlich auf einen letzten moralischen Zweck bezieht. Die rationale Klärung von Meinungsverschiedenheiten muss innerhalb der Moral möglich sein, so könnte das Argument verstanden werden; deshalb braucht es einen letzten Zweck der Moral. ${ }^{81}$ Dass Moral hier nicht einfach im umfassenden Sinne aller praktischen Fragen verstanden wird, wird eine Seite später deutlich, auf der Mill - vielleicht auch um Benthams Lehre zu entschärfen $^{82}$ - explizit sagt, der moralische Standard sei nicht der einzige Standard, nach dem menschliche Handlungen bewertet werden sollten. Es gebe außer dem moralischen Aspekt von Handlungen noch den ästhetischen und den sympathetischen. ${ }^{83}$ Der Zusammenhang ließe sich dann darstellen wie in Abbildung 4.1.

Mill führt dies am Beispiel von [Lucius Junius] Brutus aus:

The action of Brutus in sentencing his sons was right, because it was executing a law essential to the freedom of his country, against persons of whose guilt there was no doubt: it was admirable, because it evinced a rare degree of patriotism, courage, and self-control; but there was nothing loveable in it; it affords either no presumption in regard to loveable qualities, or a presumption of their deficiency. ${ }^{84}$

79 Siehe Mill, The Collected Works of John Stuart Mill, Volume VIII - A System of Logic Ratiocinative and Inductive (Books IV-VI and Appendices), S. 951 bzw. siehe Mill, „Bentham“, S. 110.

80 Siehe Mill, „Utilitarianism“, Kapitel 4.

81 Es ist „essential to the very idea of moral philosophy; is, in fact, what renders argument or discussion on moral questions possible." Mill, „Bentham“, S. 111, meine Hervorhebung.

82 Er schreibt nämlich über Bentham: „This error, or rather one-sidedness, belongs to him not as a utilitarian, but as a moralist by profession, and in common with almost all professed moralists, whether religious or philosophical: it is that of treating the moral view of actions and characters, which is unquestionably the first and most important mode of looking at them, as if it were the sole one [...]." Ebd., S. 112, Hervorhebung im Original.

83 Ebd., S. 112.

84 Ebd., S. 112, Hervorhebung im Original. Die Hervorgehobenen Wörter sollen offenbar den drei "Aspekten“ entsprechen. 


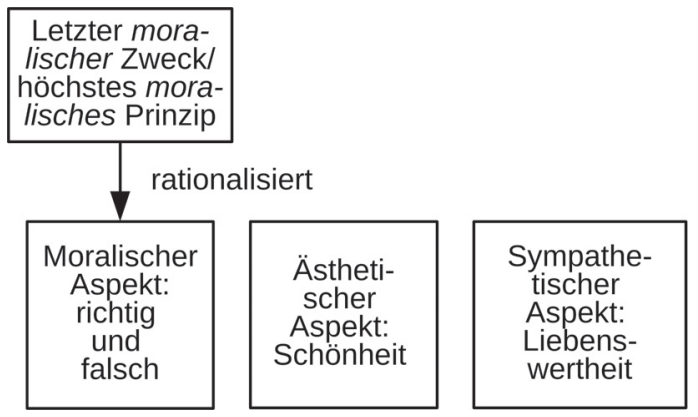

Abbildung 4.1.: Rolle des letzten Zwecks in „Bentham“

Dies führt zu einem Dilemma für Mill, nämlich zu der Frage, mit welchem Recht er den moralischen Aspekt als „unquestionably the first and most important mode of looking at them" ${ }^{\text {" } 55}$ bezeichnen kann. Wenn es wirklich so wäre, dass erstens das utilitaristische Prinzip auf den moralischen Aspekt beschränkt ist und dass zweitens die Frage des Vorranges eines Prinzips vor einem anderen im Konfliktfall nur unter Verweis auf ein höheres Prinzip entschieden werden kann, dann scheint Mill diese Bewertung nicht vornehmen zu können. Auf Brutus angewendet hieße dies (unter der Voraussetzung, dass Mill mit seiner Analyse der Situation Recht hat): Auf die Frage, ob er liebenswert oder richtig handeln sollte, gibt es keine Antwort.

Im „Utilitarianism“ scheint Mill es auf den ersten Blick ähnlich zu sehen wie in „Bentham“. Er schreibt dort in Reaktion auf den Einwand, die utilitaristische Sicht würde zu viel von uns verlangen: „, [N]inety-nine hundredths of all our actions are done from other motives, and rightly so done, if the rule of duty does not condemn them. "86 Wenn es andere Maßstäbe gibt, wieso hat der moralische Maßstab dann Vorrang? Dadurch aber, dass Mill den Einwand über die Motive rekonstruiert und nicht normativ, wird es hier komplizierter. ${ }^{87}$ Nicht alles, was wir tun, so sagt Mill hier,

85 Mill, „Bentham“, S. 112.

86 Mill, „Utilitarianism“, § 2.19 .

87 Außerdem wird es dadurch kompliziert, dass Mill den Einwand in dem relativ kurzen Absatz auf zwei weitere Weisen entkräftet: (1) Zumeinst sei es gar nicht nötig, alle Menschen zu berücksichtigen, weil die Handlung nur 
muss aus Pflichtgefühl getan werden; streng genommen sagt die utilitaristische Moral nach Mill gar nichts darüber aus, aus welchem Gefühl etwas getan werden muss, solange das, was getan wird, das utilitaristisch Richtige ist. ${ }^{88}$ Dies weicht der Frage aber eher aus, ob es neben dem utilitaristischen Bewertungsmaßstab für Handlungen keinen anderen Bewertungsmaßstab geben kann - zumindest der Person, die wissentlich aus einem der anderen Motive, von denen Mill spricht, heraus handelt, muss es ja so erscheinen, als ob es eine Perspektive der Bewertung von Handlungen gebe, die nicht mit der moralischen Perspektive identisch ist. Dann ergäbe sich aber wieder die normative Frage des Vorranges $^{89}$ - und nicht bloß eine motivationale Frage.

Mill entgeht diesem Problem im System of Logic, da er dort den utilitaristischen Maßstab an die "Art of Life" knüpft, die ihrerseits drei Teilbereiche, nämlich Moral, Klugheit und Ästhetik habe (vergleiche Abbildung 4.2). (Die ästhetische Kategorie kommt in beiden Texten vor; statt der sympathetischen Kategorie gibt es hier allerdings die Kategorie der Klugheit.) ${ }^{90}$

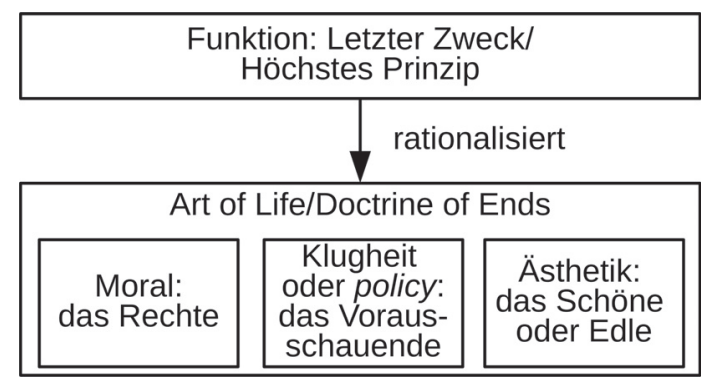

Abbildung 4.2.: Rolle des letzten Zwecks in System of Logic

wenige betreffe; (2) Handlungen, die als sekundäre Prinzipien etabliert sind (beispielsweise einen Freund verraten), könnten selbstverständlich und ohne utilitaristisches Kalkül befolgt werden. Siehe ebd., § 2.19.

88 Ebd., § 2.19 .

89 Vergleiche auch den Einwand der rationalen Egoist*in gegen Mills Beweis, den Crisp diskutiert: Crisp, Mill on Utilitarianism, S. 78.

90 Mill, The Collected Works of John Stuart Mill, Volume VIII - A System of Logic Ratiocinative and Inductive (Books IV-VI and Appendices), S. 951. 
Dies löst das Problem, wie Vorrangfragen zwischen Moral und anderen praktischen (beziehungsweise ästhetischen) Maßstäben unter der Voraussetzung geklärt werden können, dass die Rationalität eines Prinzips nur durch Ableitung aus einem höheren Prinzip gezeigt kann. Aber dieser Zusammenhang ist nicht plausibel. Unter der Voraussetzung eines sehr weiten Hedonismusbegriffs, wie ich ihn Mill in Abschnitt 5.1.2 unterstelle, könnte sicherlich gesagt werden, die Betrachtung des Schönen sei eine Form von höherer Freude oder happiness. Aber es ist eher unplausibel zu meinen, „,conduciveness to happiness of mankind, or rather, of all sentient beings" sei, was einen Gegenstand schön mache: Die Unparteilichkeit des utilitaristischen Prinzips scheint in Bezug auf Schönheit fehl am Platze. Aus dem Prinzip in der Rolle, die es in System of Logic (Abbildung 4.2) hat, könnten vielleicht Brutus (oder Williams' Gauguin ${ }^{91}$ ) ,,ableiten", ob sie liebenswert oder moralisch (beziehungsweise für oder gegen Kunst) entscheiden sollten - dies würde davon abhängen, was insgesamt mehr Freude bringt. ${ }^{92}$ Aber die praktische Vorrangfrage so zu klären ist etwas anderes als zu behaupten, ästhetischer Wert ließe sich mit dem utilitaristischen Maßstab fassen. Selbst wenn dies aber für ästhetische Werte noch irgendwie behauptet werden könnte, wird das Problem noch deutlicher im Falle der Klugheit: Was klug ist, wird eben nicht dadurch bestimmt, was "conducive[] to happiness of mankind, or rather, of all sentient beings" ist, sondern bezieht sich auf die eigene happiness.

Wir könnten die Textstelle im zweiten Bild so versuchen zu interpretieren, dass das utilitaristische Prinzip nur die Frage des praktischen Vorrangs, nicht aber die Frage, was es ist, das eine Handlung letztlich liebenswert oder einen Gegenstand schön macht, klären soll. ${ }^{93}$ Tatsächlich scheint das nahe an das ranzukommen, wie ein

91 Bernard Williams und Thomas Nagel. „Moral Luck“. In: Proceedings of the Aristotelian Society, Supplementary Volumes 50 (1976), S. 115-151.

92 Und es würde unter der Voraussetzung, dass dies im Falle von Gauguin vorher nicht feststeht, interessanterweise die Rede von ",moral luck" rechtfertigen obwohl Williams natürlich ein entschiedener Gegner des Utilitarismus gewesen ist. Siehe z. B. Bernard Williams. Morality: An Introduction to Ethics. Cambridge: Cambridge University Press, 1976, S. 82ff.

93 Ich halte es selbst nicht für sinnvoll, die Vorrangfrage von der Frage zu trennen, warum etwas gut oder schön etc. ist. Es ist hier nur als Vorschlag dazu gemeint, wie wir die Vorstellung aus dem System of Logic (Abbildung 4.2) verstehen könnten. 
sehr weiter Hedonismusbegriff bei Mill aufgefasst werden könnte. (Siehe Abschnitt 5.1.2.) Allerdings wäre Mills Kritik am Intuitionismus dann weniger überzeugend: Es stellte sich dann die Frage, warum dieser Zusammenhang nicht auch auf moralische Prinzipien zweiter Ordnung ausgedehnt werden könnte: Warum sollte es, wenn es ohnehin verschiedene praktische Maßstäbe geben kann, nicht auch verschiedene moralische Maßstäbe geben können? Falls das nicht der Fall ist, scheint unter der Voraussetzung, dass Prinzipien nur durch höhere Prinzipien begründet werden können, irgendetwas zum Beispiel für die Ästhetik oder für Prinzipien zweiter Ordnung immer noch die dem utilitaristischen Prinzip nun geraubte Rolle übernehmen zu müssen und das utilitaristische Prinzip wäre in dieser Hinsicht nicht ein Ersatz für den Intuitionismus, als den Mill es hinstellt. (Ich werde dieses Problem an zwei getrennten Stellen im Zusammenhang mit der Anwendung der Tugenden auf Mill selbst weiter diskutieren: In Kapitel 5 wird diskutiert, wie Mills Vorstellung des Guten aufzufassen ist; im ersten Teil von Kapitel 6 wird die Unparteilichkeitsannahme diskutiert.)

Dies ist aber nicht der einzige Grund, warum Mill sich eigentlich nicht darauf festlegen sollte, dass sich die Vernünftigkeit eines Zweckes oder eines Prinzips nur aus einem höheren Prinzip erweisen lässt - zumindest nicht, wenn er sich gleichzeitig darauf festlegt, dass Vernünftigkeit nur erwiesen werden kann, wenn es einen Regressstopper geben muss. Dieser muss ja auch begründet werden können. Sowohl im System of Logic als auch in „Bentham" deutet Mill an, dass er meint, dass das Prinzip gerechtfertigt werden könnte, ohne das letztlich zu tun. ${ }^{94}$ In „Utilitarianism“ beansprucht er dann tatsächlich, das utilitaristische Prinzip in seiner Rolle als Regressstopper zu begründen ${ }^{95}$ - und zwar, auch wenn es kein Beweis im gewöhnlichen Sinne sein könne, mit dem Anspruch, es als vernünftig auszuweisen. ${ }^{96}$ Damit wäre aber die Prämisse für das Argument in Frage gestellt: Die Vernünftigkeit eines Prinzips oder einer Handlung kann offensichtlich nicht nur dadurch erwiesen werden,

94 Siehe Mill, „Bentham“, S. 110 und Mill, The Collected Works of John Stuart Mill, Volume VIII - A System of Logic Ratiocinative and Inductive (Books IV-VI and Appendices), S. 951.

95 Siehe Mill, „Utilitarianism“, Kapitel 4. Siehe auch Abschnitt 6.1.2.

96 Siehe ebd., § 1.5 . 


\section{4. „Bentham"}

dass es abgeleitet („deduced“97) beziehungsweise bestimmt („determined“98) werden kann durch ein gültiges höheres Prinzip, oder auf dieses verweist („refer"99).

Es bliebe die Frage des Vorranges im Konfliktfall. Diese müsste wohl als praktisches Problem aufgefasst werden: Mill kann - nach dem der Tugend Benthams eigenen Anspruch - natürlich nicht einfach behaupten, dass es eine Lösung für Konfliktfälle gibt. Er kann behaupten, dass wir tatsächlich vor praktische Probleme gestellt sind und in solchen Fällen entscheiden müssen und wir deshalb aus praktischen Gründen, nicht aber aus theoretischen, ein solches Prinzip brauchen. ${ }^{100}$

Die Frage, ob Benthams Tugend auf soziale Praktiken angewendet ein höchstes Prinzip voraussetzt, wie Mill in „Bentham“ anzudeuten scheint, hinge, wenn Mills Argument, dass es ein höchstes Prinzip geben muss, so zu verstehen ist, aber vom praktischen Erfolg der Lösung des Konfliktes zwischen sich widersprechenden Prinzipien zweiter Ordnung ab. Dieser kann aber nach dem der Tugend Benthams eigenen Anspruch nicht einfach behauptet werden; die Notwendigkeit könnte vielleicht transzendental begründet werden - was Mill selbst aber nicht gefallen dürfte, weil ein solches Argument von der als praktisch notwendig gesetzten Möglichkeit

97 Mill, „Utilitarianism“, § 1.3.

98 Mill, The Collected Works of John Stuart Mill, Volume VIII - A System of Logic Ratiocinative and Inductive (Books IV-VI and Appendices), S. $951 \mathrm{f}$.

99 Mill, „Bentham“, S. 111.

100 Das Argument in seinen drei Formen wäre dann dem Status nach so zu verstehen, wie Korsgaard in Sources of Normativity den kategorischen Imperativ in der Universalisierungsform begründet. (Siehe Christine Korsgaard. The Sources of Normativity. Cambridge, UK: Cambridge University Press, 1996, S. 3.2.3.) Zum praktischen Status dieses Arguments, auf den es mir hier ankommt, siehe Christine Korsgaard. „Realism and Constructivism in Twentieth-Century Moral Philosophy“. In: The Constitution of Agency. Essays on Practical Reason and Moral Psychology. Oxford und andere: Oxford University Press, 2008, S. 302-326, insbesondere S. 325. Natürlich würde das nur die praktische Notwendigkeit eines solchen Prinzips zeigen, nicht aber, dass es der Utilitarismus ist, der diese Rolle füllen kann. Auf der anderen Seite erfüllt auch der Utilitarismus die Anforderung des kategorischen Imperativs (hier im Gegensatz zum moralischen Gesetz gebraucht, das Korsgaard in den Sources vom kategorischen Impertativ unterscheidet) genauso gut, wie die von ihr an dieser Stelle diskutierten Gesetze der Getriebenen, der Egoist*in u.s.w. Siehe Korsgaard, The Sources of Normativity, S. 3.2.4. 
der Konfliktlösung ausgehen müsste. Hier wären Alternativen nicht möglich. Ich habe nicht den Anspruch, hier Stellung zu beziehen. Alles, was dieser Unterabschnitt zeigen sollte, ist, dass anders als Mill andeutet, eine Festlegung auf Benthams Tugend uns noch nicht darauf festlegt, einen letzten Zweck oder ein höchstes Prinzip anzunehmen.

Zusammenfassung. In diesem Kapitel ging es darum, Benthams Tugend zu erarbeiten. Auch für Benthams Tugend stellt sich natürlich die Frage der drei Interpretationen, zumal der Aufsatz mit der Charakterisierung Benthams als kritischem Philosophen beginnt. Allerdings ist Mill hier sehr viel deutlicher, dass dies nicht das ist, was er an Bentham besonders hervorheben will. Benthams Hauptstärke liege nicht darin ein bloß „negativer“, sondern eben auch ein „positiver" Philosoph zu sein. Negative Philosoph*innen wie Hume, so meint Mill, könnten letztlich nur Inkonsistenzen oder Absurditäten in unserem Denken und unseren Praktiken aufzeigen. Ich hatte nahegelegt, dass sich darin die Rechtfertigung, hier von „kritischer Philosophie" zu sprechen, noch nicht erschöpft, obwohl das Potential der „negativen“ Seite Benthams, wie auch Mill bemerkt, Grenzen habe. (Abschnitt 4.1) Was positive Philosophie letztlich bedeutet, habe ich in Bezug auf diese Grenzen erarbeitet: „Benthams Herausforderung" habe ich die Forderung danach genannt, unsere Praktiken müssten sich nicht nur an dem Maßstab messen lassen, nicht inkonsistent und nicht absurd zu sein, sondern auch an dem Maßstab, der sich dadurch ergibt, dass wir die Dinge auch radikal anders handhaben könnten. Benthams Utilitarismus wird von Mill, so hatte ich argumentiert, dementsprechend nicht verstanden als Analyse des Wesens unserer moralischen Interaktion (beziehungsweise des Wesens der moralischen Interaktion Ende des 18. Jahrhunderts), sondern als Vorschlag für ihre Ersetzung. Die Herausforderung an die Sozialphilosophie besteht nun darin, das Gutsein oder Schlechtsein sozialer Praktiken vor dem Hintergrund, dass diese auch ganz anders sein könnten, zu erweisen. Das, was ich „Benthams philosophische Tugend" nenne, besteht darin, diese Herausforderung ernst zu nehmen und sich im Einzelfall darum zu bemühen, ihr zu begegnen. (Abschnitt 4.2.) Benthams Tugend (1) legt uns nicht auf den Utilitarismus fest, sie setzt nicht einmal voraus, dass wir uns (2) auf einen externen Maßstab zur Kritik festlegen, und sie legt uns (3) 


\section{4. „Bentham"}

auch nicht auf einen wissenschaftlichen Reduktionismus fest. Dies waren letztlich die Argumentationsziele von Abschnitt 4.3. Während Mill selbst, so hatte ich versucht zu zeigen, (1) und (3) selbst akzeptiert - zumindest unter einer bestimmten Vorstellung dessen, was wissenschaftlicher Reduktionismus beinhaltet - ist (2) etwas, das er nicht annimmt, für dessen Ablehnung er aber keine guten Gründe hat. 


\section{Zusammenfassung von Teil 1}

Teil I hatte die Aufgabe, zwei komplementäre, nicht ableitbare aber nicht exklusive sozialphilosophische Tugenden exegetisch anhand von Mills Texten „Bentham“101 und „Coleridge“102 zu erarbeiten. (In der Hauptsache erfolgte dies in den Kapiteln 3 und 4.) Ich hatte dafür argumentiert, dass beide Texte komplementär zu lesen sind; dass Bentham und Coleridge für Mill zwei Pole einer Dichotomie sind. (Abschnitt 2.4.) Aus den möglichen Antworten auf die Frage, welche von drei auf komplexe Weise zusammenhängenden Dichotomien, die Mill erwähnt, die grundlegende ist, ergeben sich drei verschiedene Interpretationen: die methodische, die gesellschaftspolitische und diejenige, die ich selbst vorgeschlagen habe, die sozialphilosophische. ${ }^{103}$ (Zum Beispiel Abschnitt 3.4.) Indem ich die Zusammenhänge zwischen den einzelnen Interpretationen aufgezeigt habe und dagegen argumentiert habe, dass die methodische oder die gesellschaftspolitische Interpretation grundlegend sein kann, habe ich für die von mir favorisierte Interpretation die sozialphilosophische argumentiert. (Siehe Abschnitte 3.4, 4.1 und 4.2.)

Der sozialphilosophischen Interpretation gemäß geht es Mill in den beiden Aufsätzen darum, zwei sozialphilosophische Tugenden vorzuschlagen. Er tut dies, indem er Charaktereigenschaften, Fähigkeiten, Schwächen von Bentham und Coleridge hervorhebt und zeigt, inwiefern der Einsatz dieser bei den beiden etwas ist, das für das Philosophieren wichtig ist. Warum sollte eine Aufgabe aus der Philosophie der Philosophie so angegangen werden? Ich hatte dafür argumentiert, dass diese Art des Schreibens über andere

101 Mill, „Bentham".

102 Mill, "Coleridge“.

103 Eine weitere Interpretation, die durch Mill in ebd., S. 125, aber in ähnlichen Worten auch in Mill, „Preface to Dissertations and Discussions“ nahegelegt wird, könnte die ideengeschichtliche Interpretation genannt werden. Die zitierten Textstellen können so verstanden werden, dass Coleridge dabei von Mill als Vertreter der Romantik, Bentham als Vertreter der Aufklärung und die Romantik als Reaktion auf die Aufklärung präsentiert wird - auch wenn Mill natürlich nicht diese Worte verwendet. Auch diese Dichotomie ist natürlich nicht logisch äquivalent mit den anderen und ich müsste daher streng genommen auch gegen sie argumentieren. Letztlich scheint es mir aber, dass die ideengeschichtlichen Bemerkungen Mills in beiden Aufsätzen insgesamt zu dürftig sind, um der ideengeschichtlichen Interpretation überhaupt eine Anfangsplausibilität zu verleihen, weswegen ich sie einfach ignoriert habe. 
Philosoph*innen vor dem Hintergrund methodischer Probleme der Philosophie der Philosophie eine legitime Methode ist. (Ich habe weder den Anspruch zu zeigen, dass es die einzige legitime Methode ist, noch will ich behaupten, dass es andere Methoden gibt.) Die Methode kann außerdem den Test der Selbstanwendung (auf die Praxis des Philosophierens) bestehen, so hatte ich versucht $\mathrm{zu}$ argumentieren, ohne dass diese Tatsache natürlich den Argumenten für die Tugenden zusätzliches Gewicht verleihen könnte. (Siehe Abschnitt 2.3.)

Coleridges sozialphilosophische Tugend besteht in der Fähigkeit und dem Interesse, dem, was eine Teilnehmer*in an einer sozialen Praxis als das, was sie in dieser Praxis als wertvoll erlebt, einen adäquaten sprachlichen Ausdruck zu verleihen. (Siehe Kapitel 3.) Eines der Beispiele, die ich dafür aufgegriffen hatte, war Coleridges Behandlung des Christentums (aus Mills Sicht): Das, was eine Christ*in im Christentum sieht, so Mills Coleridge, könne genauso wenig auf einen externen Maßstab der Vernünftigkeit reduziert werden, wie das, was eine Rechner*in als das richtige Ergebnis einer Addition sehen würde, auf externe Maßstäbe (beispielsweise soziale Autorität oder Nützlichkeit in den Naturwissenschaften) reduziert werden kann. (Siehe Abschnitt 3.3.2.) Das Beispiel ist von Mill geschickt gewählt, insofern er gleichzeitig dies zugestehen kann und seinen Atheismus behaupten kann. Dies macht deutlich: Dem einen adäquaten Ausdruck verliehen zu haben, was eine kompetente Teilnehmer*in an einer sozialen Praxis als wertvoll erlebt, bedeutet noch nicht, dass wir an der so behandelten sozialen Praxis auch teilhaben sollten. Dafür, dass wir richtiges Rechnen erlernen sollten oder beginnen sollten das Christentum zu praktizieren, müsste nämlich zusätzlich dazu, dem Witz der jeweiligen Praxis Ausdruck zu verleihen, nachgewiesen werden, dass es besser ist, teilzunehmen als nicht teilzunehmen.

Dies ist der Übergang zu Benthams Tugend. (Siehe Kapitel 4.) Nach Benthams Tugend müssen wir uns bei der Evaluation sozialer Praktiken darum bemühen, das Gute oder Schlechte an diesen anhand objektiver Kriterien zu bestimmen. Damit ist nicht nur gemeint, dass wir Argumente finden müssen, die auf eine potentielle oder reale philosophische Gegenposition eingehen; vielmehr müssen wir uns darum bemühen, das Gute (oder das Schlechte) an diesen Praktiken vor dem Hintergrund, dass unsere Praktiken auch sehr 
anders sein könnten, anhand von objektiven Kriterien zu bestimmen. Damit wird ausgeschlossen, dass Philosophie sich auf Diskussionen vor einem Hintergrund vorgegebener und nicht philosophisch hinterfragbarer Praktiken und Sprachpraktiken beschränken muss. Potentiell ist kein analytischer Zusammenhang vor dieser Art des Hinterfragens sicher: Dass (um für die Zwecke dieser Zusammenfassung ein besonders geeignetes Beispiel von meinem Betreuer zu borgen ${ }^{104}$ ) zum Beispiel reaktive und retributive Emotionen nur vor dem Hintergrund einer impliziten Freiheitsannahme verständlich werden, mag innerhalb „unserer“ (eigenen, derzeitigen) moralischen Praxis zutreffen; aber sollten wir zum Beispiel reaktive und retributive Emotionen in unseren Kindern fördern oder unterbinden? Sollten wir den Ausdruck dieser Emotionen verfeinern und ihnen Kunst und Literatur widmen - oder ihn ächten? Geht es zum Beispiel um Freiheit, reicht es vor dem Hintergrund selbst dieser bescheidenen Möglichkeiten, auf das, was „unsere Praxis“ bezeichnet, einzuwirken, nach Mills Bentham nicht, für die Annahme von Freiheit zu argumentieren, indem wir darauf hinweisen, dass Reue und Empörung implizit die Annahme von Freiheit voraussetzen und selbst die Person, die Freiheit bestreitet, Reue und Empörung bereits empfunden hat. Wir müssten uns gemäß Benthams Tugend eben auch darum bemühen, zu zeigen, dass es wirklich besser ist, solche Emotionen auszuleben und zu kultivieren.

104 Siehe Logi Gunnarsson. Vernunft und Temperament. Eine Philosophie der Philosophie. Leiden, NL u.a.: Mentis Verlag, 2020, S. 357-373. 
Teil II.

\section{Die Tugenden in Mills eigener Philosophie}




\section{Einleitung zu Teil 2}

Die vier Aufgaben des zweiten Teils. Der nun folgende Teil II meiner Arbeit hat vier Aufgaben: Die erste Aufgabe ist es, zu zeigen, dass sich Mill auch in seinen anderen philosophischen Texten teilweise explizit, häufig jedoch zumindest implizit an den philosophischen Tugenden, die ich in Teil I erarbeitet habe, orientiert. Dass „Bentham" und „Coleridge" als Betätigungen von Mill im Bereich der Philosophie der Sozialphilosophie gelesen werden, ist sehr viel plausibler, wenn es sich dabei auch um eine Philosophie der Sozialphilosophie Mills handelt.

Da ich mit dieser Arbeit aber nicht vorwiegend für eine Auslegung zweier Texte Mills, sondern auch systematisch für die darin ausgedrückten Ideale werben will, geht es zweitens darum, zu zeigen, inwiefern die Ideale fruchtbar sind. Für diese zweite Aufgabe ist es zunächst noch nicht entscheidend, dass die Ideale als Tugenden verstanden werden: Philosophische Ideale, ganz egal, wie diese letztlich beschaffen sind, müssen dazu taugen, philosophische Ansätze danach zu beurteilen, bis zu welchem Ausmaß sie diesen Idealen gerecht werden oder nicht. Ich will also nicht nur zeigen, dass Mill sich an den in Teil I beschrieben Idealen orientiert hat; ich will außerdem zeigen, dass sie einen fruchtbaren Bewertungsmaßstab für philosophische Ansätze darstellen, indem ich sie auf Mills Ansatz anwende. Es ist kein Widerspruch, einerseits ein Ideal für ein konstitutives Ideal $\mathrm{zu}$ halten und diesem andererseits trotzdem nicht immer gerecht zu werden: Dass eine philosophische Autor*in sich selbst an einer Stelle widerspricht, ist noch kein Beleg dafür, dass sie den Satz vom ausgeschlossenen Widerspruch nicht als konstitutives Ideal auch ihres philosophischen Denkens betrachtet. (Siehe dazu Abschnitt 2.2.)

Die eigentliche Stärke der beiden Ideale, um die es in dieser Arbeit geht, wird aber erst in ihrer Formulierung als Tugenden deutlich. Die dritte Aufgabe dieses Teiles ist es, das nachzuweisen. Grob gesagt wird, indem die Ideale als Tugenden formuliert werden, der Fokus auf die Philosoph*in selbst gelegt; sie wird als Philosoph*in Gegenstand des Interesses. Dies steht in Kontrast dazu, zum Beispiel den philosophischen Text oder philosophische Argumente zum Gegenstand der Ideale zu machen. Für philosophische Texte oder Argumente gibt es andere Ideale; Mills sozialphilosophischen Ideale 
sind jedoch Ideale für eine philosophierende Person. Dies mag als übergriffig empfunden werden. Zwei Dinge sind hier aber zu beachten: Erstens sollte in Teil 1 deutlich geworden sein, dass Mill mit Bentham und Coleridge durchaus so umgeht. Es sind nicht einfach ihre Texte philosophisch bedeutend, sondern Bentham und Coleridge haben für Mill besondere Fähigkeiten und Schwächen, Interessen und mangelndes Interesse und so weiter. ${ }^{105}$ Zumindest, was Mill angeht, dürfen wir uns dazu ermutigt sehen, ihn so zu behandeln, wie er andere behandelt hat. Zweitens rückt mit den philosophischen Tugenden eine Person lediglich bezüglich der Tätigkeit des Philosophierens und nicht bezüglich all den anderen Aspekten ihrer Person in den Fokus der Aufmerksamkeit. Die Übergriffigkeit ist also eingeschränkt.

Ganz analog zur Tugendethik sind dabei die Ergebnisse der tugendgeleiteten Tätigkeit natürlich nicht unwichtig: die gute Philosoph*in verfasst natürlich gute philosophische Texte. Die aus Teil I aufgeschobene Aufgabe, die nun zu bearbeiten ist, ist also, zu zeigen, dass es, wichtig ist zu verstehen, inwiefern eine Autor*in eine gute Philosoph*in war, damit wir verstehen können, was an ihrem philosophischen Text gut ist - so wie wir in der Tugendethik die Tugendhaftigkeit einer tugendhaften Handlung nur als Handlung der Tugendhaften verstehen können. ${ }^{106}$ )

105 In der Autobiographie legt Mill auch selbst Wert darauf, dass er (auch) seinen geistigen Entwicklungsprozess beschreibt: „It has also seemed to me that in an age of transition in opinions, there may be somewhat both of interest and of benefit in noting the successive phases of any mind which was always pressing forward, equally ready to learn and to unlearn either from its own thoughts or from those of others." Und natürlich ist eines der am prominentesten platzierten Beispiele dieser geistigen Entwicklung die Entwicklung seiner philosophischen Gedanken während seiner psychischen Krise in Kapitel 5 .

Seine geistige Entwicklung nachzuzeichnen ist eine von drei Aufgaben, die er am Anfang der Autobiographie, wo das Zitat herstammt, angibt; die anderen sind, mit der Darstellung seiner eigenen Erziehung einen Beitrag zum Feld der Erziehung zu liefern, und, andere Personen zu würdigen, die eine Rolle in seiner geistigen Entwicklung gespielt haben. Siehe Mill, Autobiography, S. 5.

106 Die in diesem Abschnitt hervorgehobene Strukturanalogie zwischen philosophischen und ethischen Tugenden dient nur dazu, die folgenden Erklärungen zu verdeutlichen: Mill teilt zwar mit einigen tugendethischen Ansätzen perfektionistische Züge (Siehe Piergiorgio Donatelli. „Mill's Perfectionism“. In: Prolegomena 5.2 (2006), S. 149-164, z. B.) und er vertritt auch eine in Tei- 
Diese dritte Aufgabe mag natürlich nicht nur für zu ambitioniert, sondern im Gegenteil für trivial und überflüssig gehalten werden: Innerhalb dessen, was im weitesten Sinne als neo-aristotelische Strömung der Gegenwartsphilosophie aufgefasst werden kann, ist es nicht nur im Praktischen, sondern auch im Theoretischen zu einer Wiederbelebung von Person-Idealen in Form dianoetischer Tugenden oder zumindest vernünftiger Fähigkeiten oder Vermögen gekommen. ${ }^{107}$ Wo immer das Wort "gut", so könnte eingewendet werden, Anwendung findet - sei es im Praktischen oder im Theoretischen - bedeute es letztlich zumindest implizit einen Bezug auf eine Wesenhaftigkeit, als deren hervorragendes Exemplar ein Exemplar $X$ durch die Verwendung von "gut" in Bezug auf sein Verhalten präsentiert wird: Ob das Wesenhafte, an dem $X$ dabei gemessen wird, letztlich die Flötenspieler*in, der Mensch, das wahrnehmende Wesen oder eben die Philosoph*in sei, sei für die Grundstruktur dessen, was "gut" bedeutet, unerheblich.

In dieser Arbeit soll die Analogie mit der Tugendethik jedoch nicht nahelegen, dass jedes philosophische Ideal eigentlich die Form einer Tugend hat. Dies wäre meiner Ansicht nach sogar unplausibel, ohne dass ich dafür hier argumentieren will. Was ich hier nur zeigen will, ist, dass die Ideale, die in Teil I erarbeitet werden, dazu taugen, bestimmte Werte und Schwächen eines philosophischen Ansatzes anders - adäquater - einzuordnen, wenn sie als Tugenden der Autor*in verstanden werden.

Die vierte und letzte Aufgabe ist zu zeigen, dass die Ideale wirklich Ideale sind, die es wert sind, verfolgt zu werden. Dies ist eine Aufgabe, für die letztlich nicht streng argumentiert werden kann, wie ich in Abschnitt 2.3 dargelegt hatte. Ich müsste zeigen, dass dort, wo Mill sich an den Tugenden orientiert, seine Philosophie

len beinahe aristotelische Charakterlehre (Mill, The Collected Works of John Stuart Mill, Volume VIII - A System of Logic Ratiocinative and Inductive (Books IV-VI and Appendices), Buch VI); er sagt aber natürlich, dass der moralische Wert einer Handlung einfach über ihre Konsequenzen verständlich ist und nicht dadurch, dass es die Handlung einer tugendhaften Person ist. Vgl. z. B. Mill, „Utilitarianism“, § 2.19. Dies heißt selbstverständlich nicht, dass Motive und der Charakter für Mill irrelevant wären; sie sind relevant, insofern sie zu Handlungen führen, die bestimmte Konsequenzen haben.

107 Vgl. in Bezug auf eine Analyse von Wissen in den Begriffen vernünftiger Fähigkeiten z. B. Andrea Kern. Quellen des Wissens. Frankfurt am Main: Suhrkamp, 2006. 
auch deshalb ein Beispiel guter Philosophie ist, weil er sich an den Tugenden orientiert und umgekehrt, dass seine Philosophie dort, wo er es trotz Orientierung an den Tugenden nicht schafft, diesen gerecht zu werden, seine Philosophie (zumindest unter anderem auch) deshalb defizitär ist, weil er den Tugenden dort nicht gerecht wird. Ich werde mich also (im Sinne der ersten und zweiten Aufgabe) bemühen, dafür zu argumentieren, dass Aspekte an Mills Philosophie, die ich für Beispiele guter beziehungsweise defizitärer Philosophie halte, auf die Tugenden zurückzuführen sind. Um zu erreichen, dass die Leser*in zustimmt, dass dies tatsächlich Beispiele guter und schlechter Philosophie sind, kann ich gemäß Abschnitt 2.3 nicht deduktiv argumentieren, ohne vorauszusetzen, was ich zeigen will. ${ }^{108}$ Die Leser*in als kompetente Teilnehmer*in an der philosophischen Praxis wird, so meine Hoffnung, sehen, dass etwas Entscheidendes dieser Praxis anhand der entsprechenden Beispiele aus Mills später Philosophie durch die Anwendung der Tugenden auf diese Beispiele zum Ausdruck gebracht wird. Ich bin, um einen dieser Aspekte vorwegzunehmen, also, was die Argumentation für die Tugenden angeht, epistemologisch in einer ähnlichen Situation wie Mill bei seinem Beweis des Nützlichkeitsprinzips in Kapitel 4 von „Utilitarianism"109; und ich bediene mich auch einer ähnlichen Strategie, ${ }^{110}$ einer Strategie, die, so meine ich, den beiden philosophischen Tugenden entspricht.

Der Aufbau des Teils. Diese vier Aufgaben werde ich in zwei Kapiteln bearbeiten: Das erste dieser Kapitel (Kapitel 5) liefert eine Interpretation von Mills Sozial- und Moralphilosophie ${ }^{111}$, die sich stark an Elizabeth Andersons „John Stuart Mill and Experiments in Living"112 orientiert und nur an wenigen Stellen von ihr abweicht. Diese Interpretation legt, neben den Stellen, an denen Mill sich

108 Streng genommen nicht einmal um zu erreichen, dass mir zugestimmt wird, dass das, was diese Aspekte zu guter beziehungsweise defizitärer Philosophie macht, Mills Orientierung an oder sein Verfehlen der Tugenden ist. Siehe Abschnitt 2.3.

109 Vgl. Mill, „Utilitarianism“, Kapitel 4.

110 Ich behandele diesen Beweis in Abschnitt 6.1.2.

111 Praktische Philosophie hat nach Mill einen individuellen und einen sozialen Aspekt. Siehe z. B. Mill, „Bentham“, S. 97.

112 Elizabeth S. Anderson. „John Stuart Mill and Experiments in Living“. In: Ethics 102.1 (1991), S. 4-26. 
explizit auf etwas bezieht, das als Formulierung eines der Ideale aufgefasst werden muss, sehr nahe, so behaupte ich, dass es sinnvoll ist, Mills philosophisches Tun auch implizit als durch die beiden Tugenden orientiert anzusehen. Das zweite der Kapitel dieses Teil (Kapitel 6) behandelt anhand von zwei Beispielen das Scheitern von Mill gemessen an den beiden Tugenden. Auf diese Weise bin ich den beiden ersten der oben beschriebenen Aufgaben nachgekommen.

Mills Autobiography spielt eine entscheidende Rolle in der in Kapitel 5 vorgestellten an Anderson orientierten Interpretation eines Grundzugs der Gedanken Mills und Mill beschreibt in der Autobiography seinen philosophischen Lernprozess als einen persönlichen Lernprozess seiner Meinungen und seines Charakters ${ }^{113} \cdot{ }^{114}$ Dies legt es bereits nahe, auch wenn es vielleicht noch nicht zwingend ist, die beide Ideale als Ideale für die philosophierenden Person - als Tugenden - zu verstehen. Die Art und Weise jedoch, wie Mill den Tugenden in Kapitel 5 nachkommt, nämlich als Lebensexperimente, macht deutlich, dass es sich um Tugenden handeln muss: Die Philosoph*in muss bereit sein, sich grundlegend zu ändern; sie muss gegebenenfalls bereit sein, emotionale Sensibilitäten zu entwickeln ${ }^{115}$, und versuchen, diesen sprachlichen Ausdruck zu verleihen. Damit wird ein Teil der dritten Aufgabe in Kapitel 5 erarbeitet.

Kann man den Idealen aus dem ersten Teil aber auch anders nachkommen als in Lebensexperimenten? Die Reichweite des Argumentes dafür, die Ideale als Tugenden zu verstehen, wäre dann zunächst eingeschränkt auf die Realisierung der Ideale als Lebensexperimente und es wäre offen, ob dies auch sonst gilt. Letztlich wird diese Frage auch davon abhängen, wie weit der Begriff des Lebensexperimentes gefasst wird. Irgendetwas den Lebensexperi-

113 „my opinions and my character" Mill, Autobiography, S. 145, meine Kursivsetzung. Es geht an der Textstelle, auf die sich diese Worte beziehen, um zwei wichtige Veränderungen in Mills Philosophie. Es geht hier also um Mills Charakter als Philosoph.

114 Einer der drei Gründe, die Mill dafür angibt, die Autobiography geschrieben zu haben, ist: , $[\mathrm{T}]$ here may be somewhat both of interest and of benefit in noting the successive phases of any mind which was always pressing forward, equally ready to learn and unlearn either from its own thoughts or from those of others." Ebd., S. 5. Insbesondere die „crisis in my life“, die er in Kapitel 5 behandelt, ist neben psychischer Krise nach Mill eben ein solcher philosophischer Lernprozess. Ebd., Kapitel 5.

115 In Mills Fall vor allem eine Sensibilität für Poesie. Siehe Abschnitt 5.2.2. 
menten funktional Äquivalentes muss es natürlich geben; ich werde jedoch erst in der Zusammenfassung des Teils auf diese Frage nach der Reichweite des Argumentes zurückkommen und es hier dabei belassen, die Beschränktheit der Reichweite zunächst zuzugestehen. $^{116}$

Ein zusätzlicher Vorteil daran, diese Ideale als Tugenden zu verstehen, liegt darin, dass dies eine andere und, wie ich hoffe, bessere Weise liefert, Mills philosophische Festlegungen zu verstehen: Um den Grund dafür zu ermitteln, warum Mill sich in Bezug auf ein philosophisches Problem soundso festgelegt hat, müssen wir den Komplex seiner philosophischen Ziele, Dispositionen und Fähigkeiten - seine philosophischen Tugenden - hinzuziehen; es reicht nicht festzustellen, dass er hier dies sagt, weil er an anderer Stelle jenes sagt. Dies wird insbesondere bei den Schwächen und Ambivalenzen deutlich: Für eine Denker*in, die den Anspruch hat, „equally ready to learn and unlearn either from its own thoughts or from those of others" ${ }^{\text {"117 }} \mathrm{zu}$ sein, ist es ein schlechter Grund, hier dies zu sagen, weil sie an andere Stelle jenes gesagt hat. Die Schwächen können sehr viel sinnvoller als verfehlte Versuche, den Tugenden gerecht zu werden, betrachtet werden, denn lediglich als philosophisch unhaltbare Positionen. Auch in seinen Schwächen, zumindest in denen, die ich in Kapitel 6 behandeln werde, ist Mills Verhalten von den Tugenden geleitet. Dies ermöglicht auch ein besseres Verständnis von Mills Philosophie: Die Frage, auf welche Weise wir Mills Position verbessern müssten oder wie wir eine Ambivalenz bei ihm aufösen müssten, um ihn richtig zu verstehen, können wir unter Rückbezug auf das, was Mill beim Philosophieren wichtig war, beantworten. Die Strategie besteht darin zu zeigen, dass wir Mills Position Sinn abgewinnen können, obwohl sie, so wie er sie formuliert, unhaltbar weil zum Beispiel inkonsistent - ist, und zwar vor dem Hintergrund von Mills Art und Weise der Orientierung an den Tugenden. Dies bedeutet, dass dieser Vorteil, von "Tugenden" und nicht von anders strukturierten Idealen zu sprechen, erst in Kapitel 6 deutlich wird. Diesen Vorteil deutlich zu machen ist der Rest von Aufgabe 3.

116 Der systematische Grund für die Begrenztheit des Argumentes ist die begriffliche Verschiedenheit der durch die Spannung zwischen den Idealen aufgestellten philosophischen Frage und ihrer Antwort durch Lebensexperimente.

117 Mill, Autobiography, S. 5. 
Einleitung zu Teil 2

Aufgabe vier wird, wie oben angedeutet, im gesamten Teil bearbeitet. 


\section{Mills Philosophie des Wohlergehens}

Ich halte Elizabeth Andersons Zugang zu Mills „reifer" Philosophie, wie sie ihn in „John Stuart Mill and Experiments in Living“"1 darstellt, für grundsätzlich zutreffend. Der erste Abschnitt (5.1) besteht darin, diesen Zugang vorzustellen und zu diskutieren: In Unterabschnitt 5.1.1 wird dargelegt, wie Anderson Mills Krise als philosophischen Lernprozess auffasst, und in Unterabschnitt 5.1.2 wird Mills Theorie des Guten, die nach Anderson das Ergebnis dieses Lernprozesses ist, dargestellt. Ich weiche allerdings von Anderson in der Detailfrage ab, was genau die Begründung dafür ist, Benthams Theorie des Guten zu verwerfen. Ich behaupte anders als Anderson, dass diese Begründung darin liegt, dass Bentham Coleridges Tugend nicht gerecht wird. In Unterabschnitt 5.1.3 kritisiere ich entsprechend Andersons Argument.

Der zweite Abschnitt (5.2) soll beispielhaft belegen, dass Mill sich in seiner reifen Philosophie an den beiden Tugenden orientiert. Unterabschnitt 5.2.1 soll dies für seine Philosophie des guten Lebens belegen, Unterabschnitt 5.2.2 für die damit verwandte Methode des Urteils der Erfahrenen und Unterabschnitt 5.2.3 für "On Liberty“ als Werk und insbesondere für das darin vertretene harm principle.

Mills Methode der experiments in living kann auf unterschiedlichste Weise darin scheitern, „objektiv“ zu sein, wie es Benthams Tugend verlangt. Mill waren viele Weisen zu scheitern bewusst. Der dritte Abschnitt listet diese auf und diskutiert sie. Der Sinn dieses dritten Abschnittes ist zu zeigen, dass für Mill auch das Erfüllen von Benthams Tugend keine triviale Angelegenheit ist. Wir müssen uns vor dem Hintergrund, wie wir scheitern können, bemühen Objektivität herzustellen; aber es gibt keine Methode, die den Erfolg garantiert.

Insgesamt will ich in diesem Kapitel dafür argumentieren, dass Andersons Ansatz, Mills späte Philosophie über „experiments in living" zu interpretieren, erstens sehr wichtige Gedanken von Mills später Philosophie erfasst und zweitens eine Art und Weise deut-

1 Anderson, „John Stuart Mill and Experiments in Living“. 
lich macht, wie Mill den beiden philosophischen Tugenden aus Teil I gerecht wird. Außerdem wird meine Redeweise, von Tugenden zu sprechen und nicht von anders aufgefassten Idealen, plausibilisiert. Ich will aber nicht dafür argumentieren, dass alle Gedanken von Mill sinnvollerweise so aufgefasst werden sollten; und ich will in diesem Kapitel auch nicht dafür argumentieren, dass experiments in living die einzige (oder auch nur die in irgendeiner Hinsicht beste) Möglichkeit sind, den Tugenden gerecht zu werden: Aus meinen Überlegungen in diesem Kapitel folgt nicht, dass die Akzeptanz der beiden Tugenden uns darauf festlegt, dass Philosophie in der Reflexion von Lebensexperimenten bestehen muss. Einige Gedanken zu dieser Frage werde ich erst in Kapitel 8 anstellen, aber die Frage letztlich offen lassen.

\subsection{Anderson über Mill und Experiments in Living}

Elizabeth Anderson macht den Begriff „experiments in living“ im gleichnamigen Aufsatz ${ }^{2}$, den sie aus „On Liberty“ entlehnt, ${ }^{3}$ zum zentralen Begriff ihrer Interpretation eines wichtigen Aspektes von Mills Philosophie. Die Grundidee ist, dass es zu den Bewährungsproben ethischer Ansätze in der Philosophie zählt, dass sie sich im Leben bewähren müssen. Mill selbst erklärt, „the worth of different modes of life should be proved practically “4 . Auf diese Weise werde Mills Bekenntnis zum Empirismus Rechnung getragen. Gleichzeitig sei der Anspruch, dass die Erfahrung, um die es hier geht, im Gegensatz zum Empirismus Benthams, nicht auf den Versuch hinausläuft, normatives Vokabular reduktionistisch umzuinterpretieren. ${ }^{5}$

\subsubsection{Mills philosophischer Lernprozess in seiner Krise}

Für ein Verständnis des Elementes von Mills reifer, praktischer Philosophie auf das Anderson mit Mills Phrase "experiments in living“ hinweist, bieten ihr zufolge nicht nur "On Liberty" und „Utilitarianism" entscheidende Schlüssel, sondern auch die Autobiography,

2 Anderson, ,John Stuart Mill and Experiments in Living“.

3 Vgl. Mill, On Liberty/Über die Freiheit, S. 228 und mit der Präposition „of" statt „in“ auf S. 160.

4 Ebd., S. 160.

5 Vgl. Anderson, ,John Stuart Mill and Experiments in Living“, S. 4f. 
zu deren Funktionen auch zählt, Mills eigenes Leben vor seiner ersten Krise als Versuch mit einer neuen Lebensweise ${ }^{6}$, die Benthams Philosophie entspricht, hervorzuheben. Dies entspricht dem in Fußnote 114 in der Einleitung dieses Teils bereits erwähnten Zweck der Autobiography, vor allem wenn wir Mills Beschreibung seines Verhältnisses zu Benthams Philosophie vor der Krise betrachten: Er behauptet, seine Erziehung sei bereits, bevor er mit 16 Jahren zum ersten Mal einen Text von Bentham in Händen hält, ein „Kurs in Benthamismus" gewesen ${ }^{7}$; und so ist es laut Mill kein Wunder, dass die erste Lektüre eines Textes von Bentham Mill nach eigenen Angaben sehr beeindruckt:

The "principle of utility," understood as Bentham understood it, and applied in the manner in which he applied it through these three volumes, fell exactly into its place as the keystone which held together the detached and fragmentary component parts of my knowledge and beliefs. It gave unity to my conceptions of things. I now had opinions; a creed, a doctrine, a philosophy; in one among the best senses of the word, a religion; the inculcation and diffusion of which could be made the principal outward purpose of a life. And I had a grand conception laid before me of changes to be effected in the condition of mankind through that doctrine. ${ }^{8}$

Die Textstelle belegt, was es heißt, einen ernsthaften Versuch mit einer neuen Lebensweise zu machen: Es reicht nicht, eine Position abstrakt für wahr zu halten. Die Experimentierende muss auch

6 Bruno Lemke übersetzt die zweite Stelle, an der die Phrase auftaucht, in diesen Worten. Vgl. Mill, On Liberty/Über die Freiheit, S. 229.

7 Vgl. Mill, Autobiography, S. 67. Die Kapitel 1 und 2 der Autobiography vor dieser Textstelle belegen dies sicherlich zum Teil. Neben den Methoden und Zielen der Erziehung durch seinen Vater, die Mill dort beschreibt, belegen dies auch der enge Kontakt der Familie, insbesondere des Vaters, zu Bentham und die Bewunderung für Bentham. Allerdings gibt es auch Textstellen, die dem widersprechen: So schreibt John Stuart Mill die normalerweise mit ihm selbst assoziierte höhere Bewertung geistiger gegenüber körperlichen Freuden seinem Vater zu, der diese durch seine Bewunderung für verschiedene Aspekte jeweils der stoischen, epikureischen und kynischen Schulen der Antike erworben habe (vgl. ebd., S. 49ff); weder diese Bevorzugung geistiger Freuden noch die Wertschätzung klassischer Texte für moderne praktische Probleme passt natürlich streng genommen zu einem "Kurs in Benthamismus".

8 Ebd., S. 69. 


\section{Mills Philosophie des Wohlergehens}

emotional und affektiv dahinterstehen. Im Falle von Mill ist dies durch die Erziehung geschehen. Aber ganz allgemein ist natürlich klar, dass nach einer philosophischen Position zu leben, nicht nur bedeuten kann, das zu tun, was die philosophische Position zu tun verlangt, sondern auch, sich darum zu bemühen, es aus den Einstellungen heraus zu tun, aus denen die philosophische Position dies verlangt $^{9}$ und dabei zu empfinden, was die philosophische Position zu empfinden vorgibt. Dies bietet einen ersten Hinweis darauf, dass die philosophischen Ideale zumindest in der Art und Weise, wie Mill ihnen nachkommt, auch als Ideale der Person aufgefasst werden müssen: Philosophie muss im persönlichen Leben ernst genommen werden und umgekehrt muss Philosophie sich an den entscheidenden Erfahrungen des Lebens orientieren.

Anderson hat sicherlich Recht, dass die Autobiography das Scheitern dieses Experimentes belegen soll: ${ }^{10}$ Mill gerät in eine Krise, die darin besteht, dass er die Freude am benthamitischen Projekt und seine Motivation verliert ${ }^{11}$. Er interpretiert zum Zeitpunkt der Krise diese Krise mithilfe der benthamitischen Philosophie. Danach gibt es zwei Probleme: Der junge, stark von Bentham beeinflusste Mill bringt seinen psychologischen Hedonismus, nach dem die Erwartung von eigener Lust beziehungsweise eigenem Schmerz die einzigen Motivationsquellen sind, mit seinem hedonistischen Utilitarismus, nach welchem die eigene Lust und der eigene Schmerz moralisch nicht mehr zählen als die Lust und der Schmerz jedes anderen betroffenen Wesens, durch eine Konditionierungstheorie der Erziehung in Einklang: Die einzige Möglichkeit, wie moralisches Handeln unter diesen Voraussetzungen möglich ist, ist, dass wir so konditioniert sind, dass wir Freude bei der unparteiischen Verbreitung von Freude beziehungsweise Leid bei der Verbreitung von Leid empfinden. ${ }^{12}$ Dadurch, aber, dass diese Verbindung eigener Motivation mit moralischem Handeln künstlich („artificial“) sei, erkennt

9 Dies führt zu einer Grenze des Ansatzes, auf die ich weiter unten (Abschnitt 5.3) eingehen werde und die die Freiwilligkeit solcher Experimente betrifft: Eine Person, die sich bemüht, aus Pflicht zu handeln, um die kantische Ethik auszuprobieren, handelt nicht aus Pflicht, sondern aus philosophischer Neugierde, so könnte eingewendet werden.

10 Vgl. Anderson, ,John Stuart Mill and Experiments in Living“, S. 15-20.

11 Mill, Autobiography, S. 137ff.

12 Ebd., S. 143. 
Mill, so meint er, durch seine Analysefähigkeit, die er auch seiner (benthamitischen) Erziehung zuschreibt, diese als kontingent. So meint Mill, dass seine Analysefähigkeit seine eigene moralische Motivation - und damit seinen Lebensinhalt - schwäche. ${ }^{13}$

Interessant ist, dass Mill auch bereits zum Zeitpunkt, als er sich der Bentham'schen Philosophie verpflichtet sieht, in gewisser Hinsicht personenbezogen über Philosophie denkt: Sicherlich besitzt die gute Sozialphilosoph*in Analysefähigkeit in hohem Maße. Aber, weil die gute Sozialphilosoph*in für Mill (und Bentham) auch immer eine gute Sozialreformer*in ist ${ }^{14}$ muss sie auch eine affektive (,assoziative“) Ausrichtung darauf haben, die Gesellschaft zu verbessern. Weil diese affektive Ausrichtung aber durch die Analysefähigkeit als künstlich erkannt und so geschwächt wird, muss sie bereits in der Erziehung tief verankert worden sein: In der guten Sozialphilosoph*in halten sich also wohltätige Assoziationen und Analysefähigkeit die Waage; was bei Mill schief gelaufen ist, so denkt er, ist, dass sie sich bei ihm nicht die Waage halten. ${ }^{15}$

Zum Zeitpunkt der Krise erlauben Mill diese drei Aspekte seiner benthamitischen Philosophie - die Assoziationstheorie der Erziehung kombiniert mit dem psychologischen Hedonismus einerseits und der (Hume'schen) Idee, dass Analysefähigkeit nur die Kontingenz ethischer Assoziationen zeigen könne und sie so schwäche andererseits - seine Krise zu verstehen, aber sie bieten ihm keinen Ausweg. Anderson zitiert Mill entsprechend, der im Kapitel, das seine Krise behandelt, schreibt:

13 Siehe ebd., S. 143. Zusammenfassend schreibt er: „Analytic habits may thus even strengthen the associations between causes and effects, means and ends, but tend altogether to weaken those which are, to speak familiarly, a mere matter of feeling." Ebd., S. 143.

14 Eine Kritik Mills an Whewell ist, dass er das Gebiet der konventionellen Moral genau so hinterlassen habe, wie er es vorgefunden habe: Vgl. Mill, „Whewell on Moral Philosophy“, S. 169.

15 Siehe das Zitat, zu dem Fußnote 16 gehört. An dieses schließt eine Metapher an, die die Hume'sche Psychologie, mithilfe der sich Mill zu diesem Zeitpunkt versteht, mehr als deutlich macht: „I was thus, as I said to myself, left stranded at the commencement of my voyage, with a well equipped ship and a rudder but no sail; without any real desire for the ends which I had been so carefully fitted to labour for: no delight in virtue or the general good, but also just as little in anything else." Mill, Autobiography, S. 143. 
All those to whom I looked up, were of opinion that the pleasures of sympathy with human beings, and the feelings which made the good of others and especially of mankind on a large scale the object of existence, were the greatest and surest source of happiness. I was well convinced of this, but to know that a feeling would make me happy if I had it, did not create the feeling. My education had failed, as I thought, to give me these feelings in sufficient strength to resist the dissolving influence of analysis, while the whole course of my intellectual cultivation had made precocious and premature analysis the inveterate habit of my mind. ${ }^{16}$

Mill hebt auf dieses Zitat folgend zwei wichtige Veränderungen seiner Meinungen und seines Charakters ${ }^{17}$ hervor. Die Wichtigkeit des Anstrebens nicht-hedonischer Werte (für die meisten Menschen); ${ }^{18}$ das Pflegen der inneren Kultur des Individuums. ${ }^{19}$ In einer späteren Krise wird er auch den psychologischen Hedonismus Benthams aufgeben. ${ }^{20}$

\subsubsection{Die Natur des Guten nach Mill}

Auch wenn sie dies nicht explizit macht, lässt sich die Rolle, die Versuche mit neuen Lebensweisen für Mill laut Anderson haben, differenzieren: Zum einen liefern sie beispielsweise eine Interpretation dessen, was Mill mit dem Urteil der Erfahrenen im Zusammenhang mit der Qualität von Leid und Freude meint. ${ }^{21}$ Versuche mit neuen Lebensweisen spielen auf diese Weise eine ethische Rolle innerhalb von Mills Ansatz. Darauf gehe ich in Abschnitt 5.2 weiter unten ein. Für Anderson ist aber entscheidender, was als ihre „metaethische Rolle" bezeichnet werden könnte: Nicht nur können Versuche mit

16 Mill, Autobiography, S. 143.

17 „my opinions and my character", ebd., S. 145.

18 Ebd., S. $145 \mathrm{ff}$.

19 „the internal culture of the individual“, ebd., S. $147 \mathrm{ff}$.

20 Ebd., S. 177.

21 „Mill found a basis for distinguishing pleasures in rank, for promoting ideals of excellence as intrinsically worthy ends, and for advocating the cultivation of the sentiments rather than their repression. He claimed that evidence for the superiority of one conception of the good over another can be found in experiments in living." Anderson, „John Stuart Mill and Experiments in Living", S. 14. 
neuen Lebensweisen dazu dienen, innerhalb Mills ethischer Vorstellungen bestimmte Lebensweisen vor anderen bezüglich der Qualität von Freude auszuzeichnen, sondern sie können auch dazu dienen, eine Vorstellung über die Natur des Guten zurückzuweisen: Experimente mit neuen Lebensweisen ${ }^{22}$ belegen, dass Mills Vorstellung des Guten ${ }^{23}$ Benthams Vorstellung des Guten ${ }^{24}$ überlegen ist. Damit haben sie für Anderson nicht nur eine Rolle innerhalb von Mills Ansatz, sondern sprechen für eine Vorstellung davon, worin das Gute besteht, selbst.

Ich bezeichne diesen Punkt als „metaethisch“, weil er nach dieser Skizze Mills Vorstellung von der Natur des Guten ändert. In welcher Hinsicht genau sich für Mill die Natur des Guten ändert, ist eine komplexe und schwierige Frage. Manchmal - und Mill selbst legt dies im Utilitarismus natürlich nahe ${ }^{25}$ - wird Mill so verstanden, dass er zu den für das Nutzenkalkül relevanten Faktoren der Freuden, die Bentham empfiehlt, einen weiteren hinzufügt. Doch sowohl Menschen, die Sympathien für ihn hegen als auch viele seiner Kritiker*innen bestreiten, dass dieses Verständnis so richtig sein kann. Die einen sagen, er leiste etwas viel Wichtigeres ${ }^{26}$ und die anderen weisen darauf hin, dass eine Inkonsistenz entsteht: Entweder die Qualität von Freuden lasse sich doch auf so etwas wie ihre Intensität und Nachhaltigkeit - Kriterien, die Bentham durchaus kennt reduzieren oder es seien entgegen Mills eigener Aussage eben nichthedonische Kriterien, die hinter dem unterschiedlichen qualitativen Wert der Freuden stehen. ${ }^{27}$

22 Siehe ebd., S. 15-20.

23 Siehe ebd., S. 8-15.

24 Siehe ebd., S. 6-8.

25 Siehe Mill, „Utilitarianism“, § 2.4

26 „The insistence on qualitative assessment means more than a mere rejection of Bentham's famous remark about push-pin and poetry." F. E. L. Priestly. "Introduction". In: The Collected Works of John Stuart Mill, Volume X Essays on Ethics, Religion, and Society. Hrsg. von J. M. Robson. Toronto und andere: University of Toronto Press und Routledge \& Kegan Paul, 1969, S. 78-115, S. xli. In eine ähnliche Richtung gehen zum Beispiel Fred Berger. „Mill's Concept of Happiness“. In: John Stuart Mill's Social and Political Thought. Hrsg. von G. W. Smith. Bd. 1. London und New York: Routledge, 1991, S. 117-136 und Benjamin Gibbs. „Higher and Lower Pleasure“. In: Philosophy 61.235 (1986), S. 31-59.

27 Vgl. z. B. George Edward Moore. Principia Ethica. Cambridge und andere: Cambridge University Press, 2000, § 48. Siehe Rex Martin. „A Defence of 


\section{Mills Philosophie des Wohlergehens}

Anderson selbst akzeptiert Letzteres: ${ }^{28}$ Die „Qualität von Freuden" bleibt ein irreduzibel normativer Begriff, was Anderson belegt, indem sie darauf hinweist, dass Mill Willensschwäche bezüglich der Qualität erlaubt: Wir können eine Freude als qualitativ hochwertiger bewerten, ohne dass sich dies im Sinne einer revealed preference zeigt. ${ }^{29}$ Warum bleibt Mill dann aber offiziell ethischer Hedonist? ${ }^{30}$ Eine manchmal gegebene Antwort wäre, dass Mill zu der Zeit, als er "Utilitarianism" verfasst, anders als etwa 25 Jahre vorher, als "Coleridge" und „Bentham" entstanden, nun - in der Hochphase der Viktorianischen Epoche - meint, die erhoffte gesellschaftliche Veränderung, die ihm in seinen praktischen Schriften auch immer vorschwebt, eher in hedonistischen Begriffen fassen zu können. ${ }^{31}$ Eine andere Strategie, Mill hier verständlich zu machen, besteht darin, auf Textstellen bei Mill hinzuweisen, die zeigen, dass seine Vorstellung vom Wohlergehen sehr viel komplexer ist.

So weist Anderson zum Beispiel darauf hin, dass Mills Formulierungen, nehmen wir sie wörtlich, gar nicht ausschließen, dass etwas anderes als Leid und Freude intrinsische Werte sind: Sie setzten nur voraus, dass andere intrinsische Werte, wenn sie erfahren werden, als Freude oder Leid erfahren werden. ${ }^{32}$ Eine vielleicht verwandte Art und Weise, einen qualitativen Hedonismus (als Theorie des Wohlergehens) zu plausibilisieren, liefert Roger Crisp, indem er die Fragen ,Which things make someone's life go better for them? und , [W] hat is it about these things that make them good for people?" " unterscheidet. ${ }^{33}$ Außerdem weist Anderson auf die von mir bereits in Abschnitt 4.3.2 diskutierte Stelle aus Mills Aufsatz „Bentham“ hin, in welcher deutlich wird, dass für Mill ethische Werte keineswegs die einzigen handlungsrelevanten Werte sind. ${ }^{34}$

Mill's Qualitative Hedonism“. In: Philosophy 47.180 (1972), S. 140-151 für ein Argument, dass diese Alternative zurückweist.

28 Vgl. Anderson, „John Stuart Mill and Experiments in Living“, S. 10.

29 Vgl. ebd., S. 10.

30 Für die Phrase „official ethical hedonism" vgl. ebd., S. 9.

$31 \mathrm{Zu}$ taktischen Überlegungen Mills in seinem Bekenntnis zu Bentham und dessen Hedonismus siehe z. B. Priestly, „Introduction“, xxxivf.

32 Vgl. Anderson, ,John Stuart Mill and Experiments in Living“, S. 13.

33 Vgl. Roger Crisp. Reasons and the Good. Oxford: Clarendon Press, 2008, S. 102.

34 „Every human action has three aspects: its moral aspect, or that of its right and wrong; its asthetic aspect, or that of its beauty; its sympathetic aspect, 
Alle Erklärungen haben sicher etwas für sich. Ich denke, wir können den Hauptgrund für den offiziellen ethischen Hedonismus in Mills Überzeugung sehen, dass es zum menschlichen Wohlergehen einen Zugang in der Erfahrung oder im Erleben geben muss. Diese Erklärung steht nicht in Konkurrenz zu den anderen, sondern kann als Erläuterung dienen: Mills Hedonismus besteht zumindest auf die Forderung, dass sich Lebensweisen, in denen menschliches Wohlergehen möglich ist, im Vergleich zu tatsächlich erfahrenen Alternativen, in denen das weniger der Fall ist, jedenfalls auch dadurch unterscheiden müssen, dass erstere Lebensweisen normalerweise auch als qualitativ besser erlebt werden. Der seit Moore in der Mill-Forschung eine Rolle spielende Frage, ob wir Freude oder Leid überhaupt als einheitliches Phänomen betrachten können, wäre damit ein Stück weit ausgewichen. ${ }^{35}$

Ich möchte vorschlagen, dass wir Mill letztlich einfach so verstehen sollten, dass "Freude" und „Leid“ die Wörter sind, mit denen wir die Weise, wie sich eudaimonistische Werte als Werte in unserem Erleben wiederfinden, benennen. ${ }^{36}$ Das heißt weder, dass sie ein einheitliches Phänomen im Sinne eines Typs von mentalem $\mathrm{Zu}-$ stand sein müssen, ${ }^{37}$ noch dass das Erleben von etwas als wertvoll

or that of its loveableness. The first addresses itself to our reason and conscience; the second to our imagination; the third to our human fellow-feeling. According to the first, we approve or disapprove; according to the second, we admire or despise; according to the third, we love, pity, or dislike. The morality of an action depends on its foreseeable consequences; its beauty, and its loveableness, or the reverse, depend on the qualities which it is evidence of. Thus, a lie is wrong, because its effect is to mislead, and because it tends to destroy the confidence of man in man; it is also mean, because it is cowardly [...]." Mill, „Bentham“, S. 112. Andererseits scheint Mill stellenweise nahezulegen, dass happiness der Maßstab für alle drei Handlungsbereiche sei, wie bereits in Abschnitt 4.3.2 diskutiert. Vgl zum Beispiel Mill, The Collected Works of John Stuart Mill, Volume VIII - A System of Logic Ratiocinative and Inductive (Books IV-VI and Appendices), Buch VI, Kapitel 12.

35 Moore, Principia Ethica, § 48. Siehe Crisp, Reasons and the Good, S. $101 \mathrm{f}$ für eine systematische Aufbereitung dieser Frage.

36 Dies scheint auch dem Hauptunterschied zwischen Mill und Aristoteles, den Nussbaum in „Mill between Aristotle and Bentham" hervorheben will, zu entsprechen: Siehe Martha C. Nussbaum. „Mill between Aristotle \& Bentham“. In: Daedalus 133.2 (2004), S. 60-68, S. 66.

37 Mill scheint dies stellenweise sogar explizit abzulehnen: „Neither pains nor pleasures are homogeneous, and pain is always heterogeneous with pleasure." Mill, „Utilitarianism“, § 2.8 . 
nicht fallibel sein kann. ${ }^{38}$ Dies ist vielleicht eine so schwache Bedingung, dass die Frage aufkommt, ob sie den provozierenden Titel des „Hedonismus“, den Mill für seinen Ansatz beansprucht, wirklich verdient, ${ }^{39}$ aber die Bezeichnung ist weder nichtssagend, noch selbstverständlich. ${ }^{40}$ Auch wenn ich glaube, dass in dieser schwachen Bedingung der entscheidende Grund für die hedonistische Terminologie liegt, ist es für die Zwecke meiner Arbeit nicht entscheidend, dass sich Mills Bekenntnis zum Hedonismus darin erschöpft, darauf zu bestehen, dass sich eudaimonistische Werte im Erleben über den Zeitraum eines Lebensexperimentes zeigen. Entscheidend ist lediglich, dass das, was Mill mit seinem offiziellen „Hedonismus“ meint, zumindest auch diese Funktion hat.

\subsubsection{Wie wird Benthams Philosophie durch Mills Krisenerfahrung widerlegt?}

Anderson präsentiert in ihrem Aufsatz Mills Erfahrung in der Krise mit der Theorie Benthams am Ende als strenges Argument gegen Benthams Theorie ${ }^{41}$ : Zunächst zeige Mills Krise und die Art und Weise, wie Mill die Krise überwindet, dass Benthams Theorie des ethisch Guten selbst-annullierend (self-effecating) ${ }^{42}$ ist: Benthams Theorie erkläre nicht, warum Mill in die Krise gerate und - wichtiger - sie erkläre auch nicht, warum Mill seine Krise überwinde,

38 So weist zum Beispiel Crisp darauf hin, dass das Urteil der erfahrenen Richter*innen für Mill fallibel ist. Vgl. Crisp, Mill on Utilitarianism, S. 36f. Dass das Urteil der Richter*innen fallibel ist, ist nicht der gleiche Gedanke, wie derjenige, dass das Urteil irreduzibel normativ ist, auf den ich oben mit Anderson hingewiesen habe. Ich gehe auf diesen Unterschied in Abschnitt 5.3 ein.

39 Und sicherlich stimmt auch, was Benjamin Gibbs sagt: „Mill is less than perfectly rigorous in his use of terminology, and he employs a flamboyant, oratorical style which inclines him to over-state and under-qualify his case." Gibbs, „Higher and Lower Pleasure“, S. 32.

40 Die Verwendung des Wortes ist auch nicht beispiellos: Ich glaube, sie ist sehr nahe an der Verwendung, mit der Ursula Wolf Aristoteles' erste Lustbetrachtung als „hedonistisch“ bezeichnet. Siehe Ursula Wolf. Aristoteles' „Nikomachische Ethik". 3. Aufl. Darmstadt: WBG, 2013, S. 211 und die Lustbetrachtung in Aristoteles. Nikomachische Ethik. 5. Aufl. Übersetzung von Ursula Wolf. Reinbek bei Hamburg: Rowohlt Taschenbuch Verlag, 2006, Buch VII.

41 Anderson, ,John Stuart Mill and Experiments in Living“, S. 20-23.

42 Ebd., S. 20. Den Begriff übernimmt sie aus Parfit, Reasons and Persons, S. 23. 
wenn er Dinge anstrebt, die nach Benthams Theorie nicht zu seinem Wohlergehen gehören. Damit könnte Benthams Theorie nur wahr sein, wenn Mill damit indirekt und ohne diese direkt anzustreben genau das erreicht, worin Wohlergehen nach Benthams Theorie besteht: Bloß quantitativ aufgefasste Lust und Freiheit von Schmerz. Die Theorie könnte also nur wahr sein, wenn sie uns zumindest in Fällen wie Mills empfehlen würde, einer anderen Theorie entsprechend zu handeln. ${ }^{43}$

Anderson scheint jedoch Parfit zuzustimmen, dass diese Tatsache allein noch nicht zeigt, dass eine solche Theorie nicht wahr sein kann. ${ }^{44}$ Stattdessen schlägt Anderson zwei Bedingungen vor, unter denen eine selbst-annullierende Theorie gültig sein kann: (1) Jede ethische Theorie muss handlungsleitend sein (das heißt, die selbstannullierende Theorie muss handlungsleitend sein und die Theorie, nach der sie uns zu handeln vorschlägt, muss handlungsleitend sein). (2) Eine selbst-annullierende Theorie kann nur gültig sein, wenn sie plausibel macht, warum die Theorie, die wir statt ihrer im Handeln annehmen sollten, obwohl wir sie im Handeln annehmen sollten, falsch ist. ${ }^{45}$ Diese beiden Bedingungen kann Benthams Theorie, wie Anderson zeigt, nicht erfüllen. ${ }^{46}$

Anderson gibt zu, hier ,spekulativ" für Mill zu antworten, weil er selbst dieses Problem - die Frage, ob und unter welchen Voraussetzungen die Tatsache, dass Benthams Theorie selbst-annullierend ist, gegen diese Theorie spricht - nie angesprochen habe. ${ }^{47}$ Die Spekulation erlaubt ihr jedoch ein sehr elegantes Argument zu entwickeln: ein Argument, das unter den beiden erwähnten Voraussetzungen Benthams Theorie an den eigenen Maßstäben scheitern lässt.

43 Siehe Anderson, „John Stuart Mill and Experiments in Living“, S. 16.

44 Ebd., S. 4. Wenn wir dieser Behauptung nicht zustimmten, dann wären wir nach Anderson hier bereits fertig: Eine Theorie, die uns im Handeln orientieren soll, aber scheitert, sobald wir nicht im Besitz dessen sind, auf das sich unser Handeln ausrichten soll, widerspricht ihrem eigenen Anspruch.

45 Fußnote 48 in Andersons Aufsatz macht klar, warum das wichtig ist: Ansonsten wäre ein Pluralismus, was Theorien des Guten angeht, plausibel. Da es aber nicht darum geht, Benthams Behauptung zurückzuweisen, dass Lust und Schmerz, so wie Bentham sie versteht, ethisch von Bedeutung sind, sondern darum, seine Behauptung zurückzuweisen, dass ausschließlich Schmerz und Lust in diesem Sinne von Bedeutung sind, ist die zweite Bedingung wichtig.

46 Siehe Anderson, ,John Stuart Mill and Experiments in Living“, S. 21f.

47 Siehe ebd., S. $21 \mathrm{f}$. 


\section{Mills Philosophie des Wohlergehens}

Obwohl ich glaube, dass Anderson überzeugend dafür argumentiert, dass „Experimente mit neuen Lebensweisen“ eine Schlüsselphrase im Verständnis einiger Aspekte von Mills Philosophie ist und dass es eine, vielleicht die wichtigste, Funktion seiner Autobiography ist, ein solches Experiment darzustellen, glaube ich nicht, dass es im Sinne Mills ist, hier ein strenges Argument auszuarbeiten: Benthams Theorie scheitert, so meine ich, aus Mills Sicht nicht daran, dass sie zwei plausiblen Bedingungen an selbst-annullierende Theorien $^{48}$ nicht gerecht werden kann. Mill selbst hat zum Beispiel sehr wenig dafür getan zu klären, ob wir seine eigene Theorie als selbst-annullierend verstehen sollten: Dass ethische Theorien praktisch orientieren sollen, ist ihm zwar wichtig, wie das erste Kapitel aus „Utilitarianism" zeigt. ${ }^{49}$ Und dies bedeutet in der Tat unter Andersons zweiter Voraussetzung immer eine Spannung zu selbstannullierenden Theorien. ${ }^{50}$ Auf der anderen Seite hätte sich Mill, wenn es ihm um ein strukturiertes Argument im Sinne Andersons gegangen wäre, in bestimmten Textstellen sehr viel klarer dazu verhalten müssen, ob er seine eigene Theorie als selbst-annullierend verstanden wissen will.

Dies gilt ganz besonders für Textstellen aus dem Krisenkapitel der Autobiography, die Veränderungen von Mills Meinungen und Charakter weg von Benthams und hin zu seiner eigenen, besseren Philosophie zeigen sollen. Hier zumindest müssten wir einen Anhaltspunkt finden, dass entweder Mills Theorie nicht selbst-annullierend ist oder sie, falls sie selbst-annullierend ist, mit Andersons Bedingungen an selbst-annullierende Theorien zurecht kommt. Es scheint mir aber keineswegs klar, dass die Verbesserungen seines Charakters und seiner Meinungen nach Mills eigener Einschätzung in diese Richtung gehen:

48 Oder der einen Bedingung, dass eine ethische Theorie nicht gleichzeitig selbstannullierend und handlungsleitend sein kann, aber handlungsleitend sein muss.

49 Siehe Mill, „Utilitarianism“, Kapitel 1.

50 Ich vermute, auch aufgrund der Textstellen, die ich nun besprechen werde, dass Mill sagen würde, dass die Frage, ob eine selbst-annullierende Theorie handlungsleitend sein kann, zum Teil auch eine empirische Frage ist: Bis zu einem gewissen Grade sind wir psychologisch vielleicht dazu in der Lage, zu lernen, Dinge um ihrer selbst willen anzustreben, und gleichzeitig zuzugestehen, dass das, was unser Anstreben dieser Dinge um ihrer selbst willen gut macht, Freude oder das Gefühl von Sinn u.s.w. ist. 
In the first place, they led me to adopt a theory of life, very unlike that on which I had before acted, and having much in common with what at that time I certainly had never heard of, the anti-self-consciousness theory of Carlyle. I never, indeed, wavered in the conviction that happiness is the test of all rules of conduct, and the end of life. But I now thought that this end was only to be attained by not making it the direct end. Those only are happy (I thought) who have their minds fixed on some object other than their own happiness; on the happiness of others, on the improvement of mankind, even on some art or pursuit, followed not as a means, but as itself an ideal end. Aiming thus at something else, they find happiness by the way. The enjoyments of life (such was now my theory) are sufficient to make it a pleasant thing, when they are taken en passant, without being made a principal object. Once make them so, and they are immediately felt to be insufficient. They will not bear a scrutinizing examination. Ask yourself whether you are happy, and you cease to be so. The only chance is to treat, not happiness, but some end external to it, as the purpose of life. Let your self-consciousness, your scrutiny, your selfinterrogation, exhaust themselves on that; and if otherwise fortunately circumstanced you will inhale happiness with the air you breathe, without dwelling on it or thinking about it, without either forestalling it in imagination, or putting it to flight by fatal questioning. This theory now became the basis of my philosophy of life. And I still hold to it as the best theory for all those who have but a moderate degree of sensibility and of capacity for enjoyment, that is, for the great majority of mankind. ${ }^{51}$

Es wird durch diese Textstelle (oder irgendetwas aus der Umgebung dieser Textstelle) überhaupt nicht klar, ob Carlyles „antiself-consciousness theory", so wie sie von Mill hier aufgefasst wird, als selbst-annullierende Theorie gemeint ist - und falls sie es ist, ob sie die Bedingungen Andersons erfüllt. (Und zumindest für diejenigen, die einen mäßigen Grad der Empfänglichkeit und Fähigkeit zur Freude haben, wird sie zum Zeitpunkt, zu dem er die Autobiographie verfasst, anscheinend von ihm immer noch vertreten.) In eine ähnliche Richtung geht auch eine Textstelle aus dem zwölf-

51 Mill, Autobiography, S. 145f. 


\section{Mills Philosophie des Wohlergehens}

ten Kapitel des sechsten Buches von A System of Logic, von dem Mill sagt, dass es in gewissem Sinne eine Reaktion auf die Krise ist. ${ }^{52}$ Ich will durch diese Textstellen keineswegs behaupten, dass Mill konsequent eine selbst-annullierende Theorie vertreten habe. ${ }^{53}$ Dies würde auch nicht zu dem passen, wie ich oben vorgeschlagen habe, dass wir Mills Hedonismus verstehen sollten. (Siehe 5.1.2.) Was ich mit diesen Textstellen belegen will, ist, dass es Mill in seiner Kritik an Bentham nicht darum geht, zu zeigen, dass sich ein logischer Widerspruch ergibt, wenn Benthams Theorie, Mills Krisenerfahrung und zwei Bedingungen an selbst-annullierende Theorien in Betracht gezogen werden. Wenn er das gewollt hätte, hätte er sich viel klarer zu Carlyle positioniert.

Benthams Philosophie hat aus Mills Sicht kein formales Problem dieser Art; sondern ihr Problem ist erstens, dass sie keinen Ausdruck dafür liefern kann, was Mill in seiner Krise an seinem Leben als schlecht empfindet - Mill bemüht Gedichtzeilen von Coleridge dazu in der Autobiography; ${ }^{54}$ zweitens, dass sie keinen Ausdruck dafür liefert, was Mill nach der Krise als Wert der neuen Lebensweise - er liest zum Beispiel Wordsworth - versteht. Deshalb ist Benthams Theorie eine schlechte philosophische Theorie des menschlichen Lebens und deshalb führt ein Leben nach dieser Theorie in die Krise. Dies ist aber, wie ich in Teil I versucht habe darzulegen, im Sinne von Coleridges Tugend ein Versagen der Bentham'schen Philosophie vor einem substantiellen Maßstab für Philosophie und nicht ein formales Versagen am eigenen Anspruch eine Theorie der happiness zu sein.

Diese Kritik lässt die wichtigsten Punkte von Andersons Mill-Interpretation völlig unangetastet. Vielleicht ist sie sogar nebensächlich $^{55}$ : Immerhin behauptet Anderson nicht, sie rekonstruiere

52 Siehe Mill, The Collected Works of John Stuart Mill, Volume VIII - A System of Logic Ratiocinative and Inductive (Books IV-VI and Appendices), S. 952.

53 Er ist, wie zum Beispiel Crisp bemerkt, auch kein Regelutilitarist, was eine alternative Deutung von Textstellen wie diesen ist: „In fact Mill is neither a single-level nor a self-effacing theorist; his is a multi-level view, of a particularly subtle kind." Crisp, Mill on Utilitarianism, S. 109.

54 Siehe Mill, Autobiography, S. 139 und 145.

55 Auf der anderen Seite schreibt Anderson: „If Mill had succeeded only on a practical and not on a theoretical plane, one could have charged that he had only shown that believing his theory has instrumental value, not that it was better." Mit „theoretical plane" muss - auch wenn Anderson zugibt, dass das 
Mills Argument im 5. Kapitel der Autobiography, sondern sie liefere selbst ein Argument im Sinne von Mill („on Mills behalf“) ${ }^{56}$ Für die Zwecke der Arbeit ist der exegetische Punkt aber von Bedeutung, weil ich natürlich dafür argumentieren will, dass Experimente mit neuen Lebensweisen die Art und Weise sind, wie Mill versucht, den beiden philosophischen Tugenden in seinem Leben und in seinem Werk gerecht zu werden. Benthams Theorie des Guten ist zurückzuweisen, weil sie schlechte Philosophie ist. Schlechte Philosophie ist sie nicht (oder nicht in erster Linie), weil sie argumentativ widerlegt werden kann, sondern weil (oder vor allem weil) sie einer der beiden philosophischen Tugenden nicht einmal in Ansätzen gerecht wird. In welcher Hinsicht Mills Lebensexperiment und seine aus diesem Experiment hervorgehende Philosophie den Tugenden gerecht wird, werde ich im nächsten Abschnitt deutlich machen.

\subsection{Experimente mit neuen Lebensweisen und die beiden Tugenden}

In dem folgenden Abschnitt möchte ich erläutern, inwiefern die Aspekte von Mills Philosophie, in denen Experimente mit neuen Lebensweisen eine Rolle spielen, jeweils Realisierungsversuche der Tugenden sind. Die Struktur ist hierbei natürlich jeweils ähnlich; es ist aber sinnvoll, auf die Aspekte einzeln einzugehen, weil der Wert der Tugenden sich jeweils auch daran zeigt, was mögliche Alternativen wären.

\subsubsection{Die Natur des Guten}

Experimente mit neuen Lebensweisen selbst sind noch keine Philosophie. Nicht jede*r, die sich vornimmt, nun anders zu leben, muss sich deshalb schon als Philosoph*in verstehen. Wie aber können

Argument, das sie bringt, ihr eigenes im Namen von Mill ist - die von mir hinterfragte Argumentation sein. Immerhin stellt sie diese Argumentation als Antwort auf das Problem vor, dass eine quantitative Hedonist*in, nachdem sich das Leben nach ihrer Theorie als nicht praktikabel erwiesen hat, immer noch sagen kann, dass ihre Perspektive die einzig valide Perspektive ist, von der aus sich bewertende Ansprüche rechtfertigen ließen. Siehe Anderson, „John Stuart Mill and Experiments in Living“, S. 20.

56 Siehe ebd., S. 21f. 


\section{Mills Philosophie des Wohlergehens}

Experimente mit neuen Lebensweisen Eingang in die Philosophie finden? Eine Möglichkeit, ihnen Eingang zu ermöglichen, ist die philosophische Autobiographie, die Mill wählt. Um zu verstehen, inwiefern dieser Ansatz Ausdruck der philosophischen Tugenden Mills ist, lohnt es sich, die Art Gründe, die Mill im Krisenkapitel aus der Autobiography gegen Benthams Philosophie angibt, mit den Gründen aus „Bentham ${ }^{657}$ in Bezug auf die Auseinandersetzung mit der benthamitischen Philosophie zu vergleichen. Der folgende Vergleich der jeweils unterschiedlichen Arten dieser Kritik zeigt, worum es mir geht.

Viele der inhaltlichen Punkte gegen die Philosophie Benthams werden (von der bereits angesprochenen Entschärfung der Rhetorik abgesehen, siehe Abschnitt 3.4.2) in beiden Texten angesprochen.

Allerdings überzeugt „Bentham“ nur, wenn die Leser*in bereits zugesteht, dass das, was Benthams Menschenbild etwas fehlt; und dass eine, die das behauptet, nicht nur den von Bentham kritisierten Fiktionen, die das Ziel haben, Machtverhältnisse aufrecht zu erhalten, unterliegt: Bentham selbst hat ja nach Mill gar nicht den Anspruch, unsere moralische Sprache zu analysieren und eine philosophische Theorie zu liefern, die ihrer Bedeutung gerecht wird - und Gleiches könnte sicherlich über unsere eudaimonistische Sprache gesagt werden, hätte sich Bentham dafür in gleicher Weise interessiert. Er meint, diese Aspekte unserer derzeitigen Praxis sind höchst fragwürdig und sollten nicht durch philosophische Berücksichtigung geadelt werden. Seine Philosophie, zumindest so wie Mill sie rekonstruiert, kann in dieser Hinsicht als revisionär ${ }^{58}$ bezeichnet werden: ,[H]e began de novo, laid his own foundations deeply and firmly, built up his own structure, and bade mankind compare the two. "59 Die neutrale Bilanzierung von Lust und Schmerz sind nach diesem Bild eben nicht in erster Linie als Analyse unserer Begriffe von moralisch gut und schlecht gemeint, sondern als ihr Ersatz.

57 Die Unterteilung hatte ich in Kapitel 4 vorgeschlagen.

58 Für eine Unterscheidung zwischen verschiedenen Formen des metaethischen Revisionismus in der hier gemeinten Bedeutung des Wortes siehe Christian Wendelborn. Der metaethische Relativismus auf dem Prüfstand. Berlin: De Gruyter, 2016, S. 264f. Benthams Revisionismus besteht mindestens in den Formen 2 und 3, die Wendelborn unterscheidet.

59 Mill, „Bentham“, S. 82. 
Wenn wir Benthams Moralphilosophie (auch) als Vorschlag verstehen, unsere moralische Praxis radikal zu reformieren, dann greift das Argument, diese würde das, was unsere moralischen Begriffe für uns bedeuten, nicht erfassen, nicht. ${ }^{60}$ Sobald wir zugestehen, dass wir unsere Praxis ändern könnten und zugestehen, dass es nicht gegen die Spielregeln der Philosophie ist, Änderungsvorschläge an unserer Praxis zu machen, scheinen wir auf die Weise, auf die Mill mit Bentham im zweiten Teil von „Bentham“ umgeht, Benthams ethischen Ansatz nicht mehr als falsch erweisen zu können. (Natürlich geht es darum auch nicht in „Bentham“, so wie ich den Aufsatz in Teil 1 interpretiert habe. Es geht nicht darum, einen ethischen Ansatz zurückzuweisen, sondern um die eine Hälfte von Mills Bild von guter Sozialphilosophie. Auch hier arbeitet Mill aber in einer Hinsicht empirisch: „Bentham“ und „Coleridge“ stellen sozusagen Experimente in unterschiedlichen Philosophierensweisen dar, auf die wir uns ein Stück weit einlassen müssen, um ihre Stärken und Schwächen zu erkennen.)

Gemäß Benthams Tugend müssen wir uns aber philosophisch begründet für oder gegen solche Änderungsvorschläge entscheiden. Wir müssen uns, mit anderen Worten, um eine Möglichkeit bemühen, objektive Gesichtspunkte zu finden: Die nach Benthams Tugend tugendhafte Philosoph*in betrachtet ihr philosophisches Tun nicht ausschließlich als Versuch des Ausdrucks einer bloß persönlichen philosophischen Präferenz oder eines bloß persönlichen phi-

60 In ähnlicher Weise verteidigt Peter Singer, dass er bestimmte moralische Intuitionen nicht berücksichtigt: „I have elsewhere argued against the inbuilt conservatism of this approach to ethics, an approach which is liable to take relics of our cultural history as the touchstone of morality." Peter Singer. "Utilitarianism and Vegetarianism". In: Philosophy and Public Affairs 9.4 (1980), S. 325-337, S. 326. Er bezieht sich hier auf einen anderen seiner Aufsätze, der entsprechend Rawls' Methode des Überlegensgleichgewichts, die zunächst von solchen Intuitionen ausgeht, kritisiert. Eigentlich geht es aber in dem Aufsatz, den Singer hier von sich selbst zitiert, darum, dass Rawls Sidgwick missverstehe, indem er ihm die Methode des Überlegensgleichgewichtes als allgemeine philosophische Methode zuschreibt. Es geht nur indirekt um den „eingebauten Konservativismus" dieser Methode, nämlich insofern Sidgwick der common sense Moral diese Eigenschaft zuschreibe und das Überlegensgleichgewicht nur dazu verwende, die Vertreter*in der common sense Moral vom Utilitarismus zu überzeugen, nicht aber als eigenständiges Argument für den Utilitarismus ansehe. Vgl. Peter Singer. „Sidgwick and Reflective Equilibrium“. In: The Monist 58.3 (1974), S. 490-517. 


\section{Mills Philosophie des Wohlergehens}

losophischen Temperamentes - auch wenn dies eine wichtige Rolle spielen mag. ${ }^{61}$ Als „Benthams Herausforderung“" hatte ich die Forderung bezeichnet, unsere moralische Praxis mit einer Praxis, die sehr anders ist, zu vergleichen, und eine Bewertung abzugeben, die nicht bloß Ausdruck einer subjektiven Präferenz ist. Ein solcher Versuch, eine Antwort auf Benthams Herausforderung bezüglich einer revisionären Philosophie zu finden, setzt voraus, dass wir philosophisch erfassen könnten, was es heißen würde, nach einem Vorschlag, unsere moralische Praxis radikal zu verändern, zu leben. Und das heißt, Versuche danach zu leben, müssen Eingang in die Philosophie finden können. Genau dies ist die Rolle, die die Autobiography für Mill hat.

Mein Ziel mit diesem Vergleich der Argumentationen in der $\mathrm{Au}-$ tobiographie und in „Bentham" sollte zeigen, was nötig ist, um eine Position als besser auszuzeichnen. Praktische Versuche dessen, was es heißen könnte, nach einer solchen Position zu leben, müssen Eingang in die philosophische Auseinandersetzung mit den Positionen finden können. Nur so können wir entscheiden, ob zum Beispiel eine revisionäre Position wie Benthams besser ist.

Ich werde weiter unten (Abschnitt 5.3) systematisch etwas zur Fallibilität dieser Herangehensweise sagen. Konkret aber liegt natürlich ein Einwand in Benthams Namen nahe: Vielleicht hatte seine Philosophie einfach keine faire Chance im Leben des jungen Mill, weil wir durch die Alltagsmoral so vorgeprägt sind, dass es einfach gar nicht ohne sekundäres Leid möglich ist, die Begriffe und die mit diesen Begriffen verbundenen Emotionen der herkömmlichen Moral abzustreifen. Ich denke, die Beschreibung, die Mill von seiner frühen Erziehung gibt, erfüllt auch ${ }^{62}$ die Aufgabe, diesen Einwand ein Stück weit zu zerstreuen. So legt er zum Beispiel Wert darauf, dass

61 Ich spiele an auf William James. Pragmatism and other Essays. New York: Washington Square Press, 1963, S. 6f. Für eine Deutung der Rolle des Temperamentes bei James, die in ein paar Hinsichten dem entspricht, wie ich mit Anderson Mill im Folgenden deuten werde, die aber in einigen anderen Hinsichten, so meine ich, radikaler ist, siehe Gunnarsson, Vernunft und Temperament. Eine Philosophie der Philosophie. Eine Abgrenzung dazu findet in Kapitel 8 statt.

62 Eine andere Aufgabe gibt Mill explizit an: Er will zeigen, wie wirksam frühe Erziehung ist und unter welchen Umständen (vgl. Mill, Autobiography, S. 5) - und zwar im Positiven (vgl. z. B. ebd., S. 33) wie im Negativen (vgl. z. B. ebd., S. 39 oder S. 53). 
sein Vater ihn von schädigenden äußeren Einflüssen bis zu einem gewissen Grade abgeschirmt habe. ${ }^{63}$ In Bezug auf die potentiell fest verankerte, religiöse Alltagsmoral seiner Zeit ist aber von größerer Bedeutung, was Mill über die Abwesenheit von Religion in seiner Erziehung sagt:

I am thus one of the very few examples, in this country, of one who has, not thrown off religious belief, but never had it: I grew up in a negative state with regard to it. I looked upon the modern exactly as I did upon the ancient religion, as something which in no way concerned me. It did not seem to me more strange that English people should believe what I did not, than that the men whom I read of in Herodotus should have done so. ${ }^{64}$

Mill musste die Begriffe der herkömmlichen, christlichen Moral und die damit verbundenen Emotionen nicht abstreifen, weil er diesen durch seine areligiöse Erziehung und seine Isolation in einem gewissen Sinne gar nicht ausgesetzt war. Es ist für diesen Punkt von Bedeutung, dass Mill der um ihn verbreiteten Religion mit der gleichen rein betrachtenden Einstellung begegnet wie der antiken Religion: Er glaubt nicht, dass ihm etwas fehlt, was religiöse Menschen haben. (Wenn es so etwas wie Religion für Mill gibt, so ist es Bentham selbst, sobald Mill zum ersten Mal in Dumonts Ausgabe liest. ${ }^{65}$ ) Stattdessen wird auch das Verhalten seines Vaters ${ }^{66}$ als eines durch antike Philosophie inspirierten Utilitaristen beschrieben. ${ }^{67}$ Entscheidend ist, dass es hier um das Verhalten des Vaters geht: Mill will es nicht nur als Lippenbekenntnis erscheinen lassen,

63 Beispielsweise von den Einflüssen anderer Jugendlicher. Vgl. ebd., S. 37. Aber auch, was Erwachsene angeht, scheint Mill vor allem Kontakte zu Menschen zu haben, die Bentham goutiert haben müsste. Vgl. ebd., S. 55.

64 Vgl. ebd., S. 45.

65 Vgl. ebd., S. 69.

66 Der Textstelle, in der es um die moralischen Einflüsse, die Mill durch seine Erziehung erfährt, geht, werden folgende Worte vorausgestellt: „But though direct moral teaching does much, indirect does more; and the effect my father produced on my character did not depend solely on what he said or did with that direct object, but also, and still more, on what manner of man he was." Ebd., S. 49. Es geht also darum, welche moralphilosophische Richtung das Verhalten des Vaters bestimmt.

67 Vgl. ebd., S. 49f. Wenn auch, wie bereits in Fußnote 7 erwähnt, nicht unbedingt im Sinne der reinen Lehre Benthams. 


\section{Mills Philosophie des Wohlergehens}

dass seine Erziehung, wie er im darauf folgenden Kapitel sagt, ein Kurs in Benthamismus gewesen sei. ${ }^{68}$

Auf diese Weise legt Mill nahe, dass Benthams Philosophie eine faire Chance hatte. So gut es eben möglich ist, so will Mill es uns glauben machen, hat er nach Benthams Philosophie gelebt; mit den entsprechenden Affekten - und vor allen Dingen ohne die potentiell „ideologischen" Affekte, die eine Erziehung nach der Alltagsmoral mit sich gebracht hätte. Philosophieren in dieser Hinsicht bedeutet nicht nur, kognitiv einer Theorie zuzustimmen, sondern sich auch affektiv für die entsprechende Theorie bereit zu machen - und Mill war durch seine Erziehung auf diese Weise vorbereitet. Wäre er es nicht gewesen, hätte es für den Anspruch des Versuches auf noch so bescheidene Verallgemeinerbarkeit schlecht ausgesehen. In diesem Sinne ist auch Benthams Tugend eine Tugend - zumindest in Mills Art und Weise, sie zu realisieren. Nicht nur Coleridges Tugend, sondern auch Benthams Tugend setzt voraus, dass wir uns emotional sensibilisieren oder eben desensibilisieren und wir können erfolgreich darin sein oder nicht. (Dies mag als Ergänzung zur Bemerkung aus Abschnitt 4.3.1 verstanden werden, dass wir die „Wissenschaftlichkeit", die mit Benthams Tugend einhergeht, nicht im Sinne einer Naturwissenschaft oder Ähnlichem auslegen sollten.) Dadurch, dass Mill keine emotionale Bindung an das Christentum hat, kann er Folgendes nahelegen: Die Tatsache, dass Benthams Theorie mit Mills Krise nicht umgehen kann, ist dieser Theorie selbst geschuldet und nicht der Tatsache, dass das Experiment durch verfälschende Einflüsse verpfuscht war - so bemüht sich Mill es zumindest darzustellen.

Es gibt natürlich andere gewichtige und allgemeinere Zweifel an der Gültigkeit solcher Experimente, die damit nicht aus der Welt geschafft sind. (Siehe Abschnitt 5.3.) Ich erwähne diese Punkte nur hier bereits, um zu zeigen, dass Mill das Bemühen um ein nicht bloß subjektives Kriterium zur Entscheidung für oder gegen Benthams Vorschlag einer Neugestaltung unserer moralischen Sprache sehr ernst nimmt: Eine Abneigung gegen Benthams Philosophie stammt bei Mill jedenfalls nicht aus seiner Kindheit oder Jugend.

Wie aber sieht es mit Coleridges Tugend aus? Das Kriterium für das Ausüben der Tugend wäre, dass es Mill gelingt, einen sprachli-

68 Vgl. Mill, Autobiography, S. 67. 
chen Ausdruck zu finden, der dem angemessen ist, was an der Art und Weise, nach Benthams Philosophie zu leben schlecht ist, und dem, was an Mills veränderter Art und Weise, nach der Krise zu leben, gut ist. Das heißt eine Art des Ausdrucks, die Mill und die eine Person, die wie Mill auf die jeweilige Weise gelebt hat, als angemessenen Ausdruck ihrer Erfahrungen beschreiben würde. Wie gut ihm das letztlich in diesem speziellen Fall mit der Beschreibung seiner Krise gelingt, will ich nicht entscheiden: Ich müsste lange Passagen aus der Autobiography zitieren und diskutieren, ob sie adäquate sprachliche Ausdrücke für die entsprechenden Erfahrungen sind. In Abschnitt 6.2 werde ich ein paar grundsätzliche Punkte zu Mills diesbezüglichen Versuchen machen.

Wichtig ist aber, dass sein Philosophieren als ein Versuch gesehen werden kann, genau dies zu tun: Er spricht zum Beispiel nicht rein klinisch über seine Krise, sondern bemüht sich, diese zu Erfahrungen in Beziehung zu setzen, von denen er meint, dass seine Leser*innen sie kennen: „I was in a dull state of nerves, such as everybody is occasionally liable to [...]." ${ }^{69}$ Er benutzt Gedichte (von Coleridge), um seine Krise zu beschreiben, ${ }^{70}$ und spielt auf Gedichte von Wordsworth an, um ein Beispiel davon zu geben, was ihm danach etwas bedeutet. Er beschreibt emotionale Episoden - von Suizidgedanken ${ }^{71}$ bis zur Rührung zu Tränen, beispielsweise bei der Lektüre von Jean Francois Marmontels Memoiren. ${ }^{72}$

Was die Zwecke dieser Arbeit angeht, ist vielleicht am wichtigsten: Er bemüht sich deutlich zu machen, inwiefern seine nun veränderte Philosophie mit den Erfahrungen der Krise (und späterer Krisen) umgeht: die bereits angesprochene Rolle nicht-hedonischer Werte, ${ }^{73}$ die Wichtigkeit eines breiteren Bildungsbegriffs, ${ }^{74}$ die Zurückweisung des psychologischen Hedonismus Benthams ${ }^{75}$ und neue Einflüsse anderer philosophischer Richtungen, für die er sich öffnet. ${ }^{76} \mathrm{Ob}$ dies für uns überzeugend ist, hängt natürlich davon ab, ob Mills philosophische Gedanken wirklich das treffen, was uns im Le-

69 Ebd., S. 137.

70 S. 139 und S. 145

71 Siehe Mill, Autobiography, S. 145.

72 Siehe ebd., S. 145.

73 Siehe z. B. ebd., S. 145-147.

74 Siehe z. B. ebd., S. 147-149.

75 Siehe z. B. ebd., S. 177.

76 Siehe z. B. ebd., S. 159ff. 
ben beziehungsweise in Lebensversuchen daran wichtig ist: Ist Mills Theorie vom Wohlergehen wirklich überzeugend? Ist sein perfektionistischer Bildungsbegriff sinnvoll? Entgeht Mills Modifikation der Lehre der Notwendigkeit wirklich dem, was uns am Determinismus bedrückt? Dies sind nicht der Hauptgegenstand dieser Arbeit.

Was aber, denke ich, nicht bestritten werden kann - und was den Hauptgegenstand dieser Arbeit betrifft - ist, dass Mill sich bemüht, das, was Personen, die nach den entsprechenden philosophischen Gedanken zu leben versuchen, erfahren, philosophisch so zum Ausdruck zu bringen, dass es eine ,faithful interpretation of their experience" ist. ${ }^{77}$

\subsubsection{Das Urteil der Erfahrenen}

Neben verschiedenen Leiderfahrungen bringt Mill die Bedeutung, die Literatur und besonders Poesie für ihn haben, auf den Punkt. Der Wert, den Literatur und Poesie für ihn haben, ist genauso wenig von der gleichen Art (Mill spricht von ,kinds of pleasure ${ }^{78 * \text { ) }}$ wie der Wert körperlichen Wohlbefindens, wie das Leid, das er in der Depression erlebt, von der Art körperlichen Schmerzes ist. Dies führt Mill zur Einführung seines pluralistischen Hedonismus. ${ }^{79}$ Ich hatte vorgeschlagen, dass wir das Bekenntnis zum Hedonismus zumindest so verstehen sollten, dass Mill daran festhält, dass der Wert eudaimonistischer Werte einer Person, die auf entsprechende Weise

77 Vgl. Mill, „Coleridge“, S. 119.

78 Mill, „Utilitarianism“, § 2.4.

79 Ebd., § 2.2-9. Mill geht in diesen Abschnitten so weit, eine relativ starke Form der Inkommensurabilität der Freuden und Formen des Leides zu behaupten: Er meint zum Beispiel unabhängig von der Quantität (vor allem Intensität und Dauerhaftigkeit) gebe es Formen des Leides, die wir nicht in Kauf nehmen würden, egal wie intensiv und dauerhaft das Leid ist, dass wir dafür in Kauf nehmen müssen - und analog bei Freuden. Dies ist für viele der Beispiele unplausibel. Elizabeth Anderson meint, wir müssten Mill hier schwächer verstehen. (Siehe Anderson, ,John Stuart Mill and Experiments in Living“, S. 9.) Systematisch diskutiert beispielsweise James Griffin die Plausibilität verschiedener Formen von Inkommensurabilität, insbesondere vor dem Hintergrund einer pluralistischen Auffassung von Wohlergehen. Siehe James Griffin. Well-Being. Its Meaning, Measurement and Moral Importance. Oxford: OUP, 1986, Kapitel V. 
lebt ${ }^{80}$, erlebbar sein muss. Obwohl aber Literatur und Poesie diese Bedeutung für ihn haben, ist Mill nicht der Meinung, dass er besonders rezeptiv für diese Art der Freuden ist. Er hält sich für eine unpoetische Natur und sagt über Wordsworth, der ihm viel bedeutet, entsprechend: „Compared with the greatest poets, he may be said to be the poet of unpoetical natures, possessed of quiet and contemplative tastes. But unpoetical natures are precisely those which require poetic cultivation. "81 Ebenso meint Mill, dass er zur Zeit seiner Depression für die Freuden der Musik nicht empfänglich

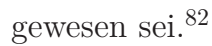

Nicht alle Menschen sind zu allen Zeiten ihres Lebens in der Lage, die Erfahrungen zu machen, die den Witz der entsprechenden Lebensweisen ausmachen. Gleiches gilt natürlich für Formen des Leides. Über Bentham schreibt Mill diesbezüglich:

He had neither internal experience nor external; the quiet, even tenor of his life, and his healthiness of mind, conspired to exclude him from both. He never knew prosperity and adversity, passion nor satiety: he never had even the experiences which sickness gives; he lived from childhood to the age of eighty-five in boyish health. He knew no dejection, no heaviness of heart. He never felt life a sore and a weary burthen. He was a boy to the last. ${ }^{83}$

Dies gilt auch allgemein. Nicht nur Mill und Bentham sind für bestimmte Formen des Leides und der Freude unsensibel, keine philosophierende Person kann die relevanten Erfahrungen jeder Art und Weise zu leben machen: „No one inquirer is all this; every inquirer is either young or old, rich or poor, sickly or healthy, married or unmarried, meditative or active, a poet or a logician, an ancient or a modern, a man or a woman [...]." ${ }^{484}$ Es scheint daher, dass wir uns

80 Es geht Mill auch in „Utilitarianism“ klar um Lebensweisen: Das Urteil der Erfahrenen wird in Bezug auf „Lebensweisen“ (,manners of existence“) im Utilitarismus eingeführt (John Stuart Mill. Utilitarianism/Der Utilitarismus. Stuttgart: Reclam, 2006, S. 29) - und wäre, bezöge es sich auf einzelne Handlungen oder gar Freuden, aufgrund der Tatsache, dass wir dann niemals niedrige Freuden wählen sollten, auch nicht plausibel.

81 Mill, Autobiography, S. 153.

82 Ebd., S. 147ff.

83 Mill, „Bentham“, S. 92.

84 Vgl. ebd., S. 90. 


\section{Mills Philosophie des Wohlergehens}

wieder zwischen den Tugenden entscheiden müssten: Die erste Möglichkeit ist, dass wir auf Objektivität setzen und uns beschränken auf die Erfahrungen, die wirklich alle Menschen unabhängig von ihren Lebenssituationen und dem Grad und der Weise der Kultivierung ihrer Fähigkeiten teilen können. Die zweite Möglichkeit ist, dass wir die Wichtigkeit, die höhere Freuden für uns haben, betonen, aber zugeben müssen, dass diese an unsere eigene kontingente Art und Weise zu leben gekoppelt sind.

Um trotzdem beiden Tugenden gerecht zu werden, bemüht Mill sich nicht nur selbst rezeptiver für zum Beispiel die ästhetischen Werte der Poesie zu werden; er öffnet auch seine Philosophie entsprechend an entscheidenden Stellen für die Erfahrungen anderer. Menschen, die Erfahrungen mit alternativen Lebensweisen gemacht haben, sind diejenigen, die Mill als erfahrene Richter*innen bezeichnet. ${ }^{85}$ Das Bild alter weißer Männer, das uns möglicherweise in den Sinn kommt, die uns ihre Meinung aufdrängen wollen, dass bei Whiskey und Zigarren geschäftliche Beziehungen in Hinterzim-

85 Natürlich nimmt Mill in Bezug auf höhere Freuden eine Antwort vorweg, für die er vielleicht selbst kein erfahrener Richter gewesen ist: „Now it is an unquestionable fact that those who are equally acquainted with, and equally capable of appreciating and enjoying, both, do give a most marked preference to the manner of existence which employs their higher faculties." Mill, „Utilitarianism“, § 2.6. Zum Beispiel Herlinde Pauer-Studer sieht Mill hier die „konventionellen Wertvorstellungen seiner Zeit“ übernehmend, insbesondere orientiere er sich „an den Idealen des Bildungsbürgertums“. Siehe Herlinde Pauer-Studer. Einführung in die Ethik. 2. Aufl. Wien: Facultas, 2010, S. 63. Auch abgesehen davon ergeben sich Probleme: Mill sagt zum Beispiel nichts dazu, ob bei Leid einfaches körperliches Leid allen Formen des Leides, die höhere Fähigkeiten voraussetzen, immer vorzuziehen ist. (Vgl. z. B. Gibbs, "Higher and Lower Pleasure", S. 46.) Außerdem ergibt sich natürlich auch für Mill ein Problem, dass bereits Bentham hatte: Ist zum Beispiel Schadenfreude, weil sie höhere Fähigkeiten involviert als die Freude an einer warmen Badewanne, wirklich vorzuziehen? Vgl. z. B. ebd., S. 46. Benjamin Gibbs meint: „Mill does not point out, as perhaps he should, that pleasures which engage the higher faculties are not necessarily high in value or importance." Vgl. z. B. ebd., S. 46. Er hat sicherlich Recht: Es geht Mill nicht darum, grundsätzlich den Vorzug von Freuden, die höhere Fähigkeiten betreffen, zu belegen, sondern darum, eine pluralistische (im oben angedeueten Sinne hedonistische) Theorie des Wohlergehens zu formulieren. Siehe auch Martin, "A Defence of Mill's Qualitative Hedonism“, S. 145. Wie auch bei der Behauptung starker Inkommensurabilität, (siehe Fußnote 79) sollten wir Mills Rhetorik hier, denke ich, abschwächen. 
mern zu pflegen eine qualitativ besonders hochwertige Freude sei, und die mit diesem Einwand verbundene Assoziation des (patriachalen) Paternalismus sind also falsch. Wenn überhaupt kommen sie weniger als andere als erfahrene Richter*innen in Betracht: Es sind ja gerade „neue und originelle“ 86 Lebensweisen, die nötig sind, damit ich wirklich andere Erfahrungen machen kann. Wer bloß der Konvention folgt, kann solche Erfahrungen nicht machen.

An der Freude, die mit Whiskey und Zigarren in Hinterzimmern verbunden ist, ist noch etwas anderes verzerrend. Der Begriff der higher pleasure scheint etwas Optionales, ja im Angesicht des gravierenden Gewichts, die ethische Fragen für uns haben, beinahe etwas Opulentes zu haben. Wenn wir uns aber ansehen, welche Erfahrungen es spezifisch sind, von denen Mill Bentham zum Beispiel im obigen Zitat vorwirft, für sie blind zu sein, sehen wir, dass dies nicht vorwiegend Freuden, sondern vor allem Leiderfahrungen sind. Leid kommt ebenso wie Freude in verschiedenen Qualitäten ${ }^{87}$ und wir können auch Mills Bemerkung über Würde, die im gleichen Abschnitt wie der Hinweis auf verschiedene Qualitäten von Leid kommt, so verstehen, dass eine Verletzung in der Würde, eine der schlimmsten, d.h. von denen, die sie erfahren haben, als am schlimmsten bewerteten, Formen des Leides mit sich bringt. Auch in der Antwort auf den Einwand, dass Glück für Menschen gar nicht erreichbar sei, betont Mill die Wichtigkeit des Leides. ${ }^{88}$ Dass eine neue und originelle Lebensweise als besser erlebt wird als eine andere, kann also auch einfach heißen, dass sie für diejenige, die sie lebt, weniger Leid oder weniger schlimmes Leid bedeutet.

\subsubsection{Das Freiheitsprinzip}

„On Liberty“, besonders das dritte Kapitel, hilft, ein Missverständnis der acht Absätze, in denen Mill in „Utilitarianism“ das Urteil der Richter*innen einführt, auszuräumen. Lesen wir Absatz 5 als

86 Siehe John Stuart Mill. „On Liberty“. In: The Collected Works of John Stuart Mill, Volume XVIII - Essays on Politics and Society Part I. Hrsg. von J. M. Robson. Toronto und andere: University of Toronto Press und Routledge \& Kegan Paul, 1977, S. 213-310, S. 281.

87 "A being of higher faculties requires more to make him happy, is capable probably of more acute suffering, and is certainly accessible to it at more points [...]." Vgl. Mill, Utilitarianism/Der Utilitarismus, S. 31.

88 Vgl. ebd., S. $38 \mathrm{ff}$. 


\section{Mills Philosophie des Wohlergehens}

Definition der Bedeutung von „Qualität" in Bezug auf Freuden, könnten wir auf die Idee kommen, Mill meine, wir sollten ein Leben vorziehen, dass eine Mehrheit der Erfahrenen als besser erlebt, auch wenn wir selbst das nicht so erleben. ${ }^{89}$ Dies wäre natürlich sehr unplausibel. ${ }^{90}$ Zum Glück macht Mill aber in "On Liberty“ klar, dass die Frage, wie weitreichend das Urteil der Erfahrenen ist, selbst eine empirische Frage ist.

The traditions and customs of other people are, to a certain extent, evidence of what their experience has taught them; presumptive evidence, and as such, have a claim to his [a human being, jt] deference: but, in the first place, their experience may be too narrow; or they may not have interpreted it rightly. Secondly, their interpretation of experience may be correct, but unsuitable to him. Customs are made for customary circumstances, and customary characters; and his circumstances or his character may be uncustomary. Thirdly [...] to conform to custom, merely as custom, does not educate or develope in him any of the qualities which are the distinctive endowment of a human being. ${ }^{91}$

Der zweite Punkt, den Mill hier macht, ist der entscheidende. Der erste Punkt, würde in die Sprache von „Utilitarianism“ zurücküber-

89 „If I am asked, what I mean by difference of quality [...], there is but one possible answer. Of two pleasures, if there be one to which all or almost all who have experience of both give a decided preference [...], that is the more desirable pleasure. If one of the two is, by those who are competently acquainted with both, placed so far above the other that they prefer it, even though knowing it to be attended with greater amount of discontent, and would not resign it for any quantity of the other pleasure $[. .$.$] , we are justi-$ fied in ascribing to the preferred enjoyment a superiority in quality." Mill, "Utilitarianism", § 2.5, meine Hervorhebung.

90 Es kann hier, anders als Mills Formulierung (siehe Fußnote 89) nahelegt, nicht wirklich um die Bedeutung von „Qualität" gehen. Vgl. z. B. Gibbs, „Higher and Lower Pleasure“, S. 47 und Martin, „A Defence of Mill's Qualitative Hedonism", S. 142. Dies wäre auch durch den von Mill selbst vorgenommenen Vergleich der Qualität mit der Intensität, die ebenso durch die Erfahrenen erfolgt, unplausibel. Intensität von Freuden bedeutet nicht, bei gleicher Qualität und Dauer vorgezogen zu werden. Das Urteil der Richter ist sicher evidentiell gemeint. Vgl. Crisp, Mill on Utilitarianism, S. 36. (Für eine Gegenposition siehe Ernest Sosa. „Mill's Utilitarianism“. In: Mill's Utilitarianism Text and Criticism. Hrsg. von James M. Smith und Ernest Sosa. Wadsworth Pub. Co, 1969.)

91 Mill, On Liberty/Über die Freiheit, S. $164 f f$. 
setzt einfach bedeuten, dass die betreffenden Personen nicht wirklich kompetente Richter*innen sind. Der zweite Punkt legt aber nahe, dass, selbst wenn sie dies sind, die Reichweite ihres Urteils nicht unbedingt jede Person abdeckt. Das Urteil von Personen mit der entsprechenden Erfahrung ist Evidenz, aber nicht zwingende Evidenz; es muss sich auch im Leben des Individuums bewähren und dies ist eine Frage der Erfahrung.

Eine pluralistische Gesellschaft ist aber nicht nur die Voraussetzung dafür, die Reichweite und Grenzen von Ansätzen zum Wohlergehen ausloten zu können (Benthams Tugend). Sie ist auch deshalb wichtig, weil die Freude oder das Leid, das mit einer Lebensweise verbunden ist, nicht mehr als solches wahrgenommen werden kann, wenn keine alternativen Lebensweisen verfügbar sind. Wir gewöhnen uns, so Mill, in diesem Fall einfach an das, was durch die Konvention vorgeschrieben wird, und die Lebensweise wird mechanisch. ${ }^{92}$ Dieser Punkt aus dem dritten Kapitel von „On Liberty“ erinnert natürlich an den gleichen Punkt über Meinungen im zweiten Kapitel, wo Mill meint, eine Meinung, der nicht gelegentlich widersprochen wird, würde zum toten Dogma und letztlich irgendwann bedeutungslos. ${ }^{93}$ Dies zeigt, dass es Mill nicht bloß um Benthams Tugend geht, sondern auch um Coleridges. Beide Tugenden sind für Mill hier verbunden: Mill meint, „Meinungen“ verlieren für uns ihre Bedeutung, und in Bezug auf soziale Praktiken verlieren wir das, was diese Praktiken wertvoll macht, aus den Augen, wenn sie unherausgefordert einfach wiederholt werden.

92 Vgl. ebd., S. 182ff. Man kann Mill mit Recht vorwerfen, dass er nicht genügend beachtet, dass die Form des Liberalismus, die er vertritt, selbst eine dominante Lebensweise ist. Vgl. z. B. Alasdair MacIntyre. Der Verlust der Tugend. Frankfurt am Main: Suhrkamp, 1995, S. 55.

93 Vgl. Mill, On Liberty/Über die Freiheit, S. 151 für die Zusammenfassung dieses Gedankens. Die Argumentation mag auf den ersten Blick so verstanden werden, als ob Mill eine frühe Version einer verifikationistischen Bedeutungstheorie vertreten würde: Um die Bedeutung einer Meinung zu erfassen, so scheint Mill sagen zu wollen, müssen wir verstehen, was es heißen würde, wenn die Meinung wahr wäre oder falsch wäre - und dafür müssen alternative Meinungen existieren. Dass Meinungen, denen nicht widersprochen wird, zu leeren Dogmen werden, ist für Mill aber keineswegs bloß eine analytische Wahrheit; es scheint auch eine psychologische Wahrheit zu sein. Auch diese zeigt sich letztlich in der Erfahrung. 
In diesem Sinne sind beide Tugenden Gegenstand von „On Liberty". Inwiefern verkörpert Mill aber selbst die Tugenden, wenn er "On Liberty" schreibt? Inwiefern ist „On Liberty" selbst eine Ausübung der philosophischen Tugenden? Dafür ist es nötig, nicht nur, was Mill sagt, in Betracht zu ziehen, sondern auch seine Absichten ${ }^{94}$ zu berücksichtigen.

Dies tut Robert Haraldsson. ${ }^{95}$ Zunächst stellt Haraldsson fest, dass es Mill um das, was manchmal das "harm-principle“ genannt wird, geht: Wir dürfen laut diesem Prinzip nur in die Lebensführung anderer Personen eingreifen, um Schaden für andere Menschen abzuwenden. Dieses Prinzip möglichst präzise zu fassen und möglichst genau auszuarbeiten, unter welchen Umständen in die Freiheit anderer Personen eingegriffen werden darf, ist aber nach Haraldson nicht die Absicht Mills. Dementsprechend dürfte es auch nach der Lektüre von „On Liberty“ viele Fälle geben, in denen Mills Leser*innen nicht klar ist, $o b$ ein Einfluss einer bestimmten Lebensweise auf eine andere Person als relevanter Schaden aufgefasst werden sollte. ${ }^{96}$ Zweitens beansprucht Mill, wie Haraldsson klarstellt, weder, dass das Schadensprinzip oder seine Formulierung desselben originell sind, noch meint er, dass viele ihm widersprechen werden. ${ }^{97}$

Beides ist auch nicht Mills Hauptinteresse, wie Haraldsson zeigt. Stattdessen befürchtet Mill laut Haraldsson, dass die Forderung nach individueller Freiheit selbst zum "toten Dogma" verkomme: Nicht, dass zu wenige Menschen Mills „very simple principle“ zustimmen, ist nach Haraldsson das Problem, sondern dass sie gleichgültig gegenüber seinem Inhalt geworden $\operatorname{sind}^{98}$ :

94 Und streng genommen auch seine Fähigkeiten.

95 Róbert H. Haraldsson. „, This all but universal illusion ... - Remarks on the question: Why did Mill write On Liberty?" In: Sats - Nordic Journal of Philosophy 5.1 (2004), S. 83-109.

96 Vgl. David Brink. "Mill's Moral and Political Philosophy“. In: The Stanford Encyclopedia of Philosophy. Hrsg. von Edward N. Zalta. Winter 2016. Metaphysics Research Lab, Stanford University, 2016, Abschnitt 3.6.

97 Siehe Haraldsson, ,., This all but universal illusion ...- Remarks on the question: Why did Mill write On Liberty?“, S. 109. Vgl. z. B. Mill, On Liberty/Über die Freiheit, S. 20, S. 42.

98 ebd., S. 160. Dass er das Prinzip auch als „truism“ (S. 42) bezeichnet, bedeutet für Mill eben, dass es beinhahe bedeutungslos geworden ist. Siehe Fußnote 93. 
Accordingly, we cannot say that Mill's goal is to get his contemporaries to agree with his principle of freedom. For one thing, they might do so already, but more importantly the agreement per se is worthless. [...] Thus a dull and torpid assent to a doctrine - even a fine doctrine for that matter, like Mill's principle of freedom - is not only worthless but can be extremely dangerous. It becomes a creed which may petrify the mind against all other influences addressed to the higher parts of our nature. ${ }^{99}$

Stattdessen gehe es Mill darum, zu zeigen, was es heißen würde, sich das Prinzip zu Herzen zu nehmen. ${ }^{100}$ Mill behandelt den Liberalismus tatsächlich in einer sehr ähnlichen Weise wie diejenige, für die er Coleridge erstens in Bezug auf das (nach Mill zu diesem Zeitpunkt leblose und dogmatische ${ }^{101}$ ) Christentum und zweitens in Bezug auf die (ebenfalls leblose und dogmatische) englische Staatskirche gelobt hatte: Das, worum es einer ernsthaft Gläubigen geht, auf den Punkt zu bringen. ${ }^{102}$

In der Sprache dieser Arbeit formuliert, bedeutet dies: In diesem Sinne verfolgt Mill in Bezug auf das Freiheitsprinzip klar Coleridges Tugend. Den Witz des Freiheitsprinzips, das, worum es einer, die diese Freiheiten kompetent ausnutzt, dabei geht, will Mill seinen Leser*innen nahebringen werden.

Mill bemüht sich auch um Benthams Tugend. Einige der Textstellen, in denen er das tut, gehören zu den problematischsten Textstellen bei Mill: Um Objektivität herzustellen verfährt Mill auch hier, indem er den Vergleich herstellt. Da die Freiheit, um die es Mill geht, aber auch eine Frage des Zusammenlebens ist, muss er dafür über Gruppen von Menschen sprechen, die seiner Meinung nach an den schlechten Folgen davon leiden, das Freiheitsprinzip nicht zu beachten. Dies mag bei den Calvinisten ${ }^{103}$ weniger schlimm sein; aber

99 Haraldsson, ,, This all but universal illusion ... - Remarks on the question: Why did Mill write On Liberty?", S. 91f.

100 Ebd., S. 92-94.

101 Mill hatte dies schon in "Coleridge" behauptet. Siehe Mill, „Coleridge“, S. 145. In „On Liberty“ widerholt er dies. Siehe Mill, „On Liberty“, S. 248f, wo das Christentum der Mehrheit der Gläubigen als Paradigma für eine leblose Überzeugung behandelt wird.

102 Mein Kapitel 3. Vgl. z. B. Mill, „Coleridge“, S. 150.

$103 \mathrm{Vgl}$. Mill, On Liberty/Über die Freiheit, S. $175 \mathrm{ff}$. 
wenn Mill zum Beispiel über China schreibt, verbreitet er letztlich kolonialistische Vorurteile. ${ }^{104}$

\subsection{Fünf Fehleranfälligkeiten von Mills Vorgehen}

Diese Verschränkung von dem, was der eudaimonistische Wert der Freiheit genannt werden könnte, der Mill wichtig ist - gesellschaftliche Freiheit ist die Voraussetzung dafür, Aussagen über das eigene Gedeihen machen zu können -, mit einer Dimension von Freiheit, die individualistisch nicht wirklich gut fassbar ist, zeigt bereits eines der Probleme von Lebensexperimenten als philosophischer Methode an. Dieser Spannung begegnet Mill mit der Zweiteilung des Kapitels:

Having said that Individuality is the same thing with development, and that it is only the cultivation of individuality which produces, or can produce, well-developed human beings, I might here close the argument: for what more or better can be said of any condition of human affairs, than that it brings human beings themselves nearer to the best thing they can be? ${ }^{105}$

Es folgt darauf der zweite Teil, der belegen soll, dass Freiheit nicht nur die Bedingung für jede*n Einzelne*n ist, ein gutes Leben $\mathrm{zu}$ führen, sondern, dass das Gewähren von Freiheit anderen gegenüber auch über die Tatsache, dass dann einzelnen Menschen und mitunter auch mir diese Freiheiten und damit die Möglichkeit auf menschliches Gedeihen eröffnet werden, hinaus wichtig ist. Dies führt Mill in Begriffen des Nutzens („some use“106) aus; und es ist weniger plausibel, dass der Wert der Freiheit so richtig erfasst wird - und damit steht auch in Frage, ob Lebensexperimente so einfach auf nicht-individuelle Fragen übertragen werden kann. Diesen Punkt und, was ich für Mills (unbefriedigende) Lösung davon halte, werde ich im nächsten Kapitel diskutieren. In diesem Kapitel bleibe ich bei der Frage des Wohlergehens.

Es gibt aber Zweifel an der Zuverlässigkeit von Lebensexperimenten selbst bezüglich eudaimonistischen Fragen. Diese will ich

104 Vgl. Mill, On Liberty/Über die Freiheit, S. $205 \mathrm{ff}$.

105 Ebd., S. 180.

106 Ebd., S. 182. 
im Folgenden explizit diskutieren - zum Teil hatte ich sie bereits implizit auf den vergangenen Abschnitten angedeutet. Obwohl diese Zweifel, so meine ich, in allen Fällen wichtige Probleme betreffen, glaube ich, dass sie letztlich keine entscheidende Argumente gegen experiments in living sind.

Die Universalität der Ergebnisse: Wie in Abschnitt 5.2 .3 bereits argumentiert, meint Mill nicht, dass die Aussage, dass eine Mehrheit der Menschen, die auf zwei verschiedene Weisen gelebt haben, eine dieser Lebensweisen entschieden bevorzugt, gleichbedeutend ist mit der Aussage, dass diese Lebensweise besser ist. Es ist lediglich letztlich ${ }^{107}$ die einzige Evidenz dafür, dass diese Lebensweise besser ist. Es könnte, wie die ebenfalls in Abschnitt 5.2.3 zitierte Textstelle belegt, auch immer sein, dass ich in entscheidender Hinsicht anders bin, als die Personen, deren Bewertungen ihrer Erlebnisse mit verschiedenen Arten und Weisen zu leben mir zugänglich sind - und dass ich, wenn ich beide Arten und Weisen zu leben ausprobieren würde, der anderen Lebensweise den entschiedenen Vorzug geben würde. Ich hatte oben geschrieben, dass die Reichweite der Ergebnisse solcher Lebensexperimente, oder die Frage, ob diese gar universell sind, für Mill selbst eine Frage der Erfahrung ist: Es kann sein, dass eine Art und Weise zu leben für Dich gut, für mich aber schlecht ist. Dies mag als zu großes Zugeständnis an den Subjektivismus empfunden werden - ein zu großes vielleicht vor dem Hintergrund von Benthams Tugend. Allerdings sind „subjektiv" und „objektiv“ notorisch mehrdeutige Begriffe. Für die Fälle dieser Fragestellung ist es sinnvoll „objektiv“ von „universell“ zu unterscheiden: Wenn „subjektiv“ in etwa heißt, dass es keinen Unterschied gibt zwischen meiner Einschätzung einer Lebensweise als gut für mich und der Frage, ob sie wirklich gut für mich ist, dann gibt es keinen Grund, warum die Tatsache, dass eine Lebensweise nicht universell besser als eine andere ist, bedeuten sollte, dass sie nicht trotzdem objektiv besser für mich sein kann: Bevor ich wirklich auf zwei verschiedene Weisen gelebt habe, mag ich einfach falsch in meiner Einschätzung liegen, dass eine Art zu leben

107 Diese Qualifikation ist wichtig: Es mag viele Gründe dafür geben, in einzelnen Situationen solche Evidenz in Frage zu stellen. Dies gilt aber nur, solange es andere Fälle der Urteile von Erfahrenen gibt - unter Umständen auch das eigene -, die hier ein Infragestellen der Evidenz plausibel machen. 
für mich besser ist. (Und wie sich in der Diskussion der anderen Fehlerquellen zeigen wird: selbst noch danach.)

Dies ist, glaube ich, wichtig, aber vielleicht reicht diese sehr bescheidene Vorstellung von „Objektivität" - die bloße Tatsache, dass es einen Unterschied macht, ob ich bloß meine, auf diese Weise zu leben sei besser für mich, oder ob es sich tatsächlich als besser herausstellt - nicht: Wie könnte zum Beispiel eine philosophische Behandlung der Frage nach dem menschlichen Gedeihen sinnvoll sein, wie sie Mill offensichtlich in der Autobiography und den zwölf Abschnitten zu einem pluralistischen Begriff von Freude und Leid im „Utilitarianism" durchgeführt hat? Denn natürlich haben solche Texte meist den Anspruch mehr zu sein als private Erkenntnisse über das eigene Gedeihen. Die Antwort ist, dass dies nicht von alleine kommt, sondern erarbeitet werden muss: Mill beispielsweise erkennt sich oder seine Situation in den Gedichten von Coleridge, die er in der Autobiography zitiert, ${ }^{108}$ und den anderen Schriften, die ihm etwas bedeuten, ${ }^{109}$ wieder. Dies erlaubt ihm, (im Einklang mit Andersons Interpretation) Benthams Theorie des Wohlergehens nicht nur für sich selbst zurückzuweisen. Sie ist damit noch nicht unbedingt universell zuwückgewiesen. Natürlich ist aber der Anspruch von Benthams Theorie, selbst eine universelle Theorie zu sein, zurückgewiesen. Aber es ist eine offene Frage, ob sie für Bentham zurückgewiesen ist, den Mill, wie bereits ausführlich dargestellt, (siehe insbesondere Kapitel 4) stellenweise so präsentiert, dass ihm einfach die Fähigkeiten zu vielen höheren Freuden und komplexeren Formen des Leides fehlen. ${ }^{110}$ Letztlich ist der Grad an Universalität also selbst eine Frage von Erfahrung. Dies führt zum nächsten Problem.

Der Erwerb von Empfänglichkeit für bestimmte Freuden und Formen des Leides: Mill ist sich sehr bewusst, dass die Empfänglichkeit für bestimmte Freuden (und sicher auch Formen des Leides) nicht einfach eine unveränderliche Tatsache über Individuen ist. Die Fähigkeit, höhere Freuden zu empfinden, muss erworben

108 Siehe Mill, Autobiography, S. 139 und S. 145.

109 Siehe ebd., S. 145.

110 Beispielhaft zur Erinnerung: Siehe Mill, „Bentham“, S. 92. 
und aufrecht erhalten werden. ${ }^{111}$ Dies verkompliziert die Frage nach der Objektivität zusätzlich - oben hatte ich nahegelegt, dass es den Tugendcharakter auch von Benthams Tugend nahelegt, weil Einstellungen und Empfänglichkeiten von der Philosoph*in gefordert werden. Eine negative Antwort auf die am Ende des letzten Absatzes aufgeworfene Frage, ob Bentham aus Mills Sicht zu höheren Freuden fähig ist, könnte beispielsweise bedeuten, dass er seine Fähigkeiten zu höheren Freuden nicht entwickelt hat, es könnte aber auch bedeuten, dass er solche Fähigkeiten nicht oder nur schwer hätte entwickeln können. ${ }^{112}$ Und natürlich ist auch vieles dazwischen möglich: Vielleicht ist der Erwerb einer Fähigkeit zu höherer Freude für die eine schwerer als für die andere; vielleicht muss die Fähigkeit für bestimmte Freuden sehr früh im Leben erworben werden und kann danach nicht mehr erworben werden, u.s.w. Dies stellt die Möglichkeit von Lebensexperimenten in Bezug auf viele Weisen zu leben ernsthaft in Frage und es lässt Zweifel daran aufkommen, wie sinnvoll der Begriff der Objektivität aus dem letzten Abschnitt noch angewendet werden kann. ${ }^{113}$

111 „Capacity for the nobler feelings is in most natures a very tender plant, easily killed, not only by hostile influences, but by the mere want of sustenance [...]." Mill, „Utilitarianism“, § 2.7.

112 Mill scheint im Falle von Bentham Ersteres zu meinen. In Bezug auf höhere Formen der Freude und des Leides meint Mill bezüglich Bentham: „[T]he quiet, even tenor of his life, and his healthiness of mind, conspired to exclude him from [internal and external experience]." Mill, „Bentham“, S. 92.

113 John McDowell fragt sich in analoger Weise in Might there be External Reasons, ob er einen externen Grund hat Zwölftonmusik zu hören. Die Idee ist, dass er jedenfalls keinen internen Grund hat und Zwölftonmusik etwas ist, für das die Hörgewohnheiten geschult werden müssen. Dies sei in etwa, was mit der Redeweise von externen Gründen gemeint sein könnte, meint McDowell; auch wenn er sich beeilt hinzuzufügen, dass wir (zumindest im Falle von Zwölftonmusik) eine Person, die diese Gründe nicht berücksichtigt, nicht als irrational bezeichnen sollten. Siehe John McDowell. „Might There Be External Reasons". In: Mind, Value and Reality. Cambridge, Mass. und London: Harvard University Press, 1998, S. 95-111, S. 107. Genau wie die Verwendung des Begriffes der Irrationalität hier seltsam wirkt, genauso seltsam ist es, in solchen Grenzfällen von Objektivität zu sprechen. (Natürlich geht es McDowell dabei nicht um „Freude“; es geht um ästhetischen Wert! Aber weder mindert das die Analogie, noch ist es unvereinbar mit meiner Minimalbedingung dessen, was „Freude“ für Mill heißt. Siehe Abschnitt 5.1.2.) 
Mill selbst scheint - bei allem Perfektionismus, der ihm zum Teil mit Recht zugesprochen wird ${ }^{114}$ - jedenfalls nicht zu meinen, dass wir um jeden Preis höhere Fähigkeiten erwerben sollten. Er reflektiert dies in Bezug auf seine Wertschätzung von Wordsworth, dem Poeten für unpoetische Naturen, ${ }^{115}$ mit denen Mill sicherlich auch sich selbst meint. Es ist plausibel, dass Mill auch durch seine Erziehung zu einer unpoetischen Natur geworden ist. ${ }^{116}$ Möglicherweise hätte Mill zu dem Zeitpunkt, zu dem er Wordsworth schätzen gelernt hat, unter großer Anstrengung noch zu einer poetischen Natur werden können; möglicherweise hätte Mill dann auch die Autor*innen schätzen können, die die Leute schätzten, aufgrund des Urteils derer er Wordsworth als „Poeten für unpoetische Naturen“ bezeichnet. Sollte er dies versuchen oder seine unpoetische Natur akzeptieren? Für solche Fragen spielt sicherlich nicht nur die Frage, was es heißen würde, eine Fähigkeit zur Perfektion auszubilden, eine Rolle, sondern auch Fragen des trade-offs zwischen verschiedenen Fähigkeiten. Vielleicht haben solche Fragen in solchen Situationen oft keine eindeutige Antwort. Mit Sicherheit sind die Antworten, dort wo es welche gibt, nicht leicht zu ermitteln. Dies ist natürlich kompatibel damit, dass es in einigen Fällen, aber sicherlich nicht grundsätzlich, sogar so sein mag, dass es unsere Entscheidung selbst ist, die eine Antwort in dieser Frage richtig macht.

Aber auch dies heißt natürlich nicht, dass dort, wo es eine Antwort auf die Frage gibt, diese Antwort sich nur darin äußern kann, dass eine Art und Weise zu leben von derjenigen, die so lebt, tatsächlich als besser erlebt wird, als eine andere Art und Weise zu leben. Es ist kein grundsätzliches Problem für experiments in living. Es zeigt nur, dass solche Antworten komplex und kompliziert und manchmal nicht eindeutig möglich sind.

114 Siehe Donatelli, „Mill's Perfectionism“.

115 Mill, Autobiography, S. 153.

116 „I had read, up to this time, very little English poetry. [...] My father never was a great admirer of Shakespeare [...]. [...] He cared little for any English poetry except Milton [...]. The poetry of the present century he saw scarcely any merit in [...]." Dabei ist zu berücksichtigen, wie stark sein Vater Mills Lektüre kontrolliert hat. Allerdings ist sein Vater nicht so benthamitisch, dass er den jungen Mill gar nicht mit Poesie in Kontakt kommen lässt. Siehe ebd., S. 19ff. 
Der soziale Kontext: Wir Leben in sozialen Kontexten und die Frage, welche Entscheidungen wir im Leben treffen können, hängt davon stark ab. An dieser Stelle ist nicht das am Anfang des Abschnittes erwähnte Problem gemeint, dass es Werte gibt, die sich nicht vollständig eudaimonistisch fassen lassen. Mit diesem Problem für Mill beschäftige ich mich im nächsten Kapitel; es ist aus theoretischer Sicht ernster. Das soll nicht heißen, dass die soziale Konstitution selbst eudaimonistischer Werte kein Problem sei, sondern dass nicht-individualistisch verstandene Güter Lebensexperimente vor eine sehr viel grundsätzlichere Art der Probleme stellen. Allerdings: Auch die Frage, was gut für ein Individuum ist, lässt sich niemals isoliert vom sozialen Kontext untersuchen. Mills höhere Freuden sind beispielsweise ausnahmslos solche, die die Existenz bestimmter sozialer Institutionen (Poesie etwa) vorraussetzen. Und natürlich ist Mill auch bewusst, dass der soziale Kontext die Möglichkeit von Lebensexperimenten stark beeinflusst und diese oft unmöglich macht: Einen sozialen Kontext herzustellen, der gegenüber möglichst vielen Arten zu leben offen ist, ist, wie bereits angedeutet (Abschnitt 5.2.3), eine der Mahnungen von „On Liberty“. Aber auch in "Utilitarianism" macht er das klar. ${ }^{117}$

Selbst, wenn wir Mill also zustimmen, dass der Benthamismus in seinem Fall eine faire Chance hatte, dürfte es in fast allen anderen Fällen deutlich weniger leicht sein: In Bezug auf die Geschlechter meint Mill zum Beispiel, dass wir uns zunächst aller Macht- und Herrschaftsbeziehungen zwischen den Geschlechtern und aller damit verbundener konventioneller Geschlechtsvorstellungen entledigen müssten. ${ }^{118}$ Es braucht nur mit Eltern gesprochen werden, die das tatsächlich in ihrer Erziehung versuchen, um festzustellen, wie schnell sie darin scheitern, ihre Kinder vor gesellschaftlichen Geschlechtsvorstellungen zu schützen. Auch das heißt natürlich nicht, dass wir uns nicht bemühen sollten!

117 Das Zitat aus Fußnote 111 geht folgendermaßen weiter: „, $[\mathrm{I}] \mathrm{n}$ the majority of young persons it speedily dies away if the occupations to which their position in life has devoted them, and the society into which it has thrown them, are not favourable to keeping that higher capacity in exercise." Mill, „Utilitarianism", § 2.7.

118 Siehe John Stuart Mill. „The Subjection of Women“. In: The Collected Works of John Stuart Mill, Volume XXI - Essays on Equality, Law, and Education. Hrsg. von J. M. Robson. Toronto und andere: University of Toronto Press und Routledge \& Kegan Paul, 1984, S. 259-340, S. $276 f$. 


\section{Mills Philosophie des Wohlergehens}

Das Problem daran ist aber nicht nur, dass aufgrund des sozialen Kontextes Lebensexperimente unmöglich gemacht werden. Dies würde nichts daran ändern, dass Lebensexperimente dort, wo sie möglich sind, und in dem Maße, in dem sie möglich sind, eine gute Methode sind. Das Problem ist auch, dass es nie leicht sein dürfte, zu entscheiden, welche Faktoren zum „Ergebnis" eines Lebensexperimentes beigetragen haben. Wie bereits erwähnt (Abschnitt 5.2.1), umgeht Mill dieses Problem, was sein eigenes benthamitisches Lebensexperiment angeht, dadurch, dass er darauf hinweist, nahezu isoliert und ohne störende Faktoren aufgewachsen zu sein. Auch die Tatsache, dass wir das Problem aber in den allermeisten Fällen nicht so umgehen können, wie Mill im Falle seines eigenen benthamitischen Lebensexperimentes diskreditiert aber Lebensexperimente selbst noch nicht. Es macht die Frage der Verallgemeinerbarkeit nur zu einer komplizierten Frage von Abwägung und Hinterfragen. ${ }^{119}$

Adaption: Gegen Wunsch- oder Interessenvorstellungen vom gedeihenden Leben werden oft „adaptive Wünsche“ angeführt; gemeint ist die zweifellos wichtige Feststellung, dass sich das, was sich Menschen wünschen oder worin ihre faktischen Interessen bestehen, an ihre Lebensumstände anpasst. Martha Nussbaum oder Amartya Sen beispielsweise bringen dieses Argument als Argument für den capability-approach vor: ${ }^{120}$ Wenn das so ist, dann taugen Wünsche oder Interessen nicht dazu, zu bestimmen, ob eine bestimmte Art zu leben selbst gedeihlich ist oder nicht.

Mill vertritt anders als Sidgwick zwar keine Wunsch- oder Interessenvorstellung des gedeihenden Lebens, aber Freude und Leid - insbesondere unter qualitativen Gesichtspunkten - sind sicher-

119 Eine stark vereinfachende Analogie aus der Mathematik wäre die der partiellen Ableitung einer Funktion mit mehreren Variablen. Obwohl eine partielle Ableitung nach einer Variablen natürlich nur etwas über die Optima einer Funktion aussagt unter der Vorraussetzung, dass die anderen Variablen sich nicht ändern, bedeutet das nicht, dass mit der partiellen Ableitung nichts über die Optima ausgesagt wäre.

120 Siehe Martha C. Nussbaum. „Der aristotelische Sozialdemokratismus“. In: Gerechtigkeit oder das gute Leben. Hrsg. von Herlinde Pauer-Studer. Frankfurt am Main: Suhrkamp, 1999, S. 24-85, S. 41-44 bzw. Sen Amartya. Commodities and Capabilities. Amsterdam etc.: North Holland, 1985. Kap. 1-6, S. 14 . 
lich ebenso anfällig für Anpassung. ${ }^{121}$ In dieser einfachen Form der Kritik trifft sie Mill natürlich nicht: Gerade weil wir uns in Freudund Leiderfahrungen so stark anpassen, kann sich letztlich nur eine Person ein primäres Urteil über zwei Weisen zu leben erlauben, die wirklich auf beide Weisen gelebt hat. Mit den beiden Beispielen (Marx' Arbeiter und analphabetische Frauen in Bangladesh), die Nussbaum anführt, um die Grenzen der Kritikmöglichkeiten einer auf Wünschen beruhenden Theorie des Wohlergehens deutlich zu machen, könnte Mill auf diese Weise also durchaus umgehen; und ebenso mit Amartya Sens Beispiel der Person, die „ill-fed, undernourished, unsheltered and ill“ ist, aber gelernt hat, „realistische“ Wünsche zu haben. ${ }^{122}$

Aber die Kritik kann verschärft werden: Es gibt sicherlich viele Fälle, in denen eine Art und Weise zu leben die Einschätzung der Freuden und des Leides einer anderen Art und Weise zu leben färbt. Der Extremfall wäre der Fall eines Sekteneintritts: Eine Person, die, zum Beispiel, immer wenn sie der Sekte beitritt, dieses Leben als besser bewertet, aber immer, wenn sie austritt, das Leben außerhalb der Sekte. Das Problem ist, dass bestimmte Arten zu leben es ausschließen, die eigene Lebensweise im Vergleich zu einer alternativen Lebensweise neutral in den Blick zu nehmen. Bestimmte Arten zu leben sind mit neutraler Betrachtung nicht vereinbar. Das trifft übrigens nicht nur auf den Extremfall des Sekteneintrittes zu; auch das Führen einer romantischen Liebesbeziehung ${ }^{123}$ und vielleicht auch die Einnahme eines moralischen Standpunktes (siehe

121 Unter Easterlin-Paradox ist eine unvorteilhafte Weise der Adaption bekannt geworden: Der Besitz bestimmter materieller Güter führen zu erhöhten Rückmeldungen auf Fragen subjektiven Wohlergehens, aber nur, solange der Besitz dieser gesellschaftlich nicht weit verbreitet sind, so Easterlins Schlussfolgerungen. Siehe Robert A. Easterlin. „Does Money Buy Happiness?“ In: The Public Interest 30 (1973), S. 3-10.

122 Siehe Nussbaum, „Der aristotelische Sozialdemokratismus“, S. 41-44 bzw. Amartya, Commodities and Capabilities, S. 14.

123 Laut Koppetsch verbleiben bestimmte geschlechtsspezifische Ungerechtigkeiten (zum Beispiel was Hausarbeit angeht) auch deshalb, weil es zum Ideal der romantischen Liebesbeziehung selbst gehört, bestimmte Formen der neutralen Bilanzierung nicht vorzunehmen. Siehe Cornelia Koppetsch. „Liebe und Partnerschaft. Gerechtigkeit in modernen Paarbeziehungen. Studien zur Soziologie intimer Beziehungen“. In: Liebe am Ende des 20. Jahrhunderts. Hrsg. von Katharina Hahn und Günter Burkart. Leske + Budrich, 1998, S. 111129. 


\section{Mills Philosophie des Wohlergehens}

Kapitel 6) sind mit bestimmten Formen der Neutralität nicht kompatibel - auch wenn es hier nicht so drastisch ist, wie im Falle der Sekte.

Hier liegt es zunächst nahe, zwei Fälle zu unterscheiden: Den Fall, in dem tatsächlich keines der Leben besser ist, und den Fall, in dem es für das Individuum einfach keinen epistemischen Zugriff ${ }^{124}$ auf diese Frage gibt. So, wie sich das Problem für Mill stellt, sind die Fälle aber nicht unterscheidbar - und das ist, so könnte gesagt werden das eigentliche Problem: Dadurch, dass die Frage, welches Leben wirklich besser ist, nur über die Bedingung, dass ein gedeihlicheres Leben zumindest auch als solches erlebt wird, gefasst wurde, ohne dass gesagt wurde, was es ansonsten umfasst, sehen beide Fälle gleich aus. ${ }^{125}$

Mill war das Problem bewusst: Innerhalb seiner Diskussion um die höheren Freuden legt er nahe, dass einige, die die Fähigkeiten zu höheren Freuden einmal erlebt hatten, die niedrigeren Freuden danach trotzdem als besser bewerten, weil sie die Sensibilitäten, die für die höheren Freuden nötig sind, verloren haben. ${ }^{126}$

Auch hier ist die Strategie, das Problem in seiner hier beschriebenen Gänze zuzugestehen, aber zu bestreiten, dass es ein grundsätzliches Problem ist. Wir wissen ja nicht a priori, sondern eben unter anderem aufgrund der Erfahrungen von Menschen, die in Sekten waren, selbst und ihrer Angehörigen, was eine solche Art zu leben bedeutet. Obwohl es also durchaus Fälle geben kann, in denen Adaption so stark ist, dass kein Urteil mehr möglich ist, ist in vielen anderen Fällen die Situation einfach sehr viel komplexer - was bedeutet, dass Urteile schwieriger, aber nicht unmöglich werden.

Experimentelles Ziel: Die Metapher des Experimentes, die ich in diesem gesamten Kapitel von Anderson übernommen habe, die diese ihrerseits einer Formulierung Mills entnimmt, ist in einigen bisher

124 Und zwar systematisch: Ein einzelnes Individuum könnte natürlich versuchen, die eigene Sicht zu korrigieren, indem es die Erfahrungsberichte anderer berücksichtigt.

125 Dies war der von mir favorisierte Vorschlag, wie wir den qualitativen Hedonismus verstehen sollten. Siehe Abschnitt 5.1.2. Damit war nicht gemeint, dass es nichts gibt, was ein Leben gedeihlicher macht, sondern lediglich, dass sich ein gedeihlicheres menschliches Leben zumindest auch dadurch auszeichnet, dass es in der Regel so wahrgenommen wird.

126 Siehe Mill, „Utilitarianism“, § 7. 
ignorierten Hinsichten problematisch. Eines der Probleme kann wie folgt gefasst werden: Wenn eine Person auf eine bestimmte Weise lebt, um ein Experiment durchzuführen, dann verfälscht das das Ergebnis. Sie hat dann eben genau nicht die Einstellungen und macht folglich auch nicht die Erfahrungen, die eine Person machen würde, die nicht in experimenteller Absicht auf diese Weise lebt. Wenn Mill - um ein sehr banales, aber ich denke leicht verständliches Beispiel zu bemühen - entscheiden würde, „mal auszuprobieren“, wie es wäre, nach Kants Moral zu leben, wäre es ihm unmöglich aus Achtung vor dem Gesetz zu handeln; er würde in Wirklichkeit immer nur aus Achtung vor dem eigenen Experiment handeln.

In diesem Sinne sind experiments in living natürlich keine Experimente: Experimente werden aus Erkenntnisinteresse - aus Neugierde - durchgeführt. Neugierde oder Erkenntnisinteresse ist aber sicherlich selten - wenn überhaupt - ein geeigneter Grund, ein experiment in living zu starten: Um wieder das banale Beispiel aus dem letzten Absatz zu bemühen: Eine Person, die versucht, aus Neugierde ihren moralischen Standpunkt gegen einen anderen einzutauschen, wird auch deshalb nicht in der Lage sein, die richtigen Einstellungen und Sensibilitäten zu entwickeln, weil moralische Neugierde ein äußerst fragwürdiges Motiv ist.

Aber die Metapher des Experimentes ist natürlich auch nicht so gemeint - weder bei Mill noch bei Anderson. Wenn Mills Erziehung als paradigmatisches experiment in living betrachtet werden kann, dann haben Lebensexperimente zunächst nichts mit Neugierde zu tun. Und auch, dass Mill nach seiner Krise versucht hat, anders zu leben und anders zu philosophieren, hat weniger mit Neugierde zu tun, als mit der Tatsache, dass das Leben, das er vorher geführt hat, für ihn nicht länger lebenswert war. Dies dürfte der Regelfall sein. Die Aufforderung in „On Liberty“, aus der die Phrase stammt, ist dementsprechend auch nicht, möglichst viel zu experimentieren, sondern dass wir Menschen die Chance geben sollten, den Wert verschiedener Lebensweisen praktisch zu ermitteln „when any one thinks fit to try them." 127

Damit will ich nicht sagen, das Wissen darum, gerade eine andere Art zu leben auszuprobieren, würde die Einstellungen und Erfahrungen der entsprechenden Personen nie in problematischer Weise

127 Mill, „On Liberty“, S. 260. 
beeinflussen: Das Wissen zu einer Avantgarde zu zählen, das Wissen, wenn nötig, in etablierte Institutionen zurückfallen zu können, das Wissen, dass es (unter Umständen) die eigene Entscheidung war, beeinflussen alle drei die Erfahrungen, die wir mit einer Art zu leben machen können. Alles, was ich sagen wollte, ist das auch diese Problematik nicht systematisch ist. Sie wäre es nur, wenn Lebensexperimente stets aus kühlem Erkenntnisinteresse durchgeführt würden und alle Einstellungen auf diese Weise hypothetisch wären. Ich will dieses fünfte Problem damit nicht herunter spielen, aber auch dieses Problem ist damit kein grundsätzliches Problem gegen einen solchen Ansatz.

All diese Punkte lassen die Frage aufkommen, ob es nicht eine bessere Art und Weise gibt, den Tugenden gerecht zu werden als durch Lebensexperimente. Und Mill selbst hat Lebensexperimente natürlich in On Liberty zunächst für praktische Fragen in Betracht gezogen. Im nächsten Kapitel werde ich mich mit anderen Versuchen Mills, beiden Tugenden gerecht zu werden, beschäftigen. Diese scheitern. Natürlich ist damit nicht gezeigt, dass Lebensexperimente die einzige Art und Weise sind, den Tugenden gerecht zu werden und ich liefere dafür im nächsten Kapitel auch kein Argument. Trotzdem kann natürlich in der Art und Weise, wie Mill meiner Meinung nach scheitert, noch einmal deutlich, was die Stärke von experiments in living als Antwort auf das durch die Spannung der Tugenden zueinander hervorgerufene Problem ist. Letztlich werde ich diese Frage offen lassen, nachdem ich in Kapitel 8 eine Abwägungen dazu vornehme.

Zusammenfassung. In Kapitel 5 ging es darum zu zeigen, wie John Stuart Mill mit seiner Theorie des menschlichen Gedeihens den beiden Tugenden gerecht wird. Ich habe dazu Elizbeth Andersons Interpretationsansatz vorgestellt und verteidigt, (Abschnitte 5.1.1 und 5.1.2) habe argumentiert, dass wir an einer für meine Interpretation entscheidenden Stelle von diesem Ansatz abweichen sollten, (Abschnitt 5.1.3) und habe schließlich anhand von drei Schlüsselstellen (Abschnitte 5.2.1, 5.2.2 und 5.2.3) gezeigt, dass Mill sich damit an beiden Tugenden orientiert und ihnen gerecht wird. Abschnitt 5.3 diente dazu, offensichtliche Einwände, von denen die meisten Mill durchaus bekannt gewesen sind, zu entkräften. In allen Fällen glaube ich, dass der jeweilige Einwand in praktischer Hinsicht 
ernstzunehmen ist, dass er aber nur zeigt, dass Mills Verfahren voraussetzt, dass wir uns dessen Fehlbarkeit vor Augen halten - nicht, dass es eine bessere Alternative gäbe.

Außerdem hoffe ich, dass in dem Kapitel deutlich geworden ist, warum es sich um philosophische Tugenden handelt: Dass sich Philosophie zumindest, sobald sie praktisch ist, im Leben zu bewähren hat, bedeutet einerseits, dass uns die Philosophie so wichtig sein muss, dass wir ihren praktischen Konsequenzen folgen; andererseits bedeutet es, dass wir die Realität unserer Leben in unseren philosophischen Betrachtungen die entscheidende Rolle spielen lassen müssen. Dies erschöpft sich nicht in Regeln oder Fähigkeiten. Es sind auch bestimmte Einstellungen zur Philosophie notwendig. (Ein weiteres Argument dafür, dass es Tugenden sein müssen, findet sich in Kapitel 7.) 


\section{Zwei Grenzen von Mills moralphilosophischem Ansatz als Versuch, den Tugenden gerecht zu werden}

In diesem Kapitel wird es weiterhin darum gehen, wie Mill die ihm von mir zugeschriebenen philosophischen Tugenden, die ich in Teil I anhand zweier mittlerer Texte Mills erarbeitet habe, in seinen reifen Texten verwirklicht. Während es im letzten Kapitel darum ging, dies anhand von einigen Motiven der Philosophie Mills, die ich für seine Stärken halte, nachzuweisen, möchte ich in diesem Kapitel auf das, was ich für einige seiner Schwächen halte, eingehen. Natürlich werde ich wie analog auch bei den Stärken im letzten Kapitel genauso in diesem Kapitel nicht auf alles eingehen können, was zum Teil berechtigterweise an der Philosophie Mills kritisiert werden könnte. Ich werde mich auf zwei grundlegende Punkte beschränken: erstens die Art und Weise, wie die Unparteilichkeit der Moral bei Mill verstanden wird (Abschnitt 6.1), welche dann letztlich zum Utilitarismus führt, und zweitens das, was gelegentlich als Mills „Psychologismus“ (Abschnitt 6.2) kritisiert wird.

In beiden Fällen, so behaupte ich, drängt die Verbindlichkeit der Tugenden für Mill diesen beim Versuch, die Spannung, die zwischen den Tugenden besteht, aufzulösen, letztlich dazu, unplausible Positionen einzunehmen. Die Bedeutung dieser unplausiblen Positionen zur Unparteilichkeit im Utilitarismus und zum Psychologismus verstehen wir besser, wenn wir sie als Versuche auffassen, beiden Tugenden gerecht zu werden. Abschnitt 6.1 soll dementsprechend zeigen, was es bedeuten würde, „Utilitarianism“ vor dem Hintergrund der beiden Tugenden zu lesen. Ich werde mich dabei letztlich mehr mit Benthams Tugend als mit Coleridges Tugend auseinandersetzen. (Nur am Anfang des Abschnittes gehe ich kurz auf Coleridges Tugend ein.) Insgesamt wird der Abschnitt 6.1 in dieser Deutung zeigen, worum es Mill bei seinem Beweisversuch des utilitaristischen Prinzips ging; warum Mill das, worum es in dem Beweis 


\section{Zwei Grenzen von Mills Philosophie}

geht, wichtig ist; und warum dies auch philosophisch ein wichtiges Anliegen ist. Außerdem werde ich behaupten, Mill scheitere letztlich an seinem Anspruch. Dies ist natürlich nicht originell: Oft wird Mills Beweis für defektiv gehalten. Wenn aber Mills Scheitern vor dem Hintergrund von dem, worum es Mill ging, verstanden wird, dann wird deutlich, was Mills Beweis leisten müsste - und vor allem, dass bestimmte Versuche, den Beweis zu rehabilitieren, ${ }^{1}$ scheitern: Diese Versuche fassen nicht, worum es Mill geht. Wir verstehen Mill also unter dieser Deutung besser.

\subsection{Unparteilichkeit im Utilitarismus}

In Abschnitt 5.3 des letzten Kapitels hatte ich Schwierigkeiten mit Mills Ansatz von Versuchen mit neuen Lebensweisen diskutiert. Die Strategie war es, zuzugeben, dass es sich jeweils um Herausforderungen handelt, dass diese aber nicht den Ansatz als solchen in Frage stellen, sondern dass innerhalb dieses Ansatzes damit umzugehen sei: Die philosophierende Person muss Sensibilitäten für solche Schwierigkeiten entwickeln - und Mill hat dies, so habe ich behauptet, auch in den meisten Fällen getan. Eine der offensichtlicheren Schwierigkeiten habe ich dabei aber aufgeschoben: Lebensexperimente lassen sich nicht trivialer Weise von Fragen des gedeihenden Lebens auf andere praktische Probleme insbesondere des Zusammenlebens übertragen - zumindest nicht, solange es keine Möglichkeit gibt, die individuellen Erfahrungen mit verschiedenen Arten des Zusammenlebens zusammenzufassen.

Benthams utilitaristisches Unparteilichkeitsprinzip ist natürlich ein Antwortversuch auf diese Frage und Mill stimmt dieser Antwort in „Utilitarianism“ zu: „Bentham's dictum, 'everybody to count for one, nobody for more than one,' might be written under the principle of utility as an explanatory commentary. "2 Wenn das so formulierte Unparteilichkeitsprinzip, so wie Mill es versteht, zum Beispiel in der Moral gültig ist und wenn Moral nichts anderes als die Förderung von happiness aus unparteiischer Perspektive ist, dann könnte Mill in gewisser Weise seinen beiden Tugenden auf

1 Beispielsweise Crisps Andeutung, Mill müsse lediglich zugeben, dass das Unparteilichkeitsprinzip im Utilitarismus eine intuitionistische Basis habe, damit der Beweis durchgehen könnte. Siehe Crisp, Mill on Utilitarianism, S. 82.

2 Mill, „Utilitarianism“, § 5.36. 
die gleiche Weise gerecht werden, wie ich es im letzten Abschnitt für die Vorstellung vom guten Leben angedeutet habe: Für Fragen der happiness haben wir philosophisch eine Antwort - und alles, was wir nun tun müssen, so scheint es, ist neben dem eigenen auch das Wohlergehen anderer Leute im Blick zu haben. Wenn also das Unparteilichkeitsprinzip gilt und Moral nichts anderes ist als die Förderung von Wohlergehen aus neutraler Perspektive, dann könnten wir sagen, Mills Experimente mit neuen Lebensweisen seien auch eine Antwort auf praktische Probleme des Zusammenlebens. Und dann wäre Mill folglich auch in diesen Fragen den beiden philosophischen Tugenden gerecht geworden. Es hängt also für die Frage, inwiefern Mill den Tugenden hier gerecht wird, viel an seinem spezifischen Unparteilichkeitsprinzip und natürlich ist seine Rolle in dieser Funktion keineswegs unproblematisch. Das utilitaristische Unparteilichkeitsprinzip will ich in diesem Abschnitt diskutieren.

Freilich sind damit, dass Mill das Unparteilichkeitsprinzip einfach von Bentham übernimmt, noch andere Probleme verbunden: So scheint Mill mit der Einführung einer pluralistischen Vorstellung vom guten Leben die Maximierung von Glück eigentlich aufgeben zu müssen. ${ }^{3}$ Es ist nicht ganz klar, was genau bei Mill an die Stelle der Maximierungsidee treten könnte, um Unparteilichkeit zu gewährleisten: Wenn wir zwischen verschiedenen Lebensweisen wählen müssen, ist es vielleicht plausibel zu sagen, es gehe nicht um Maximierung irgendeiner Art, sondern darum, ein solches Leben zu wählen, das mit der Möglichkeit der Ausübung aller menschlichen Fähigkeiten auch die damit verbundenen Freuden - das Erleben des Ausübens dieser Fähigkeiten als etwas Gutes - enthält. Wie aber auf Maximierung bei der neutralen Bilanzierung der Konsequenzen einer Handlung für alle Betroffenen verzichtet werden kann, ist nicht so einfach einzusehen. Hier soll es nicht um diese Probleme gehen, sondern um die Frage, ob es Mill gelingt, indem er seine Theorie des gedeihenden Lebens um das Unparteilichkeitsprinzip

3 Zum bereits angesprochenen Problem, wenn qualitativ unterschiedliche Freuden tatsächlich inkommensurabel sind, siehe noch einmal Griffin, Well-Being. Its Meaning, Measurement and Moral Importance, Kapitel V. Selbst aber, wenn sie nicht als in Griffins stärkstem Sinne inkommensurabel verstanden werden, ist die Möglichkeit von Maximierung natürlich nicht gewährleistet. Diese ist nur gewährleistet, wenn die Freuden vollständig quantitativ vergleichbar wären. Vgl. auch Crisp, Mill on Utilitarianism, S. $40 f$. 


\section{Zwei Grenzen von Mills Philosophie}

zu einer Theorie der Moral erweitert, den beiden Tugenden gerecht $\mathrm{zu}$ werden.

Mill scheitert, was die Unparteilichkeit im Utilitarismus angeht, am Ende an beiden Tugenden. Diese Aussage impliziert natürlich, dass Mill sich an den Tugenden orientiert, wofür ich im Folgenden argumentieren will. Das ist aber nicht alles, was ich sagen will. Wir verstehen besser, was Mill will, wenn wir sein Tun als ein Scheitern an den Tugenden verstehen: Dies können wir dann als den Versuch sehen, etwas philosophisch richtig zu machen und nicht bloß als einen Fehler. Fehler treten natürlich trivialerweise immer beim Versuch auf, etwas richtig zu machen, sonst sind sie nicht als Fehler verständlich. Mill will mit dem, was ich hier unter der Überschrift „Unparteilichkeitsprinzip“ behandele, das, was er früher den sozialen Aspekt der Moralphilosophie ${ }^{4}$ nannte, betreiben. Indem ich mit den sozialphilosophischen Tugenden spezifiziere, was das für Mill heißt, spezifiziere ich also sowohl den Fehler, als auch, was es heißen würde, es richtig zu machen.

Dazu, dass Mill sich an Coleridges Tugend orientiert, will ich, wie gesagt, nicht viel sagen. Zwar scheint, was den bloßen Aufbau von „Utilitarianism" angeht, Coleridges Tugend Priorität ${ }^{5}$ zu haben:

Before, therefore, I attempt to enter into the philosophical grounds which can be given for assenting to the utilitarian standard, I shall offer some illustrations of the doctrine itself; with the view of showing more clearly what it is, distinguishing it from what it is not, and disposing of such of the practical objections to it as either originate in, or are closely connected with, mistaken interpretations of its meaning. ${ }^{6}$

Entscheidend, um zu sehen, dass dieses Zitat einen Versuch, Coleridges Tugend gerecht zu werden, ankündigt, ist zu verstehen, was die Korrektur der "fehlerhaften Interpretationen der Bedeutung“ des utilitaristischen Prinzips leisten soll.

Die „fehlerhaften Interpretationen der Bedeutung" des utilitaristischen Prinzips, die Mill in den beiden darauf folgenden, dem „Beweis" vorausgehenden Kapiteln ausräumt, sind fast alle Versuche

4 Siehe Mill, „Bentham“, S. 98.

5 Die Priorität ist damit begründet, dass Mill glaubt, hier lägen die Haupthindernisse für die Akzeptanz des Utilitarismus. Vgl. Mill, „Utilitarianism“, § 1.6.

Ebd., § 1.6. 
zu zeigen, dass das utilitaristische Prinzip dem gerecht wird, was denjenigen, die die Einwände erheben, auf die Mill eingeht, an der moralischen Praxis wichtig ist. Was die Mills Utilitarismus zugrunde liegende Theorie des Guten angeht ${ }^{7}$, habe ich schon Einiges im letzten Kapitel (Kapitel 5) gesagt. Aber auch der Rest des Kapitels folgt einer ähnlichen Strategie: Mill greift jedes Mal einen Einwand auf, der im Wesentlichen darin besteht, der Utilitarismus könne einen Aspekt dessen, was an unserer moralischen Praxis wichtig sei, nicht erfassen; er versucht dann den Witz dieses Einwandes so zu fassen, dass er in den Utilitarismus integrierbar ist - oft indem er versucht zu zeigen, dass das, was nicht integrierbar ist, auch nicht das ist, worum es einer Person, die einen solchen Einwand formulieren würde, eigentlich geht. Dies tut er mit dem Einwand, Tugendhaftigkeit sei mit Enthaltsamkeit, nicht mit Glück verbunden und Glück im utilitaristischen Sinne sei auch nicht (zumindest nicht für viele) erreichbar; ${ }^{8}$ mit dem Einwand, Unparteilichkeit im utilitaristischen Sinne könne Menschen nicht für alle ihre Handlungen abverlangt werden, ohne sie zu überfordern; ${ }^{9}$ mit dem Einwand, Nutzenkalküle seien mit dem Wesen moralischer Überlegungen und insbesondere der Rolle moralischer Emotionen in solchen Überlegungen nicht kompatibel; ${ }^{10}$ mit dem Einwand, der Utilitarismus könne dem kategorischen Charakter einiger moralischer Regeln nicht gerecht werden, weil diese immer gebrochen werden dürften, wenn es nützlich ist: ${ }^{11}$ dem Einwand, die Art der Unparteilichkeit, die der Utilitarismus fordere, sei größeren Gefahren der ungewollten Parteilichkeit ausgesetzt als die Art der Unparteilichkeit, wie sie in anderen moralischen Systemen gefordert werde. ${ }^{12}$ Selbst mit dem, was Menschen an der dezidiert christlichen Moral schätzen, sei der Utilitarismus kompatibel. ${ }^{13}$ Auch die beiden übrigen Kapitel können in diesem Lichte gesehen werden: In Kapitel 3 versucht Mill das Gefühl der Verbindlichkeit der Moral im Utilitarismus zu fassen. ${ }^{14}$ In Kapitel 5 mit dem Thema „Justice and Utility“ geht es

7 Siehe ebd., § 2.3-2.9, diskutiert von mir in Abschnitt 5.1.2.

8 Ebd., § 2.11-2.18.

9 Ebd., § 2.19 .

10 Ebd., § 2.20-2.21.

11 Ebd., § 2.23-24.

12 Ebd., § 2.25 .

13 Ebd., § 2.23.

14 Ebd., Kapitel 3. 


\section{Zwei Grenzen von Mills Philosophie}

darum, dass der Utilitarismus auch den Witz von "Gerechtigkeit" erfassen kann, auch wenn es anders als die anderen Punkte nach dem Beweis kommt. ${ }^{15}$

Natürlich werden die meisten der Einwände auch nach Mills Zeit noch formuliert und insofern ist es fraglich, ob es Mill wirklich gelungen ist, eine moralische Theorie zu formulieren, die denjenigen, die an der moralischen Praxis teilhaben, als das erscheint, was sie für den Witz dieser Praxis halten. Ich will dies nicht im Detail diskutieren und es dabei belassen, meine Überzeugung auszudrücken, dass einige der Antworten Mills und der Modifikationen eines zu einfachen Bildes des Utilitarismus, die er vornimmt, mehr Potential haben, als ihnen häufig zugeschrieben wird. Die Ambivalenz zwischen Mills Bemühen um einen Ausdruck dessen, was an der moralischen Praxis oder einem Teil davon von Bedeutung ist, und dem Scheitern, dies mit dem Utilitarismus in Einklang zu bringen, wird zum Beispiel an einer Einschätzung Stefan Gosepaths deutlich: „Interessanterweise hat (ausgerechnet) der Utilitarist Mill eine der neben Aristoteles besten Analysen des Gerechtigkeitsbegriffs im fünften Kapitel von Utilitarismus gegeben." ${ }^{16}$ Obwohl Mill - so ist dieser Satz wohl gemeint - dem einen hervorragenden Ausdruck verleiht, worum es uns im Falle von Gerechtigkeit geht, zeigt die eingeklammerte Qualifikation „ausgerechnet“ an, dass Mill es letztlich nicht schafft, dies mit dem Utilitarismus in Übereinstimmung zu bringen. In jedem Fall hat Mill mit seinem Utilitarismus - anders als Mills Bentham, der „began de novo, laid his own foundations deeply and firmly, built up his own structure, and bade mankind compare the two"17 - auch den Anspruch, dem, um was es kompetenten Teilnehmer*innen an der moralischen Praxis dabei geht, Ausdruck zu verleihen.

Obwohl also Coleridges Tugend, was die Reihenfolge und die Anzahl der Kapitel im Utilitarismus angeht, Priorität hat, werde ich mich im Folgenden fast nur noch mit Benthams Tugend beschäftigen. Es sind letztlich die kompetenten Teilnehmer*innen an der beschriebenen moralischen Praxis, die entscheiden, ob Mill Coleridges Tugend gerecht wird. Benthams Tugend ist aber auch wich-

15 Mill, „Utilitarianism“, Kapitel 5.

16 Stefan Gosepath. Gleiche Gerechtigkeit. Frankfurt am Main: Suhrkamp, 2004, S. 48 .

17 Mill, „Bentham“, S. 82. 
tiger, denn sie ist es, der die Konkurrenztheorie, gegen die Mill den Utilitarismus im ersten Kapitel aufstellt, seiner Meinung nach nicht gerecht werden kann. Das, was Mill unter „Intuitionismus“ versteht, sofern der Intuitionismus seinen Ausgangspunkt in der konventionellen Moral und nicht in einer „Privatoffenbarung“"18 hat, mag eine Stärke darin haben, dem Ausdruck zu verleihen, was Menschen an der moralischen Praxis wichtig ist ${ }^{19}$ aber er scheitert eben darin, zu zeigen, dass es in irgendeiner Hinsicht besser ist, die Dinge dieser konventionellen Moral folgend zu tun als sie anders zu machen.

Ich werde also im Rest dieses Abschnittes (6.1) Mills Kritik am Intuitionismus bezüglich Benthams Tugend und die sich daraus ergebenden Anforderungen an seinen eigenen Versuch, Objektivität herzustellen, darstellen, (Unterabschnitt 6.1.1), dann den „Beweis“ diskutieren und letztlich kritisieren, der diese Anforderungen erfüllen soll, (Unterabschnitt 6.1.2) und schließlich dafür argumentieren, dass Mill sich dennoch an Benthams Tugend orientiert (Unterabschnitt 6.1.3) und einen Versuch unternimmt, gegenüber dem Intuitionismus etwas zu leisten, was philosophisch wichtig ist.

Die Kritik an Mills Utilitarismus wird außerdem zeigen, dass sich die Idee von Lebensexperimenten nicht auf diese Weise auf einen anderen Bereich der Philosophie, hier die Moral, übertragen lassen. Dies zeigt natürlich nicht, dass Lebensexperimente nicht in anderer Hinsicht in anderen Bereichen der Philosophie eine Rolle spielen können oder sollten. Siehe dazu Kapitel 8.

\subsubsection{Die Kritik am „Intuitionismus“}

Das erste Kapitel „Utilitarianism“ benennt die ethische Richtung, als deren Alternative Mill den Utilitarismus verstanden wissen will: Den Intuitionismus. ${ }^{20}$ Es ist wird auch klar, warum Mill das, was er unter „Intuitionismus“ versteht,${ }^{21}$ ablehnt und den Utilitarismus

18 Die Formulierung ist aus einer bereits zitierten Stelle in Mill, „Coleridge“, S. 127.

19 Mill betont dies z. B. in Bezug auf Verbindlichkeit in Mill, „Utilitarianism“, $\S 3.1$.

20 Siehe ebd., Kapitel 1.

21 Mill hat sicherlich einerseits einen weiten Begriff von Intuitionismus: Im Wesentlichen scheinen unter die Bezeichnungen alle Theorien zu fallen, die Bentham dem Prinzip der Sympathie und Antipathie zugeordnet hat, sofern sie nicht einen „moral sense" postulieren. Siehe Bentham, An Introduction to 


\section{Zwei Grenzen von Mills Philosophie}

für nötig hält, auch wenn er diesen Punkt in „Utilitarianism“ nicht betont:

According to the one opinion, the principles of morals are evident $\grave{a}$ priori, requiring nothing to command assent, except that the meaning of the terms be understood. According to the other doctrine, right and wrong, as well as truth and falsehood, are questions of observation and experience. [...] Yet they seldom attempt to make out a list of the à priori principles which are to serve as the premises of the science; [...]. They either assume the ordinary precepts of morals as of à priori authority, or they lay down as the common groundwork of those maxims, some generality much less obviously authoritative than the maxims themselves, and which has never succeeded in gaining popular acceptance. [...] I might go much further, and say that to all those à priori moralists who deem it necessary to argue at all, utilitarian arguments are indispensable. It is not my present purpose to criticize these thinkers $[\ldots]^{22}$

Der Intuitionismus läuft Gefahr, einfach die konventionelle Moral zu bestätigen oder, wenn er das nicht tut, ein fragwürdiges Prinzip zugrunde zu legen. Er ist also entweder konservativ oder, wenn er das nicht ist, lediglich Ausdruck der exzentrischen Position einer einzelnen Philosoph*in. ${ }^{23}$ Mill hält es für notwendig, dass es einen Prüfstein für unsere moralischen und politischen Überzeugungen ${ }^{24}$

the Principles of Morals and Legislation, S. II.XI-II.XIX. Wie Bentham dort postuliert aber auch Mill eine Verwandtschaft der moral sense-Theorien mit dem Intuitionismus. Siehe Mill, „Utilitarianism“, § 1.3. Siehe außerdem Mill, "Whewell on Moral Philosophy", S. 170.

22 Mill, „Utilitarianism“, § 1.3-4. Es folgt beispielhaft Kant, von dessen kategorischem Imperativ (in der Universalisierungsformel) Mill meint, dieser könne selbst großes Unrecht nur ausschließen, wenn die Folgen einer universellen Befolgung der zu prüfenden Regeln in Betracht gezogen würden. Allerdings ist dies auch im Falle der verdienstlichen Pflichten noch ein großer Unterschied zum Utilitarismus - selbst zum Regelutilitarismus - den Mill hier nicht thematisiert. Um wirklich überzeugend zu sein, hätte Mill hier außerdem zeigen müssen, dass Kant sich in der Herleitung des kategorischen Imperativs utilitaristischen Prinzipien bedienen muss.

23 Dies entspricht natürlich den bereits zitierten Gedanken aus Mill, „Whewell on Moral Philosophy“, S. 170f und Mill, „Coleridge“, S. 127.

24 Mit den Prinzipien zweiter Ordnung, (vgl. Mill, Utilitarianism/Der Utilitarismus, S. 76ff und vgl. Mill, „Bentham“, S. 111) die uns im Alltag laut Mill 
oder eben für die Prinzipien, die diesen als zugrunde liegend postuliert werden, geben muss. Dieser Prüfstein liegt für ihn im Utilitarismus und er unterstellt, dass selbst Intuitionist*innen, sofern sie in ihren moralischen Theorien nicht einfach blind die Konvention reproduzieren, (ohne es zu wissen) letztlich auf den Utilitarismus als Prüfstein zurückgreifen müssen. Sehr viel deutlicher wird dieser Punkt im Aufsatz „Whewell on Moral Philosophy“25 hervorgehoben, den ich in Abschnitt 6.1.3 diskutieren werde.

Der Utilitarismus selbst hat für Mill nicht den Status eines $a$ priori Prinzips. ${ }^{26}$ Er ordnet ihn (und sich) hier noch einmal der „induktiven“ Schule zu. ${ }^{27}$ Obwohl er aber in „Utilitarianism" die Ableitung der Moral aus Prinzipien mit einer „Wissenschaft der Moral" gleichzusetzen scheint ${ }^{28}$ und in Kapitel 1 die Darlegung des Utilitarismus mit der Notwendigkeit eines obersten Prinzips zum Zwecke einer solchen Abhandlung rechtfertigt ${ }^{29}$ - also implizit dem Anspruch einer „Wissenschaft der Moral“" zuzustimmen scheint -, ist Moralphilosophie, wie bereits erwähnt, in vieler Hinsicht für Mill

leiten, gibt es für Mill so etwas wie substantielle moralische Überzeugungen. Welche Rolle diese genau spielen, ist kompliziert: Roger Crisp schreibt: „In fact Mill is neither a single-level nor a self-effacing theorist; his is a multilevel view, of a particularly subtle kind." Vgl. Crisp, Mill on Utilitarianism, S. 109.

25 Mill, „Whewell on Moral Philosophy“.

26 Der Begriff wird bei Mill ausschließlich kritisch verwendet. Neben zahlreichen Textstellen in bereits zitierten Texten bringt Folgendes Mills Einstellungen zu solchen a priori-Argumenten zum Ausdruck: „Professedly à priori arguments are not unfrequently of a mixed nature, partaking in some degree of the $\grave{a}$ posteriori character, and may often be said to be à posteriori arguments in disguise; the à priori considerations acting chiefly in the way of making some particular à posteriori argument tell for more than its worth." Mill, „Three Essays on Religion", S. 435.

27 Mill, „Utilitarianism“, § 1.3.

28 „But both hold equally that morality must be deduced from principles; and the intuitive school affirm as strongly as the inductive, that there is a science of morals." Ebd., § 1.3. Das Problem der ,intuitiven Schule“ ist nicht, dass sie den Anspruch hat, dass Moral eine Wissenschaft ist; dieser Anspruch, so scheint Mill hier sagen zu wollen, ist wichtig. Das Problem ist, dass sie diesem Anspruch nicht gerecht werden kann, ohne implizit auf den Utilitarismus zurückgreifen zu müssen. Siehe das obige Zitat zu Fußnote 22.

29 Ebd., § 1.2. Vgl. auch Mill, The Collected Works of John Stuart Mill, Volume VIII - A System of Logic Ratiocinative and Inductive (Books IV-VI and Appendices), S. 951f. 


\section{Zwei Grenzen von Mills Philosophie}

nur in sehr übertragenem Sinne eine Wissenschaft. (Darauf habe ich bereits in Abschnitt 4.3.1 hingewiesen.)

Es könnte eingewendet werden, dass Mill nun zum Zeitpunkt der Veröffentlichung von „Utilitarianism“ seine Meinung geändert habe. Dagegen spricht allerdings, dass Mill in dem Kapitel aus A System of Logic, aus dem hier zitiert wurde, bis zum Erscheinen des Utilitarismus und darüber hinaus weitreichende Änderungen vorgenommen hat, es aber nicht für nötig hielt, diese Textstelle aus dem einleitenden Paragraphen inhaltlich zu redigieren. Wichtiger ist aber, dass Mill einen Gedanken aus diesem letzten Kapitel bezüglich der Unterscheidung von Kunst und Wissenschaft aus A System of Logic explizit übernimmt, nämlich, dass die Rechtfertigung von (letzten) Zwecken anders stattfinden müsse, als Rechtfertigungen von Wahrheit in den Wissenschaften. ${ }^{30}$ Aber während er sich in A System of Logic agnostisch darüber gibt, wie Rechtfertigungen von (letzten) Zwecken aussehen können, und seiner Verpflichtung auf den Utilitarismus (ab 1865 mit explizitem Verweis auf „Utilitarianism") lediglich Ausdruck verleiht, ${ }^{31}$ versucht er im Utilitarismus eine solche Rechtfertigung. Er schreibt, nachdem er Gedanken aus A System of Logic zu letzten Zwecken mehr oder weniger wiederholt hat und etabliert hat, dass der Nachweis des utilitaristischen Prinzips kein Beweis im gewöhnlichen und populären Sinne des Wortes ${ }^{32}$ sein kann:

30 „But though the reasonings which connect the end or purpose of every art with its means, belong to the domain of Science, the definition of the end itself belongs exclusively to Art, and forms its peculiar province. Every art has one first principle, or general major premise, not borrowed from science; that which enunciates the object aimed at, and affirms it to be a desirable object." Mill, The Collected Works of John Stuart Mill, Volume VIII - A System of Logic Ratiocinative and Inductive (Books IV-VI and Appendices), S. 949.

31 „Without attempting in this place to justify my opinion, or even to define the kind of justification which it admits of, I merely declare my conviction, that the general principle to which all rules of practice ought to conform, and the test by which they should be tried, is that of conduciveness to the happiness of mankind, or rather, of all sentient beings: in other words, that the promotion of happiness is the ultimate principle of Teleology." Ebd., S. 951.

32 „in the ordinary and popular meaning of the term“, Mill, „Utilitarianism“, $\S 1.5$. 
There is a larger meaning of the word proof, in which this question is as amenable to it as any other of the disputed questions of philosophy. The subject is within the cognizance of the rational faculty; and neither does that faculty deal with it solely in the way of intuition. Considerations may be presented capable of determining the intellect either to give or withhold its assent to the doctrine; and this is equivalent to proof. ${ }^{33}$

Wichtig ist hier, dass Mill den Anspruch erhebt, an das, was als das „rationale Vermögen“ bezeichnet werden könnte, zu appellieren; sonst könnte unter ,[c]onsiderations [...] capable of determining the intellect either to give or to withhold its assent" auch einfach Propaganda verstanden werden. Mill will überzeugen. Er will vor allen Dingen etwas liefern, von dem er meint, dass es dem Intuitionismus fehlt: eine nicht-dogmatische Grundlage für unsere moralischen Überzeugungen, nach der diese sich als richtig und falsch erweisen können und nicht einfach als richtig gesetzt sind, weil wir sie für richtig halten. ${ }^{34}$

Dafür, dass dieses Bemühen Mills um eine begründbare, wenn auch nicht im engeren Sinne „wissenschaftliche“ Grundlage für den Utilitarismus Ausdruck von Benthams Tugend ist, braucht sicherlich nicht einmal argumentiert werden. (Ich hatte genau dafür, dass Benthams Tugend keine Wissenschaftlichkeit in einem empiristischen Sinne voraussetzt, bereits in Abschnitt 4.3.1 argumentiert.) Inhaltlich geht es schließlich immer noch um genau das Thema, anhand dessen ich in Kapitel 4 das, was Mill an Bentham als Philosophen schätzt, Benthams Tugend also, ausgearbeitet hatte: Mills Kritik am Intuitionismus gleicht derjenigen, die er in „Bentham“ bei Bentham lobt - inklusive der behaupteten Verwandtschaft von

33 Ebd., $\$ 1.5$.

34 Vgl. aus dem 1851 zugefügten Absatz aus Mill, The Collected Works of John Stuart Mill, Volume VIII - A System of Logic Ratiocinative and Inductive (Books IV-VI and Appendices), S. 949: „[T]he speaker's approbation is no sufficient reason why other people should approve; nor ought it to be a conclusive reason even with himself." Vergleiche außerdem aus der Kritik an Bentham: „If he thought at all of any of the deeper feelings of human nature, it was but as idiosyncrasies of taste [...]. To say either that man should, or that he should not, take pleasure in one thing, displeasure in another, appeared to him as much an act of despotism in the moralist as in the political ruler." Mill, „Bentham", S. 96. 


\section{Zwei Grenzen von Mills Philosophie}

Subjektivismus, moral sense-Theorie und Intuitionismus ${ }^{35}$ vor dem Hintergrund, dass sie aus seiner Sicht das gleiche Problem teilen, nämlich die Immunisierung moralischer Äußerungen gegen Kritik und damit die Aufgabe des Anspruchs, Objektivität nachzuweisen.

Mill bemüht sich also, was das Nützlichkeitsprinzip angeht, um Benthams Tugend. ${ }^{36}$ Er wird ihr aber nicht gerecht, wie ich im Folgenden argumentieren werde. Dafür ist es nötig, den „Beweis“ des Utilitarismus genauer zu diskutieren.

\subsubsection{Der „Beweis“ des Utilitarismus}

Der „Beweis“ soll letztlich Benthams Tugend, das Bemühen um Objektivität vor dem Hintergrund von Alternativen, erfüllen. Die wichtigste Gegenposition ist dabei der Intuitionismus. Kapitel 2 und 3, die dem Beweis vorausgehen, und in gewissem Sinne auch Kapitel 5, das dem Beweis folgt, hatte ich so gedeutet, dass es darum geht, darin zu zeigen, dass der Utilitarismus ebenfalls das erfassen kann, was die große Stärke am Intuitionismus ist, so wie Mill diesen versteht: Einen philosophischen Ausdruck zu finden, den eine Teilnehmer*in der moralischen Praxis für das, was an dieser Praxis wichtig ist, als adäquaten Ausdruck bezeichnen könnte. Der Intuitionismus, so wie Mill ihn versteht, hat dann hier ein Heimspiel, wenn er vom konventionellen Moralbegriff ausgeht. Wenn die entsprechenden Kapitel erfolgreich sind, dann hätten wir mit dem Utilitarismus bezüglich Coleridges Tugend eine Alternative zum Intuitionismus. Das Spielfeld wäre geebnet. Nach Benthams Tugend müssen wir uns aber darum bemühen, dass die Frage, ob der Intuitionismus oder der Utilitarismus die bessere philosophische Auffassung ist, nicht „a field of interminable discussion, leading to no result "37 bleibt: Auf dem in Bezug auf Coleridges Tugend nun ebe-

35 Mill zitiert Bentham in entsprechender Weise im Kontext der Diskussion um den Intuitionismus in seiner Kritik an Whewell. Siehe Mill, „Whewell on Moral Philosophy“, S. 177f.

36 Ironischerweise anders als Bentham, der dazu knapp schreibt: „,T]hat which is used to prove every thing else, cannot itself be proved: a chain of proofs must have their commencement somewhere. To give such proof is as impossible as it is needless." Bentham, An Introduction to the Principles of Morals and Legislation, § I.XI.

37 Mill, „Bentham“, S. 83. Dagegen anzukämpfen beschreibt Mill als explizites Verdienst Benthams an der zitierten Stelle. 
nen Spielfeld muss der Utilitarismus nun in Bezug auf Benthams Tugend gewinnen.

Welche Art der Überlegung kommt aber in Frage, um zwischen Intuitionismus und Utilitarismus zu entscheiden? Mill wiederholt am Anfang von Kapitel 4 in fast den gleichen Worten, was er schon im ersten Kapitel gesagt hat: dass es sich um keinen Beweis im üblichen Sinne des Wortes handeln kann. ${ }^{38}$ Der Zugang dazu, welche Art von Überlegung Mill vorschwebt, kann in Abgrenzung zu Moores Fehldeutung erarbeitet werden. Moore hat Mills Beweis auf eine Weise missverstanden, deren Zurückweisung es ermöglicht, zu verstehen, was Mill eigentlich vorschwebt. Dies werde ich im folgenden Abschnitt tun.

\section{Von Moores Missverständnis des Beweises zum Status des Beweises nach Mill}

Vielleicht hat Moore sich dadurch in die Irre führen lassen, dass Mill das Beweiskapitel sehr klassisch aufbaut: Sein Beweisziel ist: „that happiness is desirable, and the only thing desirable ${ }^{* 39}$; nach dem kurzen Absatz nach dieser Formulierung des Beweisziels beansprucht Mill den ersten Teil gezeigt zu haben: „Happiness has made out its title as one of the ends of conduct, and consequently one of the criteria of morality ${ }^{* 40}$. Danach geht es für sieben Absätze darum, die zweite Hälfte des Ziels nachzuweisen, nämlich dass Glück das einzige Gut ist. ${ }^{41}$ Das Kapitel wirkt daher so, wie eine klassische Begriffsklärung: Erst wird gezeigt - so könnte der Aufbau verstanden werden - dass Glück (im Sinne von Freude) hinreichend für das Gute ist; dann wird gezeigt, dass Glück (im Sinne von Freude) auch notwendig ist.

Vielleicht unterstellt Moore Mill auch aus diesem Grund in seinem Beweis des utilitaristischen Prinzips ein analytisches Urteil ${ }^{42}$ über „moralisch gut" abzugeben ${ }^{43}$ obwohl sich aus analytischen

38 Mill, „Utilitarianism“, § 4.1 .

39 Ebd., § 4.2 .

40 Ebd., § 4.3.

41 Ebd., § 4.4-4.8.

42 Die Dichotomie „analytisch"-,,synthetisch" ist eine der Weisen, in der Moore seinen Gedanken fasst: „[P]ropositions about the good are all of them synthetic and never analytic;" Moore, Principia Ethica, $§ 6$.

43 Ebd., § 40-41. 


\section{Zwei Grenzen von Mills Philosophie}

Urteilen über diesen Begriff nach Moore für die Ethik nichts Interessantes - nichts Normatives jedenfalls - ableiten lasse (sondern nur aus synthetischen).$^{44}$ Bei analytischen Urteilen sei nämlich die Infragestellung durch eine Person, die die Bedeutung aller im Urteil verwendeten Begriffe versteht, nicht möglich, während synthetische Urteile diese Infragestellung ermöglichten. ${ }^{45}$ Dieser Aspekt des Argumentes der offenen Frage ist zunächst unabhängig davon, ob "gut" eine natürliche Eigenschaft ist oder nicht; wir können zum Beispiel auch den (nach Moore natürlichen) Alltagsbegriff "gelb“ nicht anhand der Wellenlänge definieren, die gelbes Licht hat. (Diese Analogie wird auch nicht dadurch aufgehoben, dass "gelb“ und "gut" in anderen Hinsichten, beispielsweise in Bezug auf die Frage, ob es sich um ein attributives oder ein prädikatives Adjektiv handelt ${ }^{46}$ nicht analog sind.) In der Tat ist etwas unklar, was Moore genau damit meint, dass "gut" über die Undefinierbarkeit hinaus, die aber auch auf „gelb“ zutrifft, keine natürliche Eigenschaft sein könne, da er den naturalistischen Fehlschluss auch dem, was er „metaphysische Ethiken“ nennt, vorwirft, die „gut" anhand von jedenfalls nicht empirisch wahrnehmbaren ${ }^{47}$, Eigenschaften definieren wollten. Entsprechend müsste der Fehlschluss nach Frankena eigentlich „Definitionsfehlschluss" heißen. ${ }^{48}$

44 Moore sagt zwar gelegentlich, wie das Zitat in Fußnote 9 belegt, es gebe gar keine wahren analytischen Aussagen über „gut", aber das scheint falsch zu sein. Beispielsweise: Gut ist das, was wir mit „gut" auszudrücken trachten. Es scheint mir, dass Moore vor allem meint, es würde nichts Normatives aus einem analytischen Urteil über „gut" folgen - ähnlich wie nach der Abbildtheorie im Tractatus keine Aussagen über die Welt durch analytische Sätze möglich sind. Z. B. Ludwig Wittgenstein. Werkausgabe in 8 Bänden - Band 1: Tractatus logico-philosophicus. Tagebücher 1914-1916. Philosophische Untersuchungen. Frankfurt am Main: Suhrkamp, 2014, S. 4.462.

45 Moore, Principia Ethica, § 13.

46 Peter Geach. „Good and Evil“. In: Analysis 17.2 (1956), S. 33-42.

47 Siehe Moore, Principia Ethica, Kapitel IV.

48 Eigentlich auch das nicht. Der nach Frankena für Moore interessanteste „Fehler" (Moore meint offenbar verschiedene nicht gleichbedeutende Dinge mit „naturalistischer Fehlschluss") liegt nämlich im Versuch, eine Definition von "gut" anzugeben. Es geht also streng genommen überhaupt nicht um einen Schluss. William Frankena. „Der naturalistische Fehlschluss“. In: Sprache und Ethik - Zur Entwicklung der Metaethik. Hrsg. von Günther Grewendorf und Georg Meggle. Frankfurt am Main: Suhrkamp, 1974, S. 83-99. 
Hier soll es nicht um eine Interpretation von Moore gehen. Es geht vielmehr darum: Angenommen Moore hätte mit seiner Deutung dieser Passage Recht und Mills „Beweis“ des utilitaristischen Prinzips wäre als deduktives Argument zu verstehen, das von (fragwürdigen) analytischen Sätzen (in fragwürdiger Weise) auf ein normatives Prinzip schließt. Dann würde Mill offensichtlich den ihm von mir unterstellten philosophischen Tugenden nicht gerecht: Wenn Mill den Anspruch hätte, „gut" einfach zu definieren, würde er, wenn die Definition die konventionelle Bedeutung von „gut" erfassen soll, dogmatisch sein; wenn die Definition bloß stipulativ sein soll, würde sie die Beweislast nicht tragen können. In jedem Fall wäre Mill keinen Schritt näher daran, Benthams Tugend nachzukommen. Moore liegt aber falsch. Um dies zu belegen, werde ich etwas genauer auf Moores Kritik eingehen. Hier sind die entscheidenden Sätze Mills, an denen Moore seine Kritik aufhängt:

The only proof capable of being given that an object is visible, is that people actually see it. The only proof that a sound is audible, is that people hear it: and so of the other sources of our experience. In like manner, I apprehend, the sole evidence it is possible to produce that anything is desirable, is that people do actually desire it. If the end which the utilitarian doctrine proposes to itself were not, in theory and in practice, acknowledged to be an end, nothing could ever convince any person that it was so. ${ }^{49}$

Moore unterstellt Mill nun, dass letzterer erstens einen analytischen Zusammenhang zwischen der Sichtbarkeit eines Objektes und der Tatsache, dass Menschen es sehen können, behaupten will, und zweitens, dass Mill in der Analogie zwischen dem Sichtbaren und dem Wünschenswerten auf diesen analytischen Zusammenhang abzielt: Wir sollen nach Moores Mill also einsehen, dass so wie „sichtbar" bedeutet, dass es von Menschen gesehen werden kann, ganz analog „wünschenswert" bedeutet, dass Menschen es sich wünschen. Entsprechend lautet seine Kritik: „The fact is that 'desirable' does not mean 'able to be desired' as 'visible' means 'able to be seen. " "50 Entsprechend meint Moore, von „guten Begierden“ zu sprechen,

49 Mill, „Utilitarianism“, § 4.3.

50 Moore, Principia Ethica, § 3.40 . 
müsse für Mill eine bloße Tautologie sein und unterstellt Mill entsprechend einen Selbstwiderspruch bei dessen Unterscheidung zwischen besseren und schlechteren Gegenständen des Begehrens, „as if, after all, what is desired were not ipso facto good, and good in proportion to the amount it is desired “51. Moore versucht sich aber gar nicht erst an einer wohlwollenden Lesart, sondern behauptet: "These are specimens of the contradictions, which, as I have tried to shew, must always follow from the use of the naturalistic fallacy " ${ }^{\text {t52 }}$. Ich glaube, beide Unterstellungen sind falsch. (1) Mill will uns nicht sagen, dass "visible" so viel wie "able to be seen" bedeutet, und (2) selbst, wenn er das wollte, geht es bei der Analogie nicht darum, die Eigenschaft einer Aussage analytisch oder definitorisch zu sein, auf eine andere Aussage zu übertragen.

(1) Zunächst sind „visible“ und „able to be seen“ unabhängig vom Status der Aussage gar nicht die Begriffe, die Mill im ersten Satz des Zitates in Verbindung bringt. Es sind die Begriffe "visible" und die Tatsache, „that people actually see it". Dies scheint (anders als das, was Moore offenbar versteht) keine Aussage über die Bedeutung von „visible" sein können: Ob Menschen etwas tatsächlich sehen, hängt auch von kontingenten Faktoren ab, wie zum Beispiel der Frage, ob gerade Menschen da sind, ihre Augen offen haben, u.s.w. Allerdings könnte eingewendet werden, dass, obwohl "visible" nicht bedeutet, dass „people actually see it", es dennoch einen analytischen Zusammenhang in dem Sinne gibt, dass, wenn Menschen ein Objekt sehen, dieses Objekt in der Tat sichtbar sein muss. Dass Menschen einen visuellen Wahrnehmungseindruck haben, kann natürlich nur als Evidenz gelten, dass es hier ein Objekt gibt und dieses sichtbar ist; aber die Formulierung „ein Objekt sehen" ist, so könnte eingewandt werden, ein Erfolgsverb, das tatsächlich ein Objekt und seine Sichtbarkeit voraussetzt. Ich glaube nicht, dass Mill hier unter Voraussetzung einer disjunktiven Wahrnehmungs-

51 Moore, Principia Ethica, § 40. Siehe das Zitat in Fußnote 34, wo Mill einen zwingenden Zusammenhang zwischen Begierden und dem Gutsein der Gegenstände der Begierden explizit ablehnt. Dies ist keine Inkonsistenz bei Mill, wie Moore zu denken scheint, sondern ein Beleg dafür, dass Mill es so, wie Moore ihn versteht, nicht gemeint haben kann. Wie im letzten Unterabschnitt (6.1.1) dargelegt, stehen Mills Überlegungen dazu, welchen Status sein Beweis hat, in deutlichem Zusammenhang zu dem Kapitel, aus dem das Zitat aus Fußnote 34 entnommen ist.

52 Ebd., § 40. 
theorie einen analytischen Satz formulieren wollte. (Wenn Mill hier auf sprachanalytische Wahrheiten hätte abzielen wollen, wäre der nächste Satz im oben zitierten Absatz gänzlich verwirrend: Wenn etwas ein „sound“ ist, dann scheint nach der gleichen Spitzfindigkeit „Hörbarkeit" schon vorausgesetzt; und weitere Beweise wären dann sinnlos.) Ich werde weiter unten einen Vorschlag machen, wie der Satz stattdessen zu lesen ist.

(2) Selbst wenn Mill aber mit dem ersten Satz eine analytische Wahrheit hat ausdrücken wollen, so ist es doch mehr als fraglich, ob es ihm in der Analogie darum geht, die Eigenschaft eines Satzes, analytisch zu sein, auf die Aussage, dass Menschen sich Wünschenswertes wünschen, zu übertragen: Er spricht in diesem Satz auch nicht mehr von "proof", sondern von „sole evidence“. Dies ist ein deutlicher Hinweis dafür, dass der Satz nicht analytisch und insbesondere nicht als Aussage über die Bedeutung von „wünschenswert" gemeint ist: Dass eine Zahl nur durch sich selbst und eins teilbar ist, ist keine Evidenz dafür, dass es sich um eine Primzahl handelt, denn dies ist, was es bedeutet, eine Primzahl zu sein; dass ein Pferd von Natur aus eine weiße Fellfarbe hat, ist keine Evidenz dafür, dass es sich um einen Schimmel handelt, sondern dies ist, was es bedeutet, ein Schimmel zu sein. Zumindest die Aussage (aber ich meine auch die Aussage zur Sichtbarkeit), dass wir davon, dass Menschen sich etwas wünschen, darauf schließen können, dass es wünschenswert sei, muss also evidentiell verstanden werden - ähnlich wie das Urteil der Erfahrenen über den qualitativen Wert der Lüste: Es ist nicht so, dass es sich hier um ein unfehlbares Kriterium handelt - Menschen wünschen sich manchmal Schlechtes und auch die Erfahrenen ziehen manchmal die niedrigere von zwei Lüsten vor -, sondern es ist so, dass die Tatsache, dass Menschen etwas wünschen, ein Indikator dafür ist, dass das, was sie sich wünschen, wertvoll ist. (Siehe Abschnitt 5.2.2.)

Wenn Moores Deutung richtig wäre, dann wäre auch nicht klar, warum Mill gleich in der Einleitung des ganzen Buches und noch einmal unmittelbar vor der problematisierten Textstelle so deutlich sagt, dass es sich nicht um einen Beweis „in the ordinary acceptation of the term" handeln könne, weil letzte Prinzipien keines Beweises fähig seien ${ }^{53}$ : Handelte es sich tatsächlich um ein dem An-

53 Mill, „Utilitarianism“, § 4.1, vgl. ebd., § 1.5. 


\section{Zwei Grenzen von Mills Philosophie}

spruch nach deduktives Argument, das von - dem Anspruch nach analytisch wahren Sätzen ausgeht, ist schwerlich etwas vorstellbar, was näher an die gängige Bedeutung des Wortes „Beweis“ kommt; es mag tatsächlich ein mangelhafter Beweisversuch sein, aber Mill müsste dann immer noch den Anspruch vertreten, etwas in der gängigen Bedeutung des Wortes „Beweis“ zu leisten, und dies tut er nicht.

Moores Deutung geht aber nicht nur gegen den Strich von Mills expliziten Bemerkungen zum Status des Beweises, sie geht auch gegen Mills metaethische Grundüberzeugung, wie er sie am Anfang des Utilitarismus darlegt: Er selbst rechnet sich, wie dargelegt, der „induktiven Schule“ der Ethik zu und grenzt diese, nachdem er die moral-sense-Theorie zurückgewiesen hat, von der ,intuitiven Schule ${ }^{\star 54}$ ab. Nach letzterer seien die Prinzipien der Moral in dem Sinne a priori evident, dass sie nichts benötigten, damit wir ihnen zustimmen, außer dass wir die Begriffe, in denen sie formuliert sind, wirklich verstehen. Diese Art zu philosophieren habe eine Tendenz, etablierte Meinungen zu rechtfertigen, indem sie letztlich in Phrasen, in denen diese Meinung Ausdruck findet, ende. ${ }^{55}$ Und es geht ja darum, wie der Utilitarismus in diesem Punkt besser als der Intuitionismus sein kann.

Moore missversteht Mills Beweis. Wie aber sollten wir den Beweis stattdessen verstehen? Ab Absatz 9 diskutiert Mill den theoretischen Status seiner Überlegungen. ${ }^{56}$ Einen Absatz später schreibt er: „[W]e have evidently arrived at a question of fact and experience,

54 Die Phrase, die Mill hier verwendet ist: „intuitive school“. (Vgl. Mill, „Utilitarianism“, § 1.3.) Birnbacher übersetzt dies mit „intuitionistische Schule“; Mill, Utilitarianism/Der Utilitarismus, S. 10f. Auch Mill verwendet gelegentlich andere Bezeichnungen: in Whewell on Moral Philosophy spricht er von der ,intuition doctrine"; ich habe mich, auch, um im Kontext der Behandlung von Moores Kritik keine illegitime Gleichsetzung und Verwirrung zu stiften, für „,intuitive Schule“ als Übersetzung entschieden.

55 Mill zählt auf: „our idea of man as man“, „all men think“, „we cannot help feeling“. Vgl. Mill, „Whewell on Moral Philosophy“, S. 193. Mill setzt dabei nicht einfach eine philosophische Ausrichtung mit einer gesellschaftlichen gleich: Er weist durchaus darauf hin, dass utilitaristische Argumente auch von Konservativen gebraucht wurden und dass die intuitive Schule im Gegenzug nicht immer das Etablierte verteidige. Er meint aber, dass sie letztlich für konservative Denker*innen besonders attraktiv sei. Vgl. ebd., S. $170 \mathrm{f}$.

56 „We have now, then, an answer to the question, of what sort of proof the principle of utility is susceptible." Mill, „Utilitarianism“, § 4.9 
dependent, like all similar questions, upon evidence. It can only be determined by practised self-consciousness and self-observation, assisted by observation of others. " ${ }^{\text {" }} 7$ Die Schritte des Beweises sind offenbar evidentiell zu verstehen. Der erste Schritt ist dann ungefähr dieser: Dass Glück ein individueller Wert ist, wird dadurch nahegelegt, dass Menschen sich Glück tatsächlich wünschen oder für gut halten. Dies ist zwar nach Mill kein zwingender (conclusi$v e)^{58}$ Schluss, aber das Ergebnis dürfte trotzdem nicht kontrovers sein. ${ }^{59}$ Wichtig, was den Status angeht: Da es im gesamten Beweis um etwas geht, dass durch Evidenz, Selbstbeobachtung, die Beobachtung von anderen u.s.w. zu beantworten ist, scheint Mill das Ergebnis seines „Beweises" und hier beispielhaft des ersten Schrittes tatsächlich im Sinne Moores als Gegenstand „offener Fragen“ zu sehen: Es ist nicht durch begriffliche Überlegungen festgelegt, dass happiness ein Wert ist. Aber dadurch, dass wir selbst und alle Menschen, von denen wir wissen, es als Wert behandeln, haben wir Evidenz dafür, wenn auch keinen zwingenden Schluss darauf, dass es sich um einen Wert handelt. Diese Überlegung zum theoretischen Status gilt auch für den Rest des Beweises.

Bevor ich mich im Folgenden näher kritisch mit einer weiteren Stufe des Beweises auseinandersetze, möchte ich diesen Gedanken, dass es sich um eine offene Frage handelt, mit Benthams Tugend in Verbindung bringen: Ich hatte bereits in Abschnitt 5.2.3 angedeutet, dass eine von Mills Begründungen für Meinungsfreiheit darin besteht, dass nur unter Bedingungen der Meinungsvielfalt Meinungen ihre Bedeutungen beibehalten könnten. Dies wiederum begründet Mill damit, dass wir, um die Bedeutung einer Meinung zu verstehen, uns der Alternativen bewusst sein müssen. Ich hatte gesagt, dass dies an den Verifikationismus erinnert, obwohl dies nicht unbedingt heißt, dass Mill im strengen Sinne Verifikationist in Bezug auf Bedeutung gewesen ist. (Siehe Abschnitt 5.2.3.) Trotzdem ist es

57 Ebd., § 4.10.

58 Vgl. Mill, The Collected Works of John Stuart Mill, Volume VIII - A System of Logic Ratiocinative and Inductive (Books IV-VI and Appendices), S. 949. Vgl. außerdem Mill, „Bentham“, S. 96. Zitate zu beiden Textstellen finden sich in Fußnote 34.

59 Vgl. Crisp, Mill on Utilitarianism, S. 73: „The suggestion that pleasure is desirable is hardly difficult to accept, and one might be forgiven for wondering why Mill thought he had to argue for it." 


\section{Zwei Grenzen von Mills Philosophie}

fraglich, ob Mill so etwas wie eine "Wissenschaft ohne Gegensatz" in Bezug auf moralische oder philosophische Fragen allgemein akzeptiert hätte, wie sie Roedl vorschwebt. ${ }^{60}$ Ebenso fraglich ist, dass er in Bezug auf die Philosophie einen Wittgenstein'schen Quietismus akzeptiert hätte. Und die Tatsache, dass die Frage „Sollten wir in moralischer Hinsicht wirklich immer und ausschließlich die Handlungen tun, die die meisten guten Konsequenzen haben?" letztlich in Moores Sinne offen ist, wäre in diesem Sinne Voraussetzung dafür, dass Benthams Tugend Anwendung findet.

Mills Strategie über „Evidenz“, „Selbstbeobachtung“ und „Erfahrung" zu gehen, ist natürlich nicht die einzige Strategie, wie moralische Fragen und Fragen nach den Grundprinzipien der Moral offene Fragen sein können. Eine andere wäre Kants Idee synthetischer Urteile a priori zu reetablieren. ${ }^{61}$ Diese Strategie scheint auch Jonathan Dancy vorzuschweben ${ }^{62}$, wobei Dancy natürlich Wert darauf legt, dass dies nicht heißt, dass moralische Urteile notwendig seien. ${ }^{63}$

\section{Der logische Status der utilitaristischen Unparteilichkeit in Mills Beweis}

Ob der Beweis aus einem oder zwei weiteren Schritten besteht, ist nicht leicht zu beantworten: Am Anfang des letzten Unterabschnittes hatte ich nahegelegt, die Rhetorik des Kapitels bestünde darin, erst zu zeigen, dass Glück (in Mills Sinne, siehe Abschnitt 5.1.2) ein Wert sei und dann zu zeigen, dass nichts anderes ein Wert sei.

60 Dies ist die Wortwahl Sebastian Roedls für seine Vorstellung von Philosophie als Wissenschaft vom Urteilen: „Wenn Urteilen selbstbewusst ist, ist diese Wissenschaft vom Urteil eigentümlich: Sie ist die Wissenschaft ohne Gegensatz. [...] Die Wissenschaft vom Urteil ist nichts anderes als die Artikulation des Selbstbewußtseins des Urteils." Sebastian Roedl. Selbstbewusstsein und Objektivität: Eine Einführung in den absoluten Idealismus. Berlin: Suhrkamp, 2019, § 3.1. Vgl. damit die bereits auf S. 91 zitierte Vorstellung Mills in Mill, The Collected Works of John Stuart Mill, Volume VII - A System of Logic Ratiocinative and Inductive (Books I-III and Appendices), S. 109.

61 Vgl. George Edward Moore. Ethics. London: Williams und Norgate, 1912, $\S 12.86$.

62 Siehe Jonathan Dancy. Ethics Without Principles. Oxford und andere: OUP, 2004, S. 146-148.

63 Vgl. auch ebd., wo er explizit Kants Begriff des synthetischen Urteils a priori verwendet. 
Damit gäbe es zwei Schritte. (Auf den Beweisteil dafür, dass nichts anderes als Glück ein (Klugheits-)Wert sei, werde ich an dieser Stelle nicht eingehen. Meine Bemerkungen in Abschnitt 5.1.2 dazu, wie Mills Hedonismus insbesondere in Bezug auf sein offensichtliches Zugeständnis, dass es nicht-hedonische Ziele gibt, zu verstehen ist, sind, so meine ich, im Großen und Ganzen in Übereinstimmung mit dem, was Mill hier dazu sagt. Die anderen Probleme mit Werten, die anscheinend trotzdem nicht reduzierbar sind auf Werte, die gut für ein Individuum sind, entstehen durch einen Zug Mills, auf den ich nun eingehe.) Allerdings fehlt diesen zwei Schritten noch ein entscheidender Gedanke des Utilitarismus, den Mill zum Beispiel in Kapitel 2 ausdrückt: „[T]he happiness which forms the utilitarian standard of what is right in conduct is not the agent's own happiness, but that of all concerned." ${ }^{4}$ Dies führt zum Beispiel Roger Crisp dazu, in seiner Diskussion des Beweises von drei Schritten zu sprechen und nicht von zweien, wie durch die Rhetorik des Buches nahegelegt. ${ }^{65}$

Im dann noch fehlenden Schritt, dem zweiten Schritt in Crisps Zählung, geht es im die Unparteilichkeitsannahme des Utilitarismus, die nach Crisp einer der Hauptstreitpunkte zwischen Mill und seinen philosophischen Gegner*innen war. ${ }^{66}$ Schritt zwei (in Crisps Zählung) besteht allerdings nur in einem einzigen Satz: „This, [dass jede Person ihr eigenes Glück wünscht] however being a fact, we have not only all the proof which the case admits of, but all which it is possible to require, that happiness is a good: that each person's happiness is a good to that person, and that the general happiness, therefore, a good to the aggregate of all persons." Angenommen, dies ist (anders als "therefore" hier nahelegt) nicht als Kompositionsfehlschluss zu verstehen, sondern als Aussage speziell über das Gute (oder das Gute als happiness verstanden): Das Gute gehört laut diesem Satz eben zu den Dingen, die (über Per-

64 Mill, „Utilitarianism“, § 2.18. Und zwar zählt das Glück jeder betroffenen Person in gleicher Weise: „Bentham's dictum, 'everybody to count for one, nobody for more than one,' might be written under the principle of utility as an explanatory commentary." Ebd., § 5.36.

65 Crisp, Mill on Utilitarianism, Kapitel 4.

66 Crisp spricht von „one of the main bones of contention between Mill and his philosophical opponents“. Ebd., S. 83. 


\section{Zwei Grenzen von Mills Philosophie}

sonen hinweg) addiert werden können. ${ }^{67}$ Und aus der moralischen Perspektive, die der Utilitarismus zeichnet, werden Handlungen danach bewertet, inwiefern sie das Glück „of all those concerned“ in unparteiischer Weise fördern. Dies ist die utilitaristische Unparteilichkeitsannahme. ${ }^{68}$ Der Satz gibt uns aber keinen Grund dafür, warum wir diese Perspektive einnehmen sollten. Damit ist nicht die Frage gemeint, warum wir moralisch sein sollten. Crisp argumentiert, dass sich Mills Argument an Menschen richtet, die Moral ernst nehmen. ${ }^{69}$ Aber der Beweis soll eine Lücke des Intuitionismus umgehen. Auch Intuitionist*innen nehmen Moral ernst: es werden Gründe gebraucht, die es besser machen, den Utilitarismus anstelle des Intuitionismus und nicht des Amoralismus anzunehmen.

Die Frage kann auch so reformuliert werden: Weiter oben hatte ich behauptet, dass der Übergang davon, dass Menschen sich Freude wünschen, dazu, dass Freude wünschenswert sei, evidentiell (und nicht analytisch) ist und daher für Mill (anders als Moore meinte) eine Antwort auf eine offene Frage im Sinne Moores ist. Es ist nicht so, dass „gut" einfach bedeutet, dass Menschen sich das so Bezeichnete wünschen; aber, dass alle Menschen sich etwas wünschen, ist Evidenz dafür, dass es gut ist - in der gleichen Weise, wie die Tatsache, dass Menschen etwas sehen, Evidenz (aber nicht zwingender Beweis) für ein sichtbares Objekt ist. (Dies ist in dem Sinne eine offene Frage, als wir uns fragen können, ob wir wirklich ein Objekt sehen, oder ob wir uns täuschen.$^{70}$ ) Dies kann nun aber für den zweiten Schritt von Mills Beweis in Crisps Zählung auch gefragt

67 Crisp, Mill on Utilitarianism, S. 77ff. Wie Mill in einem Brief klarstellt, meint er nicht, dass die Summe des Glücks aller für jede einzelne Person gut sei, sondern „nur“, dass sie gut sei, für die Summe aller Menschen. (Zitiert von ebd., S. 78.) Dies muss von Mill aber als die Perspektive verstanden werden, aus der utilitaristische Bewertungen stattfinden sollen, sonst würde sein Beweis schlicht nicht beweisen, was er zu beweisen vorgibt.

68 Über diese sagt Mill an anderer Stelle: „It is involved in the very meaning of Utility, or the Greatest-Happiness Principle. That principle is a mere form of words without rational signification, unless one person's happiness, supposed in equal degree (with the proper allowance made for kind), is counted for exactly as much as another's." Mill, „Utilitarianism", § 5.36.

69 Crisp, Mill on Utilitarianism, S. 79.

70 Natürlich ist der Zusammenhang zwischen "Ich sehe das Objekt $X^{\text {" und „ } X}$ existiert" analytisch im Sinne einer disjunktivistischen Theorie der Wahrnehmung. Aber Mill geht es weder um eine disjunktivistische Wahrnehmungstheorie, noch wäre es selbst innerhalb einer solchen Theorie plausibel, die 
werden: Ist der mit „therefore“ gekennzeichnete Schritt eine Antwort auf eine offene Frage? Beruht der Schritt davon, dass für jede Person ihre eigene Freude ein Wert ist, dahin, dass die Perspektive der Bewertung moralisch gefordert ist, die der Utilitarismus anbietet, nämlich die Perspektive, nach der wir Glück unparteiisch fördern sollten, gar auf Evidenz?

Wenn nicht, dann wäre Mill Benthams Tugend nicht gerecht geworden: Es wäre nach dieser Deutung so, dass Mill einfach meint, moralisch zu bewerten hieße so viel wie unparteiisch sein; nachdem der erste und der dritte Schritt (nach Crisps Zählung) in seinem Beweis dann gezeigt haben, dass es im Leben um Freude und Leid und nur um Freude und Leid geht, ist die Arbeit dann schon getan. Die moralische Bewertung ist nun die unparteiische Bilanzierung dieser Gegenstände. Und dies ist nach dieser Deutung keine Antwort auf eine offene Frage mehr, wenn einmal festgelegt ist, dass das, in Bezug auf was wir unparteiisch sein sollten, Freude und Leid aller ist, und wenn „moralisch" so viel wie „unparteiisch“ heißt. Die Unparteilichkeitsannahme des Utilitarismus mag dann im Sinne von Coleridges Tugend ein Ausdrucksversuch für das sein, was uns an Moral wichtig ist, sie ist aber nicht gegen Alternativen als besser erwiesen worden. (Sie ist natürlich auch kein guter Ausdruck dessen, was uns an Moral wichtig ist: Besonders, die Art der Unparteilichkeit des Utilitarismus gegenüber anderen Arten, Unparteilichkeit zu fassen, für grundlegender zu halten, wie zum Beispiel Fairness ${ }^{71}$ u.s.w. sei hier nur erwähnt.) Aber Teil des Beweises - weder im gewöhnlichen Sinne des Wortes noch in sonst irgendeinem Sinne könnte der von Crisp gezählte zweite Schritt nicht sein.

Vielversprechender wäre es vielleicht, zu überlegen, ob der zweite Schritt im Sinne einer Antwort auf eine offene Frage gemeint ist. Dann wäre die Frage, wieso Mill die (empirische) „Evidenz" nicht angibt, von der Mill meint, dass eine solche Frage „like all similar questions “72 abhängen müsste. Der eine Satz, aus dem dieser Schritt

Fallibilität von Wahrnehmung in Frage zu stellen. Und nur darum geht es bei der offenen Frage.

71 Man bedenke zum Beispiel Rawls' Kritik am Utilitarismus: Siehe John Rawls. Eine Theorie der Gerechtigkeit. Frankfurt am Main: Suhrkamp, 1979, S. 4652. Vgl. auch Crisp, Mill on Utilitarianism, S. 82.

72 Mill, „Utilitarianism“, S. 4.10. 


\section{Zwei Grenzen von Mills Philosophie}

in Crisps Zählung besteht, kann sicherlich nicht als das Liefern von Evidenz aufgefasst werden.

Könnte Mill den Status der Unparteilichkeitsannahme für den eines synthetischen Urteils a priori halten und damit daran festhalten, dass sie eine Antwort auf eine offene Frage ist? Dann wäre wahr, was Roger Crisp über Mill in Bezug auf die Unparteilichkeitsannahme des Utilitarismus sagt: Mill wäre dann Intuitionist. ${ }^{73}$ Auch dann müsste sie nach Benthams Tugend natürlich noch gegen Alternativen verteidigt werden. Mill tut dies nicht, weil er nicht glaubt, dass die intuitionistische Strategie überhaupt Benthams Tugend gerecht werden kann.

Egal, wie wir den Status des Schrittes von der Aussage, dass alle Menschen ihr eigenes Glück schätzen, zu der utilitaristischen Bewertungsperspektive, dass das aggregierte Glück aller Menschen der Maßstab moralischer Bewertung sei, also verstehen, scheint er nicht erfolgversprechend. Selbst wenn es Mill also in den anderen Kapiteln geschafft haben sollte, der Tugend Coleridges ebensogut nachzukommen wie der Intuitionismus, der hier auf den ersten Blick im Vorteil ist, selbst wenn er also gezeigt hat, dass der Utilitarismus Ausdruck dessen sein kann, was die Leser*innen seines Buches, sofern sie kompetente Teilnehmer*innen an der moralischen Praxis sind, als adäquaten sprachlichen Ausdruck dessen, worum es in dieser Praxis geht, auffassen würden, so hat er dennoch nicht gezeigt, dass der Utilitarismus gegenüber dem Intuitionismus besser ist. Er ist an Benthams Tugend gescheitert.

Dass Mill hier aber eine philosophische Tugend verfolgt und nicht einfach ein Beweisziel teilweise verfehlt, wird dann deutlich, wenn wir bedenken, worum es Mill bei seiner Kritik am Intuitionismus - und bei seinem Versuch, den Utilitarismus als Gegenmittel zu präsentieren - geht. Ich hatte angedeutet, dass Mill in „Utilitarianism" nur wenig zu den Gründen seiner Kritik am Intuitionismus sagt. Im nächsten Unterabschnitt will ich entsprechend auf eine der explizitesten Auseinandersetzungen Mills mit dem Intuitionismus im Allgemeinen und William Whewell im Besonderen, den Aufsatz „Whewell on Moral Philosophy "74 eingehen. Dies wird einerseits die Rede von einer philosophischen Tugend noch einmal plausibilisie-

73 Crisp, Mill on Utilitarianism, S. 83. Warum dies keine Möglichkeit ist, den Beweis zu retten, erläutere ich in Abschnitt 6.1.3.

74 Mill, „Whewell on Moral Philosophy“. 
ren, indem es deutlich macht, dass Benthams Vermächtnis an Mill, das Anliegen, die Moral auf eine objektive Grundlage zu stellen und so Kritik an der Alltagsmoral zu ermöglichen, zu den langfristigen philosophischen Kernanliegen Mills zählt: Der Witz des Beweisversuches, das, was aus Mills Sicht daran hängt, es so zu machen, wie er es macht, wird dann deutlich. Andererseits wird sich dadurch, dass diese Kritik Mills an zumindest den Formen des Intuitionismus, mit denen er sich auseinandergesetzt hat, wichtig ist, auch der Wert von Benthams Tugend deutlich.

\title{
6.1.3. Die Rolle des utilitaristischen Maßstabs für die Kritik an der common sense Moral
}

Roger Crisp unternimmt einen vermeintlichen Rettungsversuch von Mills Beweis, indem er Mill für den im letzten Abschnitt behandelten zweiten Schritt des Beweises einen (Mill nicht bewussten) intuitionistischen Zug unterstellt:

\begin{abstract}
Mill is after all an intuitionist. [...] [T]he impartiality assumption, which is one of the main bones of contention between Mill and his philosophical opponents, is as "a priori" as any intuitive principle. [...] His own naturalism, and his dislike of the metaphysics and the conservatism he perceived in the philosophy of writers such as Whewell, prevented his seeing that the debate between him and his opponents was ultimately about not intuitionism itself but which intuitions we should accept. ${ }^{75}$
\end{abstract}

Das Problem an dieser Einschätzung ist, dass Mill in seiner Auseinandersetzung mit Whewell, auf den Crisp hier anspielt, zeigt, dass er durchaus differenziert. ${ }^{76}$ Ich habe die Textstelle bereits in Abschnitt 3.4.2 zitiert und diskutiert. Insbesondere Hume, von dem Mill weder denkt, dass er konservativ ist ${ }^{77}$, noch dass er eine grundsätzlich schlechte Metaphysik vertritt, ${ }^{78}$ ist nach Mill im intuitionis-

75 Crisp, Mill on Utilitarianism, S. 83.

76 Mill, „Whewell on Moral Philosophy“, S. $170 f$.

77 Er wird dort explizit als Gegner der Orthodoxie aufgezählt.

78 Im System of Logic bekennt sich Mill beispielsweise explizit zu Humes Kausalitätsauffassung. Siehe Mill, The Collected Works of John Stuart Mill, Volume VIII - A System of Logic Ratiocinative and Inductive (Books IV-VI and Appendices), S. $838 \mathrm{f}$. 


\section{Zwei Grenzen von Mills Philosophie}

tischen Lager ${ }^{79}$. Das Problem am Intuitionismus und der deutschen Metaphysik, die das theoretische Äquivalent dazu sei, ${ }^{80}$ ist, dass er „, bears its testimony with equal ease for any conclusions in favour of which there is a predisposition, and is sceptical with the sceptical, and mystical with the mystical." 81 Nur, wenn die intuitionistische Moralphilosophie an konservativen Institutionen wie der englischen Universität zu Zeiten Mills ${ }^{82}$ und von konservativen Personen wie Whewell betrieben werde, sei das Ergebnis notwendig konservativ. („Coleridge“ zeigt außerdem, dass Mill durchaus bereit ist, über den Konservativismus einer Person hinwegzusehen, wenn es aus seiner Sicht etwas anderes gibt, was Bewunderung verdient.)

Ohne Frage hat Crisp also Recht, dass Mill den Konservativismus und die Metaphysik von Whewell zutiefst ablehnt. (Auch im Falle von Coleridge ist es ja umgekehrt nicht der Konservativismus oder die Metaphysik, die Mill schätzt, wie ich in Kapitel 3 argumentiert habe.) Was Mill am Intuitionismus ablehnt, erschöpft sich aber nicht darin, dass er im 19. Jahrhundert von Konservativen vertreten wurde, sondern die Tatsache, dass der Intuitionismus nach Mill nicht in der Lage ist, Benthams Herausforderung zu begegnen. Intuitionist*innen, wie Mill sie versteht, bringen zwar zum Ausdruck, was ihnen selbst als Personen oder der Mehrheit, sofern sie zu dieser zählen, oder einer privilegierten Gruppe wichtig ist, gehen aber unbegründet von der Tatsache, diesen Ausdruck gefunden zu haben, zu einem Wahrheitsanspruch über. Diese Kritik findet sich in „Coleridge“, „Whewell on Moral Philosophy“ und „Utilitarianism" 83 aber nur in „Utilitarianism" bemüht sich Mill darum, zu zeigen, dass das utilitaristische Prinzip selbst in einer besseren Po-

79 Dies ist Mills Einschätzung, auch wenn Hume, wie er heute meist verstanden wird, keine kognitivistische Auffassung der Moral hatte, sondern eine „theory of a moral sense or taste", wie Mill schreibt, vertritt. Mill, „Whewell on Moral Philosophy", S. 170f. Es wurde bereits darauf hingewiesen (nämlich in Abschnitt 3.4.2, aber auch in Fußnote 21 in diesem Kapitel), dass Mill Benthams Klassifizierung des Prinzips der Sympathie und Antipathie folgend, moral sense-Theorie und Intuitionismus für verwandt hält. Vgl. Mill, „Utilitarianism", § 1.3.

80 Mill, „Whewell on Moral Philosophy“, S. 171.

81 Ebd., S. 170.

82 Siehe ebd., S. $167 f$.

83 Siehe Mill, „Coleridge“, S. 127 bzw. siehe Mill, „Whewell on Moral Philosophy“, S. 170f bzw. siehe Mill, „Utilitarianism“, § 1.3. 
sition ist. Wenn Mill also in seiner Begründung des Utilitarismus versagt, dann ist es nicht nur so, dass der Utilitarismus in Gefahr ist, sondern auch Mills Versuch zu zeigen, dass Moralphilosophie sich neben Coleridges Tugend auch an Benthams Tugend orientieren könne. Benthams Tugend ist ein Kernanliegen, das sich durch sein ganzes Leben zieht. Der Utilitarismus ist, zumindest nach Mills reiferen Überzeugungen, lediglich die einzige Möglichkeit, diesem Kernanliegen gerecht zu werden - nicht umgekehrt ist der Utilitarismus das Kernanliegen, das nebenbei Benthams Tugend ermöglicht. Insbesondere das, was Mill unter „Intuitionismus“ versteht, kann diesem Anliegen nicht gerecht werden.

Mills Kritik daran ist aber in "Whewell on Moral Philosophy“ sehr viel ausführlicher dargestellt. Um daher zu verstehen, was der Beweis in „Utilitarianism“ leisten soll, lohnt es sich, Mills Kritik an Whewell genauer zu diskutieren. Whewell unterscheidet in den Lectures on the History of Moral Philosophy in England ${ }^{84}$, auf die sich "Whewell on Moral Philosophy" bezieht, zwischen abhängiger und unabhängiger Moral. Abhängige Moral bezieht sich auf ein externes Objekt - im Falle des Utilitarismus Freude und Leid oder happiness aller Betroffenen, während unabhängige Moral auf einem internen Prinzip oder einer internen Beziehung, wie zum Beispiel dem Gewissen, dem moralischen Vermögen, der Pflicht oder Aufrichtigkeit u.s.w. beruhten. ${ }^{85}$

Whewell führt diesen Gedanken näher aus, indem er „richtig“ und „falsch“ (und ähnliche Begriffe, wie „sollen“, welche letztlich das Gleiche bedeuteten ${ }^{86}$ ) näher analysiert. Der Gegenstand der Moral, so schließt er, lässt sich offenbar nicht nicht-zirkulär definieren:

Let us begin with the doctrine of Plato just referred to ; that Reason has a natural and rightful authority over Desire and Affection, which doctrine Butler has further illustrated. In making this principle the groundwork of morality, we seem to be guilty of an oversight; for the word rightful already involves a moral notion $[\ldots . .]^{87}$

84 William Whewell. Lectures on the History of Moral Philosophy in England. London und andere: J. W. Parker \& son, 1852.

85 Siehe ebd., S. ixf, zitiert in Mill, „Whewell on Moral Philosophy“, S. 171f.

86 Siehe Whewell, Lectures on the History of Moral Philosophy in England, S. xi.

87 Ebd., S. x. 


\section{Zwei Grenzen von Mills Philosophie}

Moral kann nicht gefasst werden, indem auf etwas außerhalb der Moral Liegendes - zum Beispiel eine außerhalb der Moral liegende Vernunft - referiert werde. Der einzige Ausweg, wie Moral dennoch vernünftig sein könne, ist letztlich, dass Vernunft selbst mit Moral identisch sei:

Having got this notion of what is right; what we ought to do; what we should do; we are already in the region of morality. What is right; what it is that we ought to do, we must have some means of determining, in order to complete our moral scheme; but whatever we so determine, we are involved in a moral system, as soon as we begin to use such words as right and ought.

Thus then we see that the supreme reason of human actions and the moral nature of them cannot be separated. The two come into our thoughts together, and are in our conceptions identical. $^{88}$

Auf den ersten Blick wirkt die Strategie ähnlich wie diejenige, die Mills Coleridge in Bezug auf die Unitarier*innen und das Christentum anwendet: Die Vernünftigkeit der Mysterien des Christentums kann sich nach Mills Coleridge, anders als die Unitarier*innen meinen, nicht durch außerhalb des Christentums liegende Maßstäbe erweisen. Für Mills Coleridge ist aber entscheidend, was praktizierende Christ*innen erleben: Die Vernünftigkeit der Mysterien des Christentums kann sich im Praktizieren des Christentums erweisen. Deswegen ist für Mills Coleridge Erfahrung entscheidend. (Siehe Abschnitt 3.3.2.) Die Parallele steht aber insofern, als sich so nicht das gesamte Christentum als vernünftig oder unvernünftig erweisen lässt. In Bezug auf die Frage „Sollten wir zum Christentum konvertieren?" braucht es nach Mill zusätzlich Benthams Tugend - zum Beispiel in Lebensexperimenten realisiert, nach denen die Erfahrungen beim Praktizieren des Christentums mit Erfahrungen anderer Weisen zu leben verglichen werden müssen. (Siehe Abschnitt 5.2.) Insofern ist zwischen Mills Coleridge und Mills Whewell ein großer Unterschied: Dort ist die entscheidende Tatsache, dass es Erfahrungen gibt, die in einer Weise an eine Art zu leben gekoppelt sind, dass sie nicht reduziert werden können auf andere Erfahrungen;

88 Whewell, Lectures on the History of Moral Philosophy in England, S. xi. 
hier gelangen wir direkt von der Unmöglichkeit, „moralisches Sollen“ unabhängig vom „vernünftigen Sollen“ definieren zu können, dahin, dass Moral und Vernunft eins sein sollen. Während Mill für ersteres viel übrig hat, hält er letzteres für Wortspielerei. ${ }^{89}$ )

Die gleiche Strategie wendet Whewell auch bei happiness an: Wie bei der Vernunft will Whewell auch bei happiness, wie bereits erwähnt, jede „Abhängigkeit" in die eine logische Richtung vermeiden: Moral darf nicht von happiness begrifflich abhängig gemacht werden; auf der anderen Seite will er auch nicht in den sauren Apfel beißen, die naheliegende Konsequenz zu schlucken, happiness habe nichts mit Moral zu tun. Folglich muss das, was happiness ist, durch die Moral bestimmt werden:

But we do not doubt of the truth of this doctrine, that right action does produce the greatest amount of human happiness; and we conceive that happiness must be so apprehended and so understood as to be consistent with this general truth. ${ }^{90}$

So radikal, wie Whewell es hier formuliert, scheint rationale Kritik an unserer derzeitigen moralischen Praxis tatsächlich fast ausgeschlossen; oder dies ist es zumindest, was Mill (Bentham hier verteidigend) kritisiert. Um Mills Kritik am Beispiel von „,purity“ einer der fünf Tugenden, die Whewell vorschlägt, ${ }^{91} \mathrm{zu}$ skizzieren: Angenommen, wir wollten sagen, es gebe keine Gründe dafür, dieser Tugend entsprechend zu handeln. Dies ist durch Whewells Schema ausgeschlossen, weil das, wofür es Gründe gibt, durch die Moral konstituiert ist. Zu sagen, eine Handlung sei eine Instantiierung der Tugend der Keuschheit, ist nach Whewell nichts anderes als einen Grund anzugeben und ihre Rationalität zu erweisen. Angenommen wir wollten sagen, dass die gesellschaftliche Akzeptanz von „,purity“ summa summarum nicht happiness, sondern Leid bei den Betroffenen erzeuge: Auch dies ist durch Whewells Schema ausgeschlossen,

89 Mill hält Phrasen wie die folgenden für höchst problematisch: „our idea of man as man“, „all men think“, „we cannot help feeling“. Vgl. Mill, „Whewell on Moral Philosophy", S. 193.

90 Whewell, Lectures on the History of Moral Philosophy in England, S. xx. Notfalls, indem wir annehmen, Gott würde die Bösen bestrafen. Siehe ebd., S. xxi.

91 Siehe ebd., S. xxif. 


\section{Zwei Grenzen von Mills Philosophie}

weil wir happiness so auffassen sollten, dass sie ebenfalls durch die Moral konstituiert ist. Zu sagen, eine Person sei im Besitz der Tugend der Keuschheit, ist nichts anderes als zu sagen, sie sei in dieser Hinsicht happy. Und so sagt Mill über Whewells Moralphilosophie, sie sei ,an apparatus for converting [...] prevailing opinions, on matters of morality, into reasons for themselves." ${ }^{\text {"2 }}$

Obwohl Mill sich gegen die Dichotomie „abhängig-unabhängig“ verwehrt ${ }^{93}$, stimmt er inhaltlich zu, dass Bentham, den er hier gegen Whewell verteidigt, nach einem externen Standard sucht, an dem sich unsere moralischen Überzeugungen messen lassen müssen. Und er zitiert Benthams kritische Bemerkung zu Positionen, die keinen externen Standard zulassen, um zu zeigen, was für Bentham an einem solchen Standard hängt:

The various systems that have been formed concerning the standard of right and wrong, may all be reduced to the principle of sympathy and antipathy. One account may serve for all of them. They consist, all of them, in so many contrivances for avoiding the obligation of appealing to any external standard, and for prevailing upon the reader to accept of the author's sentiment or opinion as a reason for itself. The phrase is different, but the principle the same. ${ }^{94}$

Der externe Standard hat bei Bentham und Mill also die Aufgabe, zu verhindern, dass wir die Meinung der Autor*in (Bentham) oder die vorherrschende Meinung (Mill) in moralischen Fragen als Gründe an sich akzeptieren müssen. Es ist in „Whewell on Moral Philosophy“ nicht ganz klar, was Mill mit der Rede von einem „externen

92 Mill, „Whewell on Moral Philosophy“, S. 169.

93 ,[T] he word independent is fully as applicable to it [den Utilitarismus] as to the intuition doctrine." Ebd., S. 172. Siehe auch die Unterscheidung zur Rolle des Utilitaristischen Prinzips, die in Abschnitt 4.3.2 diskutiert wird: Nach der Rolle, die durch Abbildung 4.1 nahegelegt wird, könnte auch der Utilitarismus in einer Hinsicht als eine „unabhängige“ Moral bezeichnet werden; nach der anderen Abbildung als eine „abhängige". Es ist aber unwahrscheinlich, dass das Zitat aus „Whewell“, in welchem Mill sagt, auch der Utilitarismus könne als unabhängige Moral bezeichnet werden, diese Frage entscheiden soll. Wahrscheinlich will Mill sich nur gegen den Reduktionismus verwehren, der mit dieser Einteilung zweifellos unterstellt werden soll. (Siehe unten.) Deutlich, was Mill genau meint, wird durch die Textstelle jedenfalls nicht.

94 Bentham, An Introduction to the Principles of Morals and Legislation, S. II.XIV. Zitiert in Mill, „Whewell on Moral Philosophy“, S. 177. 
Standard" implizieren möchte: Wie weit reicht seine Zustimmung zu Bentham in dieser Frage? Der Aufsatz ist als Verteidigung von Bentham gegen einen konservativen Intuitionisten, Whewell nämlich, konzipiert - und Mill legt hier leider keinerlei Wert darauf, seine eigene Kritik an Bentham, wie sie in der Autobiography ${ }^{95}$ oder in „Bentham“96 zum Ausdruck kommt, ins Verhältnis zu seiner Verteidigung Benthams gegen Whewell ${ }^{97}$ zu setzen.

Wenn es auch etwas unklar ist, wie weit Mills Zustimmung zu Bentham in „Whewell on Moral Philosophy" geht, ist im Gegensatz dazu aber klar, worauf die Rhetorik Whewells hinauslaufen soll: Die Rede von „unabhängiger" versus ,abhängiger" Moral und von einem „externen Standard" legt natürlich nahe, dahinter den Vorwurf des Reduktionismus zu vermuten. Und Whewells Anliegen kann vielleicht etwas wohlwollender, als Mill dies tut, in diesem Sinne gefasst werden. Mills eigene Kritik an Bentham kann aber auch als Kritik an Benthams Reduktionismus gefasst werden. (Siehe zum Beispiel Kapitel 4 und Abschnitt 5.1.2.) Insofern wäre es bedauerlich, wenn den Reduktionismus zu verteidigen die einzige Möglichkeit wäre, die Mill bleibt. Mills Kritik an Whewell und Mills Kritik an Bentham ins Verhältnis zu setzen, ist, was ich im Folgenden tun möchte, um danach die Rolle des Unparteilichkeitsprinzips wieder zu beleuchten. Es erscheint nämlich andernfalls so, als ob sich hier wieder das Problem ergibt, dass die beiden Tugenden in Spannung zueinander stehen: Benthams Tugend kann, was Moral angeht, nur - so will Whewell nahelegen - um den Preis eines Reduktionismus erkauft werden; Coleridges Tugend nur - so legt es Benthams Kritik am Prinzip der Sympathie und Antipathie nahe nur um den Preis, auf Objektivität zu verzichten. Die Frage kann also so gefasst werden: Wie kann Mill den Wert, den Bentham in einem externen Standard sieht, bewahren, ohne dass er sich der Gefahr, die Whewell in einem externen Standard sieht, aussetzt? Oder zugespitzt: Wie kann es gleichzeitig externe Standards und keine externen Standards für Moral geben?

95 Siehe Mill, Autobiography, Kapitel 5.

96 Mill, „Bentham“.

97 Whewell hatte seiner Kritik an Bentham von seinen 18 Vorlesungen aus den Lectures immerhin 6 Vorlesungen gewidmet. Siehe Whewell, Lectures on the History of Moral Philosophy in England, Lecture 13-18. 


\section{Zwei Grenzen von Mills Philosophie}

Für happiness hatte ich bereits angedeutet, wie Mill das Problem über die Lebensexperimente löst: happiness kann komparativ als evidentieller Maßstab des Gutseins verschiedener Arten zu leben herangezogen werden, ohne dass damit die Behauptung verbunden ist, höhere Freuden und höhere Formen des Leides seien etwas, das unabhängig von einer bestimmten Art zu leben, artikulierbar ist. happiness ist in diesem Sinne ein externer Standard, der gleichzeitig in einer anderen Hinsicht kein externer Standard ist. (Siehe Kapitel 5.) Das Problem an dieser Stelle ist nun, dass happiness nicht in der gleichen Weise für die Moral herangezogen werden kann: „Aber das-und-das macht mich langfristig gesehen nicht glücklich!" ist normalerweise nicht Ausdruck eines Grundes dagegen, dass das-und-das moralisch von mir gefordert sein mag. Und dies meint natürlich auch Mill.

Ich möchte im Folgenden einige Gedanken von Rosalind Hursthouse heranziehen, um eine Strategie aufzuzeigen, wie das Problem angegangen werden könnte. Dieser Strategie, so meine ich, könnte Mill zustimmen. Das Problem ist, dass Hursthouse explizit die Gerechtigkeitstugenden ausklammert. ${ }^{98}$ Mit dem Unparteilichkeitsprinzip geht es Mill aber zum Teil zumindest um etwas, das auch unter die Gerechtigkeitstugenden fällt. Die Strategie muss also erweitert werden. Wie, werde ich im Folgenden andeuten. Die von mir vorgeschlagene Erweiterung der Strategie von Hursthouse taugt, so will ich explizit klarstellen, nicht dazu, die Problemstellung, die Hursthouse sich selbst stellt, für die Gerechtigkeitstugenden zu beantworten: Hursthouse will durch die Strategie nicht nur andeuten, wie sich die Objektivität, sondern auch die Rationalität der Tugenden erweisen lasse, wobei sie unter Rationalität versteht, dass der Erwerb der Tugenden für diejenige, die die Tugenden erwirbt, gut ist. ${ }^{99}$ Ich will hier sehr viel weniger. Ich will lediglich zeigen, dass sich das Dilemma lösen lässt, das ich oben zugespitzt so formuliert hatte: Wie kann es gleichzeitig externe Standards und keine externen Standards für Moral geben?

In Bezug auf diese Frage stimmt Hursthouse John McDowell zunächst zu, dass es in einer Hinsicht keinen externen Standard für die Moral geben kann: Der Nachweis der Gültigkeit einer Tugend kön-

98 Siehe z. B. Rosalind Hursthouse. On Virtue Ethics. Oxford und andere: Oxford University Press, 1999, S. 5f.

99 Siehe ebd., S. 163. 
ne nur innerhalb einer ethischen Perspektive erbracht werden. ${ }^{100} \mathrm{Es}$ ist nicht möglich, beispielsweise auf eine moralisch neutrale Vorstellung von Glück oder Vernunft zurückzugreifen, um zu zeigen, dass der Besitz der Tugenden glücklich macht oder vernünftig ist. Trotzdem landet sie nicht bei Whewells Position - oder bei McDowells ${ }^{101}$.

100 Siehe ebd., S. 164f. Hursthouse gibt an dieser Textstelle nicht an, auf welchen Text von McDowell sie sich genau bezieht; allerdings ist es tatsächlich so, dass das Thema so prominent und so wiederkehrend bei McDowell ist, dass einen einzelnen Aufsatz auszuwählen willkürlich erschiene. In Bezug auf die für die Beschäftigung mit Mill interessante Frage, ob happiness (der Handelnden oder der Betroffenen) einen „externen“ Maßstab für Moral darstellen könnte, sind die Aufsätze John McDowell. „The Role of Eudaimonia in Aristotle's Ethics". In: Mind, Value and Reality. Cambridge, Mass. und London: Harvard University Press, 1998, S. 3-22 und John McDowell. „Eudaimonism and Realism in Aristotle's Ethics". In: The Engaged Intellect. Cambridge, Mass. und London: Harvard University Press, 2009, S. 23-40 von Bedeutung. In beiden Aufsätzen argumentiert er (trotz punktuell unterschiedlicher Schwerpunkte) gegen die Auffassung, eudaimonia könne im Rahmen von Aristoteles' Ethik als externer Maßstab für die Tugenden gehalten werden. Hinsichtlich des Vorwurfes, dies ende im ethischen Dogmatismus, den McDowell in Bezug auf Aristoteles für zutreffend hält, bezieht sich McDowell (wie auch an anderen Stellen) auf die auf „Validierung von Innen“ abzielende Metapher von Neuraths Boot - als Alternative zum „Eudaimonismus“ in puncto Validierung des ethischen Standpunkts. Vgl. ebd., S. 33-37. eudaimonia, so scheint McDowell in beiden Aufsätzen sagen zu wollen, wird durch die Tugenden konstituiert und nicht umgekehrt. (Vgl. insbesondere McDowell, „The Role of Eudaimonia in Aristotle's Ethics", S. 14-22.) Damit wäre er sehr nahe an der Position, die ich Whewell weiter oben (bei Fußnote 90) zugeschrieben habe. Allerdings scheint es mir so, dass McDowell nur zwei Alternativen zulässt, wo in Wirklichkeit mehr als zwei Alternativen möglich sind: Das Verlangen nach Validierung eines ethischen Standpunkts mithilfe der eudaimonia setzt McDowell mit dem Verlangen gleich, mit eudaimonia das, was Bernard Williams eine absolute Konzeption nennt - einen ahistorischen wissenschaftsähnlichen Maßstab der Kritik -, zu erhalten. (Siehe z. B. McDowell, „Eudaimonism and Realism in Aristotle's Ethics“, S. 37-40.) Eudaimonia ist bei ihm entweder als Teil einer absoluten Konzeption oder als Teil eines ethischen Standpunkte zu verstehen; eine dritte Möglichkeit gibt es nicht. Der Gedanke von Hursthouse, den ich hier referieren will, kann vor diesem Hintergrund so formuliert werden, dass eudaimonia auch weder noch sein kann: Weder bezieht sich eudaimonia auf etwas, das unabhängig von einem ethischen Standpunkt oder gar unabhängig von spezifischen Lebensweisen überhaupt artikulierbar wäre, noch ist eudaimonia einfach gleichbedeutend (oder equiextensional) mit einem tugendhaften Leben.

101 Hursthouse sagt über das, was Foot und McDowell zu dem hier angesprochenen Gedanken sagen würden: „I know that Foot disagrees with much of it and 


\section{Zwei Grenzen von Mills Philosophie}

Whewells Lösung würde, wie für Mill, so auch für Hursthouse bedeuten, dass sie der Anforderung, die Objektivität (in dem Sinne, wie sie in dieser Arbeit mit Benthams Tugend assoziiert wurde) zu erweisen, nicht gerecht geworden wäre:

The worry about such "validation" [from within an acquired ethical outlook] is that it cannot provide rational justifications at all, but is merely circular, doomed to be a mere rationalization of one's personal or culturally inculcated values. ${ }^{102}$.

Wie geht Hursthouse also vor, um das zu vermeiden?

Der Gedanke von Hursthouse, auf den ich eingehen möchte, beginnt mit dem, was sie als "Platons Anforderungen an Tugenden“ bezeichnet:

1. The virtues benefit their possessor. (They enable her to flourish, to be, and live a life that is, eudaimon.)

2. The virtues make their possessor a good human being. [...]

3. The above two features of the virtues are interrelated. ${ }^{103}$

Das Bekenntnis zu McDowell bedeutet zunächst, dass wir nicht gänzlich von außerhalb eines ethischen Standpunkts die Wahrheit von (1) evaluieren können. ${ }^{104}$ Dies ist natürlich nichts, dem Mill grundsätzlich widersprechen würde. In Kapitel 5 wurde das, was Mill „höhere Freuden“ oder „höhere Formen des Leides“ nennt, diskutiert. Diese sind, so hatte ich argumentiert, an Lebensweisen gekoppelt und sie können nicht evaluiert werden außer von einer

strongly suspect that McDowell would disagree with even more". Hursthouse, On Virtue Ethics, S. 163. Wie in Fußnote 100 angedeutet, folgt nicht direkt, dass McDowell Hursthouses Gedanken zurückweisen müsste; da McDowell nur die beiden in der Fußnote 100 angesprochenen Alternativen diskutiert, ist es richtiger zu sagen, dass er Hursthouses Position nicht berücksichtigt, als dass er sie ablehnt. Aber natürlich hat Hursthouse wahrscheinlich Recht damit, dass er sie ablehnen würde.

102 Ebd., S. 165.

103 Ebd., S. 167.

104 Von (2) natürlich auch nicht. Aber hier ist das trivial: „A good human being“ ist im Sinne der tugendethischen Strömung, der sich Hursthouse zurechnet, nichts anderes als das, worum es in einer ethischen Perspektive geht. 
Person, die weiß, was es heißt, auf diese Weise zu leben. Mill erwähnt auch explizit Freuden, die an eine ethische Perspektive gebunden sind, beispielsweise seine Diskussion von Würde in diesem Zusammenhang. ${ }^{105}$

Das heißt aber eben nicht, dass Glück und (ethisch) gutes Leben für Hursthouse gleichbedeutend sind; dass diejenige, die eine Tugend erwirbt und dadurch nach (2) zu einem guten Menschen wird, dadurch ipso facto auch glücklich würde: mit anderen Worten (2) ist nicht einfach eine Reformulierung von (1). ${ }^{106}$ Der in (3) von Husthouses Platon geforderte Zusammenhang ist weder Whewells Konstitutionsthese des Glückes durch die Moral, noch ist es natürlich die umgekehrte These, Moral sei letztlich durch individuelles Glück konstituiert. Die Frage ist also, wie (3) zu verstehen ist. ${ }^{107}$ Hursthouse schreibt dazu:

[T] he claim is not that the possession of the virtues guarantees that one will flourish. The claim is that they are the only reliable bet-even though, it is agreed, I might be unlucky and, precisely because of my virtues, wind up dying early or with my life marred or ruined. ${ }^{108}$

105 Siehe Mill, „Utilitarianism“, $§ 2.6$.

106 So sagt das McDowell übrigens auch nicht: Er sagt, die aristotelische Behauptung bestehe darin, dass zwei prima facie unterschiedliche Begriffe die gleiche Extension hätten. (S. 14) Trotzdem handelt es sich nach McDowell wohl nicht einfach um eine informative Identitätsbehauptung: Hier ist aber entscheidend, dass er sich vor allem dagegen wehrt, dass eudaimonia jemals Maßstab für Tugend sein könne; vielmehr müssten wir die Gleichung „the other way round" (S. 16) verstehen. Vgl. McDowell, „The Role of Eudaimonia in Aristotle's Ethics", S. 14-22. Gemeint scheint zu sein, dass der Begriff des guten Lebens konstitutiv für den Begriff der eudaimonia ist und nicht umgekehrt. Dies ist sehr nahe an Whewells oben (im Zitat zu Fußnote 90) zitierter Vorstellung von happiness und Moral.

107 In einer Hinsicht könnte auch Kant so verstanden werden, dass dem von Hursthouse geforderten Zusammenhang zustimmt. Für Kant kann es für (3) allerdings schließlich keine empirischen Belege geben; es ist aber (für Kant wie für Hursthouse) auch explizit kein analytischer Zusammenhang. Wir müssen trotzdem (mit dem Status einer transzendentalen Annahme) annehmen, dass (3) gültig ist - wenn auch nur zu praktischen Behufe. Siehe Kant, $K p V$, S. AA $110-119$.

108 Hursthouse, On Virtue Ethics, S. 172. 


\section{Zwei Grenzen von Mills Philosophie}

Tugenden (und nicht Laster) zu erwerben ist lediglich die beste Chance, glücklich zu werden. ${ }^{109}$ Dass ich durch den Besitz einer Haltung unglücklich geworden bin, ist also kein Beweis dafür, dass diese Haltung keine Tugend war. (Und ich vermute, ebenso würde Hursthouse zustimmen, dass die Tatsache, dass ich durch den Besitz einer Haltung glücklich geworden bin, kein Beweis dafür ist, dass es sich bei der Haltung um kein Laster handeln kann.) Inwiefern kann trotzdem gesagt werden, (1) stelle einen externen Standard für (2) dar?

Wir können zum Beispiel herausfinden, dass der Erwerb einer Haltung nicht die beste Chance ist, glücklich zu werden; oder wir können herausfinden, dass der Erwerb einer anderen Haltung nicht tendenziell ins Unglück führt. Dafür reicht es natürlich nicht, zu zeigen, dass einzelne Personen durch etwas, was wir für eine Tugend hielten, nicht glücklich geworden sind; beziehungsweise durch etwas, was wir für ein Laster hielten glücklich geworden sind. Aber, ob etwas die beste Chance ist, glücklich zu werden, ist dennoch keine Frage, die völlig unabhängig von solchen Erfahrungen wäre: Der Extremfall, dass noch nie eine Person durch eine bestimmte Haltung glücklich geworden ist, sollte uns entsprechend den Anforderungen daran zweifeln lassen, dass die Haltung eine Tugend ist. In Bezug auf Whewells Tugend der Keuschheit könnte sich beispielsweise herausstellen, dass sie nicht nur in einzelnen Fällen nicht zum Glück führt, sondern dass ihr Erwerb eben nicht die beste Chance auf Glück ist. Wenn wir herausfinden, dass eine Haltung nicht die beste Chance ist, glücklich zu sein, hätten wir nach dieser Idee von Hursthouse also einen Grund zu bezweifeln, dass es sich um eine Tugend handelt. In diesem Sinne ist das Glück der Handelnden ein externer Maßstab bezüglich der Haltungen, die wir für Tugenden halten, obwohl es kein externer Maßstab in dem Sinne ist, dass sich die Frage, ob eine Person glücklich ist, von außerhalb eines ethischen Standpunkts evaluieren ließe. In der zugespitzten Formulierung von oben könnte gesagt werden: Es gibt einen externen Maßstab für die Tugenden, der gleichzeitig in relevanter Hinsicht kein externer Maßstab ist.

109 Hursthouse begründet, dass dies auch von uns angenommen wird, unter anderem im Kontext der Erziehung: Guten Eltern geht es auch in der moralischen Erziehung (vorrangig) um das Gedeihen ihrer Kinder. Siehe Hursthouse, On Virtue Ethics, S. 175. 
Weiter oben hatte ich bereits angedeutet, dass ich denke, dass dieser Gedanke von Hursthouse erweitert werden muss. Eine offensichtliche Stelle, an der das der Fall ist, ist das, was Hursthouse die Gerechtigkeitstugenden nennt und, wie gesagt, ausklammert. Darüber, ob der Ansatz nur in Bezug auf diese Gerechtigkeitstugenden erweitert werden muss, möchte ich in dieser Arbeit agnostisch bleiben. Gerechtigkeitstugenden sind aber wichtig für die Frage, wie Mill im „Utilitarianism“ dem Unparteilichkeitsprinzip gerecht werden kann, weswegen ich die obigen Gedanken von Hursthouse überhaupt eingeführt habe. Die Antwort auf diese Frage wird davon abhängen, wie genau die Gerechtigkeitstugenden abgegrenzt werden. Die Strategie stößt bei Gerechtigkeitstugenden an eine Grenze, weil die bloße Tatsache, dass eine Haltung nicht die beste Chance auf Glück für mich ist, nicht bedeutet, dass sie keine Gerechtigkeitstugend ist. Oder umgekehrt: Die bloße Tatsache, dass eine Haltung normalerweise ihrer Träger*in nutzt, schließt nicht aus, dass sie ein Gerechtigkeitslaster ist - wenn eine solche Wortschöpfung in Analogie zu Gerechtigkeitstugenden erlaubt ist. (Es scheint, wie auch Mill meint, eher richtig zu sein, dass das Umgekehrte nur unter in einer bestimmten Hinsicht idealen gesellschaftlichen Bedingungen gilt. Aber Fragen der politischen Philosophie will Hursthouse nicht beantworten. ${ }^{110}$ )

Rassismus oder Sexismus zum Beispiel sind Gerechtigkeitslaster. Der Besitz entsprechender Haltungen macht in der Sprache von "Platons Anforderungen“, die oben zitiert wurden, die Besitzer*in $\mathrm{zu}$ einem schlechten Menschen. Aber es ist jedenfalls nicht offensichtlich, wenn nicht sogar unplausibel, dass die Frage, ob Rassismus oder Sexismus Laster sind, davon abhängen sollte, dass sie die Chancen auf Glück ihrer Träger*in reduzieren. Im Gegenteil: Gerechtigkeitslaster scheinen oft so aufgefasst zu werden, dass sie im Interesse der Träger*in sind: Eine rassistische Gesellschaft ist eine Gesellschaft, die Vorteile und Nachteile wie Bildungschancen, Einkommen, politische und intellektuelle Teilhabe, kulturelle Gestaltungsmöglichkeiten u.s.w. nach bestimmten, offensichtlich ungerechten Kriterien verteilt.

110 Dies sagt sie an der gleichen Stelle, an der sie auch die Gerechtigkeitstugenden aus ihrer Betrachtung ausschließt. Siehe ebd., S. 5. 


\section{Zwei Grenzen von Mills Philosophie}

Wäre es eine Möglichkeit zu sagen, dass hier ein Missverständnis vorliegt, dass die rassistische Person nicht wirklich im eigentlichen Sinne (oder zumindest nicht im richtigen Sinne ${ }^{111}$ ) glücklich ist? Hier müssten wir uns entscheiden. Eine Möglichkeit, diesen Vorschlag zu verstehen, wäre ihn im Sinne Whewells als Konstitutionsthese des Glückes zu verstehen: Was auch immer wir meinen, das eine Person vom Rassismus hat, es kann kein wahres Glück sein, weil wahres Glück durch Moral konstituiert ist und Rassismus ein Laster ist. Dies würde aber die ganze Strategie von Hursthouse in sich zusammenfallen lassen. Glück (oder Leid) wären keine Maßstäbe mehr, an denen Tugenden sich messen lassen könnten. Sie wären per definitionem von den Tugenden abhängig. Was wäre aber, wenn wir den Vorschlag anders auffassen? Die These wäre dann, dass es tatsächlich so ist, dass wenn wir ernsthaft versuchen, Prognosen anzustellen, wir feststellen, dass Rassismus dem Glück der Handelnden hinderlich ist. Dieser Vorschlag ist natürlich attraktiv: Es wäre zum Beispiel schön, wenn eine gerechtere (zum Beispiel weniger rassistische) Gesellschaft eine solche Gesellschaft wäre, in der alle und nicht nur die rassistisch Unterdrückten glücklicher sind. Selbst aber, wenn wir davon vollständig überzeugt wären, sollten wir die Frage, ob Rassismus (oder andere Gerechtigkeitslaster) wirklich Laster sind, nicht von einer solchen Prognose abhängig machen: Denn sollte sie sich als falsch herausstellen, sollten wir trotzdem nicht bereit sein zu akzeptieren, dass Rassismus oder Sexismus zum Beispiel keine Gerechtigkeitslaster wären. Es würde ein offensichtliches moralisches Übel dann von einer keineswegs offensichtlichen materialen These dazu, unter welchen Bedingungen Menschen glücklich werden können, abhängig gemacht. Wir würden zu viel auf diese Karte setzen. Beide Möglichkeiten, das Glück der Handelnden an die Gerechtigkeitstugenden zu koppeln, scheitern also. Die Strategie muss erweitert werden.

Weiter oben hatte ich bereits angedeutet, dass meine Bemerkungen dazu, wie die Strategie von Hursthouse erweitert werden müsste, nicht dazu taugen werden, einer der Problemstellungen von Hursthouse selbst gerecht zu werden, nämlich der, zu zeigen, wie Gerechtigkeitstugenden im Interesse ihrer Träger*in sein können. Aber die Strategie taugt trotzdem dazu, Objektivität, nämlich im

111 Vgl. McDowell, „Eudaimonism and Realism in Aristotle's Ethics“, 30f. 
Sinne eines externen Standards, zu gewährleisten. Leid und in geringerem Maße vielleicht auch Freude können nämlich auch zur Begründung - als externer Maßstab - für Gerechtigkeitstugenden herangezogen werden, so behaupte ich. Gemäß der Strategie von Hursthouse bedeutet das nicht, dass Gerechtigkeitstugenden in dem Sinne auf Leid und Freude reduzierbar wären, dass der Witz einer Gerechtigkeitstugend vollständig durch Freude und Leid ausdrückbar wäre. Dagegen spricht schon, dass die Rolle, die Leid (und Freude) jeweils spielt, in Wirklichkeit nicht immer dem gleichen Aggregationsprinzip folgt: Manchmal scheint es um Freude und Leid jeder einzelnen Person zu gehen; manchmal scheint nur das Leid derjenigen zu zählen, die am meisten leiden; und vielleicht zählt auch manchmal - falls die theoretischen Voraussetzungen dafür diesen Satz überhaupt zu verstehen erfüllbar sind - wie Bentham meinte, das Leid jeder Person in gleichem Maße als Grund für (oder gegen) eine Gerechtigkeitstugend. Wichtig ist nur Folgendes, was ich von der Strategie von Hursthouse übertragen möchte: Dass es kein reduktionistisches Prinzip gibt, bedeutet nicht, dass Leid keinerlei begründende Funktion haben kann.

Zweitens: Ungerecht behandelt zu werden ist in einem bestimmten Sinne natürlich selbst eine Form von Leid - und viele Formen des Leides lassen sich nicht unabhängig davon verstehen, dass wir den entsprechenden Begriff des Unrechtes haben. Dies legt uns aber nicht auf Whewells (oder McDowells) Konstitutionsthese fest: Genauso wenig wie im Besitz der Tugenden zu sein bei Hursthouse bedeutet, dadurch auch glücklich zu werden, bedeutet eine Gerechtigkeitstugend erworben zu haben, kein Leid zu hervorzubringen (oder ein Gerechtigkeitslaster erworben zu haben, Leid zu erzeugen). In diesem Sinne sind selbst die Formen des Leides, die wir nicht vollständig begreifen können, ohne auf eine Gerechtigkeitstugend selbst zu verweisen, nicht gleichbedeutend mit den Folgen eines Verstoßes gegen die Gerechtigkeitstugenden und ebenfalls in diesem Sinne bleibt Leid selbst in solchen Fällen ein externer Maßstab für einen beliebigen historisch geprägten ethischen Standpunkt.

Drittens: Es spielen auch bei Gerechtigkeitstugenden selbstverständlich nicht nur Formen des Leides eine Rolle, die in der skizzierten Weise vom Verständnis der entsprechenden Tugend abhängen.

Happiness, so wie Mill den Begriff versteht, d.h. im Sinne von Leid und Freude, ist ein externer Maßstab für ethische Standpunk- 


\section{Zwei Grenzen von Mills Philosophie}

te in dem Sinne, dass der Begriff in seiner für die begründende Funktion entscheidenden Komponente nicht vollständig durch den ethischen Standpunkt konstituiert wird. Happiness ist gleichzeitig kein externer Standpunkt, insofern „Leid“ nicht im Sinne einer absoluten Konzeption verwendet wird. Alles, was damit gemeint ist, ist dass Leid von Menschen ein Grund ist einen ethischen Standpunkt in Zweifel zu ziehen oder aber ihn beizubehalten. (Natürlich ist damit nicht gesagt, dass Leid und Freude die einzigen plausiblen Kandidat*innen für „externe“ Maßstäbe in diesem Sinne sind - sie sind es nicht und ich werde Mill in dieser Hinsicht nicht versuchen $\mathrm{zu}$ verteidigen.)

Auch wenn Mills benthamitische Version des Unparteilichkeitsprinzips die falsche Art und Weise ist, Freude und vor allen Dingen Leid anderer Menschen als externen Maßstab für moralische Standpunkte zu berücksichtigen, so kann darin doch ein legitimes Bemühen erkannt werden: Nämlich erstens einen Maßstab der Validierung (und Kritik) moralischer Standpunkte zu etablieren und zweitens die Wichtigkeit von Leid dabei herauszustellen. In eine ähnliche Richtung geht Nussbaums Einschätzung, auch wenn sie die Krise sehr anders deutet als Anderson und ich im Anschluss an Anderson. ${ }^{112}$.

\subsection{Mills praktischer Psychologismus}

Die zweite Schwäche von Mills Philosophie, die ich diskutieren will, ist das, was in Bezug auf theoretische Philosophie, insbesondere die Philosophie der Logik, im Anschluss an Edmund Husserl ${ }^{113}$ und Gottlob Frege ${ }^{114}$ manchmal „Psychologismus" genannt wird. Ich werde mich allerdings vorwiegend um die praktische Seite des, wie ich meine, gleichen Phänomens kümmern und es ebenfalls „Psychologismus" nennen. Diesbezüglich bereits angedeutet (Abschnitt 2.4) hatte ich, dass Mill sozialontologisch das ist, was Lars Udehn einen

112 Nussbaum, „Mill between Aristotle \& Bentham“.

113 Insbesondere Edmund Husserl. Logische Untersuchungen, Band 1. Halle: Niemeyer, 1913, Kapitel 3-10 beschäftigen sich mit dem Psychologismus.

114 Insbesondere bezüglich der darin enthaltenen Kritik an Mill in dieser Hinsicht siehe Gottlob Frege. Die Grundlagen der Arithmetik. Eine logisch mathematische Untersuchung. Breslau: Wilhelm Koebner, 1984. 
„psychologischen Individualisten“115 nennt: Soziale Institutionen, Praktiken und so weiter bestehen letztlich in (verbreiteten) psychischen Zuständen. ${ }^{116}$

Ziel des Abschnittes ist es, auch hier zu zeigen, dass es sinnvoll ist, den Psychologismus als einen scheiternden Versuch zu verstehen, beiden Tugenden gerecht zu werden. Ich werde im Folgenden erstens deutlich machen, warum es auf den ersten Blick so scheint, dass eine Form des Psychologismus, wie sie von Mill vertreten wird, den beiden philosophischen Tugenden gerecht wird; zweitens werde ich dafür argumentieren, dass dies aber tatsächlich nicht der Fall ist. Es geht mir also wie im letzten Abschnitt auch hier darum, wie Mills Philosophie als durch die Tugenden geleitet, aber ohne diese vollständig zu erfüllen, verstanden werden kann. Kurz vorweggenommen eignet sich die Interpretation philosophischer Fragen in den Begriffen psychischer Zustände auf den ersten Blick deshalb für beide Tugenden, weil psychische Zustände einerseits erstpersonal Bedeutung fassen zu können scheinen, wir sie aber zweitens drittpersonal als Tatsachen zuschreiben können. Mills psychologischer Individualismus erlaubt ihm dann sowohl, so scheint es, soziale Institutionen, Praktiken und so weiter in ihrer Bedeutung für das Individuum zu erfassen (Coleridges Tugend), als auch - nun dispositional als drittpersonale Tatsache betrachtet - die sozialen Auswirkungen dieser Einstellungen zu evaluieren, insbesondere, was die Interpretation institutioneller Tatsachen ${ }^{117}$ als psychische Zustände angeht.

In Teil I ist deutlich geworden, dass Mill häufig von (received) opinion schreibt, wenn es eigentlich zum Beispiel um Institutionen

115 Siehe Udehn, „The Changing Face of Methodological Individualism“, S. 482, wo Udehn explizit Mill als Vertreter einer solchen Position charakterisiert.

116 In dieser Hinsicht ist Mill mit Searle vergleichbar, auch wenn Mill anders als Searle keine Wir-Einstellungen kennt: Vgl. z. B. John Searle. Making the World Social. Oxford: Oxford University Press, 2011 oder John Searle. The Construction of Social Reality. New York: The Free Press, 1995. Dass Searle trotzdem in einer wichtigen Hinsicht individualistisch bleibt und dass dies zu philosophischen Problemen führt, die vergleichbar sind mit denen, die ich in diesem Abschnitt für Mill herausarbeiten werde, zeigt Hans-Bernard Schmid: Hans Bernhard Schmid. „Can Brains in Vats Think as a Team?" In: Philosophical Explorations 6.3 (2003), S. 201-217.

117 Der Begriff „institutionelle Tatsache“ ist natürlich ebenfalls eine Referenz auf Searle. Siehe z. B. Searle, Making the World Social, S. 10f. 


\section{Zwei Grenzen von Mills Philosophie}

oder andere soziale Entitäten geht. ${ }^{118}$ An diesem Beispiel unserer Praxis der Meinungs- oder Überzeugungszuschreibung kann deutlich gemacht werden, was damit gemeint ist, dass der Psychologismus beiden Tugenden gerecht $\mathrm{zu}$ werden scheint: $\mathrm{Ob}$ eine Person eine Überzeugung hat und sogar, welche Auswirkungen dieses Überzeugtsein hat, kann zumindest logisch völlig unabhängig von der normativen Frage, ob diese Überzeugung wahr ist, eruiert werden - und sowohl Bentham als auch Mill tun dies häufig, wie deutlich geworden sein dürfte, insbesondere, wenn es um sozialphilosophische Fragen geht. Der (oft sozial schädliche) Effekt von verbreiteten Überzeugungen ((received) opinions) kann so im Sinne von Benthams Tugend untersucht werden. Gleichzeitig geht es natürlich erstpersonal um die Frage der Wahrheit und diese Frage kann nicht bloß dispositional umgedeutet werden. Weiter unten wird deutlich, dass Mill gerade bei Wahrheit - das, worum es im Überzeugtsein geht - eine rein psychologistische Interpretation für unangemessen hält. Aber was für Überzeugungen gilt, gilt auch abgewandelt für andere psychische Zustände - und hier hat Mill fast durchgängig eine Tendenz zur psychologistischen Deutung. Durch die Möglichkeit dieser beiden Perspektiven auf psychische Zustände könnte es so scheinen, dass psychische Zustände in Bezug auf Mills Tugenden das philosophische Äquivalent eierlegender Wollmilchsäue sind: Auf der einen Seite sind Überzeugungszuschreibungen Tatsachenbehauptungen, was Benthams Tugend entgegen kommt. Auf der anderen Seite konstituieren Sie eine (bedeutungsvolle) Innenperspektive für diejenige, die sie unterhalten.

In Wirklichkeit scheitert dieser Versuch aber. Auf der abstraktesten Ebene kann das Problem so beschrieben werden: Der Versuch setzt voraus, dass die Grammatik des erstpersonalen Ausdrucks psychischer Zustände ohne weiteres in die Grammatik der drittpersonalen Zuschreibung dieser Zustände übertragbar wäre. In dieser Allgemeinheit werde ich zu diesem Thema nicht mehr viel sagen.

118 Auch hier gibt es eine Parallele zu Searle. Obwohl Searles Vorstellung kollektiver Intentionalität auch andere Zustände als Überzeugungen, so genannte „Wir-Absichten“ umfasst, (siehe John Searle. „Einige Grundprinzipien der Sozialontologie". In: Kollektive Intentionalität. Hrsg. von Hans Bernhard Schmid und David P. Schweikard. Frankfurt am Main: Suhrkamp, 2009, S. 504-533) sind seiner Meinung nach für die Konstruktion institutioneller Tatsachen lediglich Überzeugungen nötig. Siehe Searle, Making the World Social, S. 42-60. 
Ausführlich ausgearbeitet wurde dies insbesondere in Bezug auf die Frage, ob die Unterschiede zwischen der erstpersonalen und der drittpersonalen Redeweise über psychische Zustände es noch zulassen, dass wir davon reden sollten, dass wir von unseren eigenen Zuständen wissen können, so wie wir von den Zuständen anderer wissen können, von Lena Ljucovic. Sie geht auch explizit auf die Frage der Übertragbarkeit dieser Überlegungen von Überzeugungen, die in vielen Hinsichten in der Philosophie oft als paradigmatisch behandelt werden, auf andere Zustände ein. ${ }^{119}$

Ich werde mich darauf beschränken, am Beispiel moralischer Gefühle, für die sich Mill zum Beispiel in The Utility of Religion ${ }^{120}$ interessiert, zu skizzieren, inwiefern Mill diese in der beschriebenen Weise psychologistisch auffasst und warum das angesichts von Mills Tugenden ein naheliegender Fehler von Mill ist (Abschnitt 6.2.3). Die Art und Weise, wie Mill psychische Zustände hier behandelt, ist paradigmatisch dafür, wie er sie sonst behandelt. Bevor ich das jedoch tun werde, möchte ich den Vorwurf des Psychologismus im engeren Sinne, der von Husserl auch explizit auf Mill bezogen wurde, kurz erläutern (Abschnitt 6.2.1) und unter Bezug auf Mills eigene Handlungstheorie deutlich machen, inwiefern dieser Vorwurf auf das Praktische ausgedehnt werden kann (Abschnitt 6.2.2).

\subsubsection{Psychologismus im engen Sinn}

Ob Mills theoretische Philosophie oder seine Logik wirklich als psychologistisch in Husserls und Freges Sinne bezeichnet werden sollte, ist keine pauschal beantwortbare Frage. In einigen Hinsichten trifft der Vorwurf sicher zu; in anderen weniger. Psychologismus (in Bezug auf die Logik) ist die Position, dass logische Gesetze ihrem Wesen nach psychologische Gesetze (und damit empirische Gesetze ${ }^{121}$ ) sind. ${ }^{122}$ Auf den ersten Blick scheint Mill tatsächlich der paradig-

119 Lena Ljucovic. „Dissertation“. Im Erscheinen.

120 Mill, ,Three Essays on Religion“.

121 Wenn dieser Aspekt betont wird, wären auch Positionen, die logische oder andere normative Regeln als soziologische Gesetze auffassen und damit auch als empirische Tatsachen, verwandt. Siehe Fußnote 128.

122 „Die Hauptsache ist hier die Gleichsetzung der Urteilsgebilde (und dann natürlich aller ähnlichen Gebilde von Vernunftakten überhaupt) mit Phänomenen der inneren Erfahrung. Diese Gleichsetzung ist begründet durch ihr ,inneres' Auftreten im Aktbewußtsein selbst. So wären also Begriffe, Urteile, 


\section{Zwei Grenzen von Mills Philosophie}

matische Gegenstand dieser Kritik zu sein. Er schreibt über die Disziplin der Logik:

It is not a Science distinct from, and coordinate with, Psychology. So far as it is a science at all, it is a part, or branch, of Psychology; differing from it, on the one hand as a part differs from the whole, and on the other, as an Art differs from a Science. Its theoretic grounds are wholly borrowed from Psychology, and include as much of that science as is required to justify the rules of the art. ${ }^{123}$

Textstellen wie diese machen Mill nicht nur zu einem guten impliziten Ziel für Freges und Husserls Kritik; natürlich ist Mill auch explizit Gegenstand dieser Kritik. Husserl kritisiert zum Beispiel Mills, wie er meint, in diesem Sinne psychologistische Auslegung des Satzes vom ausgeschlossenen Widerspruch. Mill scheint zu behaupten, dass der Satz vom ausgeschlossenen Widerspruch auf der (von ihm behaupteten) Aversion des menschlichen Geistes dagegen, zwei sich widersprechende Sätze gleichzeitig zu unterhalten, beruhe. ${ }^{124}$ Mill scheint in diesem Sinne tatsächlich der Vorzeigedenker für psychologistische Philosophie im Sinne des Kritikgegenstandes von Husserl und Frege zu sein. Auf der anderen Seite kann Mills Theorie der Namen im krassen Gegensatz dazu auch ganz anders verstanden werden. Hier legt Mill Wert darauf, dass die Bedeutung des Namens der Gegenstand selbst sein müsse und nicht irgendeine Form von psychischem Abbild. In diesem Sinne könnten wir auch zu diesem Schluss kommen: „So far as concepts and judgments are concerned, Mill's logic is not an exemplification of what Husserl calls psychologism, but, rather, a forceful condemnation of it." "125

Auf die Theorie der Namen (und Begriffe) möchte ich hier nicht näher eingehen. Ich werde aber weiter unten (Abschnitt 7.1) auf

Schlüsse, Beweise, Theorien psychische Vorkommnisse und die Logik, wie J.

St. Mill gesagt hatte, ein ,Teil oder Zweig der Psychologie: Eben in dieser scheinbar so einleuchtenden Auffassung liegt der logischen[!] Psychologismus."

Edmund Husserl. Formale und transzendentale Logik. den Haag: Martinus Nijhoff, 1974, S. 162. Vgl. auch Husserl, Logische Untersuchungen, Band 1, S. 51.

123 Mill, The Collected Works of John Stuart Mill, Volume IX - An Examination of William Hamilton's Philosophy and of The Principal Philosophical Questions Discussed in his Writings, S. 461f.

124 Siehe Husserl, Logische Untersuchungen, Band 1, S. 81-84.

125 McRae, „Introduction“, S. xlii. 
Mills Auseinandersetzung mit Platon und "Grote's Plato" eingehen und möchte daher als Beleg für Mills partielle Opposition gegen das, was als psychologistische Vorstellung der Wahrheit verstanden werden könnte, auf eine Passage aus Mills Auseinandersetzung mit Grote (seinem Aristotle) eingehen, in welcher es um die unterschiedliche Einschätzung von Protagoras geht. Ausführlicher wird es um die Auseinandersetzung mit Grote im nächsten Kapitel (Abschnitt 7.1) gehen:

But if this was the meaning of Protagoras, it was not only paradoxically, but incorrectly expressed. It would surely be a perverse employment of language to say that if I believe two and two to make five, they really make five to me, or that, if I erroneously believe a certain person to be dead, he is really dead to me though not to other people. The truth of a belief does not consist in its being believed, but in its being in accordance with fact $[\ldots]{ }^{126}$

Bei dieser Andeutung, dass Mills Logik in einigen (Satz vom ausgeschlossenen Widerspruch) aber nicht in anderen Hinsichten (Wahrheitstheorie, Bedeutung von Namen) als mit Recht als psychologistisch einzuschätzen ist, möchte ich es hier belassen. Eine tiefergehende Erörterung der Frage, ob und in Bezug auf welche Gegenstände Mills Logik psychologistisch ist, kann ich an dieser Stelle nicht leisten. Stattdessen möchte ich, wie gesagt, die Diskussion auf die praktische Philosophie zurück lenken (Abschnitt 6.2.2). Um zu verstehen, wie Husserls und Freges Psychologismusvorwurf auf praktische Fragen anwendbar ist, ist es aber sinnvoll, zunächst eines der von ihnen ausgemachten Probleme des Psychologismus zu verstehen.

Eine, die versucht, logische Gesetze - diejenigen Dinge also, deren psychologistische Deutung in Frage steht, zum Beispiel das bereits angesprochene Gesetz vom ausgeschlossenen Widerspruch $\mathrm{zu}$ formulieren, versucht damit gültige von ungültigen Schlüssen unterscheiden. Eine, die nun sagt, logische Gesetze seien psychologische Gesetze, muss diese Unterscheidung innerhalb der tatsächlichen psychologischen Vorgänge des Schließens treffen und zwar,

126 John Stuart Mill. "Grote's Aristotle“. In: The Collected Works of John Stuart Mill, Volume XI. Essays on Philosophy and the Classics. Hrsg. von J. M. Robson. Toronto und andere: University of Toronto Press und Routledge \& Kegan Paul, 1978, S. 475-510, S. 501n. 


\section{Zwei Grenzen von Mills Philosophie}

ohne bei dieser Unterscheidung die Gesetze, um die es geht, bereits in Anspruch zu nehmen. Wir können nicht einfach alle tatsächlichen psychologischen Vorgänge des Schließens von allen Menschen zur empirischen Grundlage für logisches Schließen machen, weil es dann keine ungültigen Schlüsse mehr gäbe. Das heißt, wir müssten eine Grundlage finden, wie wir die gültigen der tatsächlichen psychologischen Vorgänge des Schließens von den ungültigen abgrenzen können. ${ }^{127}$ Es ist nicht klar, wie dies nicht-zirkulär möglich sein sollte.

Das Problem ist, anders ausgedrückt, dass die psychologischen Vorgänge so verstanden werden müssen, dass sie die Möglichkeit des logischen Fehlers nicht ausschließen dürfen. In diesem Sinne ist der Psychologismusvorwurf erweiterbar auf alle Bereiche, in denen wir davon sprechen können wollen, dass Menschen einen Fehler oder etwas falsch machen. ${ }^{128}$ Für meine Auseinandersetzung mit

127 „Wo sind die deskriptiven und genetischen Analysen, die uns berechtigen, die Denkphänomene aus zwei Klassen von Naturgesetzen zu erklären, von welchen die einen ausschließlich den Gang solcher Kausationen bestimmen, die das logische Denken hervorgehen lassen, während für das alogische Denken auch die anderen mitbestimmend sind? Ist die Bemessung eines Denkens nach den logischen Gesetzen etwa gleichbedeutend mit dem Nachweis seiner kausalen Entstehung nach eben diesen Gesetzen als Naturgesetzen?" Husserl, Logische Untersuchungen, Band 1, S. 66.

128 Der Psychologismusvorwurf ist auch anders erweiterbar. Aussagen über „unsere Praxis" werden oft in der gleichen Doppelrolle benutzt. Dies könnte in Analogie zu Psychologismus als „Soziologismus“ bezeichnet werden. Dies ist wichtig, weil ich weiter unten, wenn ich erkläre, warum der Psychologismus als (scheiternder) Versuch, den beiden philosophischen Tugenden gerecht zu werden, verstanden werden kann, nahelegen will, dass es sich beim Soziologismus ebenso verhält.

In Bezug auf die theoretische Philosophie wird eine solche Position besonders paradigmatisch von Saul Kripke in Auseinandersetzung mit Wittgensteins Problematik des Regelfolgens ausgearbeitet. Kripke, Wittgenstein on Rules and Private Language. Dass es einen Unterschied gibt zwischen dem, was ich denken soll, und dem, was ich tatsächlich denken werde, wird - am Beispiel einer Rechenaufgabe - bei Kripke erklärt mit dem Unterschied zwischen dem, was laut den mathematischen Gepflogenheiten oder der mathematischen Praxis die Antwort auf diese Rechenaufgabe ist, und dem, was ich selbst „in Isolation“ geneigt bin zu antworten. Vgl. ebd., S. 84. Dies, so mag es auf den ersten Blick erscheinen, erlaubt es, das Problem, wie es einen Unterschied zwischen mathematischer Richtigkeit und Prognose von mathematischem Verhalten geben kann, zu lösen. Der Unterschied liegt demnach im Unterschied zwischen individueller Disposition und Gruppendisposition. 
Mill werde ich im Folgenden deutlich machen, was ich mit dieser Erweiterung in Bezug auf Mills Vorstellung vom Handeln meine, und danach einige Überlegungen Mills zu moralischen Gefühlen diskutieren, was als Spezialfrage innerhalb des Psychologismus in Bezug auf Handlungen gesehen werden kann.

\subsubsection{Psychologismus in Mills Vorstellungen vom Handeln}

Innerhalb des Utilitarismus selbst, jedenfalls wie Mill ihn versteht, ist es zunächst kein Problem, einen Unterschied zwischen „richtig“ und „falsch", der sich von der entsprechenden Einschätzung unterscheidet, zu ziehen. Wir können hier sehr leicht deutlich machen, was es heißt, nicht so zu handeln, wie es der Utilitarismus vorschreibt. Es gibt hier mindestens zwei Möglichkeiten: Wir brauchen uns nur entweder eine Person denken, die wissentlich eine Handlung ausführt, die dem Utilitarismus widerspricht, oder eine Person, die zu einer falschen Einschätzung darüber gelangt, was der Utilitarismus vorschreibt. (Ersteres könnte als eine Art praktischer Fehler, letzteres als eine Art theoretischer Fehler aufgefasst werden.) Begrifflich ist die Möglichkeit, einen Fehler zu machen oder etwas falsch zu machen, hier offenbar gegeben.

Ein Problem liegt allerdings in Mills Handlungstheorie und es ist, so könnte der folgende Gedanke rhetorisch zugespitzt werden, eher die Frage, wie wir etwas richtig machen können - oder genauer gesagt, wie wir es uns vorstellen sollen, dass die utilitaristische Überlegung unser Handeln anleitet. Dies kann als allgemeines Pro-

Einen mathematischen Fehler zu machen, bedeutet, krude formuliert, dass die individuelle Disposition von der Gruppendisposition abweicht.

Dies ist natürlich nicht die Differenz, auf die es ankommt - und zwar nicht nur, weil wir uns, wenn wir es nach Rückübertragung ins Praktische oder gar als allgemeine Theorie von richtig und falsch mit einem radikalen Konventionalismus bezüglich aller Fragen von richtig und falsch, wahr und unwahr, klug und unklug abfinden müssten. Es ist in den meisten Bereichen auch nicht, was wir mit „falsch" oder einen „Fehler machen" meinen. Es ist eben auch begrifflich möglich, dass sich die meisten Menschen - oder sogar alle verrechnen. Ein entsprechender Satz ist formulierbar. Er ist nicht unsinnig. Als systematische Kritik an Kripke und als Kritik an dessen Wittgensteinexegese werden diese Punkte zum Beispiel von McDowell angeführt. John McDowell. „Wittgenstein on Following a Rule“. In: Mind, Value and Reality. Cambridge, Mass. und London: Harvard University Press, 1998, S. 221-262. 


\section{Zwei Grenzen von Mills Philosophie}

blem des Utilitarismus angesehen werden ${ }^{129}$, ich will es aber speziell als Problem von Mill behandeln. In jedem Fall ist das Problem ein Problem der Handlungstheorie oder, wenn wir so wollen, ein Problem der Kompatibilität handlungstheoretischer Überlegungen mit ethischen Überlegungen.

Den Psychologismusvorwurf von der Logik auf die Handlungstheorie zu übertragen ist keineswegs originell. Eine der umfassendsten Zurückweisungen einer psychologistischen Handlungstheorie hat Michael Thompson im zweiten Teil von Life and Action geliefert. Thompsons „naive Handlungstheorie" legt nahe, dass wir psychisches Vokabular nicht brauchen, um zu verstehen, was eine Handlung ist, und dass die kommunikative Rolle dieses Vokabulars (die Thompson natürlich nicht abstreitet) ausschließlich darin besteht, die Handelnde im Handlungsprozess, der nicht wesentlich ein psychischer Prozess ist, zu verorten. ${ }^{130}$ Und natürlich ist es, was die Kritik am Psychologismus angeht, kein Zufall, dass Thompson das Gesamtprojekt des Buches, in das die naive Handlungstheorie eingebettet ist, als Erweiterung von Freges Begriffsschrift sieht. ${ }^{131}$

Ich werde das den Psychologismus betreffende Problem zwecks Anwendung auf Mills Philosophie hier etwas anders formulieren als Thompson das tut, auch wenn der Grundgedanke, so meine ich, der gleiche bleibt.

Motivation für das, was ich für den Psychologismus in der Handlungstheorie halte, ist ein auf den ersten Blick unkontroverses Desideratum, nämlich, dass das Stellen der praktischen Frage „Was soll ich tun?"132 Konsequenzen für unser Handeln hat. ${ }^{133}$ Genauer

129 Der Utilitarismus habe einen blinden Fleck, wenn es um die „aktive“ Seite von Personen geht, argumentiert etwa Korsgaard. Siehe Christine Korsgaard. „Personal Identity and the Unity of Agency: A Kantian Response to Parfit". In: Philosophy \& Public Affairs 18.2 (1989), S. 101f.

130 Vgl. Michael Thompson. Life and Action: Elementary Structures of Practice and Practical Thought. English. Cambridge, Mass.: Harvard University Press, 2008, Teil II.

131 Vgl. ebd., Einleitung.

132 Hier und im Rest des Abschnittes ist diese Frage nicht mit moralischer Konnotation gemeint. Die Formulierung ist nicht wichtig: „Was wäre an meiner Stelle gut zu tun?“, „Was wäre klug zu tun?“, „Welche Handlung ist die Handlung, die, wenn alle Maßstäbe berücksichtigt sind, von mir zu wählen ist?" könnten alle ebenfalls eingesetzt werden.

133 Dieser „Internalismus" - das heißt die Auffassung, dass das, was durch die Nennung eines normativen Grundes herausgepickt wird, auch als Erklärung 
gesagt handelt es sich eigentlich um ein Desideratum einer philosophischen Rekonstruktion der Bedeutung dieser Frage: Die Rekonstruktion der Bedeutung dieser Frage muss es zulassen, dass die Antwort auf die Frage Konsequenzen für unser Handeln hat. Und natürlich dürfen es nicht nur irgendwelche Konsequenzen sein, sondern es sollte die Möglichkeit berücksichtigt werden, dass wir, weil wir die Frage so-und-so beantworten, so-und-so handeln. Dieses Desideratum wird zum Beispiel ausgedrückt in Donald Davidsons Idee der Rationalisierung. ${ }^{134}$

Psychologismus im obigen Sinne fängt nun, so will ich vorschlagen, dann an, wenn wir dieses Desideratum zum Anlass nehmen, zwei weitere Schritte zu unternehmen: Der erste Schritt besteht darin, die Frage „Was soll ich tun?" für ersetzbar durch eine andere Frage zu halten, nämlich die Frage nach der Prognose „Was werde ich tun?". Dies liegt nahe, weil wir ja wollten, dass unser tatsächliches Handeln von der Antwort auf die praktische Frage abhängt. Der zweite Schritt besteht darin, darauf zu bestehen, dass die zweite dieser Fragen, die Frage, was wir tun werden, irgendwie unter Rückgriff auf unser psychisches Mobiliar beantwortet werden muss. Dafür, was genau die Einstellungen sind, die für die Beantwortung beider Fragen taugen, sind äußerst technische Begrifflichkeiten erfunden worden: Davidson selbst spricht von Proeinstellungen (pro attitude), die einfach so definiert sind, dass sie alles um-

einer Handlung taugen muss - ist sicher nicht gänzlich zu bestreiten. Dies aber als Anlass zu nehmen, Gründe in eine innere Welt zu verlagern, ist die Vorstellung, die zurückgewiesen werden muss. Hinter diesem Bild steckt vielleicht die Unterstellung, dass die Kraft der Gründe gerade noch ausreiche, um so etwas Flüchtiges wie unsere psychischen Zustände zu verändern - oder umgekehrt, dass unsere Neurophysiologie, auf der unsere psychischen Zustände supervenieren würden, so komplex und feinkörnig sei, dass sie so etwas Flüchtiges wie Gründe registrieren und zu Körperbewegungen verstärken könne -, dass aber Veränderungen unserer Körper der Vermittlung unserer Psyche bedürften, um durch Gründe in Bewegung versetzt zu werden. Dieses Bild ist natürlich albern. Wenn wir daran festhalten wollen, dass Gründe Handlungen erklären können - und ich denke, das sollten wir -, dann müssen wir auch daran festhalten, dass sie das, was sie begründen, unsere Handlung, auch unvermittelt erklären können. Für eine genauere Ausführung eines entsprechenden Argumentes siehe Thompson, Life and Action, S. $117 \mathrm{f}$.

134 Siehe Donald Davidson. "Actions, Reasons, and Causes“. In: The Journal of Philosophy 60.23 (1963), S. 685-700, 685f. 


\section{Zwei Grenzen von Mills Philosophie}

fassen, was potentiell diese Doppelrolle erfüllen kann; ${ }^{135}$ Bernard Williams spricht in seiner Kritik externer Gründe von Elementen des subjective motivational set, die noch offensichtlicher das und nur das enthalten, was als Antwort auf die prognostische Frage in Frage kommt, und nutzt dies um abzugrenzen, was als Antwort auf die praktische Frage in Frage kommt; ${ }^{136}$ und Michael Smith definiert Wünsche einfach als Klasse psychischer Zustände mit der passenden Passrichtung und verschreibt sich dem, wie er es nennt, Hume'schen Standardbild menschlicher Psychologie, dass es diese und nur diese sind, die Handlungen verursachen können. ${ }^{137}$

Auch wenn die Klasse der Zustände bei Mill andere sind, ist der Gedanke der gleiche. Von Bentham übernimmt er, dass die Erwartung von Schmerz und Lust motivational wirksam sind; er erweitert Bentham aber dahingehend, dass auch unsere Dispositionen, die wir, je nach dem, ob sie mit dem Utilitarismus übereinstimmen oder nicht, als "Tugenden" oder "Laster" bezeichnen können, motivational wirksam sind. ${ }^{138}$

Das Dilemma, das entsteht, ist auch das gleiche wie im theoretischen Falle. Entweder sagen wir, die praktische Frage und die prognostische Frage hätten nichts miteinander zu tun. Dann geben wir aber das Desideratum auf, dass die praktische Frage Konsequenzen für unser Handeln hat, und stellen sie als irrelevant dar. Oder wir benutzen die prognostische Frage, um einzugrenzen, was als Antwort auf die praktische Frage in Frage kommt. Dann laufen wir aber Gefahr, begrifflich nicht mehr fassen zu können, wie es sein kann, dass wir faktisch nicht tun, was wir tun sollen. Wie auch im logischen Falle wäre die Möglichkeit, einen Fehler zu machen oder etwas falsch zu machen, nicht mehr gegeben. In dieser Hinsicht sind der Psychologismus in Bezug auf die Logik und der Psychologismus in Bezug auf die Handlungstheorie strukturanalog. ${ }^{139}$

135 Davidson, „Actions, Reasons, and Causes“, S. 685.

136 Bernard Williams. „Internal and external reasons". In: Moral Luck. Cambridge und andere: Cambridge University Press, 1982, S. 101-113, S. 102.

137 Siehe Michael Smith. The Moral Problem. Oxford und andere: Blackwell, 1994, S. 7.

138 Vgl. Mill, The Collected Works of John Stuart Mill, Volume VIII - A System of Logic Ratiocinative and Inductive (Books IV-VI and Appendices), S. 842.

139 Selbst wenn wir den Psychologismus zurückweisen, heißt das natürlich nicht, dass damit alle Rollen, die psychische Zustände in der Erklärung und Konstitution von Handlungen haben, aufgehoben wären. Wünsche und Überzeugun- 
Auch diese Frage führt bei Mill übrigens zu einer Lebenskrise, die er in der Autobiography als Problem von Freiheit und Determinismus verarbeitet und die, wie dort zu lesen ist, ${ }^{140} \mathrm{zu}$ den Überlegungen im zweiten Kapitel des letzten Buches von A System of Logic ${ }^{141}$ geführt hat. Wenn die und nur die Zustände, die für die prognostische Frage zu berücksichtigen sind, auch für die praktische Frage von Bedeutung sind, welche Rolle spielt dann überhaupt praktische Überlegung? Warum die Mühe? Entsprechend dieser Formulierung des Problems ist für Mill „Fatalismus" die Gefahr, die er mit seinem kompatibilistischen Versuch dort zu bannen versucht. Darunter versteht er die Position, dass es nichts bringt, sich um irgendetwas zu bemühen. ${ }^{142}$ Auch wenn Mill das Folgende hauptsächlich auf die soziale Nützlichkeit bezieht, könnte sogar gesagt werden, Mill sei sich bewusst, dass es sich hier, wie oben angedeutet, hauptsächlich um ein Problem der Inkompatibilität zweier Perspektiven, der erstpersonalen und der drittpersonalen, geht: „I said that it would be a blessing if the doctrine of necessity could be believed by all quoad the characters of others, and disbelieved in regard to their own." ${ }^{143}$

Mill selbst löst die persönliche Krise, die Gedanken wie diese bei ihm auslösen, indem er eine kompatibilistische Position formuliert. ${ }^{144} \mathrm{Ob}$ diese tatsächlich zeigt, dass das Gefühl von Fatalismus angesichts des Determinismus nicht angemessen ist, was Mills eigentliches Ziel in dem entsprechenden Abschnitt des System of Logic ist, will ich hier nicht beurteilen. Wichtiger ist, dass diese Antwort den Zusammenhang zwischen der praktischen Frage und der prognostischen Frage - „Was soll ich tun?" und „Was werde ich

gen gehören - wenn sie nicht einfach im Sinne Thompsons Wörter sind, mit denen wir unsere Position innerhalb des Ablaufes einer Handlung markieren - häufig beispielsweise zu einer entsprechenden Praxis oder Handlungsform dazu: In Abschnitt 2.2 hatte ich unter Verweis auf Luhmanns Begriff der generalisierten Annahmebereitschaft zwecks eines Beispiels angedeutet, dass argumentiert werden könne, sich Geld zu wünschen, gehöre zur Praxis der Geldverwendung dazu. In diesem Falle aber, haben Wünsche natürlich keine exklulsive handlungstheoretische Rolle.

140 Siehe Mill, Autobiography, S. 175ff.

141 Mill, The Collected Works of John Stuart Mill, Volume VIII - A System of Logic Ratiocinative and Inductive (Books IV-VI and Appendices), Buch VI, Kapitel 2.

142 Ebd., Buch VI, S. 839-842.

143 Mill, Autobiography, S. 177.

144 Ebd., Buch VI, S. 175ff. 


\section{Zwei Grenzen von Mills Philosophie}

tun?" - letztlich auf reine Konditionierungsprozesse abwälzt und Mill dies an anderer Stelle nicht genug war, um seine Krise zu bewältigen. ${ }^{145}$

\subsection{3. „The Utility of Religion“}

Mill beginnt den Aufsatz The Utility of Religion mit der Überlegung, dass über das Thema bisher nur wenig geschrieben wurde, weil bisher die Frage der Wahrheit der Religion im Vordergrund gestanden habe und mit der Frage der Wahrheit, zumindest im Falle einer positiven Antwort, die Frage der Nützlichkeit implizit mitbeantwortet sei. ${ }^{146}$ Er weist dann im ersten Teil des Aufsatzes $^{147}$ Argumente zurück, die die soziale Nützlichkeit der Religion etablieren sollen: Er weist zum Beispiel darauf hin, dass zwar die irdischen Sanktionen einer religiösen Gesellschaft - wie alle Sanktionen der öffentlichen Meinung - sehr effektiv seien, dass aber die spezifisch religiösen Sanktionen, zum Beispiel die Furcht vor der Hölle, nicht nur für die Kultivierung altruistischer Gefühle schädlich seien, weil sie Moral auf egoistischen Gehorsam zurückführten, sondern auch faktisch ineffektive Sanktionen seien. Übernatürliches oder spezifisch Religiöses braucht es für diese Form der Nützlichkeit also nicht. ${ }^{148}$ Auch als Quelle der Moral (in den Fällen, in denen die religiöse Moral akzeptabel ist) sei die Religion unnötig. ${ }^{149}$

Wohlwollender blickt Mill auf den Nutzen der Religion für die individuelle Kultivierung. Diesen vergleicht er er von Anfang der Diskussion dieses Gedankens im zweiten Teil an mit der Poesie:

Religion and poetry address themselves, at least in one of their aspects, to the same part of the human constitution: they both supply the same want, that of ideal conceptions grander and more beautiful than we see realized in the prose of human life. Religion, as distinguished from poetry, is the product of the craving to know whether these imaginative conceptions have realities answering to them in some other world than ours. [...] The value, therefore, of religion to

145 Siehe Mill, Autobiography, S. $141 \mathrm{f}$.

146 Siehe Mill, „Three Essays on Religion“, S. 403f.

147 Bis S. 417; die Einteilung in zwei Teile stammt von Mill selbst, siehe ebd., S. 406.

148 Siehe ebd., S. 407-415.

149 Siehe ebd., S. 415-417. 
the individual, both in the past and present, as a source of personal satisfaction and of elevated feelings, is not to be disputed. ${ }^{150}$

Mill weist direkt im Anschluss an diese Textstelle darauf hin, dass der Glaube an Übernatürliches nicht dazu notwendig sei, „höheren und schöneren" Idealen Ausdruck zu verleihen. Insofern könnte gesagt werden, dass es auch, was den zur Diskussion stehenden Nutzen der Religion für die Kultivierung des Individuums angeht, eigentlich nichts Religiöses ist, was diesen Nutzen ermöglicht. Mill entscheidet sich aber für eine andere Rhetorik und verwendet den Begriff „Religion" stattdessen auch für die ihm vorschwebende Form der Poesie, die ohne Übernatürliches auskommt:

To call these sentiments by the name morality, exclusively of any other title, is claiming too little for them. They are a real religion; of which, as of other religions, outward good works (the utmost meaning usually suggested by the word morality) are only a part, and are indeed rather the fruits of the religion than the religion itself. The essence of religion is the strong and earnest direction of the emotions and desires towards an ideal object, recognized as of the highest excellence, and as rightfully paramount over all selfish objects of desire. This condition is fulfilled by the Religion of Humanity [...]. ${ }^{151}$

Bevor ich Mills Gedanken diskutiere, möchte ich Mills Charakterisierung der religiösen Gefühle kurz beschreiben, die durch die auf säkularer Poesie beruhenden Religion der Menschheit gefördert werden sollen. Er stellt sich (unter anderem) eine Art universalisierten Patriotismus vor: Die Vorstellung ist, dass, da sich die Liebe für das eigene Land durch Erziehung und Poesie herstellen lasse, es auch möglich sein müsse, die Liebe zur Menschheit insgesamt auf diese Weise herzustellen. ${ }^{152}$ (In diesem Sinne ist die Verwendung des Wortes „Religion“ für Mills säkulare Poesie vielleicht darin begründet, dass sie im wahrsten Sinne des Wortes katholikós, das Ganze betreffend, sein soll.) Dies führte zu einer „morality grounded on

150 Siehe ebd., S. $419 f$.

151 Ebd., S. 422. Das Wort „Religion of Humanity" übernimmt er von Auguste Comte. Vgl. Michel Bourdeau. „Auguste Comte“. In: The Stanford Encyclopedia of Philosophy. Hrsg. von Edward N. Zalta. Winter 2015. 2015.

152 Mill, „Three Essays on Religion“, S. 421. 


\section{Zwei Grenzen von Mills Philosophie}

large and wise views of the good of the whole, neither sacrificing the individual to the aggregate nor the aggregate to the individual, but giving to duty on the one hand and to freedom and spontaneity on the other their proper province" ${ }^{\text {153 }}$.

Der Nutzen der religiösen Praxis oder, genauer gesagt, der Nutzen einer bestimmten Form von poetischer Praxis, die von Mill durch die Benennung "Religion of Humanity" rhetorisch hervorgehoben wird, besteht in der moralischen Verbesserung der Individuen. Damit, dass diese Verbesserung sich, wie im Zitat beschrieben, nicht auf die äußeren Werke beschränke, ist ganz im Sinne des oben angesprochenen universalisierten Patriotismus hier nur gemeint, dass das utilitaristische Gut um seiner selbst willen angestrebt wird und nicht zum Beispiel aufgrund von Sanktionen. Wenn wir Mill in Bezug auf die Wirkungen dieser hypothetischen Form von Poesie einmal glauben, und die Frage nach dem Maßstab hier ignorieren (siehe Abschnitt 4.3.2) können wir ihm vielleicht zustimmen: Eine von übernatürlichen Elementen befreite religiöse Praxis in diesem Sinne ist eine gute Sache.

Unter diesen Voraussetzungen könnte gesagt werden, Mill sei Benthams Tugend gerecht geworden. Die Strategie folgt dem oben in Bezug auf Überzeugungen nahegelegten Sinne: Bestimmte Einstellungen, „sentiments", oder ",feelings" sind nützlich; diese werden durch eine Form von Poesie gefördert, die Teil der religiösen Praxis ist, die aber auch ohne die damit sonst verbundenen übernatürlichen Elemente denkbar ist. Wir sollten uns um eine religiöse Praxis ohne übernatürliche Elemente bemühen - die Religion of Humanity. Auf das, was oben (Kapitel 4) „Benthams Herausforderung“ genannt wurde, wurde eingegangen: es ist besser, dass wir eine solche Praxis haben als eine Praxis, die diese Einstellungen nicht in uns hervorruft; und es ist besser, dass wir eine solche Praxis haben als eine Praxis, die zusätzlich problematische Überzeugungen über das Übernatürliche in uns hervorruft.

Das größere Problem damit, die religiöse oder poetische Praxis auf die so erzeugten Einstellungen (oder die so befriedigten Bedürfnisse) zu reduzieren, liegt nicht in Benthams, sondern in Coleridges Tugend. Ich vermute, dass weder die meisten religiösen Menschen den Witz der religiösen Praxis darin sehen, dass damit ihre Wün-

153 Mill, „Three Essays on Religion“, S. 421. 
sche und Emotionen auf ein höheres Ideal, das Wohl der Menschheit insgesamt etwa, gerichtet werden, noch, dass Menschen, die die Poesie, die Mill vorschwebt, schätzen, dies tun, damit dies der Fall ist. Dies ist nicht, worum es in den entsprechenden Praktiken geht und es wäre auch eine eigenartig egozentrische Art der Religionsausübung beziehungsweise Gedichtrezeption: Statt des Gedichtes oder seines Gegenstandes wären die eigenen Wünsche und Emotionen Zentrum der Aufmerksamkeit und analog wäre es bei der Religion.

Nun sagt Mill zugegebenermaßen auch nicht, dass er, indem er die Nützlichkeit der Religion für die Kultivierung des Individuums erarbeitet, einen sprachlichen Ausdruck erarbeitet, den eine kompetente Teilnehmer*in an der Praxis der Religion der Menschheit (oder einer anderen Religion) für einen adäquaten Ausdruck dessen halten könnte, worum es in dieser Praxis geht. Es geht Mill hier, so könnte argumentiert werden, eben nur um Benthams Tugend, nicht um Coleridges Tugend: Eine Praxis, die in manchen Hinsichten so ähnlich ist wie die religiöse Praxis, ist eine in bestimmter Hinsicht gute Sache. Er will aber aber nicht sagen, so könnte weiter argumentiert werden, dass Menschen, die sich an Poesie erfreuen, dies tun, damit ihre Emotionen und Wünsche auf die Menschheit als Ganzes gerichtet werden, oder dass religiöse Menschen religiös sind, damit ihre Einstellungen dadurch kultiviert werden.

Mill wäre dann dem ihm von mir zugeschriebenen Ideal von Philosophie in The Utility of Religion nicht gerecht geworden; der Aufsatz wäre nach diesem Ideal unvollständige Philosophie, aber sein Anspruch wäre eben auch nicht höher. ${ }^{154}$ Es mag sein, dass dies tatsächlich Mills einziges Ziel in dem Aufsatz ist. Unabhängig davon

154 Es könnte auch argumentiert werden, dass Mill zum Zeitpunkt des Aufsatzes - es handelt sich um einen sehr späten Aufsatz Mills, der posthum von Helen Taylor (siehe ihr Vorwort in Helen Taylor. „Introductory Notice on ,Three Essays on Religion“". In: The Collected Works of John Stuart Mill, Volume X - Essays on Ethics, Religion, and Society. Hrsg. von J. M. Robson. Toronto und andere: University of Toronto Press und Routledge \& Kegan Paul, 1969, S. 371-372) veröffentlicht wurde - meint, dass das Ideal, das in "Coleridge" und „Bentham“ als das Ideal einer Disziplin, nämlich der Philosophie, dargestellt wurde, nun auf zwei Disziplinen aufgeteilt wird: Die Philosophie und die Poesie. Dazu würde passen, dass Coleridge in der Autobiographie nur noch für seine Gedichte, nicht mehr so sehr aber für seine Philosophie gelobt wird. So schreibt Mill über Frederic Maurice: „The nearest parallel to him, in a moral point of view, is Coleridge, to whom, in merely intellectual power, apart from poetical genius, I think him decidedly superior." Mill, Autobiography, S. 161. 


\section{Zwei Grenzen von Mills Philosophie}

aber, ob Mill hier absichtlich nur einer der beiden philosophischen Tugenden gerecht wird, ermöglicht mir dies aber systematisch zu zeigen, was daran unvollständig ist.

Die Unvollständigkeit besteht, so will ich behaupten, natürlich wie im theoretischen Psychologismus darin, dass Mill die entscheidende normative Frage nicht berücksichtigt: Es gibt keine Möglichkeit mehr, zwischen besseren und schlechteren Arten und Weisen, auf die Welt Bezug zu nehmen, zu unterscheiden. (Genauer gesagt: der externe Maßstab der Nützlichkeit bestimmt, welche Zustände besser und welche schlechter sind - und dies ist zunächst unabhängig davon, welche wahr, angemessen, richtig u.s.w. sind.) Wir unterhalten psychische Zustände nicht, weil sie nützlich für unsere eigene Kultivierung oder gar sozial nützlich sind. ${ }^{155}$

Natürlich ist dies in gewisser Hinsicht Absicht: Mill sagt explizit, dass sich die Frage der Wahrheit der Religion nicht mehr in der gleichen Weise stellt, und wahrscheinlich stimmt es, dass "Wahrheit", je nach dem, was wir darunter verstehen, nicht das Wort ist, mit dem wir das, was wir an Gedichten gut finden, beschreiben würden. ${ }^{156}$ Vor diesem Hintergrund könnte Mills impliziter Ablehnung zugestimmt werden, die er dem im obigen Zitat ${ }^{157}$ angedeuteten Streben entgegenbringt, dass die poetischen Ideale einer höheren Realität entsprechen mögen, dem die Religion seiner Meinung nach nachgebe.

Selbst aber, wenn „Übereinstimmung mit den Tatsachen“ nicht der Maßstab ist, mit dem wir Gedichte und das, was sie uns bedeuten, bewerten wollen, bedeutet das nicht, dass wir stattdessen ausschließlich ihre psychischen Wirkungen bewerten können: Nicht nur könnten wir dann nicht zwischen Propaganda und Poesie un-

Das, was ich als philosophische Tugenden Mills präsentiert habe, wären in Wirklichkeit nur philosophische Tugenden des frühen Mill. Es passt aber, wie ich in Kapitel 5 argumentiert habe, nicht zum Witz der Autobiography als ganzer.

155 Soziale Erwünschtheit mag natürlich die Ursache sein; sie ist aber in der Regel nicht der Grund.

156 Natürlich könnten andere Begriffe von Wahrheit gefunden werden, die zum Beispiel nicht „,[d]as Wesen der Wahrheit [...] in der ,Übereinstimmung“ des Urteils mit seinem Gegenstand" sehen, (z. B. Martin Heidegger. Sein und Zeit. 18. Aufl. Tübingen: Max Niemeyer, 2001, § 43 und § 44) nach denen wir durchaus sagen könnten, ein Gedicht enthülle eine Wahrheit.

157 Es geht um das Zitat, zu dem Fußnote 150 gehört. 
terscheiden ${ }^{158}$; alle Gedichte mit den erwünschten psychischen Wirkungen wären gleichwertig. Es wäre außerdem so, dass das, was ein Gedicht oder ein Teil einer religiösen Praxis einer Person bedeutet, zu einem reinen Epiphänomen wird: Es gibt kein gut und schlecht bei Gedichten, lediglich gute und schlechte Wirkungen. In dieser Hinsicht hat der Psychologismus in praktischer Hinsicht das gleiche Problem wie der Psychologismus in theoretischer Hinsicht.

Um zu zeigen, was die Poesie der Religion of Humanity einer daran Teilhabenden bedeutet, müsste Mill sich mit entsprechenden poetischen Werken natürlich beschäftigen, so wie er es in der Autobiographie zumindest in Ansätzen unternimmt, (vgl. Kapitel 5) oder wie es Cora Diamond in dem bereits zitierten Aufsatz zur Tierethik tut. So aber bleibt die Religion of Humanity ein leerer sozialer Mechanismus, von dem wir uns vielleicht wünschen würden, dass andere in ihn involviert wären, in den uns selbst zu involvieren uns aber eigentlich jeder Grund fehlt, wenn wir die Einstellungen, die so hervorgebracht werden sollen, nicht ohnehin schon teilen.

Zusammenfassung. Nachdem ich in Kapitel 5 gezeigt habe, inwiefern Mills Vorstellung vom Guten sich als Verwirklichung der beiden sozialphilosophischen Tugenden verstehen lässt, ging es mir in diesem Kapitel darum, zwei Schwächen von Mill bezüglich der beiden sozialphilosophischen Tugenden aufzuzeigen: Die erste dieser Schwächen ist die Rolle und das Verständnis von Unparteilichkeit in seinem Utilitarismus und die zweite Schwäche ist seine Tendenz zum Psychologismus in manchen (wenn auch ausdrücklich nicht in allen) normativen Fragen. Dabei ging es mir in beiden Fällen darum, zu zeigen, dass Mill eine Tätigkeit, die durch die philosophischen Tugenden angeleitet wird, das Philosophieren nämlich, letztlich gemessen an diesen unzureichend ausführt. Dafür musste natürlich einerseits gezeigt werden, dass seine Tätigkeit in diesen Aspekten wirklich unter den Tugenden steht, und andererseits, dass sie ihnen nicht gerecht wird. Die Strategie dieses Kapitels war in beiden Fällen die gleiche: Ich habe mich bemüht einerseits zu zeigen,

158 Cora Diamond scheint auf eine Unterscheidung dieser Art hinauszuwollen, indem sie das Gedicht "Titmouse" von Walter de la Mare mit „Learning to be a Dutiful Carnivore" von Jane Legge, das sie als "piece of vegetarian propaganda“ bezeichnet, kontrastiert. Siehe Cora Diamond. „Eating Meat and Eating People". In: Philosophy 53.206 (1978), S. 465-479, S. 472 f. 


\section{Zwei Grenzen von Mills Philosophie}

warum es auf den ersten Blick naheliegend erscheint, den Tugenden auf die Weise gerecht zu werden, wie Mill das tut, und warum sein Anliegen diesbezüglich wichtig ist; und andererseits, warum jeweils eine der Tugenden so dennoch nicht berücksichtigt wird.

Im Falle des Unparteilichkeitsprinzips habe ich mich auf Benthams Tugend konzentriert und in Frage gestellt, dass Mill wirklich zeigen kann, dass es besser ist, der utilitaristischen Praxis als der intuitionistischen moralischen Praxis anzugehören. Gleichzeitig habe ich versucht zu zeigen, dass Mills Ziel, Kritik an unserer moralischen Praxis (auch) an externen Maßstäben zu orientieren, ein philosophisch wichtiges Ziel ist. (Ein großer Teil des Abschnittes war dem Versuch gewidmet, zu klären, was „extern“ hier bedeuten kann.)

Im Falle von Mills psychologistischer Tendenz wurde die Strategie etwas direkter verfolgt: Erstens habe ich versucht zu argumentieren, warum die Deutung normativer Begriffe als psychische Phänomene beiden sozialphilosophischen Tugenden gerecht zu werden scheint. Zweitens habe ich versucht, die Grenze dieser Strategie allgemein und beispielhaft aufzuzeigen. 


\section{Zusammenfassung von Teil 2}

In Teil I wurde eine Sicht auf Philosophie als zwei sozialphilosophische Tugenden formuliert, die Mill zumindest während er die beiden Aufsätze „Bentham“ und „Coleridge" verfasst hat, geteilt haben muss. In Teil II ging es dann darum, zu zeigen, dass die Tugenden auch später noch konstitutive Ideale, so wie ich diesen Begriff in Kapitel 2 verwende, für Mill sind: Sie sind Ideale für die Art von Philosoph, die Mill ist, auch, wenn er diesen nicht in jedem Aspekt gerecht wird. Dies ist leichter zu zeigen an den Stellen, an denen Mill den Idealen gerecht wird. Dies habe ich in Kapitel 5 für Mills Theorie des guten Lebens versucht zu tun. An den Stellen, an denen Mill den Idealen nicht gerecht wird, ist es schwerer zu zeigen, dass es sich dennoch um konstitutive Ideale im Sinne von Kapitel 2 handelt: Ich habe mich in Kapitel 6 bemüht zu zeigen, dass die Rolle, die das Unparteilichkeitsprinzip für ihn spielt, und die Funktion, die die Reduktion bestimmter normativer Fragestellungen auf psychische Phänomene bei ihm hat, jeweils auf wichtige philosophische Desiderata abzielt, die durch die Tugenden ausgedrückt werden, ohne dass die Tugenden jedoch vollständig verwirklicht würden. Damit bin ich der ersten und zweiten Aufgabe aus der Einleitung zum zweiten Teil nachgekommen.

Dass die philosophischen Ideale, um die es geht, Personideale, das, was ich Tugenden nenne, sind, ist ebenfalls deutlich geworden: Durch die Rolle, die experiments in living für Mills Art und Weise, mit den Tugenden umzugehen, haben, wird dies einerseits klar; andererseits hoffe ich, dass in Kapitel 6 deutlich geworden ist, dass wir zum Beispiel Mills scheiternden „Beweis“ für den Utilitarismus nicht einfach als das Übersehen des intuitionistischen Status einer seiner Annahmen sehen sollten, wie Crisp es meint, sondern als Versuch eines beinahe lebenslangen philosophischen Strebens danach, Philosophie nicht in ,interminable discussion, leading to no result"159 enden zu lassen. Dies ist die dritte Aufgabe aus der Einleitung zu diesem Teil gewesen.

Was die vierte Aufgabe betrifft, kann ich gemäß der methodischen Überlegungen aus Kapitel 2 lediglich hoffen, dass ich der philosophierenden Leser*in deutlich machen konnte, dass das mit den

159 Mill, „Bentham“, S. 83. 


\section{Zwei Grenzen von Mills Philosophie}

Tugenden verbundene Streben Mills tatsächlich ein wichtiges philosophisches Streben ist und nicht bloß eine eigenartige Disposition seines spezifischen Charakters.

Ich habe noch keinen Versuch unternommen, zu zeigen, dass Lebensexperimente die einzige oder die beste Art und Weise sind den beiden Tugenden gerecht zu werden, auch wenn ich glaube, dass der relative Erfolg den ich Mill in Kapitel 5 bescheinige und der Misserfolg, den ich ihm in Kapitel 6 bescheinige aud die Stärke von Lebensexperimente auf die Lösung des Problems, wie sich die Tugenden vereinigen lassen, hindeutet. Einige der Gedanken im nächsten Kapitel werden das weiter untermauern, auch wenn ich auch im nächsten Kapitel kein formales Argument diesbezüglich versuche. In Kapitel 8 werde ich die Frage aufgreifen, aber auch dort letztlich offen lassen. 


\section{Teil III.}

\section{Allgemeine Überlegungen, Abgrenzung, Ausblick}




\section{Einleitung zu Teil 3}

Im letzten Teil der Arbeit möchte ich drei noch ausstehende Aufgaben bearbeiten. Die erste dieser Aufgaben ist Mills Auseinandersetzung mit Platon, mit der ich mich in Kapitel 7 beschäftigen werde. Diese Auseinandersetzung ist deshalb von Bedeutung, weil argumentiert werden könnte, diese habe einen ähnlichen Status wie seine Auseinandersetzung mit Bentham und Coleridge in den Aufsätzen, mit denen ich mich in Teil I beschäftigt habe. Auch im Falle von Platon oder Platons Sokrates hebt Mill dessen Güte als Philosoph hervor und formuliert so implizit, wie ein guter Philosoph sein sollte. In einer Arbeit über Mills Philosophie der Philosophie darf seine Auseinandersetzung mit Platon daher nicht fehlen.

Dass die Auseinandersetzung mit Platon nicht bereits im ersten Teil stattfindet, ist trotzdem gerechtfertigt: Schon auf der Oberfläche wird deutlich, dass die Auseinandersetzung nicht zu den beiden als Komplemente gedachten Auseinandersetzungen mit Coleridge und Bentham gehört. Mit Platon setzt sich Mill in Form von Kommentarübersetzungen einiger Dialoge auseinander, von denen manche gar nicht veröffentlicht wurden und vielleicht auch nicht für die Veröffentlichung bestimmt waren. Und auch inhaltlich scheint er an Platons Sokrates viele der Dinge zu schätzen, die er auch an Bentham schätzt. Rhetorisch zugespitzt (und sicherlich nicht ganz akkurat) könnte gesagt werden, Mills unbestreitbar große Bewunderung der platonischen Dialoge ist die Bewunderung dafür, dass Platon, obwohl er so viel früher als Bentham gelebt hat, bereits ein fast so guter Philosoph war wie Bentham. Es wird also durch die Auseinandersetzung mit Platon nicht viel zu Mills Ideal der Philosophie hinzugefügt. (Genaueres in Abschnitt 7.1.)

Die Auseinandersetzung mit Platon erfolgt aber nicht nur der Vollständigkeit halber. Mill missversteht Platon, insofern er ihn mit Bentham parallelisiert, natürlich. Dieses Missverständnis auszuarbeiten ist der zweite Sinn der Diskussion von Mills Auseinandersetzung mit Platon. In der Ausarbeitung des Missverständnisses wird sich zeigen, dass die platonischen Dialoge - zumindest die Frühdialoge, auf die ich mich zwecks dieser Auseinandersetzung weitgehend beschränke - nicht nur Ähnlichkeiten zu Bentham und dem, was ich Benthams Tugend genannt habe, aufweisen, sondern auch zu Coleridges Tugend. 
Die Auseinandersetzung mit den platonischen Frühdialogen bietet sich dafür an, eine Konsequenz des Bildes von Philosophie, das durch die beiden philosophischen Tugenden gezeichnet wird, auszuarbeiten. Diese Konsequenz werde ich anhand der Frage, ob ethisches Wissen techne-Charakter hat, diskutieren, die in den Frühdialogen, insbesondere in Ursula Wolfs Lesart dieser Dialoge, prävalent ist. Ich werde argumentieren, dass die Überlegungen, die dagegen sprechen, dass ethisches Wissen techne-Charakter hat, auch dagegen sprechen, dass die Philosophie techne-Charakter hat, und dies ins Verhältnis zu den Tugenden setzen. Damit ist ein Unterschied der Philosophie zu Disziplinen, die diesen techne-Charakter haben als Konsequenz aus den Tugenden herausgearbeitet. Dieser am Ende systematische Punkt hätte vielleicht in Teil I gehört; er bietet sich aber thematisch im Rahmen der Auseinandersetzung mit Mills Platonlesart an, sodass ich ihn hier präsentieren will.

Die zweite der Aufgaben betrifft die Frage der Verallgemeinerbarkeit der sozialphilosophischen Tugenden Mills in Bezug auf andere Ansätze. Vor dem Hintergrund der Nicht-Exklusivität der Tugenden und meiner Behauptung aus Kapitel 2, dass Philosophie der Philosophie exemplarisch und paradigmatisch argumentieren muss, kann dieser Schritt nur beispielhaft erfolgen. Ich versuche dies im ersten Teil von Kapitel 8. Der zweite Teil des Kapitels ist offenen Fragen und einem Ausblick gewidmet. 


\section{Moral und Technik: Expertise in Moral und Moralphilosophie und Mills Tugenden}

Das folgende Kapitel stellt in gewisser Hinsicht eine Art Exkurs vom bisherigen Verlauf dieser Arbeit dar. Ich möchte, nachdem das letzte Kapitel vor dem Hintergrund seiner eigenen sozialphilosophischen Tugenden zwei explizite Schwächen von Mills Art und Weise, Moralphilosophie zu betreiben, deutlich gemacht hat, eine Geste in eine mögliche Richtung machen, wie es besser geht: Dies ist die Form platonischer Dialoge. Mehr als eine Geste kann es in einer Arbeit über Mills Philosophie der Sozialphilosophie natürlich nicht sein. In meiner Arbeit geht es nicht primär um Moralphilosophie, sondern um das, was ich Mills sozialphilosophische Tugenden genannt habe. Dennoch ist das Kapitel aber nicht nur ein Exkurs. Es beinhaltet auch eine andere Perspektive auf die Begründung dafür, von philosophischen Tugenden und nicht von anders strukturierten Idealen zu sprechen. Ich nehme an, dass, was ich in diesem Kapitel zu sagen versuche, voraussetzungsreicher und kontroverser sein dürfte, als es der Teil I und II waren: Sollte das dazu führen, dass die Argumentation dafür, dass es sich (über Mills eigene Auffassung und die Argumente aus Teil II hinaus) um Tugenden handeln muss, weiterhin nicht für überzeugend gehalten wird, hoffe ich, dass davon das Ergebnis, dass es sich um fruchtbare philosophische Ideale handelt, unberührt bleibt.

Was Coleridges Tugend betrifft, muss es das Ziel sein, möglichst deutlich zum Ausdruck zu bringen, worum es in der Ethik geht; und dazu gehört, was die Ethik von anderen Bereichen menschlicher Aktivität unterscheidet (oder besser: was eine ethische Betrachtungsweise menschlicher Aktivität unterscheidet von anderen praktischen Betrachtungsweisen der gleichen Bereiche). Dann muss, was Benthams Tugend angeht, gezeigt werden, dass und unter welchen Qualifikationen dies dennoch nicht in einen bloßen Dogmatismus führen muss, der darin besteht, diese Spezifik „unserer“ ethischen 


\section{Expertise und die Tugenden}

Praxis für sakrosankt zu erklären. Mein Zugang zur Fragestellung liegt in dem, was häufig als die platonischen „Frühdialoge“1 klassifiziert wird, und insbesondere auch in Ursula Wolfs Lesart dieser Dialoge $^{2}$. Warum ich mich überhaupt mit Platon auseinandersetze, erfordert vielleicht eine Begründung:

Der offensichtlichste Grund ist, dass Mill sich selbst extensiv mit Platon auseinandergesetzt hat: Er hat Kommentar-Übersetzungen ${ }^{3}$ zu neun platonischen Dialogen angefertigt und vier davon auch veröffentlicht, ${ }^{4}$ er erwähnt seine kindheitliche Beschäftigung mit den platonischen Dialogen explizit als Bestandteil seiner philosophischen und philologischen Sozialisation; ${ }^{5}$ und er hat drei Veröffentlichungen zu Platon und Grotes History of Greece rezensiert. ${ }^{6}$ Insbesondere die Rezension zu Grotes Plato ist hier interessant: Sie ist weniger eine Rezension des Werkes von Grote, sondern vielmehr eine Auseinandersetzung mit Platon und insbesondere Platons Philosophieverständnis. ${ }^{7}$ Insofern ist es fast verpflichtend auf die Aus-

1 Was diese Arbeit angeht dient diese Bezeichnung einfach für die in Band 1 befindlichen Dialoge der von Ursula Wolf herausgegeben Platonausgabe in Schleiermacher-Übersetzung. Wolf, Platon. Sämtliche Werke, Band 1. Zur Problematik dieser Festlegung, siehe unten.

2 Wolf, Die Suche nach dem guten Leben. Platons Frühdialoge.

3 John Stuart Mill. „Notes on Some of the More Popular Dialogues of Plato“. In: The Collected Works of John Stuart Mill, Volume XI. Essays on Philosophy and the Classics. Hrsg. von J. M. Robson. Toronto und andere: University of Toronto Press und Routledge \& Kegan Paul, 1978, S. 37-238.

4 Spekulationen zu den Gründen der Auswahl für Übersetzung und Veröffentlichung finden sich hier: Sparshot, „Introduction to Vol. XI“, xviiiff.

5 Siehe z. B. Mill, Autobiography, S. 25, wo die erste Auseinandersetzung mit ungefähr zwölf Jahren stattfindet. Die Wichtigkeit Platons für ihn erwähnt Mill aber immer wieder.

6 John Stuart Mill. "Grote's Plato“. In: The Collected Works of John Stuart Mill, Volume XI. Essays on Philosophy and the Classics. Hrsg. von J. M. Robson. Toronto und andere: University of Toronto Press und Routledge \& Kegan Paul, 1978, S. 375-440, John Stuart Mill. „Two Publications on Plato". In: The Collected Works of John Stuart Mill, Volume XI. Essays on Philosophy and the Classics. Hrsg. von J. M. Robson. Toronto und andere: University of Toronto Press und Routledge \& Kegan Paul, 1978, S. 239-243, John Stuart Mill. „Grote's History of Greece I". In: The Collected Works of John Stuart Mill, Volume XI. Essays on Philosophy and the Classics. Hrsg. von J. M. Robson. Toronto und andere: University of Toronto Press und Routledge \& Kegan Paul, 1978, S. 271-305.

7 Dies war von Mill so beabsichtigt, wie Sparshot anmerkt: „As its scale might suggest, Mill's review was long premediated, and he warned Grote that he 
einandersetzung Mills mit Platon in einer Arbeit über Mills Philosophie der Philosophie einzugehen: Wie auch bei Coleridge und Bentham schätzt Mill bei Platon nicht, was er für dessen substantielle Überzeugungen hält, sondern seinen Beitrag zur Verbesserung der philosophischen Praxis selbst. ${ }^{8}$ Allerdings sieht Mill Sokrates' philosophische Tugenden (beziehungsweise die Tugenden des sokratischen Platon im Gegensatz zum dogmatischen Platon, den er von diesem unterscheidet ${ }^{9}$ ) sehr analog zu denen Benthams - und zwar bis hin in die Formulierungen, die er verwendet. ${ }^{10}$ Benthams Tugend könnte aus Mills Sicht auch Sokrates' Tugend genannt werden. Mills Sicht auf Sokrates ist in dieser Hinsicht aber unplausibel: Sokrates ist nicht einfach ein etwas weniger vollkommener Bentham. Mill missversteht die sokratischen Dialoge ${ }^{11}$ Platons, wenn er meint, es gehe hier nur oder in erster Linie um eine Exemplifizierung von Benthams Tugend. Anstatt also Benthams Tugend noch einmal an Mills Bild der sokratischen Dialoge Platons herauszuarbeiten, möchte ich von dem ausgehend, was ich für ein zentrales Missverständnis in Mills Auffassung der Dialoge halte, zur oben beschriebenen Aufgabenstellung dieses Kapitels übergehen.

Der zweite Grund, sich mit den Frühdialogen zu beschäftigen, ist folgender: Ein verbreitetes Thema neuzeitlicher Philosophie ist die Auseinandersetzung mit der Befürchtung, Ethik sei vor dem Hintergrund naturwissenschaftlicher Welterklärungen eine völlig subjekti-

would be using the book as a springboard for his own considered view of Plato's achievement." Sparshot, „Introduction to Vol. XI“, S. xxxviii. Oder später noch etwas harscher: „We saw him [...] using Grote as a peg to hang his own pet notions on [...]." Ebd., S. xlxxiv.

8 Am explizitesten ist Mill vielleicht hier: Mill, Autobiography, S. 25. Aber auch in Mill, "Grote's Plato“ und Mill, „Notes on Some of the More Popular Dialogues of Plato" wird dies deutlich: Dort schreibt er in seiner Einleitung zum "Protagoras", es seien nicht die Ergebnisse, sondern der Modus des Philosophierens, um den es gehe. Siehe ebd., S. 41. Mill bezieht sich dabei stark verkürzend auf Friedrich Schleiermacher. „Über den Werth des Sokrates als Philosophen“. In: Friedrich Schleiermacher's sämmtliche Werke. Philosophische und vermischte Schriften. Zweiter Band. G. Reimer, 1838, S. 287-308.

9 Mill, „Grote's Plato“, S. 415.

10 Vgl. ebd., S. 382. Sogar das Verhältnis Hume-Bentham entspricht in Mills Darstellung ungefähr dem Verhältnis Zenon-Platon. Vgl. Mill, „Bentham“, S. 80 .

11 Gemeint ist Mills Gegensatz zu den dogmatischen Dialogen Platons. Für diese Unterscheidung Mills: siehe oben. 


\section{Expertise und die Tugenden}

ve Angelegenheit. ${ }^{12}$ Eine Strategie, diese Befürchtung zu zerstreuen, ist zu behaupten, sie finde nicht im Wesen der Ethik eine Grundlage, sondern in einem szientistischen Vorurteil. Paradigmatisch dafür ist im 20. Jahrhundert die Kritik an John Mackies Argument der Absonderlichkeit. ${ }^{13}$ „Absonderlich“, so wird Mackie entgegen gehalten, seien ethische Tatsachen nur vor dem Hintergrund einer szientistischen Ontologie. Eine besonders radikale Form des Umgangs damit ist der metaethische Naturalismus neoaristotelischer Prägung: Der Rhetorik nach wird die Behauptung des naturalistischen Fehlschlusses als Sündenfall der Philosophie ${ }^{14}$ angegriffen $^{15}$, indem die Zurückführbarkeit des ethischen Denkens auf das Denken über naturhistorische ${ }^{16}$ (oder zumindest naturteleologische) Urteile behauptet wird: Warum sollte das Ethische nicht natürlich sein? ${ }^{17}$ Es ist aber nicht nötig, ethische Naturalist*in im engeren Sinne zu

12 Dies ist zum Beispiel unter anderem eines der Elemente der Motivation der neuzeitlichen Frage nach einer Begründung der Ethik, die Korsgaard anführt. Vgl. Korsgaard, The Sources of Normativity, S. 18-89. Die Geschichte der Auseinandersetzung mit dieser Frage und ihre systematische Aufarbeitung (vgl. ebd., S. 21-89) führt Korsgaard schließlich zu einer kantischen Metaethik. Vgl. ebd., S. 90-166.

13 Das Argument richtet sich gegen das, was Mackie „objektive Werte" nennt, weil diese ontologisch seltsame Entitäten sein müssten. Vgl. John Leslie Mackie. Ethics. Inventing Right and Wrong. Harmondsworth: Penguin Books, 1977, Kapitel 1, Abschnitt 9. Dass auch in der Metaethik die ontologische Frage entscheidend sei, begründet Mackie ebenfalls - und sein Grund ähnelt Mills Kritik an den Intuitionist*innen. Vgl. ebd., Kapitel 1, Abschnitt 3.

14 Ich hatte bereits in Abschnitt 6.1.2 angedeutet, dass tatsächlich nicht ganz klar ist, worauf Moore hinaus will. Siehe insbesondere auch Frankena, „Der naturalistische Fehlschluss".

15 Zum Beispiel in Foot, „Moral Arguments“: Wenn Tatsache und Wert als voneinander logisch unabhängige Dinge definiert würden, sei es nicht verwunderlich, so zum Beispiel Foot, dass man von einer korrekten Beschreibung der Tatsachen in einer bestimmten Situation nicht auf das schließen könne, was in dieser Situation zu tun sei.

16 Thompsons Strategie ist es, zu zeigen, dass diese Urteilsform sich ganz ohne Weiteres in die Frege'sche Urteilstafel einfügen lassen könne. Thompson, Life and Action, insbesondere Teil 1. Ich vermute aber, dass besonders Teil 3 des Buches eine weitere Form ethischen Nachdenkens etablieren soll - aber auch diese sei nichts Seltsames; auch sie passe sich ein in eine erweiterte Frege'sche Urteilstafel. Siehe ebd., Teil 3.

17 Diese Seite des (neoaristotelischen) Naturalismus wird zum Beispiel von Thomas Hoffmann betont. Thomas Hoffmann. Das Gute. Berlin: De Gruyter, 2014. 
sein, um die ontologische oder epistemologische Priorität der Wissenschaften als übertriebene Entzauberung der Welt zu sehen, die in der Aufklärung ihren Ausgang genommen habe. ${ }^{18}$ Ich bin überzeugt, diese Strategie, die Wichtigkeit und Legitimität ethischen Nachdenkens vor einer wahrgenommenen szientistischen Bedrohung $\mathrm{zu}$ verteidigen, schütten das Kind mit dem Bade aus und vernachlässigen die Andersartigkeit der Ethik.

Wie kann man gegenüber dieser Strategie nun die Besonderheit der Ethik betonen (und zumindest einige Bedenken der Nonnaturalist*innen ernst nehmen), ohne sich selbst dem Vorwurf, einer szientistischen Dogmatik anzuhängen, auszusetzen - gerade vor dem Hintergrund, dass dieser Vorwurf gegenüber Mill naheliegt und sicher in gewissem Sinne auch angebracht ist? Ich meine, viele der Punkte, um die es hier geht, sind nicht neuzeitliches Dogma, sondern sie finden sich bereits in den Frühdialogen, deren Entstehung hoffentlich weit genug von unterstellbarer neuzeitlicher Wissenschaftshörigkeit entfernt ist, dass dieser Vorwurf gegenüber ihnen nicht glaubwürdig ist. ${ }^{19}$

Drittens ist auf der anderen Seite der Szientismusvorwurf - beziehungsweise, was dahinter steckt - natürlich nicht in allen Aspekten ungerechtfertigt: Er wird von McDowell ja häufig so verstanden, dass wir eine unbeteiligte Außenperspektive auf bestimmte Bereiche unseres menschlichen Tuns und Denkens nicht einnehmen können, ohne das, was diese Bereiche ausmacht, aus dem Blick zu verlieren. ${ }^{20}$ Präziser formuliert können wir die Außenperspektive auf diejenigen Bereiche unseres Tuns und Denkens nicht einnehmen, die sich einem Reduktionismus in der Hinsicht entziehen, dass eine Beschreibung

18 McDowell beschreibt das Projekt als „partial re-enchantment“. Siehe John McDowell. Mind and World. Cambridge, Mass. und London: Harvard University Press, 1996, S. 85.

19 Natürlich wird auch oft von einer „hellenistischen Aufklärung“ gesprochen, in deren Kontext die platonischen Dialoge zu verstehen seien. Siehe Wolf, Die Suche nach dem guten Leben. Platons Frühdialoge, S. 18f. In jedem Fall ist diese Aufklärung aber nicht durch den modernen Szientismus geprägt.

20 Mithilfe der Seitenblickmetapher auf Intentionalität im Allgemeinen kritisiert McDowell dies in McDowell, Mind and World, S. 153; spezifisch in Bezug auf die Ethik besonders in seiner Auseinandersetzung mit der Projektionsmetapher, die ein solcher Seitenblick ist, kritisiert er es in zum Beispiel in John McDowell. „Projection and Truth in Ethics“. In: Mind, Value and Reality. Cambridge, Mass. und London: Harvard University Press, 1998. 


\section{Expertise und die Tugenden}

aus der Außenperspektive der Bedeutung, die unsere Handlungen aus der Innenperspektive haben, niemals gerecht werden könnte. Unzweifelhaft ist dies bei der Ethik der Fall. ${ }^{21}$ Wenn der Szientismus eine Betonung von Benthams Tugend ist, dann ist die Kritik am Seitenblick eine Betonung von Coleridges Tugend. Dieser Konflikt findet sich auch in den Frühdialogen (bis in die Form hinein, siehe Abschnitt 7.3.3).

Der zweite und dritte dieser Punkte spricht also jeweils eine Form der Überbetonung einer der beiden sozialphilosophischen Tugenden an. Mit dem Bezug auf die Frühdialoge entziehe ich mich, wenn meine Auseinandersetzung mit den Frühdialogen erfolgreich ist, daher gleichzeitig dem Vorwurf, insbesondere Benthams Tugend sei eigentlich ein neuzeitliches Dogma.

Viertens erschöpft sich die Dialogform sicherlich nicht darin, authentisch die Perspektiven der Teilhaber*innen an einer Praxis zu integrieren. Auch wenn es strittig ist, worin die philosophische Relevanz der Dialogform genau besteht, ist sie sicherlich nicht irrelevant $^{22}$ - und zwar dann, wenn man bereit ist, die Dialogform, die hier als Form für die Darstellung bestimmter metaethischer Fragestellungen (was die Frühdialoge betrifft) gewählt wurde, auch als paradigmatische Form des Denkens über die Ethik, die Form der (praktischen) Philosophie also, selbst zu verstehen. Dieser Punkt beinhaltet vor dem Hintergrund der heutigen bereits in Kapitel 2 problematisierten Unterscheidung zwischen normativer Ethik und

21 Ich meine, das heißt nicht, dass es keine wissenschaftlichen Beschäftigungen mit der Ethik geben kann. Eine philosophische Beschäftigung mit der Ethik darf aber die Innenperspektive nicht ignorieren. Hier herrscht - so wie ich die Lage einschätze - weitgehende Einigkeit. Die interessante und sicherlich philosophische Frage ist die sich anschließende Frage, wie viel Kontinuität man zwischen den verschiedenen wissenschaftlichen Perspektiven und der philosophischen Perspektive erlauben will und sollte: Redet die Soziologin, wenn sie behauptet, im bildungsbürgerlichen Milieu sei Veganismus sehr viel verbreiteter als in anderen Milieus, über ein gänzlich anderes Thema als ich, wenn ich sage, Tiere zu quälen sei moralisch falsch? Oder ist es das gleiche Thema aus verschiedenen Perspektiven?

22 Beispielsweise Kenneth Sayre unternimmt einen Versuch, diese zu erfassen, der sich an den Äußerungen zur Philosophie des zwar umstrittenen, dafür aber recht expliziten siebten Briefes orientiert. Kenneth M. Sayre. „Plato's Dialogues in Light of the Seventh Letter". In: Platonic Writings/Platonic Readings. Hrsg. von Charles L. Griswold. London: Routlegde, 1988, S. 93109. 
Metaethik einen kritisierbaren Übergang. Die mögliche Kritik daran ist diese: Wenn das Thema der Frühdialoge meist als das „Wesen des Guten und des Wissens davon" charakterisiert werden kann, und wir solche Fragen und Antwortversuche, weil es philosophische Fragen und Antwortversuche sind, nicht unabhängig von den Diskursen, in denen sie entstehen, präsentieren können, ohne sie zu entstellen - und wir deshalb die Dialogform wählen sollten -, dann folgt natürlich nicht, dass dies in der normativen Ethik auch so sein sollte. Ich glaube, das in diesem Kapitel Folgende legt nahe, dass wir den Übergang vollziehen dürfen; und letztlich sogar, dass Fragen der normativen Ethik mit den (oder ohne die) gleichen Wahrheitsansprüchen beantwortet werden können wie Fragen der Metaethik. Zwischen alltäglichem ethischem Denken, der Disziplin der normativen Ethik und der Metaethik gibt es, wenn ich Recht habe, wichtige Kontinuitäten ${ }^{23}$, die, wie ich unten argumentieren will, zusätzlich die Rede von philosophischen Tugenden im Gegensatz zu anders strukturierten Idealen plausibel machen sollen. Alle vier Punkte werden im Laufe des Kapitels aufgegriffen und dabei hoffentlich klarer.

Natürlich kann ich in einem Kapitel den Frühdialogen oder Ursula Wolfs Behandlung der Frühdialoge nicht einmal in Ansätzen gerecht werden. Diesen Anspruch habe ich auch nicht. Ich werde auf drei miteinander verwandte Herangehensweisen an die Ethik und ethisches Wissen eingehen, die Mill meiner Meinung nach missversteht: Dies ist die Frage nach der Lehrbarkeit der menschlichen arete, die Frage nach Expertise im Bereich der menschlichen arete und schließlich die Frage nach der Bezahlbarkeit ethischen Rates oder ethischer Lehre. Zunächst (Abschnitt 7.1) werde ich dabei im Rahmen einer kurzen Auseinandersetzung mit Mills Platonrezeption dieses Missverständnis erarbeiten. Dann werde ich mich mit der Frage nach Expertise in beiden Tugenden einzeln und gemeinsam beschäftigen. (Abschnitt 7.2) Danach werde ich (in Abschnitt 7.3) in Anlehnung besonders an Ursula Wolf andeuten, was der Witz dieser Motive in den Frühdialogen eigentlich ist, und dies ins Verhältnis zu den Tugenden und der Frage nach Expertise in den Tugenden setzen.

23 In Abschnitt 2.3 hatte ich von einem Kollabieren der Metaebenen gesprochen. 


\subsection{Mill und Platon}

Mills Auseinandersetzung mit Platon beginnt in der Kindheit. ${ }^{24}$ Gegen Ende seines dritten Lebensjahrzehnts veröffentlicht er dann Kommentar-Übersetzungen von vier Dialogen. ${ }^{25}$ Wann diese (und weitere fünf, die er nicht veröffentlicht) entstanden sind, ist nicht ganz klar. ${ }^{26}$ Klar ist aber, dass er sich zum Zwecke der Rezension von Grotes Plato mit 59 Jahren noch einmal in Platons Dialoge einarbeitet. ${ }^{27}$

Es gibt Motive in Mills Auseinandersetzung mit Platon, die in diesem Zeitrahmen konstant bleiben: So ist es Mill ein Anliegen, Platon - und insbesondere Platons Verhältnis zu den Sophisten - vor konservativer Vereinnahmung zu bewahren. ${ }^{28}$ Bei der Veröffentlichung der Kommentar-Übersetzungen bedeutet dies, dass Mill Platon weitgehend als Gegenpol zur etablierten Meinung in Athen hinstellt. Platon, so will Mill seine Zeitgenoss*innen glauben machen, wäre im Lager der Philosophical Radicals; die Vorwürfe der Gottlosigkeit und der Verderbung der Jugend gegen Sokrates (und die Sophist*innen) seien vergleichbar mit den entsprechenden Vorwürfen gegen die Radicals. ${ }^{29}$ Das geht so weit, dass Mill sich agnostisch darüber gibt, ob Platon die Ideenlehre, die sich (seiner Meinung nach) nicht gut in dieses Bild einpasst - zumindest nicht, wenn der Empirismus der Radicals und Mills berücksichtigt wird -, wirklich vertreten hat. ${ }^{30}$

Dies scheint er später - nach erneuter Platon-Lektüre und vielleicht auch unter dem Einfluss von Grotes Plato - anders zu sehen: In „Grote's Plato“ unterscheidet er einen sokratischen Platon von

24 Siehe z. B. Mill, Autobiography, S. 25. Siehe oben.

25 Mill, "Notes on Some of the More Popular Dialogues of Plato".

26 Siehe Sparshot, „Introduction to Vol. XI“, xviiiff.

27 Siehe ebd., S. xxxviii.

28 Eine Darstellung dieser „radikalen“ Lesart Platons findet sich bei: Giovanni Giorgini. „Radical Plato: John Stuart Mill, George Grote and the Revival of Plato in Nineteenth-Century England". In: History of Political Thought 30.4 (2009), S. 617-646.

29 Mill, "Notes on Some of the More Popular Dialogues of Plato", S. 43. Vergleiche auch später: Mill, „Grote's Plato“, S. 403.

30 Er sagt zum Beispiel über die Ideen „that they were fixed opinions in his mind is more than can be affirmed, all that is certain being that his speculations tended that way". Mill, "Notes on Some of the More Popular Dialogues of Plato", S. 222. 
einem dogmatischen Platon. ${ }^{31}$ Letzterer wird von Mill auch als Alterserscheinung Platons aufgefasst. ${ }^{32}$ Der sokratische Platon allerdings bleibt für Mill ein philosophisches Vorbild: Er benutzt hier die gleiche Unterteilung zwischen negativer und positiver Philosophie, die er auch bei der Erarbeitung von Benthams Stärken in „Bentham“ benutzt hatte ${ }^{33}$ - ganz abgesehen davon, dass er Bentham in „Bentham“ explizit mit Sokrates vergleicht. ${ }^{34}$ Durch diese progressive Lesart Platons, die Mill mit Grote teilt, entsteht ein bestimmtes Bild der philosophischen Stärken und Absichten Platons. Und es ist sicher nicht von der Hand zu weisen, was Sparshot schreibt: „For that reason it seemed suitable to make a study of Plato an opportunity for speaking out on the great questions of the day. Mill, just like the transcendentalist interpreters he complains of, will let Plato inspire him but not disquiet him. “35

Besonders deutlich zeigt sich diese Vereinnahmung Platons bei Mills (und Grotes) Behandlung der Sophist*innen, wie bereits angedeutet. Mill hat unzweifelhaft Recht, dass das Bild der Sophist*innen der Korrektur bedurfte: Die Sophist*innen sind nicht radikalskeptische Denker*innen, gegen deren jugendzersetzenden Einfluss

31 Siehe Mill, „Grote's Plato“, S. 415.

32 Siehe ebd., S. 414.

33 Allerdings schreibt er diese Unterscheidung in „Grote's Plato" Grote zu. Siehe ebd., S. 382. Dies ist charakteristisch für diesen Aufsatz: Obwohl er vorgibt, eine Rezension von Grotes Plato zu sein, bezieht sich Mill fast ausschließlich dann auf Grote, wenn dieser etwas sagt, was auch Mill über Platon sagen will. Mill sagt nur sehr wenig über Grotes Text: Eine der wenigen Stellen an denen das so ist, befindet sich gegen Ende des Aufsatzes, wo Mill Grotes Verteidigung von Protagoras' (angeblich) relativistischer Wahrheitsauffassung gegen Sokrates widerspricht. Siehe ebd., S. 426ff. Dass die Bewertung von Protagoras die „einzige substantielle“ Meinungsverschiedenheit von Mill und Grote bezüglich Platon ist, meint auch Giorgini, der an dieser Einschätzung von Protagoras den Unterschied zwischen Mills elitistischer und Grotes egalitaristischer Demokratievorstellung veranschaulicht sieht: Vgl. Giorgini, „Radical Plato: John Stuart Mill, George Grote and the Revival of Plato in Nineteenth-Century England", S. 643-646.

34 Die Strukturanalogie geht nach Mill sogar noch weiter: so wie Bentham mit seiner positiven und negativen Philosophie in Hume einen Vorgänger habe, der in rein negativer Philosophie brillierte, so habe Platon (beziehungsweise Sokrates) in Zenon einen solchen negativen Vorgänger. Vergleiche Mill, "Grote's Plato", S. 382 und Mill, „Bentham“, S. 80.

35 Sparshot, „Introduction to Vol. XI“, S. xl. 


\section{Expertise und die Tugenden}

Sokrates die konservativen Institutionen verteidigt ${ }^{36}$ - jedenfalls nicht vorwiegend. In diesem Sinne ist vieles, was Mill zum Teil in Anschluss an Grote zur Rehabilitation der Sophist*innen erwähnt, sehr plausibel. ${ }^{37}$ Aber auch Mill muss natürlich zugeben, dass Platon Kritik an den Sophist*innen übt; dass sie die Antagonist*innen von Sokrates in vielen der Frühdialoge sind. ${ }^{38}$

Ich möchte für den Rest dieses Abschnittes Mills Rekonstruktion der Kritik Platons an den Sophist*innen darstellen. In Mills Text gibt es hier eine oberflächliche Spannung. Einerseits will Mill die Sophist*innen gegen konservative Schmähung verteidigen - und Platon so vor konservativer Vereinnahmung schützen. Andererseits muss er etwas zu Platons Kritik an den Sophist*innen sagen. Charakteristisch für die Art und Weise, wie Mill diese Spannung zu lösen versucht, ist folgende Passage:

It is most unjust to the Sophists to adopt, as the verdict of history upon them, the severe judgment of Plato, although from Plato's point of view they deserved it. He judged them from the superior elevation of a great moral and social reformer [...]; but, as Mr. Grote remarks, to accept his judgment of them would be like characterizing the teachers and politicians of the present time in the words applied to them by Owen or Fourier. ${ }^{39}$

36 Mill beschreibt dieses von ihm und Grote zu korrigierende Bild so: „The opinion which commonly prevails about Plato is something like the following. The Athenians, and the other Greeks, had become deeply demoralized by a set of impostors called Sophists-pretenders to universal knowledge, and adepts at disconcerting simple minds by entangling them in a mesh of words-who corrupted young men of fortune, by denying moral distinctions, and teaching the art of misleading a popular assembly. The lives and intellectual activity of Sokrates and Plato had for their chief object to counteract the doctrines and influence of these men." Mill, „Grote's Plato", S. 387.

37 In der Grote-Rezension: Siehe ebd., S. 387-394, aber auch schon vorher, z. B. Mill, „Notes on Some of the More Popular Dialogues of Plato“, S. 43.

38 In „Grote's Plato" setzt sich Mill ab Seite 399 damit auseinander. Mill, „Grote's Plato", S. 399-403.

39 John Stuart Mill. „Grote's History of Greece II“. In: The Collected Works of John Stuart Mill, Volume XI. Essays on Philosophy and the Classics. Hrsg. von J. M. Robson. Toronto und andere: University of Toronto Press und Routledge \& Kegan Paul, 1978, S. 307-337, S. 329. An anderen Stellen weist Mill auch darauf hin, dass Platon durchaus Hochachtung vor einigen Sophisten gehabt habe, z. B. Mill, „Notes on Some of the More Popular Dialogues of Plato", S. 43. 
Wie das Zitat zeigt, sieht Mill Platons Kritik an den Sophist*innen analog zur Kritik, die [Charles] Fourier oder [Robert] Owen an zeitgenössischen Politiker*innen und Lehrer*innen geübt haben könnten: Vermutlich waren Fourier und Owen nicht der Meinung, es sollte oder könne diese Berufe gar nicht geben; vielmehr erfüllen die entsprechenden Personen nicht den Standard, der an sie gelegt werden sollte. Damit übergeht Mill aber den eigentlichen Punkt in doppelter Weise: Zum einen geht es bei den Sophist*innen, wenn Platon sie kritisiert, natürlich nicht um Lehrer*innen im Allgemeinen, sondern um Lehrer*innen der menschlichen arete; zum anderen scheint die Frage der Lehrbarkeit der menschlichen arete auf etwas im Wesen der menschlichen arete hinzuweisen, so dass es sicher nicht Platons Punkt ist, dass es lediglich die Rückschrittlichkeit oder die Unvollkommenheit der Sophist*innen ist, die dagegen spricht, dass sie die Tugend lehren können. Es geht um die menschliche Tugend und darum, dass eine bestimmte Form der Auseinandersetzung mit der menschlichen Tugend - diejenige, die in einigen Dialogen als sophistisch bezeichnet wird - dieser nicht gerecht wird, und es geht nicht darum, die Unvollkommenheiten einer spezifischen, historisch realen Gruppe von Menschen nachzuweisen. ${ }^{40}$

Konkretisieren möchte ich dieses Bild des Wesens der Kritik Platons an den Sophist*innen unter den gleichen Gesichtspunkten, unter denen ich mich im nächsten Abschnitt mit den Frühdialogen selbst beschäftigen werde: Der Lehrbarkeit der menschlichen arete, der Bezahlbarkeit von Rat bezüglich der menschlichen arete und der in beiden Punkten impliziten Fragestellung, inwiefern Expertise in Fragen der menschlichen arete möglich ist. Natürlich ignoriert auch Mill diese Themen nicht, aber in seinem Versuch, die Sophist*innen zu rehabilitieren, verwischt er die Bedeutung dieser Fragen.

Dass Platon Sokrates zum Beispiel die Art und Weise, wie die Sophist*innen für die Lehrbarkeit der menschlichen arete eintreten,

40 Wie Mill eigentlich selbst bemerkt, bilden die Sophist*innen historisch gesehen in dieser Hinsicht gar keine einheitliche Gruppe (Siehe Mill, „Grote's Plato", S. 397f) und es ist natürlich eine legitime Frage, mit welchem Recht wir Sokrates aus der Gruppe der Sophist*innen als historischem Phänomen ausschließen sollten, wenn der einzige Unterschied zwischen ihm und ihnen darin besteht, dass sie weniger gut in dem waren, was sie und Sokrates machten. 


\section{Expertise und die Tugenden}

in einigen Dialogen in Frage stellen lässt, ist auch Mill klar. In den einleitenden Absätzen zu seiner Kommentarübersetzung des Protagoras spekuliert Mill beispielsweise, dass das Ziel des Protagoras sei, nachzuweisen, dass

truth could only be ascertained by that more accurate mode of sifting opinions, which the dialectic method (or that of close discussion between two persons, one of whom interrogates, and the other answers) furnishes, but which speechmaking, and the mere delivery of doctrines from master to student (the practice of the Sophists) absolutely preclude. ${ }^{41}$

Diese Gegenüberstellung dessen, was Mill die „dialektische Methode" nennt, gegenüber einer Lehrmethode, die im Redenhalten und Weitergeben von Lehrsätzen besteht, ist sicherlich Thema im „Protagoras", um den es hier geht. ${ }^{42}$ Allerdings missversteht Mill, dass es in den Dialogen nicht um eine allgemeine didaktische Richtlinie geht, sondern speziell um philosophische Fragen - im Protagoras zum Beispiel um die Lehrbarkeit der menschlichen arete. Mill hält die dialektische Methode aber ganz allgemein für wichtig, wie sich auch später in "Grote's Plato" noch einmal zeigt, wo er über den Grund für die dialektische Methode Folgendes sagt:

Yet truth, in everything but mathematics, is not a single but a double question; not what can be said for an opinion, but whether more can be said for it than against it. There is no knowledge, and no assurance of right belief, but with him who can both confute the opposite opinion, and successfully defend his own against confutation. ${ }^{43}$

Dies entspricht den Vorstellungen aus dem zweiten Kapitel von „On Liberty“, die ich bereits in Abschnitt 5.2.3 diskutiert habe:

41 z. B. Mill, „Notes on Some of the More Popular Dialogues of Plato“, S. 44.

42 Allerdings kommt es, wie auch Mill auffällt, im Protagoras zu einem interessanten performativen Widerspruch von Sokrates in Bezug auf dieses Thema, nachdem dieser den Protagoras gebeten hat, kürzer zu antworten: „[I]t is agreed, at the instance of Socrates, that Protagoras should interrogate and Socrates answer, in order that Socrates might afford a specimen of what he thought the proper mode of answering. It turned out an unhappy specimen, however, for Socrates was led by it to make as long a speech as any in the dialogue." ebd., S. 54. Dies thematisiert Mill aber ansonsten nicht weiter.

43 Mill, „Grote's Plato“, S. 411. 
Mill meint dort, dass die Bedeutung einer Meinung verblasst, wenn diese nicht herausgefordert wird. Und es passt auch gut zu den Reflexionen über den Wert seiner eigenen Erziehung im ersten Kapitel der Autobiography. ${ }^{44}$ Wahrscheinlich kann auch im Rahmen einer im weitesten Sinne konstruktivistischen Didaktik viel zugunsten der dialektischen Methode im Gegensatz zum Redenhalten und Lehrsätze-Weitergeben als Lehrmethode gesagt werden. Es spricht also nichts dagegen, mit Mill „die dialektische Methode“ ganz allgemein für eine dem bloßen Vortrag überlegene Methode zu halten.

Den Witz der Kritik an den Sophist*innen verschleiert dieser allgemeine didaktische Punkt jedoch, so wichtig er ist: Aktivität und Einbeziehung der Lernenden ist unzweifelhaft auch unverzichtbar, wenn es zum Beispiel um die Vermittlung des Biot-SavartGesetzes im Physikunterricht geht: Es müssen Fragen beantwortet, Missverständnisse aufgezeigt, Begriffe geklärt werden - und es ist sicherlich auch hilfreich im Sinne Mills, das Gesetz von Alternativen abzugrenzen, damit seine Bedeutung nicht verblasst: Es wäre auch vorstellbar, dass sich Elektronen so-und-so durch ein Magnetfeld bewegen; aber sie bewegen sich so-und-so. Wenn aber all dies geschehen ist, wenn die Lernende die Bedeutung und die Implikationen des Gesetzes verstanden hat, mag sie sich fragen, ob sie den Inhalt des Gesetzes für wahr halten sollte. Und hier ist es legitim, die Physiker*in als Expert*in zu akzeptieren und ihr zu glauben. Die Lernende könnte das Experiment auch selbst durchführen und oft ist dies in didaktischen Zusammenhängen auch sinnvoll; aber es ist nicht immer nötig und nicht immer möglich. In diesem Sinne ist "delivery of doctrines from master to student" in der Physik eben doch möglich. ${ }^{45}$ Speziell in der sophistischen Kunst ist dies, so zumindest scheint es das aber Thema des Protagoras zu sein, nicht so offensichtlich möglich - und dies übersieht Mill an den meisten Stellen.

44 „Most boys or youths who have had much knowledge drilled into them, have their mental capacities not strengthened, but overlaid by it. [...] Mine, however, was not an education of cram. [...] Anything which could be found out by thinking, I never was told, until I had exhausted my efforts to find it out for myself." Mill, Autobiography, S. 33ff.

45 Vergleiche die Abgrenzung der sophistischen Kunst zum Beispiel von der Medizin im „Protagoras“, auf den sich Mills Bemerkung bezieht. Wolf, Platon. Sämtliche Werke, Band 1, „Protagoras“, 311b. 
Dies wird auch deutlich, wird die unterschiedliche Einordnung der Mathematik bedacht: Mathematik dürfte nach Mills Zitat ${ }^{46}$ ja gerade nicht zu den Gegenständen zählen, die sich besonders paradigmatisch für eine „dialektische“ Behandlung anbieten: Wenn der Grund für die dialektische Methode derjenige ist, dass die Bedeutung einer Meinung nur vor dem Hintergrund von Gegenmeinungen deutlich gemacht werden kann, dies aber für alles gilt außer für die Mathematik, dann scheint es in der Mathematik gerade keinen Grund für die dialektische Methode zu geben. In den platonischen Dialogen, zum Beispiel im „Menon“, ist aber die Mathematik gerade paradigmatisch für die Mäeutik, die ihrerseits Grund für das, was Mill "dialektische Methode“ nennt, ist - so paradigmatisch, dass sie (mindestens) als Analogie für das kalon kagathon dienen kann. ${ }^{47}$ Mill liest die Gedanken zur Lehrbarkeit der menschlichen arete in den Frühdialogen so, als ob es generell um Lehrbarkeit ginge - und übersieht so, dass diese Gedanken dort eine Perspektive sind, unter der die menschliche arete in den Blick genommen wird.

Das gleiche Missverständnis zeigt sich auch in Mills Behandlung der Prüfung des Orakelspruchs in der "Apologie“: Mill hat zwar Recht, wenn er die Prüfung des Orakelspruchs in den Zusammenhang mit der Kritik an den Sophist*innen stellt; ${ }^{48}$ dies werde ich im nächsten Abschnitt genauer begründen. Aber auch hier ignoriert Mill, dass es nicht um Wissen schlechthin geht, sondern um Wissen vom kalon kagathon: Die Handwerker*innen haben, wie Mill richtig wiedergibt, tatsächlich Wissen. Und ebenso ist es richtig, dass sie meinen, mehr zu wissen, als sie in Wirklichkeit wissen. ${ }^{49}$ Aber dies ist trotzdem nicht der ganze Witz der Passage. Eine Schiffbauer*in,

46 Ich beziehe mich auf das Zitat, zu dem Fußnote 43 gehört.

47 Siehe Wolf, Platon. Sämtliche Werke, Band 1, „Menon“, 82b-86c: Sobald gezeigt ist, dass der Sklave die mathematische Aufgabe wie durch Wiedererinnerung lösen kann, ist die grundsätzliche Skepsis, die Menon angemeldet hatte, nämlich dass sich nicht nach etwas forschen lasse, worüber nichts gewusst sei, auch für die Tugend aus dem Weg geräumt, und Sokrates und Menon können sich diesem wieder zuwenden: „Da wir nun einig darüber sind, daß gesucht werden muss, was jemand noch nicht weiß: willst du, daß wir miteinander unternehmen zu suchen, was wohl die Tugend ist?"

48 Der Absatz behandelt "[t]he other ground of disapproval of the sophists". Mill, „Grote's Plato“, S. 402. Der erste Grund ist, dass sie Geld nehmen. Siehe ebd., S. 402.

49 Ebd., S. 403. 
die fälschlich annimmt, dass sie auch Häuser bauen kann, ist zwar sicherlich nicht besonders wissend; aber grundsätzliche handwerkliche Selbstüberschätzung ist schwerlich der Vorwurf, um den es hier gehen kann: Athen hatte sicherlich kein besonderes Problem damit, dass Schiffbauer*innen sich im Häuserbau oder als Ärzt*innen profilieren wollten. ${ }^{50}$ Es geht um eine bestimmte Selbstüberschätzung der Handwerker*innen, nämlich diejenige, dass das Wissen vom Guten so beschaffen sein muss, wie handwerkliches Wissen. Und dies ist der sehr viel spezifischere, von Mill aber leider übersehene Grund, warum es richtig ist, die Kritik an den Sophist*innen in den Kontext der Kritik an den Handwerker*innen zu stellen.

Mill lenkt - ebenso wie bei der Expertise und der Lehrtätigkeit der Sophist*innen - auch bei der Kritik an der Bezahlung der Sophist*innen die Diskussion auf eine andere Fährte, die eher Gegenwartsdiskussionen zu Mills Zeit betrifft; so fragt Mill, ob die Bezahlung von Lehrer*innen, Priester*innen, aber auch Abgeordneten u.s.w. in etablierten Institutionen nicht grundsätzlich deren Abhängigkeit von den Mächtigen fördere und ob Copyright und der damit verbundene Handel mit Ideen produktiv oder kontraproduktiv seien. ${ }^{51}$ Dies sind zwar wichtige Punkte, die zum Teil auch bei Platon angesprochen werden ${ }^{52}$, aber das zum Beispiel im „Protagoras" angesprochene Problem, wie eigentlich der Wert oder Schaden einer sophistischen Ausbildung bemessen werden könne, ${ }^{53}$ oder die von Mill ebenfalls angesprochene ${ }^{54}$, aber nicht weiter diskutierte „Ungereimtheit" der Sophisten, einerseits zu behaupten, Menschen gerecht zu machen, und andererseits sich über ausbleibende Bezahlungen zu beschweren ${ }^{55}$, haben eigentlich ein anderes Ziel: Auch hier geht es darum, technisches oder wissenschaftliches Wissen, dessen Tauschwert (verhältnismäßig) leicht bestimmbar ist, kontrastiv zu ethischem Wissen zu positionieren, bei welchem der Versuch der Be-

50 Vgl. Wolf, Platon. Sämtliche Werke, Band 1, „Protagoras“, 319b-c, wo explizit gesagt wird, dass es kein besonderes Problem ist, in solchen Fällen Hochstapelei zu erkennen.

51 Mill, „Grote's Plato“, S. 401f.

52 Wolf, Platon. Sämtliche Werke, Band 1, „Gorgias“, 463bff.

53 Siehe ebd., „Protagoras“, 312b-314b.

54 Mill, „Grote's Plato“, S. 401.

55 Siehe Wolf, Platon. Sämtliche Werke, Band 1, „Gorgias“, 519c. 


\section{Expertise und die Tugenden}

stimmung eines gerechten Tauschwerts uns in Paradoxien führt. ${ }^{56}$ Es geht nicht um die praktische Frage, ob für bestimmte oder alle Lehrtätigkeiten eine Bezahlung angemessen ist, sondern um die Implikationen einer negativen oder zumindest zweifelnden Antwort auf diese Frage im Falle der menschlichen arete. Auch hier wird der Witz der Gedanken durch Mill verdunkelt, indem er stattdessen die Frage der Bezahlung von Abgeordneten und die Frage, ob es ein Copyright geben sollte, diskutiert: Mill schreibt am Ende, er halte Platons Meinung (bezüglich Copyright und Bezahlung von Abgeordneten etc.) für falsch, ,not because nothing can be said for it, but because there is much more to be said on the opposite side ${ }^{657}$ - ganz so, als ob Platon direkt zu der konkreten Frage Stellung bezogen hätte, ob zum Beispiel Alexander Bain die Druckkosten von The Emotions and the Will durch Verkauf refinanzieren können sollte oder nicht. ${ }^{58}$

Mill verwischt in der dargestellten Weise den Unterschied zwischen Fragen nach dem kalon kagathon und anderen Formen praktischen Wissens - oder allgemeiner, den Unterschied zwischen philosophischen Fragen und anderen Formen von Wissen. Dies ist nicht nur deshalb unglücklich, weil dieser Unterschied noch einmal systematisch deutlich macht, warum für die Philosoph*in Benthams

56 Insofern hat auch Protagoras das Problem der „Ungereimtheit“ aus dem „Gorgias" nicht gelöst, wie Mill meint (siehe Mill, „Grote's Plato“, S. 401n), wenn er seine Schüler*innen den Wert der Ausbildung unter sakralem Eid selbst bestimmen lässt: Wie bestimmt sich denn der Wert der Ausbildung für sie, wenn Protagoras gleichzeitig meint, die Gerechtigkeit einer Person nütze besonders anderen Personen. Siehe Wolf, Platon. Sämtliche Werke, Band 1, „Protagoras“, 327a-c. Der Tauschwert einer Ausbildung bei Protagoras ist weiterhin unklar.

57 Mill, „Grote's Plato“, S. 402.

58 Grote und Mill haben die Veröffentlichung von Alexander Bains The Emotions and the Will möglich gemacht, indem sie ihm Garantien gegen finanziellen Verlust gegeben haben. Siehe Sparshot, „Introduction to Vol. XI“, S. lviii. Hat dies Mills Rezension von Bains Psychologie noch wohlwollender gemacht, als sie ohnehin gewesen wäre? Solche Gefahren bilden ungefähr den Hintergrund, vor dem Mill die Frage diskutiert. Aber auch hier geht es nicht um ein Problem speziell der sophistischen Kunst: Auch wenn Bain über Schiffbau geschrieben hätte und Mill dieses Buch kofinanziert hätte, hätte die Neutralität einer Rezension in Frage gestanden. Platon scheint aber nichts dagegen zu haben, dass Lehre im Schiffbau entlohnt wird, also geht Mills Diskussion am Anliegen Platons vorbei. 
Tugend und Coleridges Tugend wichtig sind und warum es sich um Tugenden handelt. Dies werde ich weiter unten zeigen. Es ist auch deshalb schade, weil es erhellend gewesen wäre, Mills Unterscheidung zwischen Kunst $\left(\operatorname{art}^{59}\right)$ und Wissenschaft, wie er sie in Kapitel 12 von Buch VI des System of Logic ${ }^{60}$ trifft, ins Verhältnis zu setzen zu den Frühdialogen. Beide Unterscheidungen liegen einerseits quer zueinander: Wie Ursula Wolf zu Recht anmerkt, sind in den Frühdialogen episteme und techne in ihrer Rolle als Gegensatz zum Wissen vom Guten, um das es geht, mehr oder weniger austauschbar. ${ }^{61}$ Auf den ersten Blick scheint Mill, wenn er genau diese Begriffe $\mathrm{zu}$ unterscheiden scheint, also auf etwas anderes hinaus zu wollen. Interessant ist aber andererseits das hierarchische Verhältnis der einzelnen Künste bei Mill: Dass das Ziel der Kardiologie, nämlich ein gesundes Herz, ein gutes Ziel ist und welche Priorität es im Verhältnis zu anderen medizinischen Zielen hat, lässt sich aus der Medizin bestimmen. Dass das Ziel der Medizin, nämlich Gesundheit, ein gutes Ziel ist, und in welchem Verhältnis es zu anderen Zielen steht, lässt sich aus dem bestimmen, was Mill dort (neben anderen Namen) Lebenskunst nennt. ${ }^{62}$ Die Lebenskunst steht aber ganz oben in der Hierarchie. Ihr Ziel lässt sich nicht mehr bestimmen, indem eine übergeordnete Kunst gefunden wird - weshalb Mill an mehreren Stellen sagt, die Art der Begründung müsse hier ganz anders sein. (Was das für Mill heißt, wurde in Abschnitt 6.1.2 und in Abschnitt 4.3 diskutiert.) Hier geht es darum, dass Mill durchaus einen Sinn für die Andersartigkeit der Frage, ob der Utilitarismus die richtige ethische Theorie ist, im Gegensatz zu anderen praktischen und theoretischen Fragen hat. Bei aller Liebe zu Platon, die

59 „It is almost superfluous to observe, that there is another meaning of the word Art, in which it may be said to denote the poetical department or aspect of things in general, in contradistinction to the scientific. In the text, the word is used in its older, and I hope, not yet obsolete sense." Mill, The Collected Works of John Stuart Mill, Volume VIII - A System of Logic Ratiocinative and Inductive (Books IV-VI and Appendices), 943n. Gemeint ist methodisch strukturierte Tätigkeit.

60 Ebd., Kapitel 12, Buch VI.

61 Siehe Wolf, Die Suche nach dem guten Leben. Platons Frühdialoge, S. 34. Zumindest in der häufig verwendeten Formel techne kai episteme.

62 Siehe Mill, The Collected Works of John Stuart Mill, Volume VIII - A System of Logic Ratiocinative and Inductive (Books IV-VI and Appendices), S. 949. 
er hat, setzt er diese Andersartigkeit aber nicht ins Verhältnis zu der Andersartigkeit, um die es in den Frühdialogen geht.

\subsection{Expert*innentum und Mills Tugenden}

Bevor ich im nächsten Abschnitt 7.3 skizzenhaft einen an Ursula Wolfs Auseinandersetzung mit den Frühdialogen angelehnten Vorschlag machen will, wie die Argumentation der Frühdialoge um diese drei Fragen herum (Lehrbarkeit, Bezahlbarkeit, Expert*innentum in der menschlichen Tugend) stattdessen rekonstruiert werden kann, möchte ich einen intuitiven Zugang dazu liefern, was eigentlich die Konsequenz der Akzeptanz der philosophischen Tugenden in Bezug auf die drei Fragen ist. Vollständig ausgearbeitet wird dies dann erst anhand der Auseinandersetzung mit den Frühdialogen im nächsten Abschnitt.

Die Behauptung die ich aufstellen will, ist, dass es die Komplementarität beider Tugenden in der Philosophie ist, die erstens Expertise - auf eine bestimmte, im nächsten Abschnitt spezifizierte Weise verstanden - hier unmöglich macht; die zweitens die systematische Erlernbarkeit in Frage stellt; und die es drittens unmöglich macht, einen Tauschwert zu bestimmen. Um diesen Punkt deutlich zu machen, möchte ich zunächst beide Tugenden einzeln betrachten und zeigen, inwiefern und unter welcher Bedingung hier die drei Fragen positiv beantwortet werden können. Ich werde dann nahelegen, dass die entscheidende Bedingung, unter der es möglich ist, in Benthams Tugend eine Expert*in zu sein, diejenige ist, dass Coleridges Tugend außen vor gelassen wird; und umgekehrt, dass die entscheidende Bedingung, unter der es möglich ist, in Coleridges Tugend eine Expert*in zu sein, diejenige ist, dass Benthams Tugend außen vor gelassen wird. Dies lege ich zunächst nur nahe und es versteht sich, dass dabei ein ganz bestimmtes Verständnis von Expertise (siehe Abschnitt 7.3) vorausgesetzt wird.

Ich werde dafür für jede der Tugenden beispielhaft beginnen und mich danach bemühen, den Punkt in Abstraktion für beide Tugenden zu formulieren. Beginnen werde ich mit dem Beispiel für Coleridges Tugend.

Wenn es darum geht, einen sprachlichen Ausdruck zu finden, der von einer kompetenten Teilnehmer*in an einer Praxis (Institution, 
Art und Weise die Dinge zu tun, u.s.w.) als adäquater Ausdruck dafür verstanden wird, worum es in dieser Praxis (Institution, Art und Weise die Dinge zu tun, u.s.w.) geht, ist klar, wer die Expert*in ist: Sie ist in die Formulierung der Tugend, so wie ich sie in dieser Arbeit bisher verwendet habe, bereits eingebaut. Es ist natürlich die kompetente Teilnehmer*in. ${ }^{63}$ Expertise hat natürlich eine soziale Komponente (die wird im nächsten Abschnitt eine Rolle spielen): Expert*innen können sich nicht bloß selbst bezüglich der Kompetenz ihrer Teilnahme an einer Praxis validieren. Aber natürlich hat die Frage, welcher Person wir eher vertrauen können, ob eine Ausdrucksform das trifft, worum es in einer Praxis geht, eine Antwort: Diejenige, die besser (länger, intensiver) daran teilgenommen hat.

Könnte solche Expertise sogar zu einer Praxis mit dem Anspruch einer techne mit einem Tauschwert erhoben werden? Vielleicht nicht in jedem Sinne des Wortes; aber diese Art der Expertise ist zumindest Grundlage für bestimmte Formen des Ratschlags: Beispiele dafür sind der Knigge oder in neuerer Zeit zahlreiche Bände zur interkulturellen Kommunikation ${ }^{64}$ im Rahmen des internationalen Handels oder internationaler Unternehmen. Die Ziele sind in beiden Fällen die gleichen, es soll einer Außenstehenden zumindest eine rudimentäre Form der Teilnahme an einer Praxis ermöglicht werden: Die Adressat*in des Knigge soll sich in „höherer" Gesellschaft bewegen können, ohne sich zu blamieren; die Unternehmer*in soll Verträge abschließen können, ohne die Vertragspartner*innen zu beleidigen. Für beides ist es wichtig, nicht nur die äußere Form der Praxis, sondern in begrenztem Umfang auch deren Bedeutung für die Teilnehmer*innen zu kennen. Erstens ist dies wichtig, um

63 In anderer Hinsicht ist es natürlich sinnvoll zu unterscheiden zwischen der bloßen Fähigkeit zu entscheiden, ob ein sprachlicher Ausdruck dem, worum es in einer Praxis geht, angemessen ist, und der Fähigkeit, einen solchen Ausdruck zu finden. Hier ist diese Frage jedoch nicht entscheidend. Sofern es Rechtfertigung und Richtigkeit in Coleridges Tugend gibt, hat sie offensichtlich mit ersterer Fähigkeit zu tun.

64 Zum Beispiel in Bezug auf China: Fred Derwin und Regis Machart, Hrsg. Intercultural Communication with China. Singapur: Springer, 2017 oder Caroline Zamor. Intercultural Trainings for German Expatriates Going to China. Hamburg: IGEL, 2008 oder Jan Selmer, Hrsg. International Management in China: Cross-Cultural Issues. London und New York: Routledge, 1998. Es sei auf Google-Books mit entsprechenden Suchbegriffen für zahlreiche weitere Beispiele verwiesen. 
bei Abweichungen vom Protokoll zu wissen, wie entscheidend der Fehler war. Zweitens sind so natürlich in Grenzen Inferenzen auf andere nicht explizit erwähnte Fälle und die Lösung von Konflikten zwischen verschiedenen Normen möglich. Hier gibt es Expertise einfach deshalb, weil es ein Erfolgskriterium gibt: Ist die Leser*in der entsprechenden Bücher nun in der Lage, an der betreffenden Praxis teilzuhaben oder nicht? Aus dem gleichen Grund lässt sich auch der Tauschwert relativ leicht (marktwirtschaftlich) bestimmen.

Wenn Coleridges Tugend so zur techne erhoben wird, dann steht natürlich oft nicht der angemessene sprachliche Ausdruck, sondern die korrekte Beschreibung der Handlungsformen im Vordergrund. Aber: nur eine, die weiß, was an den einzelnen Handlungsformen hängt, kann eine solche Beschreibung liefern. Sie ist Expert*in.

Dass in diesem Falle Benthams Tugend außen vorgelassen wird, dürfte klar sein. Das externe Interesse der Adressat*in solcher Bücher reicht als Grund aus, an der entsprechenden Praxis teilzunehmen. Ein weiterer Grund für die Teilnahme - siehe Seite 102 für Formulierung von Benthams Tugend als Streben nach einem Grund für die Teilnahme - ist nicht erforderlich. In jedem Fall kann Benthams Tugend, dort wo es um Expertise in Form von Büchern wie dem Knigge oder Unternehmensratgebern zur interkulturellen Kompetenz geht, keine Rolle spielen; sie ist extern gesetzt.

Dort, wo es wirklich auf den Ausdruck dessen ankommt, worum es in einer Praxis geht, ist allerdings nicht einmal klar, ob die darstellende Rede die richtige Form ist: In „Coleridge“ behandelt Mill Coleridge zwar als Philosophen, der in seiner Philosophie einen Ausdruck dessen, worum es in verschiedenen sozialen Praktiken geht, geliefert habe, dem eine kompetente Teilnehmer*in an einer solchen Praxis zustimmen könnte; in der Autobiography kommt diese Tugend Coleridges aber seinen Gedichten zu und Mill ist selbst derjenige, der die Adäquatheit bestimmen kann: Er befindet sich in einer Depression und er kann daher sagen, dass ihm Coleridges Gedichte aus der Seele sprechen. Hier von „Expertise“ Coleridges $\mathrm{zu}$ sprechen, mag übertrieben klingen; es ist jedenfalls eine Expertise, die nicht verdient wird, sondern deren Erwerb zum Teil vom Schicksal abhängt. (Siehe auch Abschnitt 7.3.4.)

Trotzdem ist es klar, dass es Ansprechpartner*innen gibt, deren Urteil in diesen Fällen mehr zählt als das anderer Personen. Dies zeigt sich auch bei anderen Formen des Leides, die vielleicht etwas 
offensichtlicher mit sozialen Praktiken zu tun haben: ${ }^{65}$ Was ein angemessener sprachlicher Ausdruck des Leides ist, das Sexismus, Heteronormativität, Rassismus u.s.w. erzeugen, können nur Personen entscheiden, die dieses Leid erlebt haben. Sie sind die Ansprechpartner*innen, deren Urteil mehr zählt, und sie sind - in diesem Sinne - Expert*innen. ${ }^{66}$

Wenn es auf der anderen Seite darum geht, Praktiken (Institutionen, Arten und Weisen die Dinge zu tun, u.s.w.) vor dem Hintergrund, dass es Alternativen gibt, zu bewerten, ist nicht die Person, die besonders involviert ist in eine Praxis, diejenige, die wir fragen sollten. Vielmehr ist es die Person, die eine Methode und eine Hinsicht beherrscht, anhand der die Praktiken verglichen werden können: Geht es beispielsweise darum, die Auswirkung einer Gesetzgebung auf das Bruttoinlandsprodukt zu prüfen, ist eine andere Person eine Expert*in als, wenn es darum geht, die Auswirkungen der gleichen Gesetzgebung auf $\mathrm{CO}_{2}$-Emissionen zu prüfen, oder auf das subjective well-being. Die Frage, welchen Maßstab wir wählen sollten, ist solcher Expertise extern.

65 Das soll nicht heißen, dass Depressionen sonst nichts mit sozialen Praktiken zu tun haben. Im Gegenteil: der ganze Witz der Auseinandersetzung mit Elizabeth Andersons Lesart in Kapitel 5.1 war ja, zu behaupten, Mill konstruiere seine eigene Depression als adäquate Antwort auf eine bestimmte Moralvorstellung.

66 Ich glaube der Punkt ist nicht besonders kontrovers. Es muss dabei nicht unbedingt primäre Erfahrung sein. Dies kann illustriert werden mit einem überzeugenden Gegenargument Gilligans gegen Kohlbergs Stufenmodell der Moral: Gilligan beschreibt das eindrückliche Fallbeispiel von Claire, die nachdem sie relevante Erfahrungen in Bezug auf wichtige moralische Entscheidungen (beispielsweise Abtreibung: Siehe Carol Gilligan. Die andere Stimme. München und Zürich: Piper, 1999, S. 76-78) gemacht hat, auf der Kohlberg'schen Skala fünf Jahre später abrutscht (Siehe ebd., S. 73), weil sie die Dinge nicht mehr für ganz so prinzipiell beantwortbar hält, wie es die Kohlbergskala belohnt. Ganz offensichtlich, so ist ungefähr das Argument in diesem Kapitel, ist die Kohlberg'sche Skala also zumindest einseitig, wenn sie den Erwerb (bei Gilligan hier insbesondere weiblicher) relevanter Erfahrungen, negativ einfließen lässt. Das Argument gegen Kohlberg ist überzeugend. Seine Überzeugungskraft beruht aber darauf, dass Claire, die nun erfahren hat, was es heißt, mit solchen Entscheidungen konfrontiert zu werden, zumindest in bestimmten Hinsichten besser weiß, worum es geht. Sie ist nun eher als vorher eine Person, die wir ansprechen sollten und - in diesem Sinne - eine Expertin. 
Die Beispiele zeigen auch, dass beide Arten von Expert*innentum in Spannung zueinander stehen: Wer Expert*in in der einen Hinsicht ist, ist zumindest prima facie als Expert*in in der anderen Hinsicht diskreditiert: Eine Studie zur Auswirkung bestimmter Gesetzgebungen zu $\mathrm{CO}_{2}$-Emissionen, die von einer Organisation finanziert wird, die gleichzeitig die Kultur des Autofahrens zelebriert und dem Ausdruck zu verleihen verpflichtet ist, was das Auto kulturell bedeutet, wird dadurch diskreditiert - zumindest, wenn die Unabhängigkeit der Studie nicht auf andere Weise nachgewiesen wird. Ebenso wenig würden wir auf der anderen Seite einen Versuch von Pierre Bourdieu ernst nehmen können, die Bedeutung ästhetischer Begriffe zur Beschreibung klassischer Musik aus Perspektive kompetenter Hörer*innen zu erfassen, nachdem er Die feinen Unterschiede $^{67}$ verfasst hat. Die Perspektive aus Die feinen Unterschiede korrumpiert - zumindest prima facie - die für diese Aufgabe nötige Perspektive.

Dies zeigt sich auch in der Person von John Stuart Mill und dem, was bisher dazu gesagt wurde, wie er den Tugenden gerecht wurde: Mill ist, ginge es nur um Coleridges Tugend, sicherlich nicht die Person, die wir fragen sollten, wenn es um eine Würdigung dessen, worum es in den Gedichten von Wordsworth oder von Coleridge geht, wie er es in der Autobiographie unternommen hat. Er ist sicherlich nicht die Person, die wir fragen sollten, wenn es um den Witz der Religion geht, wie er es im zweiten Teil von „The Utility of Religion" versucht hat. In beiden Fällen sollten wir Personen fragen, die in den jeweiligen Praktiken mehr zu Hause sind als er. Ginge es nur um Benthams Tugend, wäre Mill auch nicht die Person, die wir fragen sollten - zumindest nicht in seiner Funktion als Philosoph: Wie auch Bentham ist Mill stellenweise bemüht, unsere Praktiken vor dem Hintergrund zu bewerten, welche Interessen damit bedient werden; aber, dass er sich auch auf eine Innenperspektive einlässt, verstellt ihm den Blick oft. ${ }^{68}$ Wir könnten uns vor dem Hintergrund von Benthams Bewertung der Poesie und der Religion auch gut vorstellen, dass Bentham Mill in Bezug auf die

67 Pierre Bourdieu. Die feinen Unterschiede. Frankfurt am Main: Suhrkamp, 1987.

68 Ein besonders hervorstechendes Beispiel ist vielleicht in diesem Zusammenhang Mills Empfehlung eines nach Bildung gewichteten Stimmengewichts bei demokratischen Entscheidungen. 
entsprechenden Gedanken vorwerfen würde, durch seine Involvierung in poetische Tätigkeiten seine Unparteilichkeit eingebüßt zu haben - und natürlich ist dieser Vorwurf verständlich.

Trotzdem ist es genau das, was zum Beispiel Mills Auseinandersetzung mit Wordsworth in der Autobiographie lesenswert macht: Es ist, dass er damit beides will: Er will nicht nur sagen, was der Witz von Wordsworths „Ode" ist ${ }^{69}$, sondern auch, warum ein Leben, dass das enthält, worum es in dem Gedicht nach ihm (auch) geht, der Bentham'schen Alternative vorzuziehen ist.

\subsection{Lehrbarkeit und Expert*innentum als Zugang zu ethischem Wissen in den platonischen Frühdialogen}

\subsubsection{Zum Status der Auseinandersetzung mit den Frühdialogen}

Nachdem nun hoffentlich ein intuitiver Zugang zur Konsequenz der Akzeptanz beider Tugenden auf die Beantwortung der Frage nach philosophischer Expertise gegeben wurde, möchte ich die Frage nun anhand von den Frühdialogen und insbesondere von Ursula Wolfs Lesart $^{70}$ der Frühdialoge vertiefen. Dies kommt auch einer Korrektur von Mills Verständnis dieser Dialoge, das in Abschnitt 7.1 angesprochen wurde, gleich.

Ursula Wolf fasst die Frage (oder zumindest eine sehr wichtige der Fragen), um die es in den Frühdialogen geht, als Frage danach, wie das Wissen vom Guten - das Wissen um die menschliche are$t e^{71}$ - beschaffen ist. ${ }^{72}$ Heute würde diese Frage als Frage der Metaethik bezeichnet. Ich glaube aber, dass das eigentliche Problem zum Beispiel bezüglich Expertise nicht auf Metaethik beschränkt

69 Und, was nicht der Witz ist: Mill lehnt den „Platonismus“, der darin zum Ausdruck kommt - und natürlich den Begriff „Platonismus" dafür, siehe Abschnitt 7.1 - ab und schätzt etwas anderes. Siehe Mill, Autobiography, S. 151ff.

70 Siehe Wolf, Die Suche nach dem guten Leben. Platons Frühdialoge.

71 Ich werde im Folgenden, wenn ich mich auf die Dialoge inhaltlich beziehe, von der menschlichen arete reden, damit keine Verwechslung mit Mills philosophischen Tugenden, um die es in diesem Abschnitt natürlich ebenfalls geht, eintritt.

72 Siehe Wolf, Die Suche nach dem guten Leben. Platons Frühdialoge, S. 23-26. 


\section{Expertise und die Tugenden}

ist $^{73}$, sondern in der Besonderheit der philosophischen Auseinandersetzung mit beliebigen Fragen liegt - zumindest insofern diese Auseinandersetzung durch das, was ich Mills Tugenden genannt habe, strukturiert ist (siehe zu dieser Einschränkung Abschnitt 2.2, insbesondere der Punkt zur Nicht-Exklusivität).

Die Gedanken, die ich im Folgenden präsentieren werde, beziehen sich auch auf die Dialogform. Ich werde dafür argumentieren, dass sich das Spannungsverhältnis zwischen Mills philosophischen Tugenden und ihr Verhältnis zueinander als einer der Gründe für diese Form auffassen lassen. Die Dialogform bietet nämlich die Möglichkeit, rational - insofern die Dialoge nach Gründen suchende Prüfungsgespräche sind - zwischen den Tugenden zu vermitteln, ohne dass damit der Anspruch erhoben wird, es könne ein Verfahren, eine techne, angegeben werden, wie richtigerweise zwischen den Tugenden zu vermitteln sei. Könnte eine techne angegeben werden, gäbe es natürlich Expert*innen.

Selbstverständlich glaube ich nicht, dass das Spannungsverhältnis zwischen Mills Tugenden alles sind, was sich aus Sicht einer platonischen Philosophie für die Dialogform anführen lässt: Ein aus meiner Sicht plausibler Zugang zu dieser Frage findet sich im „Siebten Brief" Platons ${ }^{74}$, ganz gleich, ob er nun echt ist oder nicht. ${ }^{75}$ Natürlich ist der „Siebte Brief“" selbst eher dunkel und musste für alle möglichen Interpretationen herhalten. ${ }^{76}$ Trotzdem glaube ich,

73 Gewissermaßen könnte gesagt werden, das gilt auch so für Ursula Wolf; die Frage nach dem guten Leben ist bei ihr (und bei ihrer Platondeutung) in gewisser Hinsicht die Grundfrage der Philosophie (Siehe z. B. Ursula Wolf. Die Philosophie und die Frage nach dem guten Leben. Reinbek bei Hamburg: Rowohlt Taschenbuch Verlag, 1999, S. 22) und insofern gilt das Gesagte auch für die restliche Philosophie. Ich will hier etwas Schwächeres: Weder möchte ich diese These in dieser Arbeit vertreten noch bestreiten.

74 Siehe Kenneth Sayre für einen Vorschlag dieses Zusammenhanges: Sayre, "Plato's Dialogues in Light of the Seventh Letter".

75 Siehe ebenfalls Sayre mit einem Argument dafür, dass das nicht die entscheidendste Frage in diesem Zusammenhang ist.

76 Am berühmtesten ist die unter anderem mit den Bemerkungen aus dem "Siebten Brief" begründete Behauptung einer „esoterischen Lehre" Platons, (z. B. Konrad Gaiser. Platons ungeschriebene Lehre. Studien zur systematischen und geschichtlichen Begründung der Wissenschaften in der Platonischen Schule. Stuttgart: Klett, 1968, S. 3f) gegen die aber spricht, dass Platon explizit nicht nur von geschriebener Sprache, sondern von Sprache allgemein spricht - auch wenn Verschriftlichung vielleicht als noch schlimmer 
dass sich die Spannung zwischen den Tugenden dort wiederfindet, auch wenn, ich betone es noch einmal, dies nicht alles ist, worum es in dem Brief geht: Eines der Probleme, das die im Gegensatz zu anderen Wissensbereichen ${ }^{77}$ einen Zusammenhang zwischen fünf Elementen in den Blick nehmende Philosophie hat, ist, dass sie zwischen der Konventionalität der Wörter und Begriffe (und abgeleitet der so ausdrückbaren Wahrheiten $)^{78}$ und der Frage, wie die Dinge von dieser Konventionalität unabhängig sind, ${ }^{79}$ vermitteln muss. Ich will aber nicht behaupten, dass der Quietismus des „Siebten Briefes" aus Mills Tugenden folgt, sondern nur, dass die Dialogform eine Art und Weise ist, zwischen den Tugenden zu vermitteln: Schon aus formalen Gründen kann ein Versuch, mit der Angabe von Mills Tugenden einen sprachlichen Ausdruck von etwas zu geben, um das es neben anderen Dingen in der Philosophie geht, sich dem Quietismus der im siebten Brief offenbar angedeutet wird, ohnehin nicht vollständig anschließen, ohne einen performativen Widerspruch ${ }^{80} \mathrm{zu}$ begehen. Es gibt aber auch noch einige andere Gründe, die dagegen sprechen. ${ }^{81}$

angesehen wird. (Siehe Ursula Wolf, Hrsg. Platon. Sämtliche Werke, Band 3. 37. Aufl. Reinbek bei Hamburg: Rowohlt Taschenbuchverlag, 2013, „Siebter Brief" 341c-d.

77 Siehe ebd., "Siebter Brief" 341c.

78 Siehe ebd., „Siebter Brief“ 343b.

79 Siehe ebd., „Siebter Brief“ 342c, 343c-e.

80 Die Frage des performativen Widerspruches ist insofern interessant bezüglich der Frage der Echtheit des „Siebten Briefes", dass er, wenn man die Bemerkungen über Philosophie für Philosophie hält, es nicht stimmt, was dort behauptet wird, nämlich, dass Platon sich nie in eigener Person wahrhaft philosophisch geäußert habe; wenn der Brief hingegen nicht echt ist, ist es möglich dass diese Aussage zutreffend ist.

81 Im Anschluss an Cora Diamonds und James Conants resolute Lesart von Wittgenstein wird oft zwischen einer mystischen und einer nicht-mystischen (resoluten) Lesart des Quietismus Wittgensteins unterschieden. Nach der mystischen Lesart weist zum Beispiel Wittgensteins Tractatus auf etwas hin, das aber nicht gesagt werden kann. Nach der resoluten Lesart ist die Annahme von einem solchen etwas selbst die philosophische Illusion. Erstmalig siehe Cora Diamond. "Throwing Away the Ladder". In: Philosophy 63.243 (1988), S. 5-27. Einen Überblick über die Literatur liefert Silver Bronzo. "The Resolute Reading and Its Critics. An Introduction to the Literature". In: Wittgenstein-Studien 1.3 (2012), S. 45-80. Wenn es erlaubt ist, einen Versuch der Anwendung dieser Unterscheidung auf die Position im „Siebten Brief" zu wagen, so scheint es mir naheliegend - auch wenn ich es nicht für 
Da diese Arbeit aber keine Platonexegese ist - ein solcher Anspruch wäre vermessen -, möchte ich es bei diesen Andeutungen belassen. Ich möchte mich darauf beschränken, darauf hinzuweisen, dass die folgenden Ausführungen zu den Frühdialogen sich, obwohl sie ein viel weniger ambitioniertes Ziel haben, sich einpassen lassen in ein Gesamtbild von Platons Philosophie ${ }^{82}$, ohne dass ich hier für dieses Bild argumentieren könnte. Stattdessen werde ich die in den Frühdialogen und Wolfs Lesart dieser Dialoge wichtigen Fragen, die schon in Mills Auseinandersetzungen mit Platon angedeutet wurden, darstellen und erarbeiten. Dies waren die Fragen der Expertise, Bezahlbarkeit und Lehrbarkeit der menschlichen arete und der reflexiven Auseinandersetzung mit der menschlichen arete. Dabei werde ich versuchen nachzuweisen, dass die Spannung zwischen den Tugenden eine legitime Rekonstruktion von einem der Gründe für die aporetischen Enden der Dialoge in diesen Fragen ist.

Natürlich ist es problematisch, heutige Kategorisierungen auf antike philosophische Texte zu übertragen ${ }^{83}$ wir laufen damit Gefahr, den gleichen Fehler zu begehen, wie ich ihn Mill in Abschnitt 7.1 vorgeworfen habe. Natürlich glaube ich nicht, dass ich diesen Fehler begehe, sondern dass das, was ich sage, wirklich Teil dessen ist, um was es in den Frühdialogen geht. Warum ich überhaupt das Risiko eingehe, habe ich in der Einleitung zum Kapitel nahegelegt; es geht hauptsächlich darum, dass ich glaube, dass heutige Debatten in der Metaethik sich zu schnell auf den Hintergrund der Frage, ob der Szientismus zutreffend ist und die Naturwissenschaften die Welt vollständig beschreiben, beziehen. Obwohl dies auch eine interessante philosophische Frage ist, verdunkelt sie ein wichtiges Problem der Ethik jedoch. Genau dieser Punkt, der sonst verdunkelt wird, ist aber für das, was ich über Mills Tugenden in diesem

ausgemacht halte (vgl. als Gegenargument zum Beispiel 341e) -, dass der Quietismus im "Siebten Brief“ mystisch ist, dass der vorgebliche Autor des „Siebten Briefes" also meint, es gebe in der Philosophie etwas irgendwie aufzufassen, das aber nicht sprachlich ausdrückbar sei. Gerade die Frühdialoge, auf die ich mich im Folgenden beziehe, lassen sich aber auch nicht-mystisch lesen, so meine ich.

82 Wie es zum Beispiel von Sayre angedeutet wird: Sayre, „Plato's Dialogues in Light of the Seventh Letter".

83 Neben vielen anderen weist Elizabeth Anscombe darauf hin. Siehe G. Elizabeth M. Anscombe. „Modern Moral Philosophy“. In: Philosophy 33.124 (1958), S. 1-19, S. 1-2. 
Kapitel sagen will, entscheidend. Anders ausgedrückt: gerade weil sich bestimmte metaethische Diskurse des 20. Jahrhunderts nicht ohne Widerstände auf die Frühdialoge übertragen lassen, sind sie für mich fruchtbar. Ich versuche Kapital aus einigen der Stellen zu ziehen, wo sich die Übertragung schwierig gestaltet. Natürlich kann ich verstehen, dass es trotzdem Unbehagen dabei geben mag, Texte der Antike zur weiteren Ausarbeitung einer Position dazu, was gute Philosophie ist, die aus dem 19. Jahrhundert stammt, zu verwenden. Die Leser*innen, bei denen das der Fall ist, bitte ich daher, wenn ich zum Beispiel „Platons Sokrates“ schreibe, einfach "Sokrates nach Wolfs Platon" zu lesen und analog bei allen anderen Referenzen auf Platon oder einen entsprechenden Text - in der Hoffnung, dass die kommunikative Kluft zwischen Mill und Wolf von diesen Leser*innen als geringer empfunden wird, als die zwischen Mill und Platon.

In den Frühdialogen wird die Frage nach dem Wissen vom Guten auf verschiedene Weise thematisiert, unter anderem eben über die drei von mir bereits angesprochenen Zugangsweisen:

1. über die Frage nach der Lehrbarkeit der menschlichen arete, ${ }^{84}$

2. über die Frage nach der Bezahlbarkeit (oder anderen Formen von externen Maßstäben) von Rat oder Lehre der menschlichen arete, ${ }^{85}$

3. über die mit den beiden vorherigen Fragen verbundene Frage nach Expertise in Bezug auf die menschliche $\operatorname{arete}^{86}$.

84 Im „Protagoras“ und im „Menon“ ist dies die Ausgangsfrage für den Dialog. In der "Apologie" ist einer der zurückgewiesenen Vorwürfe an Sokrates, er gebe vor, die menschliche arete zu lehren. Diese und die anderen in dieser Aufzählung genannten Dialoge befinden sich in Wolf, Platon. Sämtliche Werke, Band 1.

85 Im „Gorgias" wird, wie bereits angedeutet, diese Frage direkt angesprochen. Im „Protagoras“ fragt Sokrates auch am Anfang den Hippokrates nach dem (externen) Wert (und der Gefahr) der sophistischen Kunst. Sie wird dort auch verglichen mit (anderen) Künsten, die einen solchen Tauschwert haben. In der „Apologie“ weist Sokrates wieder den Vorwurf zurück, Geld für seine Tätigkeit genommen zu haben.

86 Dies ist Thema in sehr vielen der Dialoge. Außer den in den letzten beiden Fußnoten bereits genannten, werde ich mich hauptsächlich auf „Hippias II“ beziehen. 
Die Grundidee in Wolfs Interpretation der Frühdialoge ist jeweils, dass, wenn es offensichtliche Unterschiede zwischen der Lehrbarkeit, der Bezahlbarkeit, der Anerkennung von Expertise in der menschlichen arete auf der einen Seite und anderen Formen des Wissens oder Könnens auf der anderen Seite gibt, dies auf einen Unterschied zwischen der menschlichen arete und diesen Formen des Wissens oder Könnens hindeutet. Dies werde ich im Folgenden versuchen auszuarbeiten. Dabei werde ich mich hauptsächlich auf Textstellen aus dem „Protagoras“, dem „Hippias II“, dem „Menon“ und dem „Ion“ und auf Ursula Wolfs Aufarbeitung dieser Dialoge beziehen.

\subsubsection{Verortung von Benthams Kritik an gängiger philosophischer Moralauffassung im Orakelspruch}

Beginnen möchte ich jedoch mit dem Orakelspruch aus der „Apologie“, erstens, weil hier die Zurückweisung einer bestimmten Form von Expertise in Sokrates zum, wenngleich manchmal missverstandenen, geflügelten Wort geworden ist; zweitens, weil in der „Apologie" sehr schnell die Ambivalenz oder Differenziertheit der Antworten auf die drei Fragen nach Expertise, Bezahlbarkeit und Lehrbarkeit deutlich wird. Der dritte Grund ist, dass auch Mill, wie im letzten Abschnitt deutlich wurde, diese Textstelle bespricht (und fehldeutet). Die nach heutigen Maßstäben fiktive Verteidigungsrede von Sokrates verteidigt ihn (unter anderem) gegen die drei Aspekte, die hier als Vorwürfe formuliert werden: Sokrates habe den Anspruch, die Jugend in der menschlichen arete zu unterrichten, so wie dies zum Beispiel Euenos tue, ${ }^{87}$ er habe den Anspruch, Expertise in okkultem Wissen und Können zu besitzen, ${ }^{88}$ wie es ihm in der Aristophanes-Komödie vorgeworfen werde, ${ }^{89}$ und er rede der Jugend ein, ihr mit diesem Wissen oder Können etwas von

87 Siehe Wolf, Platon. Sämtliche Werke, Band 1, "Apologie", 20C.

88 Vgl. die Zurückweisung dieses Anspruches mit einem von zwei im „Siebten Brief" erwähnten Missverständnissen, die mit dem Versuch eines direkten sprachlichen Ausdrucks philosophischer Thesen laut dem „Siebten Brief" unweigerlich verbunden wären: „die anderen aber [würde es] mit einem hochfliegenden und törichten Dünkel [erfüllen], als haben sie irgendwelche erhabenen Wahrheiten begriffen." Wolf, Platon. Sämtliche Werke, Band 3, „Siebter Brief", 341e.

89 Siehe Wolf, Platon. Sämtliche Werke, Band 1, „Apologie“, 19C. 
(Tausch-)Wert zu vermitteln. ${ }^{90}$ Die Ambivalenz oder Differenziertheit der Antwort, Tugend sei nicht lehrbar oder es gebe keine Expert*innen, die in der "Apologie“ deutlich wird, besteht darin, dass Sokrates natürlich sehr wohl als Lehrer oder Erzieher dargestellt wird, er einen Nutzen für die Athener*innen hat ${ }^{91}$ und eine Art Vorbild in der Suche nach der menschlichen arete sein soll - wenn auch nicht in der Weise, in der die Sophist*innen die Tugend unterrichten, sich für diesen "Nutzen“ bezahlen lassen und sich für Expert*innen im Bereich der Tugend halten. Aber ganz zweifellos, so zieht es sich durch die Frühdialoge, ist es besser, mit Sokrates in dieser Frage zu reden als zum Beispiel mit Hippias.

Gleich zu Beginn der „Apologie“ werden drei Vorstellungen vom Wesen des Wissens vom Guten zurückgewiesen - so kann die Prüfung des Orakelspruchs bei den Politiker*innen, den Dichter*innen und den Handwerker*innen jedenfalls verstanden werden. ${ }^{92}$ Die Politiker*innen, Dichter*innen und Handwerker*innen verstehen das ethisch Gute nämlich jeweils in einer Weise, die dem eigenen Tätigkeitsbereich entspricht. Sokrates' Zurückhaltung in Bezug auf eine metaethische Vorstellung macht ihn weiser als die anderen - und bestätigt damit den Orakelspruch -, weil jede der Vorstellungen, die durch die drei paradigmatischen Personengruppen repräsentiert werden, falsch ist.

Die Prüfung des Orakelspruchs ist sehr kurz und es werden daher nur drei eher allgemeine Richtungen angedeutet, wie man das Gute

90 Sokrates weist dort explizit darauf hin, dass er „in tausendfältiger Armut“ lebe und von den Söhnen „der reichsten Bürger" nicht bezahlt werde für seine Gespräche mit ihnen und nicht behaupte, ihnen etwas von (Tausch-)Wert zu vermitteln. Siehe ebd., „Apologie“, 23A-C.

91 Siehe ebd., "Apologie“, 28A-33B.

92 Dies setzt voraus, dass das kalon kagathon, das Schöne und Gute, sich in der "Apologie" (wie in den anderen Frühdialogen) hauptsächlich auf das ethisch oder praktisch Gute bezieht. Dies anzunehmen gibt es gute Gründe - nicht zuletzt die Textstellen unmittelbar vor der Prüfung, in welcher Sokrates den Vorwurf, Menschen zu guten Exemplaren ihrer Art machen wollen, zurückweist. Siehe ebd., „Apologie“, 19d-20c. Die Parallelisierung dieses Vorwurfs mit dem Vorwurf, Sokrates betreibe eine okkulte Kunst oder Wissenschaft, (siehe ebd., „Apologie“, 19b-20c) legt bereits nahe, was ich weiter unten genauer ausführen werde, dass nämlich eine Technik oder Wissenschaft davon, Menschen zu guten Exemplaren ihrer Art machen zu wollen, auch eine Art okkulte Kunst oder Wissenschaft sein müsste. Vgl. Wolf, Die Suche nach dem guten Leben. Platons Frühdialoge, S. 33-40. 
nicht verstehen sollte: Es versteht sich erstens von selbst, dass man jeweils sehr viel raffiniertere Positionen in jeder der drei Richtungen formulieren könnte. Zweitens bleibt uns Platon an dieser Stelle noch schuldig, was gegen die einzelnen Positionen spricht. Trotzdem will ich die Positionen kurz formulieren und mit den Tugenden in Verbindung bringen. Die Handwerker*innen setzen die Ethik mit anderen Bereichen unseres Handelns, in welchen unser Tun nämlich auf Wissen oder Fähigkeit beruht, gleich: sie verstehen nicht, dass unser Zugang zum Guten (die Weisheit, um die es hier geht) weder die Struktur einer episteme noch einer techne ${ }^{93}$ hat - weder ist ethische Weisheit (wissenschaftliches) Wissen, noch praktisches Wissen im Sinne von Fähigkeiten, Fertigkeiten, Kompetenzen, etc. Auf der anderen Seite besteht ethische Weisheit auch nicht einfach im für gut Halten - weder im individuellen, noch im kollektiven: Die Politiker*innen werden nämlich bei der Prüfung des Orakelspruchs einzig dadurch charakterisiert, dass sie von sich selbst und von vielen anderen für ethisch weise gehalten werden. Schließlich gibt es noch die Dichter*innen. Interessanterweise gesteht Sokrates ihnen zu, dass vieles von dem, was sie schreiben, Ausdruck des Guten ist. Allerdings qualifizieren sie sich nicht zu ethischer Weisheit, weil sie nicht in der Lage sind, transparent zu machen, warum das, was sie über Menschen in ihren Tragödien sagen, jeweils mit dem Guten (oder Schlechten) zu tun hat. Anders als die Politiker*innen stehen sie in der „Apologie“ aber nicht einfach für die Position, dass das Gute in bloßen Meinungen besteht, die nicht weiter begründet werden können. Vielmehr gibt es im Guten richtig und falsch weitgehend unabhängig davon, was Menschen meinen, aber der Weg dorthin besteht in Inspiration, deren Unerklärlichkeit im Falle der Dichter*innen diese davon disqualifiziert, sich Weisheit oder Wissen zuschreiben zu dürfen, und der anderenorts durch den Zusatz "göttlich“ charakterisiert wird. ${ }^{94}$

93 Dazu, dass episteme und techne, was diese Frage angeht, fast in den gesamten Frühdialogen als mehr oder weniger austauschbar behandelt werden, siehe Wolf, Die Suche nach dem guten Leben. Platons Frühdialoge, S. 34. Zumindest in der häufig verwendeten Formel techne kai episteme.

94 Siehe Wolf, Platon. Sämtliche Werke, Band 1, „Apologie“, 21e-22c; siehe ebd., „Ion“, 534d-535a; vgl. Wolf, Die Suche nach dem guten Leben. Platons Frühdialoge, S. 52-58. 
Auch wenn ich auf die Problematik der Rekonstruktion antiker Texte mithilfe moderner Unterscheidungen bereits hingewiesen habe, möchte ich die drei Positionen zu den beiden Tugenden ins Verhältnis setzen. Nur die Handwerker*innen beanspruchen überhaupt, die platonische Wissensdefinition zu erfüllen; nur sie erheben den Anspruch, dass ethisches Wissen nicht nur wahr, sondern auch begründet sein müsse. Auf den ersten Blick könnte also angenommen werden, sie stünden für Benthams Tugend. Coleridges Tugend könnten wir dann versuchen mit den Dichter*innen oder den Politiker*innen zu identifizieren, je nach dem, ob wir betonen, dass es um einen guten sprachlichen Ausdruck unserer Praxis geht, oder ob wir betonen wollen, dass es um einen guten sprachlichen Ausdruck unserer Praxis geht. Dass in Coleridges Tugend etwas zusammengefasst wird, was nach diesem Übertragungsversuch in den Frühdialogen differenziert wird, braucht uns in einer Hinsicht nicht zu wundern: Es wurde bereits angesprochen (zum Beispiel in Fußnote 32 aus Kapitel 4), dass Bentham alle Gegenpositionen, die seiner Meinung nach nicht der asketischen Ethik zuzuordnen sind, unter der Ethik der „Sympathie und Antipathie“ zusammenfasst - und damit auch so unterschiedliche ethische Positionen wie den intuitionistischen Kognitivismus und emotivistischen Nonkognitivismus, denen er beiden vorwirft, konventionalistisch zu sein. Die Rationalisierung dieses Zusammenfassens in den Begriffen des Orakelspruches könnte lauten: Beide ethischen Ansätze haben den Fehler, dass sie Ethik zu wenig wie die Handwerker*innen verstehen. Mill übernimmt, wie ebenfalls bereits erwähnt, (zum Beispiel Fußnote 21 in Kapitel 6) Benthams Klassifizierung und fasst dementsprechend sehr viel unter dem von ihm dafür bevorzugten Begriff des Intuitionismus. Entsprechend der Rede von den Tugenden könnten wir nach diesem Übertragungsversuch die Kritik an den drei Vorstellungen der "Apologie" also auch umdrehen und sagen: Das richtige Bild der Ethik muss ein bisschen so sein, wie das Bild der Politiker*innen, ein bisschen so, wie das Bild der Handwerker*innen und ein bisschen so, wie das Bild der Dichter*innen.

Nach diesem Bild liegt es nahe, zu meinen, die Sophist*innen, die in gewisser Weise als die Handwerker*innen (oder Wissenschaft- 
ler*innen) der Ethik dargestellt werden ${ }^{95}$, seien mit Benthams Tugend zu identifizieren. Und dies zeigt, so will ich jedenfalls im Folgenden argumentieren, warum dieser erste Übertragungsversuch, obwohl er ein paar Dinge deutlich macht, nicht ganz stimmt. Die eigentlichen Gründe dafür werde ich im Folgenden nennen. Zunächst sei aber nur darauf hingewiesen, dass dies ungefähr das konservative Bild der Sophist*innen wäre, gegen das Mills sich wehrt: Oft, so beobachtet Mill, ist es nicht Sokrates, der eine konventionelle Moralauffassung vertritt, sondern die Sophist*innen. ${ }^{96}$ Für den Anfang aber ist dieses Bild, obwohl es nicht ganz zutreffend ist, ein guter Ansatzpunkt.

\subsubsection{Der Aufbau des „Protagoras“ und Mills Tugenden}

Ich hatte gesagt, die Dialogform sei eine Möglichkeit zwischen den Tugenden rational zu vermitteln, ohne behaupten zu müssen, diese Rationalität könne dadurch nachgewiesen werden, dass eine Methode - eine techne - angegeben würde. Dies möchte ich nun im „Protagoras" aufzeigen, natürlich kann ich dabei nicht auf alles eingehen, was diesen vielschichtigen Dialog ausmacht.

Der Anlass des Gesprächs, das im „Protagoras“ von Sokrates nachträglich erzählt ${ }^{97}$ wird, ist, dass ein junger Sokrates von Hippokrates, einem noch jüngeren Athener, darum gebeten wird, bei Protagoras ein gutes Wort für ihn einzulegen, damit dieser Hippokrates in der sophistischen Kunst unterrichte. Sokrates, der sich etwas skeptisch bezüglich des Sinnes einer solchen Ausbildung gibt, erklärt sich etwas widerstrebend bereit. Da aber noch Zeit ist, befragt Sokrates den Hippokrates, was er sich unter der Ausbildung

95 In der hier diskutierten Textstelle wird das in der bereits angeführten Person des Euenos (siehe Wolf, Platon. Sämtliche Werke, Band 1, „Apologie“, 20b-c) deutlich. Später werde ich noch einige weitere entsprechende Stellen diskutieren.

96 Siehe Mill, „Grote's Plato“, S. 400-403. Dies schreibt Mill in „Utilitarianism“ beispielsweise auch über Protagoras, gegen den Sokrates die utilitaristische Lehre verteidigt habe. Siehe Mill, „Utilitarianism“, § 1.1 und etwas differenzierter in Mill, „Grote's Plato“, S. 417f. Ich selbst werde weiter unten diesen Dialog explizit ansprechen.

97 Dies ermöglicht Platon, Sokrates' (zum Teil taktische) Gedanken zur Gesprächsführung (siehe Wolf, Platon. Sämtliche Werke, Band 1, „Protagoras“, 339c, 339e) und den großen Eindruck, den Protagoras' Ausführungen zur Lehrbarkeit zunächst auf ihn machen, (328d) explizit zu machen. 
vorstellt und was er sich davon verspricht. Hippokrates, der ja offenbar noch nicht in der sophistischen Kunst unterrichtet ist, wird so gezwungen der sophistischen Kunst externe ${ }^{98}$ Gründe für die Beschäftigung mit dem Gegenstand der sophistischen Kunst auf die Weise, wie sich die Sophist*innen mit ihm beschäftigen zu geben. Keiner dieser Gründe ist natürlich überzeugend. ${ }^{99}$ Es geht sozusagen um Gründe, die nach Benthams Tugend akzeptabel wären: Sokrates weist nämlich auf die Gefahr (für die Seele) von einer bloß auf dem Erwerb einer Innenperspektive, also Konversion beruhenden Gründen für die Aufnahme einer solchen Lehrtätigkeit hin: Es gibt nämlich anders als bei anderen Produkten nicht die Möglichkeit, diese von Expert*innen überprüfen zu lassen. ${ }^{100}$

Vordergründig hat das damit zu tun, dass die Konversion die Seele betrifft, wie Sokrates ebenfalls dort sagt, und möglicherweise schon vor einer möglichen Prüfung irreparablen Schaden angerichtet hat. Andererseits wird hier sicher schon vorweggenommen, was wenig später Thema ist, nämlich dass es in Fragen der menschlichen arete, die Protagoras als den Gegenstand seiner Lehre benennt, nicht wie in anderen Bereichen Expert*innen gibt: Im Bauwesen und Schiffswesen - das sind die Beispiele, die genannt werden - ist so etwas wie Expert*innentum möglich und dies hat auch damit zu tun, dass in diesen Künsten die Produkte von Außenstehen-

98 Ich gebrauche das metaphorische Wort „extern" hier in der gleichen Weise, wie in der Formulierung „externer Maßstab" aus Abschnitt 6.1.3.

99 Siehe Wolf, Platon. Sämtliche Werke, Band 1, „Protagoras“, 311b-312e. Hippias schlägt zum Beispiel vor, ein Sophist*in sei besonders klug oder gut im Reden. Sokrates erwidert, dass dies aber auf jede Expert*in in Bezug auf ihren Gegenstand zutrifft. Hippias' Charakterisierungen der sophistischen Kunst lassen sich sicherlich auf manches Übertragen, was auch heute manchmal über die Nützlichkeit einer formalen philosophischen Bildung oder der entsprechenden Institutionen gesagt wird: Begriffliches Differenzierungsvermögen, eine kritische Einstellung gegenüber mit bloßer Autorität vorgetragenen Thesen u.s.w. Vgl. den Anfang von Raimond Gaita. „Callicles' challenge“. In: Critical Quarterly 47.1-2 (2005), S. 40-52; im Rest des Aufsatzes scheint mir Gaita zu sehr darauf zu setzen, dass der innere (der Coleridges Tugend entsprechende) Wert der universitären (auch philosophischen) Lebensform sich durch die auctoritas der so Lebenden auch den nicht so Lebenden einfach zeigen könne, wenn wir uns nur auf das besönnen, was die Universität ausmacht.

100 Siehe Wolf, Platon. Sämtliche Werke, Band 1, „Protagoras“, 313a-314c. 
den zumindest im Prinzip bewertet werden können. ${ }^{101}$ (Im letzten Abschnitt hatte ich diesen Punkt gemacht, indem ich darauf hingewiesen habe, dass „Expert*in“ in diesem Sinne einen besonderen sozialen Status bezeichnet, der gegenüber Außenstehenden gerechtfertigt werden können muss.) Die Frage nach der Bezahlung für philosophische beziehungsweise sophistische Tätigkeit als paradigmatischem externen Bewertungsmaßstab wird im Gegensatz zu anderen Dialogen im "Protagoras" nur angedeutet: es ist zwar klar, dass Hippokrates für seine Lehre bezahlen will und dies ist auch der Anlass für Sokrates' Fragen nach dem, was sich Hippokrates von Protagoras im Gegenzug verspricht ${ }^{102}$, aber Protagoras umgeht den pragmatischen Aspekt dieses Problems geschickt, indem er es der (ehrlichen) Einschätzung derer überlässt, die er ausbildet, wie viel sie ihm zahlen. ${ }^{103}$

Es wurde schon in Abschnitt 6.1.3 darauf hingewiesen, wie Benthams Tugend mit externen Bewertungsmaßstäben zusammenhängt und auch darauf, dass es nicht reicht, den Wert einer Praxis nur darin zu begreifen, was die daran Teilnehmenden als das sehen, um was es in dieser Praxis geht. Die Gefahr für Hippokrates ist, dass er schlechte (und für die Seele gefährliche) Ware erhält, ohne es zu bemerken; er wäre in der gleichen Situation wie eine Person, die Coleridges Argumentation dafür, dass die Mysterien des Christentums wesentlich für das Christentum sind, zum Anlass nimmt, von einer Atheist*in zur Christ*in zu werden. (Ausgeführt wurde dieser Gedanke in Abschnitt 3.3.2.) Bereits der Auftakt des Dialoges hat also mit Fragen zu tun, um die es auch in dieser Arbeit geht.

Der Rest des Dialoges behandelt die Frage nach der Lehrbarkeit und im Zuge dessen nach dem Wesen der menschlichen arete. Sokrates bringt am Anfang und im ersten Teil des Dialoges seinen Skeptizismus gegenüber der Kunst, die menschliche arete zu lehren, die Protagoras von sich beansprucht, zum Ausdruck, indem er

101 Siehe Wolf, Platon. Sämtliche Werke, Band 1, „Protagoras“, 319b-d. Um die Verwandtschaft der techne zur episteme herzustellen, die in Bezug auf die Frage ihrer Opposition zum Wissen vom Guten, wie bereits erwähnt, oft gleichbedeutend sind, könnte im Sinne moderner Naturwissenschaften gesagt werden, dass Expertise sich durch die Zuverlässigkeit von Vorhersagen zeigt - unter anderem aber nicht ausschließlich in der technischen Verwertbarkeit naturwissenschaftlichen Wissens.

102 Siehe ebd., „Protagoras“, 311b.

103 Siehe ebd., „Protagoras“, 328b; siehe auch Fußnote 56. 
schlicht bestreitet, dass diese gelehrt werden kann. Am Ende des Dialoges aber scheinen Sokrates und Protagoras (fast ${ }^{104}$ ) die Positionen getauscht zu haben, wie Sokrates bemerkt. ${ }^{105}$ Sie tauschen aber nicht nur die Positionen im Laufe des Dialoges, sie tauschen auch zwischenzeitig die Rollen - und zwar, weil Sokrates meint, Protagoras würde zu ausschweifend antworten. ${ }^{106}$ In diesem Teil ${ }^{107}$ ist es nicht mehr Sokrates, der das elenchos, das Prüfungsgespräch, leitet, sondern Protagoras.

Der Tausch der Gesprächsrollen ist gewissermaßen ein Bruch im Dialog. Dieser Bruch ist die Besonderheit des Aufbaus, auf die ich in diesem Abschnitt hinweisen will. In diesem Teil wird mit einer Gedichtinterpretation, so könnte gesagt werden, auch noch die dritte der Vorstellungen aus der Prüfung des Orakelspruches in der „Apologie“ angesprochen. Auf diesen Teil ${ }^{108}$ werde ich hier nicht eingehen. Wichtiger für meine Zwecke ist, was danach kommt. Der Bruch geht nämlich nicht nur einher mit dem, was einer Vertauschung der Ausgangspositionen gleichkommt, sondern auch mit einer Vertauschung legitimer Argumentationstypen und einer Vertauschung der Vorstellung des techne-Charakters der menschlichen arete und der damit verbundenen Vorstellung eines externen Maßstabes.

Im ersten Teil des Dialoges muss Protagoras natürlich verteidigen, dass die menschliche arete eine Kunst ist, weil sie als Kunst lehrbar ist. Sie ist nur anders als andere Künste, insofern ihr Ziel das friedliche Zusammenleben ${ }^{109}$ ist und so nicht (nur) ihre Besitzer*in, sondern auch alle anderen Menschen ein Interesse an der

104 Sokrates sagt nicht wirklich, dass er die Tugend nun für lehrbar hält; er sagt aber, dass, wenn es stimmt, dass Klugheit einer Messkunst (siehe unten) gleichkäme, Tugend am ehesten lehrbar wäre. Siehe ebd., „Protagoras“, 361ac.

105 Siehe ebd., „Protagoras“, 361a-c.

106 Siehe ebd., „Protagoras", 334c-338e.

107 Siehe ebd., „Protagoras", 338e-348c.

108 Siehe ebd., „Protagoras", 338e-348c.

109 Siehe ebd., „Protagoras“, 319d-323a. Im hier geschilderten PrometheusMythos, so wie Protagoras ihn erzählt, wird die Parallele deutlich: Hermes fragt, ob er Scham und Recht wie die Künste verteilen soll, die Prometheus den Menschen gegeben hat, nämlich ungleichmäßig; Zeus aber meint, dass sie dann ihr Ziel, das friedliche Zusammenleben, nicht erfüllen könnten, und entscheidet sich für eine Verteilung an alle. Wie auch die anderen Künste hat die menschliche arete einen externen Maßstab: das seinerseits für das Überleben notwendige friedliche Zusammenleben. 
Kunstfertigkeit aller anderen Menschen in dieser Kunst haben. ${ }^{110}$ Es ist im ersten Teil des Dialoges an Sokrates, den Kunstcharakter zu hinterfragen und sein „Argument" überrascht vielleicht: Es entspreche nicht der Praxis der Athener*innen, die menschliche arete so zu sehen.

Vorsichtshalber sagt Sokrates noch, dass er die Athener*innen für weise hält. Aber da er selbst Teilnehmer an dieser Praxis ist, könnte gesagt werden, dies zähle nicht viel. Es entspricht aber natürlich meiner Redeweise in der Formulierung von Coleridges Tugend von den kompetenten Teilnehmer*innen einer Praxis. Hier geht es um die staatsbürgerliche Praxis (die Teil der menschlichen arete ist) und wer hier eine kompetente Teilnehmer*in ist, besitzt daher das, worum es hier geht. Sokrates weist die These der Lehrbarkeit zur menschlichen arete also zurück, weil sie Coleridges Tugend nicht entspricht. So darf ich jetzt, glaube ich, reden, ohne dass mir etwas anderes vorgeworfen werden kann, als eine - wenn es mir vorrangig um die Dialoge ginge, zugegebenermaßen seltsame - Terminologie in den „Protagoras" einzuführen. Protagoras, so scheint es zumindest, liefert mit dem Ziel des friedlichen Zusammenlebens, das seinerseits, wie der Mythos zeigt, für das blanke Überleben notwendig ist, einen externen Maßstab für die menschliche arete und ist somit in diesem Teil des Dialoges um Benthams Tugend bemüht. ${ }^{111}$

Im anderen von mir betrachteten Teil ${ }^{112}$ argumentiert Sokrates hingegen explizit gegen die Intuitionen der Menschen ${ }^{113}$. Er vertritt dort die explizit gegen das, was die meisten Menschen denken, gerichtete These, dass niemand wissentlich das Böse tue: „Du weißt aber doch, daß die meisten Menschen [...] sagen, daß viele, welche das Bessere sehr gut erkennen, es doch nicht tun wollen, obgleich sie könnten, sondern etwas anderes tun" ${ }^{114}$ (Diese Passage wird ohne den komplexen Kontext oft zitiert, wenn es darum geht, eine

110 Siehe Wolf, Platon. Sämtliche Werke, Band 1, „Protagoras“, 327a-d.

111 In einer Hinsicht ist nach dem am Anfang dieses Unterabschnittes Gesagten natürlich auch klar, dass es sich um einen externen Maßstab handeln muss: Immerhin müsste Protagoras sonst Konversion vor der Prüfung seiner Lehre verlangen - und davor hatte Sokrates den Hippokrates ja explizit gewarnt.

112 Das heißt, wie gesagt, unter Auslassung der Gedichtinterpretation in der Mitte.

113 Im Laufe des Gesprächs nimmt er die Alltagsvorstellung „der Leute“ dennoch einmal auf, aber nur, um sie ad absurdum zu führen.

114 ,Protagoras", 352d. 
radikale Form der guise-of-the-good-These oder Position, dass Willensschwäche ein Mangel an Wissen sei, zu finden. ${ }^{115}$ ) Dass dies der Praxis der Athener*innen nicht entspricht, spielt hier keine Rolle für Sokrates. Das Argument beruht letztlich darauf, dass die menschliche arete eine techne und episteme sei, weil sie der Messkunst vergleichbar sei, „und wenn sie ein Messen ist, so ist sie notwendig eine Kunst und Erkenntnis".116

Auch Protagoras ändert seine Position zum techne-Charakter der menschlichen arete, wie spätestens aus der Schlussbemerkung von Sokrates bezüglich des Rollentauschs hervorgeht. ${ }^{117}$ Es ist es nicht ganz so offensichtlich, wie bei Sokrates, dass er bezüglich des Argumentationstyps dessen, was eine Praxis den daran Teilhabenden bedeutet, seine Einschätzung ändert. Aber die ganze Passage zur Messkunst des Guten steht im Kontext von Sokrates' Argumentation, dass alle Teile der arete, also Gerechtigkeit, Frömmigkeit, Tapferkeit ${ }^{118}$ gleichbedeutend seien. Hier hat Protagoras zumindest die Position, die den Intuitionen „der Leute" eher gerecht wird.

Sokrates' „Beweis“ der Identität beruht auf dem oben Genannten; darauf nämlich, dass die menschliche arete eigentlich (wie) die Messkunst sei. Natürlich setzt eine Messkunst zu sein voraus, dass es irgendetwas gibt, was gemessen wird. In diese Leerstelle wird Lust und Unlust von Sokrates eingesetzt - obwohl selbst innerhalb des Dialoges nicht ganz klar ist, dass Sokrates diese These selbst vertreten will. Protagoras jedenfalls will sie nicht vertreten; er sagt, „daß es einiges gibt unter dem Angenehmen, was nicht gut, und wiederum unter dem Unangenehmen einiges, was nicht böse ist [... " "119. Wieder vertritt Protagoras wohl die Position, die den Intuitionen der meisten Menschen entspricht. Daraufhin zur Einschätzung zu gelangen, der junge Sokrates habe generell den Utilitarismus (oder zumindest den Hedonismus) verteidigt und sich gegen die etablier-

115 Ich rekonstruiere das genaue Argument hier nicht. Eine ausführliche Auseinandersetzung findet sich hier: Gerasimos Santas. „Plato's Protagoras and Explanations of Weakness". In: The Philosophical Review 75.1 (1966), S. 333.

116 techne kai episteme, „Protagoras“, 357b.

117 Siehe Wolf, Platon. Sämtliche Werke, Band 1, „Protagoras“, 361a-b.

118 Siehe ebd., „Protagoras“, 330a-333d und 349d-351b. Die Argumentation von Sokrates hat hier auch einige davon unabhängige Schwächen, auf die Protagoras zum Teil auch hinweist.

119 Ebd., „Protagoras“, 351d. 


\section{Expertise und die Tugenden}

te Meinung, die durch die Sophist*innen vertreten wurde, durchgesetzt, wie Mill es tut ${ }^{120}$, geht natürlich zu weit. Aber Mill hat Recht, dass Sokrates, wenn auch nur in diesem Teil des Dialoges, Benthams Tugend gerecht wird und Protagoras Coleridges Tugend.

Was sich durch die Ausgangssituation schon angedeutet hat, wird ebenfalls durch den besonderen Aufbau des Dialoges gestützt: Es geht in dem Dialog auch um etwas, das ich in dieser Arbeit mit Benthams und Coleridges Tugend bezeichnet habe. Jeder der beiden Gesprächsteilnehmer vertritt in einem der betrachteten Teile die These, die menschliche arete habe einen externen Maßstab (das Überleben der Menschen beziehungsweise Lust und Unlust); jeder der beiden fasst sie in den Passagen, wo das so ist, als techne auf; jeder der beiden bemüht sich im jeweils anderen Abschnitt den Intuitionen zum (ethisch) Guten beziehungsweise dem Witz der ethischen Praxis gerecht zu werden.

Ich hatte gesagt, die Form des Dialoges eignet sich in besonderer Weise, um rational zwischen den Tugenden zu vermitteln, ohne ein Verfahren der Vermittlung angeben zu müssen. Jetzt dürfte deutlich geworden sein, warum das so ist: Im „Protagoras“ werden die Tugenden zu diesem Zweck auf die Gesprächspartner aufgeteilt und zwar durch den Bruch im Dialog sogar so, dass trotzdem beide beide Tugenden vertreten. ${ }^{121}$ Die Spannung zwischen Mills Tugenden

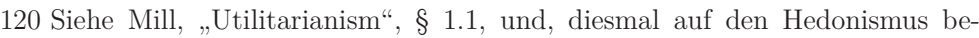
schränkt, Mill, „Grote's Plato“, S. $417 \mathrm{f}$.

121 Ich denke daher auch, dass Platon den Umgang von Sokrates mit Protagoras als respektvoller als den Umgang mit anderen Dialogpartnern intendiert hat und zwar trotz des offensichtlichen Konkurrenzverhaltens und der damit einhergehenden Feindseligkeiten in der Mitte. Darauf deutet auch hin, dass von beiden Philosophen chronologisch im Anschluss an den Dialog Wertschätzung gegenüber der Weisheit der jeweils anderen Person ausgedrückt wird: Protagoras prophezeit Sokrates am Ende, er werde „einst unter die wenigen ihrer Weisheit Berühmten“ (361e) zählen. Sokrates' Wertschätzung kommt schon im Rahmen des ansonsten erzählten Dialoges ganz am Anfang des Textes, der allerdings chronologisch auf das im Text Beschriebene folgt, zum Ausdruck: Er wird am Anfang des Textes von einem Freund, der sieht, wie er strahlt, gefragt, ob er gerade Alkibiades, einem offenbar besonders schönen Jüngling getroffen habe; ,.einen weit schöneren" antwortet Sokrates und meint damit Protagoras: „Wie sollte denn nicht, du kluger Freund, das Weisere immer als das Schönere erscheinen." Wolf, Platon. Sämtliche Werke, Band 1, „Protagoras", 309a-c. Daraufhin erzählt Sokrates von dem Treffen. Es scheint mir daher falsch zu sein, den Bruch in der Mitte und das aporetische Ende als 
drückt sich aus im aporetischen Ende des Dialoges. Philosophieren ist danach zwar der Versuch zwischen Mills Tugenden zu vermitteln, aber es ist nicht so, dass es ein Rezept dafür gäbe: Es kann nicht gesagt werden, dass wir, wenn wir es so machen wie Sokrates und Protagoras, dann eine Anleitung haben, die Spannung zwischen Mills Tugenden aufzulösen. Dass der Dialog in dieser Hinsicht, um den Tugenden gerecht zu werden, eine bessere Form ist als die philosophische Abhandlung wird deutlich, wenn wir uns vergegenwärtigen, dass es in Abhandlungen eben nicht möglich ist, einen solchen Bruch einzuführen: Die Autor*in einer Abhandlung kann nicht einfach wie Sokrates am Ende des Dialoges am Ende der Abhandlung sagen, sie habe ihre Position gewechselt.

Dies ist natürlich einer der Gründe, warum die philosophische Biographie in Bezug auf die Tugenden die Rolle spielen kann, die ich ihr in Abschnitt 5 zuschreibe. Wie auch im Dialog kann in der philosophischen Biographie die Zerrissenheit zwischen philosophi-

Anlass dazu zu nehmen zu denken, der Dialog solle lediglich oder hauptsächlich den Zusammenbruch einer Dialogsituation beschreiben, auch wenn dies in der Mitte, wenn es um Protagoras' lange Antworten und den Streit darüber geht, sicher Thema ist. Für ein solches Verständnis des Dialoges siehe: Charles L. Griswold. „Relying on Your Own Voice: An Unsettled Rivalry of Moral Ideals in Plato's Protagoras". In: The Review of Metaphysics 53.2 (1999), S. 283-307. Es ist außerdem auch nicht so, wie Griswold am Anfang nahelegt, dass es vor allem Protagoras ist, der sich aufgrund von Rivalitätsdenken nicht auf Sokrates' Form des Dialoges einlassen kann. Auch Sokrates denkt stellenweise - insbesondere nachdem er sich bereit erklärt hat, ein Beispiel für gutes Dialogverhalten für Protagoras zu sein - sehr taktisch über die Gesprächsführung. Dies erfahren wir deshalb, weil der Dialog aus Sokrates' Perspektive erzählt ist: Unmittelbar nach dem Rollentausch, in dem ja Sokrates dem Protagoras zeigen soll, wie eine Gesprächteilnehmer*in in dieser Rolle sich richtig verhält, nachdem also Sokrates' in Protagoras Situation ist und im Rahmen des elenchos geprüft wird, wehrt Sokrates eher die Fragen ab, als dass er sich darauf einlässt - und das schon nach der dritten Frage: „Zugleich aber ward mir bange, was er sagte, möchte doch etwas sein, und ich fragte Also dir erscheint es nicht so?" (339c-d) Wenig später lässt er unter dem Vorwand, dem Prodikos, einem der Umstehenden, käme es als Landsmann des Dichters, der hier interpretiert wird, am ehesten zu, diesen $\mathrm{zu}$ verteidigen, diesen antworten - explizit, weil er nicht zugeben will, dass er mehr Zeit zum Nachdenken braucht. (339e) Wenn es also um die Unfähigkeit zur Führung eines sokratischen Gespräches geht, wie Griswold meint, dann ist zumindest Sokrates dazu genauso unfähig wie Protagoras - wenigstens dann, wenn er dessen Rolle im elenchos einnehmen muss. 
schen Sichtweisen und das Bemühen um die Auflösung dieser Zerrissenheit ausgedrückt werden, ohne dass der primäre Punkt wäre, diese Zerrissenheit in einer Position oder einer Methode aufzulösen.

Ich habe bereits gesagt, dass der „Protagoras“ sehr vielschichtig ist und dass damit verbunden ist, dass natürlich mit meinem Abgleich des „Protagoras“ mit Mills Tugenden nicht der Anspruch verbunden ist, den Dialog oder auch nur das Hauptanliegen des Dialoges erfasst zu haben. Gleiches gilt für das aporetische Ende dieses Dialoges und anderer Dialoge Platons: Die Spannung zwischen Mills Tugenden kann durch das aporetische Ende deutlich gemacht werden, aber es ist vielleicht nicht die einzige Spannung oder Zerrissenheit der Philosophie, die so deutlich wird und vielleicht sind Spannung oder Zerrissenheit auch nicht die einzigen wichtigen Metaphern in diesem Zusammenhang. (Dies entspricht natürlich meiner Behauptung der Nicht-Exklusivität der beiden sozialphilosophischen Tugenden aus Kapitel 2.)

Das zweite Eingeständnis, das ich am Ende dieses Abschnittes machen muss, betrifft die Besonderheit des Aufbaus des „Protagoras": Natürlich ist es nicht in allen Dialogen so, dass sich Mills Tugenden so einfach auf beide Gesprächspartner aufteilen lassen noch dazu in einer Weise, die es ermöglicht, zu sagen, beide wären beiden Tugenden in jeweils anderen Teilen des Dialoges gerecht geworden. Trotzdem denke ich, dass sich im Folgenden zeigen wird, dass es auch in vielen anderen Dialogen um das gleiche Thema geht und dass auch dort die Dialogform bedeutet, dass Mills Tugenden jeweils aufgeteilt werden - auch wenn es in der Hinsicht nicht so systematisch geschieht wie im „Protagoras", dass die Tugenden über ganze Textabschnitte zuzuordnen sind. In diesem Zusammenhang ist vielleicht eine Klarstellung erforderlich: Ich will keinesfalls behaupten, dass Platon Begriffe von Mills Tugenden, wenn auch unter anderen Benennungen, gehabt hätte, und seine Dialoge entsprechend geplant hätte; Mills Tugenden sind Begriffe, mit denen ich nachträglich versuche, einem Problem sprachlichen Ausdruck zu verleihen, um das es auch in den Frühdialogen geht.

\subsubsection{Die Handwerker*innen und Benthams Tugend}

Ich hatte weiter oben eine provisorische Zuordnung von Mills Tugenden zu den Positionen im Orakelspruch vorgenommen. Nach 
dieser entsprechen die Handwerker*innen Benthams Tugend - ihre Position ist es, dass das Wissen vom Guten wie handwerkliches oder wissenschaftliches Wissen sein müsse und dass die Reflexionsdisziplin daher die Form einer Kunst oder Wissenschaft habe. Coleridges Tugend teilt sich nach dieser vorläufigen Zuordnung auf die Dichter*innen und Politiker*innen auf, je nachdem, welchen Aspekt wir betonen. Auch die Sophist*innen meinen, dass die Reflexionsdisziplin zur menschlichen arete eine Kunst oder Wissenschaft sein müsse, sie sind in diesem Sinne Handwerker*innen (oder Wissenschaftler*innen) der Ethik; also entsprechen sie nach dieser provisorischen Zuordnung Benthams Tugend. Die Verteilung der Rollen im "Protagoras", die ich in Abschnitt 7.3.3 ausgearbeitet habe, dürfte gezeigt haben, dass es so einfach nicht sein kann: Die Sophist*innen vertreten offenbar nicht immer Benthams Tugend und Sokrates in den Prüfungsgesprächen mit ihnen selbstverständlich nicht immer Coleridges.

Diese Komplikation will ich nun ausarbeiten. Dabei werde ich mich im überwiegenden Teil dieses Unterabschnittes auf die Handwerker*innen und die Abgrenzung von ihnen beschränken. Einmal glaube ich, dass die Position der Politiker*innen in gewisser Weise als unattraktiver erscheinen wird: Immerhin würde sie bedeuten, Ethik sei reduzierbar auf das Konventionelle. Für den Fall, dass systematische Argumente gegen diese Position verlangt werden, verweise ich auf Mills Argumente - zum Beispiel in seiner Auseinandersetzung mit Whewell. ${ }^{122}$ Es bleiben Dichter*innen und Handwerker*innen. Die Dichter*innen, so könnte angenommen werden, entsprechen ungefähr Mills Bild des Intuitionismus: Hier gibt es Wahrheitsansprüche, aber sie können nicht - oder nur durch Referenz auf mysteriöse Mechanismen, d.h. Inspiration oder Intuition - eingelöst werden. Und in der Tat kann die Kritik ähnlich rekonstruiert werden: Im „Ion“ überzeugt Sokrates den Rhapsoden Ion, dass dieser letztlich kein Wissen von dem habe, was er rezitiert, wenn er Homer rezitiert. Über Homer selbst wird keine Aussage in dem Dialog gemacht; aber, wenn die Idee göttlicher Inspiration so $\mathrm{zu}$ verstehen ist, dass sie analog zur Inspiration Ions durch Homer aufzufassen ist, dann hat auch Homer kein Wissen. Und dies ist in

122 Mill, „Whewell on Moral Philosophy“. Siehe auch Abschnitt 6.1.1. 
der Tat sehr analog zu Mills (und Benthams) Kritik am Intuitionismus. (Siehe Abschnitt 6.1.1.)

Der wichtigere Grund ist aber, dass die Zuordnung des Intuitionismus zu Politiker*innen und Dichter*innen einer Fremdbeschreibung Mills dieser Positionen entspricht - und auch Mill ist sich darüber im Klaren, dass der Anspruch ein anderer ist: Intuitionist*innen beziehungsweise diejenigen, die Mill unter diesem Label vereinigt, wollen in der Regel durchaus, dass in der Ethik Gründe angegeben werden. ${ }^{123}$ Und hier liegt der Grund, warum die Handwerker*innen von Interesse sind: Die Behauptung der Handwerker*innen entspricht der Strategie der Parallelisierung der Ethik mit anderen Sichtweisen auf menschliches Tun und Denken, die von einigen der Positionen, die Mill „intuitionistisch“ nennt, durchaus in Form einer Selbstbeschreibung geteilt werden kann. Dies will ich in diesem Anschnitt ausarbeiten und entsprechend wird die Kritik an den Handwerker*innen im Vordergrund stehen. Im Folgenden werde ich diese Strategie kurz skizzieren. Dabei werde ich in sehr groben Zügen vorgehen.

Wenn es darum geht, dass ethische Äußerungen mit Ansprüchen getätigt werden, die über den bloßen Ausdruck einer persönlichen Einstellung hinausgehen, und dass diese Ansprüche zu Recht oder zu Unrecht aufrecht erhalten werden, liegt der Vergleich mit den Wissenschaften (oder den Künsten) nahe - und natürlich ist dies auch ein Motiv in weiten Teilen der metaethischen Debatten im 20. Jahrhundert. Dem liegt zugrunde, dass es nicht besonders kontrovers zu sein scheint, dass und wie Wahrheitsansprüche wissenschaftlicher Äußerungen zu Recht oder zu Unrecht aufrecht erhalten werden können. Die Idee ist dann, dass die Ethik ein bisschen so wie die Wissenschaft (oder die Künste) ist und dass die Spezifizierung dessen, was mit „ein bisschen so wie“ gemeint ist, es erlaubt, gerechtfertigte Wahrheitsansprüche für ethische Äußerungen zu behaupten.

Obwohl es um die Behauptung einer Analogie geht, gibt es in Wirklichkeit zwei Richtungen, die eingeschlagen werden können: Einerseits kann die Analogie so verwendet werden, der Ethik die Glaubwürdigkeit der Wissenschaften zu übertragen, indem gezeigt wird, dass ethische und wissenschaftliche Diskurse ineinander über-

$\overline{123 \text { Siehe Mill, „Utilitarianism“, }} \S 1.4$. 
gehen; dass wissenschaftliche Erkenntnisse ethische Relevanz haben; vielleicht sogar, dass ethische Begriffe letztlich mithilfe wissenschaftlicher Begriffe eingeholt werden können. Auf der anderen Seite kann die Analogie das Spielfeld auch ebnen, indem gezeigt wird, dass die Wissenschaft nicht in einer besseren Situation als die Ethik ist; dass auch in der Wissenschaft kein Blick von der Seite möglich ist; dass auch hier keine absolute Konzeption möglich ist; und dann in der Konsequenz, dass, wenn wir unter diesen Umständen bereit sind, der Wissenschaft weiter Glaubwürdigkeit zuzusprechen, nichts dagegen spricht, auch der Ethik Glaubwürdigkeit zuzusprechen.

Die Richtungen der Analogie sind entscheidend für die Frage, ob die Analogie im Sinne von Benthams oder Coleridges Tugend verwendet wird: In einem Fall wird Wert darauf gelegt, dass sich auch die Ethik an externen Maßstäben zu orientieren habe; im anderen Fall wird gesagt, dass die Behauptung eines externen Maßstabes auch im Falle der Wissenschaften nicht möglich wäre. In einem Fall werden ethische Wahrheitsansprüche, um die es in beiden Fällen geht, also auf externe Maßstäbe reduziert (oder zumindest für sie geöffnet), im anderen werden sie gegen sie immunisiert. Natürlich kann eine so kurze Skizze zweier Strategien niemals fair sein gegenüber den Philosoph*innen, die sie tatsächlich verwenden - und ich habe hier auch nicht den Anspruch. Ich habe kein Interesse, in diese Frage einzugreifen, sondern ihre Gemeinsamkeit - die Analogie, egal in welche Richtung wir sie durchlaufen - in Frage zu stellen. Beiden Strategien gemein ist nämlich, dass Wahrheit und Wissen in der Ethik nicht etwas grundsätzlich anderes ist, als Wahrheit oder Wissen in den Wissenschaften oder Künsten. Ich möchte im Folgenden weitere Gedanken aus den Frühdialogen heranziehen, die diese Analogie in Frage stellen.

Die Vorstellung der Sophist*innen von der Ethik ist also mit der Vorstellung der Handwerker*innen vergleichbar: Sophist*innen sind überspitzt formuliert die Handwerker*innen der Ethik. Im Sinne der oben behaupteten mehr oder weniger vorhandenen Äquivalenz zwischen techne und episteme kann man vielleicht für uns heute auch ergänzen: Sophist*innen behaupten sich so mit Ethik zu beschäftigen wie Physiker*innen mit Physik. Sophist*innen sind also nicht nur die Handwerker*innen, sondern auch die Wissenschaftler*innen der Ethik. Ob diese Analogie immunisierend oder reduzierend 
gemeint ist, spielt zunächst keine Rolle. Weil sie Expert*innen in einer techne sind, sind sie nach eigener Auffassung als Lehrer*innen in der Ethik geeignet. Zumindest in der Art, wie die Sophist*innen Expert*innen in der Ethik sein wollen, gibt es aber in der Ethik keine Expert*innen - und für Lehrbarkeit heißt dies: In der Art und Weise, wie man Physik oder Medizin lehren oder lernen kann, kann man Ethik nicht lehren (und auch nicht lernen).

Bevor ich dieses Thema in den Frühdialogen verfolge, gilt es nun mögliche Missverständnisse aus dem Weg zu räumen: Natürlich will ich nicht bestreiten, noch glaube ich, dass es in den Frühdialogen bestritten werden soll, dass es vorkommt - und sogar häufig ist -, dass wir bei anderen Personen ethischen Rat suchen. Diejenigen, bei denen wir Rat suchen, sind aber zum Beispiel Menschen, von denen wir meinen, dass sie bereits in einer ähnlichen Situation waren wie diejenige, in der wir uns befinden: Ihre Expertise beruht auf dem Schicksal, vor ein ethisches Problem gestellt worden zu sein, das eine Entscheidung von ihnen nötig gemacht hat, und nicht darauf, den Gegenstandsbereich der Ethik besonders gut zu kennen. Dies ist nur ein Beispiel, das verdeutlichen soll, dass nicht alles, was mit „Expert*in" gemeint sein kann, ausgeschlossen ist mit dem, was hier gemeint ist. ${ }^{124}$

Das zweite Missverständnis, das aus dem Weg geräumt werden muss, ist dieses: Natürlich gibt es zum Beispiel soziologische oder psychologische Expert*innen - und diese können moralische Phänomene zum Untersuchungsgegenstand haben. Eine Soziolog*in mag die moralischen Kodizes unsere Gesellschaft besser beschreiben können als ich - und auch eine Therapeut*in mag mir im Sinne einer revealed value-Vorstellung über die Werte, an denen ich mich tatsächlich im Verhalten orientiere, unter Umständen etwas Informatives sagen. Beide könnte man problemlos als Expert*innen bezeichnen. Dass dies nicht das ist, um was es geht, sieht man spätestens daran: Sobald das, was die Psycholog*in oder die Soziolog*in mir sagen, gegen Zweifel abgesichert ist und als Tatsache behandelt werden sollte, ist es wieder eine ethische Frage, was ich damit mache. Diesen eigentlich selbstverständlichen Punkt erwähne ich nur, weil es Soziologie und Psychotherapie zur Zeit der Frühdialoge natürlich noch nicht gab und ich darauf hinweisen möchte, dass nach mei-

124 Siehe Fußnote 66. 
nem Dafürhalten Soziolog*in und Psychotherapeut*in (mehr oder weniger) problemlos den Handwerker*innen im eigentlichen Sinne zugerechnet werden können und nicht den problematisierten Sophist*innen. Sie sind Handwerker*innen und Wissenschaftler*innen der Ethik in einem völlig unproblematischen Bereich.

Ein drittes Missverständnis muss ebenso vermieden werden: Nach dem bisher Gesagten, könnte man die Auffassung vertreten, in der Ethik könne es keine Expert*innen geben, weil Ethik zum Allgemeinwissen (oder zu einem von allen erwarteten Kompetenzbereich) zähle. Genauso wenig, wie der Fragestellung, wie man Hemden mit einem Haushaltsbügeleisen am besten bügelt oder Zwiebeln mit Haushaltsmessern würfelt, eine methodisch geordnete Wissenschaft oder Kunst zugeordnet sei, sei dies eben in der Ethik der Fall. Zweifellos gibt es unzählige Handlungsbereiche, denen eine methodisch geordnete Wissenschaft oder Profession zuzuordnen übertrieben und unplausibel wäre. Nichtsdestotrotz ist dies nicht alles, was mit dem Ausschluss von Expertise an dieser Stelle gemeint ist. Wenn es zum Beispiel Protagoras im gleichnamigen Dialog, den ich ja bereits diskutiert hatte, darauf angekommen wäre, seine Fähigkeit, Zwiebeln zu scheiden oder Hemden zu bügeln, zu einer methodisch geordneten Kunst zu erheben, so würden wir dies vielleicht unnötig oder albern finden. Aber wir hätten sicherlich nicht die Art grundsätzlicher Bedenken, die Sokrates gegen dessen Anspruch, ein Experte in ethischen Fragen zu sein, formuliert.

Das letzte Missverständnis deute ich hier nur an, weil ich es später gesondert ansprechen will: Natürlich will Mill Platon als eine Art (ethisches und philosophisches) Vorbild erscheinen lassen. Sowohl in der Ethik als auch in der Disziplin, die die Ethik reflektiert gibt es Menschen, die es besser machen und Menschen, die es schlechter machen. Auch ich selbst habe ja im ersten Satz dieser Arbeit gesagt, Mill sei ein außergewöhnlich guter Philosoph. Auch dafür möchte ich Raum lassen. Ich will also nur behaupten, dass diese Möglichkeit, es besser und schlechter zu machen, nicht die Form einer Technik oder (wissenschaftlichem) Wissen hat und entsprechend nicht die Form von der Art von Expertise, die damit verbunden ist. Sie hat natürlich stattdessen die Form von Tugenden.

Dies ist auch der eigentliche Punkt, der mir in diesem Abschnitt wichtig ist. Moralisch richtig zu handeln besteht nicht oder zumindest nicht allein in der Ausübung einer Fähigkeit, Fertigkeit oder 
Kompetenz - sei diese nun einer methodisch geordneten Kunst zuzuordnen oder nicht. Ich möchte nun kurz auf die Gründe eingehen, die dagegen sprechen, Ethik als Kompetenz, Technik, Fähigkeit, Fertigkeit zu verstehen. Dies wird en passent auch deutlich machen, was hier mit Fähigkeit, Fertigkeit oder Kompetenz gemeint ist - denn wie fast immer gibt es akzeptierte Verwendungsweisen dieser Wörter, die dem, was ich hier für Ethik ausschließen will, nicht entsprechen. ${ }^{125}$ Es gibt in den Frühdialogen drei mit einander verwandte Argumente dagegen, Ethik als techne - als Fähigkeit, Fertigkeit, Kompetenz - oder episteme - wissenschaftliches Wissen - zu verstehen. Diese möchte ich um ein viertes, wieder die Lehrbarkeit betreffendes Argument ergänzen.

Das erste Argument findet sich in „Hippias II“. Hippias wird zunächst dargestellt als einer, der sich in allem, was mit Fähigkeiten zu tun hat, für den Besten hält:

Sokrates: [...] [E]s sollte mich wundern, wenn irgendeiner von denen, die sich dort [in Olympia] in Leibesübungen zeigen, so furchtlos und fest vertrauend auf seinen Leib dort hinginge zum Kampf, wie du sagst auf deinen Verstand.

Hippias: Ganz natürlich, o Sokrates, daß es mir so ergeht. Denn seitdem ich angefangen, bei den Olympischen Spielen mich im Wettkampf zu zeigen, bin ich noch auf keinen jemals getroffen, der in irgendetwas vortrefflicher gewesen wäre als ich. $^{126}$

Hippias soll paradigmatisch nicht nur für körperliche, sondern für alle Künste (Fähigkeiten, Fertigkeiten, Kompetenzen) stehen. Zunächst werden Rechnen, Messkunst und Astronomie besprochen. Aber Hippias' Können geht noch sehr viel weiter, wie Sokrates bemerkt:

$[\mathrm{D}] \mathrm{u}$ bist ja in den meisten Künsten unter allen Menschen der Weiseste, wie ich dich einmal habe rühmen hören [...].

125 Dies hat auch mit dem weiter unten angesprochenen Punkt zu tun, damit nämlich, dass der paradigmatische Fall, bei dem es darauf ankommt, dass wir intolerant in der Frage sein sollten, ob ein bestimmter Begriff und nicht ein anderer verwendet werden sollte, die Ethik ist. Stipulative Definition ist (vor allem) in der Ethik nicht erlaubt.

126 Wolf, Platon. Sämtliche Werke, Band 1, „Hippias II“, 364a. 
Du sagtest nämlich, du wärest einmal so nach Olympia gekommen, daß alles, was du an deinem Leibe hattest, deine Arbeit gewesen wäre. Zuerst der Ring, den du anhattest - damit fingst du an -, wäre deine Arbeit gewesen, so daß du also auch Steine zu schneiden verständest, und noch ein anderes Siegel deine Arbeit, und ein Badekratzer und ein Ölfläschchen, die du selbst gemacht. Hernach auch die Schuhe, die du anhattest, behauptetest du, selbst geschnitten zu haben und den Mantel gewebt und das Unterkleid, und was allen das Sonderbarste schien und der größten Geschicklichkeit Ausstellung, als du sagtest, der Gürtel deines Unterkleides sehe zwar aus wie die persischen der Vornehmen, diesen aber hättest du selbst geflochten. Überdies hättest du Gedichte bei dir gehabt, epische und Tragödien und Dithyramben, und in Prosa gar viele und mancherlei ausgearbeitete Vorträge. [...] Ich glaube aber auch noch vieles anderes vergessen zu haben. ${ }^{127}$

Zum einen macht sich Sokrates sicher hier über die Prahlerei des Hippias lustig. Zum anderen ist aber auch klar, dass pro argumentum angenommen werden soll: Dort, wo es eine Fähigkeit gibt, verfügt Hippias über diese.

Anknüpfend an die Frage, ob Odysseus oder Achilles der bessere/vortrefflichere Mensch sei, wird zunächst festgestellt, dass in jeder der Künste, die Hippias besitzt, seine Kunst ihn sowohl dazu befähigt, diese absichtlich gut auszuüben wie auch absichtlich schlecht: Lügen kann nur eine, die in dem Gebiet, über das sie spricht, auch die Wahrheit sagen könnte; ${ }^{128}$ absichtlich ein falsches Rechenergebnis bekanntgeben kann nur diejenige, die das richtige Ergebnis kennt; ein Mittel verabreichen, das den schnellen, schmerzhaften Tod einer Patientin herbeiführt, kann nur eine, die weiß, welche Mittel helfen und welche Mittel schaden. Sokrates resümiert dies, indem er Hippias bittet, eine techne (Fähigkeit, Fertigkeit,

127 Ebd., „Hippias II", 368b-e.

128 Hier gibt es eine interessante Komplikation: Sokrates scheint zu meinen, dass Achilles in der Odyssee aus guter Absicht heraus gelogen habe. Dem stimmt Hippias zwar nicht zu, aber er gibt zumindest zu, dass man aus böser Absicht die Wahrheit sagen könne: „Odysseus aber, wenn er wo die Wahrheit sagt, tut er es in böser Absicht, und wenn er lügt, ebenso." ebd., „Hippias II“, 371e. In einer Hinsicht wird hier aber, wie deutlich werden wird, ein ähnliches Problem angesprochen. 


\section{Expertise und die Tugenden}

Kompetenz) zu finden, bei der es sich anders verhält: „Gewiß, du wirst keine finden, Freund, denn es gibt keine.“129

Die Frage kann nicht nur in Bezug auf die Fähigkeiten selbst gestellt werden, sondern auch in Bezug auf die Personen, die diese besitzen: Bei allen Fähigkeiten, die es gibt, scheinen wir - dies legt der Rest des Dialoges nahe - diejenigen für die Besseren zu halten, die absichtlich ${ }^{130}$ das Schlechte zu tun in Lage sind, gegenüber denen, die das Schlechte unabsichtlich tun. Dies wird von Sokrates an einer Reihe von Beispielen durchexerziert, bevor er es auf die Seele und den Menschen überträgt. Hier wird diese Struktur unakzeptabel, weil „gut" nicht mehr im Sinne von „fähig“, sondern im Sinne von „ethisch gut" verstanden wird. ${ }^{131}$ Während diejenige, die absichtlich schlecht läuft, eben eine gute Läufer*in ist, ist diejenige, die zum Beispiel mit Absicht ungerecht ist, einfach ungerecht. Sie besitzt keine Gerechtigkeitsfähigkeit in besonders hohem Maße, gegen deren Gebrauch sie sich entscheidet. Für Hippias bedeutet das, er ist zwar dadurch, dass er alle Fähigkeiten besitzt, die man

129 Wolf, Platon. Sämtliche Werke, Band 1, „Hippias II“, 369a. Wir müssen hier Sokrates nicht vollständig zustimmen, damit der eigentliche Punkt bestehen bleibt: Vielleicht kann ein Pitcher im Baseball nicht mehr auf die gleiche Weise daneben werfen, wie eine Person, die diese Sportart nie ausgeübt hat. Und im Bereich der Sprache, die ich weiter unten diskutieren werde, mag es ähnliche Beispiele geben - auch wenn Sprache ein Grenzfall ist und es, wie weiter unten deutlich wird, darauf ankommt, was damit gemeint ist, bevor wir sagen sollten, Sprache sei eine Fähigkeit. In jedem Fall mag es durchaus Fähigkeiten geben, die unser Tun auf eine Weise strukturieren, von der wir uns nicht distanzieren können. Möglicherweise ist der Begriff der Absichtlichkeit aufgrund seiner umstrittenen Rolle in der heutigen Handlungstheorie und seiner fraglichen Beziehungen zu entsprechenden altgriechischen Wörtern, die hier natürlich relevant sind, in diesem Sinne ein unglückliches Wort. Vielleicht ist es sinnvoller, zu sagen, bei der Moralität sei das Bemühen um die richtige Ausübung normativ vorgeschrieben (und nicht nur psychologisch oder physisch unumgänglich wie im Falle des Pitchers oder von Sprachen). Weiter unten spreche ich davon, dass eine moralische Betrachtungsweise eben nicht nur konstitutiv für bestimmte Handlungen in bestimmten Situationen ist, wie dies von Fähigkeiten gesagt werden kann, sondern eben auch normativ beziehungsweise regulativ. Dies gilt weiterhin, selbst wenn es Fähigkeiten gibt, deren Erwerb es psychologisch oder physiologisch oder in einer anderen Hinsicht unmöglich macht, so zu handeln als hätten wir sie nicht.

130 Siehe Fußnote 129.

131 Vgl. Wolf, Die Suche nach dem guten Leben. Platons Frühdialoge, S. 59-63. 
besitzen kann, ein fähiger, aber dadurch noch nicht unbedingt ein guter Mensch.

Was im „Hippias II“ über Techniken gesagt worden ist, gilt, so hatte ich mehrfach angedeutet, auch für eine bestimmte Art von Wissen, nämlich episteme, und drückt sich aus in dem Sprichwort: „Wissen ist Macht". Eine Person, die über Wissen verfügt, das sich zum Beispiel für die Formulierung hypothetischer Imperative - in konstitutiver Lesart und in kausaler Lesart - verwenden lässt, hat eine Möglichkeit, absichtlich schlecht zu handeln, wo eine Person, die dieses Wissen nicht hat, dies nur unabsichtlich tun kann. Entsprechend müssen Philosoph*innen, die einen ethischen Kognitivismus vertreten wollen, der Erkennen in einer der episteme analogen Weise versteht, Urteilsexternalist*innen sein. ${ }^{132}$

Dies ist nicht nur deshalb unattraktiv, weil wir dann, selbst wenn wir es schafften, eine Person, die im Begriff ist, das Schlechte zu tun, davon zu überzeugen, dass dies das Schlechte ist, es eine völlig kontingente zweite Frage wäre, ob sie auch motiviert ist, es zu unterlassen. ${ }^{133}$ Was diese Position aber hauptsächlich unattraktiv macht, liegt nicht auf der Seite derjenigen, die wider besseres Wissen unmoralisch handelt, sondern zumindest auf den ersten Blick auf Seite derjenigen, die moralisch handelt: wir müssten nämlich bei ihr ein zusätzliches, von den Begründungen der moralischen Richtigkeit unabhängiges motivationales Element annehmen, das ungefähr die Form hat: Was auch immer das moralisch Richtige ist,

132 Z. B. Sigrún Svavarsdóttir. „Moral Cognitivism and Motivation“. In: The Philosophical Review 108.2 (1999), S. 161-219. Und bei Michael Smith ist dies eine der drei unvereinbaren Thesen des moral problem: Smith, The Moral Problem, Kapitel 1.

133 Und dies ist natürlich auch der Grund, warum Kohlberg, nachdem er moralische Entwicklung als die Entwicklung einer Fähigkeit, der moralischen Urteilskompetenz nämlich, gefasst hat, sagen muss, dass die Entwicklung der entsprechenden Handlungsbereitschaft damit noch offen ist. Lawrence Kohlberg und Daniel Candee. „Die Beziehung zwischen moralischem Urteil und moralischem Handeln“. In: Die Psychologie der Moralentwicklung. Hrsg. von Wolfgang Althof. Frankfurt am Main: Suhrkamp, 1996. Auch in Kohlbergs Modell wird Ethik im Wesentlichen mit (kognitiven) Fähigkeiten identifiziert. Lawrence Kohlberg. „Moralische Entwicklung“. In: Die Psychologie der Moralentwicklung. Hrsg. von Wolfgang Althof. Frankfurt am Main: Suhrkamp, 1996. Ich hatte bereits darauf hingewiesen, warum ich denke, dass das, was ich hier daran kritisiere, mit Gilligans einflussreicher Kritik kompatibel ist. Siehe Fußnote 66. 
ich habe eine Motivation, es zu tun. Dieser Wunsch hätte aber die Form eine Fetisches: Wir sollen das moralisch Richtige nicht deshalb tun, weil es unter einen bestimmten Begriff, den Begriff des Moralischen, fällt, sondern deshalb, weil es sich mit moralischen Gründen begründen lässt. ${ }^{134}$ Natürlich gibt es andere Möglichkeiten. McDowell zum Beispiel kann man so lesen, dass das moralische Wissen eben ein solches Wissen sei, das sich nicht ohne eine entsprechende emotionale Sensibilität erwerben lasse. ${ }^{135}$ Analog dazu könnte man auf der Seite der Fähigkeiten sagen: Die moralischen Fähigkeiten seien eben solche, deren Besitzer*in die Entscheidung darüber, ob sie sie ausüben soll oder nicht, nicht treffen kann. Auf diese Strategie werde ich eingehen, sobald ich die anderen Argumente kurz umrissen habe, die in den Frühdialogen dagegen genannt werden, dass das gute Handeln die Ausübung einer Fähigkeit sei.

Eine andere Strategie ist es, den Wechsel in der Bedeutung von „gut" beim Übergang von der Läuferin zur Seele von „fähig" zu „moralisch gut" einfach zu verneinen - oder besser gesagt: zu verneinen, dass zwischen „,ähig“ und „moralisch gut" ein Bedeutungsunterschied vorliegt. Es sei lediglich so, dass hier der Übergang von einer Personenbeschreibung, von der wir uns distanzieren können (Läuferin, Ärztin), zu einer solchen, von der wir uns nicht distanzieren können (Mensch, Seele) gemacht würde. Oder anders ausgedrückt: Während die spezifischen Fähigkeiten eben für bestimmte Teilbereiche menschlichen Handelns gelten, gibt es allgemein menschliche Fähigkeiten, zu denen die Moral zählt, die für alle Lebensbereiche gelten. Diese Fähigkeiten nennen wir zwar „Tugenden", aber es spricht nichts dagegen, dass sie ansonsten die Form von Fähigkeiten haben; statt von „Tugend der Besonnenheit" oder „Tugend der Gerechtigkeit“ könnten wir auch von „Fähigkeit zur Besonnenheit" und „Fähigkeit zur Gerechtigkeit" reden.

Zunächst lässt sich festhalten, dass in den Frühdialogen diese Ausweitung des Fähigkeitenbegriffes explizit abgelehnt wird. Dies zeigt sich zum Beispiel im „Ion“, in dem es oberflächlich um die

134 Laura Hinn nennt urteilsexternalistische Vorstellungen dieses Formates entsprechend abwertend „Zusätzliche-Wunsch-Theorien“. Siehe Laura Hinn. „Dissertation“. Im Erscheinen. Siehe auch R. Jay Wallace. „Three Conceptions of Rational Agency". In: Ethical Theory and Moral Practice 2.3 (1999), S. $217-242$.

135 Siehe zum Beispiel McDowell, „Might There Be External Reasons“. 
Frage geht, ob Rhapsodie - so wie Ion sie betreibt - eine Kunst ist. Ähnlich wie auch in der Prüfung des Orakelspruches beansprucht der Rhapsode Ion über das ethisch Gute zu reden - indem Ion Dichtungen, in denen es über das ethisch Gute geht, vorträgt und interpretiert, muss er, grob gesprochen, über das Gleiche verfügen wie die Dichter - sei es nun Inspiration oder Fähigkeit. Im „Ion“ geht es Sokrates darum zu zeigen, dass die Rhapsodentätigkeit nicht auf einer Fähigkeit beruhen kann, weil der Gegenstandsbereich einer Fähigkeit nicht so weit sein darf, dass es das menschliche Handeln als Ganzes umfasst ${ }^{136}$. Ursula Wolf fasst den Gedanken, um den es im „Ion“ geht, so zusammen:

Das begründete Wissen und das praktische Verfügenkönnen, das die techne auszeichnet, beruht also gerade auf einer scharfen Abgrenzung der Gegenstandsbereiche, die jeweils durch die Aufgabe der techne, ihr ergon bestimmt sind, das für die Ganzheit der jeweiligen techne verantwortlich ist. [...] Wie wir noch des öfteren sehen werden, steht der techne-Charakter der Politik in Frage, weil das Wohl der Polis und ihrer Bürger im ganzen, das sie zur Aufgabe hat, kein durch eine zusammenhängende Regelmenge umschriebener und von anderen abgegrenzter Gegenstand, sondern der Inhalt des gesamten ethischen Wissens ist. ${ }^{137}$

Bereits mehrfach wurde angedeutet, dass es fraglich sei, ob Ethik methodisch in dem Sinne betrieben werden könne, wie eine Wissenschaft methodisch betrieben wird. Hier nun liefert Ursula Wolf mit Sokrates einen weiteren Grund dafür: Wissenschaften und Künste können deswegen methodisch betrieben werden, weil es hier ein ergon gibt, das einen begrenzten Gegenstandsbereich individuiert und strukturiert. Ethik kann nicht in der Hinsicht methodisch be-

136 Wolf, Platon. Sämtliche Werke, Band 1, „Ion“. Noch darf er zu eng sein, was aber hier nicht von Bedeutung ist: Ion behauptet, ausschließlich Homer auslegen zu können (und in der Auslegung von Homer die Gesamtheit des menschlichen Guten zum Ausdruck bringen zu können). Ions Tätigkeit hätte nach Sokrates auch aus dem Grund nicht die Form einer Fähigkeit, sofern sie sich nicht auf andere Dichter übertragen ließe.

137 Wolf, Die Suche nach dem guten Leben. Platons Frühdialoge, S. 56f. Dass Wolf hier von ethischem Wissen spricht, soll nicht irritieren: Es wurde von ihr bereits ausgeschlossen, dass dieses Wissen die Form einer episteme habe. 
trieben werden, weil hier das ergon nicht spezifisch ${ }^{138}$ ist und der Gegenstandsbereich prinzipiell unbegrenzt. ${ }^{139}$ Dies kann man in Bezug auf verschiedene Arten und Weisen das ethische Gute zu fassen, unterschiedlich formulieren: Der kategorische Imperativ teilt eben jede meiner Handlungen ein in die Kategorien des Verbotenen, des Gebotenen und - je nach Interpretation - des Erlaubten; die Besonnenheit oder die Gerechtigkeit der Tugendhaften ist eben potentiell in jeder ihrer Handlungen gefordert; und natürlich lässt sich auch im Konsequentialismus kein Bereich von Handlungen finden, in welchem wir prinzipiell davon befreit wären, unsere Handlungen auf ihre Konsequenzen hin zu prüfen. ${ }^{140}$

Damit hängt auch der letzte Punkt zusammen, der wieder mit der Lehrbarkeit zusammenhängt, und das ist das Problem der Übung. Üben und Einüben muss in der Ethik zumindest etwas anders funktionieren, als in den Künsten und bei den Fähigkeiten. In den Künsten kann ich mich (weil es einen eingegrenzten Gegenstandsbereich und ein ergon gibt) in spezifische Situationen begeben, in denen genau die Schwäche, die ich in der entsprechenden Kunst oder Fähigkeit habe, betont wird, und durch wiederholte Versuche, die Fähigkeit in dieser Situation anzuwenden, die Fähigkeit oder Kunst verfeinern. In der Ethik funktioniert dies nur begrenzt: Dilemmageschichten im Ethikunterricht beispielsweise mögen dazu geeignet sein, die Unterschiede zwischen verschiedenen Ansätzen der normativen Ethik bezüglich ihrer tragbaren oder nicht tragbaren Implikationen klar zu machen. Zu besseren Menschen machen sie die

138 Natürlich könnte man Begriffe wie „eudaimonia“ oder ein respektvolles Zusammenleben oder Ähnliches als ergon angeben. Der Punkt wäre dann, dass dieses ergon unbestimmt bleibt (siehe Wolf, Die Suche nach dem guten Leben. Platons Frühdialoge, Kapitel 6) beziehungsweise dass die Bestimmung des ergon dort, wo es sinnvoll erscheint, nach einem solchen zu suchen, bereits selbst eine ethische Aufgabe ist.

139 Siehe dazu auch Mills Auseinandersetzung mit dieser Frage, die ich Abschnitt 4.3.2 behandele.

140 In diesem Sinne können wir, anders als dies James' transzendentale Idealistin meint, keine moralischen Ferien machen. Wir können zwar „Ferien“ von unserem Diätplan oder unserer bibliographischen Genauigkeit machen, aber nicht moralische Ferien. Vgl. James, Pragmatism and other Essays, S. 35f. Die Entscheidung zum Beispiel, jetzt einmal die Belange anderer zu ignorieren und die eigenen Interessen in den Vordergrund zu stellen, ist keine Entscheidung jetzt einmal aus dem Bereich des Moralischen auszutreten, sondern es ist selbst eine moralische Entscheidung. 
so Überlegenden aber sicherlich nicht notwendigerweise. Gleiches gilt in anderen Fällen: Wenn ich bemerkt habe, dass ich am Grab des Freundes nicht die Trauer habe empfinden können, die angebracht gewesen wäre, und sich die darauf folgende Beileidsbekundung falsch und oberflächlich angefühlt hat, dann ist das Rezept hierfür nicht, einfach häufiger zu Beerdigungen von geliebten Menschen zu gehen. Wenn ich merke, dass ich ein Problem habe, mich zu entschuldigen, wenn ich Menschen auf eine persönliche Weise verletzt habe, dann ist das Rezept auch hier nicht, Menschen einfach häufiger zu verletzen, um zu üben, mich zu entschuldigen.

Ich würde durchaus zugestehen, dass zum Beispiel Literatur etwas sein kann, in dem wir tragische Situationen nachempfinden können, ohne dass wir sie selbst erleben müssen. Wichtig scheint mir aber, dass dies nicht die gleiche Struktur und Funktion hat, wie Übung im Falle von Fähigkeiten. Zum Beispiel kann auch Abstumpfen gegenüber oft zur Kenntnis genommenem Unrecht als eine Art Übung gesehen werden.

Dies alles soll nicht bedeuten, dass Übung nicht doch eine Rolle in bestimmten Bereichen des Ethischen - solchen Bereichen vielleicht, in denen es auch um Selbstdisziplinierung geht - spielen kann. Ich wollte nur darauf hinweisen, dass ihre Rolle mittelbarer oder auf kompliziertere Weise mit der eigenen Verbesserung zu tun hat, als dies bei den Fähigkeiten der Fall ist.

Ich nehme an, viele würden trotz dieser Gedanken im Anschluss an den „Hippias II" daran festhalten wollen, dass der Bereich des Ethischen genauso wie alle anderen praktischen Bereiche über Fähigkeiten strukturiert sei. Ich hatte bereits gesagt, ich will keine Worte sanktionieren und Begriffe aus dem weiten Begriffsfeld der Fähigkeiten werden ja tatsächlich in sehr viel weiterer Weise gebraucht als meine verhältnismäßig strenge Eingrenzung nahelegt: Wir reden von der Stromleitfähigkeit von Kupfer, von der spezifischen Wärmekapazität von Wasser, von der Leidensfähigkeit von Tieren. Und natürlich wurde auch gesagt, der Mensch sei ein Tier, das zur Vernunft oder zur Moral oder zur Kultur oder zur Empfindung höherer Freuden als Schweine fähig sei. Genauso reden wir in Bezug auf einzelne Menschen auch in moralischer Hinsicht manchmal von Fähigkeiten: „Er war des Mitgefühls unfähig."

In vielen dieser Fälle könnte vielleicht „Disposition“ an Stelle von „Fähigkeit“ verwendet werden. Den Begriff „Fähigkeit“ zu wählen 
erfüllt aber natürlich eine rhetorische Funktion: Kupfer kann dazu benutzt werden, Stromkabel herzustellen und Wasser für Heizungen. Davon zu reden, dass Tiere zur Empfindung von Leid fähig sind, analogisiert das Leid von Tieren mit dem, was beim Menschen „kognitive Fähigkeiten“ genannt wird. Und natürlich macht es auch rhetorisch einen Unterschied, ob ich von der Disposition des Menschen zur Vernunft oder von seiner Fähigkeit zu derselben rede. Das Wort „Fähigkeit“ deutet hier (neben anderen Dingen) zumindest auch an, dass es sich um eine gute Disposition handelt. „Gute Disposition" kommt natürlich auch sehr nahe an das, was Aristoteles in der Nikomachischen Ethik beschreibt, wenn er die psychologische Kategorie, der die Tugenden zuzuordnen sind, von Fähigkeiten abgrenzt. ${ }^{141}$ Natürlich ist die Güte der Vernunft nicht wirklich nicht-zirkulär auf den Punkt zu bringen - genauso wenig wie die Güte der Moral oder der Kultur. Aber es ist selbstverständlich trotzdem gänzlich unproblematisch an der Redeweise festzuhalten, der Mensch sei zur Vernunft oder zur Moral fähig.

Natürlich folgt aus der Aussage, dass der Mensch in diesem Sinne zur Moral fähig ist, nicht, dass meine Moralität eine Fähigkeit (oder eine Menge von Fähigkeiten) von mir ist. Aber auch dies mag man weiterhin behaupten wollen. Hier spielt vielleicht eher der Wunsch nach handlungstheoretischer Eleganz eine Rolle: Es wäre sicher sehr schön, wenn wir für jede Handlung (und jede Sichtweise auf Handlungen als Handlungen) sagen könnten, dass sie durch Unterordnung unter eine Fähigkeit strukturiert sei, die mit den Begriffen des Gelingens und Misslingens (die wiederum durch das ergon bestimmt werden) den einzigen Maßstab zur Verfügung stellt, nach dem diese Handlung bewertet werden kann. Ich kann dieses Motiv durchaus verstehen. Der Punkt, den man in diesem Fall nicht aus den Augen verlieren sollte, ist aber, dass es sich dann um Fähigkeiten einer in entscheidender Hinsicht anderen Form handeln muss, als die Fähigkeiten der alltäglicheren Form, die unsere anderen Handlungen strukturieren: Moralische Fähigkeiten müssten Fähigkeiten sein, (1) deren Ausübung oder nicht-Ausübung ${ }^{142}$ nicht der Ent-

141 Siehe Aristoteles, NE, 1105b-1106a.

142 In vielen Fällen ist es vielleicht sinnvoller von deren besserem oder schlechterem, bemühterem oder gleichgültigerem Ausführen zu sprechen: Das Argument bleibt das gleiche. 
scheidung der Handelnden unterliegt, ${ }^{143}$ (2) die anders als andere Fähigkeiten keinen eingegrenzten Gegenstandsbereich haben, weil sie (3) kein bestimmbares ergon haben, und die deswegen (4) nicht durch methodisches Üben erworben werden können. Zusätzlich können wir unter Bezug auf das Vorherige sagen: Es müsste sich um Fähigkeiten handeln, denen (5) prinzipiell keine methodische Kunst oder Wissenschaft zuzuordnen wäre, sodass es (6) keine Expertinnen für diese Fähigkeiten geben kann, die den Kunstverständigen in den anderen Künsten entsprechen würden. Wenn es trotz dieser Unterschiede weiterhin Gründe dafür gibt, zu sagen, die Struktur einer Handlung als moralischer Handlung sollte so verstanden werden wie die Struktur einer Handlung als Ausübung einer Fähigkeit, spricht meines Erachtens nichts dagegen ${ }^{144}$. Ich selbst werde aber an den wenigen Stellen, in denen der Fähigkeitenbegriff im Rest dieses Textes noch vorkommt, diesen in der strengeren Variante verstehen, also so, dass (1) Fähigkeiten jeweils zwei Handlungsmöglichkeiten

143 Weil wir nicht sagen wollen, die absichtlich ungerecht Handelnde habe eine größere Fähigkeit zum gerechten Handeln, als die versehentlich ungerecht Handelnde. Wenn wir dies nämlich sagten, müsste zur gerecht Handelnden etwas über die Fähigkeit Hinausgehendes dazu kommen - und die gerechte Handlung wäre nicht mehr bloß durch Fähigkeiten strukturiert.

144 Inhaltlich meine ich aber gibt es zumindest ein Grund, den Begriff der Fähigkeit für etwas anderes zu reservieren: es ist sinnvoll, zu sagen, dass mit dem Aufzeigen einer moralischen Kategorie für Handlungen auch in diesem Fall das Gegenteil - die moralisch schlechte Handlung - konstituiert wird. Nur wer diese praktische Kategorie besitzt, kann ihr wissentlich entgegen handeln: Nur wer weiß, wie man Menschen respektvoll behandelt, kann zum Beispiel effizient beleidigen. (Man könnte so weit gehen zu sagen, dass das, was Judith Butler in Burning Acts, Injurious Speech (Judith Butler. „Burning Acts, Injurious Speech". In: Excitable Speech. A Politics of the Performative. New York, London: Routledge, 1997, S. 43-69) beschreibt, sogar die Bedingungen von Habermas für kommunikativen und nicht instrumentellen Sprachgebrauch erfüllt: Zumindest bestimmte Formen der hate speech funktionieren eben dadurch, dass sie in ihrer illokutionären Rolle als hate speech verstanden werden - die Perlokution wird in diesen Fällen also nicht verschleiert, indem die Rede eine illokutionäre Form hat, die der Perlokution widerspricht, wie dies beim instrumentellen Sprachgebrauch nach Habermas der Fall ist. Wer hate speech verwendet, hat nicht einfach einen Mangel an Fähigkeiten.) Von Fähigkeiten zu reden würde demnach der Handelnden auch hier beide Handlungsmöglichkeiten eröffnen. Gerade also, weil wir den Begriff der Fähigkeit gut verwenden können, um die mit moralischen Kategorien taktisch Verfahrende zu beschreiben, sollten wir den Begriff nicht verwenden, um die moralisch Handelnde zu beschreiben. 
ihrer Besitzerin konstituieren; dass (2) Fähigkeiten sich auf einen eingeschränkten Gegenstandsbereich beziehen, der (3) durch ein ergon strukturiert ist; dass (4) Fähigkeiten durch Übung erworben werden können; dass (5) für Fähigkeiten zumindest im Prinzip eine methodische Kunst gefunden oder erfunden werden könnte - selbst wenn dies für einige Fähigkeiten albern erscheinen mag; und dass (6) es daher für alle Fähigkeiten, die es in diesem Sinne des Wortes gibt, im Prinzip Expert*innen und professionsmäßige Lehrer*innen geben kann.

Was ist aber mit begrifflichen Fähigkeiten? Auf der Ebene dichter moralischer Begriffe - auf der Ebene unserer Bewertungen also - spiegelt sich das, was für Handlungen gesagt wurde, natürlich. Diesen Punkt hatte ich in Fußnote 125 versucht zu machen, indem ich sehr abkürzend gesagt hatte, stipulative Definition sei in der Ethik nicht erlaubt. Gemeint ist folgende Entsprechung: Weiter oben ging es um die im „Hippias II“ thematisierte Feststellung, dass „absichtlich schlecht Handeln" zwar im Falle von Fähigkeiten auf die Fähigkeit der Handelnden schließen lässt, dass es aber, wenn „schlecht" im Sinne von „moralisch schlecht" verstanden wird, natürlich nicht auf die Tugendhaftigkeit der Handelnden geschlossen werden kann. Dem entspricht etwas auf der Ebene der Begriffe und zwar, dass die Anwendung eines moralischen Bewertungsbegriffes auf eine Handlung von der Sprecherin als nicht optional verstanden wird. Es macht einen Unterschied, ob der Völkermord an den Herero als solcher bezeichnet wird, oder ob ich von tragischen Ereignissen spreche. Umgekehrt wurde der Slogan von Teilen der Friedensbewegung, Soldaten seien Mörder, unter dem Ausdruck von Empörung von den Öffentlichkeitsabteilungen der Berufsarmeen verschiedener Länder zurückgewiesen; es wurde nicht einfach gesagt: Wir haben eine andere ebenso zulässige Ebene der Beschreibung der Handlungen. Man kann dies unter Verweis auf Anscombe ${ }^{145}$ auch so sagen: Während das Wesen einer Handlung als Handlung auch von der Beschreibungsweise der Handlung durch die Handelnde abhängt, legt die Sprecher*in einer moralischen Äußerung darauf Wert, dass eine bestimmte Beschreibungsweise nicht optional ist und bei der Bestimmung des Wesens der Handlung eine Rolle spielen muss.

145 Vgl. G. Elizabeth M. Anscombe. Intention. Cambridge, Massachusetts und andere: Harvard University Press, 2000, § $23 \mathrm{ff}$. 
Es wurde in Bezug auf dichte Begriffe in diesem Zusammenhang häufig darauf hingewiesen, dass sich die evaluative Komponente nicht von der beschreibenden trennen lasse. Eine, der Grausamkeit vorgeworfen wird, kann nicht einfach sagen, sie teile die Beschreibung, lehne aber die daraus resultierende Bewertung ab: Sie kann Grausamkeit nicht stipulativ zu einem Begriff mit bloß deskriptivem Gehalt umdefinieren, bloß weil dies ihren Kommunikationsinteressen entspricht, sondern sie muss, wenn sie der Bewertung entgehen will, das Urteil, die Handlung sei grausam, zurückweisen. So zumindest sieht es die Vorwerfende. Eine bestimmte - nämlich die richtige $^{146}$ - Beschreibungsweise einer Handlung in moralischer Hinsicht ist nicht optional, ganz gleich welche anderen Handlungsbeschreibungen bezüglich der Handlung auch immer sonst noch wahr oder sinnvoll sein mögen.

Man mag also sagen, dass dichte moralische Bewertungsbegriffe nicht bloß konstitutiv für unsere moralische Praxis der Bewertung sind, sondern auch gleichzeitig normativ oder regulativ - und zwar in einer Weise, die sich nicht einfach in Komponenten aufteilen lässt, weil wir sonst im Urteilsexternalismus landen würden. Diese Doppelrolle mag mit dem Wort „kategorisch“ bezeichnet werden. Das Problem, dass moralische Güte sich nicht als Ausdruck einer Fähigkeit verstehen lässt, lässt sich auf der Seite der Begriffe entsprechend so formulieren: Lediglich die konstitutive Rolle, die unsere dichten Begriffe für unsere moralische Bewertungspraxis spielen, lässt sich mit einigem Recht auf unsere Fähigkeit zur Verwendung dieser Begriffe zurückführen. Und mit dieser Fähigkeit geht die Fähigkeit zum taktischen Gebrauch dieser Begriffe einher: Ich kann nun, um das obige Beispiel zu wiederholen, sagen „Soldaten sind Mörder", auch wenn mir klar ist, dass ich damit eigentlich eine rhetorische Zuspitzung eines von mir wahrgenommen Missstandes betreibe.

$146 \mathrm{Zu}$ diesem Punkt passt auch eine Beobachtung aus dem „Protagoras“, die unter dem Begriff der Einheit der Tugend gefasst wird. Dort wird behauptet, dass eine Handlung nicht gleichzeitig Ausdruck eines Lasters und einer Tugend sein könnte. Auf dichte Begriffe übertragen würde dies bedeuten: Die Verwendung eines negativ-bewertenden moralischen dichten Begriffes schließt die Verwendung eines positiv-bewertenden moralischen dichten Begriffes aus. Meiner Einschätzung nach stimmt dies, was unsere Alltagspraxis angeht, nicht völlig, aber es gibt durchaus Tendenzen, die in diese Richtung gehen. Siehe Wolf, Platon. Sämtliche Werke, Band 1, „Protagoras“, 328d-333d. 
Ihre normative Rolle hingegen - das heißt, dass mit dem Begriff der Grausamkeit die Auffassung einer Handlung als moralisch verwerflich vorgeschrieben ist - lässt sich nicht so einfach auf eine Fähigkeit zurückführen. Ich halte mich zum Beispiel für fähig, um mich noch einmal auf Whewell zu beziehen, den Begriff der Keuschheit ${ }^{147}$ zu verstehen und auch anwenden zu können - ich könnte ihn zum Beispiel in einer fiktiven Erzählung der Teilnehmerin an einem Amerikanischen purity ball als Erklärung für ihre Teilnahme an einer solchen Veranstaltung in den Mund legen. Ich könnte ihn gegenüber Menschen, die Keuschheit als moralische Kategorie verwenden auch taktisch einsetzen. Ich weiß also auch, dass von einer, die den Begriff der Keuschheit (oder den der Unkeuschheit) verwendet, eine moralische und damit auch emotionale Bewertung abgegeben wird, und ich bin auch bereit zuzugestehen, dass ich das, was sie sagen will, wenn sie etwas als „keusch“ bezeichnet, nicht verstehen würde, würde ich die Bewertung, die sie dabei abgibt, überhören. In diesem Sinne lässt sich die evaluative Komponente tatsächlich nicht von der deskriptiven trennen.

Dies ist aber auch schon alles, was ich sagen kann, wenn ich meine Fähigkeit, einen dichten bewertenden Begriff zu verwenden, auf ihre konstitutive Rolle für mein Verwenden dieses Begriffes beschränke. Dies kommt mir bei „keusch“ natürlich gerade recht; aber auch nur, weil der Begriff der Keuschheit ein Begriff ist, mit dem ich keinerlei moralische Bewertung verbinde. Die Frage, ob ich den Begriff der Keuschheit (oder der Unkeuschheit) ganz generell verwenden und die moralische Bewertung akzeptieren sollte, wenn ich sehe, dass eine Verwendung hier regelgemäß wäre, wird mit der Fokussierung auf seine konstitutive Rolle für den Kommunikationserfolg eben nicht beantwortet. Von dichten moralischen Begriffen, von denen ich meine, dass sie wirklich zum Beispiel moralische Missstände ausdrücken, hingegen würde ich sagen, dass ich einen Begriff, wenn ich sehe, dass seine Verwendung hier regelgemäß wäre, auch anwenden und die moralische Bewertung akzeptieren muss. Sie gelten kategorisch und das heißt, dass bei ihnen konstitutive Rolle und regulative Rolle nicht getrennt werden können (wenn wir nicht im Urteilsexternalismus landen wollen).

147 Siehe Abschnitt 6.1.1. 
Natürlich wird auch von anderen Fällen im Bereich des Begrifflichen gesagt, hier seien die Regeln, um die es geht, sowohl normativ als auch konstitutiv zu verstehen. Dies sei eben doch kein Alleinstellungsmerkmal der Ethik. Besonders fundamental ist hier vielleicht die Logik: Man kann vielleicht sagen, der Satz vom ausgeschlossenen Widerspruch sei normativ und gleichzeitig konstitutiv für unser Denken. Dies bin ich bereit zu akzeptieren. Ich hatte auch nicht gesagt, es handele sich hier um ein Alleinstellungsmerkmal der Ethik, sondern es unterscheide die Ethik von einem anderen Modell menschlichen Handelns. ${ }^{148}$ Wenn logische Regeln in dieser Hinsicht ethischen Regeln ähneln, heißt das nur, dass auch hier von logischen Fähigkeiten (oder gar einer Fähigkeit, sich selbst nicht zu widersprechen) zu reden, problematisch ist. In der Tat gäbe es für logische Fähigkeiten auch die anderen Probleme eines nicht bestimmbaren ergon, eines nicht eingrenzbaren Gegenstandsbereiches, ein Problem zu fassen, was es heißen könnte, logische Fähigkeiten systematisch einzuüben. Und dies hieße auch, dass wir in der Metalogik einen Teil $^{149}$ der gleichen Probleme hätten wie in der Metaethik..$^{150}$

148 Im „Menon“ wird die Mathematik, die in einer Hinsicht vielleicht auch ähnlich ist, als Beispiel genannt: Wolf, Platon. Sämtliche Werke, Band 1, „Menon“, $81 \mathrm{c}-86 \mathrm{c}$.

149 Natürlich nicht alle! Es gibt wichtige Unterschiede zwischen Ethik und Logik oder Mathematik. Wie Korsgaard richtig bemerkt, gibt es eine Form des Motivationsproblems, das sich für die Logik oder die Mathematik in der Hinsicht nicht stellt. Korsgaard fasst das so: „But people do not regulate their actions, love, hate, live, kill, and die for mathematical truths." Korsgaard, The Sources of Normativity, S. 12.

150 Zum Beispiel: Sollen wir aufgrund der Tatsache, dass wir in der Logik „wahr“ und „falsch" verwenden können (nämlich, zum Beispiel, indem wir Tautologien und Antilogien bezeichnen - oder ganz allgemein die Logik oder Grammatik einer Redeweise zu formulieren trachten), einen quietistischen logischen Realismus vertreten? Oder sollten wir Expressivist*innen sein und sagen, indem wir das, was wir für die logischen Regeln unserer Alltagssprache halten, auf den Punkt zu bringen suchen, trachten wir danach, etwas von dem auszudrücken, was wir tun, wenn wir uns einer bestimmten Redeweise bedienen verbunden vielleicht mit einem emphatischen: „Lasst uns das so-und-so machen!"? Dies sollen nur Beispiele sein. Was die in dieser Arbeit vertretene Position angeht, bin ich mir zwar nicht ganz sicher, wie fruchtbar eine sozialphilosophische Auseinandersetzung mit der Logik ist; aber insofern wir eine solche unternehmen wollten, müssten wir uns zusätzlich zum logischen Expressivismus - dem Versuch, den Witz dieser Regeln auszudrücken - darum bemühen, deutlich zu machen, warum es gut ist, diesen Regeln zu folgen. 


\section{Expertise und die Tugenden}

Die Kritik aus den Frühdialogen daran, Moral ausschließlich in Begriffen von Fähigkeiten zu verstehen, trifft in diesem Sinne auch das, was Mill „den Intuitionismus" nennt, zumindest wenn dieser mit einem bestimmten Anspruch vertreten wird - dem Anspruch, ethische Äußerungen könnten mit den Wissenschaften und Künsten analogen Wahrheitsansprüchen vertreten werden und mit den damit verbundenen Formen von Expertise, Methoden von Lehrbarkeit und Möglichkeiten, einen Tauschwert zu bestimmen. Dass diese Kritik auch auf das, was Mill "Intuitionismus“ nennt, zutrifft, habe ich anhand der Frühdialoge, wie sie im Rahmen von Ursula Wolfs Lesart ausgearbeitet wurden, gezeigt, indem ich darauf hingewiesen habe, dass sie sich auch auf Gedanken wie die von Whewell anwenden lässt. Aber natürlich ist das Thema in der Philosophie nicht unbekannt. Zu Recht oder zu Unrecht wird sie zum Beispiel gegen McDowells Idee der zweiten Natur erhoben. ${ }^{151}$

Für mich war dieser längere Exkurs wichtig, um zu zeigen, dass die Kritik der Frühdialoge an den Sophist*innen, die Ethik beziehungsweise ihre Reflexionsdisziplin als techne und entsprechend ihren Erwerb durch Individuen als Fähigkeiten aufzufassen, nicht bedeutet, dass die Sophist*innen automatisch mit Benthams Tugend

Wie auch analog in der Ethik (siehe Abschnitt 6.1.3) kann ein Maßstab, anhand dessen sich zum Beispiel die Regel des ausgeschlossenen Widerspruches als gut erweist, in einer Hinsicht der Logik nicht extern sein. Dies ist in der Logik sicher noch offensichtlicher: Wir werden die logischen Regeln immer schon zu beachten haben, um einen solchen Maßstab zu formulieren. Aber es ist trotzdem nicht so, dass zum Beispiel mit der Aussage offensichtlich nichts gesagt sei, die Regel des ausgeschlossenen Widerspruchs sei nötig, um die Praxis der feststellenden Rede zur Entscheidung zwischen praktisch relevanten theoretischen Aussagen aufrechterhalten zu können.

151 Vgl. z. B. Georg W. Bertram. „Was heißt es, Kunst als paradigmatische Praxis der zweiten Natur zu begreifen?" In: Deutsche Zeitschrift für Philosophie 66.3 (2018), S. 362-382. Er wirft McDowell vor, dass bei ihm das kritische Potential des Begriffes der zweiten Natur verloren gehe. (S. 362) Interessanterweise ist es bei Bertram die Kunst (bei Mill war es die Poesie), die es ermöglicht, einen begrifflichen Rahmen zu hinterfragen oder in einem anderen Lichte zu sehen: „Die transformatorischen Impulse, die Kunstwerke freisetzen, resultieren daraus, wie sie Praktiken herausfordern. [...] In der Auseinandersetzung mit Kunstwerken wissen wir grundsätzlich nicht genau, wie wir hören oder sehen, wie wir uns bewegen oder wie wir sprechen sollen. Wie ist ein Gemälde von Édouard Manet angemessen zu sehen? Wie kann man zur Musik von Prince angemessen tanzen? Welche Worte sind angemessen, um das filmische Geschehen in Lars von Triers Melancholia zu artikulieren?" (S. 370f) 
identifiziert werden müssten. Mill hat also Recht, wenn er darauf hinweist, dass Sokrates, auch wenn einer seiner zentraleren Vorwürfe an die Sophist*innen ist, sie würden sich als Expert*innen in der Ethik verstehen, damit nicht ausschließlich auf Coleridges Tugend festgelegt ist: Dieser Vorwurf läuft nicht darauf hinaus, Philosophie könnte nichts tun, als sprachlicher Ausdruck unserer Praxis zu sein.

Zusammenfassung. In Kapitel 7 ging es mir um Mills Auseinandersetzung mit Platon. Mill schreibt Platon im Rahmen seiner radikalen ${ }^{152}$ Lesart eine inhaltlich ähnliche Rolle für die Philosophiegeschichte zu wie Bentham; in diesem Sinne ist die radikale Lesart aber falsch. Abschnitt 7.1 diente der Erarbeitung der Missverständnisse Mills bezüglich dreier zusammenhängender zentraler Themen: der Frage nach dem Status einer Expert*in bezüglich der menschlichen arete, der Frage der Lehrbarkeit der menschlichen arete und der Frage des Tauschwerts für Lehre und Expert*innenrat bezüglich der menschlichen arete. Abschnitt 7.2 hatte die Aufgabe, die Annahme einer Spannung zwischen den beiden sozialphilosophischen Tugenden in Bezug auf diese drei Themen systematisch zu motivieren. In Abschnitt 7.3 habe ich erstens dann die Problematik in Bezug auf diese drei Themen in den Frühdialogen angedeutet; zweitens habe ich dafür argumentiert, dass die Spannung zwischen den Tugenden ein Teil dessen ist, worum es auch in den Frühdialogen in Bezug auf diese drei Themen geht, auch wenn ich eingestanden habe, dass damit die Behandlung dieser Fragen in den Frühdialogen nicht philosophisch erschöpft ist; und drittens habe ich gezeigt, dass die Problematik der drei Themen sich nicht bloß für philosophische Positionen ergibt, die sich an Benthams Tugend orientieren, sondern für alle Positionen, die beinhalten, Wissen in philosophischen Gegenstandsbereichen sei vergleichbar mit Wissen in naturwissenschaftlichen oder technischen Gegenstandsbereichen - egal in welcher Richtung dieser Vergleich intendiert ist. Die Überbetonung jeder der beiden Tugenden geht mit der Behauptung einer Art der Expertise, Lehrbarkeit und Bezahlbarkeit der philosophischen Auseinandersetzung mit der menschlichen arete einher, die

152 Giorgini, „Radical Plato: John Stuart Mill, George Grote and the Revival of Plato in Nineteenth-Century England". 
aber dadurch, dass die andere Tugend dabei vernachlässigt wird, nicht haltbar ist.

Ich habe bereits angedeutet, dass dies eine weitere Perspektive auf den Grund dafür ist, warum die beiden philosophischen Ideale als Tugenden aufgefasst werden sollten. Dies werde ich nun sehr kurz explizit ausformulieren. Ich habe in diesem Kapitel nahegelegt, dass einzeln betrachtet beide Ideale als Fähigkeiten verstanden werden können. Obwohl Fähigkeiten auch Personideale sind, geht damit noch nicht einher, dass wir die Ideale unbedingt als Personideale verstehen müssen, denn Fähigkeiten implizieren alles andere, was in den Frühdialogen damit verbunden ist: Es wäre im Prinzip möglich sie zu einer techne zu erheben, ihre Prinzipien zu formulieren, Expert*innen auszubilden und zu bezahlen u.s.w. In diesem Sinne könnte, wenn wir die beiden Ideale einzeln betrachten, auch gesagt werden, jedes der beiden Ideale sei eine Menge von Prinzipien, eine abstrakte Forschungsmethodik, eine Technik. Philosophisch werden die Ideale aber erst in ihrer Komplementarität (Abschnitt 2.2). Sie stehen dann in Spannung zueinander und für die Auflösung dieser Spannung gilt all das nicht: Die Aufösung dieser Spannung ist keine Fähigkeit; ihr ist keine techne zuzuordnen; es gibt Expert*innen nicht in dem gleichen Sinne; und es ist völlig unklar, wie ihr Tauschwert bestimmt werden sollte. ${ }^{153}$

Philosophisch werden die Ideale erst in der Festlegung der Philosoph*in auf beide miteinander in Spannung stehenden Ideale: In diesem Sinne sind sie als philosophische Ideale notwendig Personideale. Die Philosoph*in versucht beiden Idealen gerecht zu werden, ohne dabei eines aus dem Blick zu verlieren. Ich hatte nahegelegt, dass dies in zwei philosophischen Textformen sogar explizit gemacht werden kann: dem Dialog und der philosophischen Biographie. Keine der Sophist*innen mit ihren Theorien, mit denen Sokrates zum Beispiel spricht, ist (notwendig) eine Philosoph*in. Aber Platon ist ein Philosoph, indem er Dialoge formuliert, in denen die Spannung zwischen den Idealen, die ihn umtreiben, - auf die Dialogpartner*innen mehr oder weniger verteilt - zum Ausdruck kommt, und zwar

153 Ich halte es eigentlich für selbstverständlich, aber ich sage es, um mir nicht unnötig Widerspruch einzuhandeln: Es folgt daraus natürlich nicht, dass es keine Berufsphilosoph*innen geben sollte. Es folgt nur, dass wir die Rechtfertigung dafür, dass es Berufsphilosoph*innen gibt, nicht in ihrem marktwirtschaftlichem Nutzen sehen dürfen. 
ohne, dass Platon am Ende selbst Stellung bezieht für die eine oder andere Position. In der Biographie ist ähnliches möglich: Hier kann, wie ich versucht habe deutlich zu machen, ein durch beide Tugenden geleiteter Lernprozess beschrieben werden - in Mills Fall durch philosophische Krisen und philosophische Lösungsversuche für diese Krisen. Die Spannung zeigt sich in diesem Fall als Kampf mit sich selbst.

Natürlich gibt es andere Formen: Ideengeschichtliche Ausführungen können beispielsweise die gleiche Funktion haben. Und selbstverständlich muss es nicht immer darum gehen, die Spannung selbst auf den Punkt zu bringen. Im institutionellen Diskurs können wir der Spannung auch mit systematischen Thesen begegnen in dem Vertrauen darauf, dass diese von anderen geprüft und kritisiert werden. Wir müssen nicht immer danach streben, Philosophie als Individuum zu betreiben. Es ist aber dann nicht unwichtig - und dies sei bezüglich des Tugendcharakters als Letztes festgehalten - dabei nicht aus den Augen zu verlieren, was Philosophie als Ganzes ist. 


\section{Abgrenzung, offene Probleme, Ausblick}

In den letzten sieben Kapitel habe ich versucht, einen Beitrag Mills zur Philosophie der Philosophie auszuarbeiten und zu verteidigen. Mills Beitrag ist in der Hinsicht implizit, dass Mill nicht Teil einer im engeren Sinne akademischen Debatte über „Philosophie der Philosophie" war; er ist aber durchaus explizit in dem Sinne, dass es Mill um gutes und schlechtes Philosophieren geht. Mill selbst hat zu diesem Thema die Auseinandersetzung mit philosophischen Vorbildern gewählt; und obwohl meiner Auseinandersetzung mit Mill methodisch ein ähnlicher Status zugeschrieben werden kann (siehe Abschnitt 2.3), habe ich mich bemüht, die Stärken und Schwächen, die Mill an seinen philosophischen Vorbildern ausmacht, zwei komplementären philosophischen Tugenden zuzuordnen und zu zeigen, wie Mill selbst in seinem philosophischen Handeln von der Spannung zwischen diesen Tugenden geleitet ist.

Ich hoffe, dass deutlich geworden ist, dass Mill auch deshalb ein guter Philosoph ist, weil er mit dieser Spannung zeitlebens - oder um genauer zu sein: mindestens seit seiner ersten Krise - kämpft. Die Revisionen seiner philosophischen Positionen, die Mill im Laufe seines Lebens vornimmt, können insofern nicht deshalb als Verbesserungen gesehen werden, weil sie näher an der philosophischen Wahrheit oder methodisch klarer wären, sondern weil sie jeweils aus der Einsicht darein entspringen, einer der beiden Tugenden nicht wirklich gerecht geworden zu sein. (Damit ist natürlich nicht gemeint, dass wir jede von Mills Revisionen seiner Positionen als Verbesserung sehen müssen.)

In einer Hinsicht bin ich dem ersten Satz meiner Arbeit damit gerecht geworden. Selbst wenn ich aber aus Sicht meiner Leser*in erfolgreich darin war, zu zeigen, dass Mill in diesem Sinne und auch aus diesen Gründen ein guter Philosoph war, sind noch viele Fragen offen. Diese Fragen möchte ich in diesem Kapitel ansprechen. In manchen Fällen werde ich eine Antwort andeuten; in manchen Fällen werde ich lediglich das Problem anerkennen. 
Die erste Frage ist, inwiefern das hier Gesagte auf andere philosophische Ansätze ausgedehnt werden kann. Diese Frage werde ich sehr beispielhaft in Abschnitt 8.1 angehen. Zweitens gibt es einige Detailfragen insbesondere in Bezug auf die Kritik, die ich an Mill in Kapitel 6 geübt habe. Die damit verbundenen Fragen werde ich in Abschnitt 8.2 andeuten.

\subsection{Verhältnis zu anderen Ansätzen}

Wie verallgemeinerbar sind Mills Tugenden? Auf der einen Seite ist mit Mills Tugenden natürlich der Anspruch verbunden, verallgemeinerbar in dem Sinne zu sein, dass sie dazu taugen, philosophische Stärken und Schwächen nicht nur von Mill, sondern auch von anderen Ansätzen deutlich zu machen. Auf der anderen Seite sollte deutlich geworden sein, dass nach Mills eigenem Ansatz ein Pluralismus philosophischer Vorstellungen ein Wert ist. Zwischen verschiedenen philosophischen Vorstellungen kann, wenn wir Mills Tugenden auf die Philosophie der Philosophie selbst anwenden und ernst nehmen, nicht rein argumentativ entschieden werden. Es muss, um Mills/Andersons Phrase aus Kapitel 5 abzuwandeln, experiments in philosophizing geben.

Damit habe ich nun endlich auch die Begründung für die Qualifikation der "Nicht-Exklusivität" aus Kapitel 2 gegeben: Mills sozialphilosophische Tugenden selbst können sich nur als sinnvoll erweisen vor dem Hintergrund einer Pluralität von philosophischen Ansätzen. Mills Philosophie der Philosophie könnte, um diesen Gedanken rhetorisch etwas zuzuspitzen, in diesem Sinne Exklusivität nur zum Preis der Selbstauslöschung beanspruchen. (Mill ist der Forderung nach Pluralität natürlich durch die Wahl seiner Vorbilder gerecht geworden: Jedes seiner beiden Vorbilder hatte eigentlich eine andere Vorstellung von Philosophie, deren Stärken und Schwächen Mill beispielhaft hervorgehoben hat, und aus deren Synthese sich das, was ich Mills sozialphilosophische Tugenden genannt habe, ergeben.)

Trotzdem hatte ich auf den vorhergehenden Seiten natürlich bereits zur Erläuterung der Position Mills Vergleiche mit anderen Philosoph*innen gezogen - teilweise in kritischer, teilweise in zustimmender Absicht. Darauf werde ich nun explizit eingehen. 


\subsubsection{Hegelianische Ansätze: Jaeggi}

In diesem Abschnitt möchte ich mich mit Rahel Jaeggis Idee der immanenten Kritik ${ }^{1}$ auseinandersetzen. Immanente Kritik soll nach Jaeggi sowohl Schwächen der externen Kritik als auch Schwächen der internen Kritik vermeiden. ${ }^{2}$ Es liegt daher nahe, zu vermuten, hier gehe es um etwas Ähnliches, wie in den Tugenden: Benthams Tugend könnte als externe Kritik verstanden werden und, obwohl ich nicht sicher bin, ob wir die Möglichkeit der Kritik, die mit Coleridges Tugend einhergeht, als interne Kritik verstehen sollten, macht Mill selbst den Punkt, dass Coleridges Tugend eine bestimmte Form von Kritik, die sich auf das Wesen oder die Idee einer Institution bezieht, möglich macht. ${ }^{3}$ Ob diese Art durch Coleridge inspirierter Kritik, so wie Mill sie auffasst, am Ende in Jaeggis Terminologie immanente oder interne Kritik ist, will ich hier nicht entscheiden: In jedem Fall gibt es Parallelen zwischen Jaeggis Ansatz und dem, was ich Mill zuschreibe, die ich hier ausarbeiten will.

Jaeggi steht in meiner Arbeit aber auch beispielhaft für hegelianische Ansätze. Immerhin könnte gesagt werden, Mill habe die "Germano-Coleridgians", wie er sie nennt, einfach nicht verstanden; Hegel habe das Problem, vor das sich Mill gestellt sieht, nicht nur (und zwar deutlich vor Mill) anerkannt ${ }^{4}$, sondern hegelianische Ansätze hätten auch die Ressourcen, es zu lösen.

Dem will ich eingeschränkt zustimmen und diese eingeschränkte Zustimmung in diesem Unterabschnitt am Beispiel von Rahel Jaeggis Kritik von Lebensformen auf den Punkt bringen. Ich hatte bereits gesagt, dass Coleridges Tugend eher auf Mills Bild von Coleridge beruht, als auf Coleridge selbst und das Mill durchaus keine wohlwollende Sicht auf Hegel selbst oder die sonstige deutsch-

1 Siehe Jaeggi, Kritik von Lebensformen.

2 Siehe ebd., S. 277.

3 Siehe Mill, „Coleridge“, S. 150, vgl. Abschnitt 3.4.

4 Zum Beispiel gelegentlich so formuliert, dass die wahre Philosophie zwischen den Polen von Skeptizismus und Dogmatismus stehen muss: „Ohne [...] die Einsicht, [...] daß es also eine Philosophie gibt, die weder Skeptizismus noch Dogmatismus und also beides zugleich ist, können alle die Geschichten und Erzählungen und neuen Auflagen des Skeptizismus zu nichts führen." G. W. F. Hegel. „Verhältnis des Skeptizismus zur Philosophie“. In: Jenaer Kritische Schriften II. Hrsg. von Hans Brockard und Hartmut Buchner. Hamburg: Meiner, 1983. 
sprachige Tradition hatte, aus der Coleridge entspringt. Dies heißt natürlich nicht, dass Mills sozialphilosophischen Tugenden damit kritisiert wären; es heißt nur, dass wir Mill als Interpreten hegelianischer Ansätze nicht allzu ernst nehmen sollten.

Meine Zustimmung wird aber eingeschränkt sein: Ich versuche $\mathrm{zu}$ zeigen, dass es ein paar paradigmatische praktische Probleme gibt, in Bezug auf die es vorteilhafter ist, die Problemstellung so zu formulieren, wie Mill es tut. Damit will ich nicht sagen, dass es unmöglich ist, solche Probleme in Jaeggis Terminologie zu rekonstruieren, sondern dass sich Mills Beschreibung in Bezug auf bestimmte Probleme eher anbietet.

In einer anderen Hinsicht leisten Mills Tugenden aber viel weniger als Jaeggis immanente Kritik. Ich werde eine Perspektive anbieten, unter der wir das verstehen können: Ich werde behaupten, dass wir Jaeggi so verstehen können, dass sie Mills Tugenden gerecht wird, allerdings unter einer spezifischeren Fragestellung. Was diese Fragestellung angeht, leistet Jaeggi damit sehr viel mehr als Mill; ihr Ansatz ist aber für Probleme, die diese Spezifik überschreiten, dadurch auch weniger allgemein. Natürlich ist dies nur eine Perspektive und es gäbe sicherlich andere Möglichkeiten, immanente Kritik mit Mills Tugenden ins Verhältnis zu setzen. Wenn sich diese Perspektive (oder natürlich eine andere Möglichkeit, immanente Kritik mit Mills Tugenden ins Verhältnis zu setzen) aber als fruchtbar herausstellt, wäre ein Beispiel ${ }^{5}$ gegeben, wie wir Mills Tugenden auf andere Ansätze übertragen können.

\section{Darstellung „immanente Kritik“}

Die Problemstellung, die Jaeggi am Anfang ihres Buches angibt, ist die Frage, wie über die bloß formale Kritik kantischer oder habermasianischer Ansätze hinaus Kritik an Lebensformen - das heißt, an sehr umfassend verstandenen Arten und Weisen, wie das gesellschaftliche Leben und Zusammenleben organisiert ist ${ }^{6}$ - möglich

5 Aufgrund der oben noch einmal angesprochenen Nicht-Exklusivität der Tugenden kann Übertragbarkeit nur für einzelne Beispiele gezeigt werden; und aufgrund der methodischen Problemstellung der Philosophie der Philosophie (Abschnitt 2.3) kann sie nur mithilfe der Auseinandersetzung mit einzelnen Ansätzen gezeigt werden.

6 Wie sie Lebensformen genau versteht und wie der Begriff der Lebensform zum Beispiel vom Begriff der sozialen Praxis abzugrenzen ist, beschreibt Jaeggi in 
ist. ${ }^{7}$ Das Problem dieser Ansätze ist, wie Jaeggi sagt, dass sie die Wahl der Lebensform, insofern sie nicht gegen die formalen Prinzipien verstößt, zur reinen Geschmacksfrage machen, über die philosophisch wie auch praktisch nichts weiteres gesagt werden kann. Damit ist auch klar, dass es bei der Frage, wie Kritik möglich ist, darum geht, wie begründete Kritik möglich ist: Es gehe um die $R a$ tionalität von Lebensformen, wie Jaeggi schreibt. ${ }^{8}$

Die Problemstellung kann ergänzt werden durch Spezifikation dessen, was Rationalität hier bedeutet, indem Jaeggis Diskussion zweier Kritikformen - interne Kritik und externe Kritik - in Betracht gezogen wird. (Die Vermeidung der Probleme beider dieser Kritikformen ist natürlich schließlich das Ziel von Jaeggis eigener Idee der immanenten Kritik. ${ }^{9}$ ) Interne Kritik ist bei Jaeggi lediglich die Kritik der Art und Weise, wie wir wirklich leben, vor dem Hintergrund der Normen, die unserer Lebensform intern sind. ${ }^{10} \mathrm{Um}$ Steuerhinterziehung kritisieren zu können, brauchen wir nicht die ganze Marktwirtschaft in Frage zu stellen. Dass Steuerhinterziehung schlecht ist, ist aus den Normen, die mit unserer Lebensform verbunden sind, direkt ableitbar.

Was aber, wenn wir fundamentalere Kritik üben wollen, wenn also die Normen selbst zur Disposition stehen - und wenn wir diese nicht bloß formal, zum Beispiel auf Widerspruchsfreiheit hin, kritisieren wollen? Hier liegt es nahe, einen externen Maßstab zu suchen. ${ }^{11}$ Ein für Jaeggi entscheidender Nachteil jedoch, den externe Kritik hat, wird deutlich, wenn sie die Vorteile interner Kritik diskutiert: Diese teilen sich auf in die von Jaeggi als „systematisch“

den Kapiteln 1 und 2 ihres Buches. Jaeggi, Kritik von Lebensformen. Ich werde, wie ich es auch im Rest dieser Arbeit getan habe, versuchen, in Bezug auf die Frage, welche Entitäten wir genau zur Grundlage der Sozialphilosophie machen sollten, soweit dies möglich ist, neutral bleiben. Damit will ich nicht sagen, dass diese Frage grundsätzlich nicht wichtig ist, sondern nur, dass sie, um zu verstehen, was mit Mills Tugenden gemeint ist, nicht entscheidend ist.

7 Siehe ebd., S. 9ff.

8 Siehe ebd., S. 13.

9 Siehe ebd., Kapitel 6.

10 Siehe ebd., S. $263 f f$.

11 Jaeggi diskutiert die verschiedenen Varianten externer Kritik explizit nicht. Sie deutet aber an, was sie darunter versteht: Sowohl Kritik einer „Beobachterin eines fremden Landes", sowie Nagels „Blick von nirgendwo", als auch (unter anderem vermutlich neo-aristotelische) Versuche, Kritik aus anthropologischen Tatsachen abzuleiten, fallen darunter. Siehe ebd., S. 261f. 
bezeichnete Frage, wie ein solcher Maßstab begründet werden kann, und die verwandte von ihr als ,praktisch-pragmatisch“ bezeichnete Frage, wie andere Personen davon überzeugt werden können, die Kritik zu akzeptieren. ${ }^{12}$ Das Problem gibt es bei interner Kritik nicht. Diesem Vorteil interner Kritik steht natürlich der Nachteil gegenüber, bestimmten Bedürfnissen fundamentalerer Kritik nicht gerecht werden zu können ${ }^{13}$ : Wir wollen oft auch die Normen, die unserer Lebensform intern sind, selbst kritisieren.

Die angekündigte Spezifikation dessen, was Rationalität in Jaeggis Problemstellung bedeutet, kann anhand dessen, was nach Jaeggi die Vorteile interner Kritik sind, also auf folgende Weise gefasst werden: Die Rationalität der Kritik an Lebensformen, die in dem Buch gefunden werden soll, muss sich so erweisen können, dass diese von den Kritisierten (zumindest prinzipiell) einsehbar ist - und zwar aus systematischen Gründen (weil dann die Notwendigkeit der Begründung eines externen Maßstabes entfallen kann ${ }^{14}$ ) und aus praktisch-pragmatischen Gründen (weil Kritik nur so effektiv sein kann). Die Bedingung der Einsichtigkeit der Kritik durch die Kritisierten bezeichne ich als „zusätzliche Spezifikation“, weil sie auch bestritten werden kann: Es ist zum Beispiel behauptet worden, dass es Gründe für eine Person geben kann, zu deren Einsicht ihr die Fähigkeiten oder Sensibilitäten fehlen, ohne dass deshalb gesagt werden könnte, es handelte sich nicht um gute Gründe. Zwar mag der Erwerb der Sensibilitäten und Fähigkeiten, die nötig sind, um die Gründe einzusehen, mit einigem Recht als „Konversion“ zu einem neuen ethischen Standpunkt in Bernard Williams Sinne bezeichnet werden. Aber dass der Weg von einem ethischen Standpunkt zu einem anderen nicht immer auf Einsicht beruht, bedeute nicht, so zum Beispiel McDowell oder Gunnarsson, dass nicht einer der Standpunkte besser ist oder dass es keine Gründe für den einen oder den anderen gebe. Es gibt lediglich keine solchen Gründe, die einer neutralen Beobachter*in, die keinen der Standpunkte teilt, zugänglich wären - oder zumindest sind es nicht diese Grün-

12 Siehe Jaeggi, Kritik von Lebensformen, S. $268 \mathrm{f}$.

13 Siehe ebd., S. $269 f$.

14 Siehe ebd., S. $268 f$. 
de, auf die es ankommt. ${ }^{15}$ In diesem Sinne handelt es sich bei dem, was nach Jaeggi die Vorteile interner Kritik sind, um eine zusätzliche Anforderung an das, was mit der Rationalität (der Kritik) von Lebensformen nachgewiesen werden muss. Kritik an Lebensformen muss sich in einem Sinne als rational erweisen können, der von den unter dieser Lebensform Lebenden einsehbar ist.

Wie schafft es Jaeggi nun mit ihrer Vorstellung von immanenter Kritik, die Vorteile interner Kritik gegenüber externer Kritik zu bewahren, ohne die Nachteile in Kauf nehmen zu müssen? Jaeggis Ausführungen zur immanenten Kritik können nach eigenem Anspruch als Formulierung dessen gesehen werden, was es heißen kann, in Hegels Sinne „seinem Begriff nicht [zu] entsprechen“" ${ }^{16}$ Jaeggis Idee ist, dass die Veränderung von Lebensformen (zumindest oft, siehe unten) als Lernprozess aufgefasst werden kann. Der Anspruch auf Rationalität, die dem Gedanken eines Lernprozesses im Gegensatz zu einem bloßen Veränderungsprozess inne ist, wird von Jaeggi so eingeholt, dass eine neue Lebensform als eine Instanz zur Lösung eines Problems aufgefasst wird. Den Witz der Lebensform zu verstehen beinhaltet dann, das Problem zu verstehen, das zu lösen die Lebensform eine Strategie ist. ${ }^{17}$

Vor diesem Hintergrund kann nun gesagt werden, dass eine Lebensform daran scheitert, ein Problem zu lösen. Und, was wichtig ist: auch die internen Normen der Lebensform können sich als ungeeignet dazu herausstellen, das Problem zu lösen. Immanente Kritik in diesem Sinne kann also vor dem Hintergrund, dass Lebensformen als Problemlösungsstrategien aufgefasst werden, über interne Kritik hinausgehen und auch die fundamentalen Normen, die zu einer Lebensform gehören, kritisch in Frage stellen.

Was ein Problem ist, wird bei Jaeggi absichtlich weit gefasst und ich werde nicht auf alles eingehen, was sie dazu sagt. Wichtig scheint mir aber das zu betonen, was Jaeggi zu Problemen in Abgrenzung zu Bedürfnissen sagt: Probleme ergeben sich nicht unabhängig von

15 Siehe zum Beispiel McDowell, „Might There Be External Reasons“ oder siehe Gunnarsson, Making Moral Sense - Beyond Habermas and Gauthier, Kapitel 14.

16 Siehe Jaeggi, Kritik von Lebensformen, S. 182-193.

17 Siehe ebd., S. 200. 
Lebensformen; sie sind zum Beispiel nicht darauf reduzierbar, dass bloße Grundbedürfnisse nicht befriedigt werden ${ }^{18}$ :

„Probleme“, wie ich sie in diesem Zusammenhang verstehe, sind also erstens kulturell spezifisch und historisch wie sozial formiert. [...] Probleme in Bezug auf Lebensformen sind zweitens grundlegend normativ verfasst in dem Sinne, dass sie sich [...] nicht einfach als Behinderungen oder Störquellen eines bestimmten Vollzuges per se darstellen, sondern als Problematischwerden in Bezug auf eine ethisch vordefinierte Problembeschreibung [...]. Drittens sind Probleme dann immer auch Resultate bereits versuchter Problemlösungen; sie sind Probleme, die aus dem Versuch, Probleme zu lösen, entstanden sind $[\ldots]^{19}$

Insofern kann die Geschichte der Veränderungen der Lebensformen als (rationaler) Lernprozess verstanden werden: Jede Lebensform muss als Problemlösungsinstanz für ein Problem aufgefasst werden, das sich seinerseits aus (ethisch gefassten) Problemen ergibt, die sich innerhalb (vorgängiger) Lebensformen ergeben. Insofern sich neue Probleme ergeben oder eine Lebensform den ihr gestellten Problemen nicht gerecht wird, gibt es Anlass zu immanenter Kritik, und so weiter.

\section{Gemeinsamkeiten und Unterschiede}

Hier deuten sich einige Gemeinsamkeiten natürlich schon an. Im Folgenden möchte ich den Vergleich systematisieren, indem ich zwischen dem, was ich "Mills Tugenden" genannt habe, und Mills Versuch, diesen Tugenden gerecht zu werden, unterscheide. Diese Unterscheidung entspricht natürlich der Unterscheidung zwischen Teil I und Teil II dieser Arbeit. „Mills Tugenden“ sind Ausdruck einer Vorstellung von Philosophie, die ich in dieser Arbeit verteidigen möchte. Mills Versuch diesen Tugenden gerecht zu werden, habe ich explizit nicht vorbehaltlos verteidigt: ich habe in Kapitel 5 angedeutet, wenn wir ihn richtig verstehen, ist Mills Strategie, den Tugenden gerecht zu werden, plausibel für Vorstellungen des gelingenden Lebens und für den Zusammenhang von Vorstellungen

18 Siehe Jaeggi, Kritik von Lebensformen, S. $204 \mathrm{ff}$.

19 Siehe ebd., S. 205. 
des Wohlergehens mit dem gelingenden Leben. Was aber diejenigen Aspekte der Moral angeht, die sich auf andere Personen beziehen - insbesondere Fragen der Gerechtigkeit - sind, so hatte ich in Kapitel 6 nahegelegt, Mills Strategien weniger überzeugend, obwohl sie eine wichtige Leerstelle aufdecken und wir Leid als Maßstab für moralische Vorstellungen nicht ohne Weiteres aufgeben sollten. Die Art und Weise, wie Leid genau im Utilitarismus eine moralische Rolle spielt, ist, so hatte ich behauptet, aber unzureichend.

Damit ist nicht gesagt, dass Lebensexperimente, je nach dem, wie weit wir den Begriff fassen, nicht die Antwort sind. Aber es ist damit gesagt, dass wir mit den benannten und offensichtlichen Schwächen der Übertragung auf andere Bereiche als das gute Leben umgehen müssen. Indem ich im Folgenden zwischen der Problemstellung (Mills Tugenden) und den Lösungen (z.B. Mills experiments in living) unterscheide, und einen anderen Ansatz dahingehend prüfe, inwiefern sie der Problemstellung gerecht wird, wird auch deutlich werden, welche Elemente eine Lösung haben muss. Die Frage, ob wir dann jeden Ansatz, der diese Elemente aufweist, unter die Überschrift des „Lebensexperimentes“ subsumieren, ist dann zweitrangig.

In diesem Sinne könnte also gesagt werden, die Tugenden stellten eine philosophische Herausforderung dar, der Mills eigener Ansatz gerecht werden muss. In gleicher Weise können wir natürlich auch bei Jaeggi zwischen der Problemstellung („Wie kann Kritik an Lebensformen in der qualifizierten Weise als ,rational' verstanden werden?") und ihrer Lösung für dieses Problem, der immanenten Kritik, unterschieden werden.

Es wurde bereits am Anfang dieses Unterabschnittes (8.1.1) auf die Ähnlichkeiten zwischen den Problemstellungen Mills und Jaeggis hingewiesen. Natürlich geht es Jaeggi vordergründig nicht um die Frage, was Philosophie ist, sondern um die Frage, wie rationale Kritik in der qualifizierten Weise möglich ist. Trotzdem sind hier viele Motive ähnlich - Kritik gehört natürlich gerade für Denker*innen, die der kritischen Theorie zuzuordnen sind, zum Kern des Wesens der Philosophie. ${ }^{20}$ Insofern geht es auch bei Jaeggi zumindest implizit um die Frage, wie Philosophie möglich ist. Hier könnte na-

20 Vgl. Max Horkheimer. "Traditionelle und kritische Theorie“. In: Traditionelle und kritische Theorie. 6. Aufl. Frankfurt am Main: Fischer, 2005, S. 205-259. 
türlich auch ein Unterschied vermutet werden, den ich bereits in Abschnitt 2.2 angesprochen habe, der aber nun in Abgrenzung etwas klarer formuliert werden kann: Jaeggi kann, insbesondere vor dem Hintergrund, wie sie die „Vorteile und Grenzen interner Kritik" formuliert" ${ }^{21}$, so verstanden werden, dass Kritik das eigentliche Ziel (zumindest des Gegenstandes ihres Buches, vielleicht auch der Philosophie grundsätzlich) ist. Alle anderen Charakterisierungen würden, so verstanden, diesem Ziel untergeordnet.

In der vorläufigen Qualifizierung des Status' der Tugenden (Abschnitt 2.2) hatte ich jedoch behauptet, die beiden Tugenden Mills seien logisch nicht voneinander abhängig. Der Grund, warum wir Coleridges Tugend brauchen, ist nach Mill nicht, weil wir erst dann Benthams Projekt einer radikalen Kritik umsetzen können. Der Grund ist, dies hatte ich versucht in Kapitel 3 zu zeigen, dass Mill meint, es gebe bestimmte menschliche Erfahrungen, die zu machen nur innerhalb einer bestimmten Weise zu Leben möglich ist. (Dies hat mir dann eine Interpretation dessen, was mit höheren Freuden und höheren Formen des Leides in "Utilitarianism" gemeint ist, die mit bestimmten Arten und Weisen zu leben verbunden seien, ermöglicht, Kapitel 5.) Diese Erfahrungen als menschliche Erfahrungen, als das, was menschlich zu leben im Rahmen einer bestimmten Art und Weise zu leben bedeutet, zu berücksichtigen, ist aus eigenem Recht ein komplementärer Teil dessen, was die Philosophie tun sollte. Coleridges Tugend ist somit eigenständiger Teil einer durch beide Tugenden charakterisierten Vorstellung von Philosophie.

Ich will diesen Unterschied in der Begründung der Pole, zwischen welchen die Problemstellung bei Mill und Jaeggi jeweils besteht, aber auch nicht überbetonen. Erstens gibt es in den Konsequenzen keinen großen Unterschied und zweitens wird spätestens bei der Lösung klar, dass meine vorläufige Charakterisierung Jaeggis zu undifferenziert ist: Auch im Falle von Jaeggis immanenter Kritik kann der Witz einer Lebensform als Instanz einer Problemlösung nicht losgelöst von unseren Arten und Weisen zu leben betrachtet werden. ${ }^{22}$ Wie bereits erwähnt, sind die Probleme, die gelöst werden sollen, nicht einfach Fragen, wie biologische Bedürfnisse befriedigt

21 Siehe Jaeggi, Kritik von Lebensformen, S. 268ff. Um eine Darstellung dieser habe ich mich im letzten Abschnitt bemüht.

22 Siehe ebd., S. 205, ausführlicher zitiert weiter oben in diesem Unterabschnitt (8.1.1). 
werden können, sondern sie stellen sich innerhalb der ethischen Verfasstheit einer Lebensform.

Nicht nur bei der Problemstellung, auch bei den Lösungen gibt es natürlich Gemeinsamkeiten. Sowohl Mill als auch Jaeggi legen großen Wert darauf, dass das jeweils die Kritik rationalisierende Element - höhere Formen des Leides und der Freude im ersten Fall, Probleme einer Lebensform im zweiten Fall - nicht unabhängig von einer bestimmten Art zu leben erfasst werden kann; dass aber andererseits dies nicht bedeutet, dass nicht die Vorteile externer $\mathrm{Kritik}^{23}$ beziehungsweise die Vorteile eines externen Maßstabes damit verbunden sein können. Ich hatte das für Mill auf die Formel gebracht, dass Mill beansprucht einen Maßstab zu geben, der gleichzeitig extern und nicht-extern ist, Abschnitt 6.1.3. Bei Jaeggi zeigt sich das in der aus (vorhergehenden) Lebensformen hervorgehenden Verfasstheit der Probleme, die Lebensformen zu lösen beanspruchen.

Mills Lösung, die ich mit Anderson unter dem Schlagwort „experiments in living" zusammengefasst habe, hat in der spezifischen Weise, wie Mill sie vertritt, wie ich in Kapitel 6 versucht habe zu begründen, große Schwächen, wenn es nicht mehr um die bloß individuellen Aspekte des gelingenden Lebens geht. Das, was Jaeggi dazu sagt, was es heißt, dass eine Lebensform problematisch wird, erscheint mir plausibler, als das Problematischwerden wie bei Mill auf individuelle Erfahrungen von Freude und Leid zurückzuführen. Zur Rettung Mills könnte jedoch gesagt werden, dass auch in Jaeggis Fall das Problematischwerden einer Lebensform eine empirische Frage ist, die in irgendeiner Form als schlecht erlebt werden muss, sich jedenfalls nicht a priori zeigen kann. Es erfüllt also die Minimalbedeutung von Hedonismus aus Kapitel 5. Aber es handelt sich sicherlich nicht um die Art von individuellen Erfahrungen, die Mill in seiner Autobiographie beschreibt, oder die durch exzentrische Lebensentwürfe, wie er sie in „On Liberty“ verteidigt, gemacht werden können. Bestimmte Fragen können so individualistisch, wie Mill es tut, nicht beantwortet werden und hier hat Jaeggi, was die Lösungen angeht, sicherlich einen plausibleren Ansatz als Mill.

In diesem Sinne könnten wir versucht sein, Mills Tugenden als Charakterisierung der Philosophie zu verstehen aber seine eigenen

23 Beziehungsweise die Vermeidung der Nachteile bloß interner Kritik. 
Versuche, diesen Tugenden gerecht zu werden, durch Jaeggis Begriff der immanenten Kritik zu ersetzen. Im Folgenden möchte ich argumentieren, dass wir dies nicht tun sollten. Es gibt Probleme, die sich mithilfe der Tugenden formulieren lassen, für die, so meine ich, immanente Kritik nicht die beste Antwort ist. Selbst, wenn wir uns auf den Standpunkt stellen, dass diese Probleme letztlich nicht lösbar sind, sollten wir die entsprechenden Problemstellungen nur aufgeben, wenn wir wirklich zeigen können, dass es sich im strengen Sinne um Scheinprobleme handelt. In diesem Sinne sind Mills sozialphilosophische Tugenden, weil sie Merkmale der Philosophie sind, allgemeiner als Jaeggis Lösung. Um dies zu zeigen, möchte ich drei miteinander zusammenhängende Grenzen aufzeigen.

Immanente Kritik setzt voraus, dass Geschichte als Lernprozess aufgefasst werden kann, der sich an sukzessiven Problemen und deren Lösungen orientiert. Jaeggi weist zwar darauf hin, dass ihr Ansatz nicht voraussetzt, dass die Frage, inwiefern unsere Lebensform eine Problemlösungsinstanz ist, allen daran Teilnehmenden jederzeit klar sein muss; ${ }^{24}$ aber immanente Kritik setzt dennoch voraus, dass die Kritiker*in irgendwie erkennen kann, inwiefern unsere Lebensform Teil eines historischen Lernprozesses ist. Damit verbunden erscheint es mir wichtig, dass wir hier von einem "Erkennen“ reden können: Es mag zwar eine gewisse hermeneutische Offenheit darin geben, wie genau wir die Geschichte rekonstruieren, um diesen Lernprozess sichtbar zu machen, aber es kann nicht völlig willkürlich sein. Es muss Richtig und Falsch geben, ansonsten wäre auf die skeptische Herausforderung in Jaeggis Problemstellung, dass nämlich Kritik an unseren Lebensformen, die über rein formale Kritik hinausgeht, am Ende auf bloßen Geschmacksurteilen beruht, nicht eingegangen. Die Konsequenz auf Jaeggis Position ist daher folgende: Die Geschichte, die zu unserer Lebensform führt, muss nicht nur als Lernprozess aufgefasst werden, sie muss in diesem Sinne auch tatsächlich ein Lernprozess sein. Zweitens muss dieser Lernprozess für die Kritisierenden auch zumindest im Prinzip erkennbar sein, damit rationale Kritik möglich ist.

Beides mag oft der Fall sein; es scheint mir aber nicht klar, dass es immer der Fall sein muss. (Mill selbst meint, wir müssen aktiv dafür sorgen, dass Geschichte ein Lernprozess ist; dass

24 Siehe Jaeggi, Kritik von Lebensformen, S. $206 \mathrm{f}$. 
dies aber nicht automatisch gegeben sei. ${ }^{25}$ ) Ein vielleicht extremes Beispiel sind katastrophale Ereignisse: Den Zusammenbruch der Bronzezeit-Gesellschaften oder die Auslöschung wichtiger Teile der Gesellschaften der amerikanischen Ureinwohner durch Krieg, neue Krankheiten u.s.w. können, so meine ich, nur in dem Sinne als rational bezeichnet werden, in dem jeder Prozess, auch wenn wir nur eine bloß kausale Beschreibung haben, als rational bezeichnet werden kann. Dass beispielsweise der Zusammenbruch der aztekischen Herrschaftsstrukturen eine rationale Antwort auf Cortés' Angriffe gewesen sei, ist ähnlich plausibel wie zu sagen, dass zu sterben eine rationale Antwort auf einen Hirntumor ist.

Der Punkt hier ist nicht, dass solche extremen Ereignisse besonders häufig sind. Wenn aber rationale und bloß kausale Deutung historischer Ereignisse in dieser Weise in Konflikt stehen können, dann stellt sich die Frage, ob nicht auch in anderen geschichtlichen Entwicklungen nur Teile der Erklärung diese Entwicklungen richtiger Weise als Lernprozess in Erscheinung treten lassen. ${ }^{26}$ Es ist, so meine ich, genauso optimistisch anzunehmen, dass die Geschichte notwendig ein Lernprozess ist, wie es wäre, wenn eine Person annähme, dass persönliche Veränderungen notwendig einen Lernprozess darstellen - zumal, wenn es, wie angedeutet, darum gehen muss, dass es hier Richtig und Falsch gibt: Wir dürfen nicht nur irgendwie die Ereignisse so deuten, dass sie einen Lernprozess darstellen. Wenn immanente Kritik also die einzig legitime Form der Kritik (oder der Reaktion auf Mills Tugenden) wäre, dann würde das bedeuten, dass wir ausgerechnet diejenigen Aspekte unserer Lebensform, die sich nicht besonders plausibel als Lernprozess beschreiben lassen, nicht rational kritisieren können!

Ein zweites Problem dabei, von einem kontinuierlichen, historischen Lernprozess auszugehen, ist, dass bestimmte Probleme, die die Frage betreffen, wessen Tradition hierfür die Grundlage bilden soll, nicht berücksichtigt werden. Viele von uns dürften liberale Verfassungen, seien sie nun geschrieben oder ungeschrieben, und die Tradition, in der diese stehen, für eine geeignetere Grundlage für eine Rechtsordnung halten als zum Beispiel die Scharia. Effektiv

25 Siehe Mill, „On Liberty“, S. 238.

26 Oder, dass sie sich als Lernprozess einer herrschenden Gruppe und nicht der Lebensform als ganzer verstehen lassen; dass also Macht der kausale Faktor ist. 
verdanken sie ihre Geltung sicherlich der faktischen Macht: Die entsprechenden Rechtsordnungen sind in den jeweiligen Gesellschaften breit genug akzeptiert, institutionell genug geschützt und materiell gut genug ausgestattet - und die Tatsache, dass sie das sind, kann historisch erklärt und sicherlich zumindest zum Teil auch als Lernprozess aufgefasst werden. Ihre effektive Geltung können wir aber kaum als Grund angeben, wenn es um die Frage geht, warum die Rechtsordnungen, die als Ergebnisse eines Lernprozesses unserer Geschichte aufgefasst werden können, besser sind als Rechtsordnungen, die Ergebnisse eines Lernprozesses einer anderen geschichtlichen Entwicklung sind. „Weil sie Resultat eines Lernprozesses unserer Geschichte sind" ist keine Antwort, wenn die Fragende eine andere historische Tradition als die ihre identifiziert. ${ }^{27}$

Hier gibt es, soweit ich sehe, zwei Strategien: Die erste ist, zuzugestehen, dass immanente Kritik für Fragen dieses Typs keine Antwort liefert und sie damit als Kritikform auf Personen zu beschränken, die sich im Großen und Ganzen mit der gleichen geschichtlichen Entwicklung identifizieren. Die zweite wäre, von einer globalen Geschichte auszugehen, in welcher sich der Konflikt zwischen verschiedenen partikularen Traditionen innerhalb der universellen Geschichte selbst als Problem darstellt, das wiederum von einer reformierten Lebensform zu lösen wäre. Vielleicht ist dieser Gedanke attraktiv. Auf der anderen Seite läuft diese Strategie Gefahr, einen der Hauptvorteile der immanenten Kritik, nämlich ihre Verbundenheit mit einer partikularen Lebensform, aufzugeben: Erhält immanente Kritik die Vorteile interner Kritik (ohne ihre Nachteile in Kauf nehmen zu müssen) eigentlich noch aufrecht, wenn der

27 Ich will damit keineswegs behaupten, dass es grundsätzlich keine Antwort auf solche Fragen geben kann. Vielmehr möchte ich auf das Problem hinweisen, das durch die Rolle der Geschichte als Lernprozess im Rahmen der immanenten Kritik hervorgerufen wird. Nach Mill müssten wir uns für die Beantwortung dieser Fragen an Personen wenden, die verstehen, was es heißt, nach verschiedenen Lebensformen, die verschiedenen historischen Traditionen entspringen, zu leben. Dann können wir Hoffnung auf eine Antwort haben, auch wenn dadurch natürlich nicht garantiert wird, dass es eine eindeutige und unkomplizierte Antwort gibt. Die Probleme, die damit seinerseits verbunden sind, habe ich bereits erwähnt; hier dient diese Bemerkung nur dazu, dafür zu argumentieren, immanente Kritik nicht von Vornherein als einzige Möglichkeit, Mills Tugenden gerecht zu werden, aufzufassen. Siehe Abschnitt 5.3. 
Referenzpunkt nicht eine Partikulargeschichte, sondern eine Globalgeschichte ist?

Der dritte Punkt, den ich nennen möchte, ist, dass das, was ich oben die „zusätzliche Spezifikation der Rationalitätsbedingung“ genannt habe, eine zu starke Einschränkung ist. Wir sollten zumindest nicht theoretisch ausschließen, dass es die Möglichkeit gibt, dass eine Art und Weise zu leben besser oder schlechter als eine andere ist, ohne dass es die Möglichkeit gibt, dass es einen rationalen, argumentativen Weg von der einen zur anderen Lebensform gibt. Natürlich sollten wir diese Qualifikation der Rationalitätsbedingung nicht leichtfertig aufgeben: In verschiedenen Ansätzen ist damit die Hoffnung verbunden, Streitigkeiten argumentativ und ohne Gewalt zu lösen. Allerdings ist die Alternative „Argumente oder Gewehre" natürlich nicht die einzige: Thomas Kuhn, für dessen Struktur wissenschaftlicher Revolutionen sich das Problem in ähnlicher Weise stellt, legt nahe, dass wissenschaftliche Paradigmen zum Beispiel dann aufgegeben werden, wenn ihre Vertreter*innen allmählich aussterben $^{28}$, dass es jedenfalls keine wissenschaftlichen Nachweise für den Vorrang eines Paradigmas vor einem anderen geben könne. ${ }^{29}$ Trotzdem und trotz der Tatsache, dass die Güte der Wahl eines neuen wissenschaftlichen Paradigmas nicht mit den Mitteln des alten nachgewiesen werden kann, verwehrt er sich gegen einen Relativismus. ${ }^{30}$ Dies gilt umso mehr für die Ethik. Konversion muss nicht gewaltgeprägt beruhen und darauf, dass es zwischen den Extremen der streng deduktiven Schlussfolgerung und schamloser Propagan-

28 Er zitiert zumindest Max Planck in dieser Hinsicht zustimmend. Siehe Thomas Kuhn. Die Struktur wissenschaftlicher Revolutionen. 2. Aufl. Frankfurt am Main: Suhrkamp, 1976, S. 162.

29 Siehe ebd., S. 159.

30 „My views, it is said, make of theory choice 'a matter for mob psychology' [...] The debates surrounding such choices must, my critics claim, be for me 'mere persuasive displays without deliberative substance.' Reports of this sort manifest total misunderstanding, and I have occasionally said as much in papers directed primarily to other ends." Thomas Kuhn. „Objectivity, value judgment, and theory choice". In: Arguing about Science. Hrsg. von Alexander Bird und James Ladyman. London und New York: Routledge, 2013, S. 74-86, S. 74 . 
da wichtige (und ethisch relevantere) Zwischentöne gibt, hat Cora Diamond hingewiesen. ${ }^{31}$

Das heißt natürlich nicht, dass wir uns nicht um das, was durch die zusätzliche Spezifikation ausgedrückt wird, bemühen sollten, wo es möglich ist. Wir sollten lediglich nicht von vornherein ausschließen, dass es Fälle gibt, in denen wir eine Lebensform als besser (und in der unqualifizierten Weise als rationaler) bezeichnen wollen, ohne dass wir sie in der qualifizierten Weise als rationaler bezeichnen wollen würden. Dies gilt nicht nur für den dritten dieser Punkte, sondern auch für die anderen beiden: Ich will nicht sagen, dass ein Ansatz wie Jaeggis mit diesen Punkten nicht irgendwie umgehen könnte; ich will lediglich sagen, dass diese Art von Problemen dafür sprechen, Mills sozialphilosophische Tugenden unabhängig von einer spezifischen philosophischen Position für fruchtbar zu halten.

\subsubsection{Ausdehnung auf weitere Ansätze?}

Ich möchte davon Abstand nehmen, die Ausdehnung von Mills Tugenden auf weitere philosophische Ansätze zu demonstrieren, obwohl ich glaube, dass die Tugenden sehr häufig eine Rolle spielen: Eine solche Ausdehnung läuft immer Gefahr, als gezwungen wahrgenommen zu werden und ich glaube, dass ich nicht viel dadurch gewinnen würde, weil ich die Nicht-Exklusivität der Tugenden aus internen Gründen zugestehen muss. Sie folgt aus der Selbstanwendung der philosophischen Tugenden auf die Philosophie der Philosophie. Dass die Tugenden trotzdem oft eine Rolle spielen, bedeutet nämlich nicht, dass sie das vorrangige Organisationsmerkmal dieser Ansätze sind. Jaeggis Ansatz zu untersuchen erschien mir sinnvoll, weil Mills Tugenden selbst mit Coleridge ein hegelianisches Element enthalten, und ich zeigen wollte, dass es sinnvoll ist, die Tugenden auch unabhängig von bestimmten geschichtsphilosophischen Annahmen zu formulieren. Im letzten Kapitel habe ich mich außerdem um eine Lesart der platonischen Frühdialoge vor dem Hintergrund der Tugenden bemüht; der Grund war hier, dass Mill sich selbst extensiv mit Platon auseinandergesetzt hat und in der Korrektur des Bildes von Platon, das Mill hatte, die sozialphiloso-

31 Cora Diamond. „Anything but argument“. In: Philosophical Investigations 5.1 (1982), S. 23-41. 
phischen Tugenden tatsächlich deutlicher werden, als dies bei Mills eigener Auseinandersetzung mit Platon der Fall war.

\subsection{Offene Probleme und Ausblick}

Besonders eine Frage, die diese Arbeit offen lassen muss, möchte ich im Folgenden ansprechen, weil sie sich vielleicht aufdrängt: Könnte der Gedanke der experiments in living nicht einfach sehr viel einfacher auf nicht-individuelle Fragen ausgeweitet werden, als Mill dies tut? Zunächst werde ich kurz erläutern, warum sich diese Frage aufdrängt, um dann zu erklären, was mit der Frage gemeint ist.

In Kapitel 6 hatte ich Mills Utilitarismus kritisiert. Dieser ist aber ein Versuch eine Position zum Wohlergehen, der gegenüber ich in Kapitel 5 weniger kritisch war, zu verallgemeinern. Wenn wir über das, was Anderson im Anschluss an Mill experiments in living nennt, einen philosophischen Zugang zum gelingenden Leben erhalten und wir zeigen könnten, dass es in der Moral im engeren Sinne (zum Beispiel im Sinne von Gerechtigkeit) auch um das gelingende Leben - allerdings aus neutraler Perspektive betrachtet - geht, dann wären wir auch in der Moral im engeren Sinne den Tugenden gerecht geworden.

Nun stimmt es zwar, so hatte ich versucht nahezulegen, dass es in der Moral auch um das gelingende Leben geht, und ebenso, dass es nicht (zumindest nicht nur) um das gelingende Leben der Handelnden geht; ebenso richtig ist es, dass dies einen Maßstab für Moralvorstellungen darstellt, der zwar nicht von außerhalb eines ethischen Standpunkts aus ersichtlich ist, der aber gleichzeitig nicht begrifflich auf diesen reduzierbar ist. Obwohl Mill also in diesen Punkten zuzustimmen ist und der Utilitarismus, wenn wir diese Eigenschaften betonen, als Versuch aufgefasst werden kann, beiden Tugenden gerecht zu werden, bedeutet das natürlich nicht, dass darum nun seinerseits Moral im engeren Sinne auf eine Theorie des Wohlergehens und das ein oder andere neutrale Aggregationsprinzip reduziert werden kann. Diese Art und Weise, die Idee der experiments in living auf die Moral zu übertragen, schlägt, so hatte ich in Kapitel 6 gesagt, fehl.

Dies ist aber natürlich nicht die einzige Art und Weise, wie dieser Gedanke übertragen werden könnte. Eine vielleicht auf den ersten 
Blick sogar naheliegendere Strategie wäre es, den Gedanken der experiments in living direkt zum Beispiel auf zum Beispiel Gerechtigkeitsvorstellungen zu übertragen. Dies bietet sich vor allem an, wenn „Hedonismus" in der minimalen Weise verstanden wird, die ich in Kapitel 5 nahegelegt habe: Wenn die Minimalvorstellung von Hedonismus, die Mill mindestens vertreten muss, damit die Idee der experiments in living plausibel ist, darin besteht, dass normalerweise und vor dem Hintergrund all der bereits erwähnten Grenzen (siehe zum Beispiel Abschnitt 5.3) eine gelingendere Art und Weise zu leben von einer, die kompetent an zwei Lebensweisen teilgehabt hat, auch als eine gelingendere Art und Weise zu leben erlebt wird, steht der Übertragung vielleicht nicht so viel im Weg. Es wird dabei ja zum Beispiel gar nicht gesagt, auf welche Weise dieses „als besser Erleben" stattfindet, mit welchen Zuständen und Emotionen es verbunden ist u.s.w.

Die Frage, die sich für die direkte Übertragung stellt ist also: Ist es ebenso plausibel wie beim Wohlergehen, dass ein besserer moralischer Standpunkt zu einer moralischen Frage auch als ein besserer moralischer Standpunkt zu dieser Frage erlebt wird von einer die ernsthaft darum bemüht war, zwei oder mehr moralische Standpunkte einzunehmen? Natürlich ist, wenn die Gedanken in Abschnitt 6.1.3 zutreffend sind, ausgeschlossen, dass „besser" hier „besser für die Handelnde" heißen kann. Das einzige, was zählen könnte wäre, dass die entsprechende Perspektive als „moralisch besser" erlebt wird - und wir sollten vermutlich hier ebenso wie bei der Minimaldefinition von Hedonismus, die ich vorgeschlagen habe, darauf verzichten, dies an eine spezifisch moralische Erfahrung zu koppeln: Genauso wenig, wie meine Minimalhedonist*in darauf festgelegt ist, Freude und Leid als homogene Zustände aufzufassen, genauso wenig sollten wir den Gedanken, dass es so etwas gibt, wie etwas als „moralisch besser" zu erleben, an die Annahme einer bestimmten homogenen Art von Zuständen oder Erkenntnisfähigkeiten koppeln.

Es gibt noch einen zweiten Grund, warum sich diese Art es zu sehen, aufdrängen könnte: In Kapitel 7 habe ich Gilligan mit ihrem Fallbeispiel „Claire" zitiert und meine Zustimmung, dass es unkontrovers sein dürfte, dass wir nicht annehmen sollten, dass Claire, nachdem sie die von Gilligan beschriebenen Erfahrungen gemacht hat, nun die moralische Urteilskompetenz abgesprochen 
werden sollte, wie es die Kohlbergskala nahelegt. Es liegt, wie Gilligan überzeugend darlegt, viel näher zu sagen, Claire sehe die Dinge jetzt besser als zuvor. ${ }^{32}$ Wir hätten so auch die Möglichkeit, die in Kapitel 7 angedeutete Frage nach Expertise in moralischen Fragen zu beantworten, ohne dass wir behaupten müssten, diese Expertise könne wie eine techne methodisch erworben werden.

Und natürlich könnte, wenn die Ausweitung der Idee, dass experiments in living Teil des Philosophierens sind, auf die Moral möglich ist, auch darüber nachgedacht werden, sie auf weitere Bereiche der Philosophie auszudehnen - und so eine sehr allgemeine Antwort auf die Frage zu erhalten, wie wir beiden philosophischen Tugenden gerecht werden können. Ohne dass er dies freilich in den Kontext von Mills beiden Tugenden stellt, kann Logi Gunnarssons Auseinandersetzung mit dem jungen William James in diese Richtung verstanden werden. ${ }^{33}$

Trotz all dieser Punkte möchte ich, wie angedeutet, die Frage in dieser Arbeit offen lassen. Einige der Argumente, die dagegen sprechen, habe ich in anderem Kontext bereits in Kapitel 6 erwähnt: Moral scheint in einer qualitativ anderen Weise sozial zu sein als Wohlergehen ${ }^{34}$, was die Rolle zumindest individuell verstandenen Erlebens in Frage stellt. Einige der Gegenargumente lassen sich vielleicht auch auf ähnliche Weise entkräften, wie die entsprechenden Argumente gegen experiments in living als Methode für das Wohlergehen. Der Grund, warum ich mich enthalten will, sind entsprechend nicht die Probleme, die mit der Rolle von Erfahrung in der Moral oder moralischer Expertise ${ }^{35}$ verbunden sind, als vielmehr

32 Siehe Gilligan, Die andere Stimme, S. 73-78. Siehe Fußnote 66 in Kapitel 7.

33 Gunnarsson, Vernunft und Temperament. Eine Philosophie der Philosophie.

34 Natürlich spielt, wie mehrfach betont, auch bei Wohlergehen der soziale Kontext eine entscheidende Rolle: $\mathrm{Ob}$ wir überhaupt auf eine bestimmte Weise leben können und ob wir auf eine bestimmte Weise ein gelingendes Leben führen können, hängt stark davon ab, in welcher Gesellschaft wir leben. Für die nachträgliche Antwort auf die Frage aber, ob so-und-so gelebt zu haben Teil meines Glückes war oder nicht, scheint die Einschätzung anderer weniger entscheidend als für die gleiche nachträgliche Frage, ob so-und-so $\mathrm{zu}$ leben moralisch vertretbar ist. Wir müssen nicht so weit gehen wie Habermas und behaupten, dass die Einschätzung anderer (im idealen Diskurs) mit-konstitutiv ist für solche Fragen, um dieser Behauptung zuzustimmen.

35 Für eine Diskussion einiger Einwände gegen moral testimony siehe Alison Hills. „Moral Testimony and Moral Epistemology“. In: Ethics 120.1 (2009), S. 94-127. Hills argumentiert schließlich dafür, dass moral testimony in Bezug 
die Tatsache, dass ich es zwar für prima facie plausibel halte, dass gelingendere Arten des Lebens normalerweise auch als gelingender erlebt werden, während es mir nicht so offensichtlich erscheint, dass dies auch bei der Moral und bei anderen philosophischen Fragen der Fall ist.

Damit bleibt auch die Frage offen, ob es eine allgemeine Art und Weise gibt, den beiden philosophischen Tugenden systematisch gerecht zu werden. Dies ist insofern kein bedauerliches Ergebnis für mich, als es dann umso sinnvoller ist, Mills Philosophie der Philosophie anhand der beiden philosophischen Tugenden deutlich zu machen.

auf moralisches Wissen möglich ist, dass es aber oft eher auf moralisches Verständnis ankomme, das nicht allein durch die Akzeptanz des Urteils anderer erreicht werden könne. 


\section{Literatur}

Amartya, Sen. Commodities and Capabilities. Amsterdam etc.: North Holland, 1985. Kap. 1-6.

Anderson, Elizabeth S. „John Stuart Mill and Experiments in Living“. In: Ethics 102.1 (1991), S. 4-26.

Anschutz, R. P. „The Logic of J. S. Mill“. In: Mill - A Collection of Critical Essays. Hrsg. von Jerome Schneewind. London u.a.: Palgrave Macmillan, 1968, S. 46-83.

Anscombe, G. Elizabeth M. Intention. Cambridge, Massachusetts und andere: Harvard University Press, 2000.

- „Modern Moral Philosophy“. In: Philosophy 33.124 (1958), S. 119.

Aristoteles. Nikomachische Ethik. 5. Aufl. Übersetzung von Ursula Wolf. Reinbek bei Hamburg: Rowohlt Taschenbuch Verlag, 2006. Bentham, Jeremy. An Introduction to the Principles of Morals and Legislation. Oxford: Clarendon Press, 1907.

- Deontology together with A Table of the Springs of Action and The Article on Utilitarianism. Oxford und andere: Oxford University Press, 1983.

- „The Book of Fallacies“. In: The Works of Jeremy Bentham, Volume II. Hrsg. von John Bowring. London: Simpkin, Marshall \& Co., 1843, S. $374-487$.

Berger, Fred. „Mill's Concept of Happiness“. In: John Stuart Mill's Social and Political Thought. Hrsg. von G. W. Smith. Bd. 1. London und New York: Routledge, 1991, S. 117-136.

Bertram, Georg W. „Was heißt es, Kunst als paradigmatische Praxis der zweiten Natur zu begreifen?" In: Deutsche Zeitschrift für Philosophie 66.3 (2018), S. 362-382.

Bourdeau, Michel. „Auguste Comte“. In: The Stanford Encyclopedia of Philosophy. Hrsg. von Edward N. Zalta. Winter 2015. 2015.

Bourdieu, Pierre. Die feinen Unterschiede. Frankfurt am Main: Suhrkamp, 1987.

Brink, David. „Mill's Moral and Political Philosophy“. In: The Stanford Encyclopedia of Philosophy. Hrsg. von Edward N. Zal- 
ta. Winter 2016. Metaphysics Research Lab, Stanford University, 2016.

Bronzo, Silver. „The Resolute Reading and Its Critics. An Introduction to the Literature“. In: Wittgenstein-Studien 1.3 (2012), S. $45-80$.

Butler, Judith. „Burning Acts, Injurious Speech“. In: Excitable Speech. A Politics of the Performative. New York, London: Routledge, 1997, S. 43-69.

Coleridge, Samuel Taylor. On the Constitution of Church and State. London: William Pickering, 1839. URL: https://archive.org/ details/cu31924105501906 (besucht am 05. 05. 2019).

Crisp, Roger. Mill on Utilitarianism. London und New York: Routledge, 1997.

- Reasons and the Good. Oxford: Clarendon Press, 2008.

Dancy, Jonathan. Ethics Without Principles. Oxford und andere: OUP, 2004.

Davidson, Donald. „Actions, Reasons, and Causes“. In: The Journal of Philosophy 60.23 (1963), S. 685-700.

- "Mental Events". In: Essays on Actions and Events. Philosophical Essays Volume I. Oxford und andere: Oxford University Press, 2001. Kap. 11, S. 207-225.

Derwin, Fred und Regis Machart, Hrsg. Intercultural Communication with China. Singapur: Springer, 2017.

Diamond, Cora. „Anything but argument“. In: Philosophical Investigations 5.1 (1982), S. 23-41.

- „Eating Meat and Eating People“. In: Philosophy 53.206 (1978), S. $465-479$.

- "Throwing Away the Ladder“. In: Philosophy 63.243 (1988), S. 527.

Donatelli, Piergiorgio. „Mill's Perfectionism“. In: Prolegomena 5.2 (2006), S. 149-164.

Easterlin, Robert A. „Does Money Buy Happiness?“ In: The Public Interest 30 (1973), S. 3-10.

Foot, Philippa. „Moral Arguments“. In: Mind 67.268 (1958), S. 502513.

- „Morality as a System of Hypothetical Imperatives“. In: The Philosophical Review 81.3 (1972), S. 305-316.

- Natural Goodness. Oxford: Clarendon Press (OUP), 2001. 
Frankena, William. „Der naturalistische Fehlschluss“. In: Sprache und Ethik - Zur Entwicklung der Metaethik. Hrsg. von Günther Grewendorf und Georg Meggle. Frankfurt am Main: Suhrkamp, 1974, S. 83-99.

Frege, Gottlob. Die Grundlagen der Arithmetik. Eine logisch mathematische Untersuchung. Breslau: Wilhelm Koebner, 1984.

Gaiser, Konrad. Platons ungeschriebene Lehre. Studien zur systematischen und geschichtlichen Begründung der Wissenschaften in der Platonischen Schule. Stuttgart: Klett, 1968.

Gaita, Raimond. „Callicles' challenge“. In: Critical Quarterly 47.1-2 (2005), S. 40-52.

Geach, Peter. „Good and Evil“. In: Analysis 17.2 (1956), S. 33-42.

Gibbs, Benjamin. „Higher and Lower Pleasure“. In: Philosophy 61.235 (1986), S. 31-59.

Gilligan, Carol. Die andere Stimme. München und Zürich: Piper, 1999.

Giorgini, Giovanni. „Radical Plato: John Stuart Mill, George Grote and the Revival of Plato in Nineteenth-Century England". In: History of Political Thought 30.4 (2009), S. 617-646.

Gosepath, Stefan. Gleiche Gerechtigkeit. Frankfurt am Main: Suhrkamp, 2004.

Griffin, James. Well-Being. Its Meaning, Measurement and Moral Importance. Oxford: OUP, 1986.

Griswold, Charles L. „Relying on Your Own Voice: An Unsettled Rivalry of Moral Ideals in Plato's Protagoras". In: The Review of Metaphysics 53.2 (1999), S. 283-307.

Gunnarsson, Logi. Making Moral Sense - Beyond Habermas and Gauthier. Cambridge et al.: Cambridge University Press, 2003.

- Vernunft und Temperament. Eine Philosophie der Philosophie. Leiden, NL u.a.: Mentis Verlag, 2020.

Habermas, Jürgen. „Diskursethik - Notizen zu einem Begründungsprogramm". In: Moralbewusstsein und kommunikatives Handeln. Frankfurt am Main: Suhrkamp, 1983, S. 53-125.

- ,Taking Aim at the Heart of the Present". In: Foucault - A Critical Reader. Hrsg. von David C. Hoy. Oxford und andere: Basil Blackwell, 1986, S. 103-108.

Hamilton, Andy. „Conservatism“. In: The Stanford Encyclopedia of Philosophy. Hrsg. von Edward N. Zalta. Fall 2015. 2015. 
Haraldsson, Róbert H. ,., This all but universal illusion ... - Remarks on the question: Why did Mill write On Liberty?" In: Sats Nordic Journal of Philosophy 5.1 (2004), S. 83-109.

Hegel, G. W. F. „Verhältnis des Skeptizismus zur Philosophie“. In: Jenaer Kritische Schriften II. Hrsg. von Hans Brockard und Hartmut Buchner. Hamburg: Meiner, 1983.

Heidegger, Martin. Sein und Zeit. 18. Aufl. Tübingen: Max Niemeyer, 2001.

Hills, Alison. „Moral Testimony and Moral Epistemology“. In: Ethics 120.1 (2009), S. 94-127.

Himmelfarb, Gertrude. On Liberty $\&$ Liberalism: The Case of John Stuart Mill. New York: Knopf, 1974.

Hinn, Laura. „Dissertation“. Im Erscheinen.

Hoffmann, Thomas. Das Gute. Berlin: De Gruyter, 2014.

Horkheimer, Max. „Die gesellschaftliche Funktion der Philosophie“. In: Traditionelle und kritische Theorie. 6. Aufl. Frankfurt am Main: Fischer, 2005, S. 270-290.

- „Traditionelle und kritische Theorie". In: Traditionelle und kritische Theorie. 6. Aufl. Frankfurt am Main: Fischer, 2005, S. 205259.

Hursthouse, Rosalind. On Virtue Ethics. Oxford und andere: Oxford University Press, 1999.

Husserl, Edmund. Formale und transzendentale Logik. den Haag: Martinus Nijhoff, 1974.

- Logische Untersuchungen, Band 1. Halle: Niemeyer, 1913.

Jacobs, Jo Ellen. The Voice of Harriet Taylor Mill. Bloomington: Indiana University Press, 2002.

Jaeggi, Rahel. Kritik von Lebensformen. Berlin: Suhrkamp, 2014.

James, William. Pragmatism and other Essays. New York: Washington Square Press, 1963.

Kant, Immanuel. Grundlegung zur Metaphysik der Sitten. Hamburg: Felix Meiner, 1999.

- Kritik der praktischen Vernunft. Hamburg: Meiner, 2003.

- Kritik der reinen Vernunft. Hamburg: Meiner, 1956.

Kern, Andrea. Quellen des Wissens. Frankfurt am Main: Suhrkamp, 2006.

Kohlberg, Lawrence. „Moralische Entwicklung“. In: Die Psychologie der Moralentwicklung. Hrsg. von Wolfgang Althof. Frankfurt am Main: Suhrkamp, 1996. 
Kohlberg, Lawrence und Daniel Candee. „Die Beziehung zwischen moralischem Urteil und moralischem Handeln". In: Die Psychologie der Moralentwicklung. Hrsg. von Wolfgang Althof. Frankfurt am Main: Suhrkamp, 1996.

Koppetsch, Cornelia. „Liebe und Partnerschaft. Gerechtigkeit in modernen Paarbeziehungen. Studien zur Soziologie intimer Beziehungen“. In: Liebe am Ende des 20. Jahrhunderts. Hrsg. von Katharina Hahn und Günter Burkart. Leske + Budrich, 1998, S. 111-129.

Korsgaard, Christine. „Personal Identity and the Unity of Agency: A Kantian Response to Parfit". In: Philosophy 83 Public Affairs 18.2 (1989).

- „Realism and Constructivism in Twentieth-Century Moral Philosophy“. In: The Constitution of Agency. Essays on Practical Reason and Moral Psychology. Oxford und andere: Oxford University Press, 2008, S. 302-326.

- The Sources of Normativity. Cambridge, UK: Cambridge University Press, 1996.

Kripke, Saul. Wittgenstein on Rules and Private Language: An Elementary Exposition. Cambridge, Massachusetts: Harvard University Press, 1984.

Kuhn, Thomas. Die Struktur wissenschaftlicher Revolutionen. 2. Aufl. Frankfurt am Main: Suhrkamp, 1976.

- „Objectivity, value judgment, and theory choice". In: Arguing about Science. Hrsg. von Alexander Bird und James Ladyman. London und New York: Routledge, 2013, S. 74-86.

Ljucovic, Lena. „Dissertation“. Im Erscheinen.

Luhmann, Niklas. Die Gesellschaft der Gesellschaft. 1. Aufl. Bd. 1. Frankfurt am Berlin: Suhrkamp, 1998.

MacIntyre, Alasdair. Der Verlust der Tugend. Frankfurt am Main: Suhrkamp, 1995.

Mackie, John Leslie. Ethics. Inventing Right and Wrong. Harmondsworth: Penguin Books, 1977.

Martin, Rex. „A Defence of Mill's Qualitative Hedonism“. In: Philosophy 47.180 (1972), S. 140-151.

McDowell, John. „Eudaimonism and Realism in Aristotle's Ethics“. In: The Engaged Intellect. Cambridge, Mass. und London: Harvard University Press, 2009, S. 23-40. 
McDowell, John. „Might There Be External Reasons“. In: Mind, Value and Reality. Cambridge, Mass. und London: Harvard University Press, 1998, S. 95-111.

- Mind and World. Cambridge, Mass. und London: Harvard University Press, 1996.

- „Projection and Truth in Ethics“. In: Mind, Value and Reality. Cambridge, Mass. und London: Harvard University Press, 1998.

- "The Role of Eudaimonia in Aristotle's Ethics“. In: Mind, Value and Reality. Cambridge, Mass. und London: Harvard University Press, 1998, S. 3-22.

- „Wittgenstein on Following a Rule“. In: Synthese 58 (1984).

- "Wittgenstein on Following a Rule“. In: Mind, Value and Reality. Cambridge, Mass. und London: Harvard University Press, 1998, S. 221-262.

McRae. „Introduction“. In: The Collected Works of John Stuart Mill, Volume VII - A System of Logic Ratiocinative and Inductive (Books I-III and Appendices). Hrsg. von J. M. Robson. Toronto und andere: University of Toronto Press und Routledge \& Kegan Paul, 1974, S. xxi-xlviii.

Mill, John Stuart. „Bentham“. In: The Collected Works of John Stuart Mill, Volume X-Essays on Ethics, Religion, and Society. Hrsg. von J. M. Robson. Toronto und andere: University of Toronto Press und Routledge \& Kegan Paul, 1969, S. 78-115.

- "Coleridge". In: The Collected Works of John Stuart Mill, Volume $X-$ Essays on Ethics, Religion, and Society. Hrsg. von J. M. Robson. Toronto und andere: University of Toronto Press und Routledge \& Kegan Paul, 1969, S. 119-164.

- "Grote's Aristotle“. In: The Collected Works of John Stuart Mill, Volume XI. Essays on Philosophy and the Classics. Hrsg. von J. M. Robson. Toronto und andere: University of Toronto Press und Routledge \& Kegan Paul, 1978, S. 475-510.

- "Grote's History of Greece I". In: The Collected Works of John Stuart Mill, Volume XI. Essays on Philosophy and the Classics. Hrsg. von J. M. Robson. Toronto und andere: University of Toronto Press und Routledge \& Kegan Paul, 1978, S. 271-305.

- "Grote's History of Greece II". In: The Collected Works of John Stuart Mill, Volume XI. Essays on Philosophy and the Classics. Hrsg. von J. M. Robson. Toronto und andere: University of Toronto Press und Routledge \& Kegan Paul, 1978, S. 307-337. 
- „Grote's Plato“. In: The Collected Works of John Stuart Mill, Volume XI. Essays on Philosophy and the Classics. Hrsg. von J. M. Robson. Toronto und andere: University of Toronto Press und Routledge \& Kegan Paul, 1978, S. 375-440.

- „Notes on Some of the More Popular Dialogues of Plato“. In: The Collected Works of John Stuart Mill, Volume XI. Essays on Philosophy and the Classics. Hrsg. von J. M. Robson. Toronto und andere: University of Toronto Press und Routledge \& Kegan Paul, 1978, S. 37-238.

- "On Liberty“. In: The Collected Works of John Stuart Mill, Volume XVIII - Essays on Politics and Society Part I. Hrsg. von J. M. Robson. Toronto und andere: University of Toronto Press und Routledge \& Kegan Paul, 1977, S. 213-310.

- On Liberty/Über die Freiheit. Deutsch/Englisch. Stuttgart: Reclam, 2009.

- „Preface to Dissertations and Discussions“. In: The Collected Works of John Stuart Mill, Volume X-Essays on Ethics, Religion, and Society. Hrsg. von J. M. Robson. Toronto und andere: University of Toronto Press und Routledge \& Kegan Paul, 1969, S. 493-494.

- The Collected Works of John Stuart Mill, Volume I - Autobiography and Literary Essays. Hrsg. von John M. Robson und Jack Stillinger. Toronto und andere: University of Toronto Press und Routledge \& Kegan Paul, 1981.

- The Collected Works of John Stuart Mill, Volume IX - An Examination of William Hamilton's Philosophy and of The Principal Philosophical Questions Discussed in his Writings. Hrsg. von J. M. Robson. Toronto und andere: University of Toronto Press und Routledge \& Kegan Paul, 1979.

- The Collected Works of John Stuart Mill, Volume VII - A System of Logic Ratiocinative and Inductive (Books I-III and Appendices). Hrsg. von J. M. Robson. Toronto und andere: University of Toronto Press und Routledge \& Kegan Paul, 1974.

- The Collected Works of John Stuart Mill, Volume VIII - A System of Logic Ratiocinative and Inductive (Books IV-VI and Appendices). Hrsg. von J. M. Robson. Toronto und andere: University of Toronto Press und Routledge \& Kegan Paul, 1974.

- The Collected Works of John Stuart Mill, Volume XIV - The Later Letters of John Stuart Mill 1849-1873 Part I. Hrsg. von 
Francis E. Mineka und Dwight N. Lindley. Toronto: University of Toronto Press, 1972.

Mill, John Stuart. „The Subjection of Women“. In: The Collected Works of John Stuart Mill, Volume XXI - Essays on Equality, Law, and Education. Hrsg. von J. M. Robson. Toronto und andere: University of Toronto Press und Routledge \& Kegan Paul, 1984, S. 259-340.

- "Thoughts on Parliamentary Reform". In: The Collected Works of John Stuart Mill, Volume XIX - Essays on Politics and Society Part 2. Hrsg. von J. M. Robson. Toronto und andere: University of Toronto Press und Routledge \& Kegan Paul, 1977, S. 311-340.

- „Three Essays on Religion“. In: The Collected Works of John Stuart Mill, Volume X-Essays on Ethics, Religion and Society. Hrsg. von J. M. Robson. London: Routledge und Kegan Paul, 1969.

- "Two Publications on Plato". In: The Collected Works of John Stuart Mill, Volume XI. Essays on Philosophy and the Classics. Hrsg. von J. M. Robson. Toronto und andere: University of Toronto Press und Routledge \& Kegan Paul, 1978, S. 239-243.

- „Utilitarianism“. In: The Collected Works of John Stuart Mill, Volume $X$-Essays on Ethics, Religion, and Society. Hrsg. von J. M. Robson. Toronto und andere: University of Toronto Press und Routledge \& Kegan Paul, 1969, S. 203-259.

- Utilitarianism/Der Utilitarismus. Stuttgart: Reclam, 2006.

- „Whewell on Moral Philosophy“. In: The Collected Works of John Stuart Mill, Volume X-Essays on Ethics, Religion, and Society. Hrsg. von J. M. Robson. Toronto und andere: University of Toronto Press und Routledge \& Kegan Paul, 1969, S. 167-201.

Miller, Dale E. „Harriet Taylor Mill“. In: The Stanford Encyclopedia of Philosophy. Hrsg. von Edward N. Zalta. Metaphysics Research Lab, Stanford University, 2018.

Moore, George Edward. Ethics. London: Williams und Norgate, 1912.

- Principia Ethica. Cambridge und andere: Cambridge University Press, 2000.

Nagel, Thomas. What Does It All Mean? A Very Short Introduction To Philosophy. Oxford und andere: Oxford University Press, 1987. 
Nussbaum, Martha C. „Der aristotelische Sozialdemokratismus“. In: Gerechtigkeit oder das gute Leben. Hrsg. von Herlinde PauerStuder. Frankfurt am Main: Suhrkamp, 1999, S. 24-85.

- „Mill between Aristotle \& Bentham“. In: Daedalus 133.2 (2004), S. 60-68.

Parfit, Derek. Reasons and Persons. New York: Oxford University Press, USA, 1986.

Pauer-Studer, Herlinde. Einführung in die Ethik. 2. Aufl. Wien: Facultas, 2010.

Priestly, F. E. L. „Introduction“. In: The Collected Works of John Stuart Mill, Volume X-Essays on Ethics, Religion, and Society. Hrsg. von J. M. Robson. Toronto und andere: University of Toronto Press und Routledge \& Kegan Paul, 1969, S. 78-115.

Rawls, John. Eine Theorie der Gerechtigkeit. Frankfurt am Main: Suhrkamp, 1979.

Rescorla, Michael. „Rationality as a Constitutive Ideal“. In: A Companion to Donald Davidson. Oxford und andere: Wiley Blackwell, 2013. Kap. 27, S. 472-488.

Robson, J. M., Hrsg. The Collected Works of John Stuart Mill, Volume XI. Essays on Philosophy and the Classics. Toronto und andere: University of Toronto Press und Routledge \& Kegan Paul, 1978.

Roedl, Sebastian. Selbstbewusstsein und Objektivität: Eine Einführung in den absoluten Idealismus. Berlin: Suhrkamp, 2019.

Salmon, Wesley. Four Decades of Scientific Explanation. Pittsburgh: University of Pittsburgh Press, 1990.

Santas, Gerasimos. „Plato's Protagoras and Explanations of Weakness". In: The Philosophical Review 75.1 (1966), S. 3-33.

Sayre, Kenneth M. „Plato's Dialogues in Light of the Seventh Letter". In: Platonic Writings/Platonic Readings. Hrsg. von Charles L. Griswold. London: Routlegde, 1988, S. 93-109.

Schleiermacher, Friedrich. „Über den Werth des Sokrates als Philosophen“. In: Friedrich Schleiermacher's sämmtliche Werke. Philosophische und vermischte Schriften. Zweiter Band. G. Reimer, 1838, S. 287-308.

Schmid, Hans Bernhard. „Can Brains in Vats Think as a Team?“ In: Philosophical Explorations 6.3 (2003), S. 201-217.

Schopenhauer, Arthur. Preisschrift über das Fundament der Moral. Hamburg: Meiner, 1979. 
Searle, John. „Einige Grundprinzipien der Sozialontologie“. In: Kollektive Intentionalität. Hrsg. von Hans Bernhard Schmid und David P. Schweikard. Frankfurt am Main: Suhrkamp, 2009, S. 504533.

- Making the World Social. Oxford: Oxford University Press, 2011.

- The Construction of Social Reality. New York: The Free Press, 1995.

Selmer, Jan, Hrsg. International Management in China: Cross-Cultural Issues. London und New York: Routledge, 1998.

Singer, Peter. „Sidgwick and Reflective Equilibrium“. In: The Monist 58.3 (1974), S. 490-517.

- „Utilitarianism and Vegetarianism“. In: Philosophy and Public Affairs 9.4 (1980), S. 325-337.

Smith, Michael. The Moral Problem. Oxford und andere: Blackwell, 1994.

Sosa, Ernest. „Mill's Utilitarianism“. In: Mill's Utilitarianism Text and Criticism. Hrsg. von James M. Smith und Ernest Sosa. Wadsworth Pub. Co, 1969.

Sparshot, Francis. „Introduction to Vol. XI“. In: The Collected Works of John Stuart Mill, Volume XI. Essays on Philosophy and the Classics. Hrsg. von J. M. Robson. Toronto und andere: University of Toronto Press und Routledge \& Kegan Paul, 1978, S. vii-lxxv.

Stevenson, Charles Leslie. „Die emotive Bedeutung ethischer Ausdrücke". In: Sprache und Ethik - Zur Entwicklung der Metaethik. Hrsg. von Günther Grewendorf und Georg Meggle. Frankfurt am Main: Suhrkamp, 1974, S. 116-139.

Sullivan, Eileen. „Liberalism and Imperialism: J. S. Mill's Defense of the British Empire". In: Journal of the History of Ideas 44.4 (1983), S. 599-617.

Svavarsdóttir, Sigrún. „Moral Cognitivism and Motivation“. In: The Philosophical Review 108.2 (1999), S. 161-219.

Szapuová, Mariana. „Mill's Liberal Feminism: Its Legacy and Current Criticism“. In: Prolegomena 5.2 (2006), S. 179-191.

Taylor, Helen. „Introductory Notice on ,Three Essays on Religion““. In: The Collected Works of John Stuart Mill, Volume X - Essays on Ethics, Religion, and Society. Hrsg. von J. M. Robson. Toronto und andere: University of Toronto Press und Routledge \& Kegan Paul, 1969, S. 371-372. 
Thompson, Michael. Life and Action: Elementary Structures of Practice and Practical Thought. English. Cambridge, Mass.: Harvard University Press, 2008.

Udehn, Lars. „The Changing Face of Methodological Individualism". In: Annual Review of Sociology 28 (2002), S. 479-507.

Wallace, R. Jay. "Three Conceptions of Rational Agency“. In: Ethical Theory and Moral Practice 2.3 (1999), S. 217-242.

Weber, Max. Wirtschaft und Gesellschaft. Grundriß der verstehenden Soziologie [1921]. Tübingen: Mohr, 1976.

Wendelborn, Christian. Der metaethische Relativismus auf dem Prüfstand. Berlin: De Gruyter, 2016.

Whewell, William. Lectures on the History of Moral Philosophy in England. London und andere: J. W. Parker \& son, 1852.

Williams, Bernard. „Internal and external reasons“. In: Moral Luck. Cambridge und andere: Cambridge University Press, 1982, S. 101113.

- Morality: An Introduction to Ethics. Cambridge: Cambridge University Press, 1976.

Williams, Bernard und Thomas Nagel. „Moral Luck“. In: Proceedings of the Aristotelian Society, Supplementary Volumes 50 (1976), S. $115-151$.

Wittgenstein, Ludwig. Werkausgabe in 8 Bänden - Band 1: Tractatus logico-philosophicus. Tagebücher 1914-1916. Philosophische Untersuchungen. Frankfurt am Main: Suhrkamp, 2014.

Wolf, Ursula. Aristoteles' "Nikomachische Ethik". 3. Aufl. Darmstadt: WBG, 2013.

- Die Philosophie und die Frage nach dem guten Leben. Reinbek bei Hamburg: Rowohlt Taschenbuch Verlag, 1999.

- Die Suche nach dem guten Leben. Platons Frühdialoge. Reinbek bei Hamburg: Rowohlt Taschenbuch Verlag, 1996.

- Hrsg. Platon. Sämtliche Werke, Band 1. 32. Aufl. Reinbek bei Hamburg: Rowohlt Taschenbuchverlag, 2011.

- Hrsg. Platon. Sämtliche Werke, Band 3. 37. Aufl. Reinbek bei Hamburg: Rowohlt Taschenbuchverlag, 2013.

Zamor, Caroline. Intercultural Trainings for German Expatriates Going to China. Hamburg: IGEL, 2008. 
